\title{
THERM 5 / WINDOW 5 NFRC Simulation Manual
}

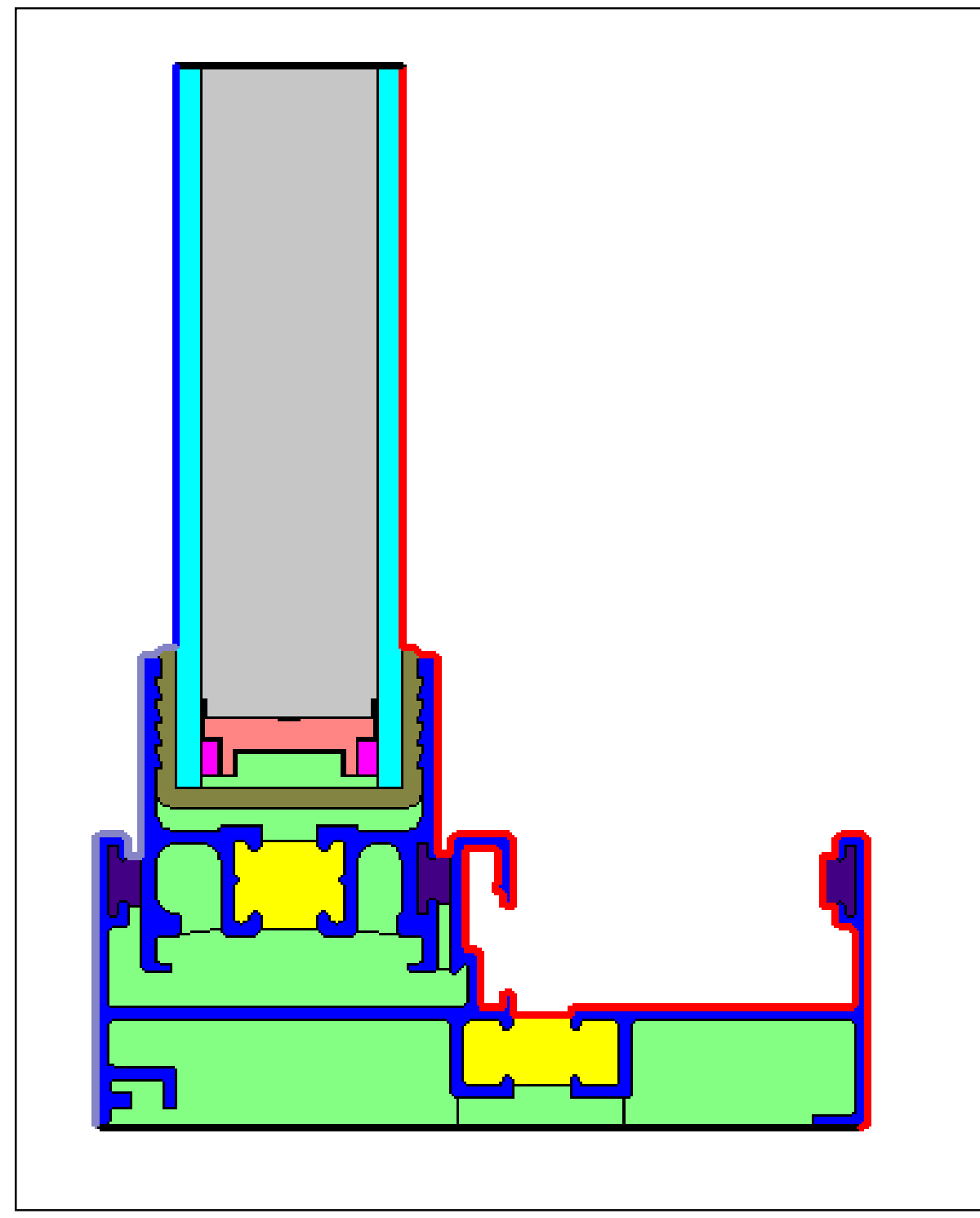

Lawrence Berkeley National Laboratory

JUNE 2003

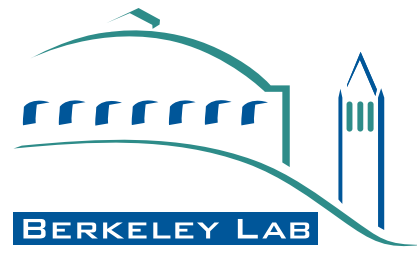


DISCLAIMER

This document was prepared as an account of work sponsored by the United States Government. While this document is believed to contain correct information, neither the United States Government nor any agency thereof, nor The Regents of the University of California, nor any of their employees, makes any warranty, express or implied, or assumes any legal responsibility for the accuracy, completeness, or usefulness of any information, apparatus, product, or process disclosed, or represents that its use would not infringe privately owned rights. Reference herein to any specific commercial product process, or service by its trade name, trademark, manufacturer, or otherwise, does not necessarily constitute or imply its endorsement, recommendation, or favoring by the United States Government or any agency thereof, or The Regents of the University of California. The views and opinions of authors expressed herein do not necessarily state or reflect those of the United States Government or any agency thereof, or The Regents of the University of California. 


\title{
THERM 5 / WINDOW 5 NFRC Simulation Manual
}

\author{
Robin Mitchell, Christian Kohler, and Dariush Arasteh \\ Windows and Daylighting Group \\ Building Technologies Department \\ Environmental Energy Technologies Division \\ Lawrence Berkeley National Laboratory \\ Berkeley, California 94720 \\ http://windows.lbl.gov/software/software.html \\ John Carmody \\ College of Architecture and Landscape Architecture \\ University of Minnesota \\ Minneapolis, Minnesota \\ Charlie Huizenga \\ Center for Environmental Design Research \\ University of California \\ Berkeley, California \\ Dragan Curcija \\ Carli, Inc \\ Amherst, Massachusetts
}

June 2003

(c) Regents of the University of California

This work was supported by the Assistant Secretary for Energy Efficiency and Renewable Energy, Office of Building Technology, Building Technologies Program of the U.S. Department of Energy under Contract No. DE-AC03$76 S F 00098$. 



\section{Contents}

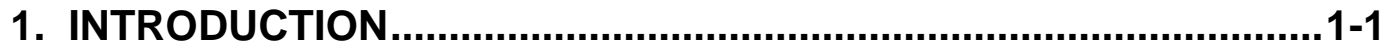

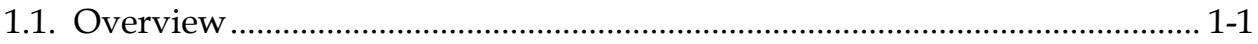

2. FENESTRATION PRODUCTS ….......................................................

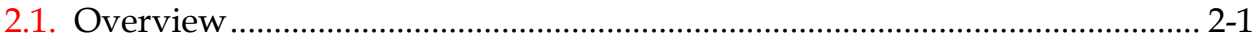

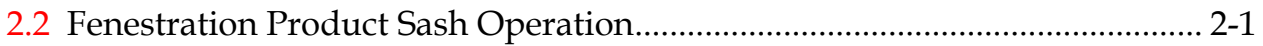

2.2.1. Projected or Hinged Windows............................................................. 2-2

2.2.2. Sliding Windows............................................................................ 2-2

2.2.3. Sliding Glass Doors ................................................................... 2-2

2.2.4. French Doors and Folding Patio Doors ……………….................... 2-2

2.2.5. Skylights and Roof Windows............................................................ 2-2

2.3 Performance Implications of Basic Fenestration Types .................................. 2-3

2.3.1. Hinged Windows.............................................................................. 2-3

2.3.2. Sliding Windows............................................................................. 2-3

2.3.3. Sliding Glass and French Doors........................................................ 2-4

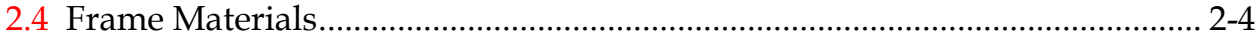

2.4.1. Wood Frames ........................................................................... 2-4

2.4.2. Aluminum Frames...................................................................... 2-4

2.4.3. Vinyl Frames ................................................................................... 2-5

2.4.4. Fiberglass and Engineered Thermoplastics...................................... 2-5

2.4.5. Wood Composites............................................................................ 2-5

2.4.6. Hybrid and Composite Frames......................................................... 2-5

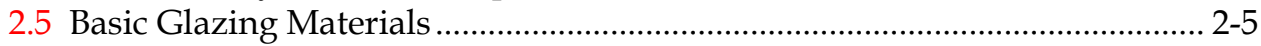

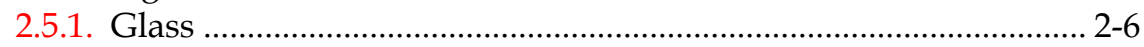

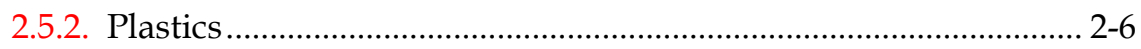

2.6 Improved Glazing Products ..................................................................... 2-7

2.6.1. Tinted Glazing.............................................................................. 2-7

2.6.2. Reflective Coatings and Films................................................... 2-8

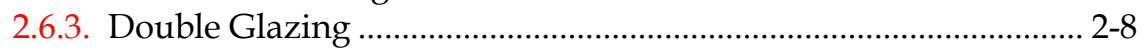

2.6.4. Glass Coatings and Tints in Double Glazing ................................. 2-8

2.6.5. Gap Width in Multiple Glazed Units ............................................. 2-9

2.6.6. Divided Lights....................................................................................... 2-9

2.6.7. Special Products ............................................................................... 2-10

2.6.8. Multiple Panes or Films ................................................................. 2-10

2.6.9. Low-Emittance Coatings................................................................ 2-11

2.6.9.1. Coating Placement ............................................................... 2-12

2.6.9.2. Coating Types.......................................................................... 2-12

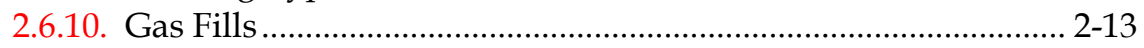

2.7 Thermally Improved Edge Spacers ............................................................ 2-14 
3. FENESTRATION HEAT TRANSFER BASICS................................3-1

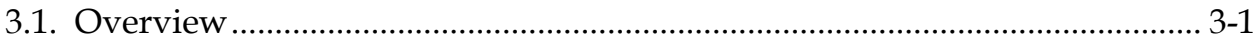

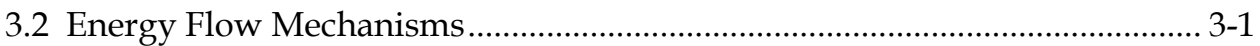

3.3 Temperature Driven Heat Transfer ………................................................ 3-2

3.3.1. Conduction ……........................................................................... 3-3

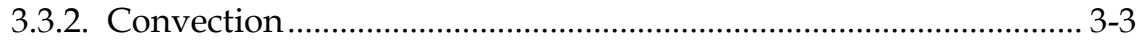

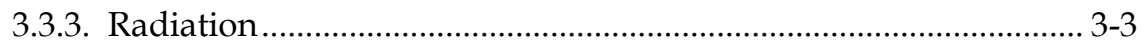

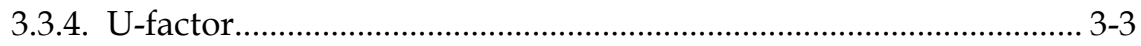

3.3.4.1. Total Product U-factor............................................................. 3-4

3.3.4.2. Center-of-Glazing U-factor ............................................................. 3-4

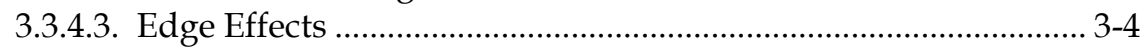

3.3.4.4. Frames and Sashes..................................................................... 3-4

3.3.4.5. Overall U-factor .......................................................................... 3-4

3.4 Solar Heat Gain and Visible Transmittance .............................................. 3-5

3.4.1. Determining Solar Heat Gain.......................................................... 3-5

3.4.2. Solar Heat Gain Coefficient (SHGC) ............................................. 3-6

3.4.3. Visible Transmittance..................................................................... 3-6

3.5 Condensation Resistance ............................................................................

3.5.1. Impact of Glazing Type and Spacers on Condensation................. 3-8

3.5.2. Condensation Resistance ............................................................. 3-8

3.5.3. Outdoor Condensation ................................................................... 3-9

3.5.4. Condensation Between Glazings ...................................................... 3-9

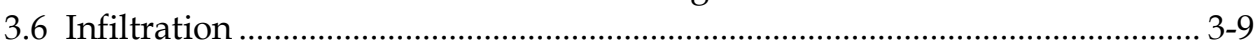

3.7 Solar Properties of Glazing Materials....................................................... 3-10

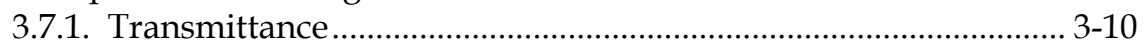

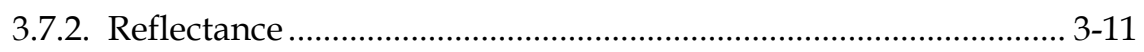

3.7.3. Absorptance ............................................................................ 3-12

3.8 Infrared Properties of Glazing Materials (Emittance) ...................................... 3-12

4. SUMMARY OF ALGORITHMS..................................................4-1

4.1. THERM and WINDOW Algorithms ............................................................. 4-1

4.1.1. WINDOW …………………....................................................... 4-1

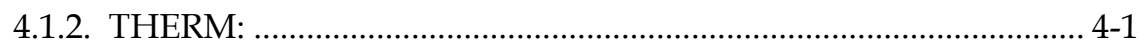

4.2 WINDOW Computational Method ............................................................ 4-1

4.3 THERM Computational Methods.................................................................... 4-3

4.4 Total Product Calculations .............................................................................. 4

4.4.1. U-factor......................................................................................... 4-4

4.4.2. Solar Heat Gain Coefficient (SHGC) .............................................. 4-5

4.4.3. Visible Transmittance..................................................................... 4-5

4.4.4. Condensation Resistance ................................................................... 4-6

5. MODELING CENTER-OF-GLAZING WITH WINDOW ....................5-1

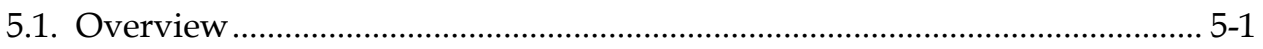

5.2 Glass Library …....................................................................................... 5-2

5.3 Glazing System Library -- Center-of-Glazing U-factor ................................... 5-4

5.3.1. Environmental Conditions ........................................................... 5-7

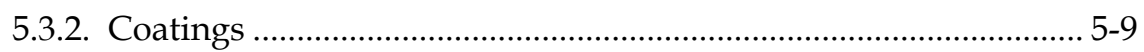

5.3.3. Gas Fill........................................................................................ 5-10

5.3.4. Laminated Glass / Applied Films ................................................. 5-11 


\section{MODELING FRAME AND EDGE HEAT TRANSFER WITH THERM6-1}

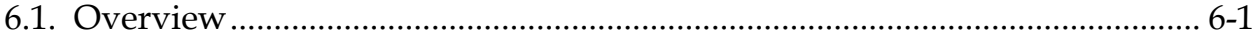

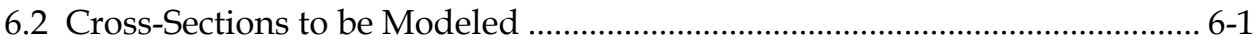

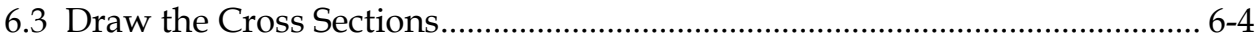

6.3.1. Getting Started - Drawings and DXF Files ................................... 6-4

6.3.2. File Preferences -- Cross-Section Type .............................................. 6-4

6.3.3. Cross-Section Orientation.............................................................. 6-5

6.3.4. What is Not Modeled ...................................................................... 6-6

6.3.4. What is Not Modeled .................................................................. 6-7

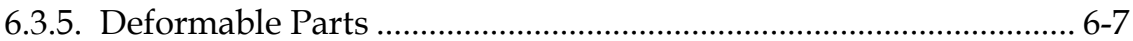

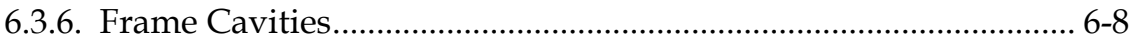

6.3.7. Slightly Ventilated Exterior Cavities ............................................. 6-13

6.3.8. Modeling Sloped and Curved Surfaces ....................................... 6-14

6.3.9. Modeling Sloped Sills.................................................................. 6-15

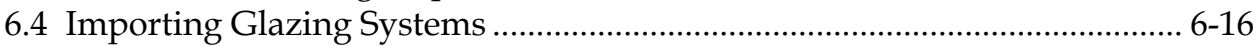

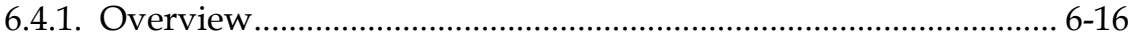

6.4.2. Inserting Glazing Systems ........................................................ 6-17

6.4.3. Adding the Radiation Enclosure Model ........................................ 6-18

6.4.4. Multiple Glazing Options ............................................................ 6-18

6.4.5. Condensation Resistance ........................................................... 6-21

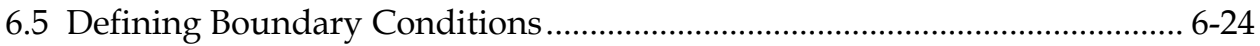

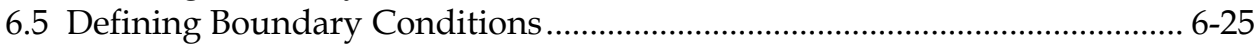

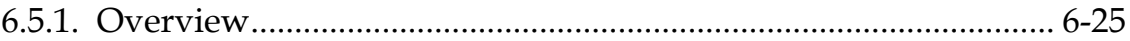

6.5.2. Assigning Boundary Conditions and U-factor Surface Tags...... 6-27

6.5.3. Voids, Overlaps and Bad Points ..................................................... 6-31

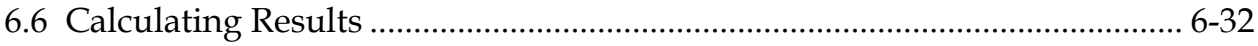

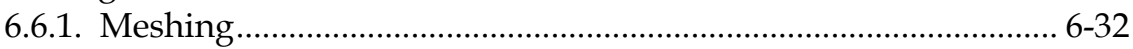

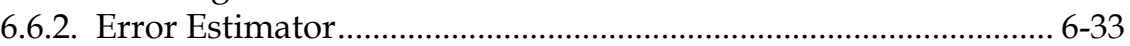

6.6.3. Required Settings for NFRC Simulations ....................................... 6-34

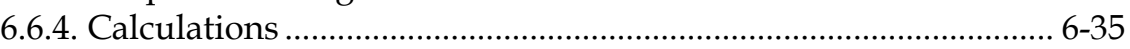

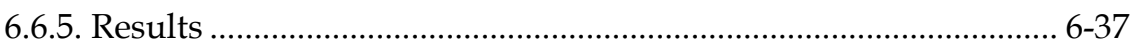

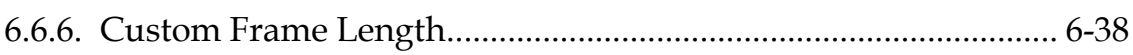

6.6.7. Importing Results into WINDOW ................................................. 6-39

7. TOTAL PRODUCT CALCULATIONS USING WINDOW ................. 7-1

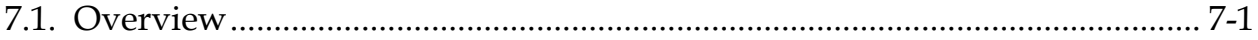

7.2 Frame and Edge U-factors from THERM …….............................................. 7-1

7.3 Center-of-Glazing U-factors from WINDOW ............................................ 7-2

7.4 Overall Product U-factor, SHGC, VT, and CR Calculations .......................... 7-3

7.4.1. Individual Product SHGC and VT (SHGC 0 \& 1, VT 0 \& 1) ......... 7-5

7.4.2. Whole Product U-factor With Dividers .............................................. 7-8

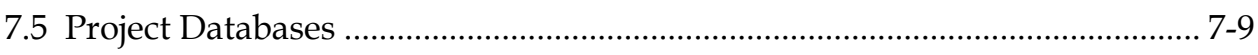

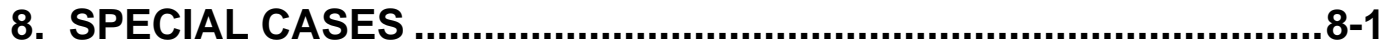

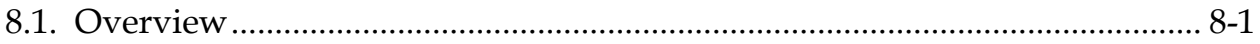

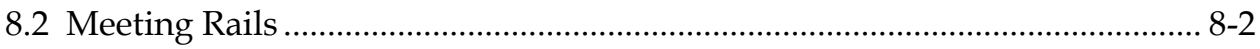

8.2.1. Modeling Meeting Rails ................................................................... 8-2

8.2.2. Steps for Meeting Rail U-factor Calculation................................... 8-3 
8.2.3. Steps for Meeting Rail Condensation Resistance Calculation ...... 8-8

8.3 Internal Dividers (Suspended Grilles) .......................................................... 8-10

8.3.1. Modeling Steps............................................................................. 8-10

8.3.3. Gas Filled Glazing Systems ........................................................... 8-18

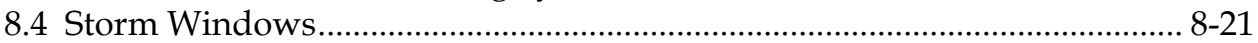

8.4.1. Modeling Steps............................................................................... 8-21

8.4.2. Storm Window Example.................................................................. 8-23

8.4.3. Steps for Storm Window Condensation Resistance Calculation 8-27

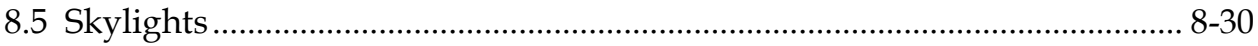

8.5.1. Skylight Modeling Steps ............................................................... 8-30

8.5.2. Skylight Mounting Details.......................................................... 8-32

8.5.3. Example Flush Mounted Skylight Problem ................................... 8-33

8.6 Modeling Tubular Daylighting Devices ........................................................... 8-43

8.6.4. Example Tubular Devices Problem.................................................. 8-47

8.6.5. Example: Tubular Device Problem With the Double-Glazed

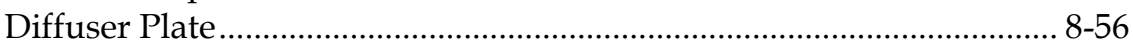

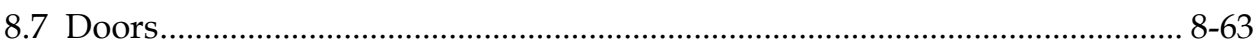

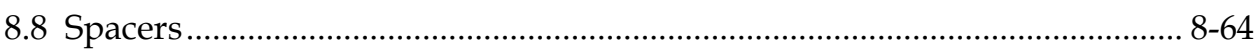

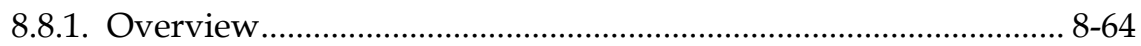

8.8.2. Linking Glazing Cavity properties (imported from WINDOW) for

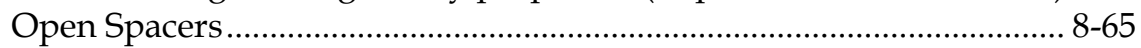

8.9 Non Continuous Thermal Bridge Elements .............................................. 8-66

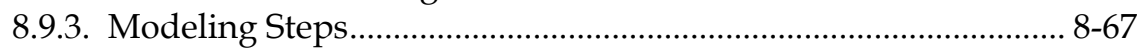

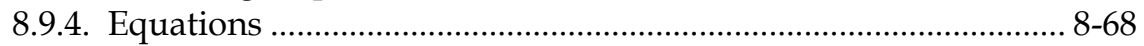

8.9.5. Example 1: Bolts in Curtain Wall.................................................. 8-69

8.9.6. Example 2: Thermally slotted cross-section ................................. 8-72

8.9.6. Example 2: Thermally slotted cross-section ................................ 8-73

8.9.7. Example 3: Skip-and-debridge:....................................................... 8-77

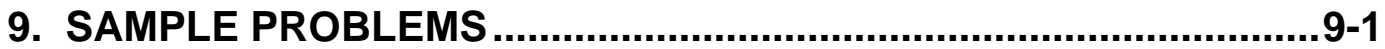

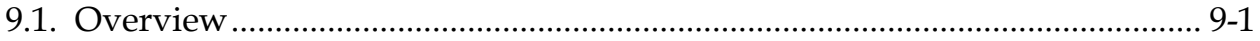

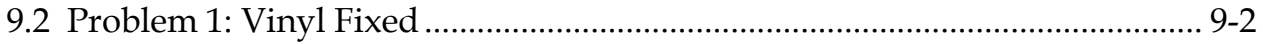

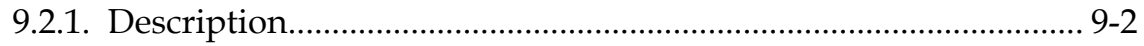

9.2.2. Glazing Matrix ................................................................................ 9-2

9.2.3. Center-of-glazing Modeling (WINDOW) ....................................... 9-3

9.2.4. Edge-of-glazing and Frame Modeling (THERM) for U-factor ..... 9-4

9.2.5. Total Product U-Factor..................................................................... 9-9

9.2.6. Individual Product SHGC and VT using SHGC 0 \& 1

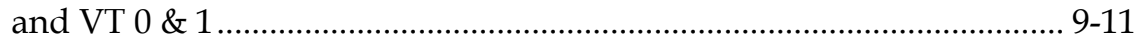

9.2.7. Drawings Vinyl Fixed Window ……….......................................... 9-12

9.3 Problem 2: Aluminum Horizontal Slider Window ........................................ 9-14

9.3.1. Description................................................................................. 9-14

9.3.2. Glazing Matrix ................................................................................. 9-14

9.3.3. Center-of-glazing Modeling (WINDOW) ..................................... 9-15

9.3.4. Edge-of-glazing and Frame Modeling (THERM) .......................... 9-16

9.3.5. Total Product U-Factor.................................................................. 9-25

9.3.6. Individual Product SHGC and VT using SHGC 0 \& 1

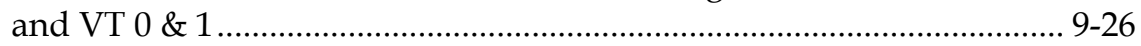

9.3.7. Drawings for Aluminum Horizontal Slider Window.................. 9-27

9.3.7. Drawings for Aluminum Horizontal Slider Window................... 9-27 


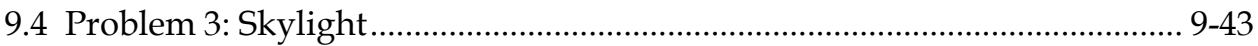

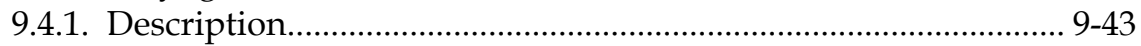

9.4.2. Glazing Matrix ............................................................................... 9-43

9.4.3. Center-of-glazing Modeling (WINDOW) ..................................... 9-44

9.4.4. Edge-of-glazing and Frame Modeling (THERM) ......................... 9-45

9.4.5. Total Product U-Factor...................................................................... 9-49

9.4.6. Individual Product SHGC and VT using SHGC 0 \& 1

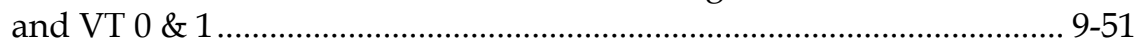

9.4.7. Skylight Drawings........................................................................ 9-51

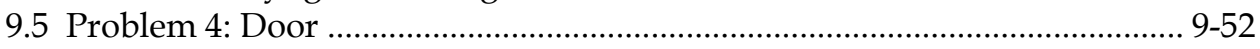

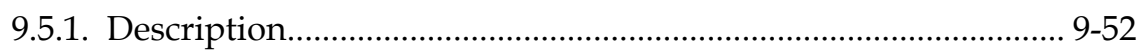

9.5.2. Glazing Matrix ................................................................................ 9-52

9.5.3. Center-of-Glazing Modeling (WINDOW) .................................... 9-53

9.5.4. Edge-of-Glazing and Frame Modeling (THERM) ......................... 9-54

9.5.5. Total Product U-Factor, SHGC and VT ........................................ 9-61

9.5.7. Wood Stile and Rail Door Drawings............................................... 9-61

10. ACKNOWLEDGEMENTS ...........................................................

APPENDIX A. The Application of ISO 15099 to

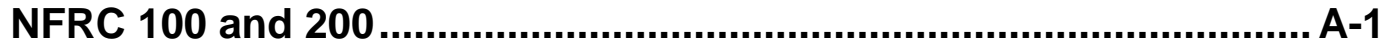

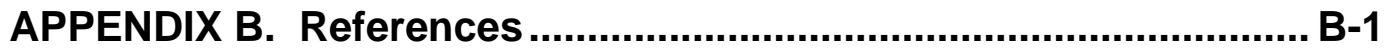





\section{INTRODUCTION}

\subsection{Overview}

This document, the THERM 5 /WINDOW 5 NFRC Simulation Manual, discusses how to use the THERM and WINDOW programs to model products for NFRC certified simulations and assumes that the user is already familiar with those programs. In order to learn how to use these programs, it is necessary to become familiar with the material in both the THERM User's Manual and the WINDOW User's Manual.

In general, this manual references the User's Manuals rather than repeating the information.

If there is a conflict between either of the User Manual and this THERM 5 / WINDOW 5 NFRC Simulation Manual, the THERM 5 / WINDOW 5 NFRC Simulation Manual takes precedence.

In addition, if this manual is in conflict with any NFRC standards, the standards take precedence. For example, if samples in this manual do not follow the current taping and testing NFRC standards, the standards not the samples in this manual, take precedence. 



\section{FENESTRATION PRODUCTS}

\subsection{Overview}

Much of the information in this chapter is taken from Residential Windows: A Guide to New Technologies and Energy Performance by John Carmody, Stephen Selkowitz, and Lisa Heschong.

Although glazing materials are the focus of much of the innovation and improvement in fenestration products, the overall performance of any unit is determined by the complete fenestration product assembly. The assembly includes the operating and fixed parts of the product frame as well as associated hardware and accessories. These are defined and illustrated at the beginning of this section. The next two sections address the different options available for sash operation and new advances in frame materials designed to improve product energy efficiency. Proper installation is an important aspect of their performance as well. The final section of this chapter discusses other installation issues.

\subsection{Fenestration Product Sash Operation}

There are numerous operating types available for fenestration products. Traditional operable types include the projected or hinged types such as casement, awning, and hopper, and the sliding types such as doubleand single-hung and horizontal sliding. In addition, the current market includes storm windows, sliding and swinging patio doors, skylights and roof-mounted (i.e., sloping) windows, and systems that can be added to a house to create bay or bow windows, miniature greenhouses, or full sun rooms.

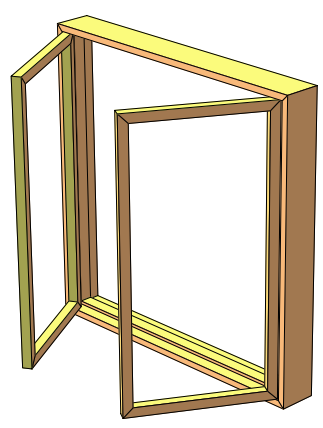

Casement

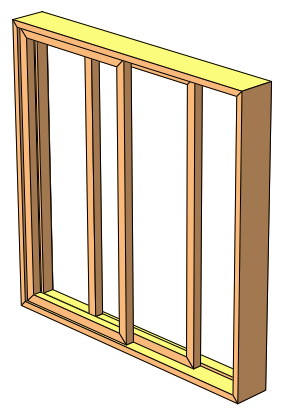

Horizontal slider

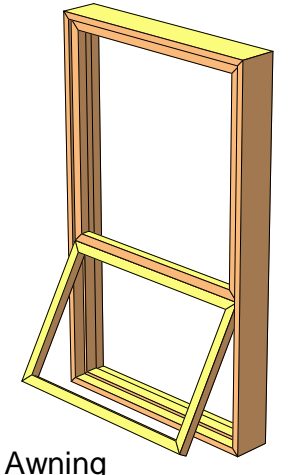

Awning

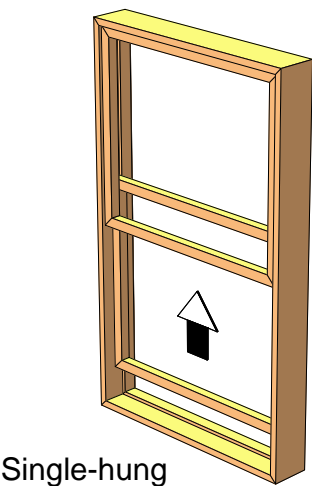

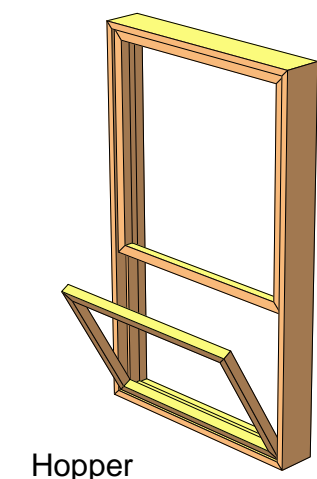

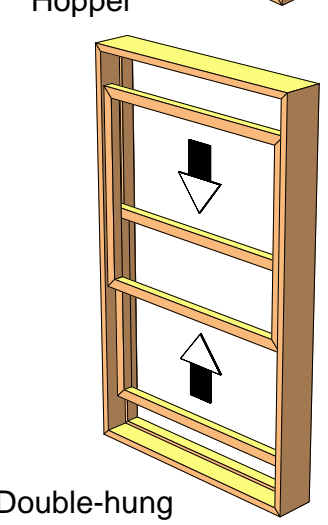

Figure 2-1. Fenestration Operator Types 


\subsubsection{Projected or Hinged Windows}

Hinged windows include casements, awnings, and hoppers - hinged at the side, top, and bottom, respectively. Some manufacturers also make pivoting and combination windows that allow for easier cleaning of the exterior surfaces. Hinged windows, especially casements, project outward, providing significantly better ventilation than sliders of equal size. Because the sash protrudes from the plane of the wall, it can be controlled to catch passing breezes, but screens must be placed on the interior side. Virtually the entire casement window area can be opened, while sliders are limited to less than half of the window area.

\subsubsection{Sliding Windows}

Sliders are the most common type of windows and include horizontal sliders and single-hung and doublehung windows. Ventilation area can vary from a small crack to an opening of one-half the total glass area. Screens can be placed on the exterior or interior of the window unit.

In double-hung or double-sliding units, both sashes can slide. In double-sliding units, the same net amount of glass area can be opened for ventilation as in single sliders, but it can be split between the top and bottom or two ends of the window for better control of the air flow.

\subsubsection{Sliding Glass Doors}

Sliding glass doors (patio doors) are essentially big sliding windows. As extremely large expanses of glass, patio doors exaggerate all of the issues related to comfort and energy performance. Since the proportion of glass to frame is very high for a glass sliding door, the selection of high-performance glass can have significant benefits.

\subsubsection{French Doors and Folding Patio Doors}

French doors and folding glazed doors are growing in popularity. A basic double French door consists of two hinged doors with no center mullion, resulting in a 1.5 to $1.8 \mathrm{~m}$ (5- to 6-foot) wide opening. Folding doors are typically made of pairs of hinged doors, so that a double folding door with two pairs of doors can create an opening of $3.7 \mathrm{~m}$ (12 feet) or more.

\subsubsection{Skylights and Roof Windows}

The vast majority of skylights are permanently fixed in place, mounted on a curb above a flat or sloped roof. However, hatch-style skylights that can be opened with an extended crank, push latch, or remote control motor are becoming more common. Some skylights have a domed profile made of one or two layers of tinted or diffusing plastic.

A roof window is a hybrid between a skylight and a standard window. They have become increasingly popular as homeowners and designers seek to better utilize space in smaller houses by creating habitable rooms under sloping roofs. They are glazed with glass rather than plastic and are available with most of the glazing and solar control options of standard windows. Both fixed and operable versions are available, and the operable roof windows can be opened manually or by a motorized system. In addition, some manufacturers offer special venting mechanisms that allow some ventilation air flow without actually opening the window. Operable skylights or vents allow hot air that rises to the ceiling level to be effectively exhausted from the space.

Skylights and roof windows present a special case for insulating around windows because they are typically set into the thickest, most heavily insulated framing in the house, and they must also meet much more stringent conditions for shedding water. In order to create a positive water flow around them, skylights are commonly mounted on "curbs" set above the roof plane. These curbs, rising 15 to 30 centimeter (6 to 12 
inches) (s) above the roof, create additional heat loss surfaces, right where the warmest air of the house tends to collect. Ideally, they should be insulated to the same level as the roof. In practice, it is often difficult to achieve insulation levels much above R-11. Some manufacturers provide curbs prefabricated out of a rigid insulating foam, which can be further insulated at the site.

Roof windows, mounted in a sloping roof, often include a metal flashing system. If this metal flashing is in contact with a metal window frame, it can create additional surfaces for conducting heat. Thus, as with thermally broken aluminum windows, care should be taken to ensure a thermal separation between the cold outer metal surfaces and metal parts of the window frame that are exposed to the warm interior air.

\subsection{Performance Implications of Basic Fenestration Types}

There are subtle performance differences between a fixed and operable fenestration product that fills an identical rough opening. The fixed unit will typically have a smaller fraction of frame and proportionately more glass than the similar operable unit. Thus, fixed products with high-performance glass will have a better, lower U-factor, but a higher SHGC due to a smaller frame area and larger glass area. Fixed products have very low infiltration rates, but then they also do not provide natural ventilation and do not satisfy building code requirements for fire egress.

For operating fenestration products, the type of operation has little direct effect on the U-factor or SHGC of the unit, but it can have a significant effect on the air infiltration and ventilation characteristics. Operation can be broken into two basic types: sliding products and hinged products. The comments below are a general characterization of American fenestration products; however, they may not apply to a specific product made by a given manufacturer.

\subsubsection{Hinged Windows}

Hinged windows such as casements, awnings, and hoppers generally have lower air leakage rates than sliding windows from the same manufacturer because the sash closes by pressing against the frame, permitting the use of more effective compression-type weatherstripping. In most types, the sash swings closed from the outside, so that additional external wind pressure tends to push the sash more tightly shut. Hinged windows require a strong frame to encase and support the projecting sash. Also, because projectingtype sashes must be strong enough to swing out and still resist wind forces, the stiffer window units do not flex as readily in the wind. In addition, hinged windows have locking mechanisms that force the sash against the weatherstripping to maximize compression. These design details tend to reduce air infiltration of hinged windows in comparison to sliders.

\subsubsection{Sliding Windows}

Sliding windows, whether single-hung, double-hung, or horizontal sliders, generally have higher air leakage rates than projecting or hinged windows. Sliding windows typically use a brush-type weatherstripping that allows the sash to slide past. This type is generally less effective than the compression gaskets found in projecting windows. The weatherstrip effectiveness also tends to be reduced over time due to wear and tear from repeated movement of the sliding sash. The frames and sashes of sliding units can be made with lighter, less rigid frame sections since they only need to support their own weight. This lightness may permit the sliding frames to flex and can allow more air leakage under windy conditions. Manufacturers can choose to engineer greater stiffness in their products by design and material selection.

Slider window performance can also be improved with latching mechanisms that compress the sash to the fixed frame and by the addition of compression weatherstripping at the head and sill of double-hung windows or the end jamb of horizontal sliders. 


\subsubsection{Sliding Glass and French Doors}

As previously noted, sliding doors are essentially big sliding windows. However, they are more complicated because of their size and weight and because the sill is also a door threshold, which must keep water out while allowing easy passage of people and objects. The threshold is typically the most difficult part of the frame to weatherstrip effectively.

French doors benefit from being much more like traditional doors than sliding doors. French doors can use weatherstripping and operating hardware designed for similar nonglazed doors. However, when there are large openings with multiple hinged doors, it is more difficult to positively seal the joints between door leaves and to create the stiffness that will resist infiltration.

\subsection{Frame Materials}

The material used to manufacture the frame governs the physical characteristics of the fenestration product, such as frame thickness, weight, and durability, but it also has a major impact on the thermal characteristics of the product. Increasingly, manufacturers are producing hybrid or composite sash and frames, in which multiple materials are selected and combined to best meet the overall required performance parameters. Thus, a simple inspection of the inner or outer surface of the frame is no longer an accurate indicator of the total material or its performance. Since the sash and frame represent from 10 to 30 percent of the total area of the fenestration unit, the frame properties will significantly influence the total product performance.

\subsubsection{Wood Frames}

Wood fenestration products are manufactured in all configurations, from sliders to swinging windows. Wood is favored in many residential applications because of its appearance and traditional place in house design.

A variation of the wooden product is to clad the exterior face of the frame with either vinyl or aluminum, creating a permanent weather-resistant surface. Clad frames thus have lower maintenance requirements, while retaining the attractive wood finish on the interior.

From a thermal point of view, wood-framed products perform well. The thicker the wood frame, the more insulation it provides. Wood-framed fenestration products typically exhibit low heat loss rates.

However, metal cladding, metal hardware, or the metal reinforcing often used at corner joints can degrade the thermal performance of wood frames. If the metal extends through the fenestration product from the cold side to the warm side of the frame, it creates a thermal short circuit, conducting heat more quickly through that section of the frame.

\subsubsection{Aluminum Frames}

The biggest disadvantage of aluminum as a fenestration product frame material is its high thermal conductance. It readily conducts heat, greatly raising the overall U-factor of a fenestration unit. Because of its high thermal conductance, the thermal resistance of an aluminum frame is determined more by the amount of surface area of the frame than by the thickness or the projected area, as with other frame materials. Thus, an aluminum frame profile with a simple compact shape will perform much better than a profile with many fins and undulations.

In cold climates, a simple aluminum frame can easily become cold enough to condense moisture or frost on the inside surfaces of fenestration product frames. Even more than the problem of heat loss, the condensation problem spurred development of a more insulating aluminum frame.

The most common solution to the heat conduction problem of aluminum frames is to provide a "thermal break" by splitting the frame components into interior and exterior pieces and use a less conductive material 
to join them. There are many designs available for thermally broken aluminum frames. The most prevalent technique used in residential fenestration products is called "pouring and debridging." The frame is first extruded as a single piece with a hollow trough in the middle. This is filled with a plastic that hardens into a strong intermediate piece. The connecting piece of aluminum is then milled out, leaving only the plastic to join the two halves of aluminum. Functionally, the resulting piece is cut, mitered, and assembled like a simple aluminum extrusion. Thermally, the plastic slows the heat flow between the inside and outside. There are other manufacturing techniques for producing a thermal break, but the thermal results are similar.

\subsubsection{Vinyl Frames}

Plastics are relative newcomers as fenestration product frame materials in North America. Vinyl, also known as polyvinyl chloride (PVC), is a versatile material with good insulating value.

The thermal performances of vinyl frames is roughly comparable to wood. Large hollow chambers within the frame can allow unwanted heat transfer through convection currents. Creating smaller cells within the frame reduces this convection exchange, as does adding an insulating material. Most manufacturers are conducting research and development to improve the insulating value of their vinyl fenestration product assemblies.

\subsubsection{Fiberglass and Engineered Thermoplastics}

In addition to vinyl fenestration products two other polymer-based technologies have become available, fiberglass and thermoplastics. Frames can be made of glass-fiber-reinforced polyester, or fiberglass, which is pultruded into lineal forms and then assembled into fenestration products. These frames are dimensionally stable and have good insulating value by incorporating air cavities (similar to vinyl).

\subsubsection{Wood Composites}

Most people are familiar with composite wood products, such as particle board and laminated strand lumber, in which wood particles and resins are compressed to form a strong composite material. The wood fenestration product industry has now taken this a step further by creating a new generation of wood/polymer composites that are extruded into a series of lineal shapes for frame and sash members. These composites are stable, and have the same or better structural and thermal properties as conventional wood, with better moisture resistance and more decay resistance. They can be textured and stained or painted much like wood. They were initially used in critical elements, such as sills and thresholds in sliding patio doors, but are now being used for entire units. This approach has the added environmental advantage of reusing a volume of sawdust and wood scrap that would otherwise be discarded.

\subsubsection{Hybrid and Composite Frames}

Manufacturers are increasingly turning to hybrid frame designs that use two or more of the frame materials described above to produce a complete fenestration product system. The wood industry has long built vinyland aluminum-clad products to reduce exterior maintenance needs. Vinyl manufacturers and others offer interior wood veneers to produce the finish and appearance that many homeowners desire. Split-sash designs may have an interior wood element bonded to an exterior fiberglass element.

\subsection{Basic Glazing Materials}

Two basic materials are used for fenestration product glazing: glass, which is by far the most common, and plastics, which have many specialized applications. 


\subsubsection{Glass}

Traditionally, fenestration products have been made of clear glass. Most residential-grade clear glass today is produced with the float technique in which the glass is "floated" over a bed of molten tin. This provides extremely flat surfaces, uniform thicknesses, and few if any visual distortions. The glass has a slight greenish cast, due to iron impurities, but this is generally not noticeable except from the edge. An even higher-quality glass with reduced iron content eliminates the greenness and also provides a higher solar energy transmittance. This is commonly called "water-white glass."

Obscure glasses still transmit most of the light but break up the view in order to provide privacy. This effect is generally achieved either with decorative embossed patterns or with a frosted surface that scatters the light rays.

By adding various chemicals to glass as it is made, glass can be produced in a wide variety of colors. Glass colors are typically given trade names, but the most frequently used colors can be generally described as clear, bronze, gray, and blue-green. After clear glass, the gray glasses are most commonly used in residential construction, as they have the least effect on the perceived color of the light. Tinted glass is discussed later in this chapter.

The mechanical properties of glass can be altered, as well as its basic composition and surface properties. Heat-strengthening and tempering make glass more resistant to breakage. Heat-strengthened glass is about twice as strong as standard glass. Tempered glass is produced by reheating and then quickly chilling the glass. It breaks into small fragments, rather than into long, possibly dangerous shards. Laminated glass is a sandwich of two outer layers of glass with a plastic inner layer that holds the glass pieces together in the event of breakage. Fully tempered and laminated glass is required by building codes in many door and fenestration product applications.

\subsubsection{Plastics}

Several plastic materials have been adapted for use as glazing materials. Their primary uses are fenestration products with special requirements and skylights.

The following list of plastic glazing materials covers the major types of plastic glazing materials and compares their general properties:

- Clear acrylic is widely available and relatively inexpensive. It is available in various tints and colors. It has excellent visible light transmittance and longevity. However, it is softer than glass, which makes it vulnerable to scratching.

- Frosted acrylic is like clear acrylic, except that it diffuses light and obscures the view. It comes in varying degrees of light transmittance. Most bubble skylights are made of frosted acrylic.

- Clear polycarbonate is like acrylic sheet, but it is harder and tougher, offering greater resistance to scratching and breakage. It is more expensive than acrylic.

- Fiber-reinforced plastic is a tough, translucent, flexible sheet material with good light-diffusing properties. Short lengths of fiberglass are embedded in a polymer matrix to form flat or ribbed sheets. Stiff, insulating, translucent panels are created by bonding double layers to a metal frame and adding fiberglass insulation. It is also formed into corrugated sheets as a translucent roofing material. Surface erosion may shorten its useful life.

- Extruded multicell sheet, usually made with acrylic or polycarbonate plastic, is a transparent or tinted plastic extruded into a double- or triple-wall sheet with divider webs for stiffness, insulating value, and light diffusion.

- Polyester is a thin film used to carry specialized coatings and/or to divide the air space between two layers of glass into multiple air spaces. Highly transparent, it is protected from abuse and weathering by 
the two exterior glass layers. It can also be used in tinted or coated forms as film that is glued to the inner surface of existing fenestration products for retrofitting applications.

\subsection{Improved Glazing Products}

There are three fundamental approaches to improving the energy performance of glazing products:

1. Alter the glazing material itself by changing its chemical composition or physical characteristics. An example of this is tinted glazing. The glazing material can also be altered by creating a laminated glazing.

2. Apply a coating to the glazing material surface. Reflective coatings and films were developed to reduce heat gain and glare, and more recently, low-emittance and spectrally selective coatings have been developed to improve both heating and cooling season performance.

3. Assemble various layers of glazing and control the properties of the spaces between the layers. These strategies include the use of two or more panes or films, low-conductance gas fills between the layers, and thermally improved edge spacers.

Two or more of these approaches may be combined. Each of these improvements to the glazing is discussed below

\subsubsection{Tinted Glazing}

Both plastic and glass materials are available in a large number of tints. The tints absorb a portion of the light and solar heat. Tinting changes the color of the fenestration product.

Tinted glazings retain their transparency from the inside, so that the outward view is unobstructed. The most common colors are neutral gray, bronze, and blue-green, which do not greatly alter the perceived color of the view and tend to blend well with other architectural colors. Many other specialty colors are available for particular aesthetic purposes.

Tinted glass is made by altering the chemical formulation of the glass with special additives. Its color changes with the thickness of the glass and the addition of coatings applied after manufacture. Every change in color or combination of different glass types affects transmittance, solar heat gain coefficient, reflectivity, and other properties. Glass manufacturers list these properties for every color, thickness, and assembly of glass type they produce.

Tinted glazings are specially formulated to maximize their absorption across some or all of the solar spectrum and are often referred to as "heat-absorbing." All of the absorbed solar energy is initially transformed into heat within the glass, thus raising the glass temperature. Depending upon climatic conditions, up to 50 percent of the heat absorbed in a single layer of tinted glass may then be transferred via radiation and convection to the inside. Thus, there may be only a modest reduction in overall solar heat gain compared to other glazings.

There are two categories of tinted glazing: the traditional tints that diminish light as well as heat gain, and spectrally selective tints that reduce heat gain but allow more light to be transmitted to the interior. The traditional tinted glazing often forces a trade-off between visible light and solar gain. For these bronze and gray tints, there is a greater reduction in visible light transmittance than there is in solar heat gain coefficient. This can reduce glare by reducing the apparent brightness of the glass surface, but it also reduces the amount of daylight entering the room.

To address the problem of reducing daylight with traditional tinted glazing, glass manufacturers have developed new types of tinted glass that are "spectrally selective." They preferentially transmit the daylight portion of the solar spectrum but absorb the near-infrared part of sunlight. This is accomplished by adding special chemicals to the float glass process. Like other tinted glass, they are durable and can be used in both monolithic and multiple-glazed fenestration product applications. These glazings have a light blue or green 
tint and have visible transmittance values higher than conventional bronze- or gray-tinted glass, but have lower solar heat gain coefficients. Because they are absorptive, they are best used as the outside glazing in a double-glazed unit. They can also be combined with Low-E coatings to enhance their performance further.

\subsubsection{Reflective Coatings and Films}

As the solar heat gain is lowered in single-pane tinted glazings, the visible light transmission drops even faster, and there are practical limits on how low the solar heat gain can be made using tints. If larger reductions are desired, a reflective coating can be used to lower the solar heat gain coefficient by increasing the surface reflectivity of the material. These coatings usually consist of thin metallic layers. The reflective coatings come in various metallic colors (silver, gold, bronze), and they can be applied to clear or tinted glazing (the substrate). The solar heat gain of the substrate can be reduced a little or a lot, depending on the thickness and reflectivity of the coating, and its location on the glass.

As with tinted glazing, the visible light transmittances of reflective glazings are usually reduced substantially more than the solar heat gain.

\subsubsection{Double Glazing}

Storm windows added onto the outside of window frames during the stormy winter season were the first double-glazed fenestration products. They reduce infiltration from winter winds by providing a seal around all the operating sash and they improve the insulating value of the glazing as well.

When manufacturers began to experiment with factory-sealed, double-pane glass to be installed for yearround use, they encountered a number of technical concerns, such as how to allow for different thermal movement between the two panes, how to prevent moisture from forming between the panes and condensing on an inaccessible surface, and how to allow for changes in atmospheric pressure as the assembly was moved from factory to installation site. These issues have been successfully addressed over the years with a variety of manufacturing techniques and material selections.

When double-glass units first came on the market, the two glass layers were often fused around the perimeter to make a permanently sealed air space. In recent years, however, spacers and polymer sealants have largely replaced glass-to-glass seals, and have proven sufficiently durable for residential applications. The layers of glass are separated by and adhere to a spacer, and the sealant, which forms a gas and moisture barrier, is applied around the entire perimeter. Normally, the spacer contains a desiccant material to absorb any residual moisture that may remain in the air space after manufacture. Sealed insulating glass units are now a mature, well proven technology. Designs utilizing high-quality sealants and manufactured with good quality control should last for decades without seal failure.

\subsubsection{Glass Coatings and Tints in Double Glazing}

Both solar reflective coatings and tints on double-glazed fenestration products are effective in reducing summer heat gain; however, only certain coatings contribute to reducing winter heat loss, and tints do not affect the heat loss rates at all. It is possible to provide reflective coatings on any one of the four surfaces, although they are usually located on the outermost surface or on the surfaces facing the air space. Coating location can also depend on the type of coating. Some vacuum-deposited reflective coatings must be placed in a sealed air space because they would not survive exposure to outdoor elements, finger prints, or cleaning agents. Pyrolytic coatings that are created with a high-temperature process as the glass is formed are extremely hard and durable and can be placed anywhere. Each location produces a different visual and heat transfer effect. Other advanced coatings such as low-emittance and spectrally selective coatings are normally applied to double-glazed or triple-glazed fenestration products. These applications are discussed later in this chapter. 
Double-pane units can be assembled using different glass types for the inner and outer layers. Typically, the inner layer is standard clear glass, while the outer layer can be tinted, reflective, or both. The solar heat gain coefficient is reduced because the tinted glass and clear glass both reduce transmitted radiation. In addition, this design further reduces solar heat gain because the inner clear glass, the gas fill, and any Low-E coating keep much of the heat absorbed by the outer glass from entering the building interior.

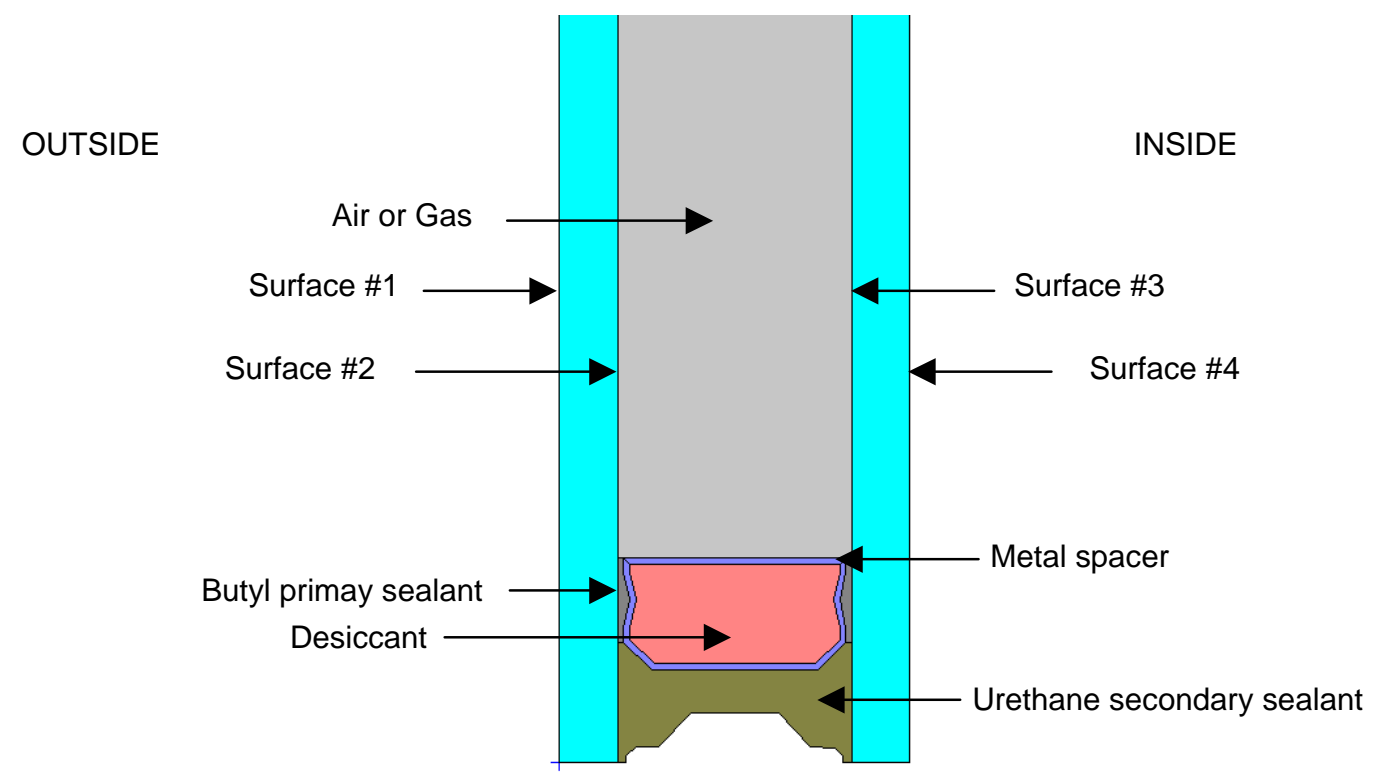

Figure 2-2. Insulating Glass Unit (IGU).

\subsubsection{Gap Width in Multiple Glazed Units}

Fenestration product manufacturers have some flexibility to reduce heat transfer by selecting the best gap width between two or more glazings. The air space between two pieces of glass reaches its optimum insulating value at about 1/2-inch $(12 \mathrm{~mm})$ thickness when filled with air or argon. As the gap gets larger, convection in the gap increases and slowly increases heat transfer. Below $3 / 8$ inch $(9 \mathrm{~mm})$, conduction through the air gap increases and the U-factor rises more rapidly. Krypton gas has its optimum thickness at about $1 / 4$ inch $(6 \mathrm{~mm})$, so that if smaller air gaps are required, for example in a three-layer fenestration product whose overall exterior dimensions are limited, krypton may be the best selection, although it is also more costly.

\subsubsection{Divided Lights}

Manufacturers have been struggling with the problem of many homeowners' preference for traditional, divided light fenestration products, which have many small panes separated by thin bars called muntins. With single-pane glass, true divided lights actually improved the thermal performance of the fenestration product because the wood muntins had a higher insulating value than the glass. Some manufacturers have introduced "true divided light" insulated units, in which traditional-looking muntins hold small, individual, insulated panes. However, these are expensive and difficult to fabricate with insulated glass and have greater thermal losses due to the number of edges, which now have metal in them.

A second option is to produce a single, large sealed glass unit with "muntins" glued to the inside and outside surfaces, while a grid is placed in the middle of one large insulated unit, giving the visual effect of divided lights. This reduces fabrication costs but does not reduce resistance to heat flow if the muntins in the middle are metal and if they touch both lights of glass. 
A third option, which is more energy efficient, is to build a large-pane insulated unit that has snap-on or glued-on grilles to simulate the traditional lights.

The energy performance of the simple snap-on grid will be similar to a unit without any mullions; however, the true divided lights will result in greater heat transfer because of the additional edges.

\subsubsection{Special Products}

Glass blocks present a very special case of double glazing. They provide light with some degree of visual privacy. Plastic blocks, which have a lower U-factor than glass, are also available. However, when installed, the necessary grouting reduces the energy efficiency. Also, metal mesh and steel reinforcing bars, used between blocks to provide structural stability, provide thermal bridges which also reduce energy efficiency.

Plastic glazings are available in a number of configurations with double layers. Double-glazed acrylic bubble skylights are formed with two layers separated by an air space of varying thickness, ranging from no separation at the edges to as much as 3 inches $(7.6 \mathrm{~cm})$ at the top of the bubble. The average separation is used to calculate the effective U-factor.

Multicell polycarbonate sheets, which can be mounted with the divider webs running vertically or horizontally, are available. The divider webs increase the effective insulating value of the glazing by reducing convection exchange within the cells, especially when they are mounted horizontally.

\subsubsection{Multiple Panes or Films}

By adding a second pane, the insulating value of the fenestration product glass alone is doubled (the U-factor is reduced by half). As expected, adding a third or fourth pane of glass further increases the insulating value of the fenestration product, but with diminishing effect.

Triple- and quadruple-glazed fenestration products became commercially available in the 1980s as a response to the desire for more energy-efficient products. There is a trade-off with this approach, however. As each additional layer of glass adds to the insulating value of the assembly, it also reduces the visible light transmission and the solar heat gain coefficient, thereby reducing the fenestration product's value for providing solar gains or daylighting. In addition, other complications are encountered. Additional panes of glass increase the weight of the unit, which makes mounting and handling more difficult and transportation more expensive.

Because of the difficulties discussed above, it is apparent there are physical and economic limits to the number of layers of glass that can be added to a fenestration product assembly. However, multiple-pane units are not limited to assemblies of glass. One popular innovation is based on substituting an inner plastic film for the middle layer of glass. The plastic film is very lightweight, and because it is very thin, it does not increase the thickness of the unit. The glass layers protect the inner layer of plastic from scratching, mechanical abuse, corrosion, weathering, and visual distortions caused by wind pressure. Thus, the strength and durability of plastic as a glazing material are no longer issues when the plastic is protected from physical abuse and weathering by inner and outer layers of glass. The plastic films are specially treated to resist UV degradation and they are heat shrunk so they remain flat under all conditions.

The plastic inner layer serves a number of important functions. It decreases the U-factor of the fenestration product assembly by dividing the inner air space into multiple chambers. Units are offered with one or two inner layers of plastic. Secondly, a Low-E coating can be placed on the plastic film itself to further lower the U-factor of the assembly. Also, the plastic film can be provided with spectrally selective coatings to reduce solar gain in hot climates without significant loss of visible transmittance. The performance of multiple-pane fenestration product assemblies with low-emittance coatings and gas fills is described in the following sections. 


\subsubsection{Low-Emittance Coatings}

The principal mechanism of heat transfer in multilayer glazing is thermal radiation from a warm pane of glass to a cooler pane. Coating a glass surface with a low-emittance material and facing that coating into the gap between the glass layers blocks a significant amount of this radiant heat transfer, thus lowering the total heat flow through the fenestration product. The improvement in insulating value due to the Low-E coating is roughly equivalent to adding another pane of glass to a multipane unit.

The solar spectral reflectances of Low-E coatings can be manipulated to include specific parts of the visible and infrared spectrum. A glazing material can then be designed to optimize energy flows for solar heating, daylighting, and cooling.

With conventional clear glazing, a significant amount of solar radiation passes through the fenestration product, and then heat from objects within the house is reradiated back through the fenestration product. For example, a glazing design for maximizing solar gains in the winter would ideally allow all of the solar spectrum to pass through, but would block the reradiation of heat from the inside of the house. The first LowE coatings were designed to have a high solar heat gain coefficient and a high visible transmittance to transmit the maximum amount of sunlight into the interior while reducing the U-factor significantly.

A glazing designed to minimize summer heat gains but allow for some daylighting would allow visible light through, but would block all other portions of the solar spectrum, including ultraviolet light and nearinfrared, as well as long-wave heat radiated from outside objects, such as paving and adjacent buildings, as shown in Figure 2-3. These second-generation Low-E coatings were designed to reflect the solar nearinfrared, thus reducing the total solar heat gain coefficient while maintaining high levels of light transmission . Variations on this design (modified coatings and/or glazings) can further reduce summer solar heat gain and control glare.

There are three basic types of Low-E coatings available on the market today:

1. High-transmission Low-E:

These Low-E glass products are often referred to as pyrolitic or hard coat Low-E glass, due to the glass coating process. The properties presented here are typical of a Low-E glass product designed to reduce heat loss but admit solar gain.

2. Moderate-transmission Low-E:

These Low-E glass products are often referred to as sputtered (or soft-coat products) due to the glass coating process. (Note: Low solar gain Low-E products are also sputtered coatings.) Such coatings reduce heat loss and let in a reasonable amount of solar gain.

3. Low-transmission Low-E:

These Low-E products are often referred to as sputtered (or soft-coat) due to the glass coating process. (Note: Moderate solar gain Low-E products are also sputtered coatings.) This type of Low-E product, sometimes called spectrally selective Low-E glass, reduces heat loss in winter but also reduces heat gain in summer. Compared to most tinted and reflective glazings, this Low-E glass provides a higher level of visible light transmission for a given amount of solar heat reduction.

The type and quality of Low-E coating will affect not only the U-factor, but also the transmittance and solar heat gain coefficient of a glass. All these properties (U-factor, VT, and SHGC) need to be taken into consideration in characterizing a particular glazing product. 


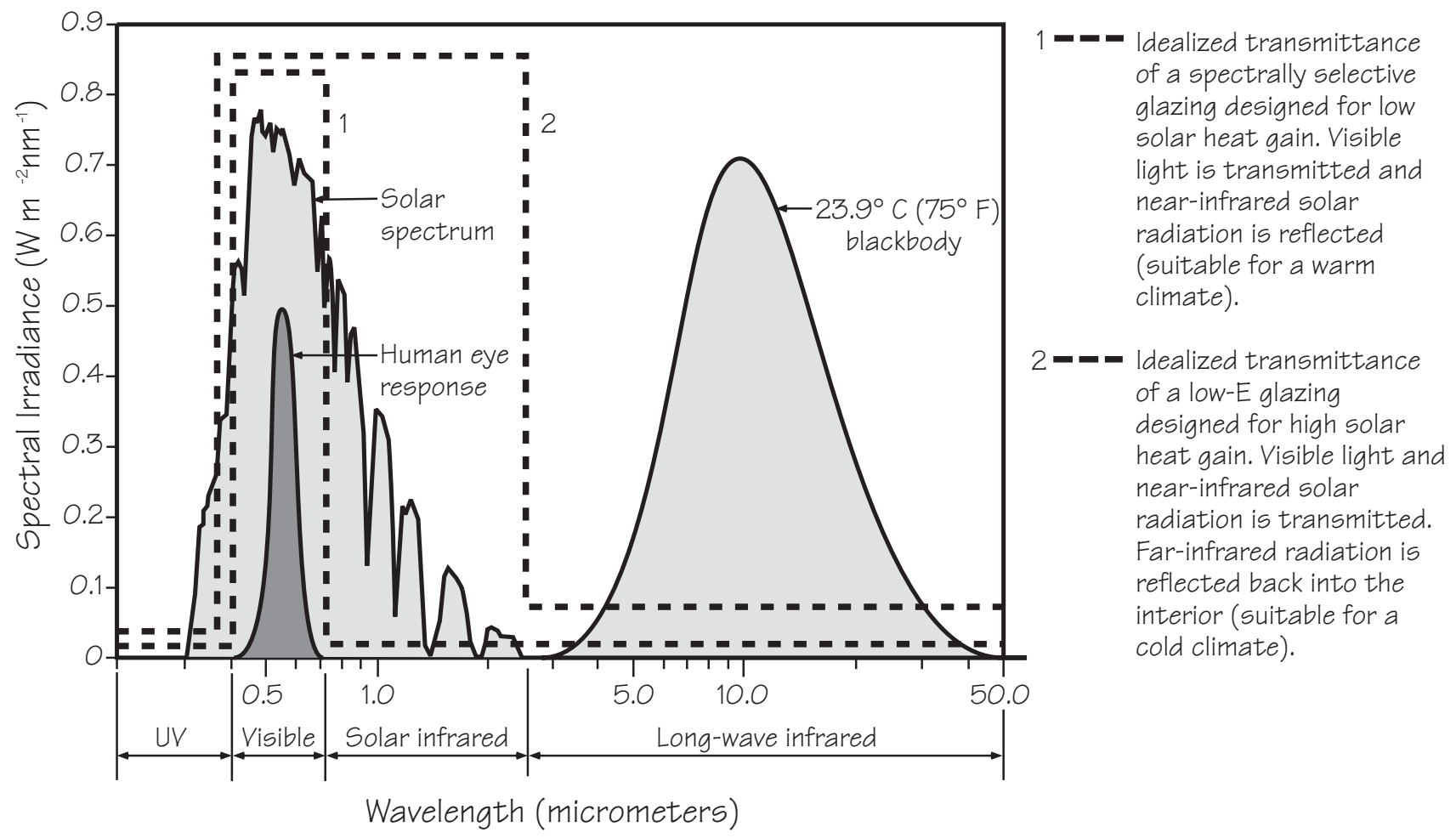

Figure 2-3. Ideal spectral transmittance for glazings in different climates. (Source: "Sensitivity of Fenestration Solar Gain to Source Spectrum and Angle of Incidence." ASHRAE Transactions 10, R. McCluney, June 1996).

\subsubsection{Coating Placement}

The placement of a Low-E coating within the air gap of a double-glazed fenestration product does not significantly affect the U-factor but it does influence the solar heat gain coefficient (SHGC). That is why, in heating-dominated climates, placing a Low-E coating on the \#3 surface (outside surface of the inner pane) is recommended to maximize winter passive solar gain at the expense of a slight reduction in the ability to control summer heat gain. In cooling climates, a coating on the \#2 surface (inside surface of the outer pane) is generally best to reduce solar heat gain and maximize energy efficiency. Manufacturers sometimes place the coatings on other surfaces (e.g., \#2 surface in a heating climate) for other reasons, such as minimizing the potential for thermal stress. Multiple Low-E coatings are also placed on surfaces within a triple-glazed fenestration product assembly, or on the inner plastic glazing layers of multipane assemblies referred to as superwindows (discussed later in this chapter), with a cumulative effect of further improving the overall Ufactor.

\subsubsection{Coating Types}

There are two basic types of Low-E coatings - sputtered and pyrolytic, referring to the process by which they are made. The best of each type of coating is colorless and optically clear. Some coatings may have a slight hue or subtle reflective quality, particularly when viewed in certain lighting conditions or at oblique angles.

A sputtered coating is multilayered (typically, three primary layers, with at least one layer of metal) and is deposited on glass or plastic film in a vacuum chamber. The total thickness of a sputtered coating is only $1 / 10,000$ of the thickness of a human hair. Sputtered coatings often use a silver layer and must be protected from humidity and contact. For this reason they are sometimes referred to as "soft coats." Since sputtering is a low-temperature process, these coatings can be deposited on flat sheets of glass or thin plastic films. While sputtered coatings are not durable in themselves, when placed into a sealed double- or triple-glazed assembly 
they should last as long as the sealed glass unit. Sputtered coatings typically have lower emittances than pyrolytic coatings. They are available commercially with emittance ratings of $\mathrm{e}=0.20$ to as low as $\mathrm{e}=0.04(\mathrm{e}=$ 0.20 means that 80 percent of the long-wavelength radiant energy received by the surface is reflected, while e $=0.04$ means 96 percent is reflected). For uncoated glass, $\mathrm{e}=0.84$, which means only 16 percent of the radiant energy received by the surface is reflected.

A typical pyrolytic coating is a metallic oxide, most commonly tin oxide with some additives, which is deposited directly onto a glass surface while it is still hot. The result is a baked-on surface layer that is quite hard and thus very durable, which is why this is sometimes referred to as a "hard coat." A pyrolytic coating can be ten to twenty times thicker than a sputtered coating but is still extremely thin. Pyrolytic coatings can be exposed to air, cleaned with normal cleaning products, and subjected to general wear and tear without losing their Low-E properties.

Because of their greater durability, pyrolytic coatings are available on single-pane glass and separate storm windows, but not on plastics, since they require a high-temperature process. In general, though, pyrolytic coatings are used in sealed, double-glazed units with the Low-E surface inside the sealed air space. While there is considerable variation in the specific properties of these coatings, they typically have emittance ratings in the range of $\mathrm{e}=0.20$ to $\mathrm{e}=0.15$.

A laminated glass with a spectrally selective Low-E sputtered coating on plastic film sandwiched between two layers of glass offers the energy performance of single-pane, spectrally selective glass and the safety protection of laminated glass. However, in this configuration, since the Low-E surface is not exposed to an air space, there is no effect on the glazing U-factor.

\subsubsection{Gas Fills}

Another improvement that can be made to the thermal performance of insulating glazing units is to reduce the conductance of the air space between the layers. Originally, the space was filled with air or flushed with dry nitrogen just prior to sealing. In a sealed glass insulating unit air currents between the two panes of glazing carry heat to the top of the unit and settle into cold pools at the bottom. Filling the space with a less conductive gas minimizes overall transfer of heat between two glass layers.

Manufacturers have introduced the use of argon and krypton gas fills, with measurable improvement in thermal performance. Argon is inexpensive, nontoxic, nonreactive, clear, and odorless. The optimal spacing for an argon-filled unit is the same as for air, about $1 / 2$ inch $(12 \mathrm{~mm})$. Krypton has better thermal performance, but is more expensive to produce. Krypton is particularly useful when the space between glazings must be thinner than normally desired, for example, $1 / 4$ inch $(6 \mathrm{~mm})$. A mixture of krypton and argon gases is also used as a compromise between thermal performance and cost.

Filling the sealed unit completely with argon or krypton presents challenges that manufacturers continue to work on. A typical gas fill system adds the gas into the cavity with a pipe inserted through a hole at the edge of the unit. As the gas is pumped in, it mixes with the air, making it difficult to achieve 100 percent purity. Recent research indicates that 90 percent is the typical concentration achieved by manufacturers today. Some manufacturers are able to consistently achieve better than 95 percent gas fill by using a vacuum chamber. An uncoated double-pane unit filled with 90 percent argon gas and 10 percent air yields a slightly more than 5 percent improvement in the insulating value at the center of the glass, compared to the same unit filled with air. However, when argon and krypton fills are combined with Low-E coatings and multipane glazings, more significant reductions of 15 to 20 percent can be achieved. Since the Low-E coating has substantially reduced the radiation component of heat loss, the gas fill now has a greater proportional effect on the remaining heat transfer by convection and conduction. 


\subsection{Thermally Improved Edge Spacers}

The layers of glazing in an insulating glass (IG) unit must be held apart at the appropriate distance by spacers. The spacer system must provide a number of additional functions in addition to keeping the glass units at the proper dimension:

- accommodate stress induced by thermal expansion and pressure differences;

- provide a moisture barrier that prevents passage of water or water vapor that would fog the unit;

- provide a gas-tight seal that prevents the loss of any special low-conductance gas in the air space;

- create an insulating barrier that reduces the formation of interior condensation at the edge.

Older double-pane wood fenestration products used a wood spacer that could not be hermetically sealed and thus was vented to the outside to reduce fogging in the air gap. Modern versions of this system function well but, because they are not hermetically sealed, cannot be used with special gas fills or some types of Low-E coatings. Early glass units were often fabricated with an integral welded glass-to-glass seal. These units did not leak but were difficult and costly to fabricate, and typically had a less-than-optimal narrow spacing. The standard solution for insulating glass units (IGUs) that accompanied the tremendous increase in market share of insulating glass in the 1980s was the use of metal spacers, and sealants. These spacers, typically aluminum, also contain a desiccant that absorbs residual moisture. The spacer is sealed to the two glass layers with organic sealants that both provide structural support and act as a moisture barrier. There are two generic systems for such IGUs: a single-seal spacer and a double-seal system.

In the single-seal system, an organic sealant, typically a butyl material, is applied behind the spacer and serves both to hold the unit together and to prevent moisture intrusion. These seals are normally not adequate to contain special low-conductance gases.

In a double-seal system, a primary sealant, typically butyl, seals the spacer to the glass to prevent moisture migration and gas loss, and a secondary backing sealant, often silicone, provides structural strength. When sputtered Low-E coatings are used with double-seal systems, the coating must be removed from the edge first ("edge deletion") to provide a better edge seal.

Since aluminum is an excellent conductor of heat, the aluminum spacer used in most standard edge systems represented a significant thermal "short circuit" at the edge of the IGU, which reduces the benefits of improved glazings. As the industry has switched from standard double-glazed IGUs to units with Low-E coatings and gas fills, the effect of this edge loss becomes even more pronounced. Under winter conditions, the typical aluminum spacer would increase the U-factor of a Low-E, gas fill unit slightly more than it would increase the U-factor of a standard double-glazed IGU. The smaller the glass area, the larger the effect of the edge on the overall product properties. In addition to the increased heat loss, the colder edge is more prone to condensation.

Fenestration product manufacturers have developed a series of innovative edge systems to address these problems, including solutions that depend on material substitutions as well as radically new designs. One approach to reducing heat loss has been to replace the aluminum spacer with a metal that is less conductive, e.g., stainless steel, and change the cross-sectional shape of the spacer. Another approach is to replace the metal with a design that uses materials that are better insulating. The most commonly used design incorporates spacer, sealer, and desiccant in a single tape element. The tape includes a solid, extruded thermoplastic compound that contains a blend of desiccant materials and incorporates a thin, fluted metal shim of aluminum or stainless steel. Another approach uses an insulating silicone foam spacer that incorporates a desiccant and has a high-strength adhesive at its edges to bond to glass. The foam is backed with a secondary sealant. Both extruded vinyl and pultruded fiberglass spacers have also been used in place of metal designs.

There are several hybrid designs that incorporate thermal breaks in metal spacers or use one or more of the elements described above. Some of these are specifically designed to accommodate three- and four-layer glazings or IGUs incorporating stretched plastic films. All are designed to interrupt the heat transfer pathway at the glazing edge between two or more glazing layers. 
Warm edge spacers have become increasingly important as manufacturers switch from conventional double glazing to higher-performance glazing. For purposes of determining the overall fenestration product Ufactor, the edge spacer has an effect that extends beyond the physical size of the spacer to a band about 63.5 $\mathrm{mm}$ (2.5 inches) wide. The contribution of this $63.5 \mathrm{~mm}$ (2.5-inch) wide "glass edge" to the total fenestration product U-factor depends on the size of the product. Glass edge effects are more important for smaller fenestration products, which have a proportionately larger glass edge area. For a typical residential-size window ( 0.8 by 1.2 meters , 3 by 4 feet), changing from a standard aluminum edge to a good-quality warm

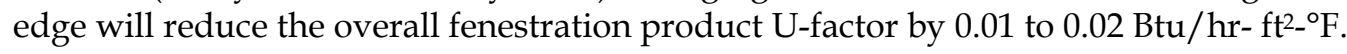





\section{FENESTRATION HEAT TRANSFER BASICS}

\subsection{Overview}

Much of the information in this chapter is taken from the book Residential Windows: A Guide to New Technologies and Energy Performance by John Carmody, Stephen Selkowitz, and Lisa Heschong. Additional information can also be found in the ASHRAE 2001 Handbook of Fundamentals.

\subsection{Energy Flow Mechanisms}

Overall energy flow through a fenestration product is a function of:

- Temperature Driven Heat Transfer: When there is a temperature difference between inside and outside, heat is lost or gained through the fenestration product frame and glazing by the combined effects of conduction, convection, and radiation. This is indicated in terms of the U-factor of a fenestration assembly.

- Solar Gain: Regardless of outside temperature, heat can be gained through fenestration products by direct or indirect solar radiation. The amount of heat gain through products is measured in terms of the solar heat gain coefficient (SHGC) of the glazing.

- Infiltration: Heat loss and gain also occur by infiltration through cracks in the fenestration assembly. This effect is measured in terms of the amount of air (cubic feet or meters per minute) that passes through a unit area of fenestration product (square foot or meter) under given pressure conditions. In reality, infiltration varies with wind-driven and temperature-driven pressure changes. Infiltration also contributes to summer cooling loads in some climates by raising the interior humidity level.

The 2001 ASHRAE Handbook of Fundamentals contains the following equation for calculating the energy flow through a fenestration product (assuming no humidity difference):

$$
q=U_{t} * A_{p f}\left(t_{o u t}-t_{\text {in }}\right)+\left(S H G C_{t} * A_{p f} * E_{t}\right)
$$

Where:

$$
\begin{array}{ll}
q & =\text { instantaneous energy flow, } \mathrm{W}(\mathrm{Btu} / \mathrm{h}) \\
U_{o} & =\text { overall coefficient of heat transfer }(\mathrm{U} \text {-factor }), \mathrm{W} / \mathrm{m}^{2}-\mathrm{o} \mathrm{K}\left(\mathrm{Btu} / \mathrm{h}-\mathrm{ft}^{2}-\mathrm{o} \mathrm{F}\right) \\
t_{\text {in }} & =\text { interior air temperature, }{ }^{\circ} \mathrm{C}\left({ }^{\circ} \mathrm{F}\right) \\
t_{\text {out }} & =\text { exterior air temperature, }{ }^{\circ} \mathrm{C}\left({ }^{\circ} \mathrm{F}\right) \\
A_{p f} & =\text { Total projected area of fenestration, } \mathrm{m}^{2}\left(\mathrm{ft}^{2}\right) \\
S H G C_{t} & =\text { overall solar heat gain coefficient, non-dimensional } \\
E_{t} & =\text { incident total irradiance, } \mathrm{W} / \mathrm{m}^{2}-\mathrm{o} \mathrm{K}\left(\mathrm{Btu} / \mathrm{h}-\mathrm{ft}^{2}-\mathrm{o} \mathrm{F}\right)
\end{array}
$$

This equation shows that the properties of U-factor, SHGC, and infiltration are major factors which determine the energy flow through a fenestration product. For this reason, the NFRC rating system rates the U-factor, 
SHGC, and air infiltration of products, and the NFRC 100, NFRC 200, and NFRC 400 documents define the procedures for calculating these values for the total product.

\subsection{Temperature Driven Heat Transfer}

Fenestration product heat loss/gain due to temperature is a combination of three modes of heat transfer:

1. Conduction (heat traveling through a solid material, the way a frying pan warms up) through glazing, frame elements, and air or other gases in the glazing gap

2. Convection (the transfer of heat by the movement of gases or liquids, like warm air rising from a candle flame) through air layers on the exterior and interior fenestration product surfaces and between glazing layers

3. Radiative heat transfer (the movement of heat energy through space without relying on conduction through the air or by movement of the air, the way you feel the heat of a fire) between glazing layers, or between IG units and interior or exterior spaces.

Solar radiation absorbed by glazing layers will influence these processes, while solar radiation transmitted by the glazing system serves as a heat gain to the space. These three modes of heat transfer are shown schematically in Figure 3-1. Heat flows from warmer to cooler bodies, thus from inside to outside in winter, and reverses direction in summer during periods when the outside temperature is greater than indoors.

The amount of heat transfer due to these three processes in quantified by its $\mathrm{U}$-factor $\left(\mathrm{W} / \mathrm{m}^{2}-\mathrm{o} \mathrm{C}\right.$ or Btu/h- $\mathrm{ft}^{2}$ ${ }^{\circ}$ F). The inverse of heat flow, or resistance to heat transfer, is expressed as an R-value. NFRC's rating system quantifies and predicts U-factors.

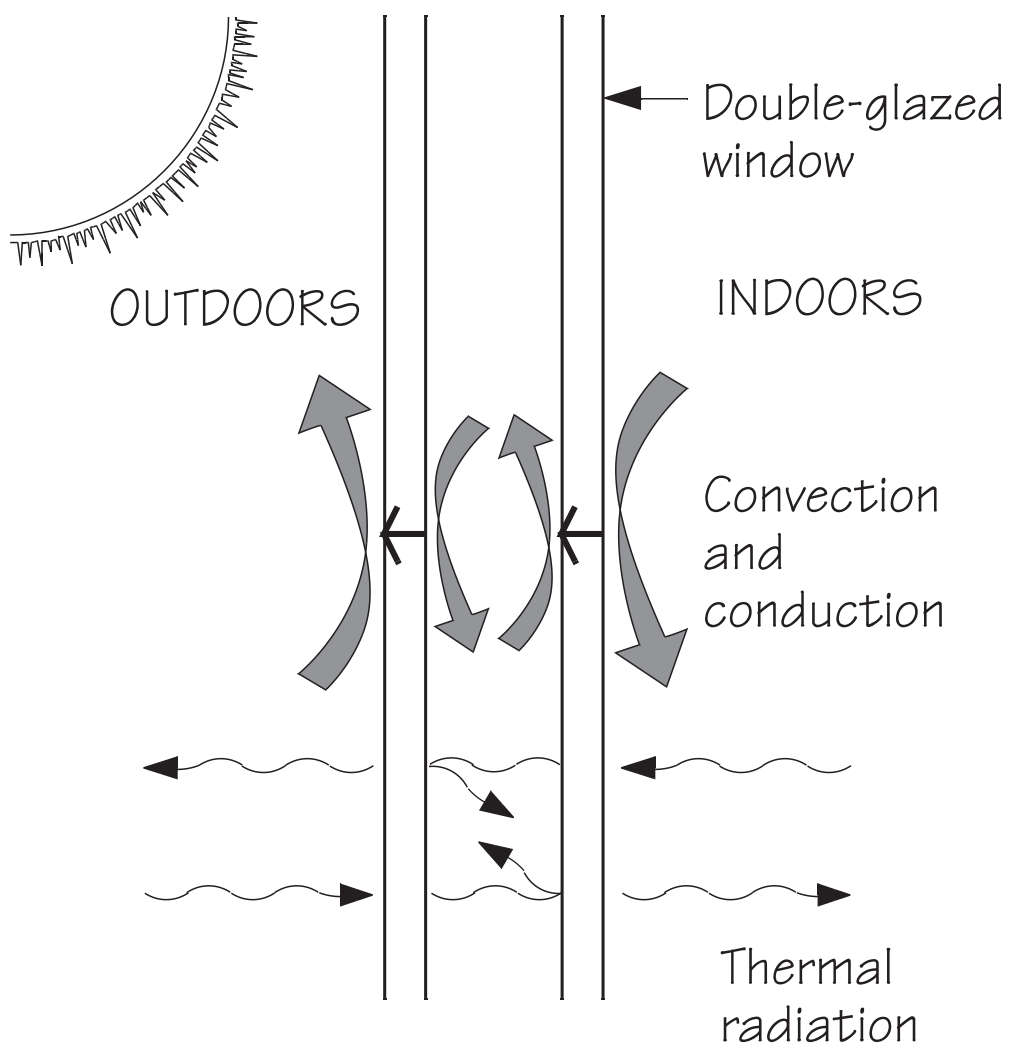

Figure 3-1. Mechanisms of heat transfer in a fenestration product. 


\subsubsection{Conduction}

Compared to a well-insulated wall, heat transfer through a typical older fenestration product is generally much higher. A single-glazed fenestration product has roughly the same insulating qualities as a sheet of metal-most of the insulating value comes from the air layer on each surface of the glass. Such a product can be considered a thermal hole in a wall and typically has a heat loss rate ten to twenty times that of the wall. A product with such a poor insulating value allows heat to flow out of a space almost unimpeded. If the temperature inside is $21^{\circ} \mathrm{C}\left(70^{\circ} \mathrm{F}\right)$ and outside is $-18^{\circ} \mathrm{C}\left(0^{\circ} \mathrm{F}\right)$, the glass surface of a single-glazed product would be about $-8^{\circ} \mathrm{C}\left(17^{\circ} \mathrm{F}\right)$ - cold enough to form frost on the inside of the glass.

\subsubsection{Convection}

Convection affects the heat transfer in many places in the assembly: the inside glazing surface, the outside glazing surface, inside frame cavities, and inside any air spaces between glazings. A cold interior glazing surface chills the air adjacent to it. This denser cold air then falls to the floor, starting a convection current which is typically perceived as a "draft" caused by leaky fenestration products. One remedy for this situation is to install a product with lower heat loss rates that provides a warmer glass surface.

On the exterior, a component of the heat transfer rate of a fenestration product is the air film against the glazing surface. As wind blows across the product (causing convection), and the insulating value of this air film diminishes which contributes to a higher rate of heat loss. Finally, when there is an air space between layers of glazing, convection currents can facilitate heat transfer through this air layer. By adjusting the space between the panes of glass, as well as choosing a gas fill that insulates better than air, double-glazed fenestration products can be designed to minimize this effect.

\subsubsection{Radiation}

All objects emit invisible thermal radiation, with warmer objects emitting more than colder ones. Hold your hand in front of an oven window and you will feel the radiant energy emitted by that warm surface. Your hand also radiates heat to the oven window, but since the window is warmer than your hand, the net balance of radiant flow is toward your hand and it is warmed. Now imagine holding your hand close to a singleglazed window in winter. The window surface is much colder than your hand. Each surface emits radiant energy, but since your hand is warmer, it emits more toward the window than it gains and you feel a cooling effect. Thus, a cold glazing surface in a room chills everything else around it.

\subsubsection{U-factor}

The U-factor (also referred to as U-value) is the standard way to quantify insulating value. It indicates the rate of heat flow through the fenestration product. The U-factor is the total heat transfer coefficient of the fenestration system, in $\mathrm{W} / \mathrm{m}^{2}{ }^{\circ} \mathrm{C}\left(\mathrm{Btu} / \mathrm{hr}-\mathrm{ft}^{2}{ }^{\circ}{ }^{\circ} \mathrm{F}\right)$, which includes conductive, convective, and radiative heat transfer for a given set of environmental conditions. It therefore represents the heat flow per hour, in Watts (Btu per hour) through each square meter (square foot) of fenestration product for a $1^{\circ} \mathrm{C}\left(1^{\circ} \mathrm{F}\right)$ temperature difference between the indoor and outdoor air temperature. The smaller the U-factor of a material, the lower the rate of heat flow. The total R-value, which measures thermal resistance, is the reciprocal of the total Ufactor $(\mathrm{R}=1 / \mathrm{U})$.

The U-factor depends on the thermal properties of the materials in the fenestration product assembly, as well as the weather conditions, such as the temperature differential between indoors and outside, and wind speed. NFRC has standardized the exterior conditions (called environmental conditions) for U-factor calculations for product ratings using the following temperatures and wind speeds:

- Wind Speed: $5.5 \mathrm{~km} / \mathrm{hr}(12.3 \mathrm{mph})$

- Indoor air temperature: $21^{\circ} \mathrm{C}\left(70^{\circ} \mathrm{F}\right)$ 
- Outdoor air temperature: $-18^{\circ} \mathrm{C}\left(0^{\circ} \mathrm{F}\right)$.

Skylights and roof fenestration products are simulated at a 20 degree slope from horizontal.

\subsubsection{Total Product U-factor}

The U-factor of a total fenestration assembly is a combination of the insulating values of the glazing assembly itself, the edge effects that occur in the insulated glazing unit, and the insulating value of the frame and sash.

\subsubsection{Center-of-Glazing U-factor}

The U-factor of the glazing portion of the fenestration unit is affected primarily by the total number of glazing layers, the dimension separating the various layers of glazing, the type of gas that fills the separation, and the characteristics of coatings on the various surfaces. The U-factor for the glazing alone is referred to as the center-of-glass U-factor.

\subsubsection{Edge Effects}

A U-factor calculation assumes that heat flows perpendicular to the plane of the fenestration product. However, fenestration products are complex three-dimensional assemblies, in which materials and cross sections change in a relatively short space.

For example, metal spacers at the edge of an insulating glass unit have much higher heat flow than the center of the insulating glazing, which causes increased heat loss along the outer edge of the glazing. The relative impact of these "edge effects" becomes more important as the insulating value of the rest of the assembly increases.

\subsubsection{Frames and Sashes}

The heat loss through a fenestration product frame can be quite significant: in a typical 1.2 by $0.9 \mathrm{~m}\left(4^{\prime}\right.$ by $\left.3^{\prime}\right)$ double-hung wood frame product, the frame and sash can occupy approximately 30 percent of the product area.

In a frame with a cross section made of one uniform, solid material, the U-factor is based on the conduction of heat through the material. However, hollow frames and composite frames with various reinforcing or cladding materials are more complex. Here, conduction through materials must be combined with convection of the air next to the glazing and radiant exchange between the various surfaces.

Furthermore, frames rarely follow the same cross section around a fenestration product. For example, a horizontal slider has seven different frame cross sections, each with its own rate of heat flow.

\subsubsection{Overall U-factor}

Since the U-factors are different for the glazing, edge-of-glazing zone, and frame, it can be misleading to compare U-factors if they are not carefully described. In order to address this problem, the concept of a total fenestration product U-factor is utilized by the National Fenestration Rating Council (NFRC). A specific set of engineering assumptions and procedures must be followed to calculate the overall U-factor of a fenestration unit using the NFRC method. In most cases, the overall U-factor is higher than the U-factor for the glazing alone, since the glazing remains superior to the frame in insulating value.

The U-factor of a product is calculated with the product in a vertical position. A change in mounting angle can affect its U-factor. 


\subsection{Solar Heat Gain and Visible Transmittance}

The second major energy performance characteristic of fenestration products is the ability to control solar heat gain through the glazing. Solar heat gain through fenestration products tends to be the single most significant factor in determining the air-conditioning load of a residential building. The intensity of heat gain from solar radiation can greatly surpass heat gain from other sources, such as outdoor air temperature or humidity.

Visible transmittance (VT) is an optical property that indicates the amount of visible light transmitted through the glazing. Although VT does not directly affect heating and cooling energy use, it is used in the evaluation of energy-efficient fenestration products and therefore is discussed following the solar heat gain section.

The origin of solar heat gain is the direct and diffuse radiation coming directly from the sun and the sky or reflected from the ground and other surfaces. Some radiation is directly transmitted through the glazing to the space, and some may be absorbed in the glazing and then indirectly admitted to the space. Sunlight is composed of electromagnetic radiation of many wavelengths, ranging from short-wave invisible ultraviolet, to the visible spectrum, to the longer, invisible near-infrared waves. About half of the sun's energy is visible light; the remainder is largely infrared with a small amount of ultraviolet. This characteristic of sunlight makes it possible to selectively admit or reject different portions of the solar spectrum. While reducing solar radiation through fenestration products is a benefit in some climates and during some seasons, maximizing solar heat gain can be a significant energy benefit under winter conditions. These often conflicting directives can make selection of the "best" product a challenging task. See Section 2.6.9 for a more detailed discussion of these properties of fenestration products.

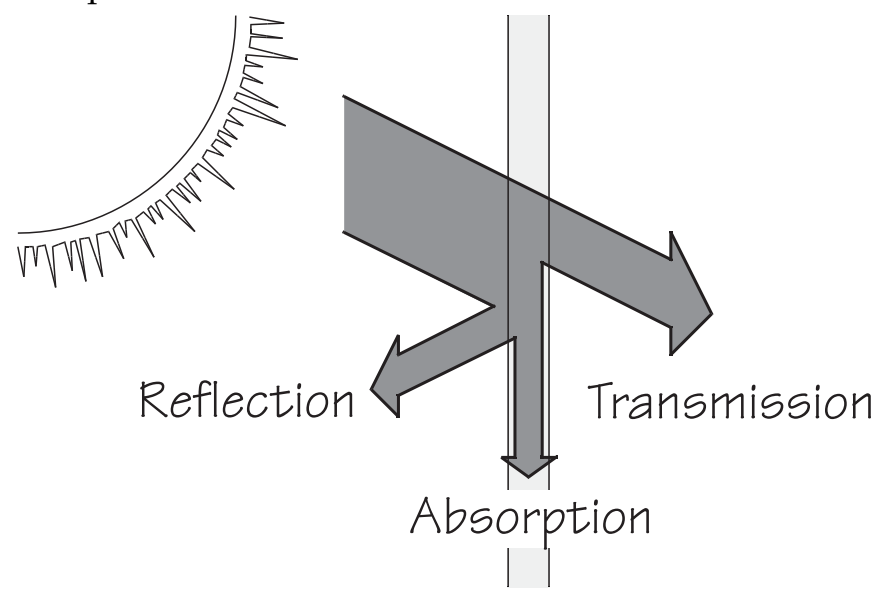

Figure 3-2. A glazing systems properties of reflection, transmission and absorption determine what happens to solar gain.

\subsubsection{Determining Solar Heat Gain}

There are two means of indicating the amount of solar radiation that passes through a fenestration product. These are solar heat gain coefficient (SHGC) and shading coefficient (SC). In both cases, the solar heat gain is the combination of directly transmitted radiation and the inward-flowing portion of absorbed radiation (Figure 3-3). However, SHGC and SC have a different basis for comparison or reference. The SHGC value is calculated for NFRC rating and certification. SHGC replaces SC because it more correctly accounts for angledependent effects. SC represents the ratio of solar heat gain through the system relative to that through3 $\mathrm{mm}$ (1/8-inch ) clear glass at normal incidence. 


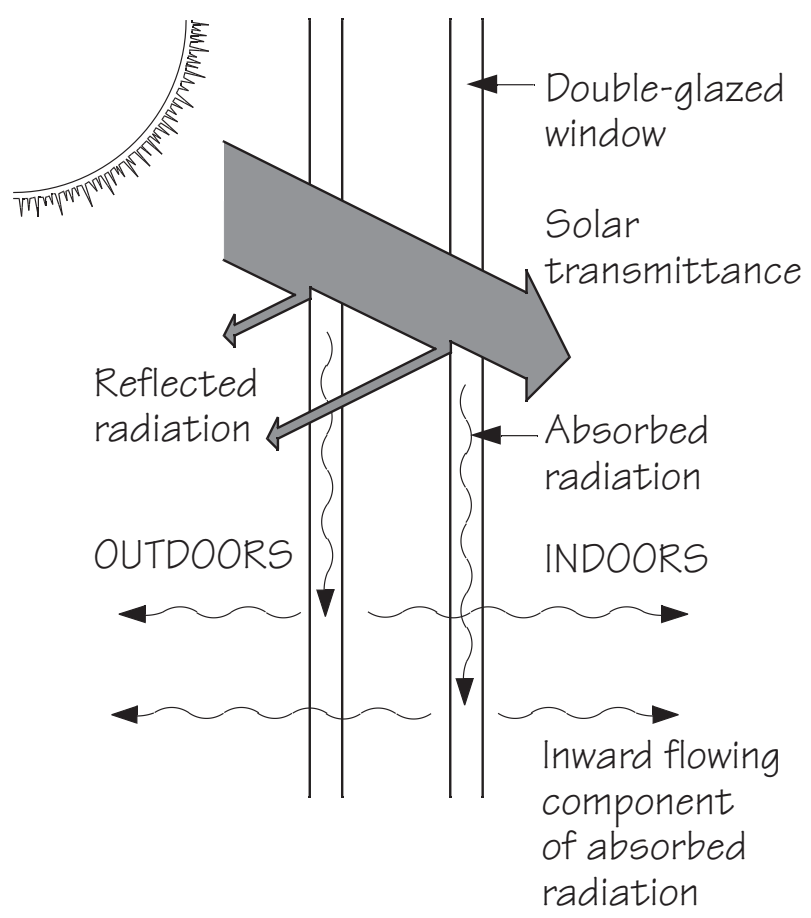

Figure 3-3. A glazing systems properties of reflection, transmission and absorption determine what happens to solar gain.

\subsubsection{Solar Heat Gain Coefficient (SHGC)}

The solar heat gain coefficient (SHGC) represents the solar heat gain through the fenestration system relative to the incident solar radiation. Although SHGC can be determined for any angle of incidence, the default and most commonly used reference is normal incidence solar radiation. NFRC rated SHGC's are at $0^{\circ}$ incidence. The SHGC refers to total fenestration product system performance and is an accurate indication of solar gain under a wide range of conditions. SHGC is expressed as a dimensionless number from 0 to 1.0. A high SHGC value signifies high heat gain, while a low value means low heat gain.

\subsubsection{Visible Transmittance}

Visible transmittance is the amount of light in the visible portion of the spectrum that passes through a glazing material. This property does not directly affect heating and cooling loads in a building, but it is an important factor in evaluating energy-efficient fenestration products. Transmittance is influenced by the glazing type, the number of layers, and any coatings that might be applied to the glazings. These effects are discussed in more detail later in this chapter in conjunction with a review of various glazing and coating technologies. Visible transmittance of glazings ranges from above 90 percent for water-white clear glass to less than 10 percent for highly reflective coatings on tinted glass.

Visible transmittance is an important factor in providing daylight, views, and privacy, as well as in controlling glare and fading of interior furnishings. These are often contradictory effects: a high light transmittance is desired for view out at night, but this may create glare at times. These opposing needs are often met by providing glazing that has high visible transmittance and then adding attachments such as shades or blinds to modulate the transmittance to meet changing needs.

NFRC reports visible transmittance as a rating on the label. Note that NFRC's rating is a whole product rating that combines the effect of both glazing and frame. There are many cases where the transmittance of glazing alone will be required, so it is important to make sure that the appropriate properties are being compared. 
In the past, products that reduced solar gain (with tints and coatings) also reduced visible transmittance. However, new spectrally selective tinted glasses and selective coatings have made it possible to reduce solar heat gain with little reduction in visible transmittance.

\subsection{Condensation Resistance}

Condensation has been a persistent and often misunderstood problem associated with windows. In cold climates, single-glazed windows characteristically suffer from water condensation and the formation of frost on the inside surface of the glass in winter. The surface temperature of the glass drops below either the dew point or frost point of the inside room air.

Excessive condensation can contribute to the growth of mold or mildew, damage painted surfaces, and eventually rot wood trim. Since the interior humidity level is a contributing factor, reducing interior humidity is an important component of controlling condensation.

Condensation can also be a problem on the interior surfaces of window frames. Metal frames, in particular, conduct heat very quickly, and will "sweat" or frost up in cool weather. Solving this condensation problem was a major motivation for the development of thermal breaks for aluminum windows.

Infiltration effects can also combine with condensation to create problems. If a path exists for warm, moisture-laden air to move through or around the window frames, the moisture will condense wherever it hits its dew point temperature, often inside the building wall. This condensation can contribute to the growth of mold in frames or wall cavities, causing health problems for some people, and it encourages the rotting or rusting of window frames. Frames must be properly sealed within the wall opening to prevent this potential problem.

Condensation can cause problems in skylights and roof windows as well as typical windows. "Leaky" skylights are frequently misdiagnosed. What are perceived to be drops of water from a leak are more often drops of water condensing on the cold skylight surfaces. A skylight is usually the first place condensation will occur indicating too much moisture in the interior air. Insulating the skylight well and providing adequate air movement assists in reducing condensation. Also, the use of more highly insulating glazing with a well-designed frame can help solve this problem. In many systems, a small "gutter" is formed into the interior frame of the skylight where condensate can collect harmlessly until it evaporates back into the room air.

The NFRC Special Publication NFRC 500 UG: User Guide to NFRC 500 contains more information about condensation resistance. 


\subsubsection{Impact of Glazing Type and Spacers on Condensation}

Figure 3-4 indicates condensation potential for four glazing types at various outdoor temperature and indoor relative humidity conditions. Condensation can occur at any points that fall on or above the curves. As the Ufactor of windows improves, there is a much smaller range of conditions where condensation will occur. Figure 3-4 must be used with caution, since it shows condensation potential for the center of glazing area only (the area at least $63.5 \mathrm{~mm}$ (2.5 inches) from the frame/glazing edge). Usually condensation will first occur at the lower edge of the product where glazing temperatures are lower than in the center.

As Figure 3-4 shows, double-glazed products create a warmer interior glazing surface than single-glazing, reducting frost and condensation. The addition of low-E coatings and argon gas fill further reduce condensation potential. The triple-glazed product with low-E coatings has such a warm interior surface that condensation on any interior surfaces may be eliminated if humidity levels are maintained at reasonable levels.

Condensation for typical glazing types occur at points in the following shaded areas on the graph.

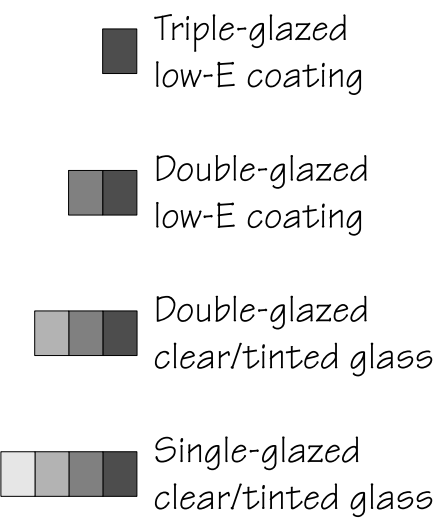

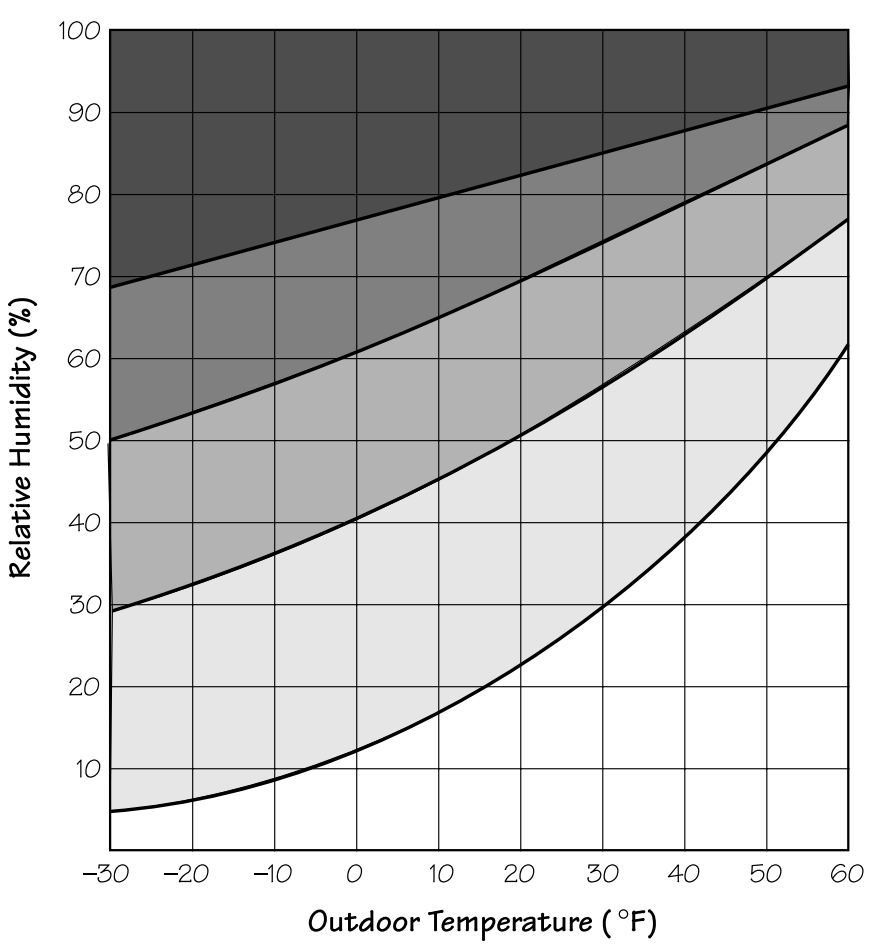

Figure 3-4. Condensation potential on glazing (center of glazing) at various outdoor temperature and indoor relative humidity conditions.

Condensation forms at the coldest locations, such as the lower corners or edges of an insulated product even when the center of glazing is above the limit for condensation. Generally, as the insulating value of the glazing is improved, the area where condensation can occur is diminished. Condensation potential increases as the outdoor temperature is lowered and the indoor relative humidity increases.

\subsubsection{Condensation Resistance}

NFRC has developed a Condensation Resistance (CR) value for rating for how well a fenestration product can resist the formation of condensation on the interior surface of the product at a specific set of environmental conditions. The CR calculation method is defined in the NFRC 500: Procedure for Determining Fenestration Product Condensation Resistance Values. 


\subsubsection{Outdoor Condensation}

Under some climate conditions, condensation may occur on the exterior glazing surface of a window. This is more likely to occur on higher-performance windows with low-E coatings or films, and low-conductance gas fills that create very low U-factors. By preventing heat from escaping from the interior, the exterior surfaces of the window approach outside air temperature. These exterior temperatures may be below the exterior dew point causing condensation on the exterior glazing surfaces. This is most likely to happen when there is a clear night sky, still air, and high relative humidity, in addition to the right temperature conditions. Like other dew formed at night, exterior window condensation will disappear as surfaces are warmed by the sun. It is the excellent thermal performance of well-insulated glazing that creates the condition where the outer glazing surface can be cold enough to cause condensation to form.

\subsubsection{Condensation Between Glazings}

A more annoying problem can arise with double-pane winodws: Condensation between the panes. Moisture can migrate into the space between the panes of glass and condense on the colder surface of the exterior pane. This condensation is annoying not only because it clouds the view and stains th e interior surfaces, but because it may mean that the glazing unit must be replaced (if it is a sealed insulating glazing unit). In a nonsealed unit, simpler remedies may correct the situation.

Factory-sealed insulated glazing utilizes a permanent seal to prevent the introduction of moisture. The void may be filled with air or dry gases, such as argon. A desiccant material in the edge spacer between the panes is used to absorb any residual moisture in the unit when it is fabricated or any small amount that might migrate into the unit over many years. These windows will fog up when moisture leaking into the air space through the seals overwhelms the ability of the dessiccant to absorb it. This could happen early in the window's life (the first few years) if there is a manufacturing defect, or many decades later because of diffusion through the sealant. Quality control in manufacture, sealant selection, window design, and even installation can influence the rate of failure. Once a sealed window unit fails, it is not generally possible to fix it, and the sealed unit must be replaced. Moisture in the unit is also likely to reduce the effectiveness of low-E coatings and suggests that gas fills may be leaking out. Most manufacturers offer a warranty against sealedglazing failure which varies from a limited perior to the lifeimte of the window.

When condensation occurs between glazings in a nonsealed unit, there are several possible remedies. Most manufacturers who offer nonsealed double glazing include a small tube connecting the air psace to the outside air, which tends to be dry during winter months.Check to be sure that the inner glazing seals tightly to the sash, and clear the air tube if it has become obstructed, Insome cases, reducing interior room humidity levels may help alleviate the problem.

\subsection{Infiltration}

Infiltration is the leakage of air through cracks in the building envelope. Infiltration leads to increased heating or cooling loads when the outdoor air entering the building needs to be heated or cooled. Fenestration products and doors are typically responsible for a significant amount of the infiltration in homes. In extreme conditions, depending on the type and quality, infiltration can be responsible for as much heat loss or gain as the rest of the product. The level of infiltration depends upon local climate conditions, particularly wind conditions and microclimates surrounding the house. Typically, U-factor and SHCG effects far outweigh infiltration effects. Tight sealing and weatherstripping of the fenestration product, including sash, and frames is of paramount importance in controlling infiltration.

High quality fixed fenestration products help to reduce infiltration because they are easier to seal and keep tight. Operable fenestration products are necessary for ventilation, but they are also more susceptible to air leakage. Operable units with low air leakage rates are characterized by good design and high-quality 
construction and weatherstripping. They also feature mechanical closures that positively clamp the product shut against the wind. For this reason, compression-seal products such as awning, hopper, and casement designs are generally more effectively weatherstripped than are sliding-seal products. Sliding products rely on wiper-type weatherstripping, which is more subject to wear over time and can be bypassed when it flexes under wind pressure.

\subsection{Solar Properties of Glazing Materials}

Three things happen to solar radiation as it passes through a glazing material. Some is transmitted, some is reflected, and the rest is absorbed. These are the three components of solar that determine many of the other energy performance properties of a glazing material, such as the solar heat gain coefficient and shading. Manipulating the proportion of transmittance, reflectance, and absorptance for different wavelengths of solar radiation has been the source of much recent innovation in fenestration energy performance.

Visible light is a small portion of the electromagnetic spectrum (see Figure 2-3). Beyond the blues and purples lie ultraviolet radiation and other higher-energy short wavelengths, from $\mathrm{X}$ rays to gamma rays. Beyond red light are the near-infrared, given off by very hot objects, the far-infrared, given off by warm roomtemperature objects, and the longer microwaves and radio waves.

Glazing types vary in their transparency to different parts of the spectrum. On the simplest level, a glass that appears to be tinted green as you look through it toward the outside will transmit more sunlight from the green portion of the visible spectrum, and reflect/absorb more of the other colors. Similarly, a bronze-tinted glass will absorb the blues and greens and transmit the warmer colors. Neutral gray tints absorb most colors equally.

This same principle applies outside the visible spectrum. Most glass is partially transparent to at least some ultraviolet radiation, while plastics are commonly more opaque to ultraviolet. Glass is opaque to far-infrared radiation but generally transparent to near-infrared. Strategic utilization of these variations has made for some very useful glazing products.

The basic properties of glazing that affect solar energy transfer are:

- Visible transmittance

- Reflectance

- Absorptance

Each is described below.

\subsubsection{Transmittance}

Transmittance refers to the percentage of radiation that can pass through glazing. Transmittance can be defined for different types of light or energy, e.g., "visible light transmittance," "UV transmittance," or "total solar energy transmittance." Each describes a different characteristic of the glazing. Visible transmittance is the total fenestration product system's transmittance across the visible portion of the solar spectrum.

Although VT can be determined for any angle of incidence, the default and most commonly used reference is normal incidence solar radiation. Transmission of visible light determines the effectiveness of a type of glass in providing daylight and a clear view through the fenestration product. For example, tinted glass has a lower visible light transmittance than clear glass.

With the recent advances in glazing technology, manufacturers can control how glazing materials behave in these different areas of the spectrum. The basic properties of the substrate material (glass or plastic) can be altered, and coatings can be added to the surfaces of the substrates. For example, a product optimized for daylighting and for reducing heat gains should transmit an adequate amount of light in the visible portion of the spectrum, while excluding unnecessary heat gain from the near-infrared part of the electromagnetic spectrum. 
On the other hand, a product optimized for collecting solar heat gain in winter should transmit the maximum amount of visible light as well as the heat from the near-infrared wavelengths in the solar spectrum, while blocking the lower-energy radiant heat in the far-infrared range that is an important heat loss component. These are the strategies of spectrally selective and low-emittance coatings, described later in the chapter.
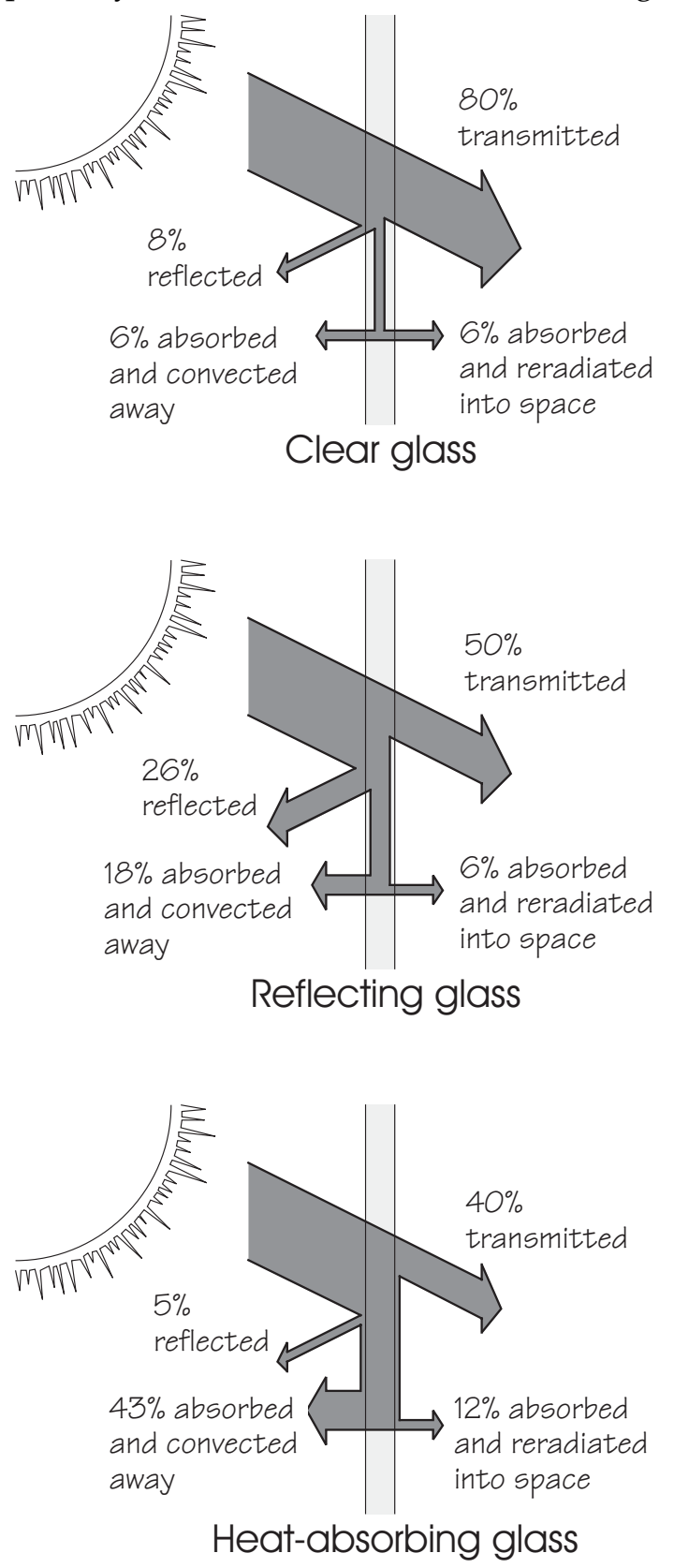

Figure 3-5. Different glass types have different characteristics for the amount of solar radiation reflected, transmitted, absorbed, and re-radiated.

\subsubsection{Reflectance}

Just as some light reflects off of the surface of water, some light will always be reflected at every glass surface. A specular reflection from a smooth glass surface is a mirror-like reflection similar to when you see an image of yourself in a store window. The natural reflectivity of glass is dependent on the quality of the glass surface, the presence of coatings, and the angle of incidence of the light. Today, virtually all glass manufactured in the 
United States is float glass and has a very similar quality with respect to reflectance. The sharper the angle at which the light strikes, however, the more the light is reflected rather than transmitted or absorbed. Even clear glass reflects 50 percent or more of the light striking it at incident angles greater than about 70 degrees. (The incident angle is formed with respect to a line perpendicular to the glass surface.)

Coatings can often be detected by careful examination of a reflected bright image, even if the coating is a transparent low-E coating. Hold a match several inches from a fenestration product at night and observe the reflections of the match in the glass. You will see two closely spaced images for each layer of glass, since the match reflects off the front and back surface of each layer of glass. A wider spacing between the two sets of pairs of images occurs with a wider air space between the glass panes. A subtle color shift in one of the reflected images normally indicates the presence of a low-E coating.

The reflectivity of various glass types becomes especially apparent during low light conditions. The surface on the brighter side acts like a mirror because the amount of light passing through the fenestration product from the darker side is less than the amount of light being reflected from the lighter side. This effect can be noticed from the outside during the day and from the inside during the night. For special applications when these surface reflections are undesirable (i.e., viewing merchandise through a store window on a bright day), special coatings can virtually eliminate this reflective effect.

The reflectivity of glass can be increased by applying various metallic coatings to the surface. Early processes used a liquid alloy of mercury and tin to create mirrors. A silvering process developed in 1865 improved the performance of mirrors. Today, mirror-like surfaces can be created by using vacuum-deposited aluminum or silver, or with a durable pyrolytic coating applied directly to the glass as it is manufactured. Thick coatings can be fully reflective and virtually opaque; a thinner coating is partially reflective and partially transmitting.

Most common coatings reflect all portions of the spectrum. However, in the past twenty years, researchers have learned a great deal about the design of coatings that can be applied to glass and plastic to reflect only selected wavelengths of radiant energy. Varying the reflectance of far-infrared and near-infrared energy has formed the basis for low-emittance coatings for cold climates, and for spectrally selective low-emittance coatings for hot climates.

\subsubsection{Absorptance}

Energy that is not transmitted through the glass or reflected off of its surfaces is absorbed. Once glass has absorbed any radiant energy, the energy is transformed into heat, raising the temperature of the glass.

Typical 1/8-inch ( $3 \mathrm{~mm}$ ) clear glass absorbs only about 4 percent of incident sunlight. The absorptance of glass is increased by adding to the glass chemicals that absorb solar energy. If they absorb visible light, the glass appears dark. If they absorb ultraviolet radiation or near-infrared, there will be little or no change in visual appearance. Clear glass absorbs very little visible light, while dark tinted glass absorbs a considerable amount. The absorbed energy is converted into heat, warming the glass. Thus, when these "heat-absorbing" glasses are in the sun, they feel much hotter to the touch than clear glass. They are generally gray, bronze, or blue-green and are used primarily to lower the solar heat gain coefficient and to control glare. Since they block some of the sun's energy, they reduce the cooling load placed on the building and its air-conditioning equipment. Absorption is not the most efficient way to reduce cooling loads, as discussed later.

\subsection{Infrared Properties of Glazing Materials (Emittance)}

When heat or light energy is absorbed by glass, it is either convected away by moving air or reradiated by the glass surface. This ability of a material to radiate energy is called its emissivity. Fenestration products, along with all other household objects, typically emit, or radiate, heat in the form of long-wave far-infrared energy. This emission of radiant heat is one of the important heat transfer pathways for a fenestration product. Thus, reducing the product's emission of heat can greatly improve its insulating properties. 
Standard glass has an emittance of 0.84 over the long wavelength portion of the spectrum, meaning that it emits 84 percent of the energy possible for an object at its temperature. It also means that for long-wave radiation (where there is no transmittance) striking the surface of the glass, 84 percent is absorbed and only 16 percent is reflected. By comparison, low-E glass coatings have an emittance as low as 0.04 . This glazing would emit only 4 percent of the energy possible at its temperature, and thus reflect 96 percent of the incident longwave infrared radiation. 



\section{SUMMARY OF ALGORITHMS}

\subsection{THERM and WINDOW Algorithms}

This section is a brief overview of the calculational algorithms found in THERM and WINDOW. Much more detailed documentation is available and is referenced in the appropriate parts of this discussion. The algorithms in both programs are based on ISO 15099, with the exceptions documented in Appendix A of this manual.

\subsubsection{WINDOW}

The WINDOW program calculates:

- Center-of-glazing properties of a glazing system

- Total product area-weighted properties (based on previously calculated center-of-glazing properties, frame, edge-of-glazing, divider, and divider-edge properties calculated in THERM)

WINDOW algorithms are documented in two publications:

- "WINDOW 5: Program Description, A PC Program for Analyzing the Thermal Performance of Fenestration Products", R. Mitchell, C. Kohler, D. Arasteh, John Carmody, C. Huizenga, Dragan Curcija, LBNL-44789 DRAFT, June 2001;

\subsubsection{THERM:}

The THERM program calculates:

- Frame and edge-of-glazing properties, the results of which are imported into WINDOW where the total product properties are calculated.

\subsection{WINDOW Computational Method}

Heat transfer across a fenestration product is a function of both the temperature difference between the inside and outside and the incident solar radiation on the product. In order to evaluate heat transfer through a specific product, its configuration and physical dimensions must be specified. This includes the glazing properties (visible, total solar and infrared optical properties, and thermal conductivity), the gap gas (air or low-conductivity gas) thermophysical properties, spacer and frame characteristics, and environmental conditions.

Fenestration product heat transfer through the center-of-glazing area is primarily a one-dimensional process. It is analyzed by breaking down the glazing system cross section into an assembly of nodes and calculating the heat transfer between each node. Under steady-state conditions, the temperatures of the nodes are such that the net energy flux entering each node is equal to that leaving each node. To perform the energy balance, WINDOW models the user-defined glazing system as a one-dimensional, steady-state resistance network, shown in Figure 4-1. An iterative solution method is then used to converge upon the correct temperature distribution. From this temperature distribution, any desired performance index can be calculated. 


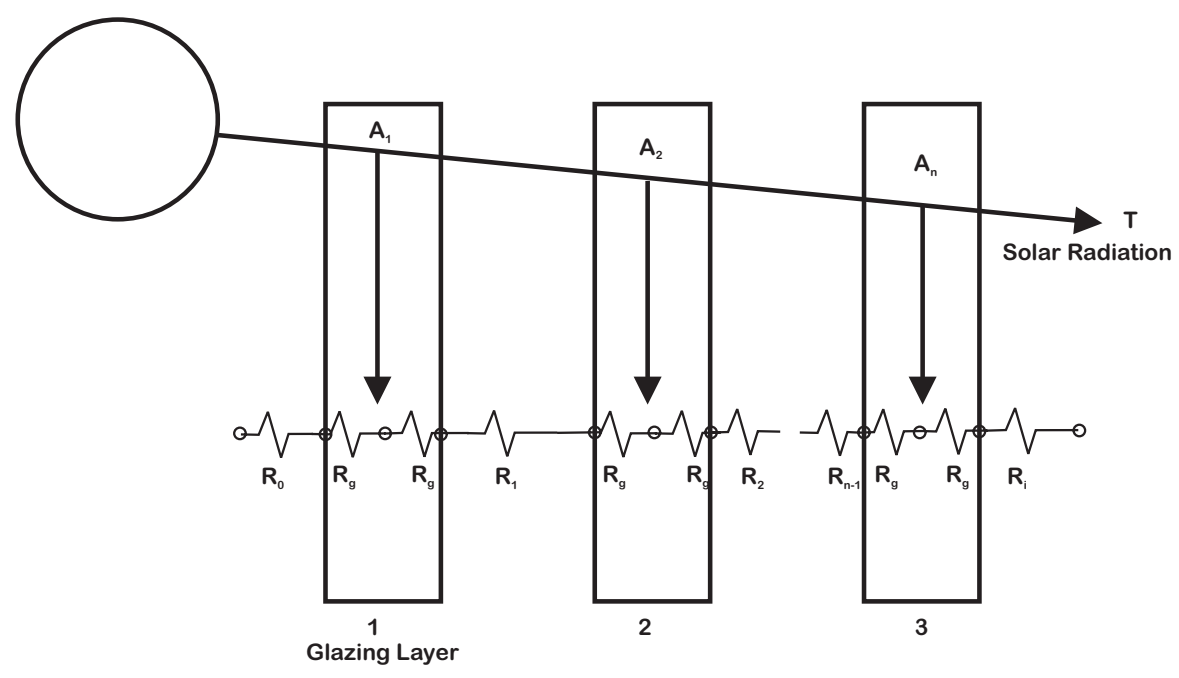

Figure 4-1. Resistance network used to model center-of-glazing heat transfer in WINDOW.

Two temperature nodes are assigned to each glazing layer (exterior and interior surface), along with outside air and inside air temperature nodes. The resistance between each node equals the inverse of the sum of the radiative and conductive/convective heat transfer coefficients. The temperature-dependent conduction/convection and effective-radiation heat transfer coefficients for the outward-facing and inwardfacing surfaces and for the gas-filled gaps are calculated from the temperature distribution. The heat transfer coefficients between the nodes within the solid materials simply depend on the conductivity of the materials.

Conductive/convective heat transfer coefficients are calculated based on empirical relationships. The outside film coefficient depends on the windspeed and the direction from which the wind is blowing. The inside film coefficient is a function of the difference between the inward-facing surface temperature, the inside temperature, and the height of the fenestration product. Gap heat transfer coefficients are computed from empirical equations for the Nusselt number. The Nusselt number relates the temperature difference between the surfaces bounding the gap, and width, height and thermophysical properties of the gap. Fenestration product tilt is also accounted for in all conductive/convective correlations.

The radiative energy flux leaving each surface is calculated from the Stephan-Boltzmann law using the surface infrared hemispherical emissivity and temperature. The net radiative flux between radiating nodes divided by the associated temperature difference gives an effective radiation heat transfer coefficient.

To accurately model glazing systems with multiple spectrally selective glazings (i.e., glazings with solaroptical properties which vary by wavelength, such as many low-emissivity coatings), a multi-band model is used in WINDOW. In this model, WINDOW calculates the transmittance and reflectance for the glazing layer or the glazing system wavelength by wavelength, and then weights the properties by the appropriate weighting functions to obtain the total solar, visible, thermal infrared properties, as well as the damageweighted transmittance and the transmittance between 0.30 and 0.38 microns. To use the multi-band model, WINDOW needs a spectral data file for each glazing layer. These data files are updated and maintained by LBNL and available from NFRC. If some of the glazing layers in a glazing system do not have a spectral data file, WINDOW assumes a flat spectral behavior of the glazings without the spectral data files, based on their stated visible and solar properties. For NFRC certification simulation, the NFRC-approved spectral data files must be used (indicated by .a \# symbol in the WINDOW Glass Library). 


\subsection{THERM Computational Methods}

Figure 4-2 shows the steps involved in a THERM analysis.

\section{Model is defined}

(See Finlayson 1998)

- Geometry is drawn

- Material properties assigned

- Boundary Conditions assigned

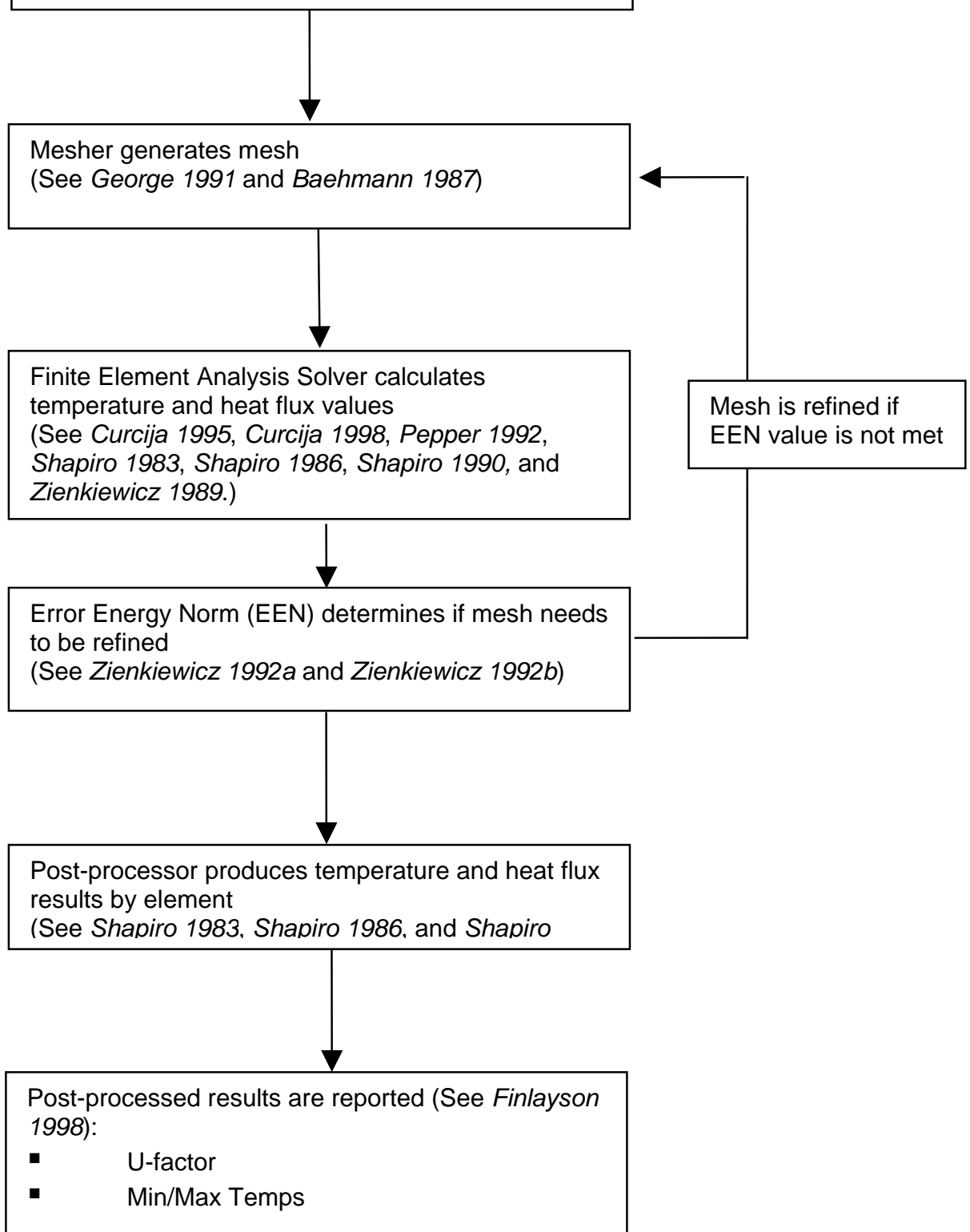

Figure 4-2. THERM program calculation procedures flow chart. 


\subsection{Total Product Calculations}

The total fenestration product properties for U-factor, SHGC and VT are based on an area-weighted average of the product's component properties which are:

- the center-of-glazing properties of the glazing system

- the frame

- edge-of-glazing

- divider

- Edge-of-divider

The frame edge and divider edge properties depend on the center-of-glazing properties of the associated glazing system.

This area-weighted total product value can be calculated using the WINDOW program, or other calculation tool such as a spreadsheet. This procedure for this area-weighted calculation is:

1. Multiply the component property by the component area

2. Sum these area-weighted component properties

3. Divide the area-weighted sum by the total projected area of the product

The operator types (fixed, vertical slider, horizontal slider, casement) determine which components (head, jamb, sill and meeting rail) are required to calculate the whole product area-weighted values.

\subsubsection{U-factor}

The whole-product area weighted U-factor calculation, shown below, is documented in Equation 4 in "NFRC 100: Procedures for Determining Fenestration Product U-factors".

$$
U_{t}=\frac{\left[\sum\left(U_{f} * A_{f}\right)+\sum\left(U_{d} * A_{d}\right)+\sum\left(U_{e} * A_{e}\right)+\sum\left(U_{d e} * A_{d e}\right)+\sum\left(U_{c} * A_{c}\right)\right]}{A_{p f}}
$$

Where:

$$
\begin{aligned}
& \mathrm{U}_{\mathrm{t}} \quad=\text { Total product U-factor, } \mathrm{W} / \mathrm{m}^{2} \mathrm{o}^{\mathrm{o}} \mathrm{K},\left(\mathrm{Btu} / \mathrm{hr}-\mathrm{ft}^{2} \mathrm{o}^{\mathrm{o}} \mathrm{F}\right) . \\
& \mathrm{A}_{\mathrm{pf}} \quad=\text { Projected fenestration product area, } \mathrm{m}^{2}\left(\mathrm{ft}^{2}\right) \text {. } \\
& \mathrm{U}_{\mathrm{f}} \quad=\text { Frame U-factor, } \mathrm{W} / \mathrm{m}^{2}-\mathrm{o} \mathrm{K},\left(\mathrm{Btu} / \mathrm{hr}-\mathrm{ft}^{2}-\mathrm{o} \mathrm{F}\right) \text {. } \\
& \mathrm{A}_{\mathrm{f}} \quad=\text { Frame area, } \mathrm{m}^{2}\left(\mathrm{ft}^{2}\right) \text {. } \\
& \mathrm{U}_{\mathrm{d}} \quad=\text { Divider U-factor, } \mathrm{W} / \mathrm{m}^{2} \mathrm{-o} \mathrm{K},\left(\mathrm{Btu} / \mathrm{hr}-\mathrm{ft}^{2}-\mathrm{o} \mathrm{F}\right) \text {. } \\
& \mathrm{A}_{\mathrm{d}} \quad=\text { Divider area, } \mathrm{m}^{2}\left(\mathrm{ft}^{2}\right) \text {. } \\
& \mathrm{U}_{\mathrm{e}} \quad=\text { Edge-of-glazing U-factor, } \mathrm{W} / \mathrm{m}^{2}{ }^{-} \mathrm{o} \mathrm{K},\left(\mathrm{Btu} / \mathrm{hr}-\mathrm{ft}^{2}-\mathrm{o} \mathrm{F}\right) \text {. } \\
& \mathrm{A}_{\mathrm{e}} \quad=\text { Edge-of-glazing area, } \mathrm{m}^{2}\left(\mathrm{ft}^{2}\right) \text {. } \\
& \mathrm{U}_{\mathrm{de}} \quad=\text { Edge-of-divider U-factor, } \mathrm{W} / \mathrm{m}^{2} \mathrm{o}^{\circ} \mathrm{K},\left(\mathrm{Btu} / \mathrm{hr}-\mathrm{ft}^{2}-\mathrm{o} \mathrm{F}\right) \text {. } \\
& \mathrm{A}_{\mathrm{de}} \quad=\text { Edge-of-divider Area, } \mathrm{m}^{2}\left(\mathrm{ft}^{2}\right) \text {. } \\
& \mathrm{U}_{\mathrm{c}} \quad=\text { Center-of-glazing U-factor, } \mathrm{W} / \mathrm{m}^{2}-\mathrm{o} \mathrm{K},\left(\mathrm{Btu} / \mathrm{hr}-\mathrm{ft}^{2}-\mathrm{o} \mathrm{F}\right) \text {. }
\end{aligned}
$$


$\mathrm{A}_{\mathrm{c}} \quad=$ Center-of-glazing area in $\mathrm{ft}^{2}\left(\mathrm{~m}^{2}\right)$.

\subsubsection{Solar Heat Gain Coefficient (SHGC)}

The total solar heat gain coefficient is determined by an area-weighted average of contributions from the transparent and the opaque elements in the fenestration product. The SHGC is a function of the solar transmittance, the solar absorptances of each layer and the inward flowing fraction of thermal energy. The SHGC is calculated for each component of the product separately. See ISO 15099 for detailed algorithm documentation

All the transparent regions (center-of-glazing, edge-of-glazing, and edge-of-divider) have the same SHGC. Once the SHGC of the opaque elements is determined the total SHGC is calculated as the area-weighted average of the SGHC through the transparent and the opaque portions of the fenestration product as shown below.

$$
S H G C_{t}=\frac{\left[\left(S H G C_{f} * A_{f}\right)+\left(S H G C_{d} * A_{d}\right)+\left(S H G C_{e} * A_{e}\right)+\left(S H G C_{d e} * A_{d e}\right)+\left(S H G C_{c} * A_{c}\right)\right]}{A_{p f}}
$$

Where:

$$
\begin{aligned}
& \mathrm{SHGC}_{\mathrm{t}} \quad=\text { Total product SHGC (dimensionless). } \\
& \mathrm{A}_{\mathrm{pf}} \quad=\text { Projected fenestration product area, } \mathrm{m}^{2}\left(\mathrm{ft}^{2}\right) \text {. } \\
& \mathrm{SHGC}_{\mathrm{f}}=\text { Frame SHGC (dimensionless). } \\
& \mathrm{A}_{\mathrm{f}} \quad=\text { Frame area in, } \mathrm{m}^{2}\left(\mathrm{ft}^{2}\right) \text {. } \\
& \mathrm{SHGC}_{\mathrm{d}} \quad=\text { Divider SHGC (dimensionless). } \\
& \mathrm{A}_{\mathrm{d}} \quad=\text { Divider area in, } \mathrm{m}^{2}\left(\mathrm{ft}^{2}\right) \text {. } \\
& \mathrm{SHGC}_{\mathrm{e}} \quad=\text { Edge-of-glazing SHGC (dimensionless). } \\
& \text { SHGC }_{\mathrm{e}} \quad=\text { Edge-of-glazing area in, } \mathrm{m}^{2}\left(\mathrm{ft}^{2}\right) \text {. } \\
& \text { SHGC }_{\mathrm{de}} \quad=\text { Edge-of-divider SHGC (dimensionless). } \\
& \mathrm{SHGC}_{\mathrm{de}} \quad=\text { Edge-of-divider Area in, } \mathrm{m}^{2}\left(\mathrm{ft}^{2}\right) \text {. } \\
& \mathrm{SHGC}_{\mathrm{c}} \quad=\text { Center-of-glazing SHGC (dimensionless). } \\
& \mathrm{A}_{\mathrm{c}} \quad=\text { Center-of-glazing area, } \mathrm{m}^{2}\left(\mathrm{ft}^{2}\right) \text {. }
\end{aligned}
$$

For NFRC rating purposes, Section 6 of NFRC 200 shall be followed to obtain SHGC values.

\subsubsection{Visible Transmittance}

The whole-product area weighted visible transmittance calculation is shown below.

$$
V T_{t}=\frac{\left[\left(V T_{f} * A_{f}\right)+\left(V T_{d} * A_{d}\right)+\left(V T_{e} * A_{e}\right)+\left(V T_{d e} * A_{d e}\right)+\left(V T_{c} * A_{c}\right)\right]}{A_{p f}}
$$

Where:

$$
\begin{array}{ll}
\mathrm{VT}_{\mathrm{t}} & =\text { Total product VT (dimensionless) } \\
\mathrm{A}_{\mathrm{pf}} & =\text { Projected fenestration product area, } \mathrm{m}^{2}\left(\mathrm{ft}^{2}\right) \\
\mathrm{VT}_{\mathrm{f}} & =\text { Frame VT (dimensionless). } \\
\mathrm{A}_{\mathrm{f}} & =\text { Frame area, } \mathrm{m}^{2}\left(\mathrm{ft}^{2}\right) . \\
\mathrm{VT}_{\mathrm{d}} & =\text { Divider VT (dimensionless). }
\end{array}
$$




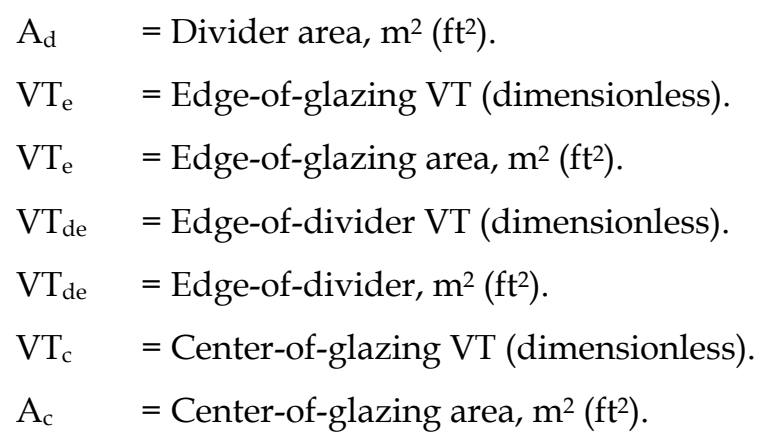

For opaque components (all known frames and dividers) the component visible transmittance $\left(\mathrm{VT}_{\mathrm{f}}, \mathrm{VT}_{\mathrm{d}}\right)$ are zero. Also note that, as defined by NFRC 200, visible transmittance $\mathrm{VT}_{\mathrm{c}}=\mathrm{VT}_{\mathrm{e}}=\mathrm{VT}_{\mathrm{de}}$

For NFRC rating purposes, Section 6 of NFRC 200 shall be followed to obtain VT values.

\subsubsection{Condensation Resistance}

The whole-product Condensation Resistance calculation is implemented in WINDOW according to NFRC 500: Procedure for Determining Fenestration Product Condensation Resistance Values. 


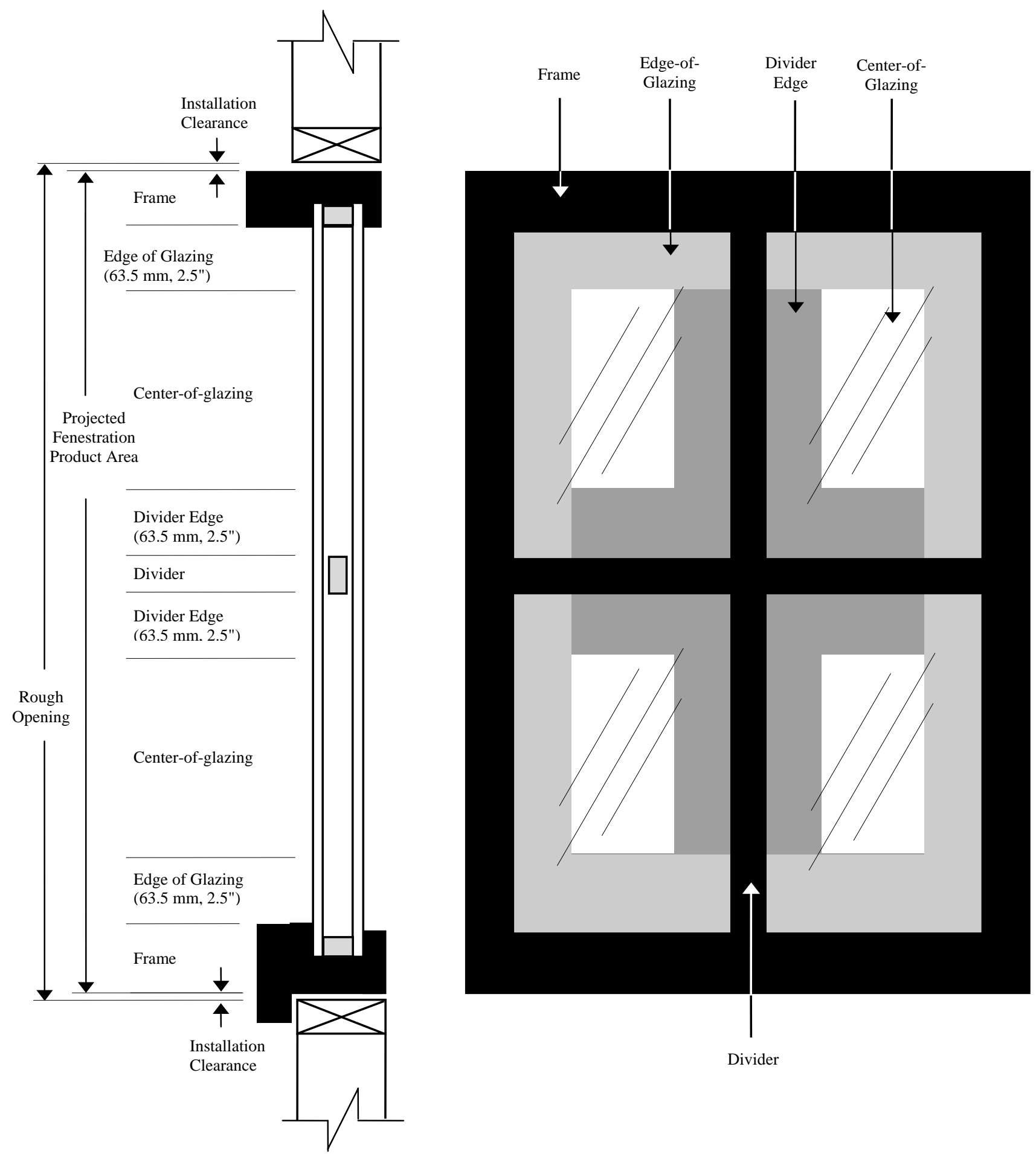

Figure 4-3. Components for the whole product area-weighted calculation. The view on the left is a section, and the view on the right is an elevation. 
The following figure is from NFRC 100 and shows in detail how each section of the product is area-weighted. The WINDOW program implements this scheme for area-weighting.

\section{Figure 1: Fenestration Product Schematic-Vertical Elevation}

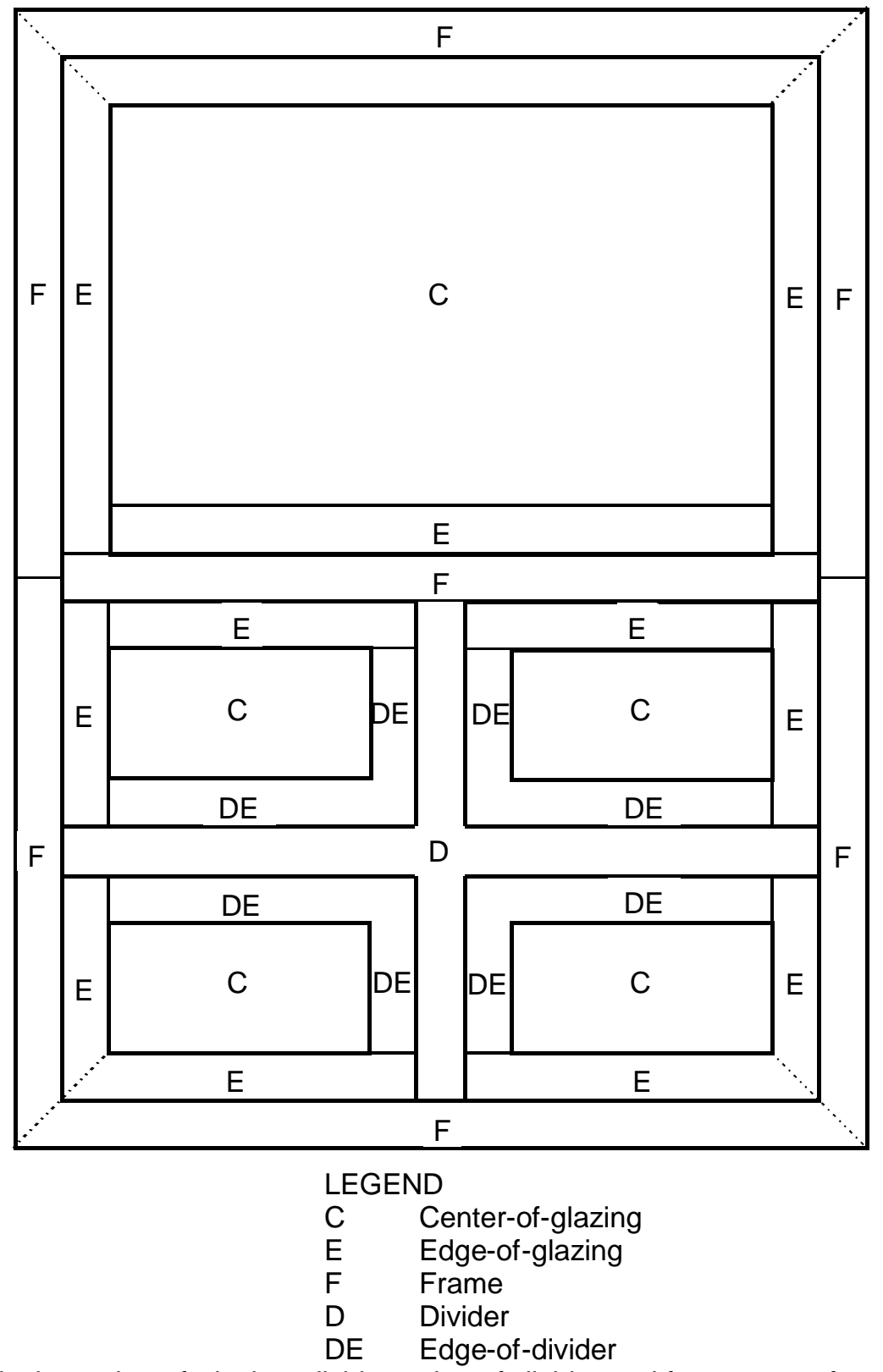

Center-of-glazing, edge-of-glazing, divider, edge-of-divider and frame areas for a typical fenestration product. Edge-of-glazing and edge-of-divider areas are $63.5 \mathrm{~mm}$ (2.5 in.) wide. The sum of these component areas equals the total projected fenestration product area.

Figure 4-4. Schematic for whole product area-weighting from the NFRC 100 document. 


\section{MODELING CENTER-OF-GLAZING WITH WINDOW}

\subsection{Overview}

The WINDOW program calculates the center-of-glazing U-factor $\left(\mathrm{U}_{\mathrm{c}}\right)$, Solar Heat Gain Coefficient $\left(\mathrm{SHGC}_{\mathrm{c}}\right)$, Visible Transmittance $\left(\mathrm{VT}_{\mathrm{c}}\right)$ and Fading Resistance $(\mathrm{FR})$ according to the following standards:

- NFRC 100: Procedure for Determining Fenestration Product Thermal Properties

- NFRC 200: Procedure for Determining Fenestration Product Solar Heat Gain Coefficients at Normal incidence

- NFRC 300: Procedure for Determining Solar Optical Properties of Simple Fenestration Product

- NFRC 500: Procedure for Determining Fenestration Product Condensation Resistance Values

- ISO 15099: Thermal Performance of Windows, Doors and Shading Devices - Detailed Calculations

The WINDOW User's Manual, WINDOW 5: Program Description, A PC Program for Analyzing the Thermal Performance of Fenestration Products contains detailed information about how to use the program, and can be used to become familiar with the program before reading this manual.

For NFRC simulations, the procedure for calculating the center-of-glazing U-factor in WINDOW is:

- Verify that the Glass Library entries are from the currently approved International Glazing Data Library associated with the Optics program (the following website contains current updates: http:// windows.lbl.gov/software and click on the Optical Data Library link)

- Create a glazing system for the product to be modeled which is composed of entries from the Glass and Gas Libraries

- This Glazing System can then be imported into THERM to calculate the frame and edge-of-glazing values

- The Glazing System is also used in WINDOW when constructing the whole product in the Window Library.

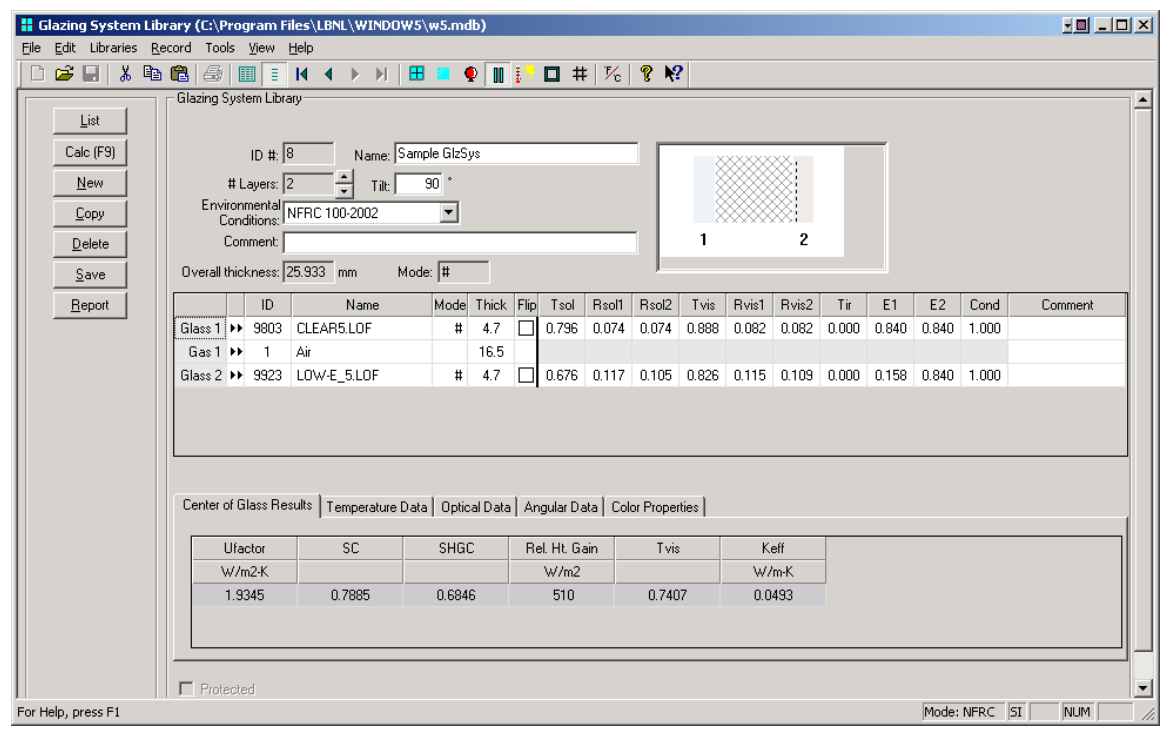

Figure 5-1. WINDOW Glazing System Library Detailed View. 


\subsection{Glass Library}

The Glass Library contains individual glass layers that can be used to construct glazing systems. For NFRC simulations, the NFRC approved glass layers from the International Glazing Database found in the Optics program shall be used. WINDOW is shipped with the currently approved International Glazing Database, but the library is updated frequently so check the website mentioned in Section 5.1 for updates. The data in the International Glazing Database

is determined according to the NFRC 300 procedure. When the NFRC approved glass data is used, a \# symbol appears in the Mode fields of both the Glass Library (shown in Figure 5-2) and the Glazing System Library (shown in Figure 5-1). All certification simulations must use the most current NFRC-approved International Glazing Database (IGDB).

The Glass Library imported from the International Glazing Database contains glass products of specific manufacturers, as well as several entries for generic uncoated products, indicated by the Manufacturer field being set to "Generic". The values for these generic entries are not measured properties from any specific glass products, but are averaged spectral data from at least two samples.

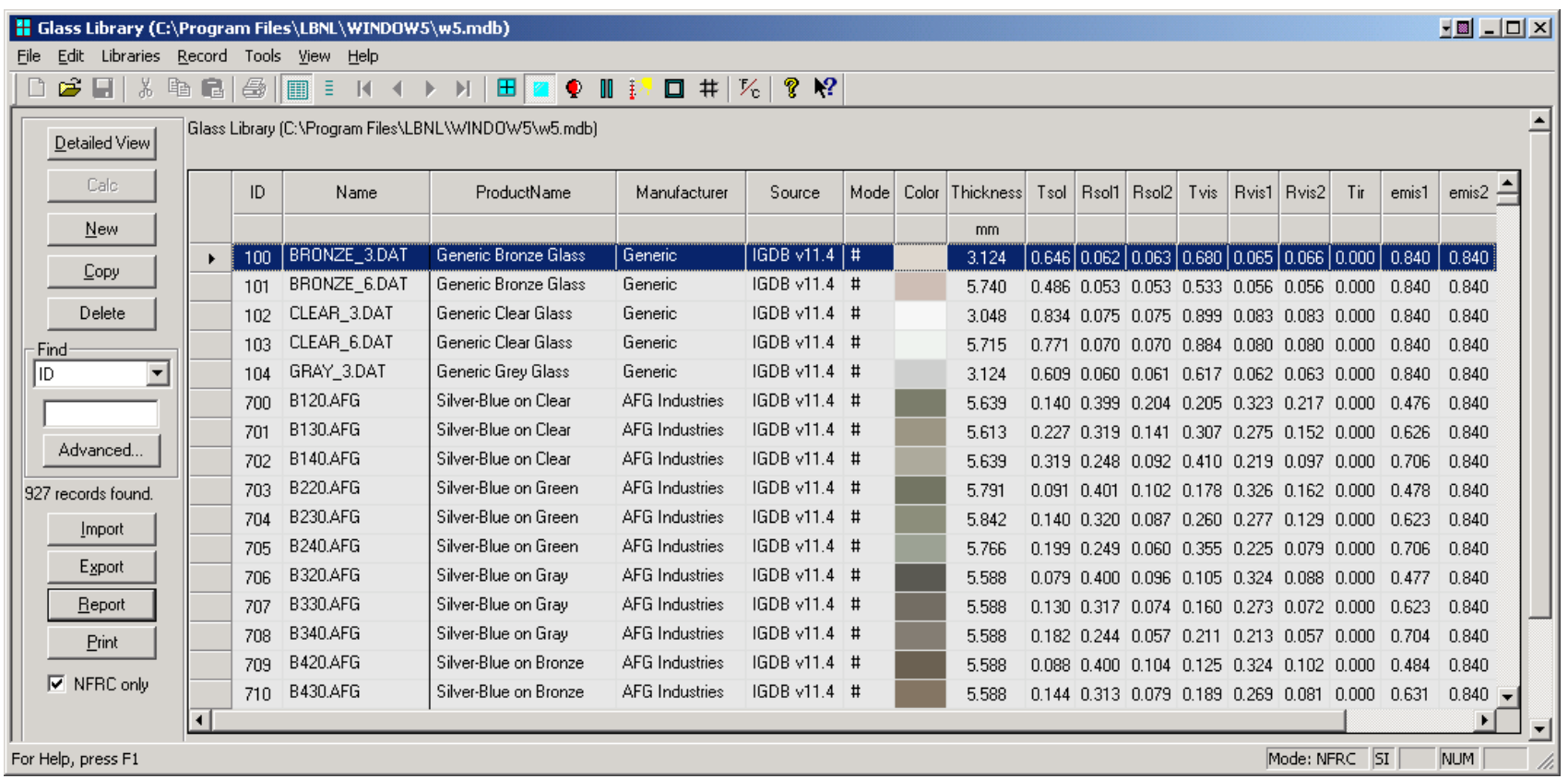

Figure 5-2. WINDOW Glass Library.

On the left hand side of the screen, of particular interest is the NFRC only checkbox; if checked, only the records with a "\#" in the Mode column will be displayed, which are the records certified for NFRC simulations.

Detailed View Click on this button to see all the information about the currently highlighted record.

The optical properties defined for each glass entry are listed below:

ID

Name

Product Name
The unique ID associated with this record. For records whose Source is "Optics", this ID is the "NFRC ID" from the International Glazing Database. For records whose Source is "User", this ID is assigned automatically by WINDOW but can be overwritten by the user as long as it is unique.

The name of the glass layer. If the record was imported from the International Glazing Database, this name will automatically come from that database.

The Product Name field from the International Glazing Database. 
Manufacturer The name of the glass manufacturer. If the record was imported from the International Glazing Database, this name will automatically come from that database.

Source Source of the glass record. Current options are:

- Optics5 v 11.4: Indicates that the data was imported from the International Glazing Database, with the database version number, in this example, 11.4. These records will have the spectral data information from the International Glazing Database.

- User: Indicates that the data was created when the user copyied an existing record into a new record. User defined records will not have associated spectral data values.

Mode

An identifier to determine if the glass layer is approved by NFRC. Only records with "\#" in this field can be used for NFRC simulations.

Color A graphic representation of the color of the glass.

Thickness Glass thickness. Units: $\mathrm{mm}$ (SI); inches (IP).

Tsol Solar transmittance of the glazing layer.

Rsol1

Rsol2

Tvis

Rvis1

Rvis2

Solar reflectance of the glazing layer, exterior-facing side.

Solar reflectance of the glazing layer, interior-facing side.

Visible transmittance of the glazing layer.

Visible reflectance of the glazing layer, exterior-facing side.

Tir

emis1

Visible reflectance of the glazing layer, interior-facing side.

emis2

Thermal infrared (longwave) transmittance of the glazing layer.

Infrared (longwave) emittance of the glazing layer, exterior-facing side

Infrared (longwave) emittance of the glazing layer, interior-facing side 


\subsection{Glazing System Library -- Center-of-Glazing U-factor}

The center-of-glazing U-factor is determined in the WINDOW Glazing System Library. A glazing system is created by specifying layers of glass from the Glass Library, as well as the gas fill material between the layers, such as air or argon. Detailed instructions about creating a glazing system can be found in the WINDOW 5 User's Manual. A sample glazing system library entry is shown in Figure 5-3.

When defining a glazing system, the number of glass layers (\# Lay), the Tilt, and the Environmental Conditions (Env Cond) must be specified. For NFRC certification calculations the Environmental Conditions have restricted values, as discussed in the next section. Also, the glass layers must be from the Glass Library using approved records from the International Glazing Database, indicated by a \# value in the Mode field of the glass layer record, as shown in Figure 5-3.

Note: to see the U-factor value to four decimal places, click on File/Preferences menu choice and in the Calculation Options tab, set the Display Precision field to" 4 "

For NFRC certification calculations, the NFRC 100-2001 Environmental Conditions choice must be used.

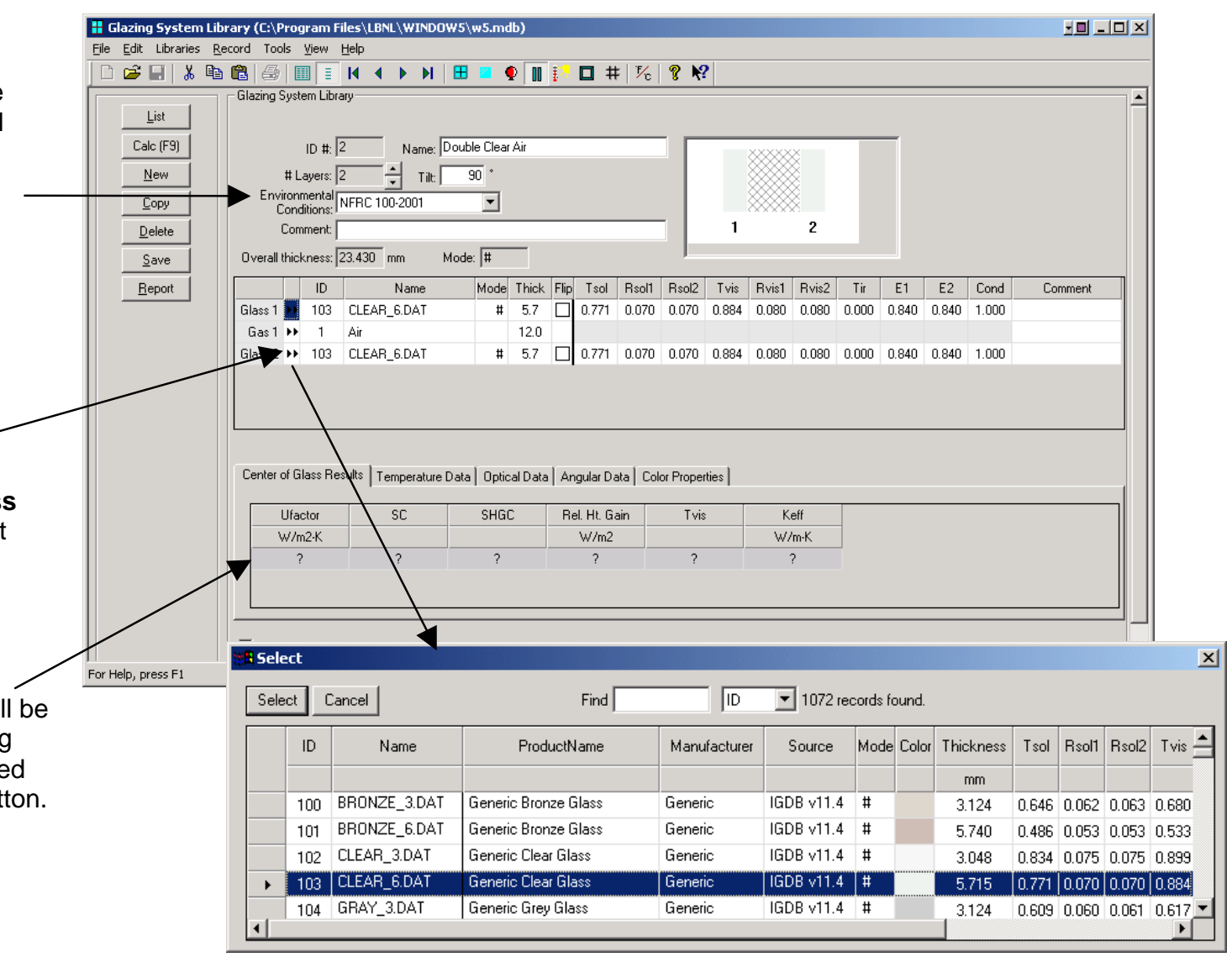

Click on the double arrow to access the Glass Library to select a layer.

Figure 5-3. Selecting glass layers in the Glazing Systems Library. 


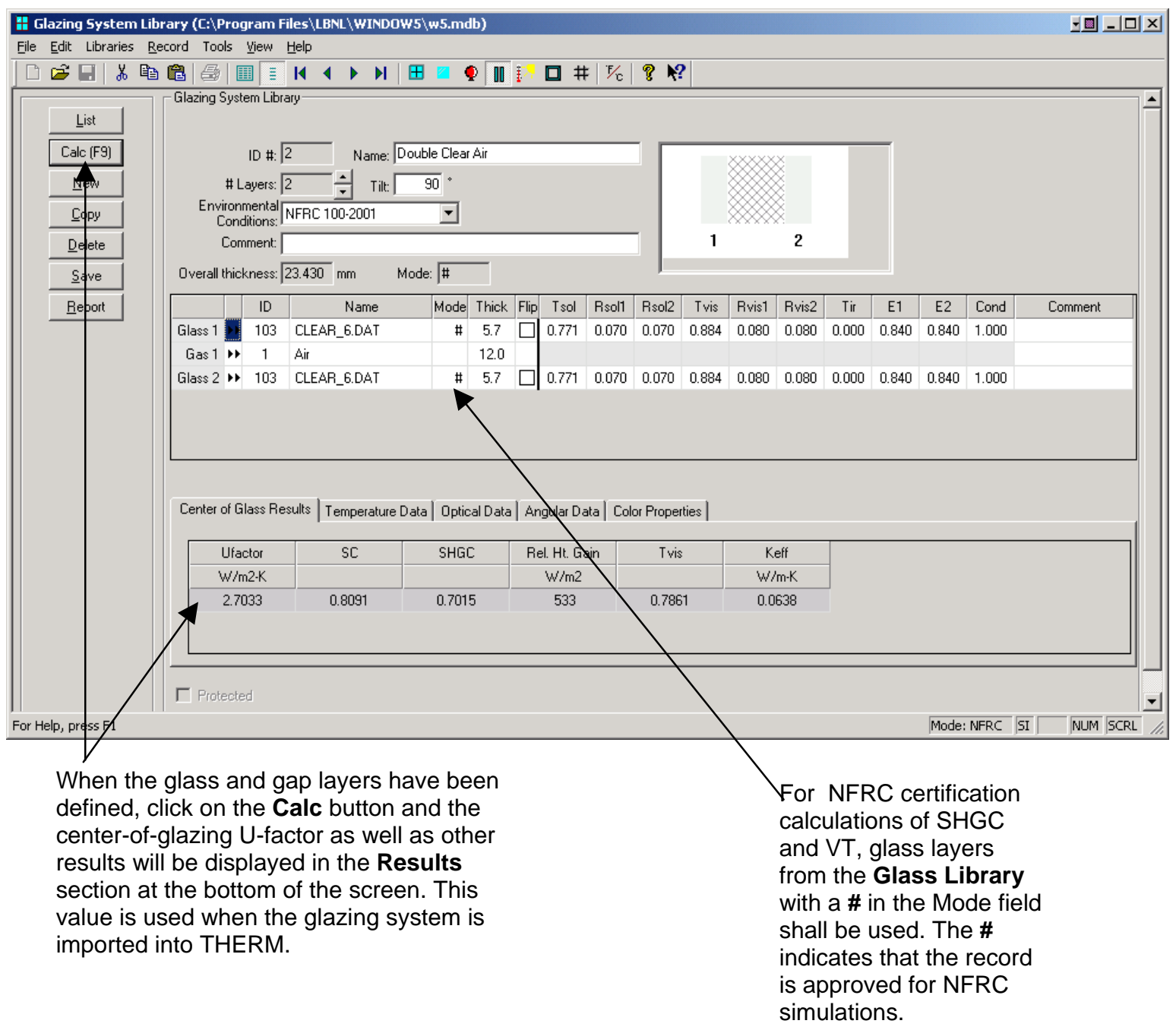

Figure 5-4. Calculating results in the Glazing Systems Library.

The Center of Glass Results tab at the bottom of the screen shows the results for the glazing system. The Ufactor results are based on a default glazing system height of one meter. This center-of-glazing U-factor value will be recalculated in the Window Library to reflect the true height of the product being modeled. (See Section 7, "Total Product Calculations", for more information). 
All the Glazing System Library records can be seen in the List View, access by clicking the List button from the Glazing System Library Detailed View.

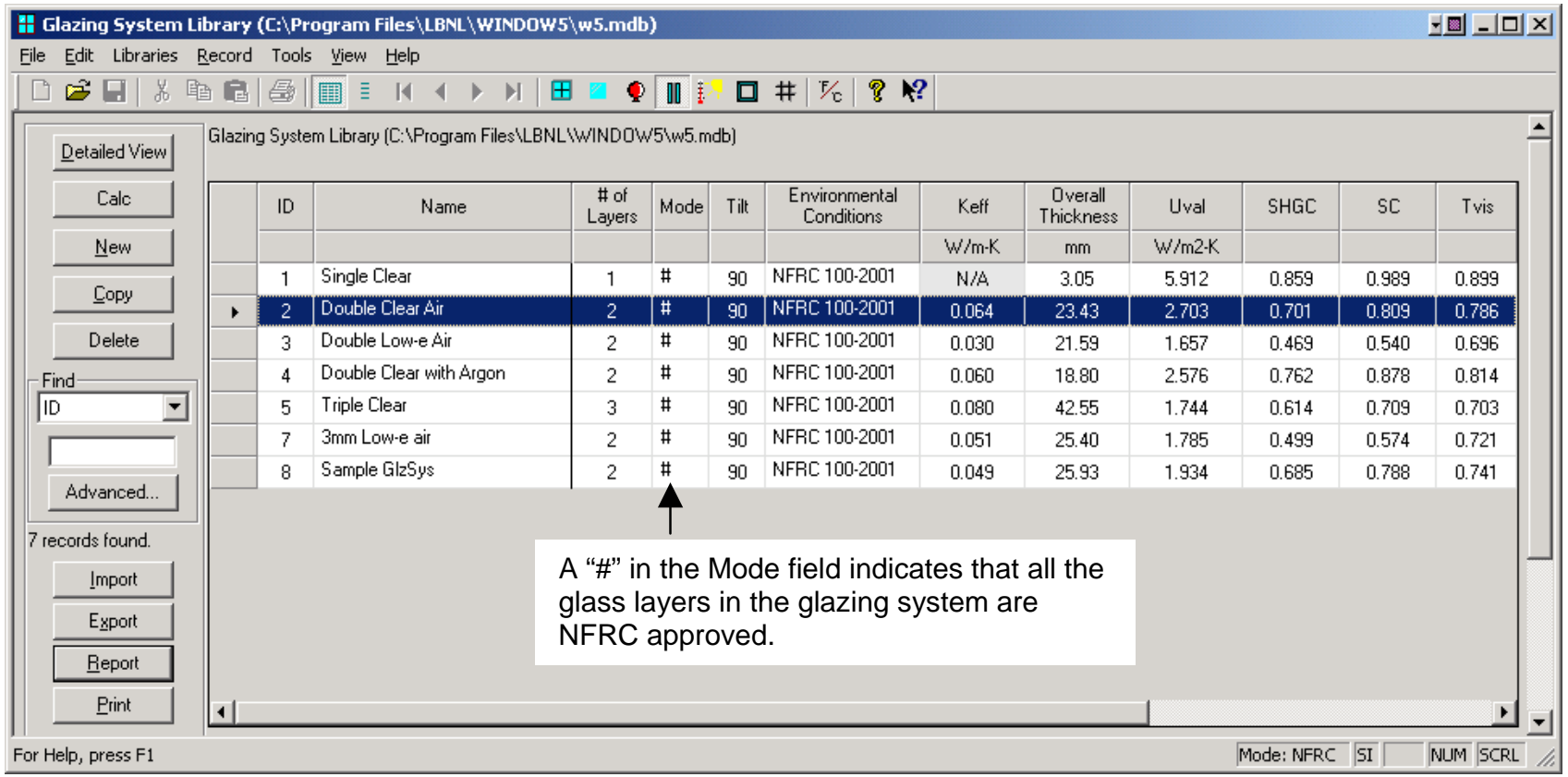

Figure 5-5. The List View of the Glazing System Library shows all the glazing systems. 


\subsubsection{Environmental Conditions}

When defining the glazing system for use in an NFRC certified simulation, the NFRC 100-2001 choice must be used for the Environmental Conditions (Env Cnd) parameter, which contains the parameters defined by NFRC 100. Figure 5-4 shows the NFRC 100-2001 choice in the WINDOW Environmental Conditions Library. Table 5-1 lists the values for the U-factor calculation and Table 5-2 lists the values for the Solar Heat Gain Coefficient calculation.

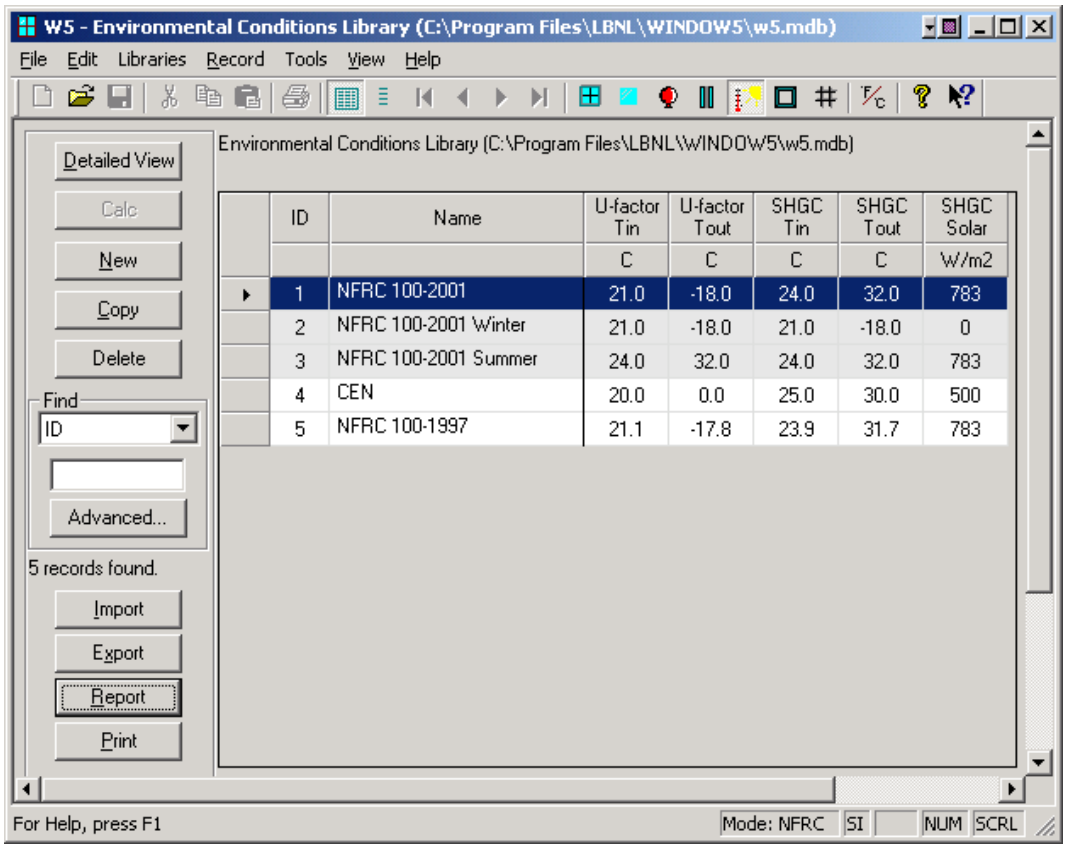

Figure 5-6. WINDOW Environmental Conditions Library List View.

Use the NFRC 100-2001 Environmental Conditions Library in WINDOW for NFRC center-of-glazing simulations

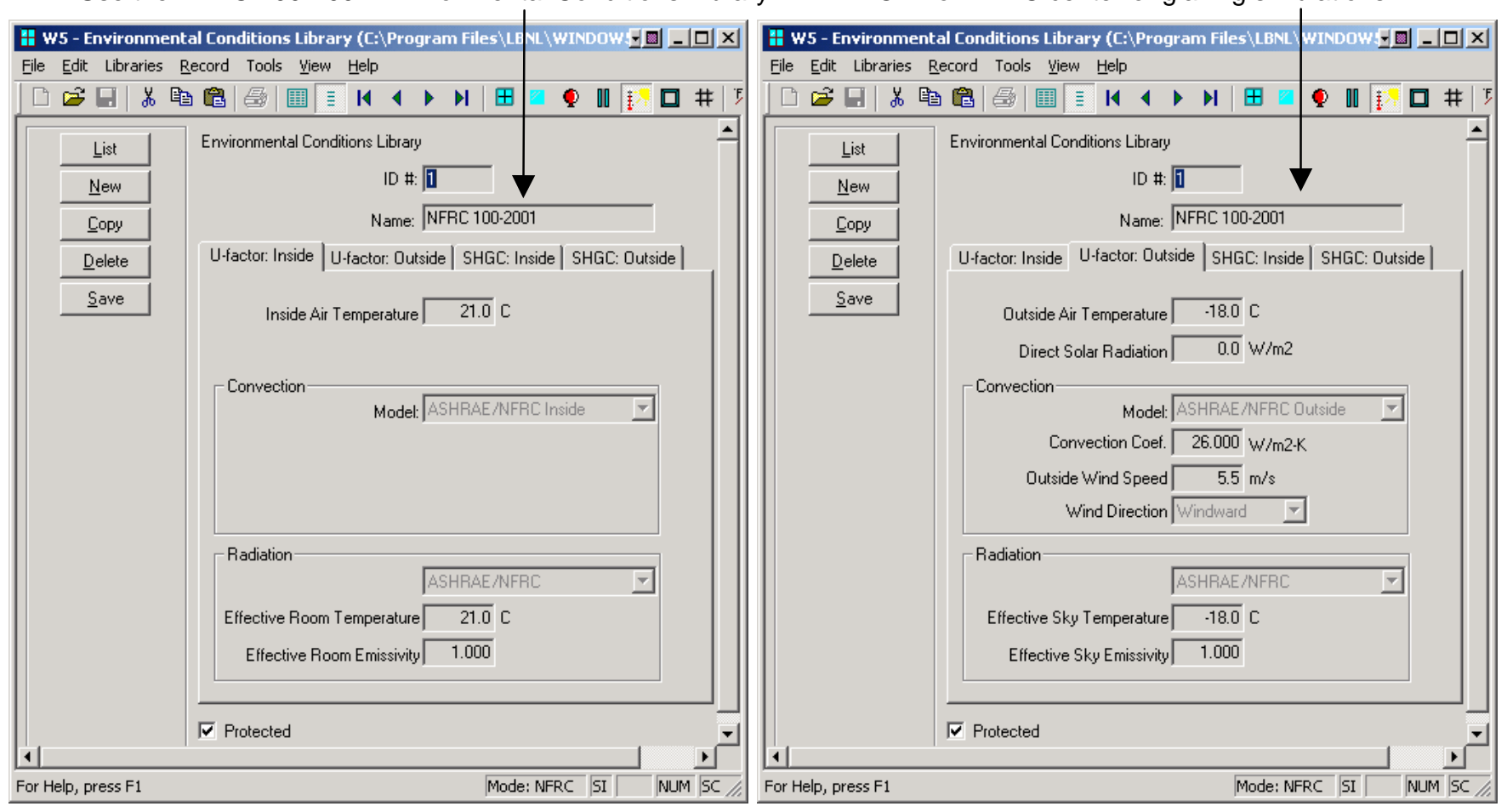

Figure 5-7. WINDOW Environmental Conditions Library Detailed View of U-factor settings. 
Table 5-1. Environmental Conditions for NFRC Simulations for U-factor calculations.

\begin{tabular}{|l|l|l|}
\hline Variable & SI Units & IP Units (reference only) \\
\hline Outside Temperature & $-18^{\circ} \mathrm{C}$ & $0 \circ \mathrm{F}$ \\
\hline Inside Temperature & $21^{\circ} \mathrm{C}$ & $70^{\circ} \mathrm{F}$ \\
\hline Wind Speed & $5.5 \mathrm{~m} / \mathrm{s}$ & $12.3 \mathrm{mph}$ \\
\hline Wind Direction & Windward & Windward \\
\hline Direct Solar & $0 \mathrm{~W} / \mathrm{m}^{2}$ & $0 \mathrm{Btu} / \mathrm{hr}^{-\mathrm{ft}^{2}}$ \\
\hline Sky Temperature (Tsky) & $-18^{\circ} \mathrm{C}$ & $0 \circ \mathrm{F}$ \\
\hline Sky Emissivity (Esky) & 1.00 & 1.00 \\
\hline
\end{tabular}

Use the NFRC 100-2001 Environmental Conditions Library in WINDOW for NFRC center-of-glazing simulations

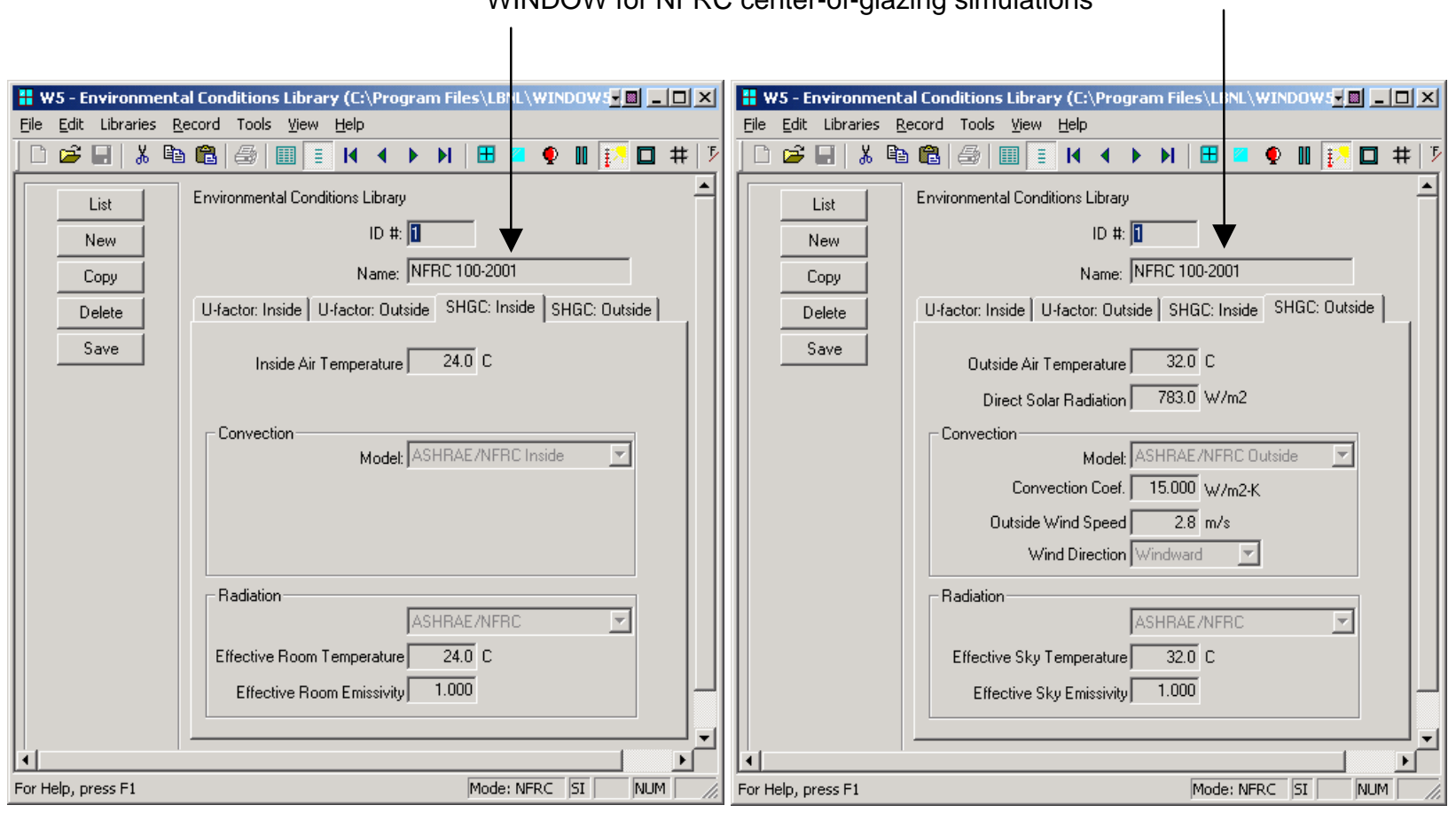

Figure 5-8. WINDOW Environmental Conditions Library - Settings for SHGC.

Table 5-2. Environmental Conditions for NFRC Simulations for SHGC and VT calculations.

\begin{tabular}{|l|l|l|}
\hline Variable & SI Units & IP Units \\
\hline Outside Temperature & $32^{\circ} \mathrm{C}$ & $89^{\circ} \mathrm{F}$ \\
\hline Inside Temperature & $24^{\circ} \mathrm{C}$ & $75^{\circ} \mathrm{F}$ \\
\hline Wind Speed & $2.75 \mathrm{~m} / \mathrm{s}$ & $6.15 \mathrm{mph}$ \\
\hline Wind Direction & Windward & Windward \\
\hline Direct Solar & $783 \mathrm{~W} / \mathrm{m}^{2}$ & $248.2 \mathrm{Btu} / \mathrm{hr}^{-\mathrm{ft}^{2}}$ \\
\hline Sky Temperature (Tsky) & $32^{\circ} \mathrm{C}$ & $89{ }^{\circ} \mathrm{F}$ \\
\hline Sky Emissivity (Esky) & 1.00 & 1.00 \\
\hline
\end{tabular}


It is possible to make new environmental conditions with specific conditions specified, in order to evaluate the design of a product. However, only the pre-defined NFRC 100-2001 shall be used for NFRC rating purposes.

\subsubsection{Coatings}

The location of coatings on a glass layer can affect the center-of-glazing U-factor and therefore the whole product calculation, so it is important to specify the location correctly. When using a glass entry from the WINDOW Glass Library, if the coating is not on the correct surface, the glass layer shall be flipped. To flip a glass layer, while on a glass layer in Edit mode, click on the Flip checkbox, and the glass surfaces will be flipped, as indicated by the dashed line in the graphic display of the glazing system.

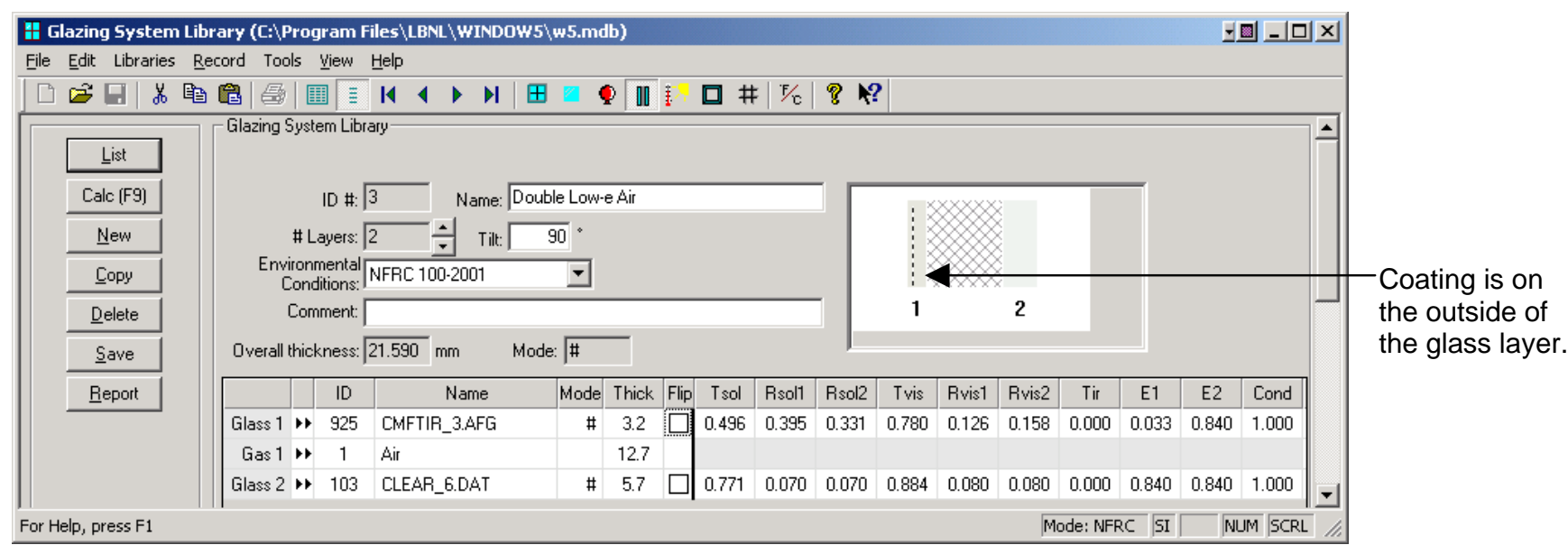

Click on the Flip checkbox and the glass

layer will be flipped so that the coating is on the inside of the glass layer.

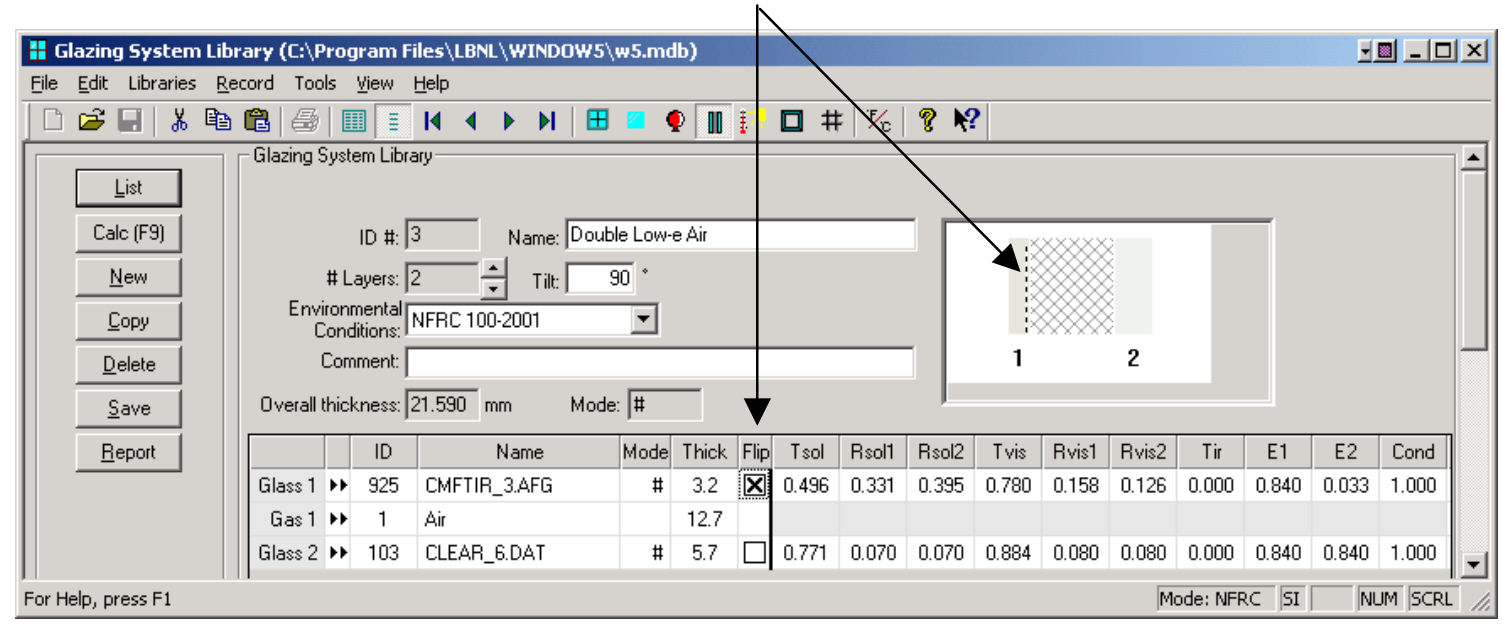

Figure 5-9. Use the Flip checkbox to flip the coatings on a glass layer. 


\subsubsection{Gas Fill}

The gas used to fill the gap between the glass layers makes a significant contribution to the center-of-glazing U-factor, although it does not have much affect on the Solar Heat Gain Coefficient. For NFRC certified simulations, the gas to be shipped in the fenestration product shall be modeled. When creating a glazing system in WINDOW, choose the appropriate gas fill from the Gas Library, which contains the maximum gas fills that can be used, as shown in the figure below. Custom gas mixtures can be defined in the Gas Library. See the WINDOW User's Manual for more details about making a new gas mixture in the Gas Library.

When a gas is used to fill the gap between glass layers, there is always a mixture of the gas and air. The amount of air mixed in is dependent on many factors including the method used to fill the gap, either evacuated chamber filling, two-probe filling with a concentration sensor, or single-probe timed filling. Table 5-3 shows the maximum gas concentrations that can be achieved with each method. For NFRC certification simulations, the simulator shall request the gas-filling technique and the gas concentration for their product from the manufacturer.

Table 5-3. Gas Concentrations based on Filling Technique.

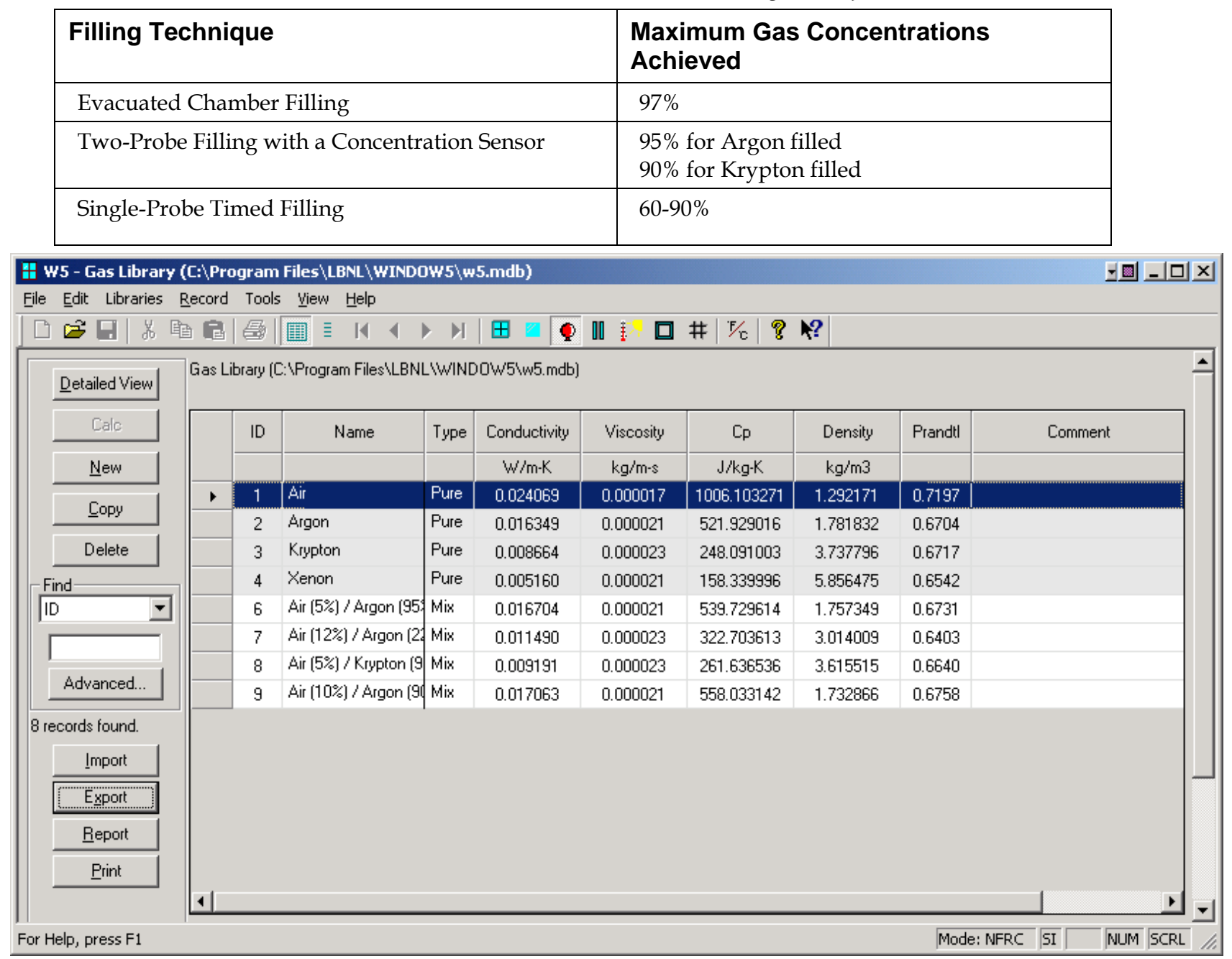

Figure 5-10. WINDOW Gas Library. 


\subsubsection{Laminated Glass / Applied Films}

To model laminated glass or glass with applied films in WINDOW, use NFRC approved spectral data to create a record in the WINDOW Glass Library which can then be used as a glazing layer in the Glazing System Library.

\subsection{Solar Heat Gain Coefficient and Visible Transmittance}

The document NFRC 200: Procedure for Determining Fenestration Product Solar heat Gain Coefficients at Normal Incidence contains the rules and definitions for calculating the Solar Heat Gain Coefficient and Visible

Transmittance for products. Consult NFRC 200 to determine how to group products for these calculations, as well as algorithm documentation.

In WINDOW, the center-of-glazing Solar Heat Gain Coefficient (SHGC) and Visible Transmittance (VT) are automatically calculated in the Glazing System Library in the Center of Glass Results tab, as shown in the figure below.

In addition, these values are calculated for the whole product and for the NFRC SHGC 0 and 1 and VT 0 and 1 cases in the Window Library, as explained in Chapter 7 of this manual.

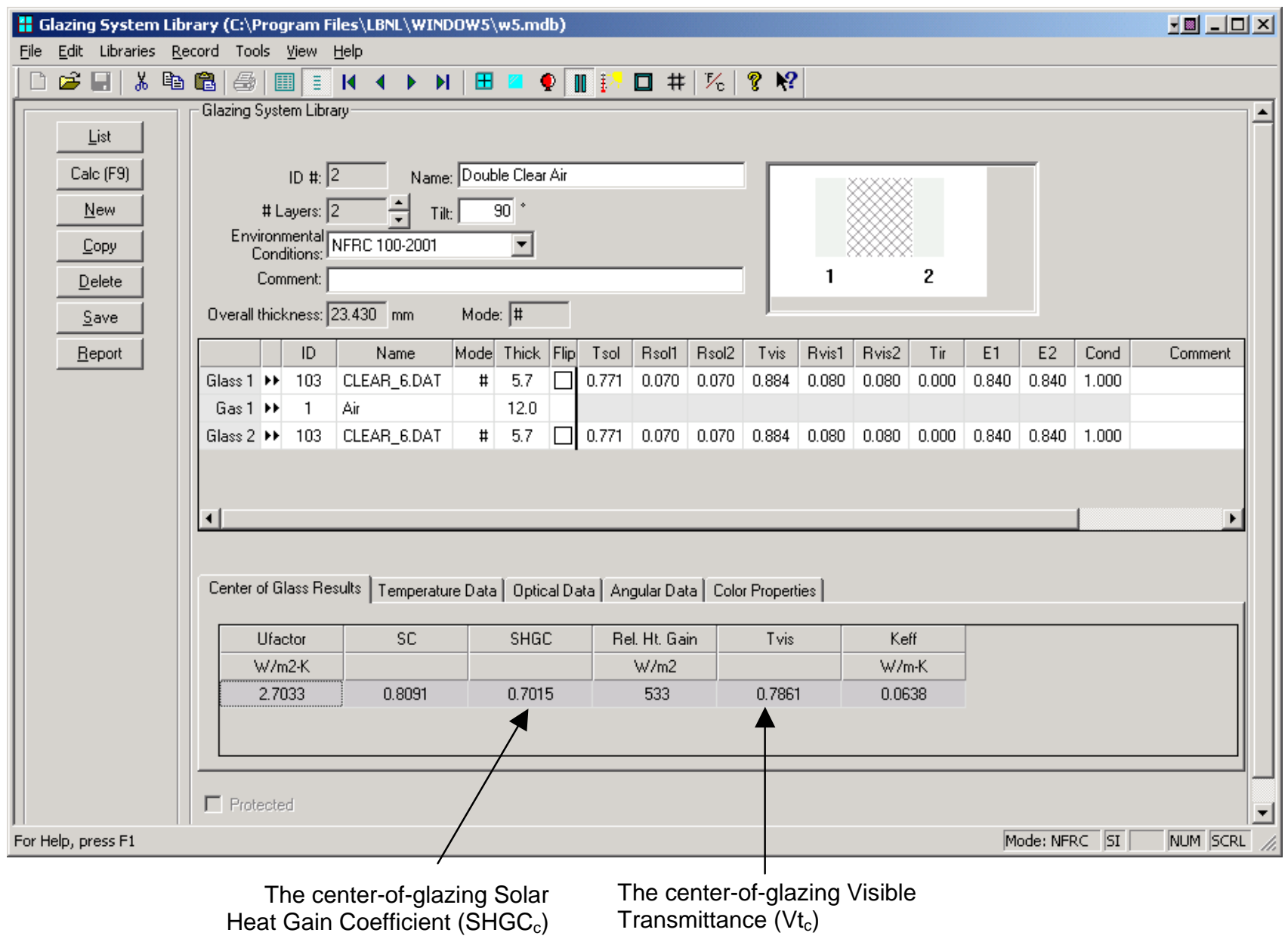

Figure 5-11. The center-of-glazing SHGC, VT and Fading Resistance are calculated in the WINDOW Glazing System Library. 



\section{MODELING FRAME AND EDGE HEAT TRANSFER WITH THERM}

\subsection{Overview}

In order to calculate the total product U-factor, the U-factor must be determined for the following components, and then these values must be area-weighted for the whole product:

- Center-of-glazing values from WINDOW

- Frame and edge-of-glazing values from THERM

The NFRC 100: Procedures for Determining Fenestration Product $U$-factors is the definitive source for the methodology for calculating U-factors, and should be used for situations not covered in this manual. In addition, NFRC Technical Interpretations supplement the NFRC 100 document, and these should also be consulted when questions arise.

\subsection{Cross-Sections to be Modeled}

The fenestration product $b$ eing modeled must be divided into the cross-sections which completely define the heat transfer through the product. THERM is used to calculate the U-factor values for the edge-of-glazing as well as the frame components (sill, head, jamb, meeting rail, and divider).

The operator type of the fenestration product, as well as the configuration of each component of the frame, will determine the number of cross sections that must be modeled. Table 6-1 lists the minimum and maximum cross sections that must be modeled for each operator type, and Figure 6-1 illustrates where the cross sections must be taken. The maximum assumes that each frame cross section is unique and therefore must be modeled. (Note that significant operating hardware may require more cross sections to be simulated. See Section 6.3.3, "What is Not Modeled"). If there are some cross sections of the frame which are identical, such as a fixed window with the same cross section for the jambs, then only one cross section needs to be modeled for the jamb. However, the new modeling assumptions for cavities when modeling Condensation Resistance, based on ISO 15099, require that different cross sections be modeled for head, sill, and jambs if those cross sections contain frame cavities. If only U-factor is being modeled, the head and the sill can be combined into one calculation if the geometries for each are the same.

Although all the Product types listed in Table 1 of NFRC 100 are not listed here in Table 6-1 or Figure 6-1, these four basic categories cover all the geometries in NFRC 100. 
Table 6-1. Cross sections to be modeled

\begin{tabular}{|c|c|c|c|c|}
\hline \multirow{2}{*}{$\begin{array}{l}\text { Operator } \\
\text { Type }\end{array}$} & \multicolumn{3}{|c|}{ Number of Cross Sections to Model } & \multirow[b]{2}{*}{ Cross Sections } \\
\hline & \multicolumn{2}{|c|}{ Minimum } & \multirow{3}{*}{ Maximum } & \\
\hline Single Lite & CR \& U Calc & U only Calc & & \multirow{2}{*}{$\begin{array}{ll} & \text { Head } \\
\quad & \text { Jamb (1 or 2) }\end{array}$} \\
\hline $\begin{array}{l}\text { (Fixed, } \\
\text { Picture, } \\
\text { Transom, } \\
\text { Casement, } \\
\text { Awning, etc) }\end{array}$ & $\begin{array}{l}3 \\
\text { If Jambs are the same }\end{array}$ & $\begin{array}{l}2 \\
\text { If Head and Sill are the } \\
\text { same }\end{array}$ & & \\
\hline $\begin{array}{l}\text { Two Lite } \\
\text { Vertical } \\
\text { (Vertical } \\
\text { Slider) }\end{array}$ & $\begin{array}{l}5 \\
\text { If Left and Right } \\
\text { Upper Jambs are the } \\
\text { same, and Left and } \\
\text { Right Lower Jambs } \\
\text { are the same }\end{array}$ & $\begin{array}{l}4 \\
\text { If Head and Sill are the } \\
\text { same }\end{array}$ & 7 & $\begin{array}{ll}\text { - } & \text { Head Fixed or Vent } \\
\text { - } & \text { Jamb Fixed \& Vent } \\
\text { - } & \text { Sill Fixed \& Vent } \\
\text { - } & \text { Meeting Rail }\end{array}$ \\
\hline $\begin{array}{l}\text { Two Lite } \\
\text { Horizontal } \\
\text { (Horizontal } \\
\text { Slider, } \\
\text { Sliding Patio } \\
\text { Door) } \\
\end{array}$ & 7 & $\begin{array}{l}5 \\
\text { If Head and Sill are the } \\
\text { same }\end{array}$ & 7 & $\begin{array}{ll}\text { - } & \text { Head Fixed \& Vent } \\
\text { - } & \text { Jamb Fixed \& Vent } \\
\text { - } & \text { Sill Fixed \& Vent } \\
\text { - } & \text { Meeting Rail }\end{array}$ \\
\hline $\begin{array}{l}\text { Two-lite } \\
\text { casement, } \\
\text { (Window } \\
\text { Wall, Curtain } \\
\text { Wall) }\end{array}$ & $\begin{array}{l}4 \\
\text { If Left and Right } \\
\text { Head are the same, } \\
\text { Left and Right Sills } \\
\text { are the same, and } \\
\text { Left and Right Jambs } \\
\text { are the same. }\end{array}$ & 4 & 7 & $\begin{array}{ll}\text { - } & \text { Head Fixed \& Vent } \\
\text { - } & \text { Jamb Fixed \& Vent } \\
\text { - } & \text { Sill Fixed \& Vent } \\
\text { - } & \text { Meeting Rail }\end{array}$ \\
\hline
\end{tabular}




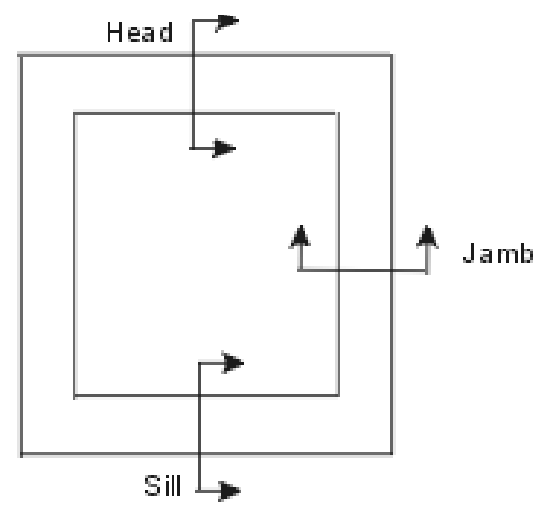

Fixed

(Max. 4, Min 3 cross sections)

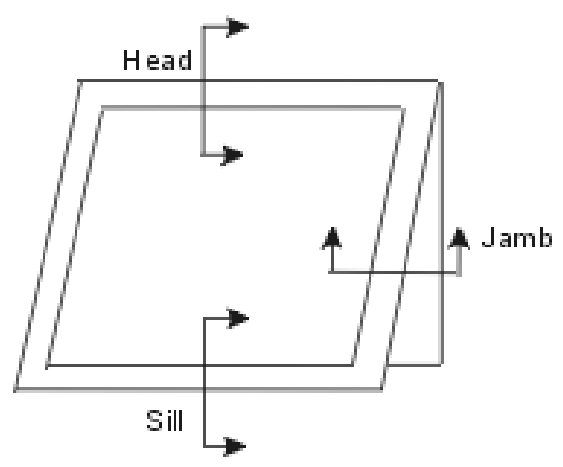

Projecting/Awning

(Max. 4, Min 3 cross sections)

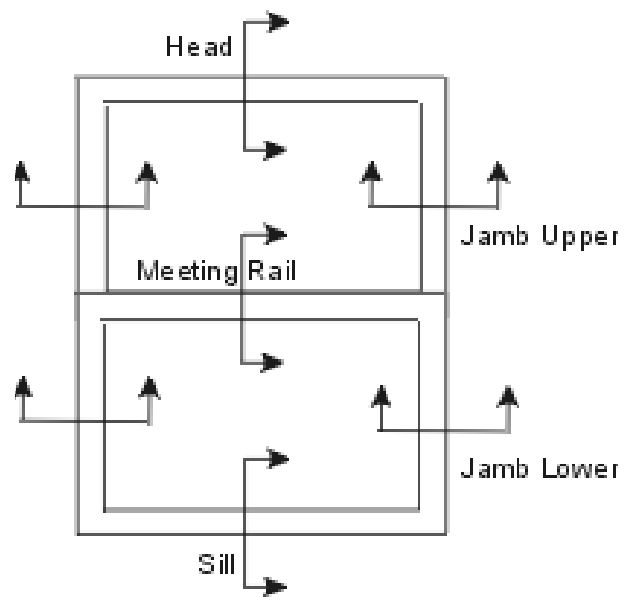

Vertical Slider

(Max. 7, Min 5 cross sections)

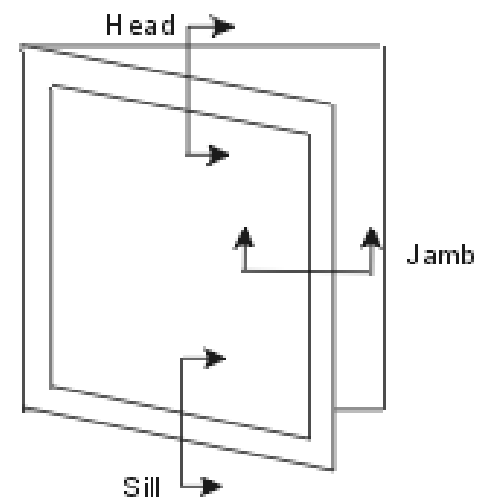

Casement

(Max. 4, Min 3 cross sections)

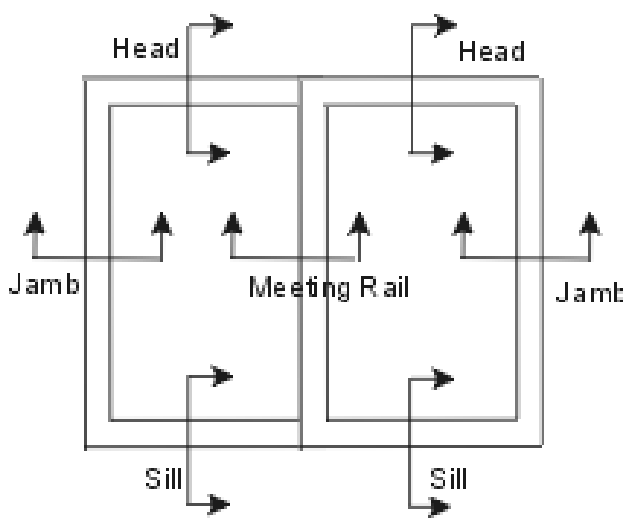

Horizontal Slider (Max. 7, Min 5 cross sections)

Figure 6-1. Minimum and maximum cross sections to be modeled based on operator type. 


\subsection{Draw the Cross Sections}

After the number of cross sections to be modeled has been determined, draw them in THERM. The Section 5, "Drawing Cross-Section Geometry" in the THERM User's Manual contains detailed instructions about how to draw cross sections. This section of the THERM NFRC Simulation Manual contains information pertaining specifically to the NFRC procedures.

\subsubsection{Getting Started - Drawings and DXF Files}

In order to reproduce accurate cross sections in THERM, it is necessary to use dimensioned drawings and/or DXF files. Section 5.2, "Importing a DXF or Bitmap File as an Underlay" in the THERM User's Manual.

If only dimensioned drawings are available (assembly drawings and die drawings), and there are no DXF files, it is still possible to create a cross-section in THERM. Section 5.5, "Drawing Using the Keyboard (Numeric Cursor Positioning)" of the THERM User's Manual.

\subsubsection{File Preferences -- Cross-Section Type}

It is extremely important to specify the Cross Section Type in the THERM File Properties dialog box (accessed from the File/Properties menu). The Cross Section Type, in combination with the glazing system orientation, will determine how many of the parameters in the the ISO 15099 simulation models are implemented, such as the frame cavity gravity vector. This setting will appear in the WINDOW Frame Library in the Type field, and it also appears in the lower right-hand corner of the main THERM screen.

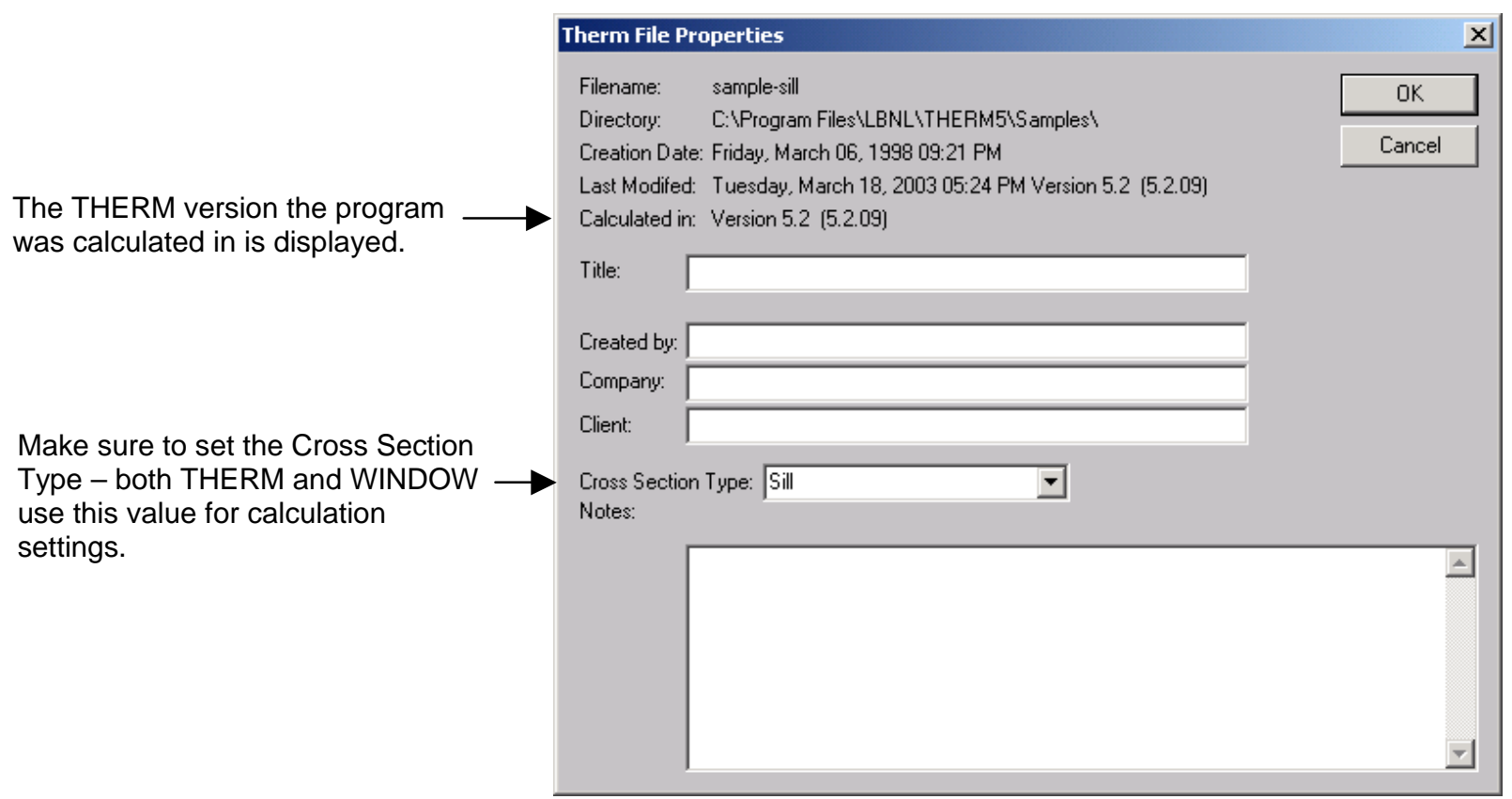

Figure 6-2 The Cross Section Type value from the THERM File Properties is used by the ISO 15099 models to determine the gravity vector and also in WINDOW to determine the method for calculating the Condensation Resistance values. 


\subsubsection{Cross-Section Orientation}

For NFRC simulations using THERM, all cross sections shall be oriented in a vertical direction, i.e., all the glazing systems must be pointing either up or down, not horizontally. (The exception is for skylights, which are modeled at a 20 degree tilt; see Section 8.5 for a discussion of modeling skylights). When creating the cross section, the frame components may be drawn horizontally if that is easiest (i.e., if the DXF file is drawn that way), but before importing the glazing system, rotate the cross section so that the glazing will come in either up or down.

So, for example, jamb cross sections can be drawn horizontally, but they must be rotated into a vertical position for modeling. Figure 6-3 shows how a DXF file might be drawn. The horizontal jamb and meeting rail sections can be drawn horizontally as in the DXF file until the point of importing the glazing system, at which time they must be rotated 90 degrees. For heads, the glass should face down, for sills and jambs the glass should face up, with the inside surfaces of the model facing to the right and the exterior surfaces facing to the left.

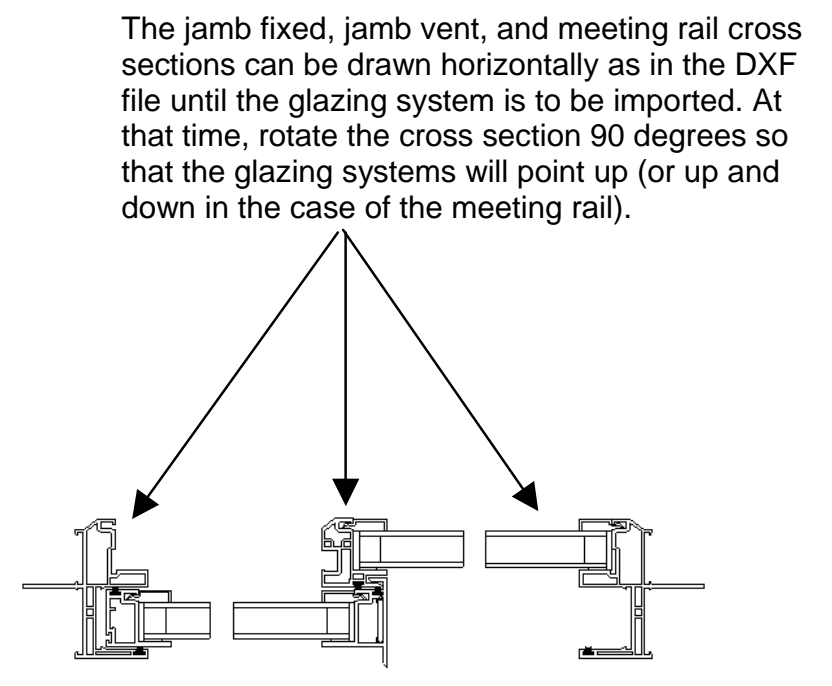

Figure 6-3. DXF file with horizontal cross sections that must be rotated 90 degrees before importing glazing systems and simulating. 


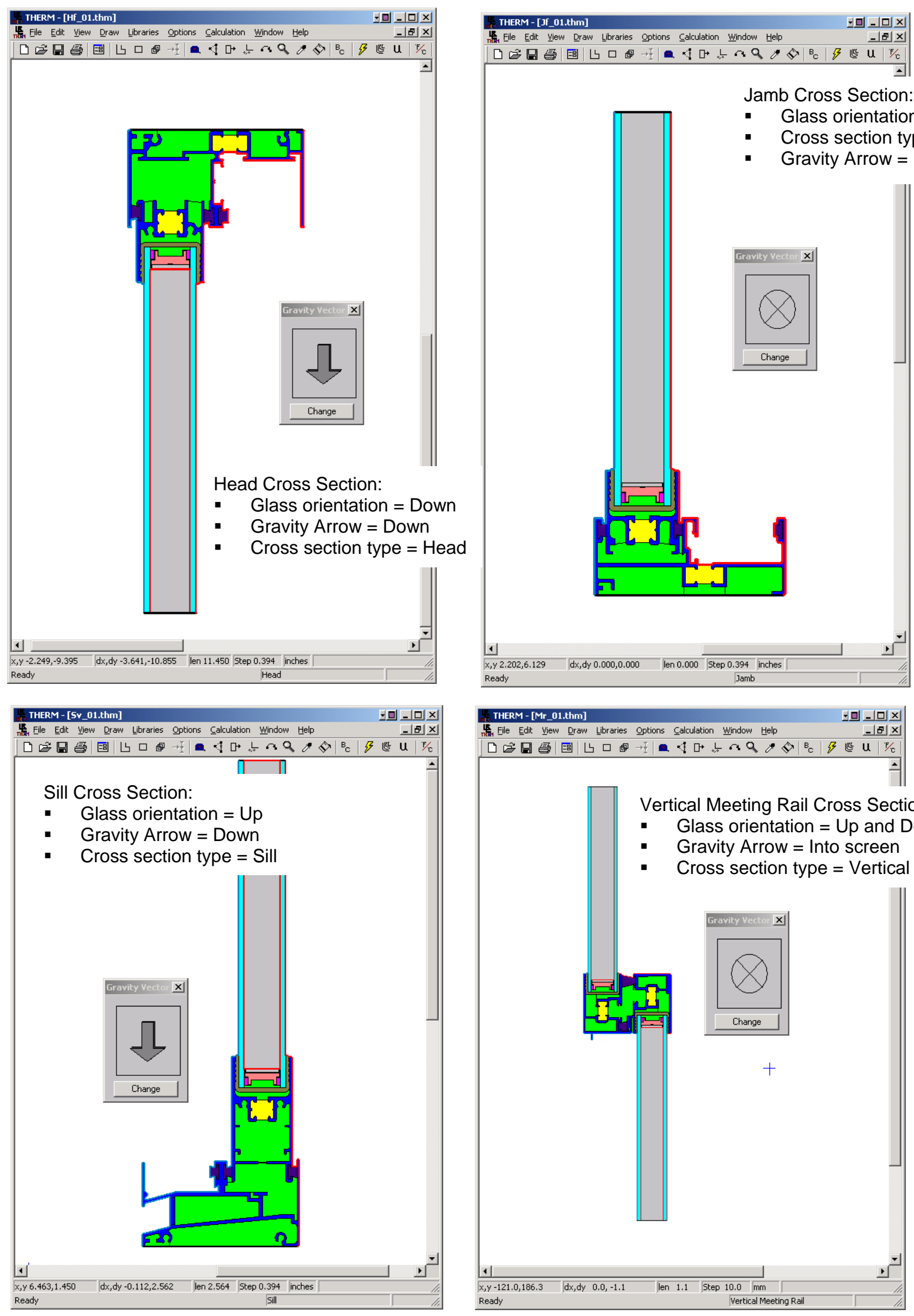

Figure 6-4 Sample cross sections from a horizontal slider showing the cross section orientation, cross section type label in the lower right corner of the screen, and gravity arrow orientation.. 


\subsubsection{What is Not Modeled}

NFRC 100 specifically excludes certain options on fenestration products which are not modeled in THERM. These options include:

- Screens

- Optional Interior trim

- Removable grilles applied to the interior or exterior surface of the glass (i.e., snap-on grilles). Grilles or dividers between glass layers must be modeled under some circumstances, as discussed in Section 8.3, "Modeling Internal Dividers."

- Optional jamb, head, and sill extensions

- Interior or exterior shading devices

- Nailing flanges which can be removed from a fenestration product, and which are removed for testing. These may be vinyl flanges on wood windows, or flanges on vinyl or aluminum windows designed to be removed for some installations. Permanent nailing flanges that would be in place during a test shall be modeled.

In addition, as stated in NFRC 100, "including but not limited to screws and bolts in curtainwalls and pourand-debridge thermal breaks which are not full debridged, shall be simulated". For the time being, components that do not have to be modeled include:

- Hinges

- Locks

- Balances

- Non-continuous Operator Hardware

- Weep Holes

- Setting Blocks

- Shear Blocks

- Corner Keys

\subsubsection{Deformable Parts}

Because deformable parts, such as glazing clips, weatherstripping and other snap-in parts, are often drawn in the undeformed states in DXF files and assembly drawings (many times in the DXF files they overlap other parts), simulator judgement is still required to ensure that these parts are modeled in a way that results in accurate heat transfer results. For example, there are several instances when small air gaps can be replaced with solid materials, such as in sweeps and seals. 


\subsubsection{Frame Cavities}

For NFRC Simulations, frame cavities are modeled using the ISO 15099 Cavity Model. There are default items in the Material Library that can be used to model frame cavities:

- Frame Cavity NFRC 100-2001: to be used for all interior frame cavities

- Frame Cavity Slightly Ventilated NFRC 100-2001: to be used for frame cavities open to the exterior. See Section 6.3.7, Vented Exterior Cavities, for a complete description of when to apply these cavities.

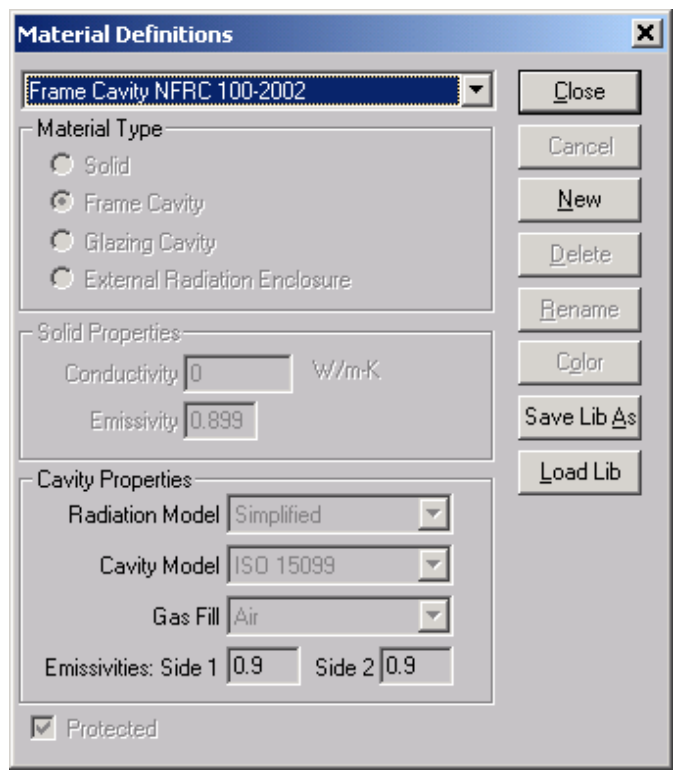

Figure 6-5. Default frame cavity material for NFRC Simulations.

As shown in the figure above, the Frame Cavity NFRC 100-2001 material has the following characteristics:

- Radiation Model: Simplified

- Cavity Model: ISO 15099

This cavity model is an implementation of the ISO 15099 standard, and will cause THERM to automatically calculate the cavity wall temperatures, emissivities and heat flow direction.

- Gas Fill: Air

- Emissivities:

The default emissivities that are included in this frame cavity material are essentially irrelevant because THERM will recalculate and override them during the simulation of the cross section. However, when either the "Frame Cavity NFRC 100-2001" or the "Frame Cavity Slightly Ventilated NFRC 100-2001", the frame cavity surfaces will be outlined in red. Double-clicking on one of these red surface segments will display a dialog box that can be edited. This allows the emissivity values assigned by the program to be overridden. The edited values will then be what the program uses for the simulation.

THERM also makes the following assumptions about the frame cavity:

- Default frame cavity height: 1 meter

- Gravity vector: based on Cross Section Type (see Section 6.3.2, "Cross Section Type", and Section 6.3.3, "Cross Section Orientation") 
If the frame cavity model is set to "ISO 15099", which it is for Frame Cavity NFRC 100-2001 the emissivities, temperatures and heat flow direction will be automatically calculated by the program during simulation. Therefore, the default values that appear for individual frame cavities before the simulation do not need to be edited. These automatic calculations are computed as follows:

- Heat Flow Direction: The calculated heat flow direction will depend on the Cross Section Type setting, accessed from the File/Properties menu. It also depends on the orientation of the glazing system, ie, whether it is imported up, down, left or right. The "gravity vector" can be displayed using the View/Gravity Arrow menu. (See the THERM User's Manual for a discussion of the gravity vector). The assumptions for each type of cross section are the following:

- Head: Cross Section Type = Head, glass imported facing down, gravity vector is pointing down

- Sill: Cross Section Type = Sill, glass imported facing up, gravity vector is pointing down

- Jamb: Cross Section Type = Jamb, glass imported facing up, gravity vector is pointing into the screen

- $\quad$ Meeting Rail: Cross Section Type = Meeting Rail, glass imported facing up and down, gravity vector is pointing into the screen for a horizontal slider and gravity vector is pointing down for a vertical slider.

- Temperature: The program calculates the temperatures of the cavity walls based on the surrounding material temperatures, and determines the heat flow direction based on the temperature differences. It is not necessary to change the initial default cavity temperatures before the simulation. After the simulation, the calculated temperatures can be viewed by double clicking on a frame cavity.

- Emissivity: The program determines the emissivities of the frame cavity walls based on the emissivity values of adjacent materials. If there are materials with different emissivities in the walls perpendicular to the heat flow direction, the program will area-weight the emissivity values. After the simulation, the calculated emissivities can be viewed by double clicking on a frame cavity. 
When the cross section has been simulated, double click on a frame cavity to view the resulting heat flow direction, temperatures, and emissivities automatically calculated by the program, as shown in the figure below.

The information in this dialog box is from a frame cavity in a THERM file that has been simulated, so the values for Nusselt (Nu), Heat Flow Direction, Temperature and Emissivity (for Side 1 and Side 2) are not the default values, but have been automatically calculated by the program.

Select the "Frame Cavity NFRC 100-2001" choice from the Material Library pulldown list. Because the Cavity Model for that material is set to "ISO 15099", the heat flow direction, emissivities, and temperatureswill be automatically calculated.

The emissivity values are associated with the materials adjacent to the cavities, and they are automatically calculated by the program during the simulation. If there are materials adjacent to the cavity which have different emissivities, the program will area-weight the final values, as in this example.

Therefore, it is not necessary to check the emissivity of every frame cavity before the simulation because what is displayed are default values that are not particularly relevant.

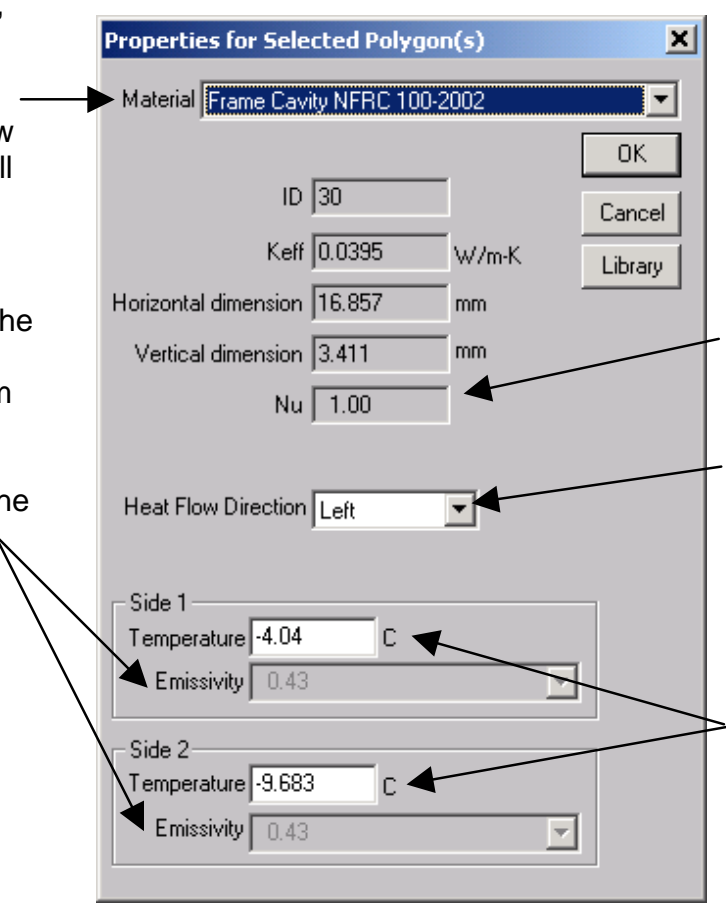

The Nusselt number $(\mathrm{Nu})$ is calculated before the simulation based on default temperatures, but will be recalculated based on the actual cavity temperatures during the simulation.

The Heat Flow Direction is automatically calculated by the program; therefore it is not necessary to check it for every cavity before the simulation.

The Temperatures are automatically calculated by the program.

Figure 6-6. Select the Frame Cavity choice from the Material Library that matches the emissivity for both sides of each frame. 
THERM bases the convection in the frame cavity on rectangularizton of the cavity according to ISO 15099 specifications. The current NFRC procedure is to break up cavities that are separated by a connection, or "throat", less than or equal to $5 \mathrm{~mm}$ (0.20 inches). However, if a frame cavity has a Nusselt number less than or equal to 1.20 before simulating, it is not necessary to break it up into smaller cavities. The Nusselt number can be displayed by double clicking on the frame cavity, which displays the Properties dialog box. The horizontal and vertical dimensions of the bounding rectangle, which determine the Nusselt number, are also shown in this dialog box.

Note: Before a file is calculated, the Nusselt number is based on the default temperatures and emissivities for the frame cavity. There is a slight chance that the Nusselt number of a cavity before a calculation is less than or equal to 1.20, and after the calculation is greater than 1.20. In this case, the cavity does not need to be broken up.

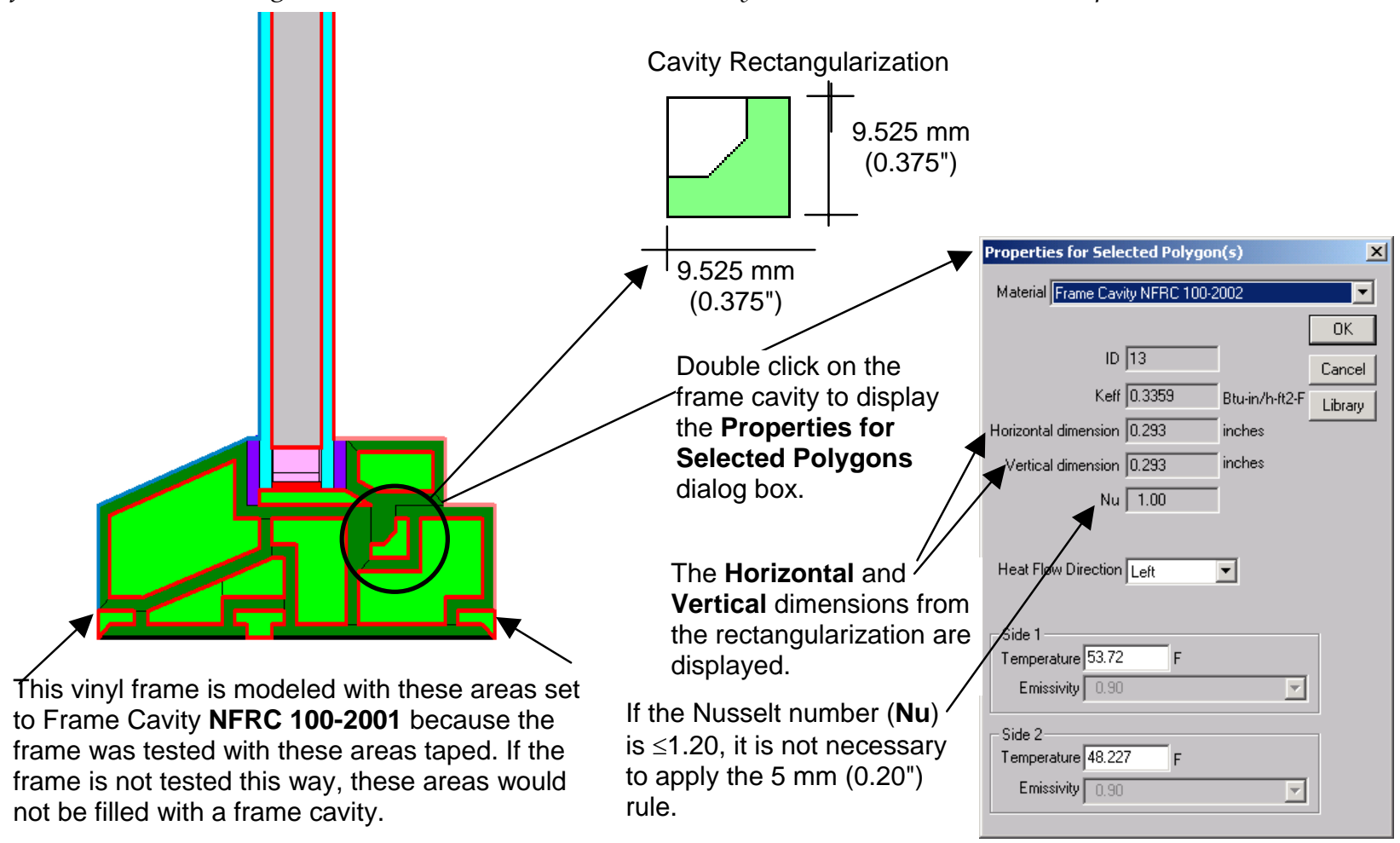

Figure 6-7. THERM uses the ISO 15099 rectangularization specification to model the thermal characteristics of cavities.

The best method for determining whether a frame cavity with a $5 \mathrm{~mm}\left(0.20^{\prime \prime}\right)$ throat will have to be divided up, and how to divide it if necessary, is the following:

1. Verify that there are throats (constrictions) in the frame cavity less than or equal to $5 \mathrm{~mm}(0.20$ inches). If there are no throats, do not break up the cavity. A throat is defined as any place in a frame cavity where two walls, two points, or a wall and a point in the cavity are at or within $5 \mathrm{~mm}(0.20$ inches) of each other.

2. Check the Nusselt Number of the undivided cavity. If the Nusselt Number is less than or equal to 1.20, the simulator shall not break the cavity into smaller polygons.

3. If the Nusselt Number is greater than 1.20, the simulator shall identify all cavities that are less than or equal to $5 \mathrm{~mm}$ (0.20 inches) in both opening and depth and define them as separate cavities.

4. Re-check the Nusselt number of the remaining large cavity. If it is less than or equal to 1.20, no further action is necessary. If the number is greater than 1.20 , the simulator shall: 
a. Identify throats (constriction) in the cavity that are less than $5 \mathrm{~mm}(0.20$ inches) and working from the largest part of the cavity to the smallest, break-up the larger cavity into smaller cavities, splitting first horizontally, then vertically, and finally (as a last resort) diagonally.

b. Each time the cavity is broken, the simulator shall re-check the Nusselt number of the new cavities. If the Nusselt number in the remaining cavity/cavities is less than or equal to 1.20, no further action is necessary. If the Nusselt number for the remaining cavity/cavities is greater than 1.20, proceed, again, with step 4 until no further constrictions can be defined.

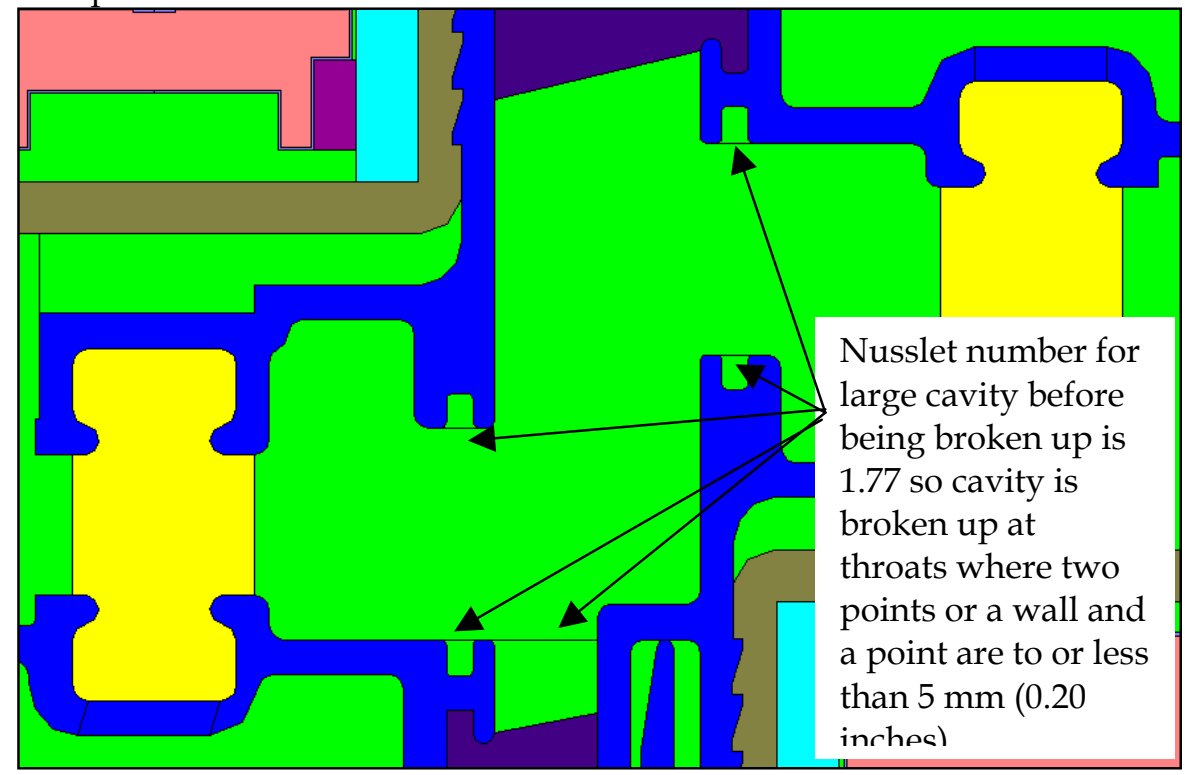

Figure 6-8. Breaking up frame cavities.

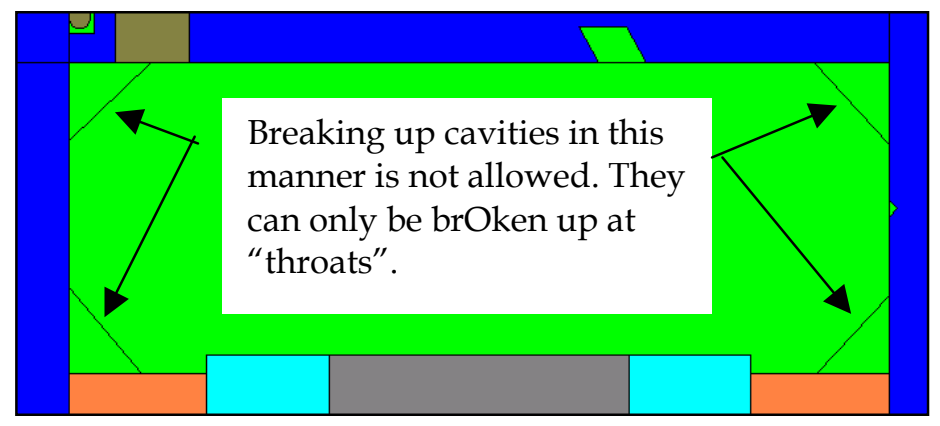

Figure 6-9. Breaking up frame cavities in this manner is not allowed. 


\subsubsection{Slightly Ventilated Exterior Cavities}

For NFRC simulations, air cavities that are open to the exterior within a frame section, such as the air cavity under the sash of a casement window that is vented to the exterior, shall be modeled according to ISO 15099, Section 6.7.1 which states that cavities greater than $2 \mathrm{~mm}$ but equal to or less than $10 \mathrm{~mm}$ shall be modeled as slightly ventilated air cavities. The THERM Material Library has a default material for this case, called "Frame Cavity Slightly Ventilated NFRC 100-2001", which will be used to fill the entire cavity. The figure below illustrates this.

Model a cavity open to the

exterior environment as

slightly ventilated when:

$$
d \geq b
$$

and

$2 \mathrm{~mm}<\mathrm{b} \leq 10 \mathrm{~mm}$

For cavities $<2 \mathrm{~mm}$, use the standard "Frame Cavity NFRC 100-2001"

For cavities $>10 \mathrm{~mm}$, do not model a frame cavity

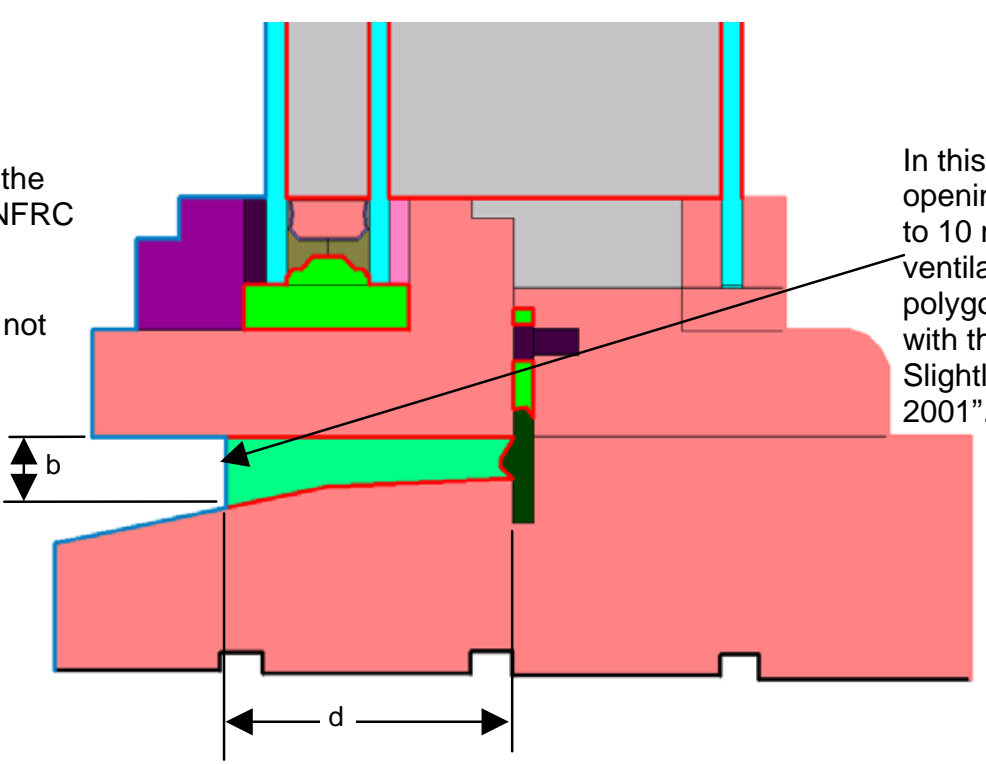

Figure 6-10. Slightly Ventilated exterior cavities .

If during testing, the product is sealed at the inside surface with tape, those cavities must be simulated with the standard "Frame Cavity NFRC 100-2001" material. 


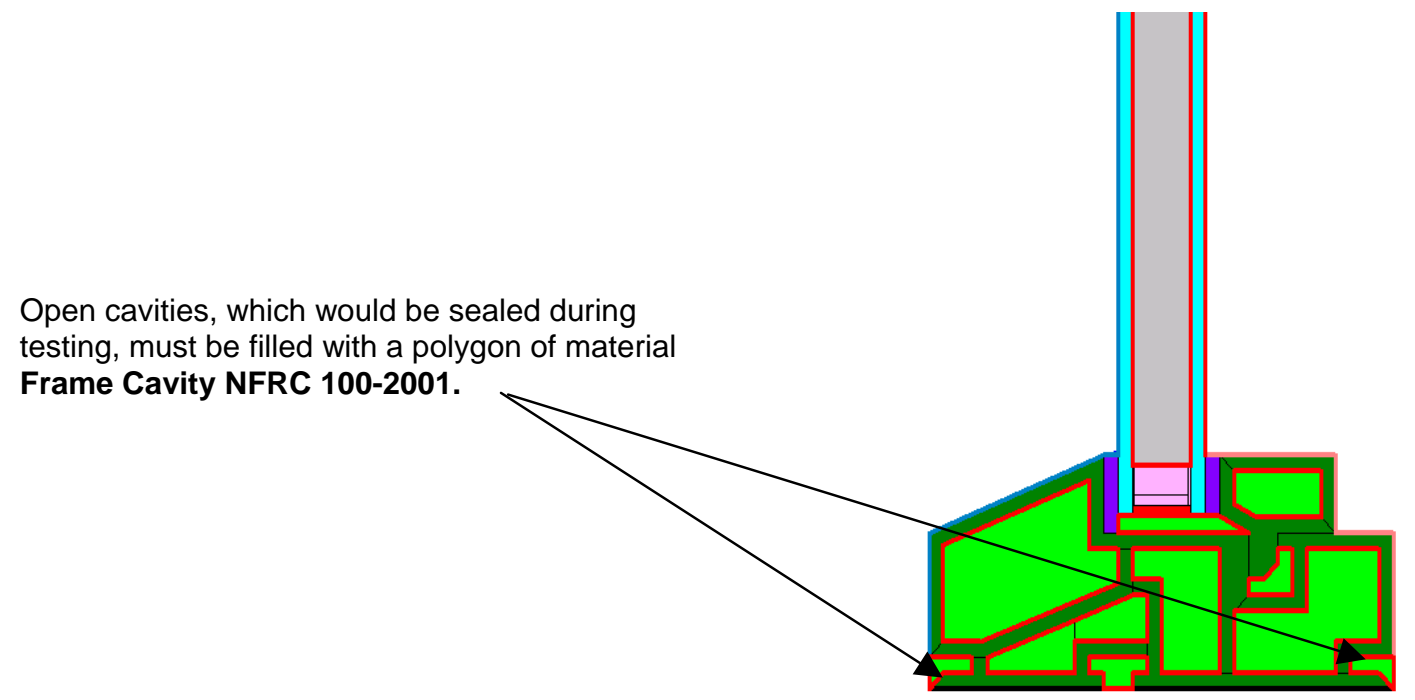

Figure 6-11. Cavities open to the interior are filled with Frame Cavity NFRC 100-2001 material .

\subsubsection{Modeling Sloped and Curved Surfaces}

Because the exact geometry of sloped surfaces can be modeled in THERM, there is no need to change the boundary conditions for sloped surfaces.

For curved surfaces, THERM approximates curves with line segments. The number of line segments used in the curve will determine how close the final model is to the original geometry of the product. When importing a DXF file, the number of line segments is set using the Arc to Polygon setting in the Options menu. See Section 10, "Interpreting Arcs" in this manual for a detailed discussion of this procedure. In general, an Arc to Polygon setting of 45 degrees for small rounds and fillets in extrusions will give great enough accuracy without introducing unnecessary detail. For larger curved details, all points on the represented line must be within $5 \mathrm{~mm}$ of the actual line or curve. The averaged distance (for all points) between the represented line and the actual line or curve must be not greater than $2.5 \mathrm{~mm}$ (ISO 15099 Standard). 


\subsubsection{Modeling Sloped Sills}

A product with a sloped sill must be modeled in the same way that it was tested. When a vertical sliding or fixed window is tested, the air space underneath the sloped portion of the sill is filled with expanded polystyrene insulating material. For the simulation of the sill section this window must have the insulating material modeled underneath the sloped portion of the sill as well, as shown in the figure below. The bottom surface of the insulating material will have an adiabatic boundary condition applied. The surface of the insulating material that faces to the interior will have a boundary condition applied that is the same as the condition applied to the frame above the insulating material. The U-factor surface tag for the interior facing surface of the insulating material will be defined as Frame, which ensures the correct projected frame dimension will be used in the area weighting of the total window U-factor.

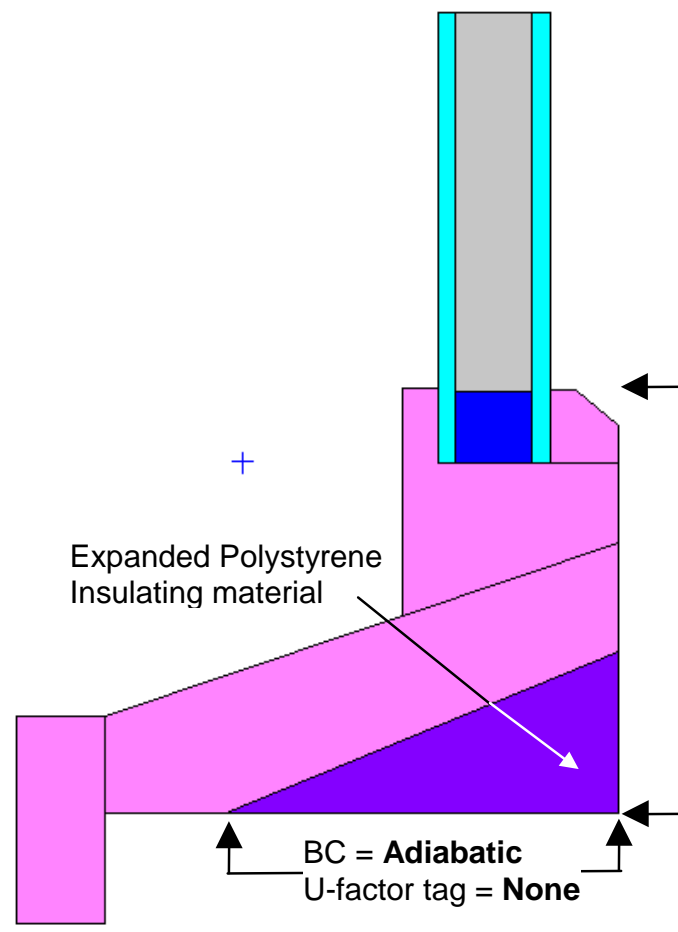

Boundary condition is the same for all edges facing in.

The insulating material is added so that the full frame height is used by the program.

U-factor tag $=$ Frame

Figure 6-12. Model the sloped sill as it was tested, with insulating material under the sill. 


\subsection{Importing Glazing Systems}

When the frame cross section has been drawn, and rotated into a vertical orientation if necessary, the next step is to import a glazing system. Section 5.9, "Inserting a Glazing System" in the THERM User's Manual contains detailed information about this step. The discussion in this manual is added information about glazing systems.

\subsubsection{Overview}

For NFRC simulations, a glazing system shall be imported into THERM from the Glazing System Library in the WINDOW program. When THERM imports the glazing system from the WINDOW library, it obtains the following information from the glazing system:

- emittance of the glazing surfaces

- the effective conductivity of the glazing cavity

- the interior and exterior boundary conditions of the glazing system

As discussed in Section 6.5, glazing systems should always be imported into the cross section in a vertical (either up or down) orientation (it may be necessary to rotate the cross section in order to do this). The figure below shows where the Locator should be positioned based on the orientation of the glazing system when it is imported. The orientation of the glazing system determines in part the gravity vector orientation so it is extremely important to model it correctly.

NOTE: Although in general it is best to rotate and flip cross sections before the glazing systems are imported and boundary conditions are defined, the exception is skylights, which are modeled at a tilt. In that case, import the glazing system in a vertical orientation, define the boundary conditions, and then rotate the entire cross section.

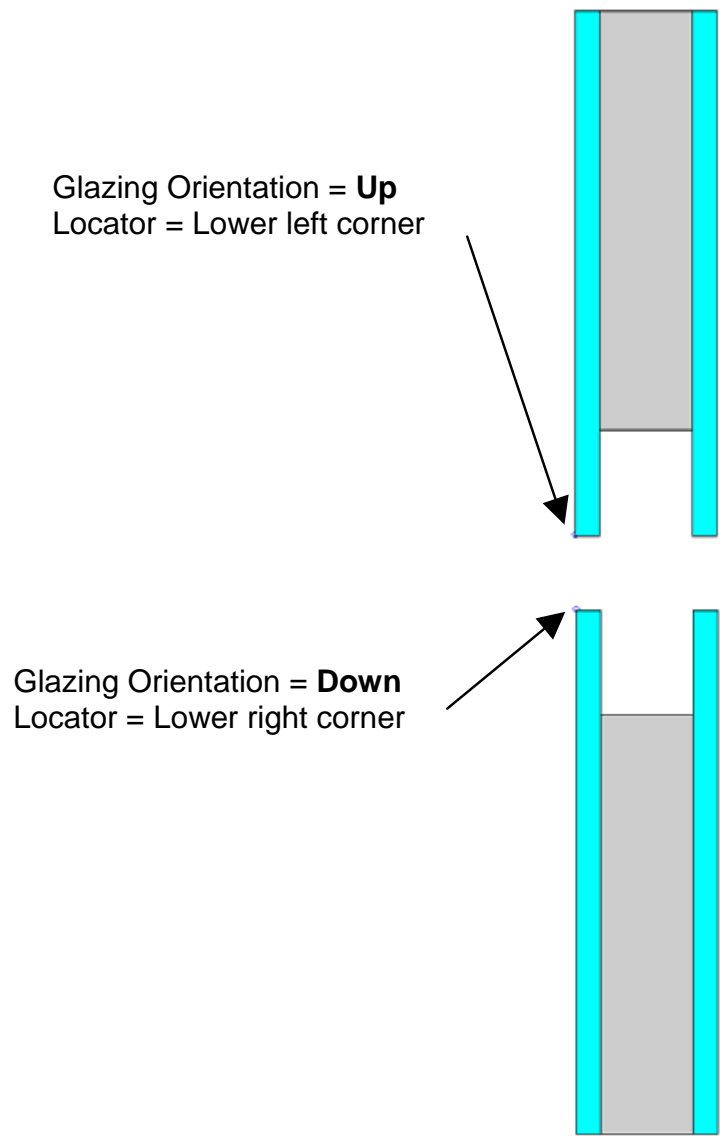

Figure 6-13. The Locator position when importing glazing systems in different orientations. 


\subsubsection{Inserting Glazing Systems}

The THERM User's Manual contains a detailed description about how to insert glazing systems into a THERM file for U-factor and Condensation Resistance calculations. The figure below is a brief overview of the steps.

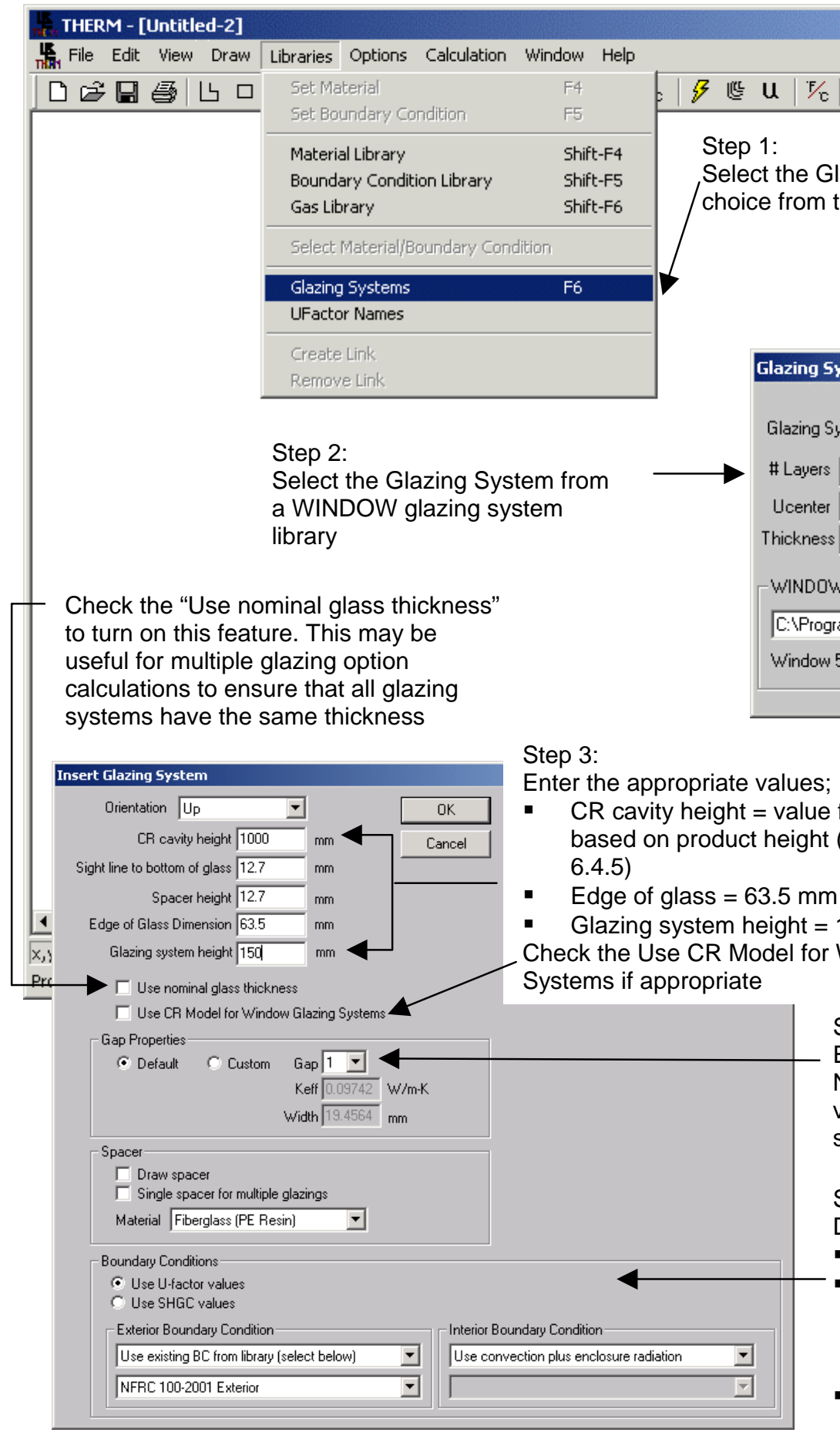

Step 4

Enter the appropriate Gap Properties. For NFRC simulations, "Default", which uses the values directly from WINDOW, is the correct setting

Step 5:

Define the Boundary Conditions:

- Click on "Use U-factor values"

Exterior Boundary Conditions:

- Set to "Use existing BC from Library", and set the value to "NFRC 100-2001 Exterior"

- Interior Boundary Condition:

- Set to "Use convection plus enclosure radiation", which sets the boundary condition by default to "AutoEnclosure"

Figure 6-14. Inserting a Glazing System into a cross section. 
The NFRC modeling requirements when inserting a glazing system are:

- CR cavity height: See table 6-2, Section 6.4.5 for values. Only used if the Condensation Resistance model is activated.

- Edge of Glass: $63.5 \mathrm{~mm}(2.5$ inches)

- Glazing System Height: $150 \mathrm{~mm}$ (6.0 inches) for all products

- Exterior Boundary Condition: Set to "Use existing BC from library" and select "NFRC 100-2001 Exterior". This will be applied automatically by the program on the exterior surfaces of the glazing system and frame.

- Interior Boundary Condition: Set to "Use convection plus enclosure radiation". This will cause the program to automatically calculate the boundary conditions for the glazing system to the

"Autoenclosure" radiation model, which does not require the Radiation Enclosure geometry to be drawn.

The nominal thickness feature, turned on by checking the "Use nominal glass thickness" when importing a glazing system, can be useful for multiple glazing options, where THERM expects all the glazing system options to have identical thicknesses. This feature is explained in more detail in the THERM User's Manual.

\subsubsection{Adding the Radiation Enclosure Model}

In accordance with NFRC 100-2001, the THERM radiation enclosure model shall be used for all simulations.

In THERM 5.0, it is not necessary to draw the enclosure geometry. If the glazing system was imported with the boundary condition set to "Use convection plus enclosure radiation", THERM will automatically assign boundary conditions to the glazing system that have the Radiation Model set to "Autoenclosure". This type of boundary condition models the radiation enclosure without the enclosure geometry being drawn. A more detailed description is found in Section 6.5, "Boundary Conditions" in this manual.

See the THERM User's Manual for more details about the Autoenclosure feature, and information about drawing the enclosure geometry for the case where more than one radiation enclosure is desired.

\subsubsection{Multiple Glazing Options}

THERM allows multiple glazing options to be associated with a glazing system. This feature can be useful for simulations where many different glazing systems are to be modeled in the same frame cross section.

To use this feature, follow these steps:

1. Determine the frame cross sections to be modeled: The glazing options for a frame cross section must be identical in their geometry, including overall thickness, cavity thickness, spacer height, and sight line dimension. Therefore, the first step is to determine the number of frame cross sections that must be defined in THERM for each set of glazing options.

2. Create and Simulate the Base Case File: For each frame/glazing option set, create a THERM file with the complete frame cross section and one of the glazing options. Create the boundary conditions for this model, making sure to check the "Use convection plus enclosure radiation" when importing the glazing system, and simulate it to make sure that the geometry is correct. This will be the "base case" file, and will contain no results once the multiple glazing options have been defined and simulated.

3. Define the glazing options: There are two ways to define the glazing options in a file:

- From the Calculation menu, select the Glazing Options menu choice. OR

- Double click on the glazing system geometry, and click on the Glazing Options button in the Glazing System Info dialog box. 


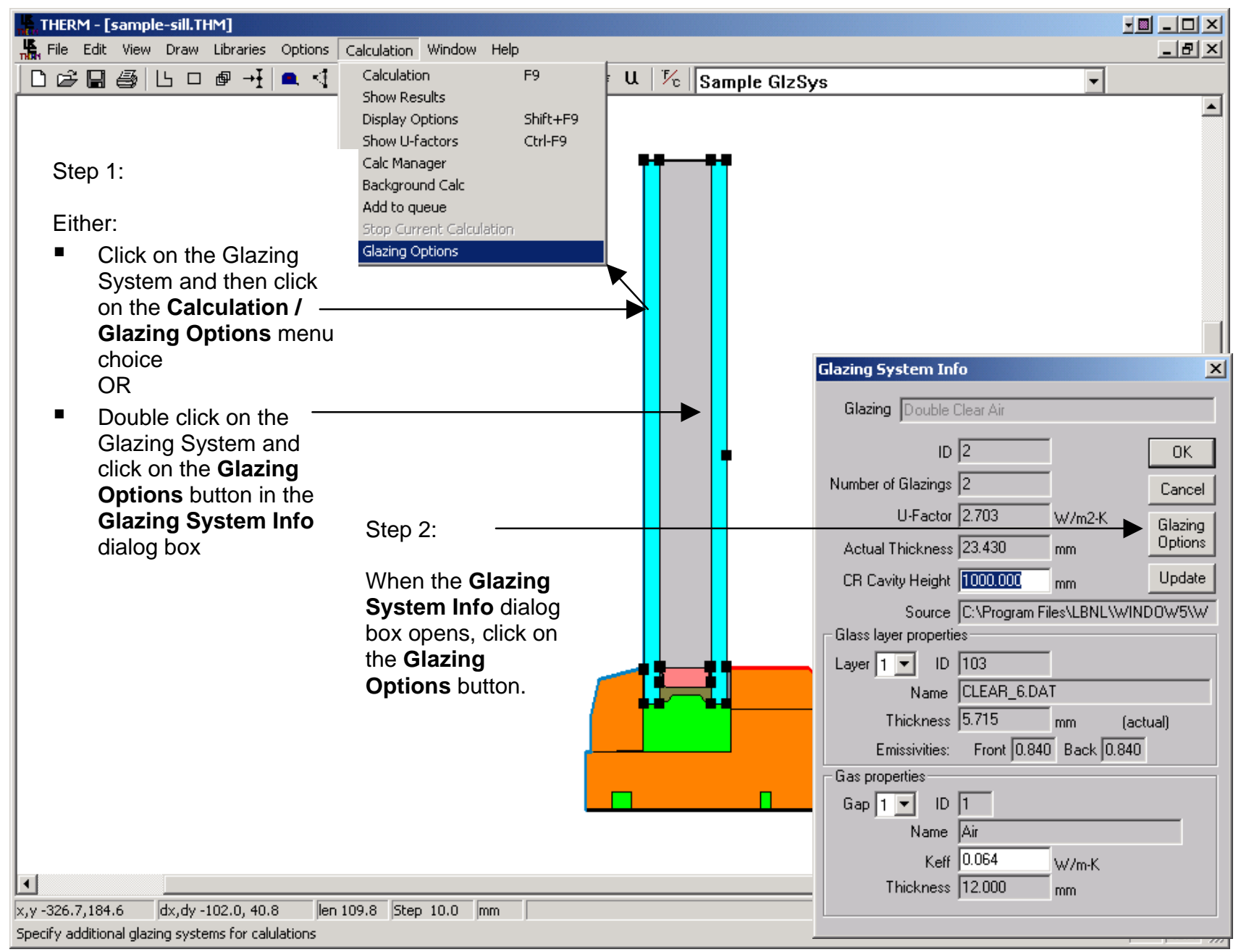

Figure 6-15. Define multiple glazing options.

- Add glazing options: From the Glazing System Options dialog box, click on the Add button to see a list of all the glazing systems in the currently selected Glazing System Library that have the same overall thickness and glass layer thickness as the base case glazing system. (Note: Use the nominal glass feature if necessary to make all the glazing systems a uniform thickness.) Click on the glazing systems in the Add Glazing Options dialog box that are to be associated with this cross section (use Shift-click or Ctrl-click to select multiple glazing systems) Click OK when all are selected.

4. THERM will make a separate file for each glazing option, and in the Glazing System Options dialog box, click on the radio button choice to determine how the program will automatically name each file. The options are either that the program will append the Glazing System ID (as a 3 character number, such as 001, 002) or Name to the base case filename.

5. Boundary Conditions: THERM will automatically calculate the boundary conditions for each separate glazing system option. 
Click on the Add button in the Glazing System Options dialog box.

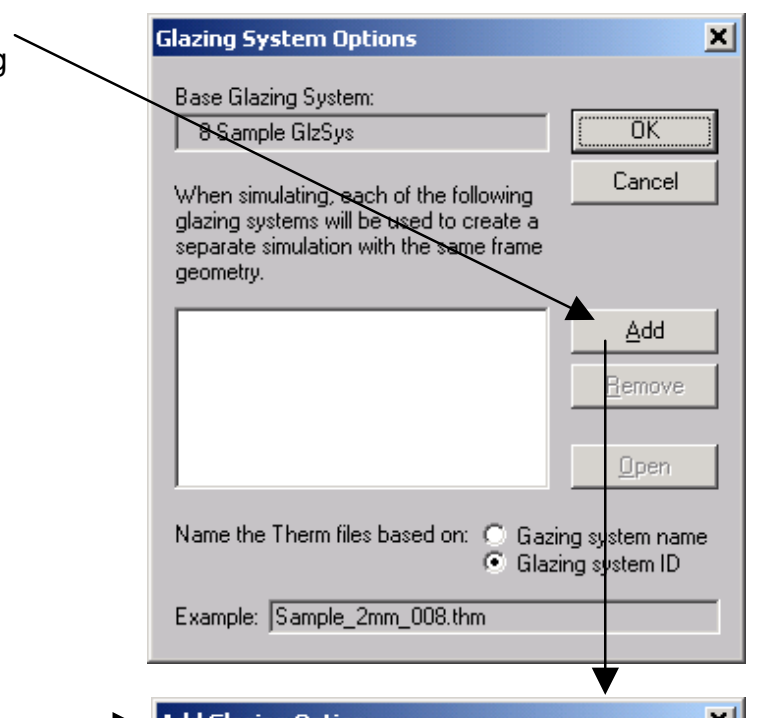

The Add Glazing Option dialog box shows all the glazing systems which match the Base Glazing System in terms of glass layer thickness and overall thickness.

Select individual glazing systems (use Shift-click and Ctrl-click) or click on Select AlI, and click the OK button.

Click on the Browse to select a different WINDOW Glazing Svstem Librarv.

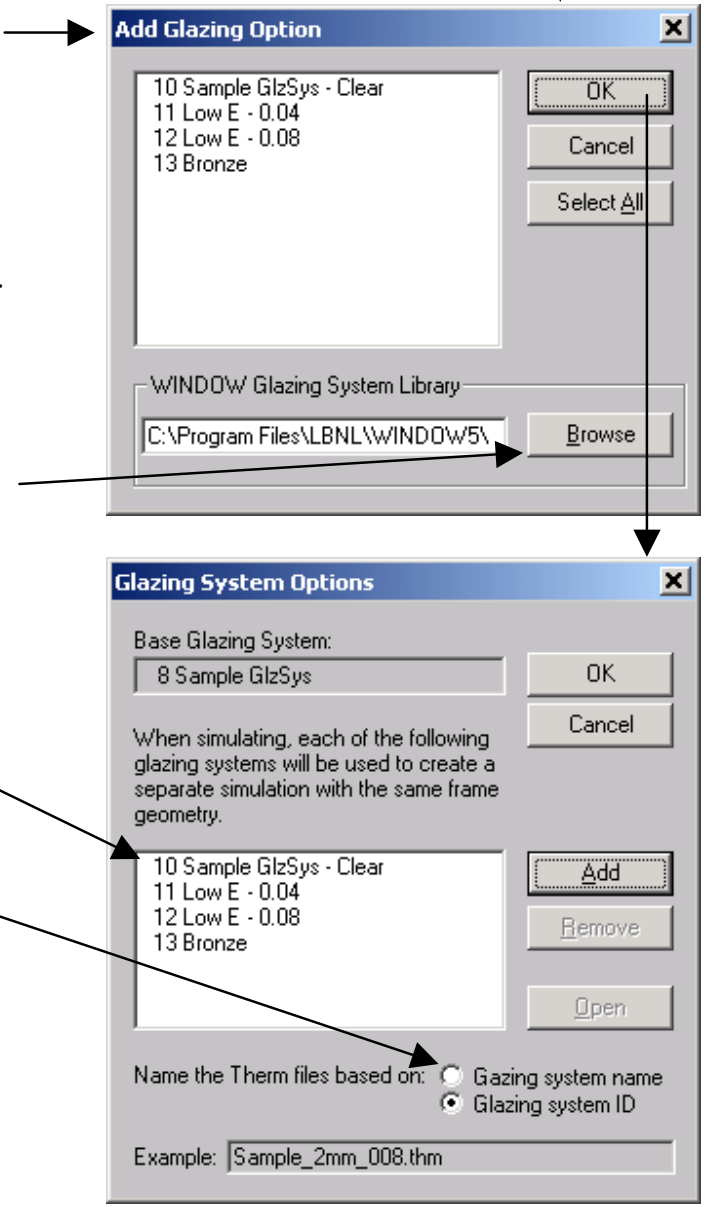

Figure 6-16. Select the glazing systems to be associated with the base case file.

6. Calculate the Results: the file can now be simulated, or more files can be created and all the files simulated through the Calc Manager. This is explained in more detail in Section 6.6, "Calculating the Results". 


\subsubsection{Condensation Resistance}

There are two ways to activate the Condensation Resistance model in THERM:

- When importing the glazing system, check the "Use CR Model for Window Glazing System" checkbox.

- In the Options menu, Preferences choice, Therm File Options tab, check the "Use CR Model for Glazing Systems"

When inserting the glazing system into the model, as discussed in Section 6.4.2, the value for the "CR cavity height" shall come from the table below.

Table 6-2. Default Values for Actual Glazing Height Modeling for Condensation Resistance

\begin{tabular}{|l|l|}
\hline Real Product Height & $\begin{array}{l}\text { Default Glazing Height for Condensation Modeling } \\
\text { (input as the "CR cavity height" in THERM) }\end{array}$ \\
\hline $2000 \mathrm{~mm}$ & $1900 \mathrm{~mm}$ \\
\hline $1500 \mathrm{~mm}$ (vertical slider) & $675 \mathrm{~mm}$ per sash \\
\hline $1500 \mathrm{~mm}$ (non-vertical slider) & $1400 \mathrm{~mm}$ \\
\hline $1200 \mathrm{~mm}$ & $1100 \mathrm{~mm}$ \\
\hline $600 \mathrm{~mm}$ & $500 \mathrm{~mm}$ \\
\hline
\end{tabular}

Activating the Condensation Resistance model in THERM will cause boundary conditions to be drawn inside the glazing system cavity, as shown in the figure below. When this model is simulated, THERM will automatically calculate both the U-factor results and CR temperature data that will be be used, when this profile is imported into WINDOW, to generate the overall Condensation Resistance value for the whole product. During the simulation, two simulations will appear at the bottom of the screen, the first for the Ufactor results and the second for the CR results.

The Condensation Resistance model is not used for vertical cross sections, such as a Jamb, a Vertical meeting rail, or a Vertical Divider. Even if the CR model is activated (i.e., the "Use CR Model for Window Glazing System" is checked) for these Cross Section Types, THERM will not perform the CR simulation for that file. 
THERM generates temperature data from both the CR and U-factor simulations which WINDOW uses to calculate the frame CR values (shown in the Frame Library Detailed View) and also the whole product CR values (shown in the Window Library Detailed View). When the CR model is not appropriate for the whole product CR calculations, WINDOW will use the temperatures from the THERM U-factor calculation instead, as shown below.

\begin{tabular}{|c|c|c|}
\hline $\begin{array}{l}\text { Product Type } \\
\text { (From THERM) }\end{array}$ & $\begin{array}{l}\text { WINDOW } 5 \text { Whole Product Tilt } \\
\text { (input in Window Library } \\
\text { Detailed View) }\end{array}$ & $\begin{array}{l}\text { Temperatures Used by } \\
\text { WINDOW5 for whole product CR } \\
\text { calculations }\end{array}$ \\
\hline $\begin{array}{l}\text { Horizontal elements: } \\
\text { Sill, Head, Horizontal Meeting } \\
\text { Rail, Horizontal Divider }\end{array}$ & 90 & $\mathrm{CR}$ \\
\hline $\begin{array}{l}\text { Horizontal and Vertical elements: } \\
\text { - Sill, Head, Horizontal } \\
\text { Meeting Rail, Horizontal } \\
\text { Divider; } \\
\text { - Jamb, Vertical Meeting } \\
\text { Rail, Vertical Divider }\end{array}$ & $\geq 0, \leq 20$ & U-factor \\
\hline $\begin{array}{l}\text { Vertical elements: } \\
\text { Jamb, Vertical Meeting Rail, } \\
\text { Vertical Divider }\end{array}$ & 90 & U-factor \\
\hline $\begin{array}{l}\text { Horizontal and Vertical elements: } \\
\text { - Sill, Head, Horizontal } \\
\text { Meeting Rail, Horizontal } \\
\text { Divider; } \\
\text { - Jamb, Vertical Meeting } \\
\text { Rail, Vertical Divider } \\
\end{array}$ & $>20,<90$ & CR not calculated \\
\hline
\end{tabular}


The application of these rules happens when WINDOW 5 performs a calculation in the Window Library. It is up to the simulator to have provided WINDOW with the appropriate simulations in THERM in order for WINDOW to be able to calculate the result. For example, if there are no CR results for a Sill or Head THERM file modeled in a window with a 90 tilt, then WINDOW will not calculate a whole product CR value because the needed CR temperature data does not exist. A message, shown below, will indicate which cross sections are missing the CR data.

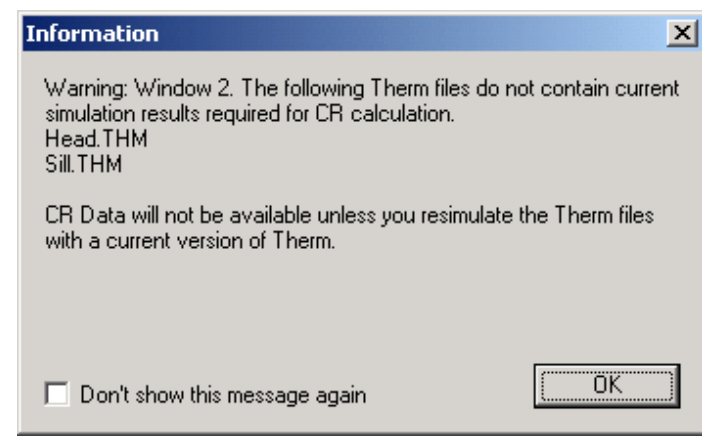

Figure 6-17. WINDOW5 will display a message if it determines that CR temperature data is not available from the THERM file.

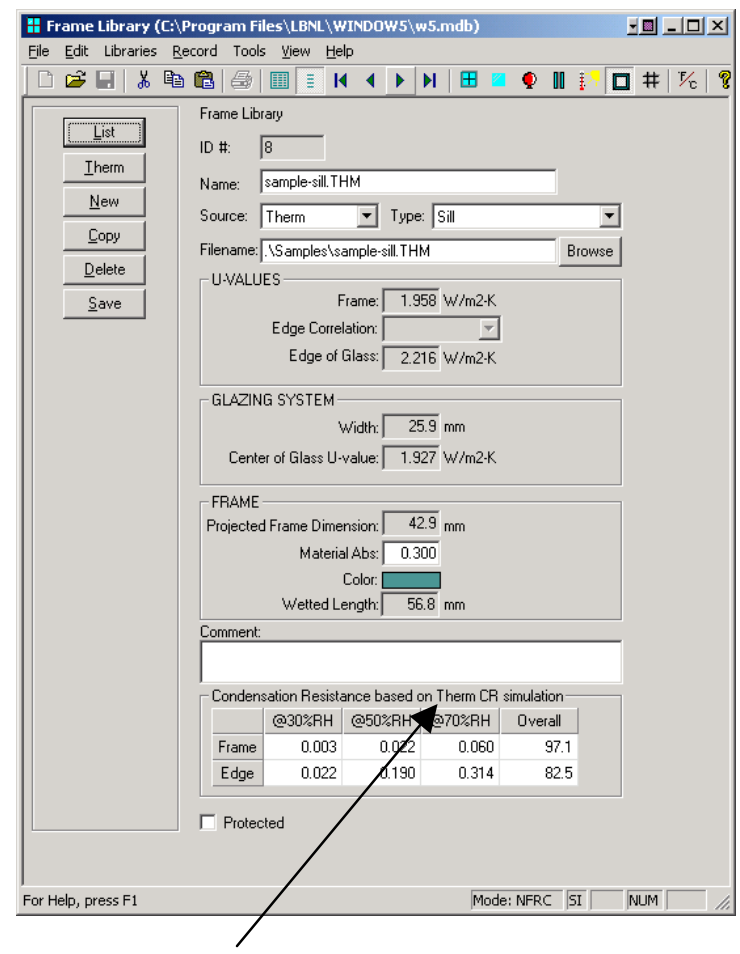

For Product Type $=$ Sill, not rotated, the $\mathrm{CR}$ calculation will be based on the THERM CR simulation

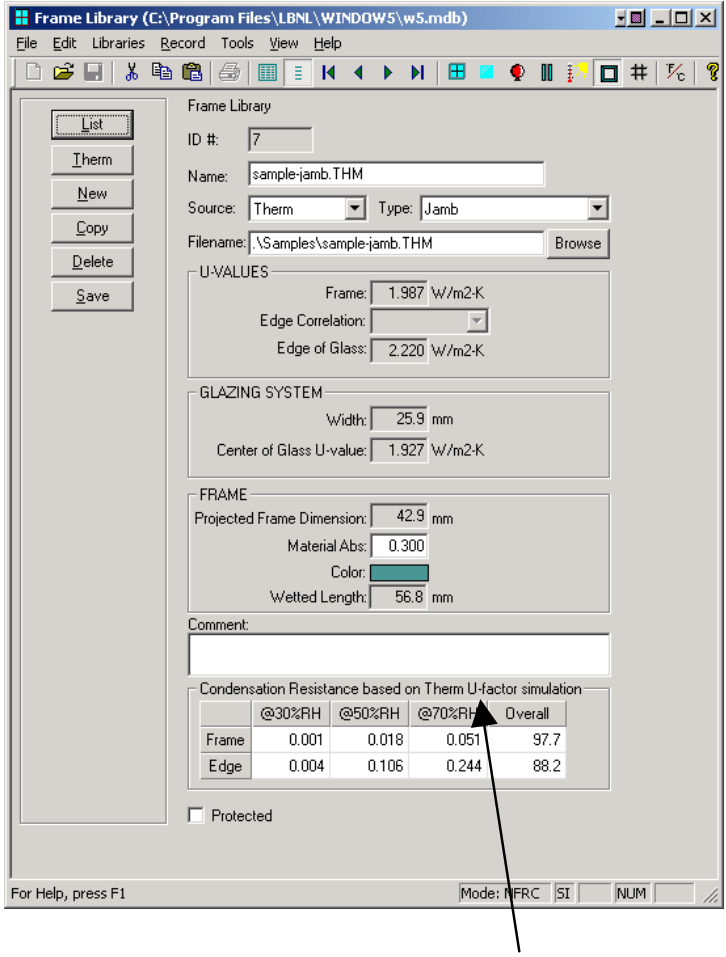

For Product Type = Jamb, not rotated, the CR calculation will be based on the THERM Ufactor simulation

Figure 6-18. WINDOW5 Frame Library Detailed View displays the type of THERM temperature data to be used with the CR calculation, either U-fator or CR. 


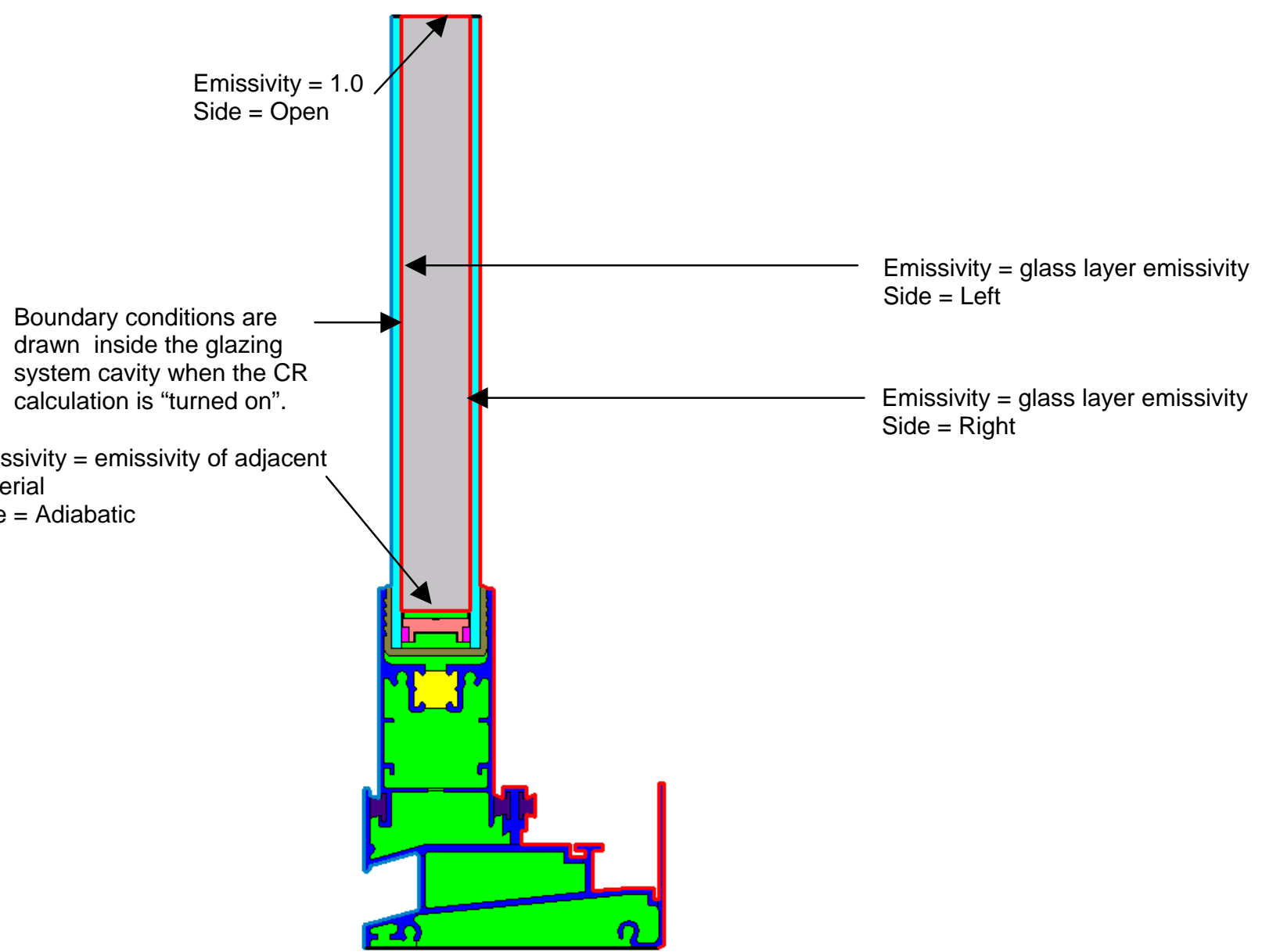

Figure 6-19. Modeling the profile with the CI model turned on. 


\subsection{Defining Boundary Conditions}

Chapter 6 of the THERM User's Manual contains a detailed explanation of how to define boundary conditions in a model. The information found in this manual is supplemental to that discussion.

\subsubsection{Overview}

Boundary conditions must be defined for all the surfaces on the perimeter of the models, as well as at the surfaces adjacent to the radiation enclosure. These boundary conditions define the temperatures and film coefficients for each element of the perimeter. Different boundary conditions are defined for the surfaces on the interior and the exterior of the cross section. Surfaces which are assumed to have no heat transfer are assigned Adiabatic boundary conditions.

There are three main categories of boundary conditions:

- Interior: An interior boundary condition is used for all interior surfaces, and assumes that the surface is exposed to natural convection, and the heat transfer coefficient used depends on the temperature of the surface, which is a function of the material.

- For Glazing Systems: The interior glazing system boundary conditions should be set to the boundary condition that is associated with the glazing system imported from WINDOW. (WINDOW calculates the center-of-glazing surface temperatures, and THERM uses these values to automatically calculate the edge-of-glazing boundary conditions for glazing systems imported from WINDOW).At the time the glazing system is imported from WINDOW, set the Interior Boundary Condition pulldown to "Use Convection plus enclosure radiation", which will cause the program to set the glazing system interior boundary condition Radiation Model to "AutoEnclosure". THERM will then model the cross section with the radiation enclosure feature (which is required by NFRC for all simulations) without the geometry of Radiation Enclosure being drawn. The boundary condition is named "<filename>:<glazing system name> U-factor Inside Film". Double click on an interior boundary conditions to see its characteristics, as shown in the figure below.

- For Frame Elements: Set all non-glazing system interior boundary conditions according to the material, from the following predefined boundary conditions (which all have the Radiation Model set to "Autoenclosure"), one set for cross sections at a $90^{\circ}$ tilt and another set for cross sections at a $20^{\circ}$ tilt. )If there are frame materials that fall into more than one category, apply the boundary condition of the predominant material to all the frame elements):

- Interior Aluminum Frame (Convection only)

- Interior Thermally Broken Frame (Convection only)

- Interior Thermally Improved Frame (Convection only)

- Interior Wood/Vinyl Frame (Convection only)

- Interior (20 tilt) Aluminum Frame (Convection only)

- Interior (20 tilt) Thermally Broken Frame (Convection only)

- Interior (20 tilt) Thermally Improved Frame (Convection only)

- Interior (20 tilt) Wood/Vinyl Frame (Convection only)

- Exterior: The "NFRC 100-2001 Exterior" exterior boundary condition is used for all exterior surfaces, including the glazing system, and assumes that the surface is exposed to a $5.5 \mathrm{~m} / \mathrm{sec}(12.3 \mathrm{mph})$ air velocity, which correcponds to a film coefficient of $26 \mathrm{~W} / \mathrm{m}^{2}-{ }^{\circ} \mathrm{C}\left(4.58 \mathrm{Btu} / \mathrm{h}-\mathrm{ft}^{2}-{ }^{\circ} \mathrm{F}\right)$.

- Adiabatic: The adiabatic boundary condition is used for any surface assumed to have no heat flow. This is used for the top of the glazing system at the boundary between the edge-of-glazing and the center-ofglazing, because the assumption is that the heat transfer between the two sections are independent of 
each other. Adiabatic is also used for the bottom of the frame that would sit in the mask wall of the thermal chamber during testing.

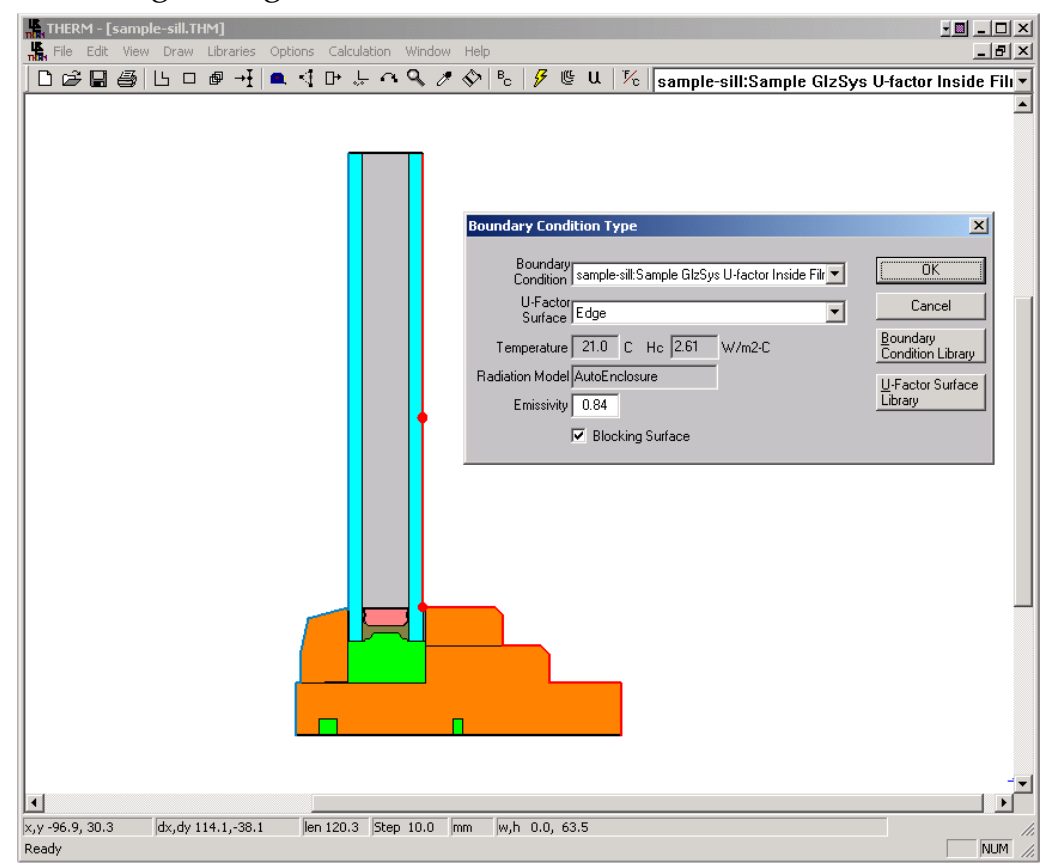

Figure 6-20. Double click on a boundary condition segment to see its characteristics..

THERM has a Boundary Condition Library (accessed from the Library/Boundary Conditions menu, or by double clicking on a boundary condition) which has the standard boundary conditions defined by NFRC, shown in Table 6-3, as well as Adiabatic. By default, the exterior boundary conditions are blue, interior boundary conditions are red, and the adiabatic boundary condition is black.

Table 6-3. Boundary condition definitions

\begin{tabular}{|c|c|c|c|c|c|}
\hline \multirow{3}{*}{ Boundary Condition } & \multirow{3}{*}{$\begin{array}{l}\text { Radiation } \\
\text { Model }\end{array}$} & \multicolumn{4}{|c|}{ Convective Film Coefficient } \\
\hline & & \multicolumn{2}{|c|}{ Tilt $=90^{\circ}$} & \multicolumn{2}{|c|}{ Tilt $=20^{\circ}$} \\
\hline & & $\mathrm{W} / \mathrm{m}^{2}-{ }^{\circ} \mathrm{K}$ & Btu/h-ft ${ }^{2}{ }^{\circ} \mathrm{F}$ & $\mathrm{W} / \mathrm{m}^{2}-{ }^{\circ} \mathrm{K}$ & Btu/h- $-\mathrm{ft}^{2}-{ }^{\circ} \mathrm{F}$ \\
\hline NFRC 100-2001 Exterior & Blackbody & 26 & 4.578 & 26 & 4.578 \\
\hline $\begin{array}{l}\text { Interior Aluminum Frame } \\
\text { (convection only) }\end{array}$ & AutoEnclosure & 3.29 & 0.579 & 4.94 & 0.869 \\
\hline $\begin{array}{l}\text { Interior Thermally Broken } \\
\text { Frame (convection only) }\end{array}$ & AutoEnclosure & 3.00 & 0.528 & 4.38 & 0.771 \\
\hline $\begin{array}{l}\text { Interior Thermally } \\
\text { Improved Frame } \\
\text { (convection only) }\end{array}$ & AutoEnclosure & 3.12 & 0.549 & 4.60 & 0.81 \\
\hline $\begin{array}{l}\text { Interior Wood/Vinyl Frame } \\
\text { (convection only) }\end{array}$ & AutoEnclosure & 2.44 & 0.429 & 3.38 & 0.595 \\
\hline $\begin{array}{l}\text { WINDOW Glazing System } \\
\text { boundary condition } \\
<\text { filename }>\text { :<glazing system } \\
\text { name> U-factor Inside Film }\end{array}$ & AutoEnclosure & \multicolumn{4}{|c|}{$\begin{array}{l}\text { Depends on the WINDOW calculations for the imported } \\
\text { glazing system }\end{array}$} \\
\hline
\end{tabular}




\subsubsection{Assigning Boundary Conditions and U-factor Surface Tags}

Boundary conditions for a cross section are created in THERM by pressing the Boundary Condition toolbar button, clicking on the Draw/Boundary Conditions menu option, or pressing the F10 key.

THERM will automatically assign boundary conditions, both interior and exterior, to a glazing system imported from WINDOW, as discussed above. However, the frame boundary conditions must be assigned in most cases.

- NFRC 100-2001 Exterior for the entire exterior surface of the glazing system and frame

- Adiabatic for bottom of the frame

- Interior Frame Components: The appropriate interior convection-only boundary condition for all other interior surfaces, from the choices in Table 6-3, found in the THERM Boundary Condition Library.

The technique for doing this is described in detail in Chapter 6, "Defining Boundary Conditions" of the THERM User's Manual.

In THERM, in addition to assigning boundary conditions to a boundary segment, the U-factor Surface Tags must also be assigned for each boundary condition. The U-factor Surface Tags, which are selected from the U-factor Names library, are used by THERM to calculate the component U-factors. For a THERM U-factor calculation that will be used in WINDOW, it is necessary to use the following U-factor Surface Tags, with the same capitalization (WINDOW will not recognize any other values):

- Frame: Use this tag for all interior boundary conditions that are part of the frame U-factor calculation, including the boundaries of the glazing system below the sight line. See NFRC 100, "Figure 2, Fenestration Product Schematic -- Vertical Section". THERM will automatically assign the U-factor tag of Frame to the portion of the glazing system that is below the sight line, based on the Site Line to Bottom of Glass value entered when the glazing system is inserted from WINDOW.

- Edge: Use this tag for all interior boundary conditions that are to be used in the edge-of-glazing calculation. THERM will automatically assign the U-factor tag of Edge based on the dimension entered in the Edge of Glass Dimension when importing the glazing system, which for NFRC modeling should be $63.5 \mathrm{~mm}$ ( 2.5 inches). This dimension will be added above the sight line, defined by the Sight line to bottom of glass dimension when importing the glazing system.

- None: Use this tag for the $86.5 \mathrm{~mm}$ (3.5 inches) of glazing system that is modeled above the Edge-ofglazing, and for the glazing system exterior boundary condition. This is the default U-factor tag automatically assigned by THERM for all surfaces except the glazing system.

- SHGC Exterior: Use this tag for all exterior non-glazing surfaces. This tag is used to calculate the wetted length of the exterior frame to be used in WINDOW for the Solar Heat Gain Coefficient (SHGC) calculation.

The Blocking Surface checkbox should be checked for frames, but not for glazing systems.

To change the boundary condition for one boundary segment, double click on the segment, or single click and press Enter, and the Boundary Condition Type dialog box will appear. This dialog box allows specification of both the boundary condition and the U-factor tag at the same time. To change the boundary condition for multiple boundary segments, $\mathrm{cl}$ ick on the first segment and double click (or single click and press Enter) on the last segment in a counterclockwise direction. The choices made in the Boundary Condition Type dialog box will then be applied to all the selected boundary segments. It is also possible to change the boundary condition definition for boundary segments using the Select Material/BC toolbar button. Click on the Eye Dropper tool, click on a boundary segment whose definition is to be duplicated to other segments, then click on the boundary segment(s) which need that boundary condition definition. The program will 
assign both the boundary condition and U-factor tag from the copied segment. There is also one level of "undo" for boundary conditions, accessed through the Edit/Undo file menu choice.

If the interior boundary conditions do not have the Radiation Model set to "AutoEnclosure" (double click on the boundary condition to open the Boundary Condition Type dialog box which displays the setting), they can be easily changed to that model. To change the Radiation Model, go to the Boundary Condition Library, either through the Library menu, Boundary Condition Library choice, or by double clicking on a boundary condition segment and clicking on the Boundary Condition Library button. In the Boundary Condition Library, under the Radiation Model, click on the "Automatic Enclosure Model" radio button.

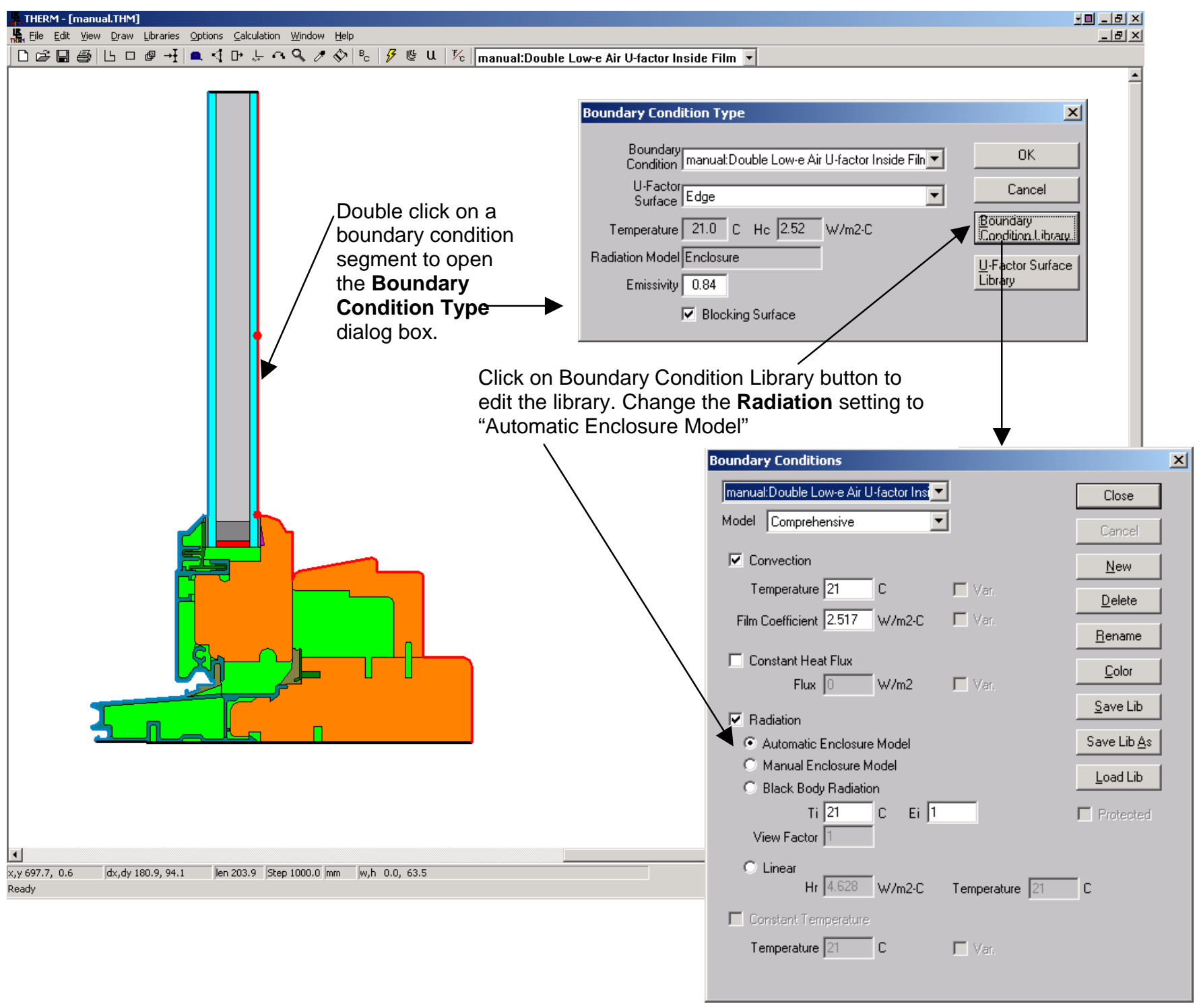

Figure 6-21. Make sure that the interior boundary conditions have set to "AutoEnclosure". 
When boundary conditions are generated for a model, the program keeps them in the model until they are regnerated. If the boundary conditions are regenerated, the program will display a dialog box with three choices for how the program should generate them, as shown below.

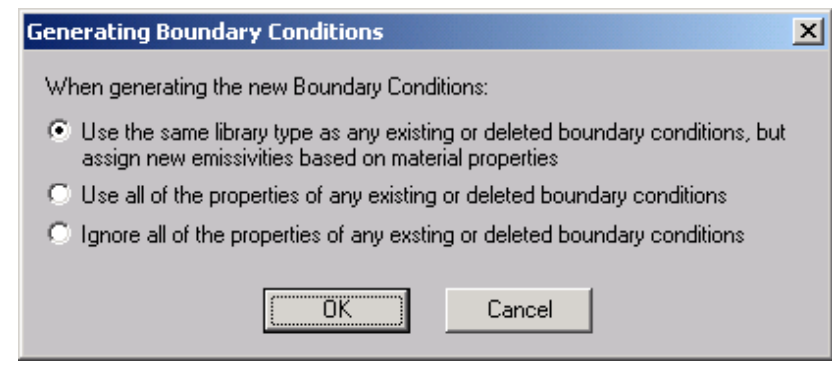

Figure 6-22. The dialog box displayed when boundary conditions are regenerated.

In general, pick the first option in "Generating Boundary Conditions" dialog box, which will pick up the emissivities of the materials in the model. However, do not select this option if you have edited the emissivities of material boundary conditions, such as frame cavities, by hand.

All the boundary conditions can be deleted by deleting one boundary segment.

Boundary conditions and U-factor tags are stored in libraries, and new entries can be defined for both using the Libraries menu choice. See Section 6.2, "Assigning Boundary Condition Definitions" in the THERM User's Manual for more detailed information.

Occasionally THERM loses track of the glazing system boundary conditions. If this happens they can be reassigned from the Boundary Condition Library. 


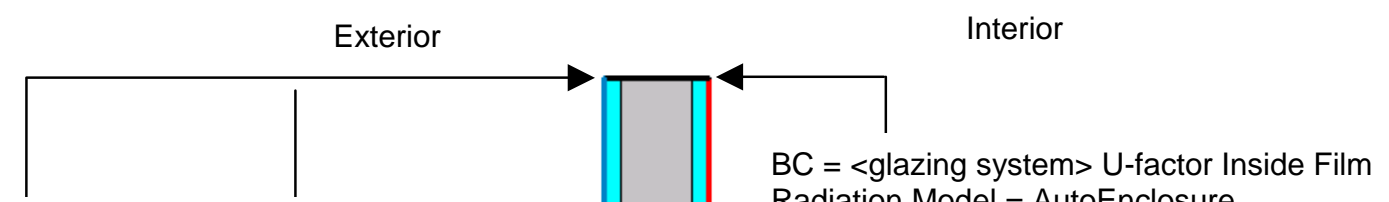

$B C=$ NFRC 100-2001 Exterior

U-factor tag $=$ None
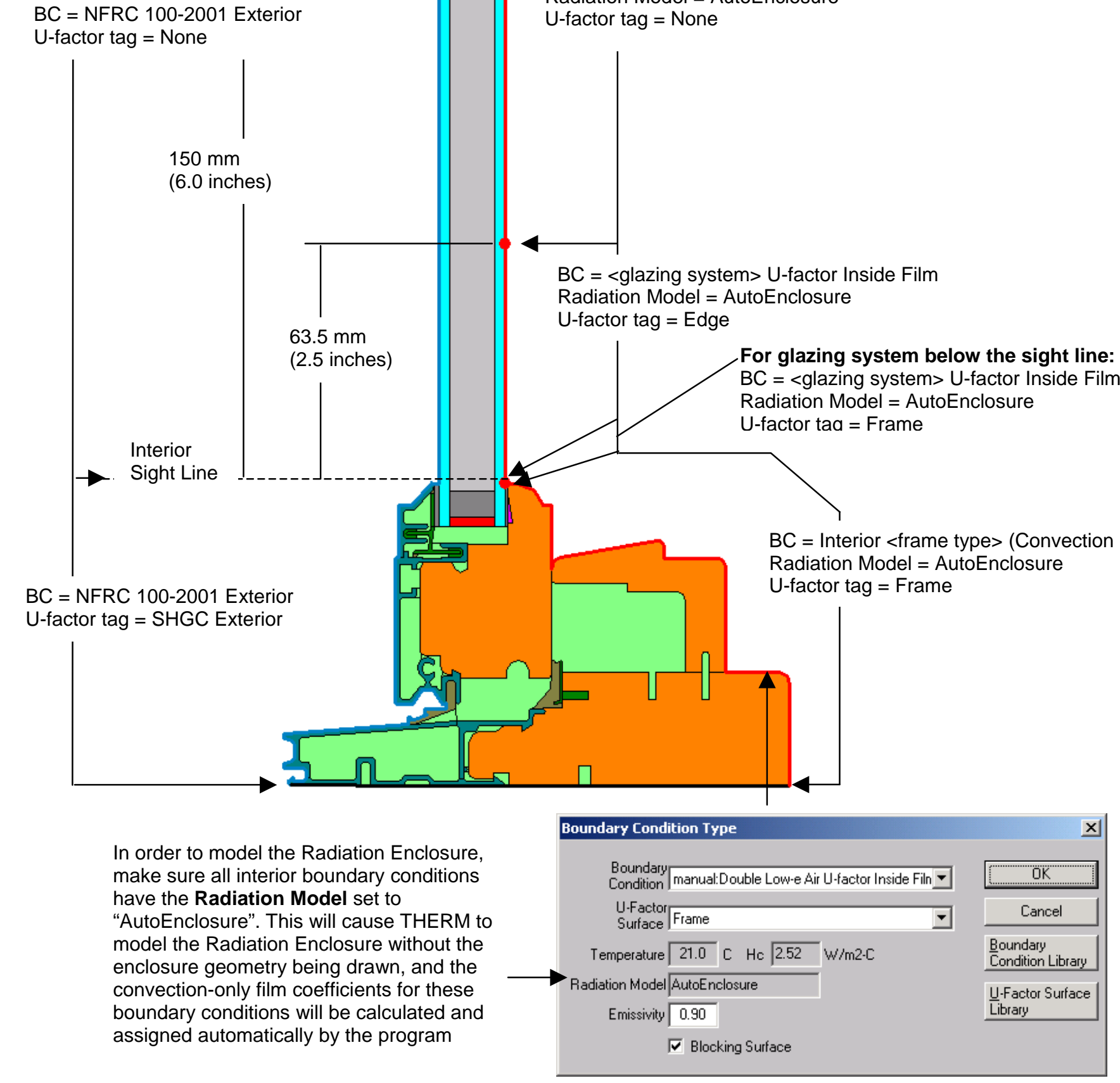

Radiation Model $=$ AutoEnclosure

U-factor tag $=$ None

Figure 6-23. Standard boundary condition assignments for most models. 


\subsubsection{Voids, Overlaps and Bad Points}

In order to perform a simulation, the geometry of the cross section must be correctly defined, i.e., there must not be any voids, overlapping polygons, or points that cannot be resolved by THERM. See Section 6.3, "Error Detection in THERM" in the THERM User's Manual.

While drawing, there are a number of features in THERM to help avoid creating voids, overlapping polygons, and bad points. The Always Check for Overlapping Polygons feature (in

Options/Preferences/Drawing Options) is the main tool used to avoid drawing overlaps and voids. This option can be turned off, but this is NOT recommended for most situations. The View Voids and Overlaps (from the Draw menu) feature is also a good tool for visual inspection to find voids and overlapping polygons in a cross section.

In addition to the features that attempt to prevent the creation of bad geometry during the drawing process, a number of error checks are performed when THERM calculates the boundary conditions in order to make sure the model geometry is correct.

For example, when the boundary conditions are calculated THERM identifies all points within $0.1 \mathrm{~mm}(0.004$ inches) and $0.01 \mathrm{~mm}$ (0.0004 inches) of each other (points closer together than $0.01 \mathrm{~mm}$ are automatically merged). A message box appears saying that there are points closer than the program tolerance, as shown in Figure 6-20. In general, select the option Automatically adjust points within tolerance option, in which case THERM will fix all of the points automatically. This now works even for models with thin films in glazing systems. If you are unsure about whether to let the program adjust the points automatically, select the Mark the points but don't adjust them option, which causes THERM to draw red circles in the problem areas. Then you can examine all the circled points and fix them if appropriate, or regenerate the boundary conditions and let the program automatically adjust them. If the marked points are actual details in the drawing that need to be kept, they do not have to be fixed, although they might cause meshing problems during the simulation because of too much detail. Once all the circled areas in the drawing have been examined, press the Boundary Conditions toolbar again, and if there are still "bad points", select the Automatically adjust points within tolerance option, in which case THERM will fix all of the points automatically. (Having THERM adjust the points is an "all or nothing" proposition -- which is why all the points should be examined first before having the program adjust them. Also keep in mind that the automatic fixing of points cannot be reversed by THERM.)

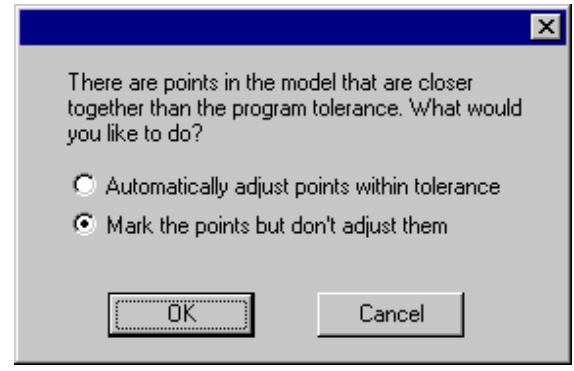

Figure 6-24. Message when trying to define boundary conditions indicating that there is a problem in the geometry.

The best way to fix points that are too close together is to delete or move the offending point. Many times, the point in question is in all the adjacent polygons, so it is best to delete the point in all the polygons at the same time by selecting all the polygons using the Shift key, then delete the point; it will be deleted from all the selected polygons.

THERM also marks Voids and Overlaps by checking for "lonely edges" -- two adjacent edges with a different number of points on each edge. THERM marks voids and overlapping polygons with red circles also. One way to eliminate these two conditions is by "jiggling" the points -- moving the points away from the adjacent polygon, then moving them back within the sticky distance and letting the program "snap" them to the 
polygon. Another way is to delete the offending polygon, and use the Fill Void toolbar button to create it again, which means that THERM will automatically align all points and ensure that no voids are created.

THERM can also find invalid polygons caused by points collapsing on each other, creating a zero-area extension. In this case both the point at the tip of the extension and the extra point at its base must be deleted.

Turning off the Material Color and the Boundary Conditions from the View menu can sometimes help to find the problems.

THERM will simulate a problem with bad points, because in some cases details are drawn that give bad points but which need to be modeled.

If changes are made to polygons on the boundary of the cross section, the boundary conditions must be generated again. If all the corrections are on internal polygons, the boundary conditions will not be automatically deleted. However, in this case, it is still a good idea to press the Boundary Conditions toolbar button again, to ensure that all points on all surfaces have boundary segments defined between them.

\subsection{Calculating Results}

When the boundary conditions have been correctly defined for the cross section, the model can be simulated. Begin the calculation by either clicking on the Lightning Bolt toolbar button, selecting the Calculation/Calculation menu option, or pressing F9.

See Chapter 7 in the THERM User's Manual for detailed information about the calculation procedures, and the results produced by THERM.

\subsubsection{Meshing}

The first step performed by THERM in the simulation is to generate a mesh from the geometry on which the thermal analysis is based. This mesh is generated automatically, and a summary of how it works is found in "Appendix C" in the THERM User's Manual.

The one variable in the mesh generation that the user can control is the Quadtree Mesh Parameter, which determines how fine the mesh is. The larger the mesh parameter value, the smaller the largest element in the mesh is. The NFRC required default value for the mesh parameter is 6, as shown in Table 6-4.

Occasionally the mesh generator cannot create a closed mesh with the geometry that has been drawn, usually created because of extremely fine detail in the cross section. THERM circles the point(s) where the mesher failed. If this happens, first:

- Rerun the problem with a higher mesh parameter value. This causes the program to generate a finer mesh, and results in longer run times. The default mesh parameter value is 6 . Mesh parameter values greater than 8 are rarely needed for single cross sections, but may be needed for full height product simulations. Options/Preferences/Simulation has a setting to "Automatically increment mesh parameter". If this option is checked the program will increase the mesh parameter until the problem meshes.

If this doesn't work:

- Simplify any unnecessarily complicated details. Keep in mind that the circled point often lies on the boundary between two polygons; the detail that is causing the problem could be anywhere in those two polygons and is often not at the place that is circled. One method to try is to break the problem polygon into multiple polygons, while keeping the geometry otherwise unchanged. Another option is to delete unnecessary points, such as in curves, because each point generates a mesh node. 


\subsubsection{Error Estimator}

THERM has a built-in error estimator, and automatically refines the mesh in the areas where it is needed. The details of the error estimation algorithm are found in "Appendix C" of the THERM User's Manual. The error estimator returns the Percent Error Energy Norm, which is related to the gradient of heat flux (energy). If the returned value is greater than the target value, THERM refines the mesh in areas with a high rate of change in the heat flux.

The default value for the Percent Error Energy Norm in THERM to 10\%, as shown in Figure 6-19. This setting forces THERM to do the error estimator calculation and return a value for the error estimate (recorded with the U-factor results) and the program may or may not refine the mesh, depending on the result of the Error Energy Norm. If an error message is displayed saying that the error energy norm cannot be reached, increase the Maximum Iterations value (set by default to three iterations). An Error Energy Norm of less than $10 \%$ is equivalent to an uncertainty of less than $1 \%$ in the U-factor. 


\subsubsection{Required Settings for NFRC Simulations}

In the THERM File Options tab found in the Options/Preferences menu, the following values must be set:

Table 6-4. Options/Preferences settings for THERM NFRC modeling

\begin{tabular}{|l|l|}
\hline Setting & Value for NFRC Modeling \\
\hline Quad Tree Mesh Parameter & 6 or greater \\
\hline Maximum \% Error Energy Norm & $10 \%$ \\
\hline Run Error Estimator & must be checked \\
\hline
\end{tabular}

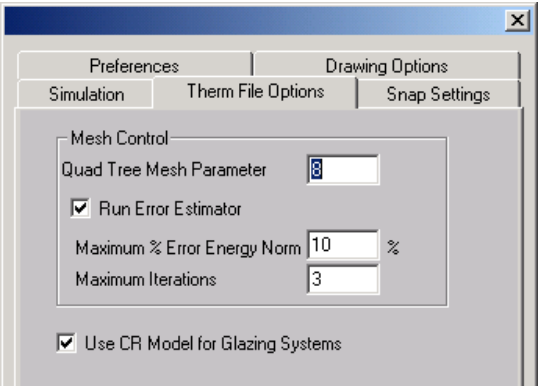

Figure 6-25. NFRC required THERM File Options settings.

In the Simulation tab found in the Options/Preferences menu, the Relaxation Parameter can be set to 1.0, and the feature to "Automatically adjust relaxation parameter" should be set, as shown in the figure below.

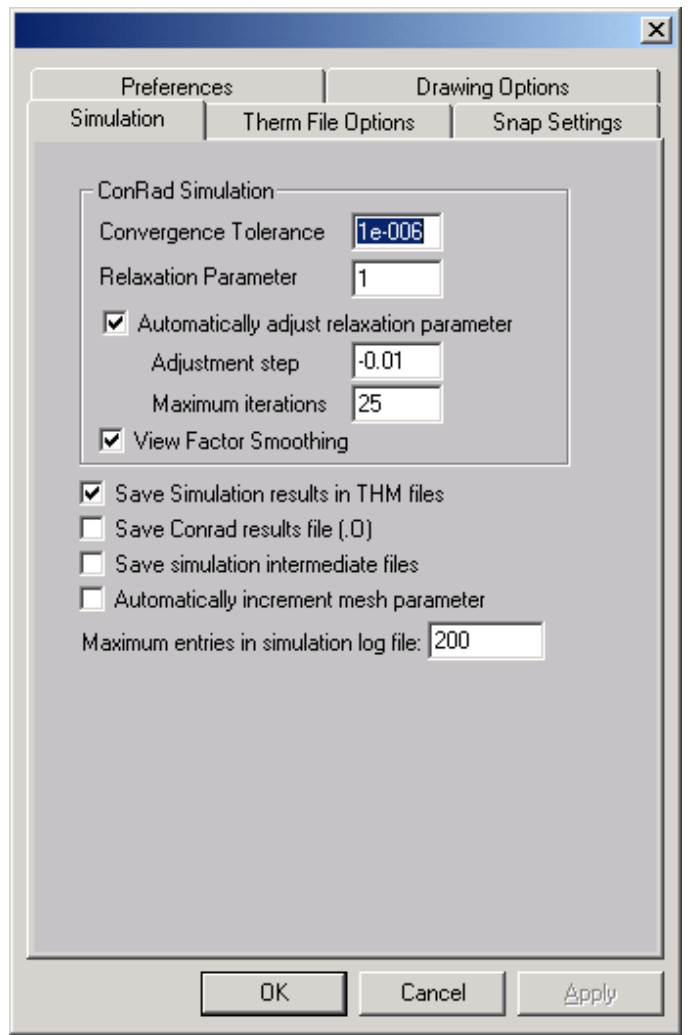

Figure 6-26. Simulation option settings. 


\subsubsection{Calculations}

When a calculation is started, THERM can do the calculations in the background while another model is being worked on. There is also a Calculation Manager, accessed from the Calculation/Calc Manager menu option that allows the submittal of many files at one time to be calculated in the order they are added. The Calc Manager Log shows whether the jobs ran or not. Section 7.2, "Calculations," in the THERM User's Manual contains detailed information about the Calc Manager.

\section{Calculating Multiple Glazing Options}

If a base case file has been created with multiple glazing options (see Section 6.4.3, "Multiple Glazing Options"), when the file is simulated using the Calc toolbar, the Calculation/Calculation menu option, or the F9 shortcut key, the Glazing Option Simulation dialog box will appear with the following options, as shown in Figure 6-20:

- Create the glazing option files and perform all simulations: This choice is the default, and it creates and runs all the THERM files for the multiple glazing options defined in the base case file.

- Simulate the current file (base case) only: This choice is useful when setting up the base case file to make sure there are no geometry errors before running the entire multiple glazing options.

- Create the glazing option files but do not perform the simulations: This choice will cause the program to make individual THERM files for each of the glazing options associated with the base case file, with either the Glazing System ID or Name appended on to the base case filename, but it will not simulate the files. These files can then be opened and simulated individually, or simulated through the Calc Manager.

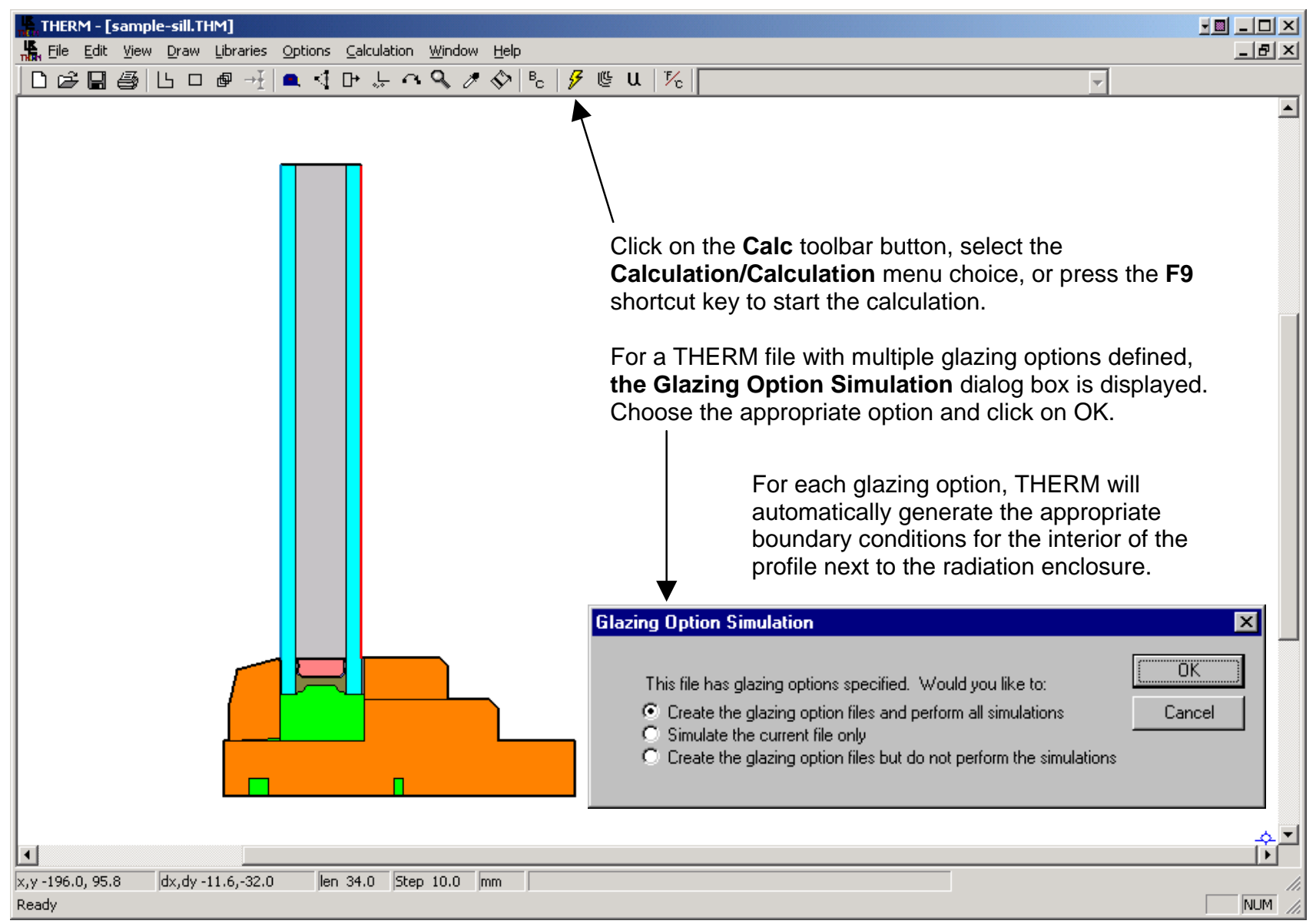

Figure 6-27. When a file with multiple glazing options is calculated, THERM gives several calculation options. 
Multiple base case files with multiple glazing options in each file can be added to the Calc Manager, as shown in the figure below. The Calc Manager does not ask the three simulation-option questions; it implements the first choice, which is that to make all the files and then simulate them. The Calculation Log can be viewed to see the status of the runs. The information in the Calculation Log can be sorted by clicking on a column heading.

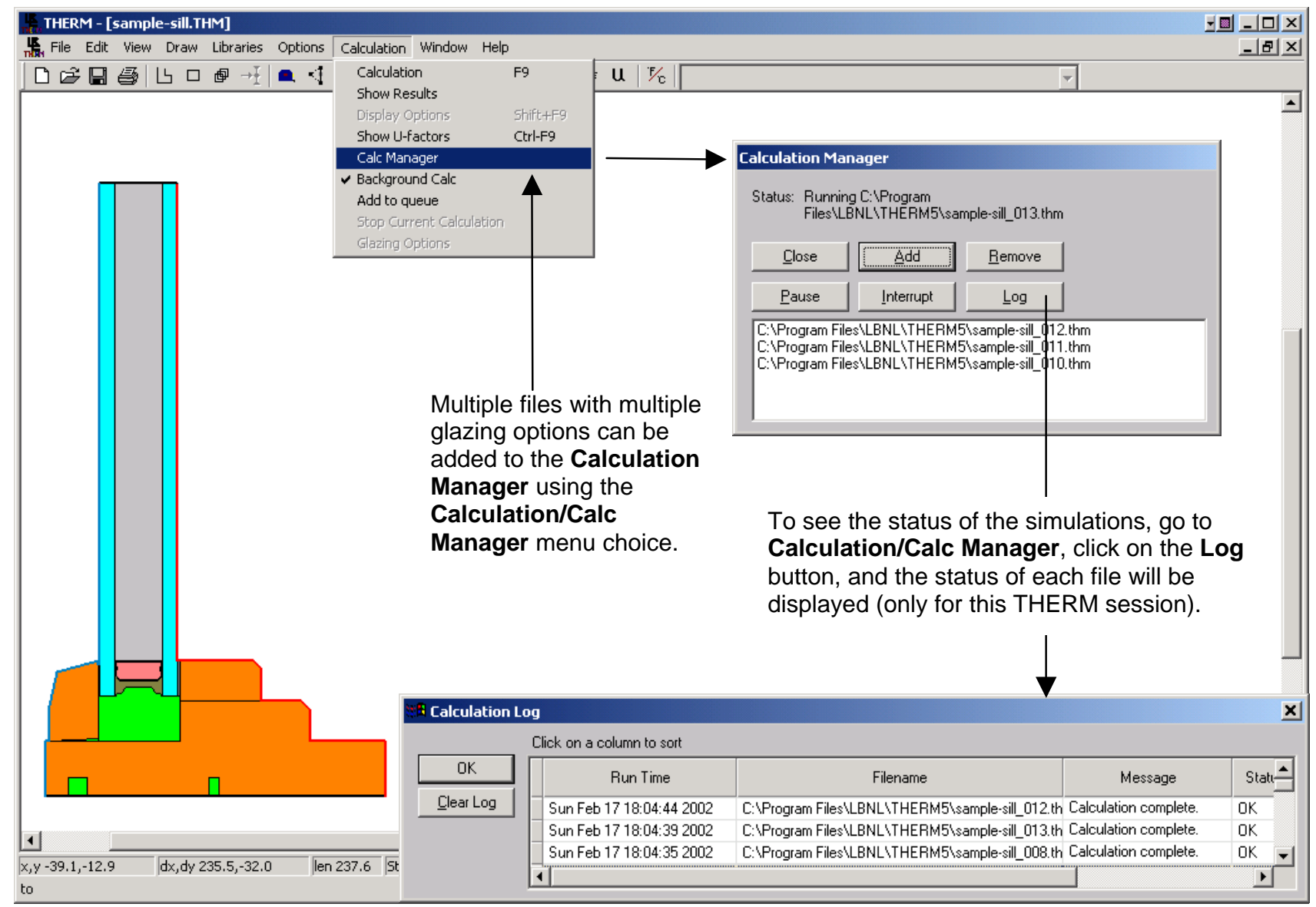

Figure 6-28. The Calc Manager can be used to simulate multiple files with multiple glazing options in each file. 


\subsubsection{Results}

When the calculation is finished, the U-factors (shown in the figure below) can be viewed using the Calculation/Show U-factor menu choice or the Show U-factor toolbar button. $u$

In addition, the graphic display of the results can be controlled using either the Calculation/Show Results menu choice or the Show Results toolbar button. 幽

The U-factors can be viewed for Projected X, Projected Y, Total Surface Length, or a Custom Frame Length. U-factors are generally calculated in the Projected $\mathrm{Y}$ direction, but there are a few special cases where the Custom Frame Length feature must be used for an NFRC simulation. (See the following discussion in Section 6.6.6, "Custom Frame Length" for an example using Custom Frame Length.)

The SHGC Exterior U-factor is meaningless - it is a result of the SHGC Exterior tag, which is used by WINDOW to calculated the frame exterior wetted length for the ISO 15099 SHGC value.

Frame and Edge $U$-factors are reported. These values will be used by WINDOW to calculate the total product U-factor.

\% Error Energy Norm must be less than $10 \%$.

\section{U-Factors}

Uractors

(1)
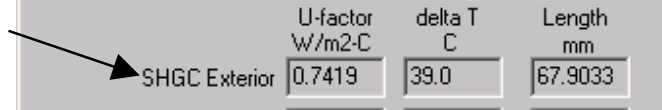

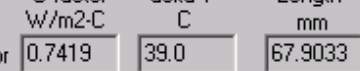

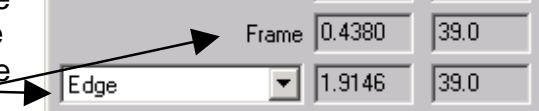

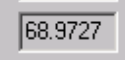

naw

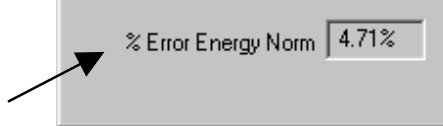

U-factors are generally calculated in the Projected $Y$ dimension.

Figure 6-29. U-factor results from the Calculation/Show U-factors menu choice.

Local temperatures can be displayed at the cursor (from the View/Temperature at Cursor menu choice) and average temperatures can be displayed with the tape measure (from the Options/Preferences menu, Drawing Options tab, check the Tape Measure Average Temperature checkbox).

THERM has a report which summarizes all the details of a THERM file, including the polygon ID numbers for all cavities. This report can be viewed and printed from the File/Report menu choice.

THERM files (*.THM) can be saved without the detailed temperature data by selecting the Options/Preferences menu, going to the Simulation tab, and unchecking Save Simulation results in THM files. The THERM file will then contain only the geometry and boundary conditions used to run the simulation, the U-factor results, and the report, thus making the file much smaller. 


\subsubsection{Custom Frame Length}

There is a case where THERM will not calculate the project frame length correctly. This happens when a section of an adiabatic boundary "overlaps" a boundary with a U-factor tag in the projected dimension over which the length is being calculated. The figure below illustrates this. This incorrect frame length will cause the U-factor over the projected length to be calculated incorrectly. The U-factors results dialog box has a feature which allows a custom frame length to be entered, in order for THERM to calculate the correct Ufactor. This situation happens in some skylight files, although it potentially could happen in any model. The figure below describes the steps required to use the Custom Frame Length feature.

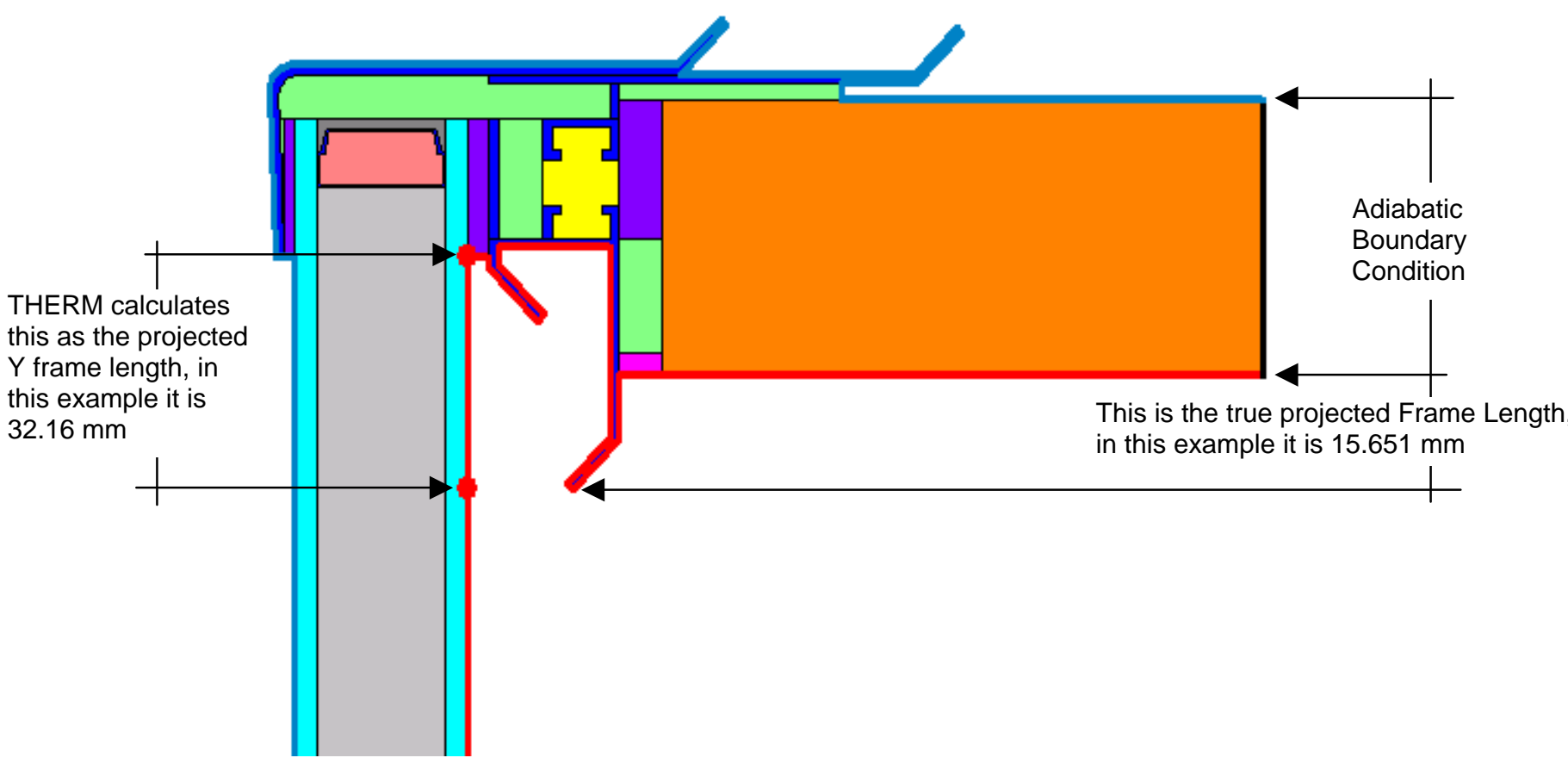

Figure 6-30 Example of a case where THERM does not calculate the projected $Y$ frame length correctly because of an adiabatic boundary condition that "overlaps" an interior boundary condition.

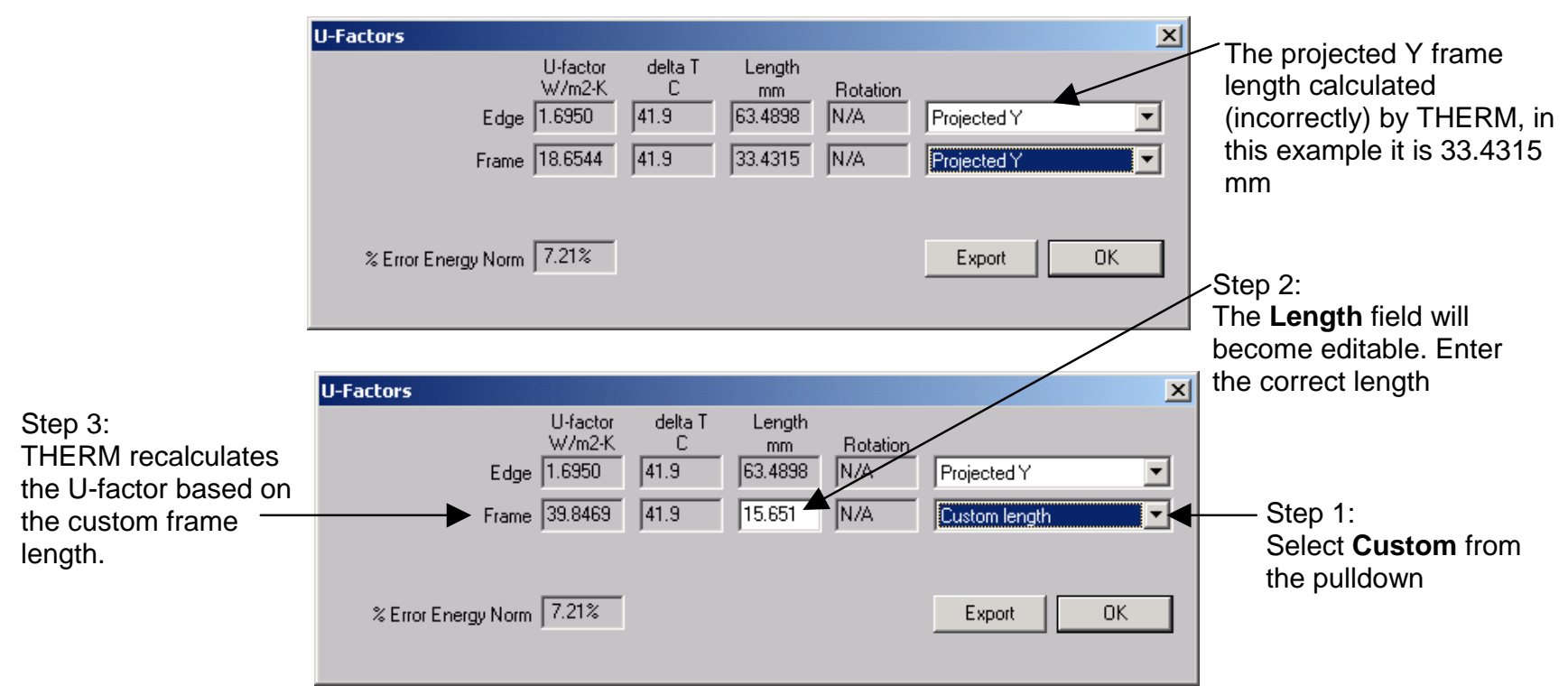

Figure 6-31 The Custom Frame Length feature allows the correct frame length to be entered. 


\subsubsection{Importing Results into WINDOW}

Because the U-factors and temperatures from individual THERM cross sections will be used for the whole product calculations in WINDOW, it is necessary to import the THERM files with U-factor and simulation temperature results into the WINDOW program. This is explained in detail in the WINDOW User's Manual, Section 4.7.3, "Importing THERM Files". The basic steps are:

- Create the THERM models, calculate the results, and save and close the files.

- Open WINDOW and go to the Frame Library List View.

- Click on the Import button, select the "Therm Files" option from the pulldown, then THERM files to import (select multiple files using either Shift or Ctrl keys).

- WINDOW will ask how to number the new Frame Library records, either sequentially starting from the last existing record, or overwriting existing records.

- WINDOW will add each THERM file to the Frame Library as a separate record, assigning the THERM file name as the record name by default.

- If necessary, set (in WINDOW File/Preferences/Calculation Options) the frame Absorptivity to 0.30 or 0.50 , according to the NFRC 200 specifications.

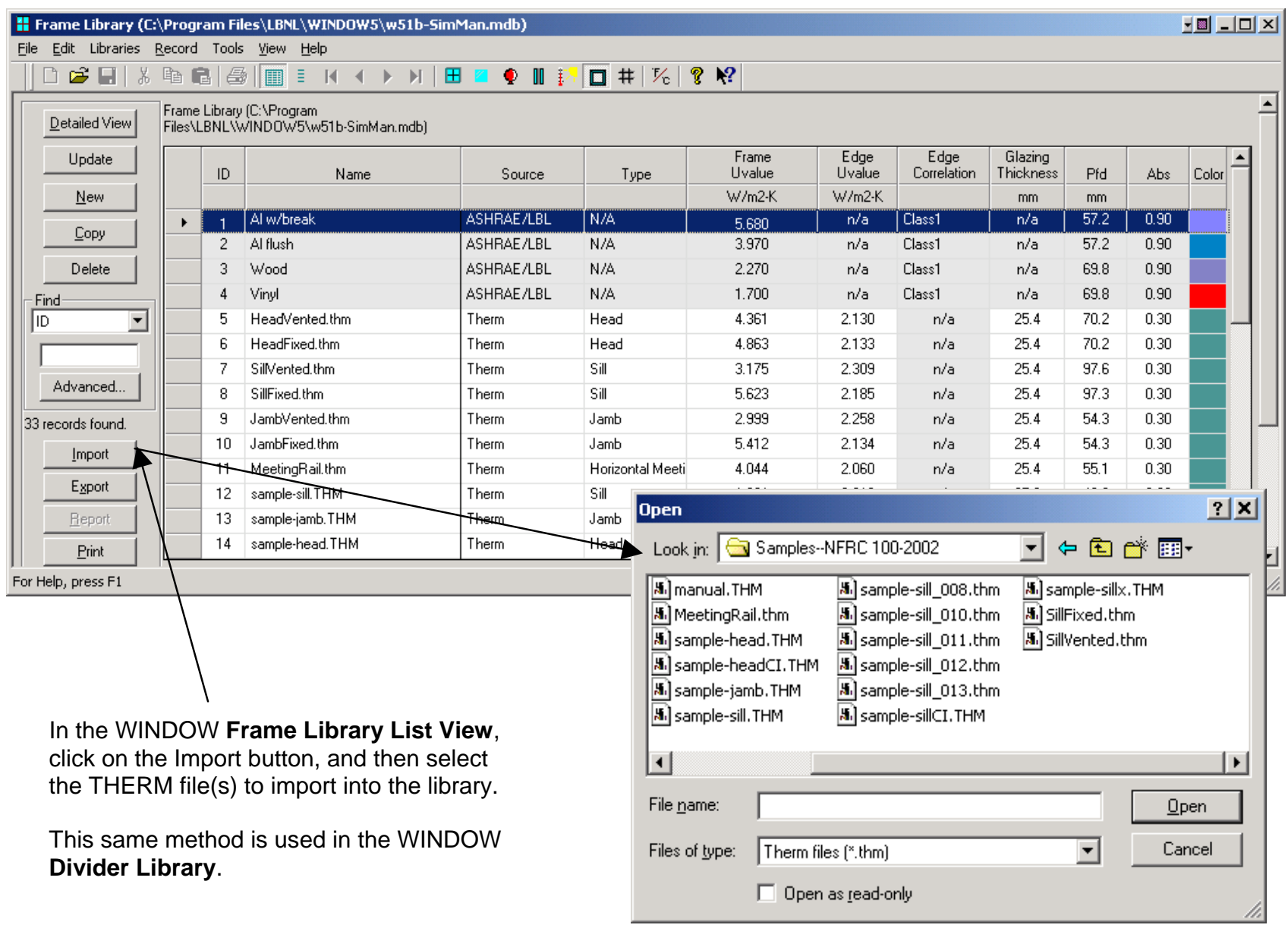

Figure 6-32 Importing THERM files into the WINDOW Frame Library. 



\section{TOTAL PRODUCT CALCULATIONS USING WINDOW}

\subsection{Overview}

WINDOW determines the total product U-factor according to NFRC 100 and ISO 15099 by calculating an area-weighted average of the U-factors of the product components and accounting for product height:

- Frame and edge values for each cross section (frames and dividers) calculated in THERM: The Ufactors are area-weighted based on the projected area on a plane parallel to the glass (the Projected $\mathbf{Y}$ in THERM if the cross section is modeled vertically), not the total surface area of the frame and edge.

- Center-of-glazing values from WINDOW

WINDOW calculates the total product area-weighted solar heat gain coefficient (SHGC) and visible transmittance (VT)according to the NFRC 200 procedures and the total product Condensation Resistance (CR) according to the NFRC 500 procedures.

\subsection{Frame and Edge U-factors from THERM}

As discussed in Section 6.6.7, "Importing Results into WINDOW", THERM files are imported into the WINDOW Frame and Divider Libraries in order to calculate the whole product values in WINDOW.

When the THERM files are imported into the WINDOW Frame and Divider Libraries, they can be used in whole product calculations. The Source field in the Frame Library indicates whether the files were imported from THERM, as shown in the following figure.

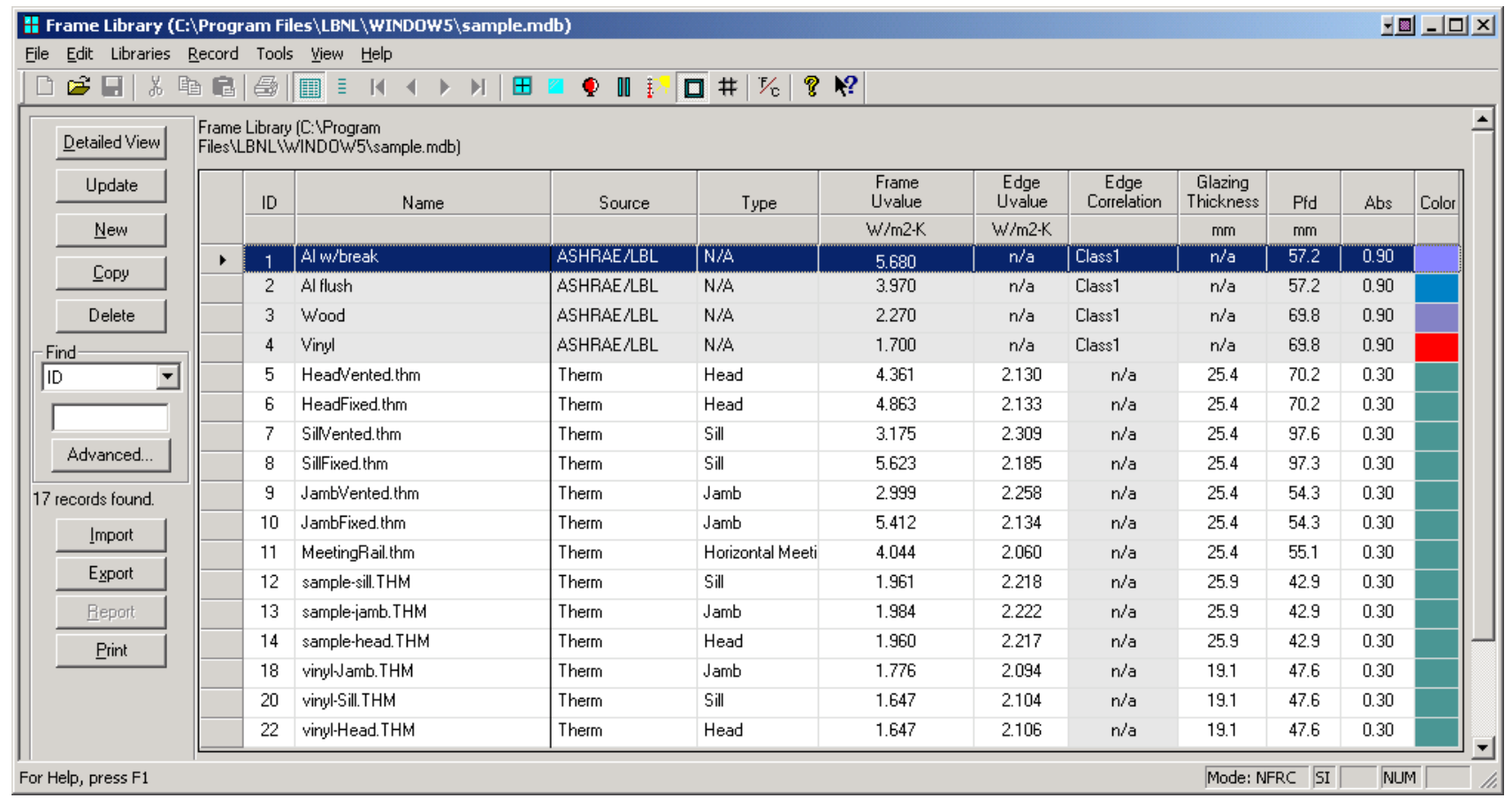

Figure 7-1. WINDOW Frame Library with records imported from THERM . 


\subsection{Center-of-Glazing U-factors from WINDOW}

The WINDOW User's Manual contains detailed information about calculating the center-of-glazing U-factors.

For an NFRC simulation, create glazing systems that represent all the glazing types to be modeled from the glass matrix provided by the manufacturer. In WINDOW, access the Glazing System Library List View either from the Libraries/Glazing System menu, by clicking on the Glazing System Library toolbar button, or by pressing $\mathbf{F 5}$.

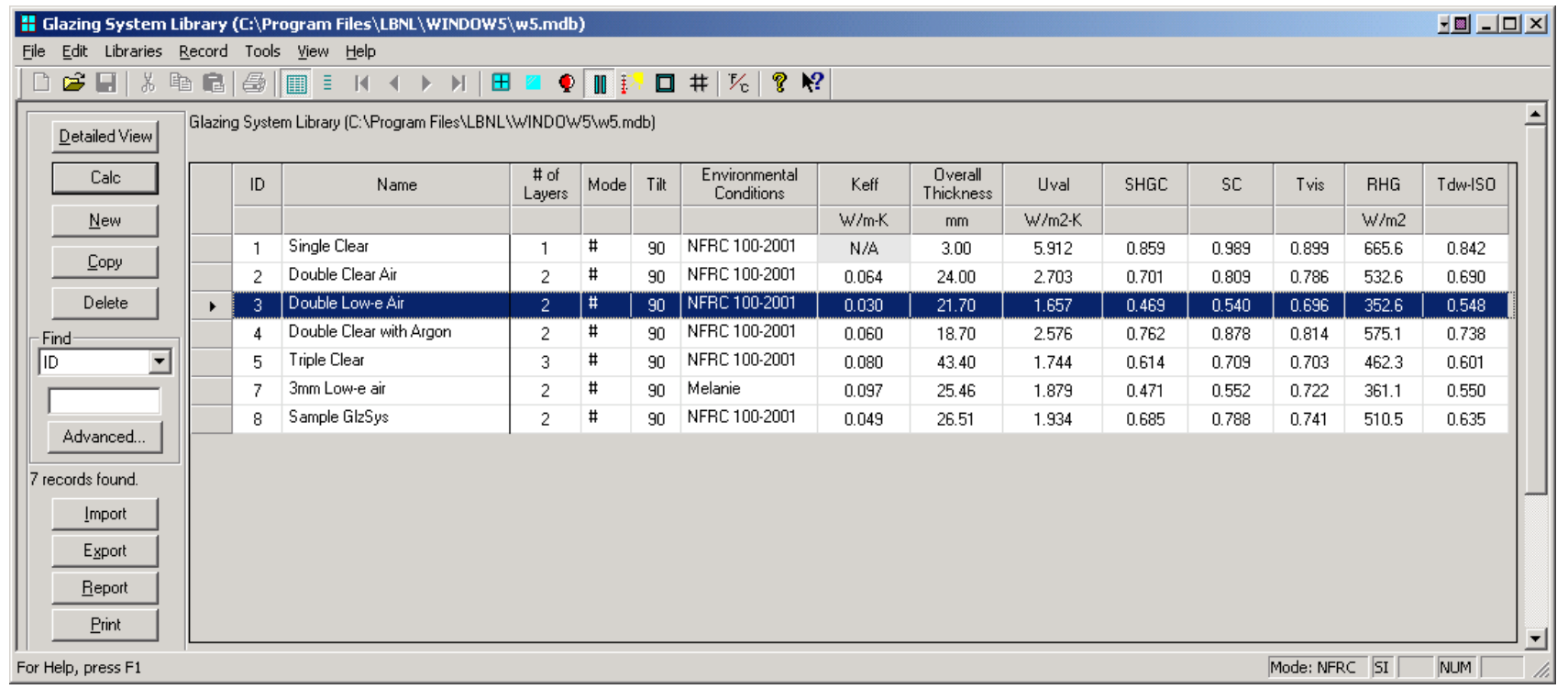

Figure 7-2. WINDOW Glazing System Library .

For NFRC certified simulations, use the currently approved International Glazing Database (IGDB) spectral data, imported into the WINDOW Glass Library. Glazing Systems that use approved spectral data will have a "\#" in the Mode field, as shown in the figure above.

The U-factors shown in the Glazing System Library are based on a on meter default height. When these glazing systems are used in whole products, in the Window Library, the center-of-glazing U-factors will be recalculated based on the actual product height specified in the Window Library. Therefore, the U-factors in the Glazing System and Window Libraries will probably be slightly different. 


\subsection{Overall Product U-factor, SHGC, VT, and CR Calculations}

When the THERM results have been imported into the WINDOW Frame and Divider Libraries, and the needed glazing systems have been defined in the Glazing System Library (also needed for the THERM simulations), the whole product values for U-factor, SHGC, VT and CR can be calculated in the Window Library. Calculating these values is explained briefly below and in more detail in the WINDOW User Manual.

In the Window Library Detail View, set the appropriate values on the left-hand side of the screen, which depend on the type of fenestration product. The Mode field should be set to "NFRC", and the Type field should be set to the appropriate choice for the fenestration product being modeled. The complete list of choices can be viewed by clicking on the double arrow next to the Type pulldown list. The choices with the Size field set to "NFRC" are the official NFRC sizes. The Environmental Conditions field should be set to "NFRC 100-2001".

On the right-hand side of the screen, for each cross section component, select the appropriate records from from the Frame and Divider libraries. When the frame cross sections and the glazing systems are specified, click on the Calc button and WINDOW will calculate the total product U-factor, SHGC, VT and CR, shown in the lower left corner of the main screen. In addition, for obtaining NFRC rating values, the program calculates the $\mathrm{SHGC}_{0}, \mathrm{SHGC}_{1}, \mathrm{VT}_{0}$ and $\mathrm{VT}_{1}$, discussed in detail in the following section.

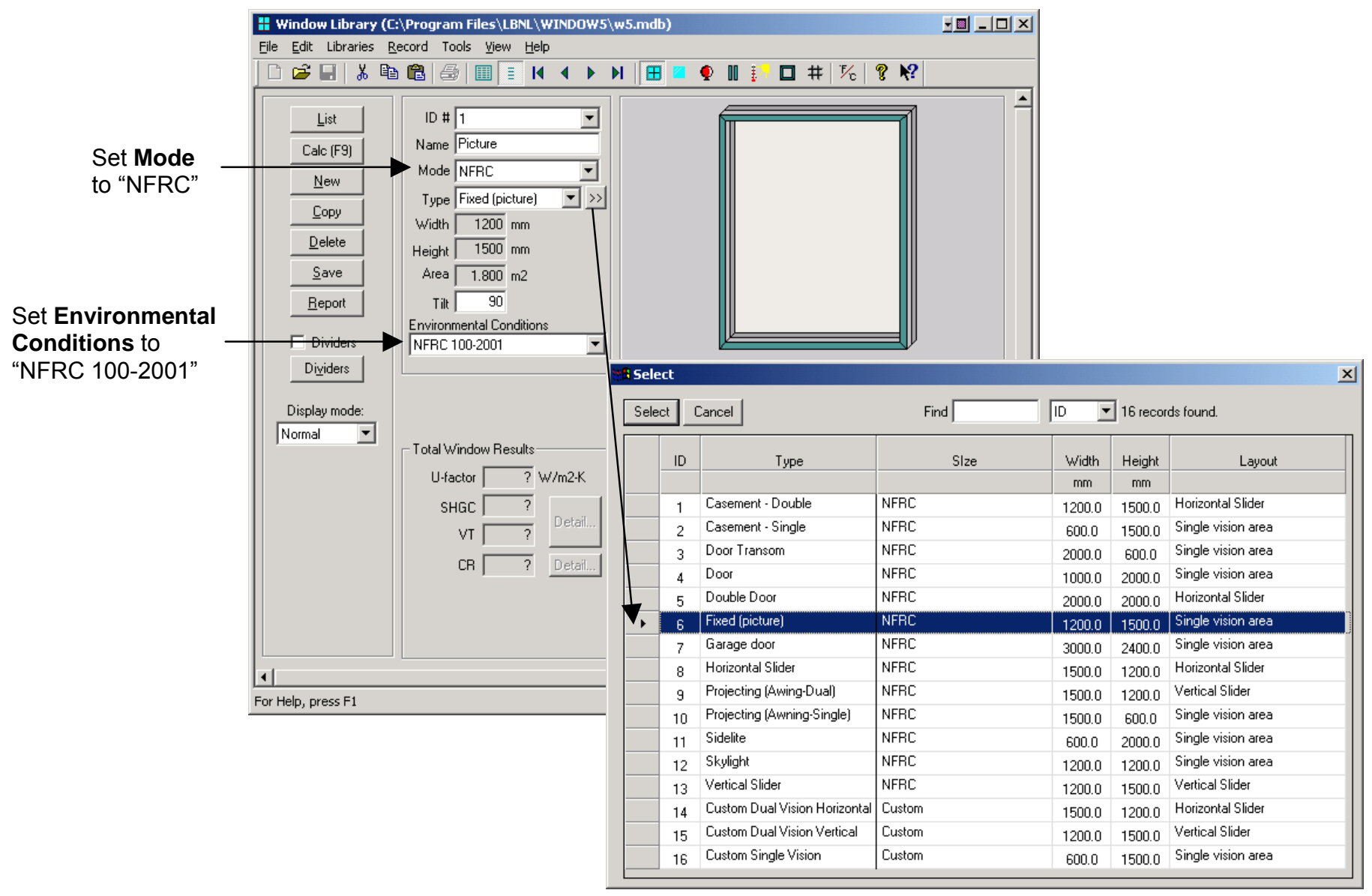

Figure 7-3. Window Library Detail View where the whole product is defined. 
Once the values have been calculated, the results are displayed in the Total Window Results section, as shown below. The Detail button next to the SHGC and VT results can be used to view the $\mathrm{SHGC}_{0}, \mathrm{SHGC}_{1}$, $\mathrm{VT}_{0}$ and $\mathrm{VT}_{1}$ values, and the Detail button next to the $\mathrm{CR}$ result is used to view the intermediate values used to determine the overall $\mathrm{CR}$ result.

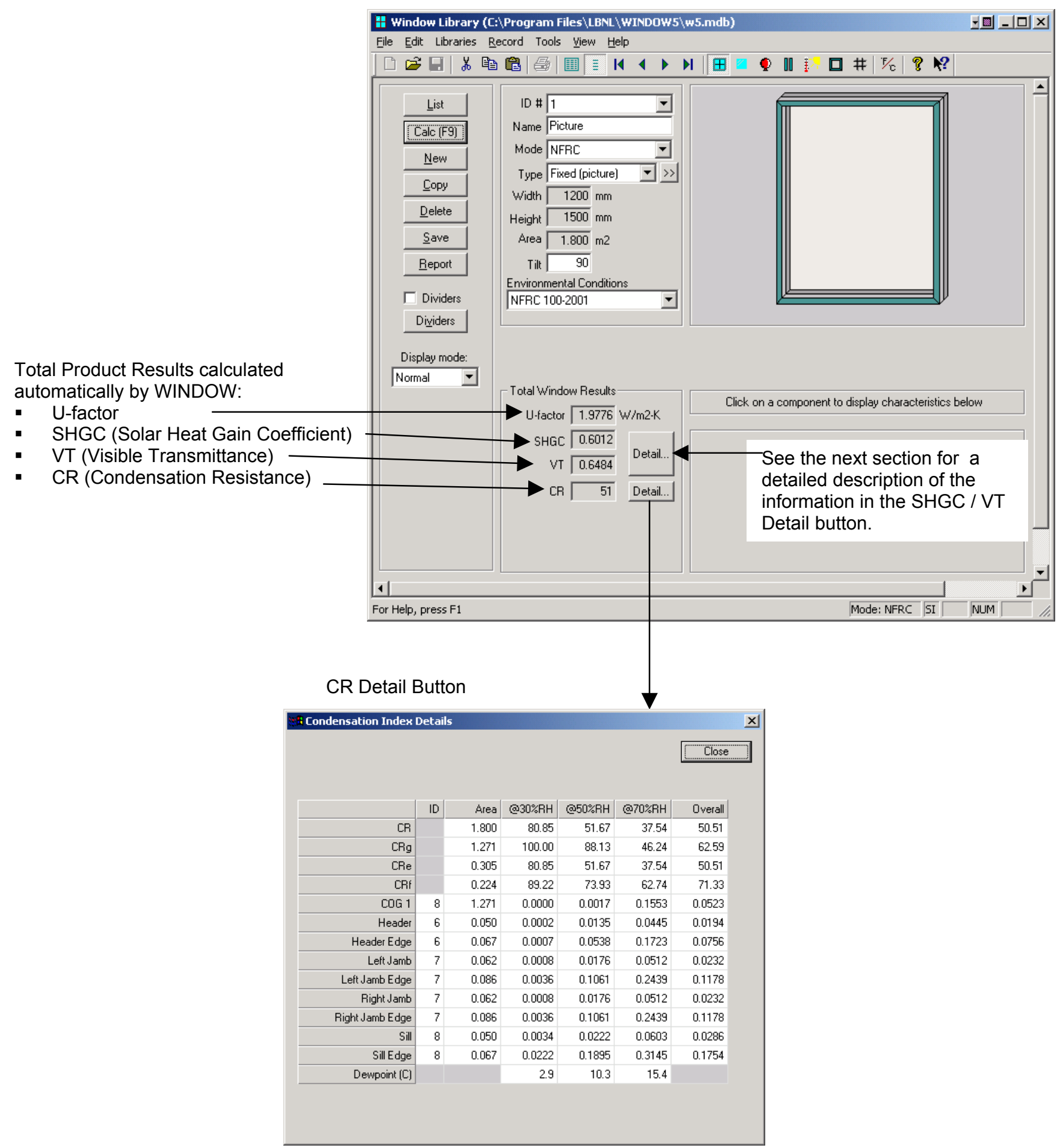

Figure 7-4. Window Library Detail View where the whole product results are calculated and displayed. 


\subsubsection{Individual Product SHGC and VT (SHGC 0 \& 1, VT 0 \& 1)}

It is not necessary to calculate the SHGC and VT for all indivual products and associated model sizes. NFRC 200 Section 6.1.1 outlines a procedure for determining $\mathrm{SHGC}_{0}, \mathrm{SHGC}_{1}, \mathrm{VT}_{0}, \mathrm{VT}_{1}$ values which can then be used to calculate the SHGC and VT of any individual product. This procedure is used to obtain NFRC rating values.

These values are calculated in WINDOW for the best glazing option modeled with the highest frame and edge U-factor frame, as outlined in NFRC 200, Section 6.1.1 (a). The values calculated from that one case are then used to calculate the SHGC for any other glazing options using Equations 3 and 4 in NFRC 200 -- do not use the $\mathrm{SHGC}_{0}, \mathrm{SHGC}_{1}, \mathrm{VT}_{0}, \mathrm{VT}_{1}$ from WINDOW5 for every glazing option, just for the "best" (lowest centerof-glass) glazing option in the product matrix.

The values are calculated for the product using the best glazing system, for three cases:

- No dividers

- Dividers $<25.4 \mathrm{~mm}\left(1^{\prime \prime}\right)$, modeled at $19 \mathrm{~mm}\left(0.75^{\prime \prime}\right)$

- Dividers $\geq 25.4 \mathrm{~mm}\left(1^{\prime \prime}\right)$, modeled at $38 \mathrm{~mm}\left(1.50^{\prime \prime}\right)$

WINDOW automatically calculates these values for all records in the Window Library, using the U-factor for the default divider as defined in NFRC 200. The values can be displayed by clicking on the Details button next to the whole product results for SHGC and VT, as shown in the figure below.

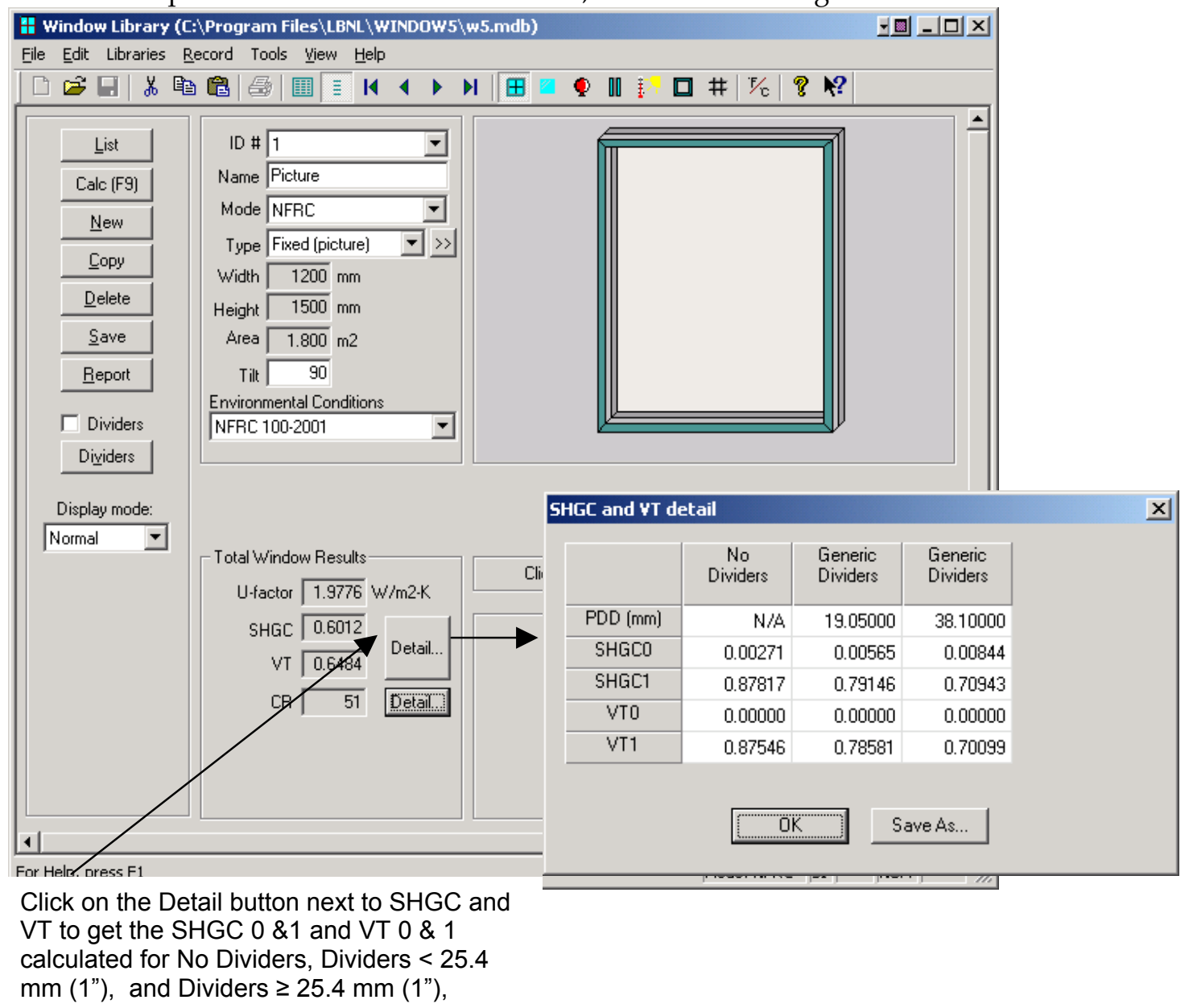

Figure 7-5. Click on the Detail button to get the SHGC $0 \mathcal{E} 1$ and VT 0 \& 1 results. 
If the product has real dividers, the program will calculate the SHGC 0 \& 1 and VT 0 \& 1 values for the generic dividers as well as for the actual dividers, as shown below. For NFRC simulations, the default divider results should always be used.

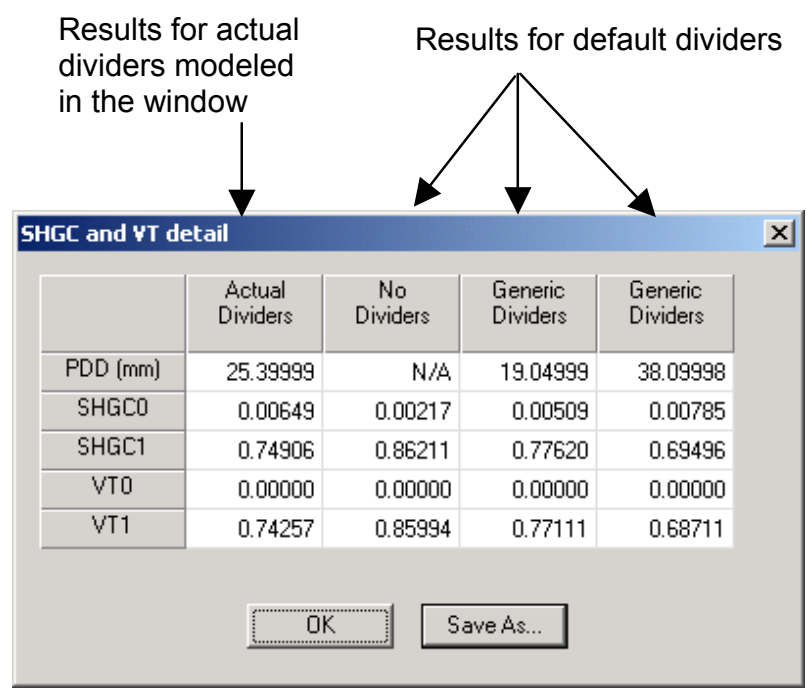

Figure 7-6. The SHGC 0 \& 1 and VT 0 \& 1 results are shown for the actual dividers if they are modeled. 


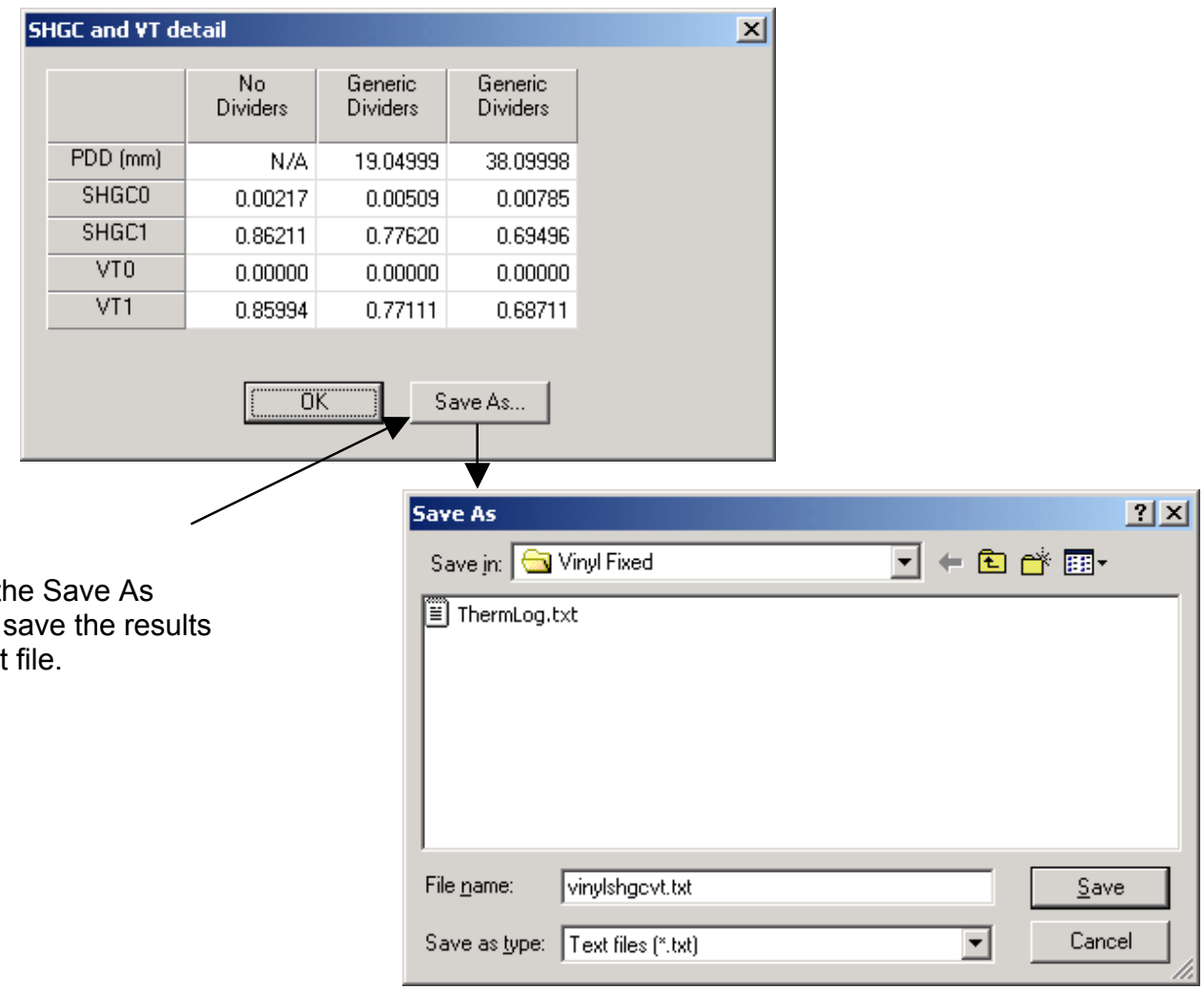

Click on the Save As button to save the results into a text file.

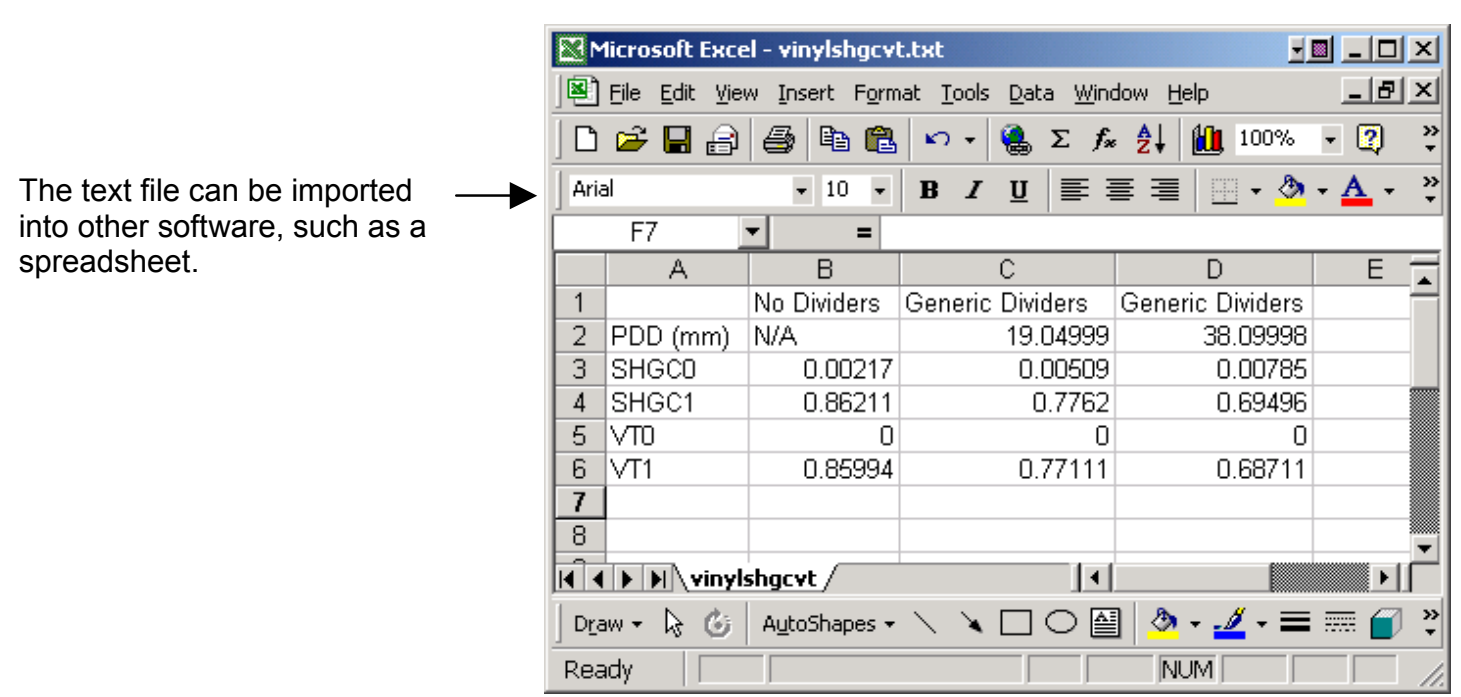

Figure 7-7. The SHGC 0 \& 1 and VT 0 \& 1 results can be saved to a text file which can be imported into other applications, such as a spreadsheet or word processing program. 


\subsubsection{Whole Product U-factor With Dividers}

The generic dividers used to calculate the $\mathrm{SHGC}_{0}, \mathrm{SHGC}_{1}, \mathrm{VT}_{0}, \mathrm{VT}_{1}$ values are NOT used to calculate the whole product U-factor, if the whole product is manufactured with dividers. In that case, the actual divider geometry is modeled in THERM, the THERM file is imported into the WINDOW Divider Library, and used in a record (different from those used for the $\mathrm{SHGC}_{0}, \mathrm{SHGC}_{1}, \mathrm{VT}_{0}, \mathrm{VT}_{1}$ calculations) in the Window Library. This is shown in the following figures.

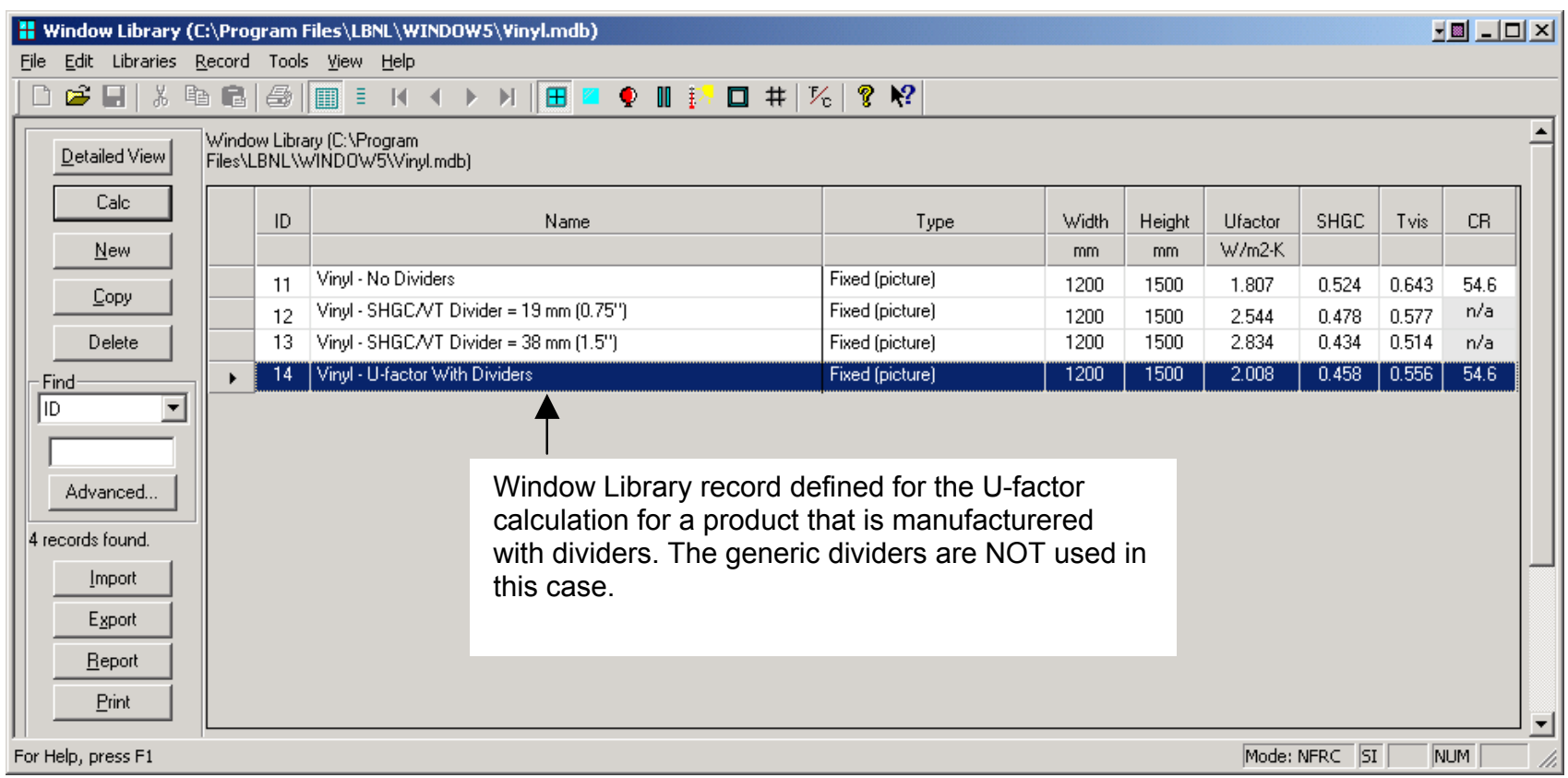

Figure 7-8. Window Library List View for the "U-factor with Dividers" case.

The Window Library Detailed View shows that the divider used for this product is from a THERM file, rather than one of the generic dividers.

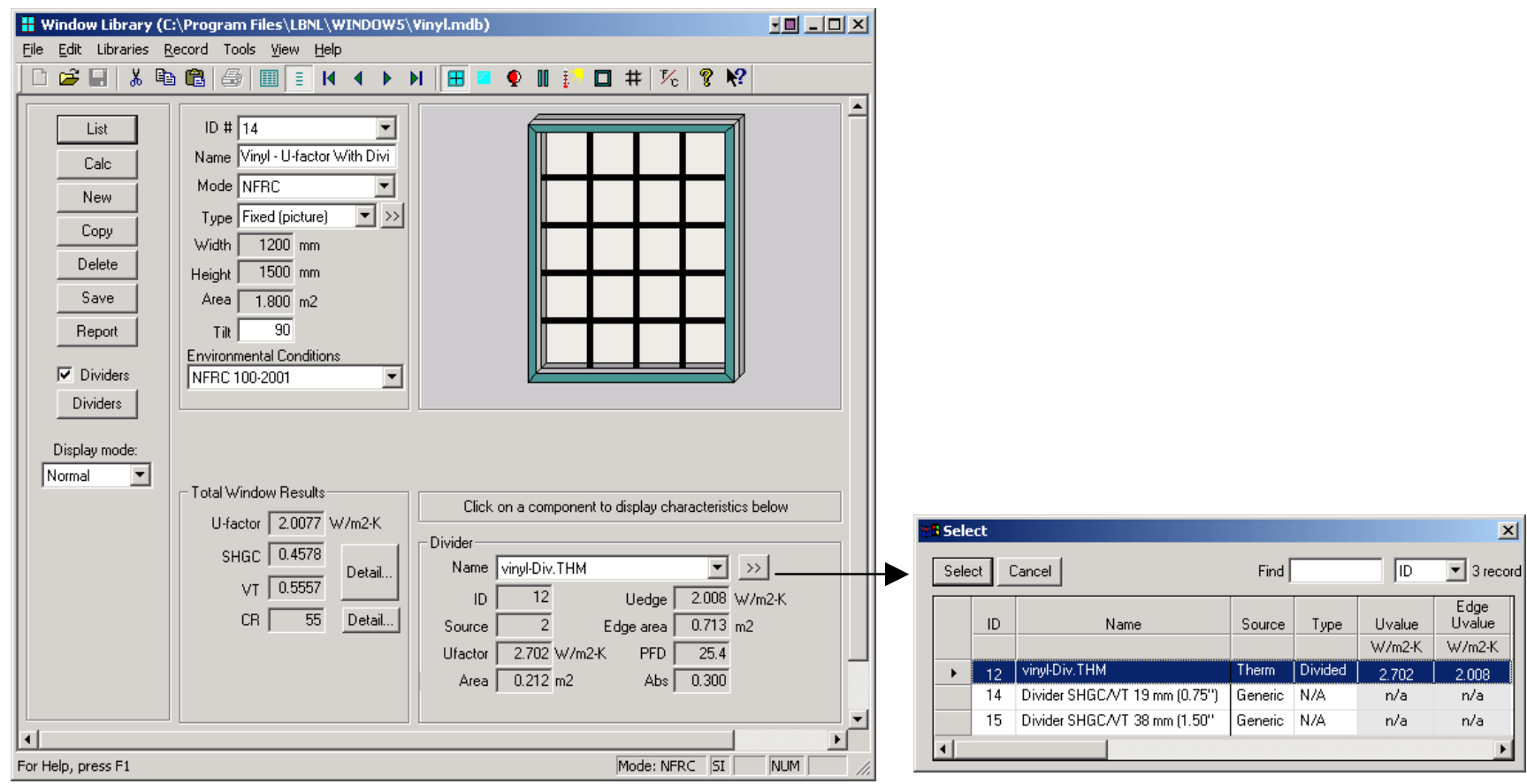

Figure 7-9. Window Library List View for the "U-factor with Dividers" case. 


\subsection{Project Databases}

It is possible to make a much smaller WINDOW5 project database by saving only the records in each library that are used in the defined glazing systems and windows. For example, saving a project database in this fashion would mean that only the glass layers referenced by the glazing system would be saved in the glass library, rather than the entire glass library that is installed with the program. The database size reduction is significant - a database with the entire Glass Library records is $15+\mathrm{MB}$, while a database with only the referenced glass layers can be less than $1 \mathrm{MB}$.

The steps to save a WINDOW database in this manner are the following:

- Select the Library "level" that the export should start from:

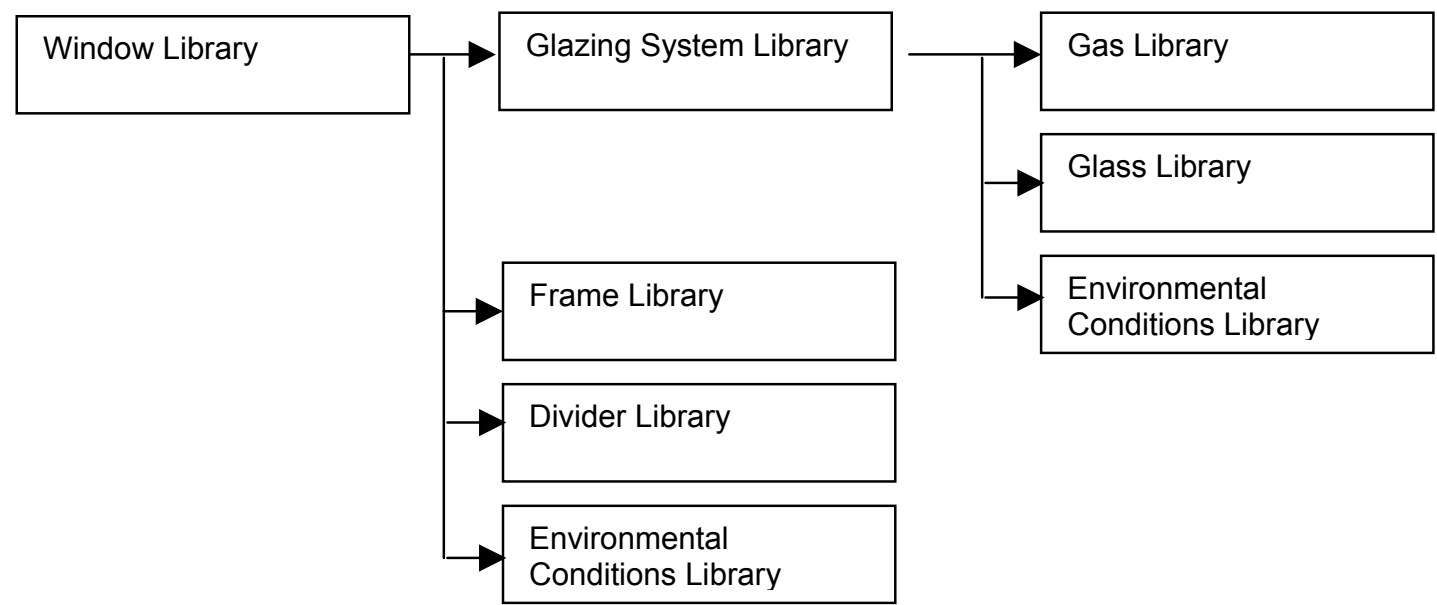

Figure 7-10. The database heirarchy in the WINDOW project database.

Starting from the Window Library will mean that records from all the other libraries that are used either directly or indirectly from those libraries will be included in the project database:

- Glazing System Library: All the glazing systems referenced in the Window Library entries

- Frame Library: All the frames referenced in the Window Library entries

- Divider Library: All the dividers referenced in the Window Library entries, if dividers are modeled.

- Glass Library: All the glass layers referenced from the glazing systems used in the Window Library entries

- Gas Library: All the gases referenced from the glazing systems used in the Window Library entries

- Environmental Conditions Library: All the environmental conditions referenced from the glazing systems and the Window Library entries.

Starting from the Glazing System Library will mean that records from the other libraries used to define the glazing systems will be written into the project database, and no other entries:

- Glazing System Library: All the glazing systems referenced in the Window Library entries

- Glass Library: All the glass layers referenced from the glazing systems used in the Window Library entries

- Gas Library: All the gases referenced from the glazing systems used in the Window Library entries 
- Environmental Conditions Library: All the environmental conditions referenced from the glazing systems and the Window Library entries.

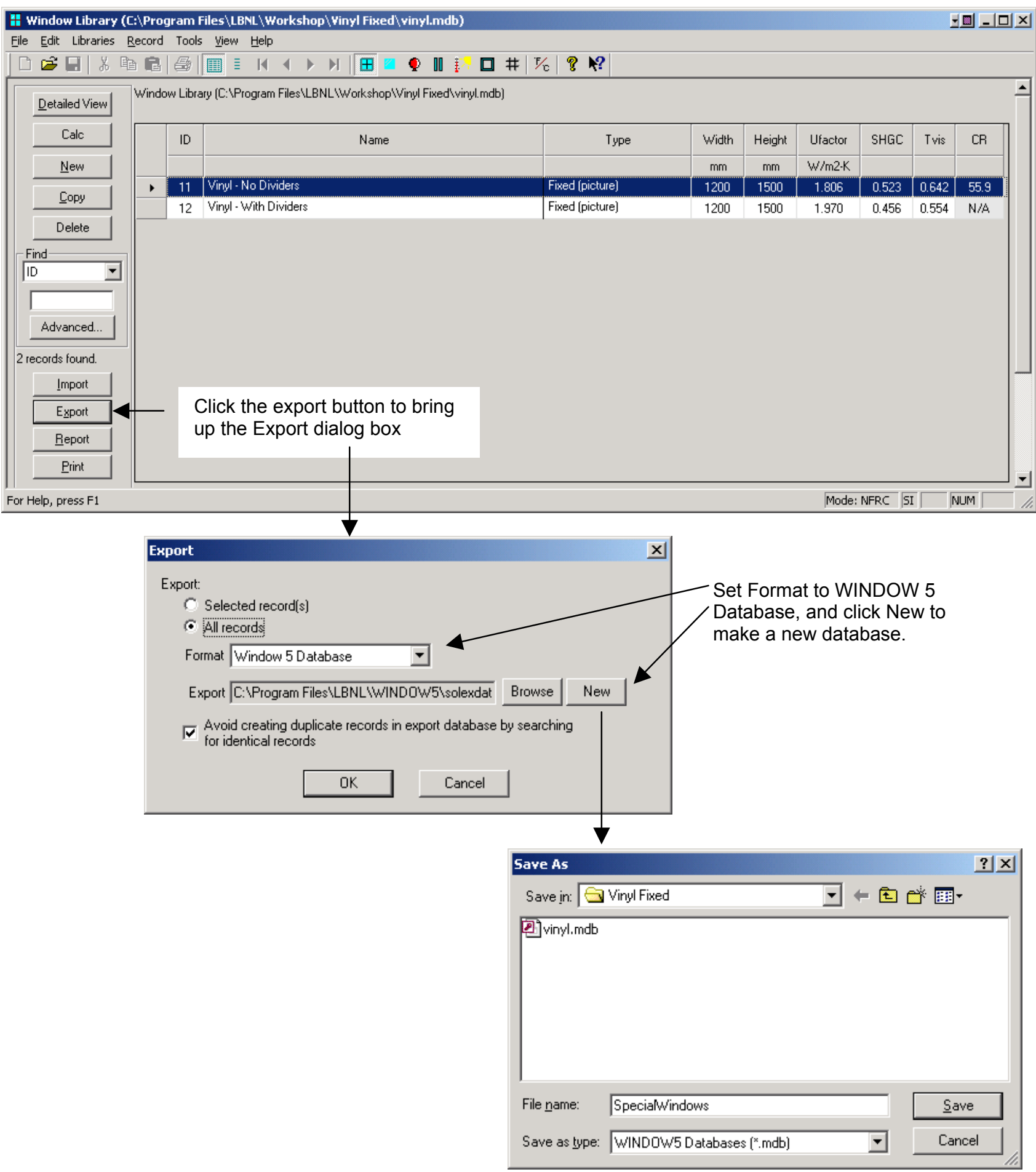

Figure 7-11. Export only referenced records into a new WINDOW5 database. 


\section{SPECIAL CASES}

\subsection{Overview}

The following special cases are covered in this section:

8.2. Meeting Rails

page $8-2$

8.3. Dividers

page $8-10$

8.4. Storm Windows

page 8-21

8.5. Flat Skylights

page $8-29$

8.6. Tubular Skylights

page $8-42$

8.7. Doors

page 8-61

8.8. Spacers

page 8-62

8.9. Non Continuous Thermal Bridge Elements

page 8-64

8.10. Garden Windows

page $8-77$ 


\subsection{Meeting Rails}

Meeting rail cross sections are the stiles or rails that meet in the middle of a sliding window. In this manual, the term "meeting rail' is used generically to describe meeting rails, meeting stiles, interlock stiles, interlocking stiles, sliding stiles, check rails, and check stiles.

\subsubsection{Modeling Meeting Rails}

When modeling a meeting rail, both the sashes and their associated glazing systems are modeled. Figure 8-1 shows an example of the meeting rail from an aluminum horizontal slider.

Creating the cross section for a meeting rail is no different than any other model in THERM. A few things to keep in mind are:

- Two glazing systems are imported, one facing up and one facing down

- Interior boundary conditions for each of the glazing systems are labeled with the Edge U-factor tag, and the program averages the values for both to derive one Edge U-factor.

- Model the meeting rail with the glazing systems facing up and down (see Section 6.3.2, "Cross Section Orientation" in this manual). If the DXF file is drawn with them in a horizontal position, draw the frame cross section, and then rotate it before inserting the glazing system.

The following discussion lists the steps for making a cross section with two glazing systems and assigning the correct boundary conditions.

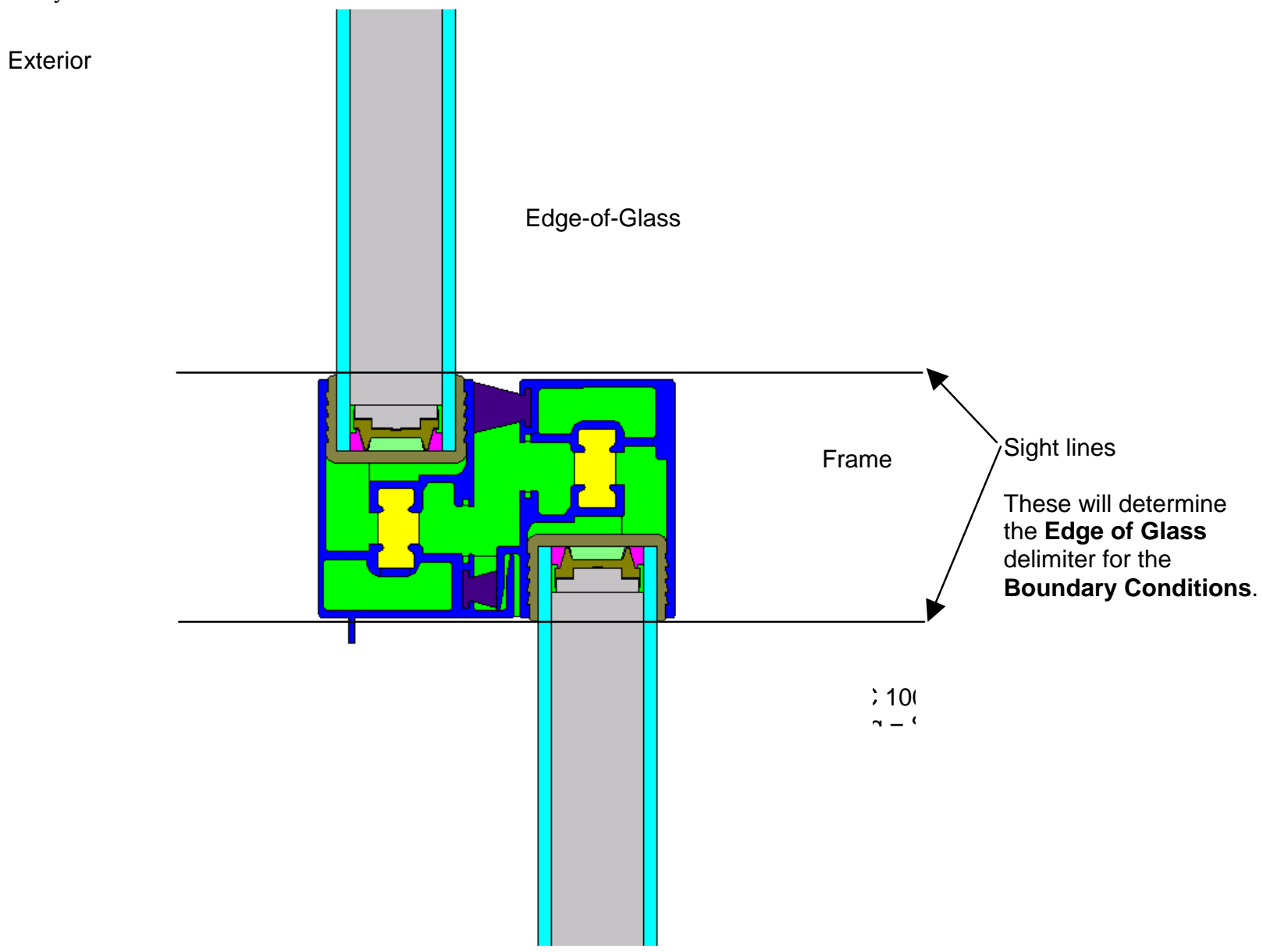

Figure 8-1. Meeting rail cross section. 


\subsubsection{Steps for Meeting Rail U-factor Calculation}

1. Using dimensioned drawings or a DXF file, create the cross section for the frame portion of the meeting rail. In Figure 8-2, the frame for the aluminum horizontal slider meeting rail has been created.

Step 1:

Draw the frame portion of the meeting rail cross section, including both sash elements, and the sweeps between them.

Define the air between the sashes as Frame Cavity NFRC 100-2001.

Sash 1

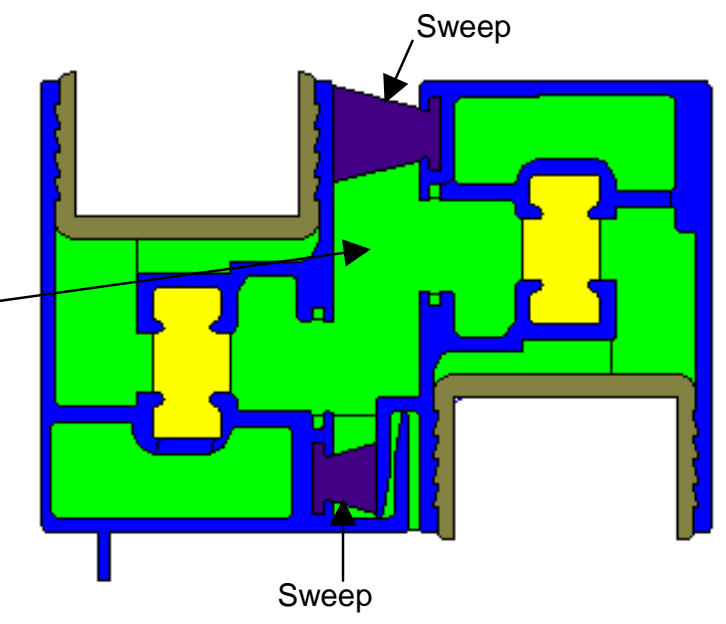

Sash 2

Figure 8-2. Frame portion of meeting rail cross section.

2. Position the Locator (using the Draw/Locator menu choice, or pressing Shift F2) in the lower left corner of the frame where the first glazing system will be inserted, as shown in Figure 8-3.

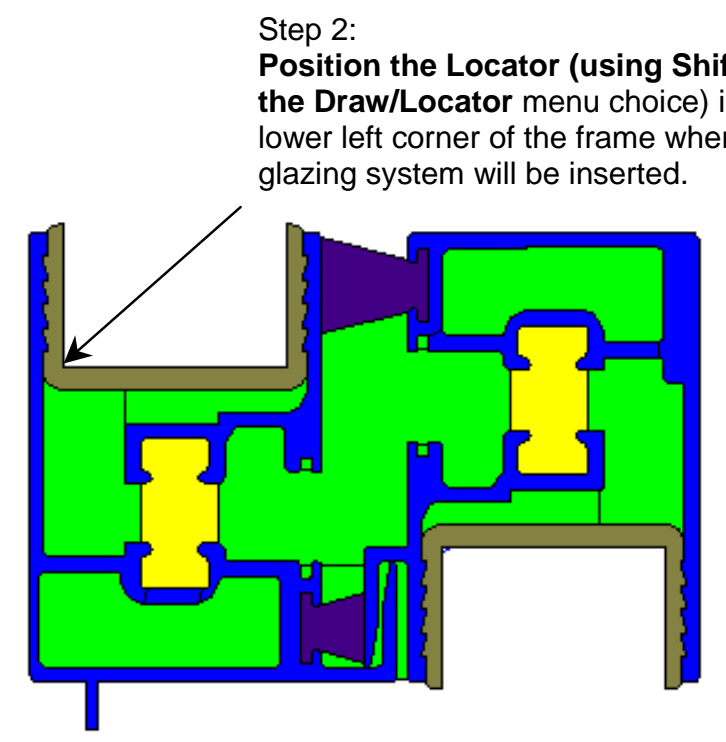

Figure 8-3. Position the Locator for the first glazing system. 
3. Using the Libraries/Glazing Systems menu option (or the $\mathbf{F 6}$ key), insert the upper glazing system, as shown in Figure 8-4. In this example, the spacer will be copied and pasted into the cross section later. Add a spacer and use the Material Link (Library/Create Link) to link the glazing system cavity conductivity with adjacent cavities in a spacer which is open to the glazing system cavity, if necessary.

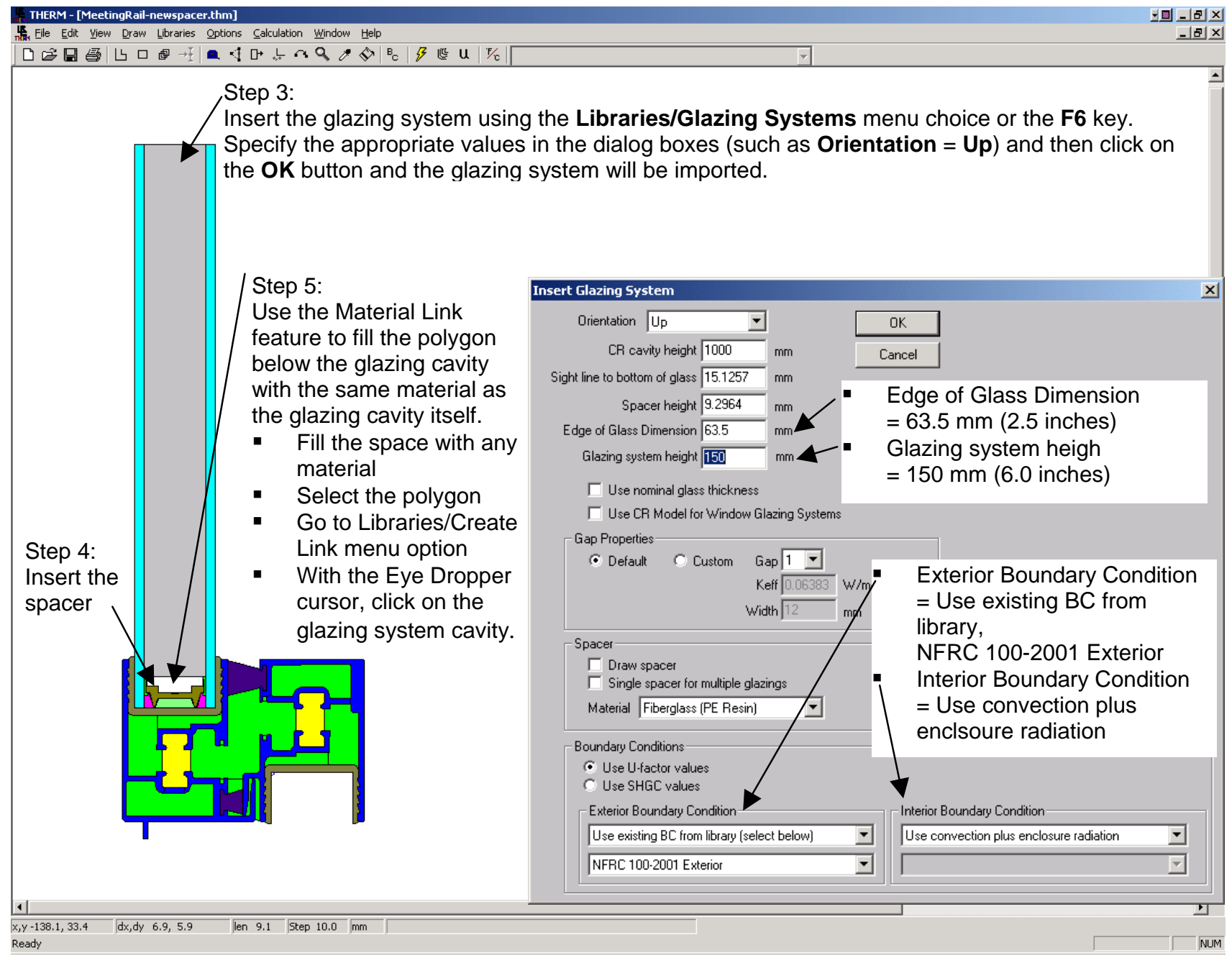

Figure 8-4. Insert the first glazing system. 
4. Reposition the locator to the upper left corner for the $2^{\text {nd }}$ glazing system.

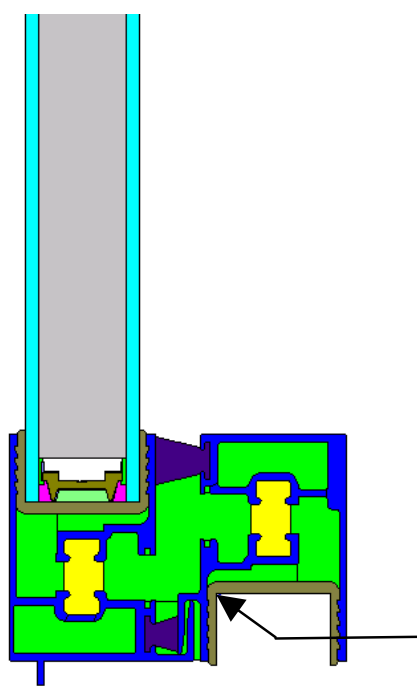

Step 4:

Reposition the Locator in the upper left corner of the frame for the $2^{\text {nd }}$ glazing

Figure 8-5. Reposition the Locator for the $2^{\text {nd }}$ glazing system.

5. Insert the $2^{\text {nd }}$ glazing system, setting the Orientation to "Down", and entering the correct values for Sight line to bottom of glass and Spacer height.

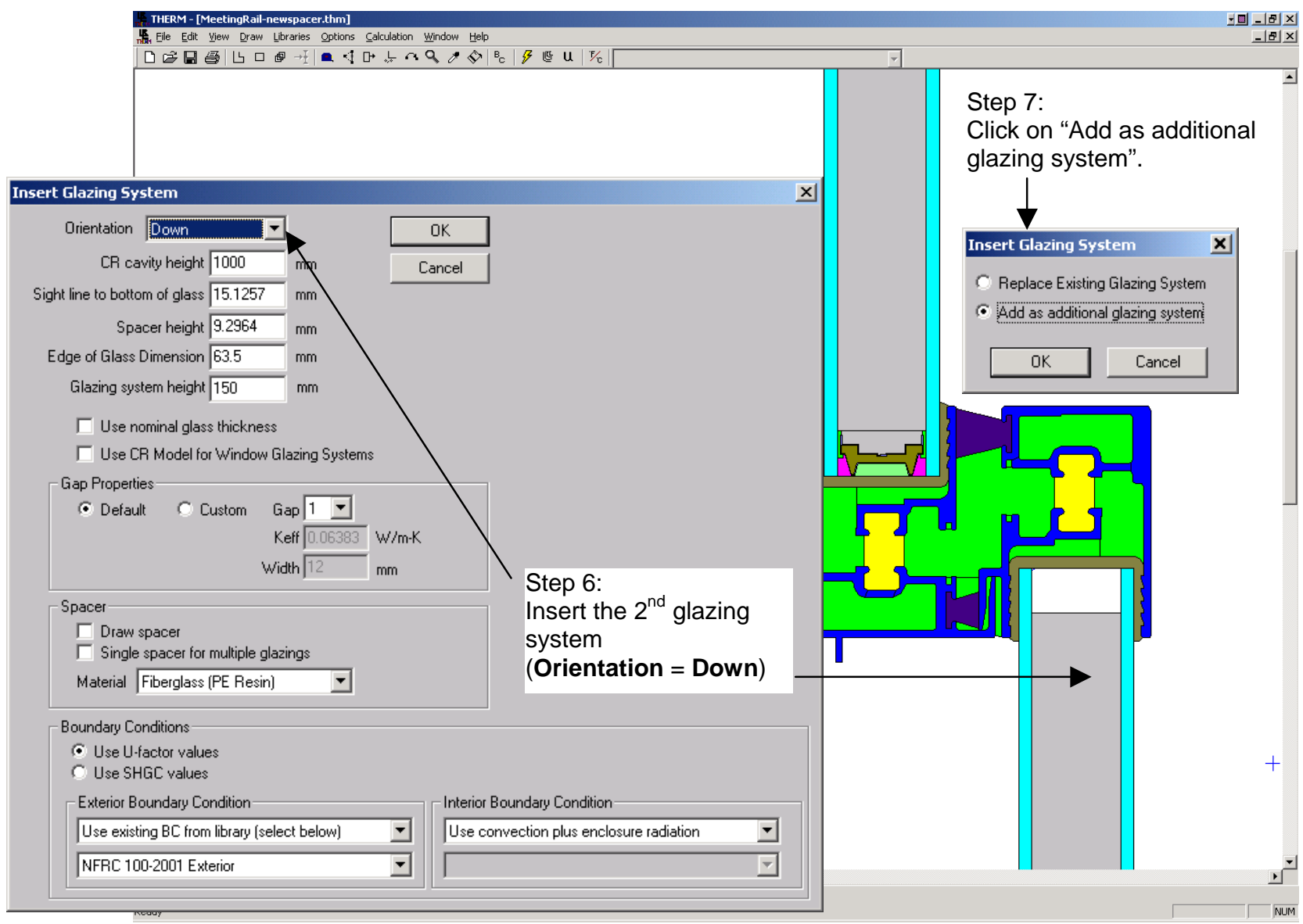

Figure 8-6. Insert the $2^{\text {nd }}$ glazing system. 
6. Add spacers and create materials linked to the glazing system cavity if necessary.

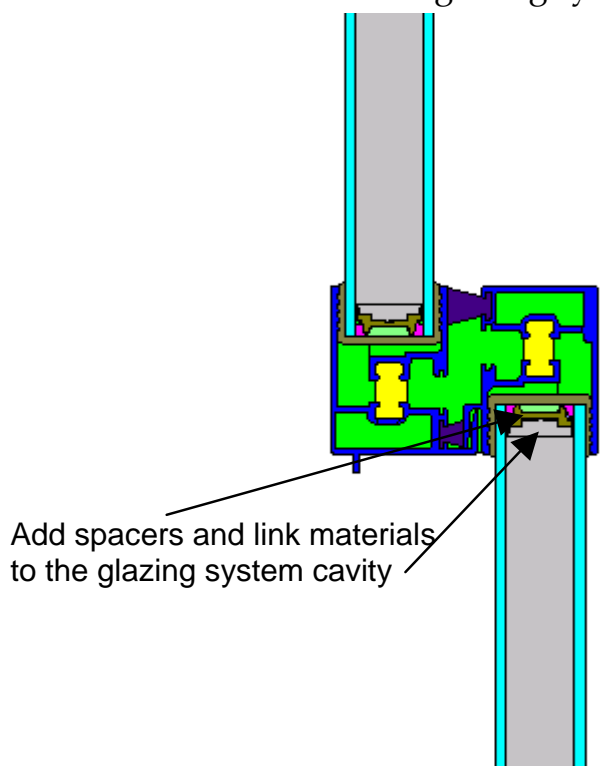

Figure 8-7. Add custom spacers. 
7. Define the boundary conditions by pressing the Boundary Conditions toolbar button, or clicking on the Draw/Boundary Conditions menu choice, or pressing the F10 key. Make sure that the interior boundary conditions are set to Radiation Model = "AutoEnclosure". Assign the Edge U-factor tag to each of the interior glazing system boundary conditions, as shown in Figure 8-8.

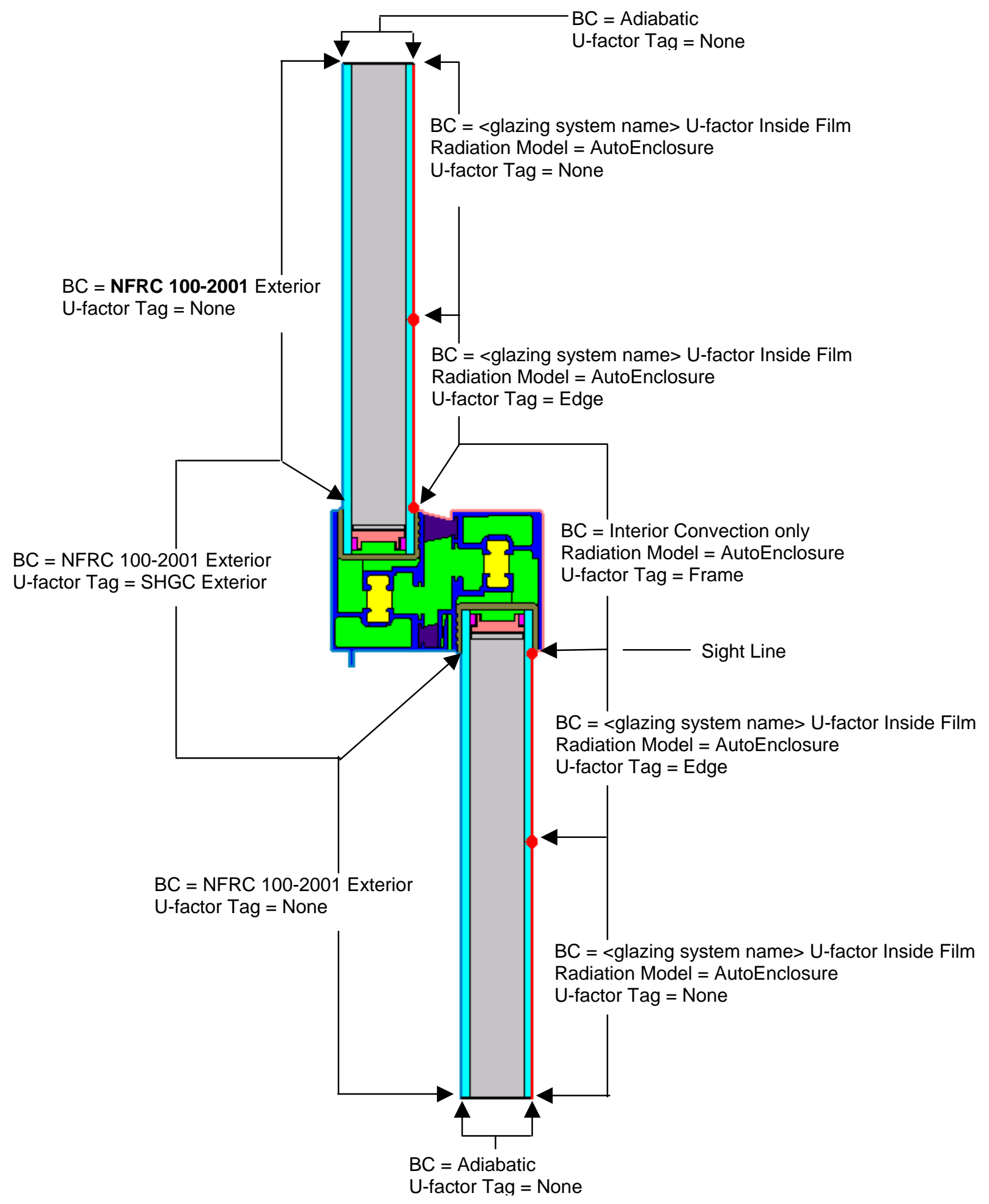

Figure 8-8. Define the Boundary Conditions for the meeting rail. 
8. Run the simulation, by pressing the Calc toolbar button, clicking on the Calculation/Calculation menu choice, or pressing the F9 key. The U-factor results are calculated for the Frame and Edge U-factor tags, as shown in the figure below.

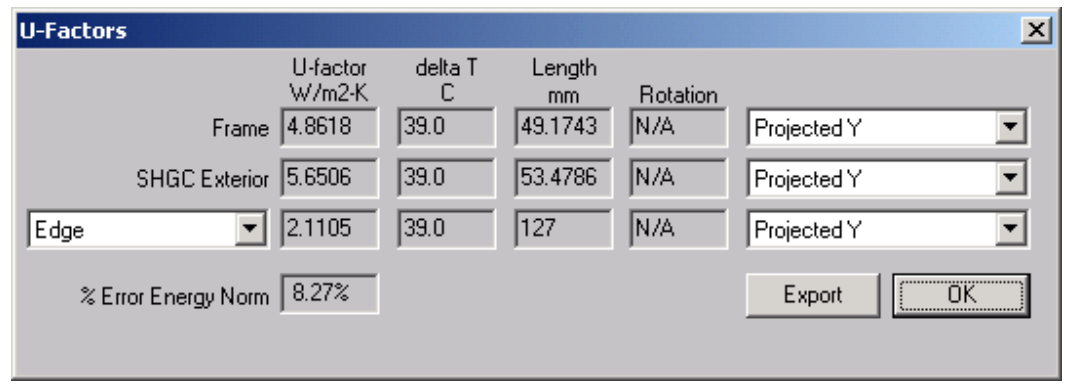

Figure 8-9. Calculate the results.

9. Import the THERM file into the WINDOW Frame Library.

\subsubsection{Steps for Meeting Rail Condensation Resistance Calculation}

The Condensation Resistance model is only appropriate for horiztonal meeting rails (found in vertical sliding products) - THERM will not calculate the Condensation Resistance for a file with the Cross Section Type set to "Vertical Meeting Rail".

There are two methods for calculating the Condensation Resistance information in THERM, which will be used in WINDOW to calculate the total Condensation Resistance of the product:

- Check the "Use CR Model for Window Glazing System" checkbox when importing a glazing system OR

- In the Options menu, Preferences choice, THERM File Options tab, check the "Use CR Model for Glazing Systems", as shown in the figure below.

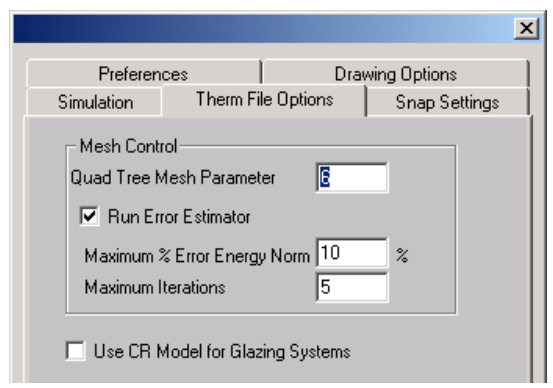

Figure 8-10 In Options/Preferences/Therm File Options, check the "Use CR Model for Glazing Systems" checkbox.

When the CR model has been "turned on", red boundary conditions will appear inside the glazing system, and the following steps should be taken to simulate the file:

1. Check the emissivities of these boundary conditions. They should be the following:

- Emissivity of the surrounding surface, such as 0.84 for standard glass, 0.90 for most frame materials, 0.20 for metal, and so forth.

- 1.0 for the adiabatic (open end) of the glazing cavity.

- Actual cavity height per Table 6-2, Section 6.4.5

2. Simulate the model. The program will calculate both U-factor results and the Condensation Resistance results. 
3. Import the results into the WINDOW Frame Library and use the meeting rail file to create the whole product in the Window Library as applicable.

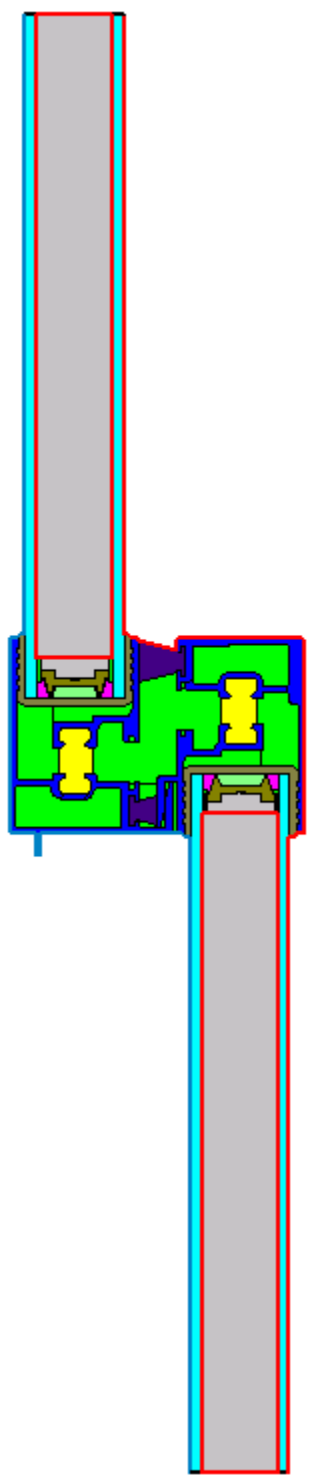

Figure 8-11 Red boundary conditions will appear inside the glazing system when the Condensation Resistance feature is activated. Check the emissivities of each of these boundaries. Note that Condensation Resitance is only modeled for horizontal meeting rails (such as in a double hung window). 


\subsection{Internal Dividers (Suspended Grilles)}

The criteria for when dividers are modeled can be found in NFRC 100, Section 1.4.4, "Simplifications to a Product Line". The discussion below describes the methodologies in WINDOW and THERM for modeling dividers when that criteria is met.

\subsubsection{Modeling Steps}

The modeling steps in THERM5 and WINDOW5 are the same for all divider shapes and all possible gas fills, in contrast to modeling steps in previous versions of THERM. These steps are the following:

In WINDOW:

- No new work is required, because the same glazing system that is used to model the rest of the product is used in the divider model.

In THERM:

- The new ISO 15099 modeling assumptions would theoretically warrant modeling horizontal and vertical dividers separately. However, a conservative simplificiation is to model all dividers, including horizontal ones, as vertical dividers. Therefore, only one divider model is created in THERM and referenced in WINDOW.

- Set the Cross Section Type to "Vertical Divider" for all dividers.

- Insert the glazing system twice, once facing up, with a spacer height defined as the same height as the divider height, and once facing down with the spacer height set to zero.

- NOTE: Because all dividers are modeled as "Vertical Dividers" the CR model is not run in THERM for these files. However, WINDOW will still calculate a whole product $C R$ value when these dividers are used in a product, by using the U-factor temperatures for the dividers.

- Draw the true geometry of the divider in the upper glazing system, in the "spacer" area.

- Depending on the fill of the glazing system, assign the appropriate frame cavity material to the cavities between the glazing system and the divider, as well the cavity inside the divider, as follows:

- For air-filled dividers: Assign "Frame Cavity NFRC 100-2001" material

- For gas-filled dividers: Create a new material in the Material Library that is identical to the "Frame Cavity NFRC 100-2001" material, except that the gas used in the glazing system, found in the Gas Library, is referenced in the Gas Fill field. Assign this new material to the cavities in the divider. (See the example below)

- Assign Boundary Conditions.

- Simulate the results.

- Import the file into the WINDOW Divider Library. Reference the Divider as appropriate from the Window Library when constructing the whole product.

When modeling glazing options with caming, which are treated in a similar fashion to dividers, the NFRC default caming can be used. 


\subsubsection{Air Filled Glazing Systems}

The modeling steps for a divider with an air-filled glazing system are explained in detail in the following pages.

In THERM:

1. Set the Cross Section Type to "Vertical Divider".

2. Import the glazing system for the divider, which is the same glazing system as the rest of the product, with the following settings:

- Orientation $=U p$

- Actual Cavity height $=1000 \mathrm{~mm}$ (39 inches)

- Sight line to bottom of glass = height of the divider (in this example it is $19.05 \mathrm{~mm}$ [0.75 inches])

- Spacer height $=$ height of the divider (in this example it is $19.05 \mathrm{~mm}[0.75$ inches)

- Edge of Glass Dimension $=63.5 \mathrm{~mm}$ (2.5 inches)

- Glazing System Height: $150 \mathrm{~mm}$ (6.0 inches)

- $\quad$ Draw spacer $=$ Not checked
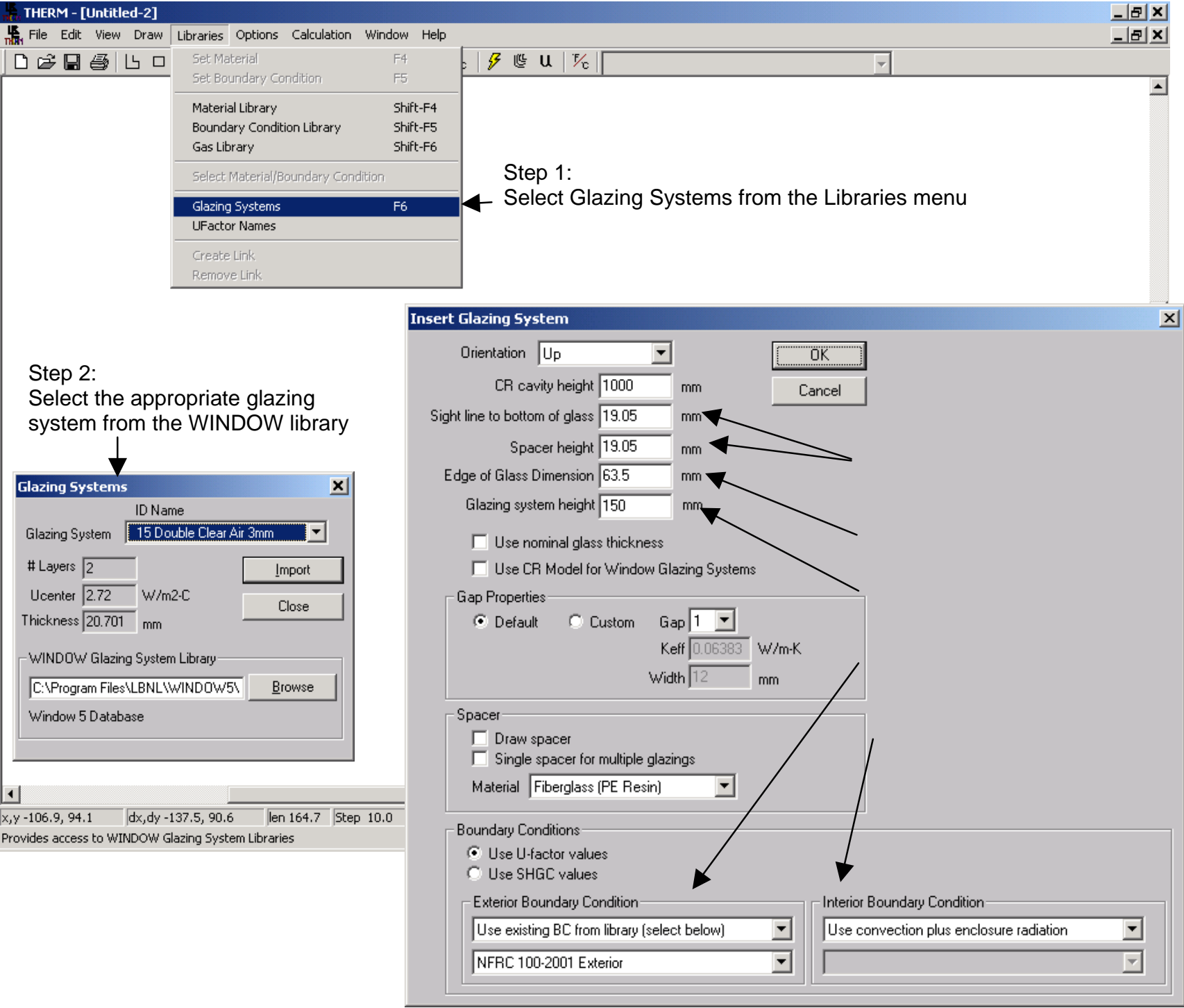

Figure 8-12. Import the first glazing system. 
3. Import the glazing system again as an additional glazing system, below the first one (the locator does not have to be moved), but facing down this time. Use the following settings for this glazing system:

- Orientation $=$ Down

- Actual Cavity height $=1000 \mathrm{~mm}$ (39 inches)

- Sight line to bottom of glass $=0$

- Spacer height $=0$

- $\quad$ Edge of Glass Dimension $=63.5 \mathrm{~mm}(2.5$ inches $)$

- Glazing System Height: $150 \mathrm{~mm}$ (6.0 inches)

- $\quad$ Draw spacer $=$ Not checked

Insert the glazing system as an Additional Glazing.

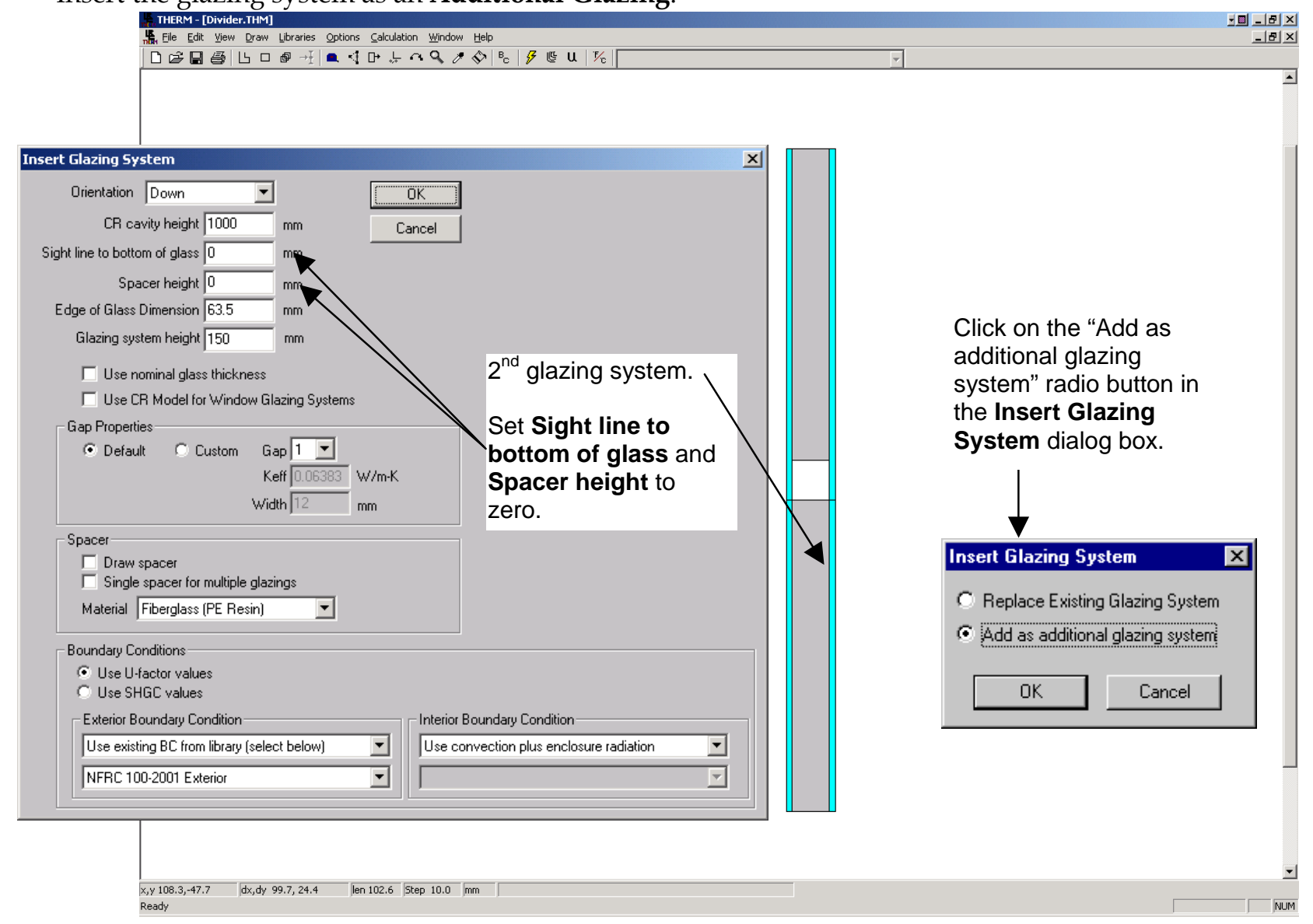

Figure 8-13. Import the second glazing system as an additional glazing system, facing down. 
4. Draw (or copy and paste from another THERM file) the polygons in the cavity that represent the divider. The figure below shows the divider for this example drawn with the material set to Aluminum Alloy

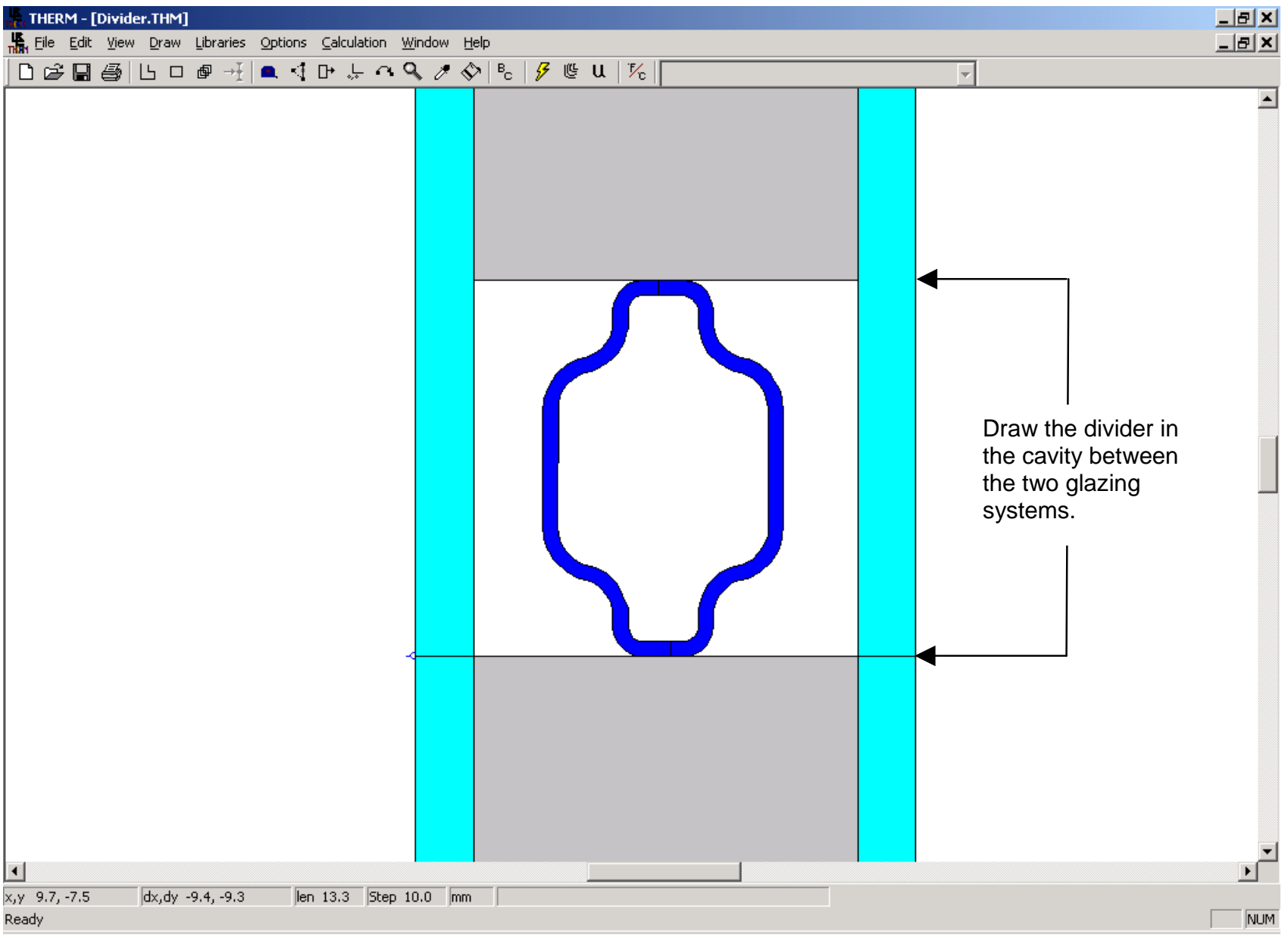

Figure 8-14. Draw the polygons to represent the divider. 
5. Fill the cavities between the divider and the glass layers and inside the divider with the material "Frame Cavity NFRC 100-2001". Divide the cavities up according to the $5 \mathrm{~mm}$ rule as necessary.

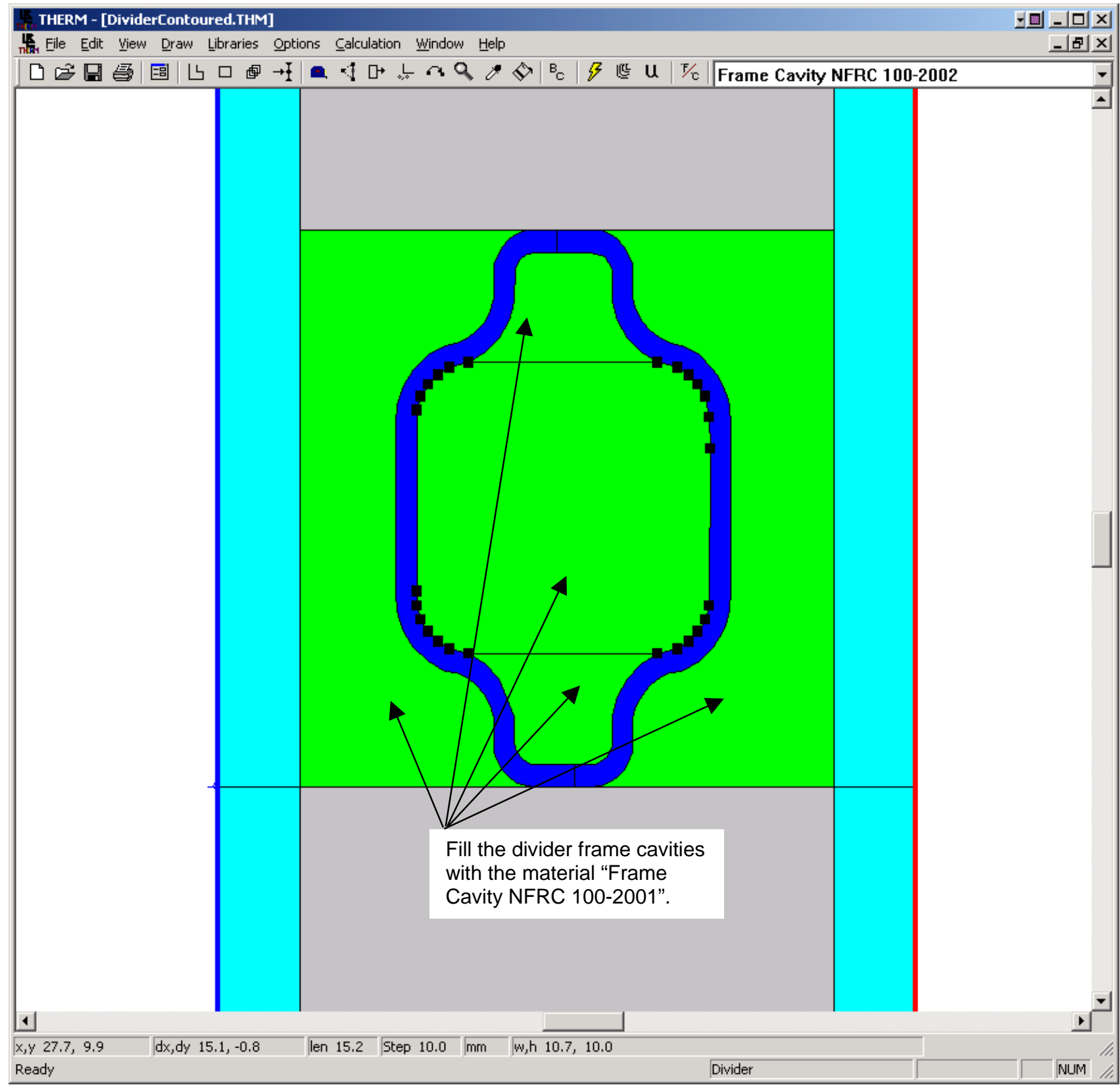

Figure 8-35 Fill the divider frame cavities with Frame Cavity NFRC 100-2001. 
6. Define the boundary conditions, using the "AutoEnclosure" choice for the Radiation Model.

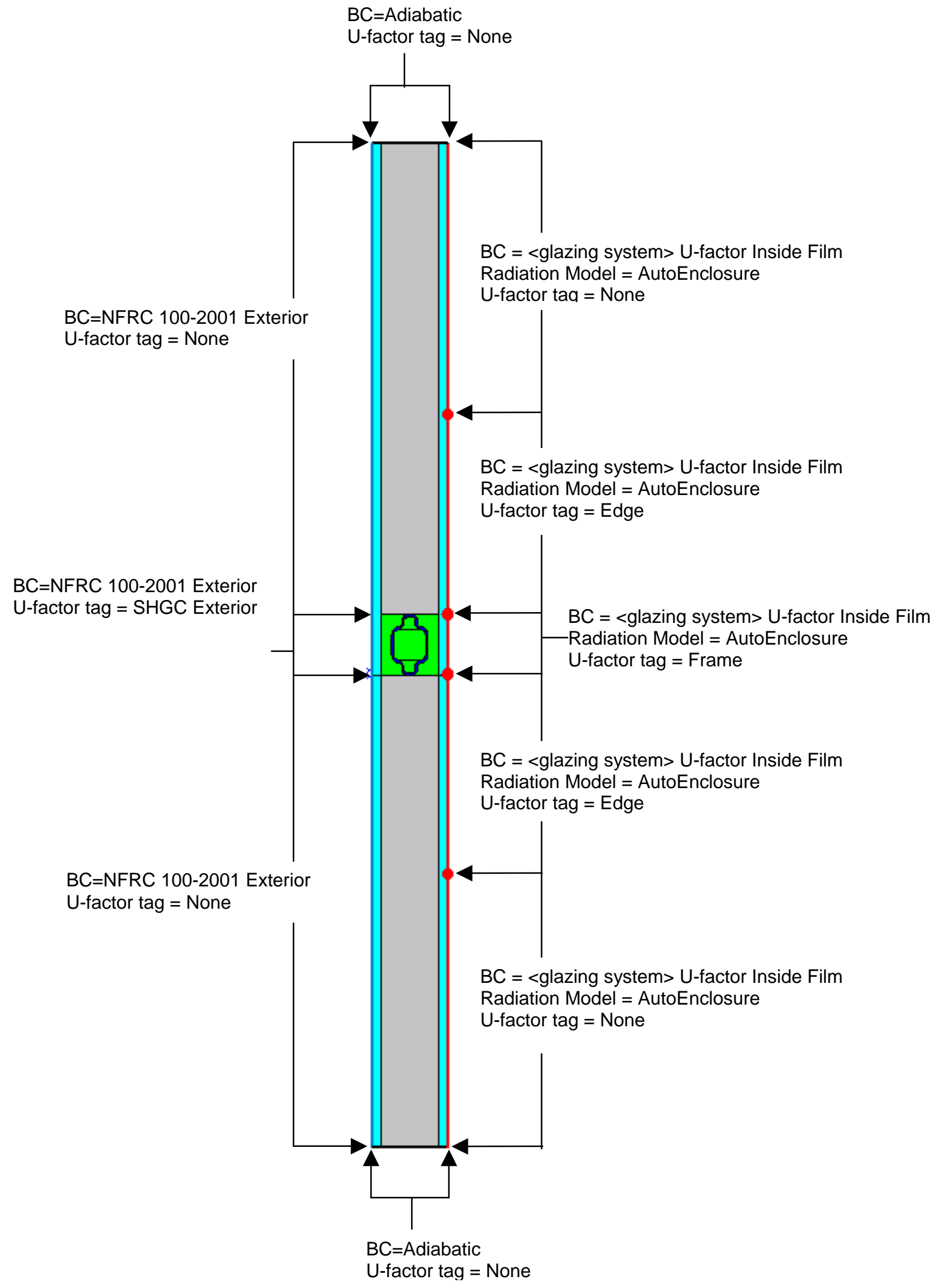

Figure 8-15 Assign the boundary conditions. 
7. Calculate the results.

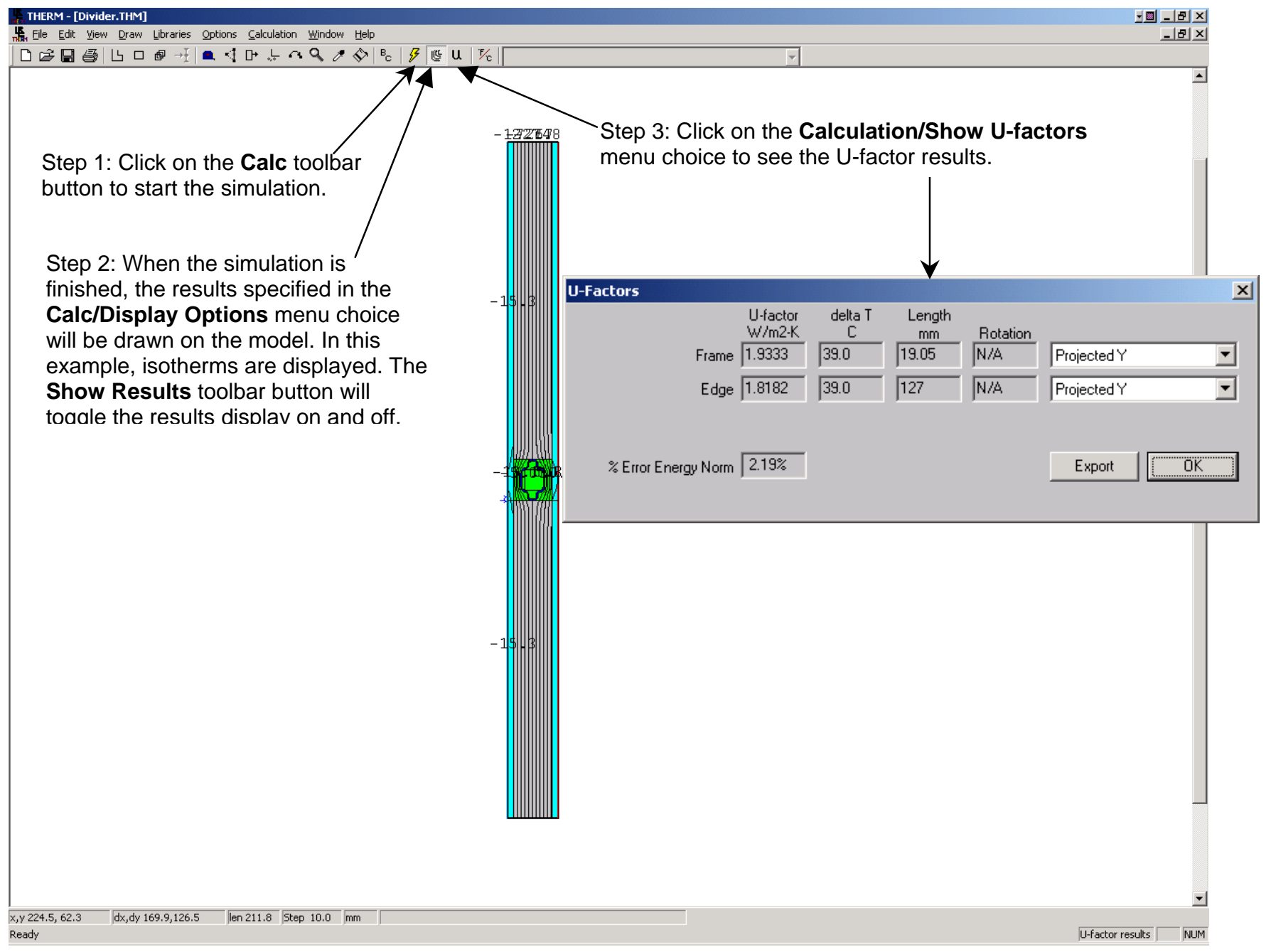

Figure 8-16 Calculate the results.

8. Save the file using the File/Save As menu choice. 
9. Import the results to the WINDOW Divider Library, as shown below. See Section 4.7.3, "Importing THERM files" in the WINDOW User's Manual for more information about importing THERM files.

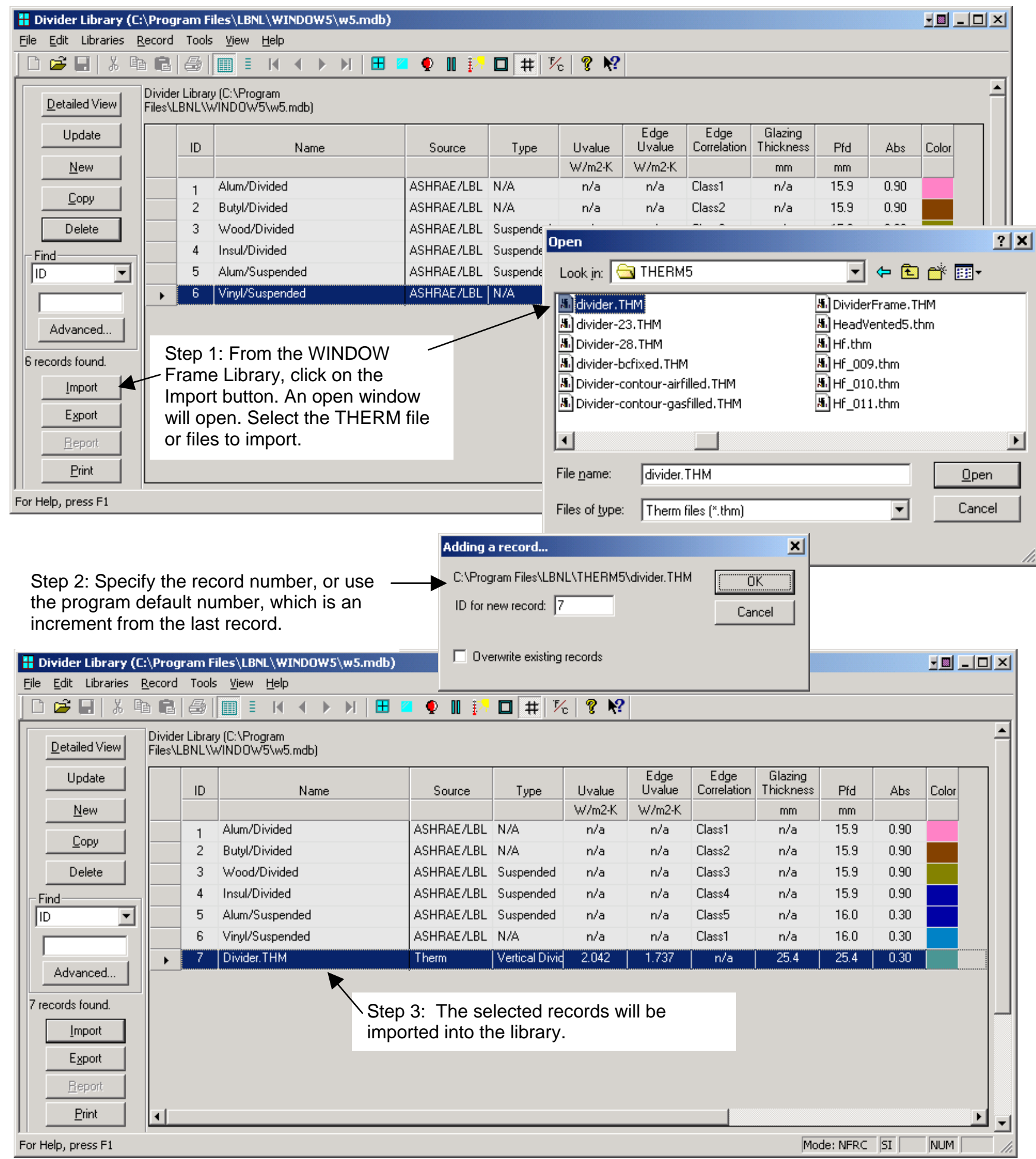

Figure 8-17 Import the THERM file into the WINDOW Divider Library.

10. Use the new divider in the calculation of the complete product values in the main screen of WINDOW. 


\subsubsection{Gas Filled Glazing Systems}

If the glazing system being modeled with a divider is gas-filled, it is necessary to model the divider with the same gas fill as the glazing system. This means a new material must be defined for the gas-filled frame cavities around and inside the divider.

The THERM Gas Library contains entries for standard gases, as well as examples of gas mixtures. These gases are not made in THERM; they are made in the WINDOW Gas Library and then imported into the THERM Gas Library. When the gas mixtures have been imported into THERM, they can be referenced from a new frame cavity material for the divider model, as shown below.

1. Create the gas mixture in the WINDOW Gas Library. Presumably it already exists for the product glazing system model. See Section 4.6, "Gas Library" in the WINDOW User's Manual for details about creating new entries in the Gas Library.

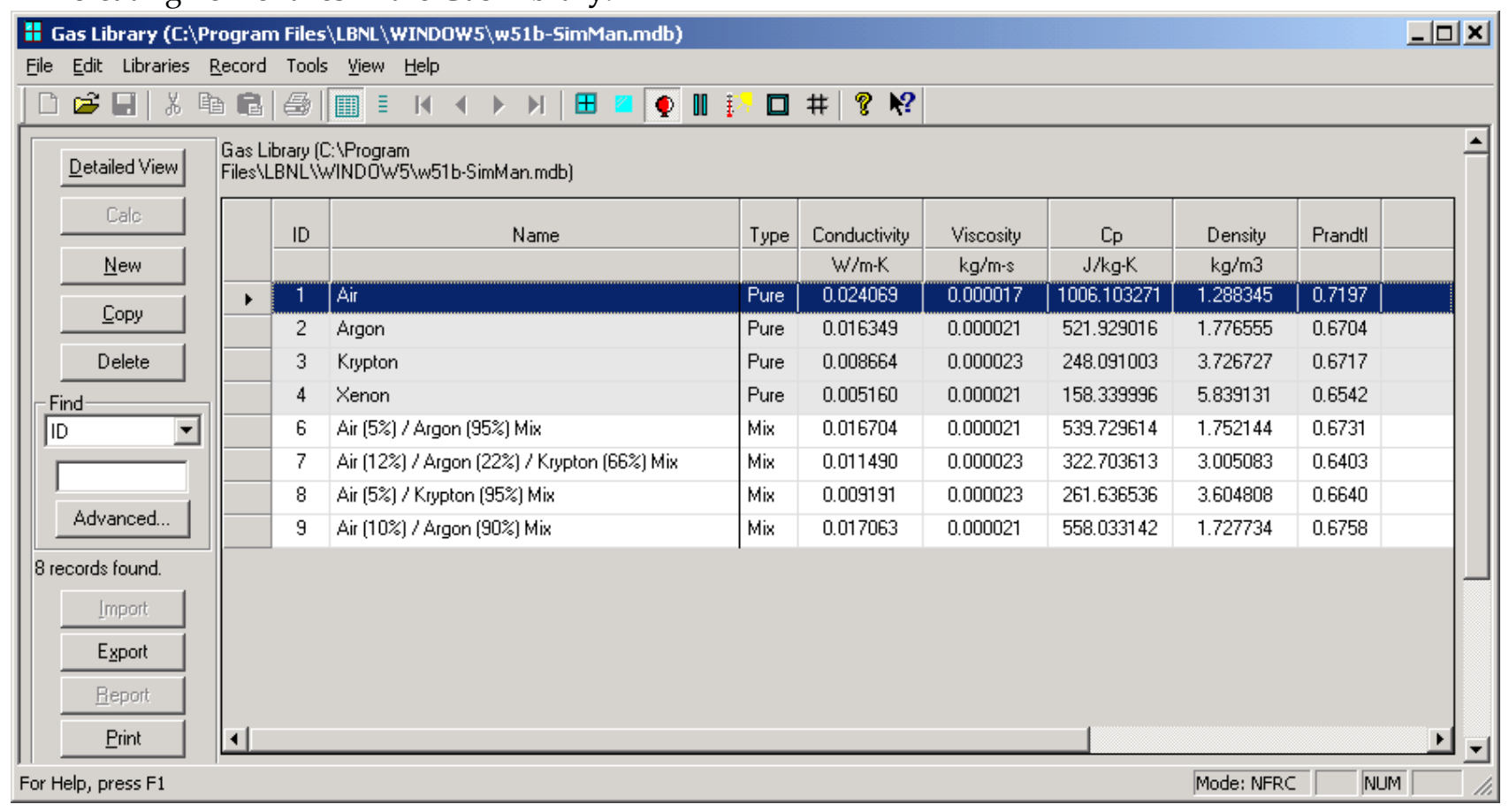

Figure 8-18 Make the necessary gas mixture in the WINDOW Gas Library. 
2. Import the WINDOW gas mixture into the THERM Gas Library, if it is not already there.

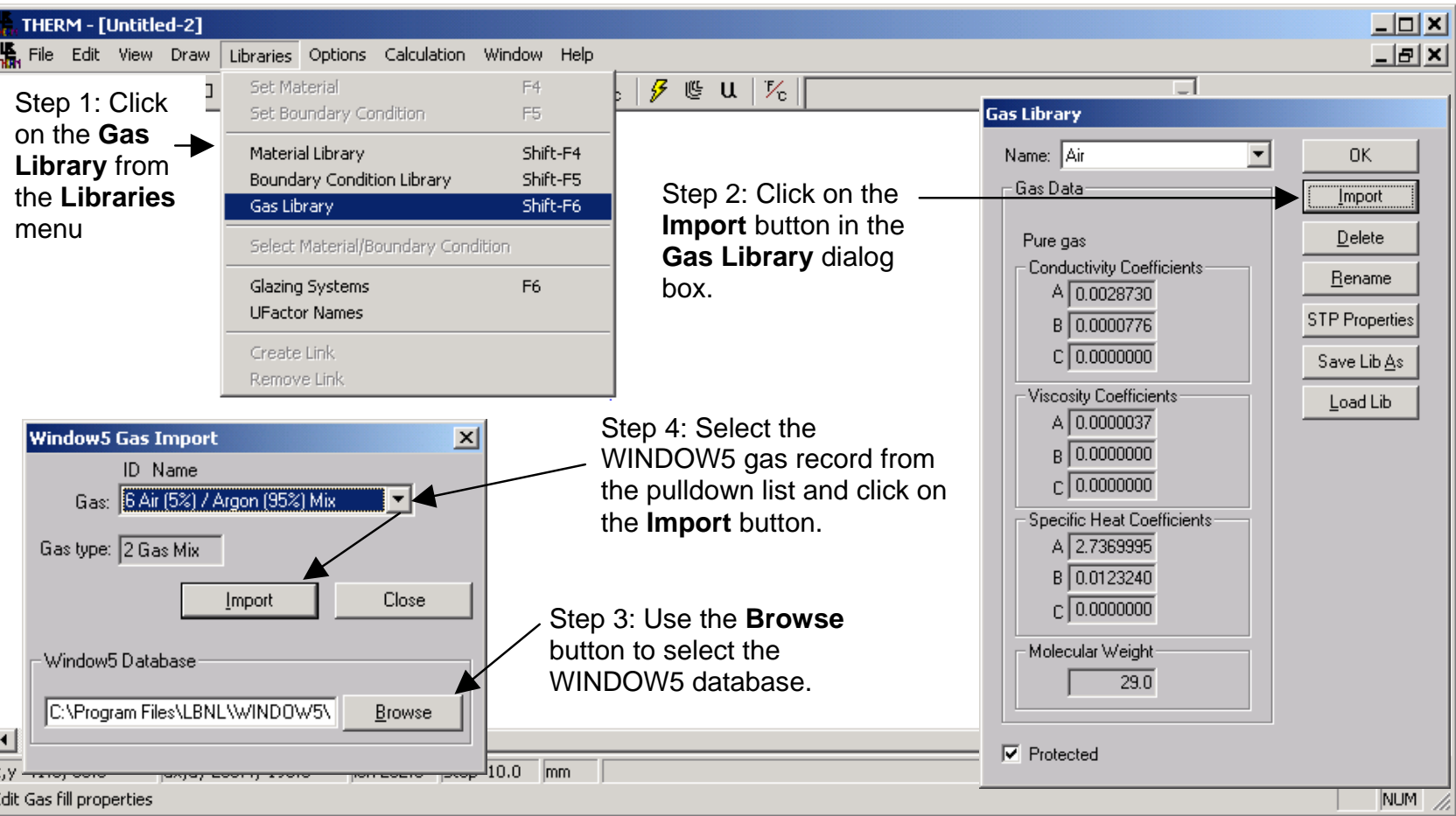

Figure 8-19 Import the gas mixture enntries into the THERM Gas Library.

3. Make a new frame cavity material in the THERM Material Library based on "Frame Cavity NFRC 1002001" but with the Gas Fill field set to the correct gas mixture from the Gas Library.

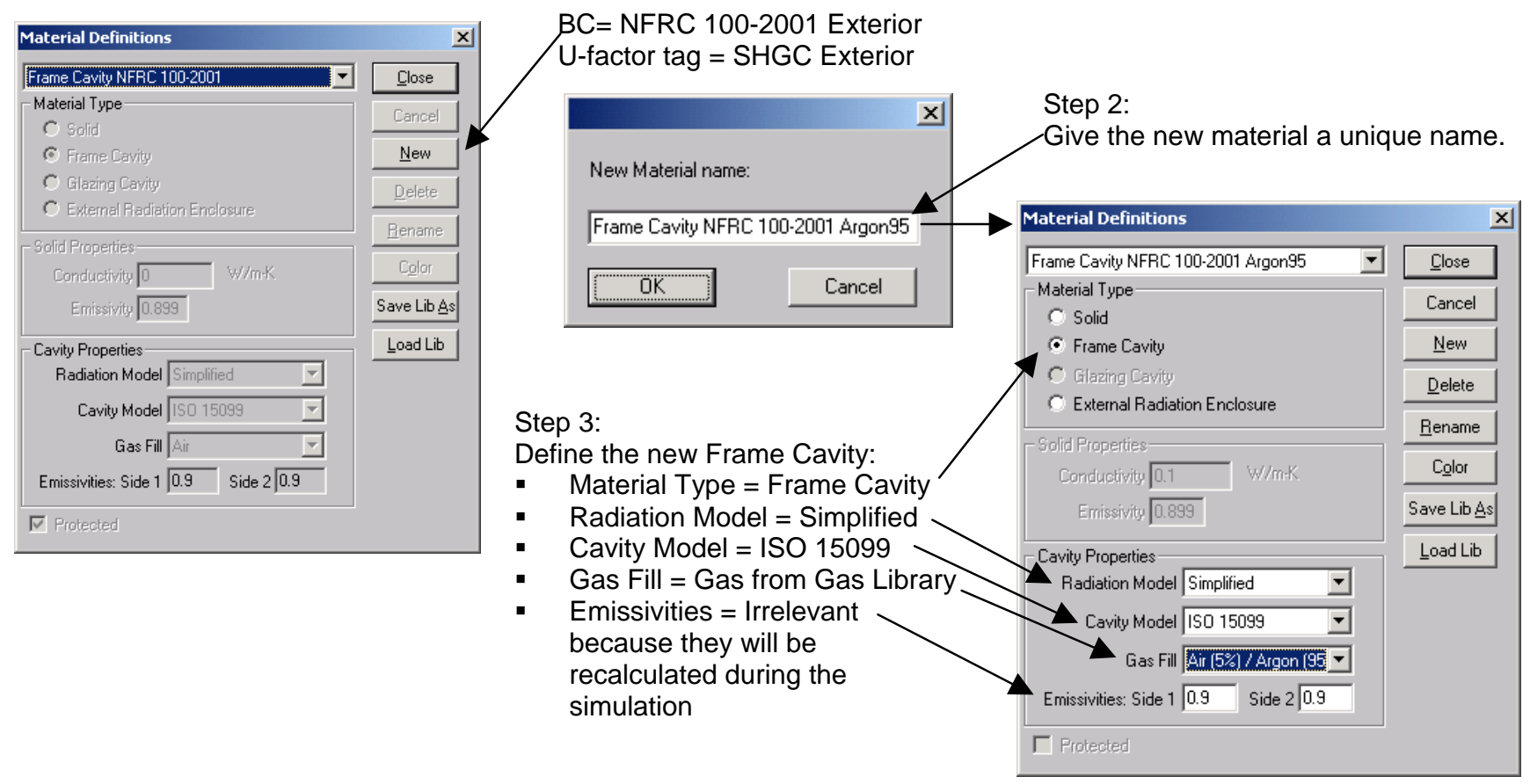

Figure 8-20 Import the gas mixture enntries into the THERM Gas Library. 
4. Use this new frame cavity material in the divider model cavities.

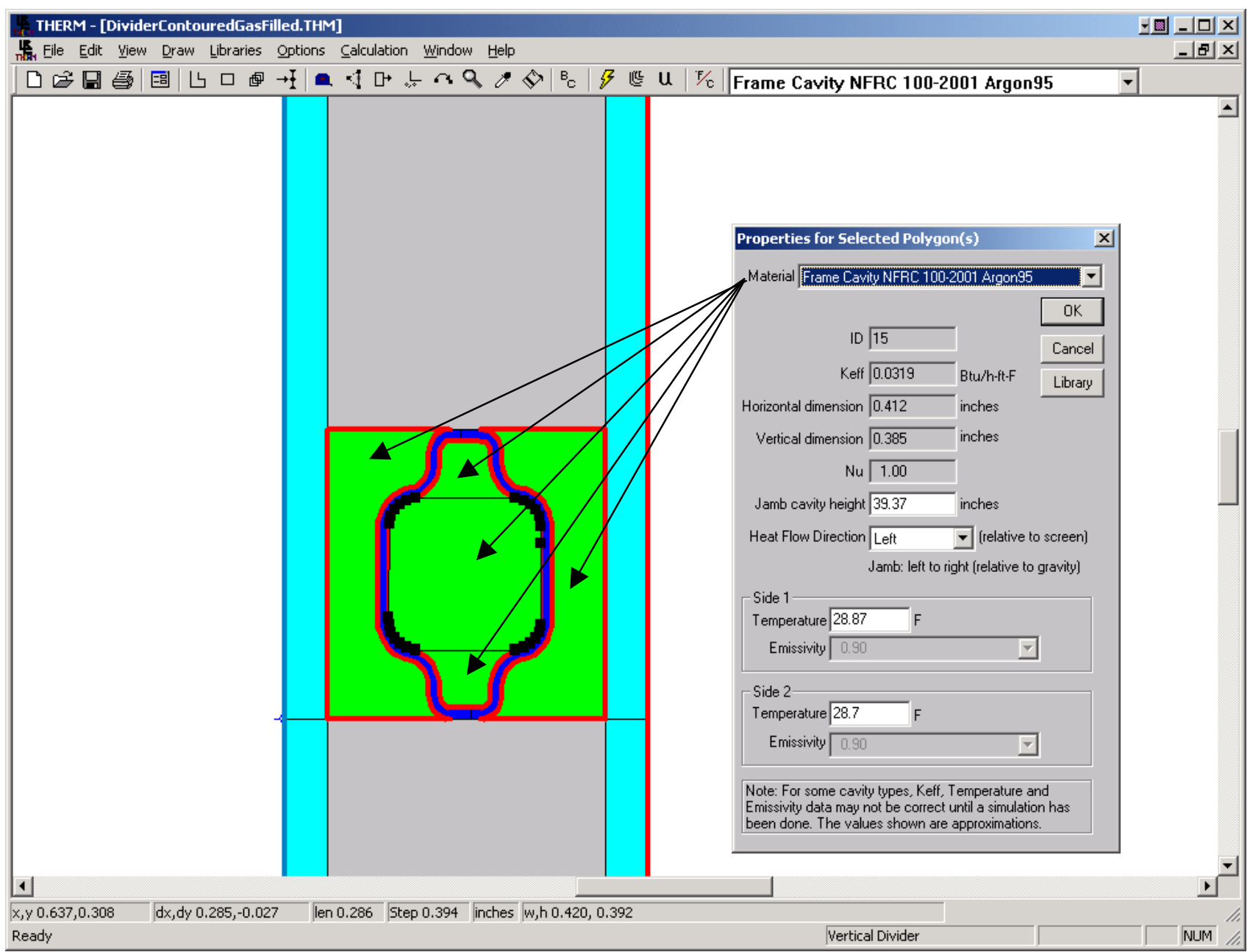

Figure 8-21. Use the new Frame Cavity material to fill the divider cavities. 


\subsection{Storm Windows}

Storm windows present a modeling problem different from most insulated glass (IG) units, because the spacing between the IG unit and the storm window is usually quite large, as shown in the figure below. As with all other product modeling, all relevant cross sections (head, sill, jambs, meeting rails and dividers) must be modeled in THERM.

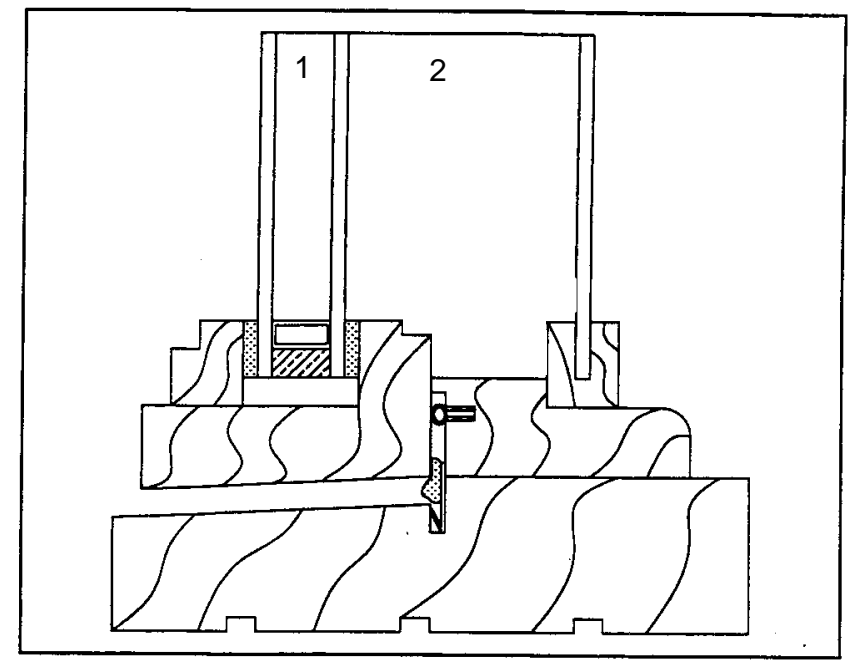

Figure 8-22 Product with an interior storm window.

\subsubsection{Modeling Steps}

Follow the steps below to model storm windows. These steps are discussed in more detail in the following sections.

If the product is NOT a single or double hung (i.e., it is a casement, fixed, picture, transom, awning, etc), do the following:

In WINDOW:

- Create a three-layer glazing system with the correct spacing between each of the glass layers in WINDOW.

In THERM

- Draw the frame components for the product in THERM.

- Import the glazing system into THERM

Edge of Glass Dimension $=63.5 \mathrm{~mm}(2.5 \mathrm{inch})$

Glazing System Height $=150 \mathrm{~mm}(6.0 \mathrm{inch})$.

- Fill the air cavity below the glazing system and use the Library/Create Link feature to link that air cavity to the glazing cavity.

- Assign the boundary conditions

Exterior Boundary Condition = Use existing BC from library, select “NFRC 100-2001 Exterior", and assign the SHGC Exterior U-factor tag to the exterior frame components

Interior Boundary Condition = Use "convection plus enclosure radiation" for Glazing System, use appropriate "convection only" frame boundary condition for the frame components.

- Simulate the problem 
If the product IS a single or double hung (i.e., a vertical or horizontal slider), where there will be a different gap width between the glazing system and the storm window for different frame profiles, do the following:

In WINDOW:

- Create three three-layer glazing systems as follows:

- One glazing system with a gap width between the glazing and the storm window that is the average of the gaps of the entire product.

- Two glazing systems, with the correct spacing between each of the glazing system and the storm window, for each of the frame profiles that will be modeled in THERM.

\section{In THERM}

- Draw the frame components for the product in THERM.

- Import the glazing systems with the actual gap widths into the appropriate frame profiles with the following settings:

Edge of Glass Dimension $=63.5 \mathrm{~mm}(2.5 \mathrm{inch})$

Glazing System Height $=150 \mathrm{~mm}$ (6.0 inch).

- Edit the Keff values for each glazing system cavity to match that of the first "average-gap" glazing system made in WINDOW. Do this by double clicking on the glazing system.

- Fill any air cavity between the bottom of the glazing system and the top of the frame profile as necessary, and use the Library/Create Link feature to link that air cavity to the glazing cavity.

- Assign the boundary conditions

Exterior Boundary Condition = Use existing BC from library, select "NFRC 100-2001 Exterior", and assign the SHGC Exterior U-factor tag to the exterior frame components

Interior Boundary Condition = Use "convection plus enclosure radiation" for Glazing System, use appropriate "convection only" frame boundary condition for the frame components.

- Simulate the problem

In WINDOW:

- Import the THERM frame profiles that have the correct geometry for the glazing systems. Copy each record and edit the glazing system thickness to match the thickness of the "average-gap" glazing system in WINDOW.

- In the Window Library, create a product that is made of the frame records that have the "average-gap" glazing system thickness and the center-of-glazing defined as the "average-gap" glazing system defined first.

- Calculate the overall product values from this combination of components. 


\subsubsection{Storm Window Example}

The following example problem, based on the product in Figure 8-23, is explained in detail in the following discussion.

\subsubsection{Create Glazing System in WINDOW:}

1. Make a glazing system consisting of three layers of glass, with the dimensions of the glazing cavity for the first gap, and the correct dimension from the glass to the storm window for the second gap.

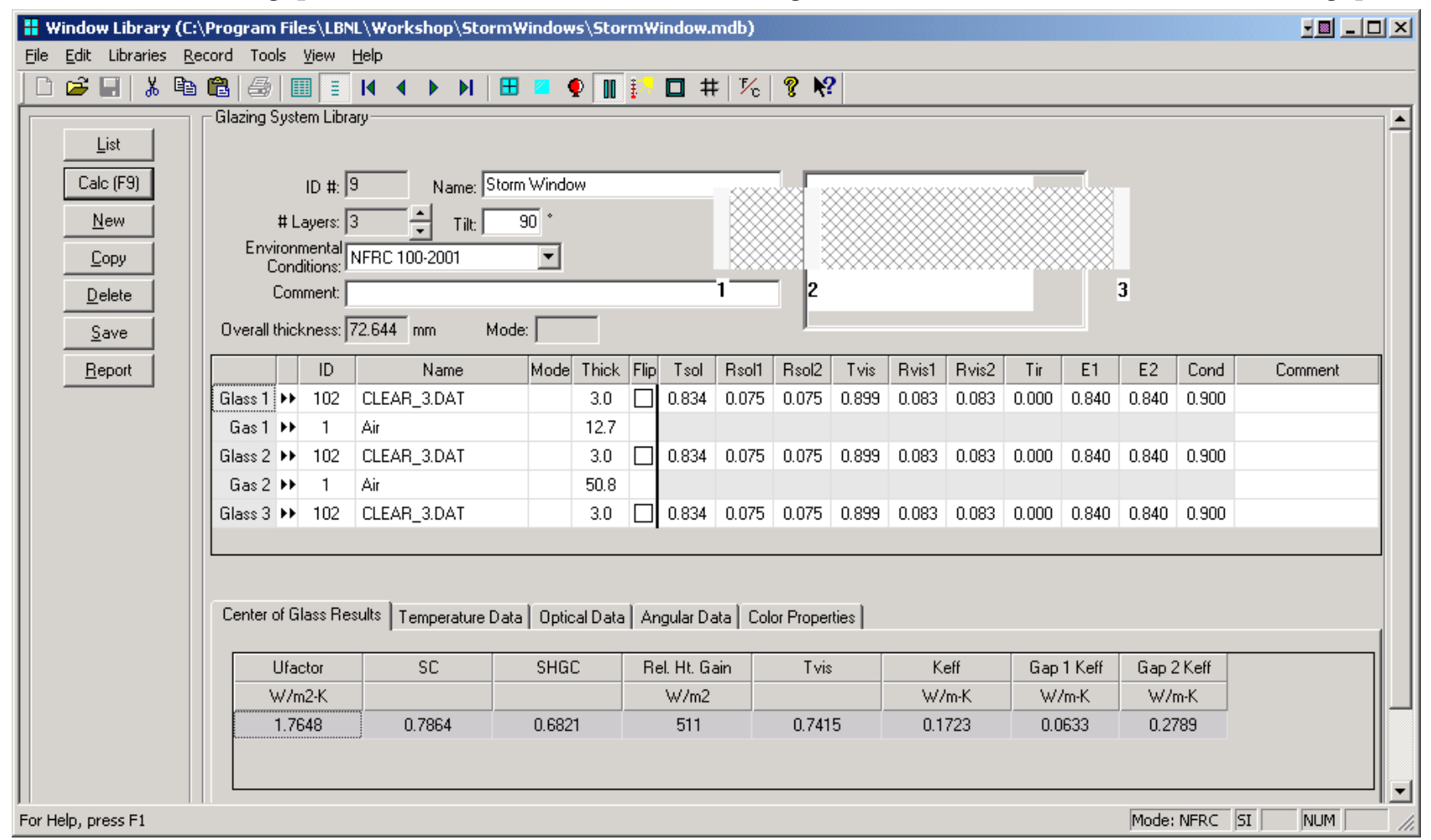

Figure 8-23 Make a triple glazed glazing system with a large gap width between the IG and the storm window. 


\subsubsection{Calculate U-factor in THERM}

The steps for importing the glazing system into THERM are explained in more detail below.

1. Draw the required frame cross sections (such as head, sill, jambs, meeting rails, and dividers)

2. From the File/Properties menu, select the appropriate Cross Section Type, such as "Sill", "Head", "Jamb", and so forth.

3. Import the glazing system with the correct storm window cavity dimensions (created in WINDOW), in this case the glazing system with the 2 " gap.

Edge of Glass Dimension $=63.5 \mathrm{~mm}(2.5 \mathrm{inch})$

Glazing System Height $=150 \mathrm{~mm}$ (6.0 inch).

Exterior Boundary Condition = Use existing BC from library, select "NFRC 100-2001 Exterior"

Interior Boundary Condition $=$ Use convection plus enclosure radiation for glazing system, and appropriate "convection only" boundary condition for the interior frame components.

THERM - [Stormwindow thm]

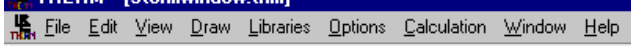

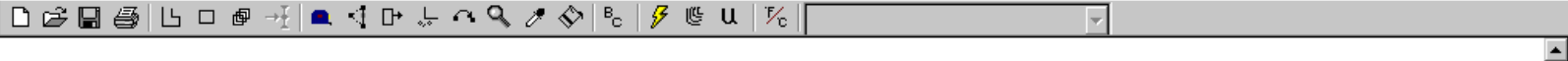

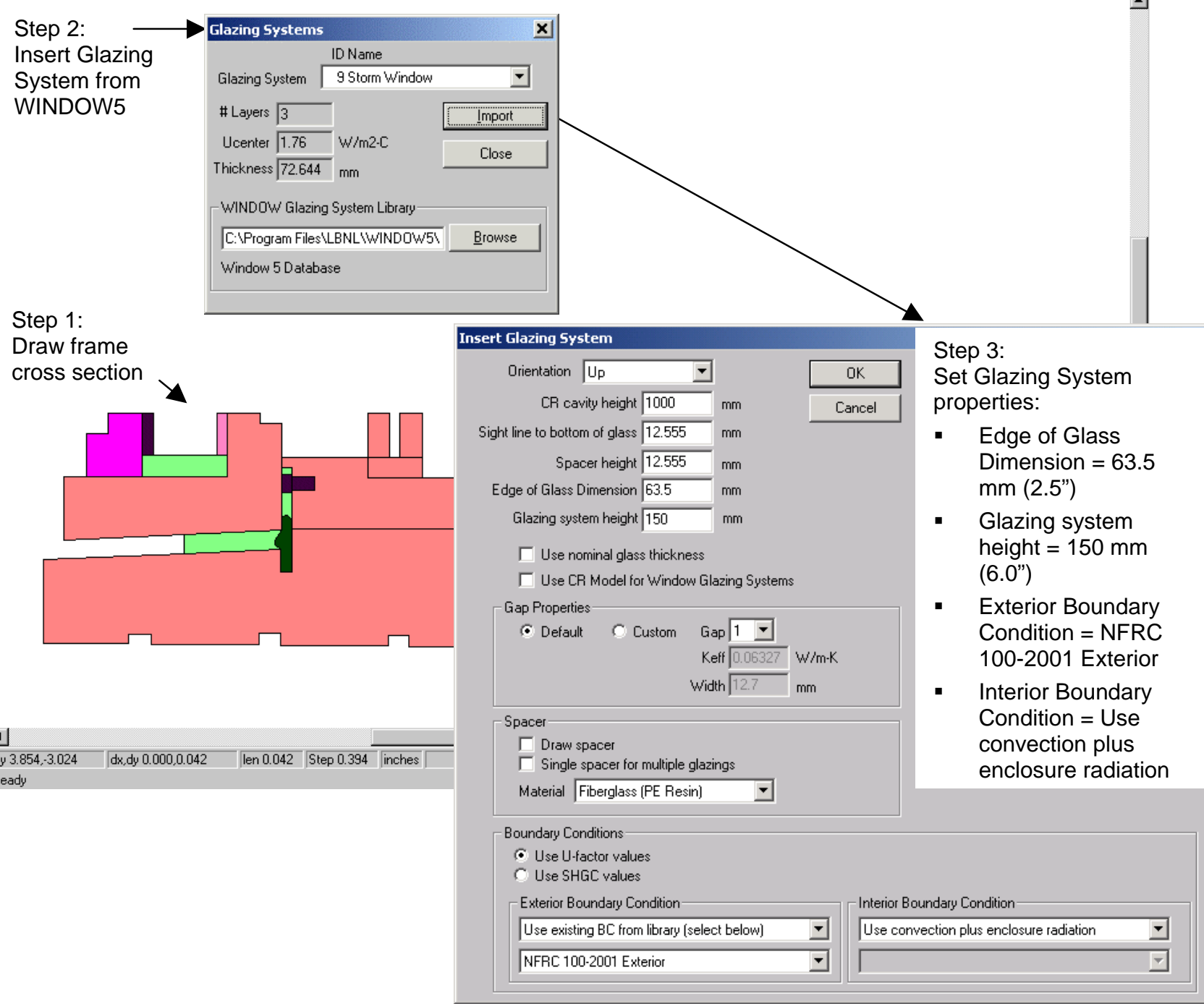

Figure 8-24 Insert the glazing system. 
4. If necessary (as in this example because there is a gap between the bottom of the glazing cavity and the frame), use the material linker to create a separate polygon and link the properties to the 2 " glazing cavity.

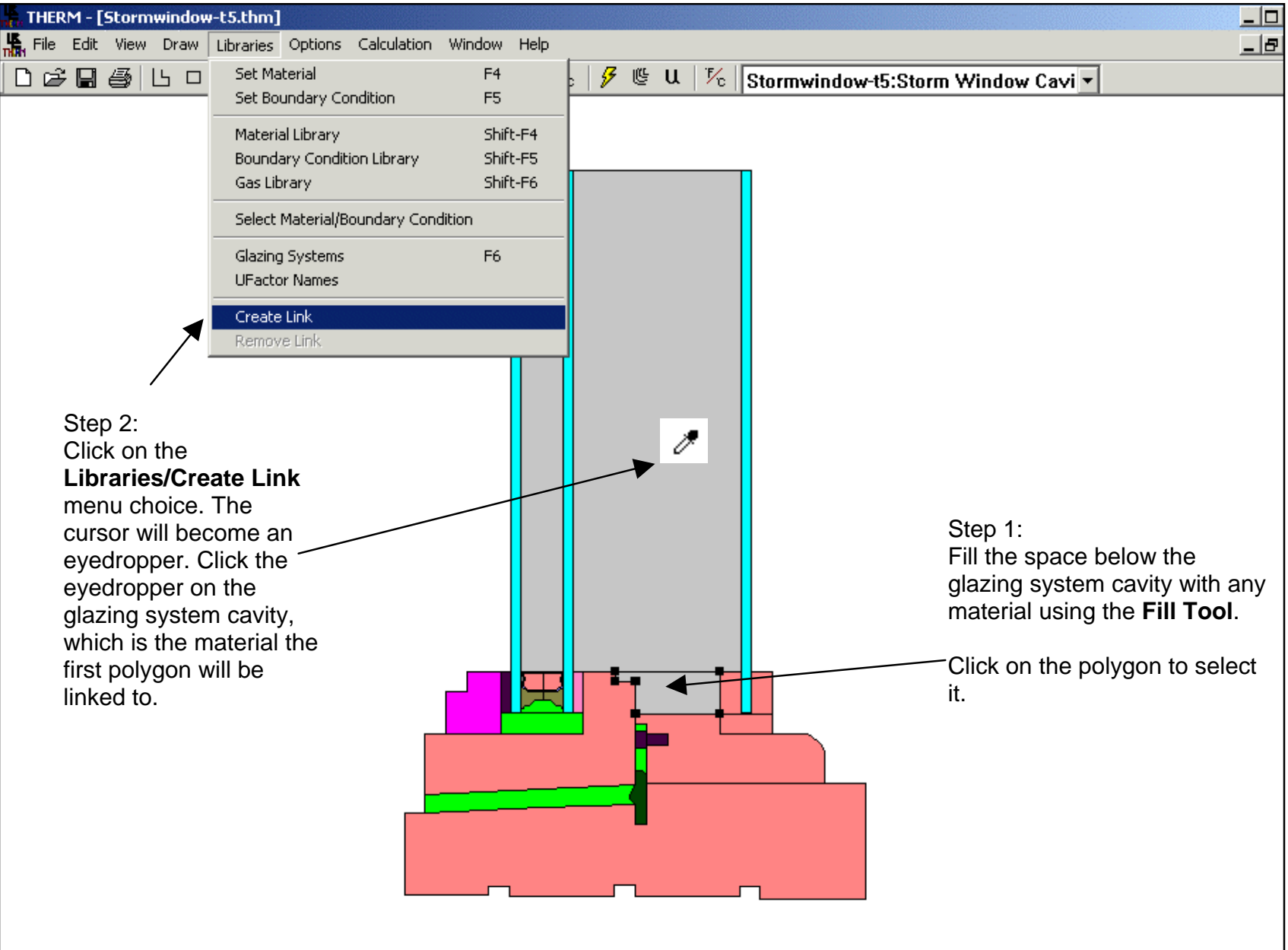

Figure 8-25 If needed, create a material link between the glazing system cavity. 
5. Generate the Boundary Conditions by pressing the $\mathbf{B C}$ toolbar button. The figure below shows the boundary conditions for one storm window cross section. Make sure that the interior boundary conditions have the Radiation Model set to "AutoEnclosure".

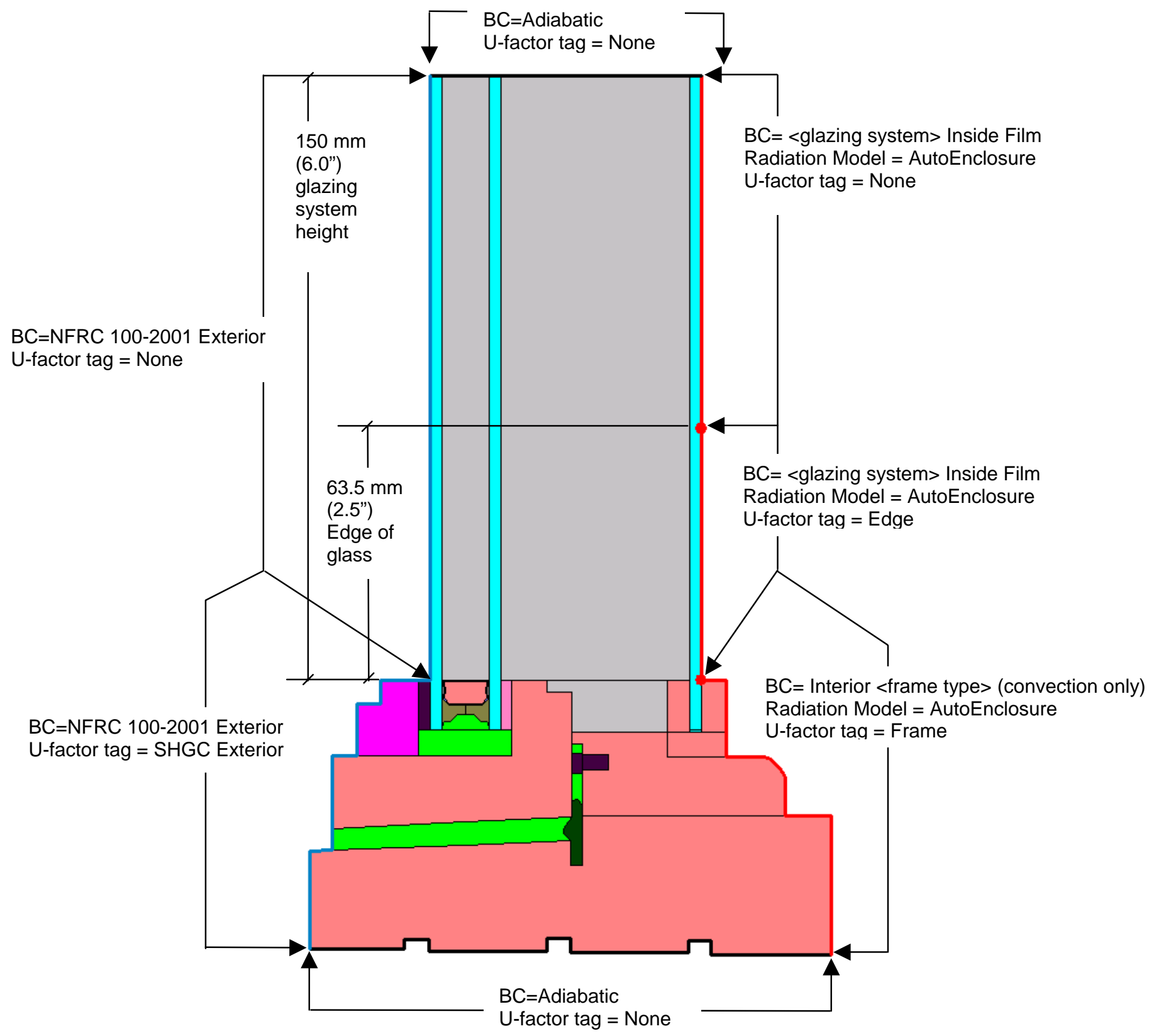

Figure 8-26 Define the boundary conditions. 
6. Simulate the problem and save the file.

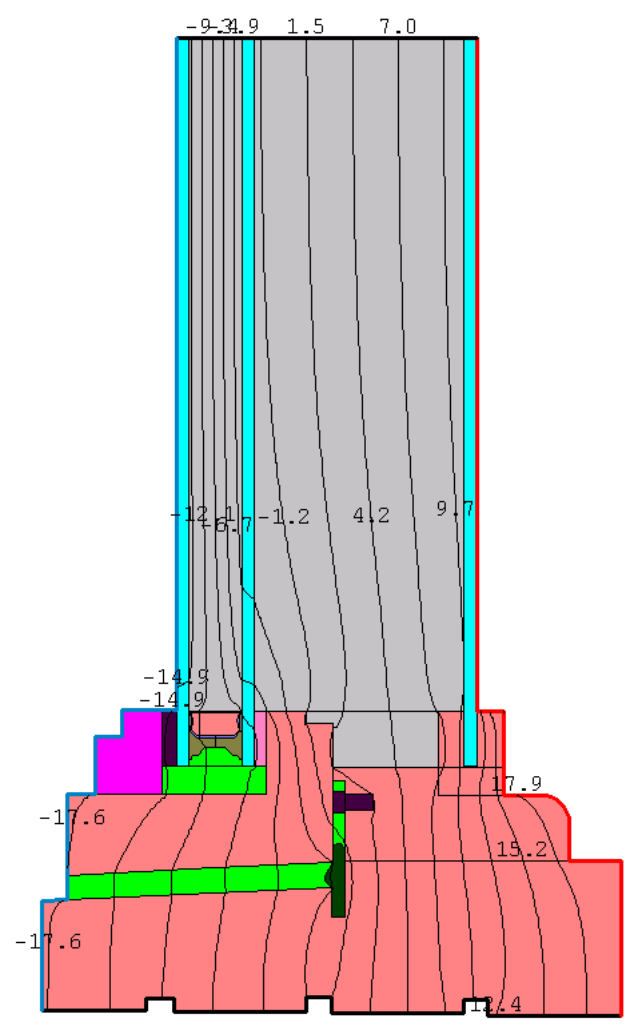

Figure 8-27 Simulate the file.

\subsubsection{Steps for Storm Window Condensation Resistance Calculation}

The Condensation Resistance model is only appropriate for horizontal frame components such as Head and Sill elements - THERM will not calculate the Condensation Resistance for a file with the Cross Section Type set to "Jamb" or "Vertical Meeting Rail".

There are two methods for calculating the Condensation Resistance information in THERM, which will be used in WINDOW to calculate the total Condensation Resistance of the product:

- Check the "Use CR Model for Window Glazing System" checkbox when importing a glazing system OR

- In the Options menu, Preferences choice, THERM File Options tab, check the "Use CR Model for Glazing Systems", as shown in the figure below.

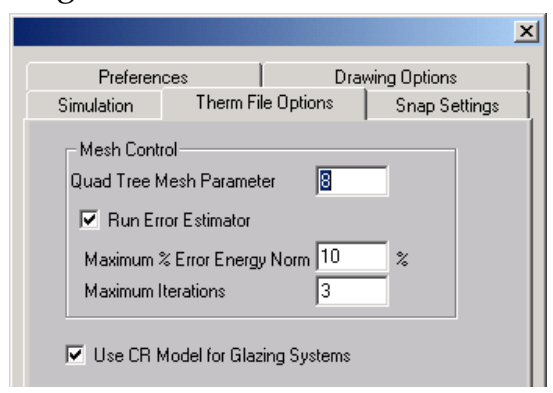

Figure 8-28 In Options/Preferences/Therm File Options, check the "Use CR Model for Glazing Systems" checkbox. 
When the CR model has been "turned on", red boundary conditions will appear inside the glazing system, and the following steps should be taken to simulate the file:

1. Check the emissivities of these boundary conditions. They should be the following:

- Emissivity of the surrounding surface, such as 0.84 for standard glass, 0.90 for painted metal and most other frame materials, 0.20 for mill finish metal, and so forth.

- 1.0 for the adiabatic (open end) of the glazing cavity.

2. Simulate the model. The program will calculate both U-factor results and the Condensation Resistance results if the CR model is checked.

3. Import the results into the WINDOW Frame Library and use the file to create the whole product in the Window Library as applicable.

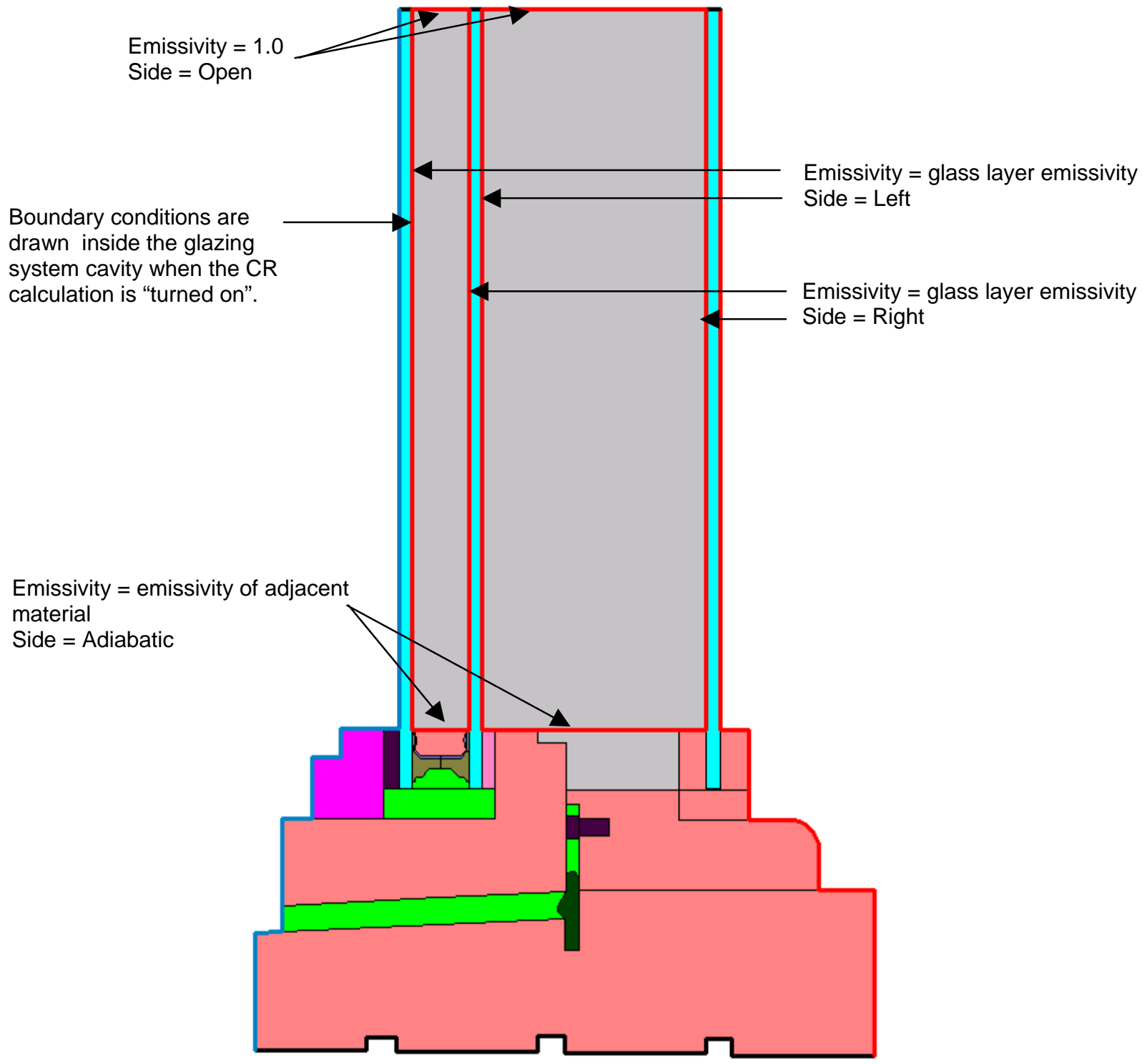

Figure 8-29 Red boundary conditions will appear inside the glazing system when the CondensationResistance option is turned on. Check the emissivities of each boundary condition. 


\subsubsection{Calculate the Total Product Values in WINDOW}

The following discussion explains how to model the whole product values for the storm window in WINDOW.

- Import the THERM files into the WINDOW Frame Library.

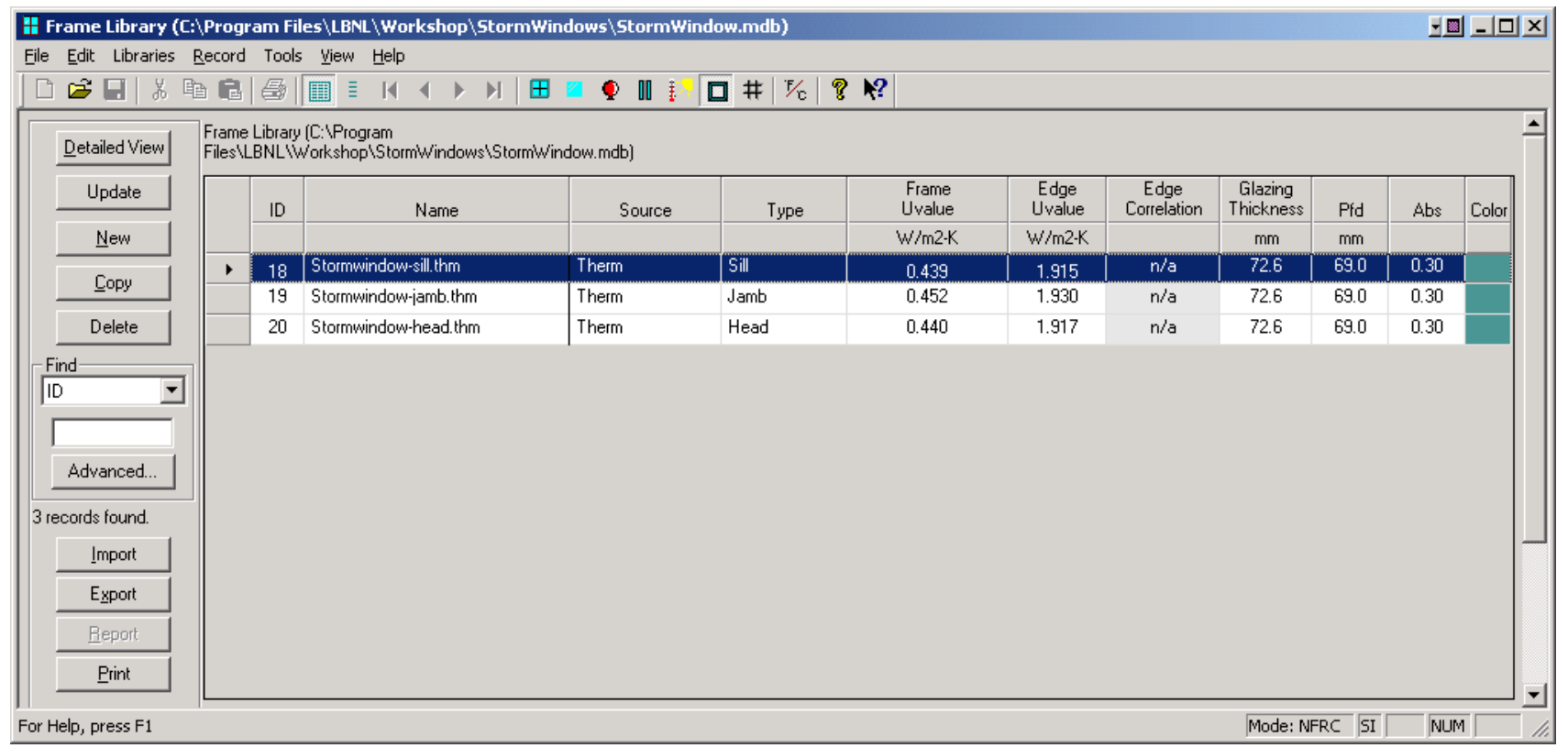

Figure 8-30 Import the storm window THERM files .

- In the WINDOW Window Library, construct the storm window from the THERM files and the glazing system previously defined, and calculate the total product values.

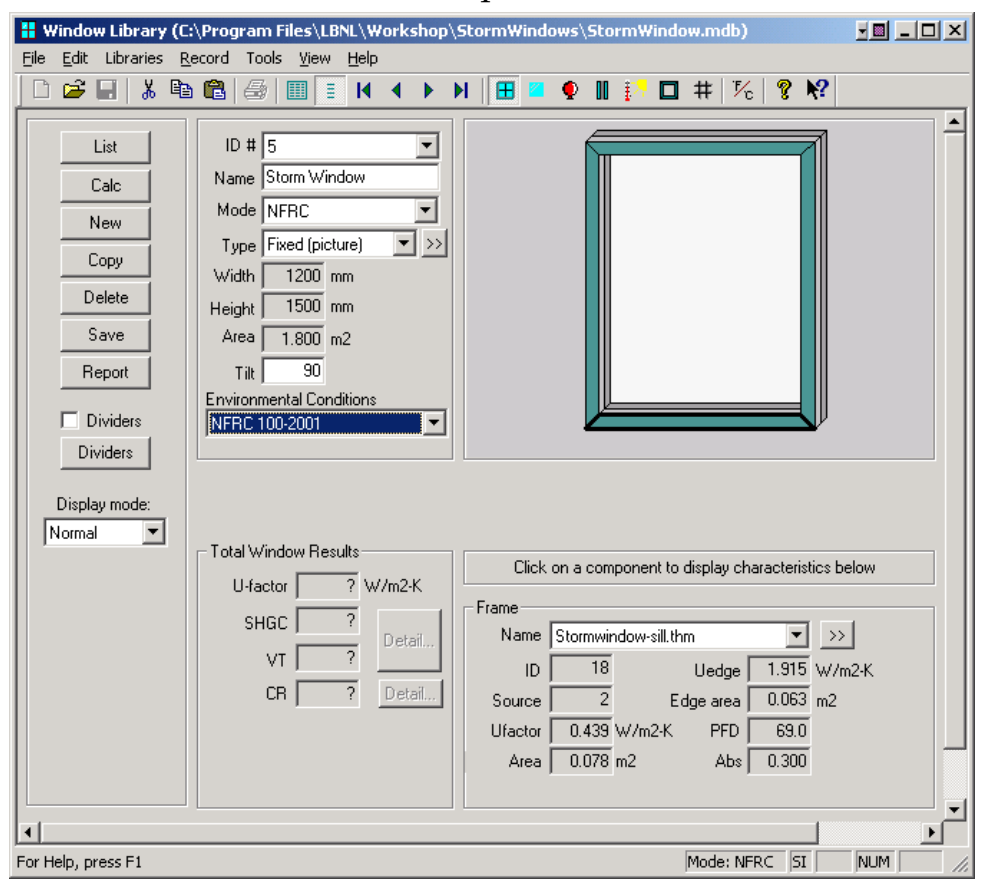

Figure 8-31 Storm window created in the Window Library to obtain total product results. 


\subsection{Skylights}

This section discusses the modeling procedures for skylights, which are modeled in sections in a similar manner to other products, rather than as full-height products as suggested in the THERM 2.1a NFRC Simulation Manual. In addition, in accordance with NFRC 100, skylights are modeled at a $20^{\circ}$ slope from horizontal.

\subsubsection{Skylight Modeling Steps}

The steps for modeling a skylight are as follows:

\section{In WINDOW:}

- Create the skylight glazing system in WINDOW:

- Set Tilt to "20" degrees

\section{In THERM:}

- Draw the required frame cross sections in THERM, for example a head, sill, and jambs if they are all different, untilted. Because the tilt of the jambs will be in the z-direction, which is not possible to display in the two dimensional viewing of THERM, they will be drawn vertically and the gravity vector oriented properly to reflect the tilt in the z-direction.

- Do not use the Condensation Resistance Model on any of the THERM skylight cross sections. WINDOW will calculate the $C R$ value based on the temperatures from the U-factor results. (Even if the THERM cross sections are modeled with CR enabled, WINDOW will use the U-factor temperature results rather than the $C R$ temperature results when calculating the whole product $C R$ value).

- Set the Cross Section value in File/Properties as follows:

- For Sill: set Cross Section to "Sill", Gravity Vector should face "Down"

- For Head: set Cross Section to "Head", Gravity Vector should face "Down"

- For Jambs: set Cross Section to "Sill", set Gravity Vector to "Right"

- The Frame Cavity height is not used by the program for the skylight cross sections, as long as the Types are defined properly as shown above, so the default value of $1000 \mathrm{~mm}$ can be left unchanged.

- Insert the glazing system from WINDOW into the frame cross sections with the the Edge of Glass Dimension field set to $150 \mathrm{~mm}$ (6.0 inches). The CR cavity height field can be set to any value (you can leave it set to the default of $1000 \mathrm{~mm}$ ) because the U-factor temperatures not the CR temperatures will be used in WINDOW to calculate the overal CR value).

- Insert the Sill glazing system with orientation up

- Insert the Head glazing system with orientation down

- Insert the Jamb glazing system with orientation up

- Assign the boundary conditions. Interior Boundary conditions have the following settings:

- Radiation Model set to "AutoEnclosure"

- Frame Boundary Conditions: set to the appropriate "Interior (20 tilt) ..." choices

- Tilt the cross section 20 degrees from horizontal:

- For a Sill or Head, rotate the entire model 70 degrees clockwise

- For Jambs, do not rotate the model at all 
- Simulate the skylight cross sections and save them.

- View the U-factor for the cross section, and make sure the "Projected in Glass Plane" is selected from the Projection pulldown list, as shown in the figure below. This will ensure that the projection will be correct for the tilted cross section.

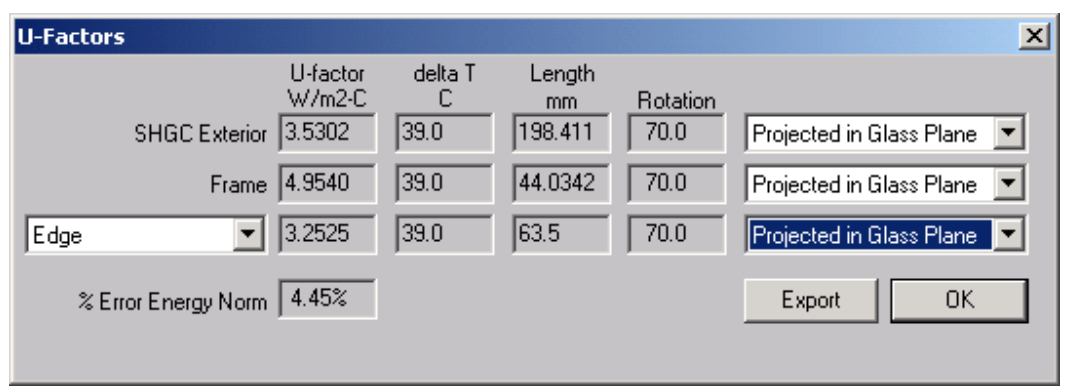

Figure 8-32 Make sure the "Projected in Glass Plane" projection option is selected for the tilted cross section.

- Import the components into the WINDOW Frame Library (and Divider Library if appropriate)

- Constuct the whole product in the WINDOW Window Library to get the overall product results. 


\subsubsection{Skylight Mounting Details}

There are two ways that skylights can be mounted into a roof system, either flush-mounted or curb-mounted. Figure 8-33 and 8-34 show these two different mounting styles. Each mounting style has a slightly different definition of the adiabatic boundary condition, and each will have a different projected frame length. The rules for modeling can be found in NFRC 100 and the NFRC Technical Interpretations. To model curb mounted skylights, if the projected frame height is zero, define a Frame U-factor Surface Tag $0.25 \mathrm{~mm}(0.01$ inches) up the interior of the glass, which will result in a non-zero frame height.

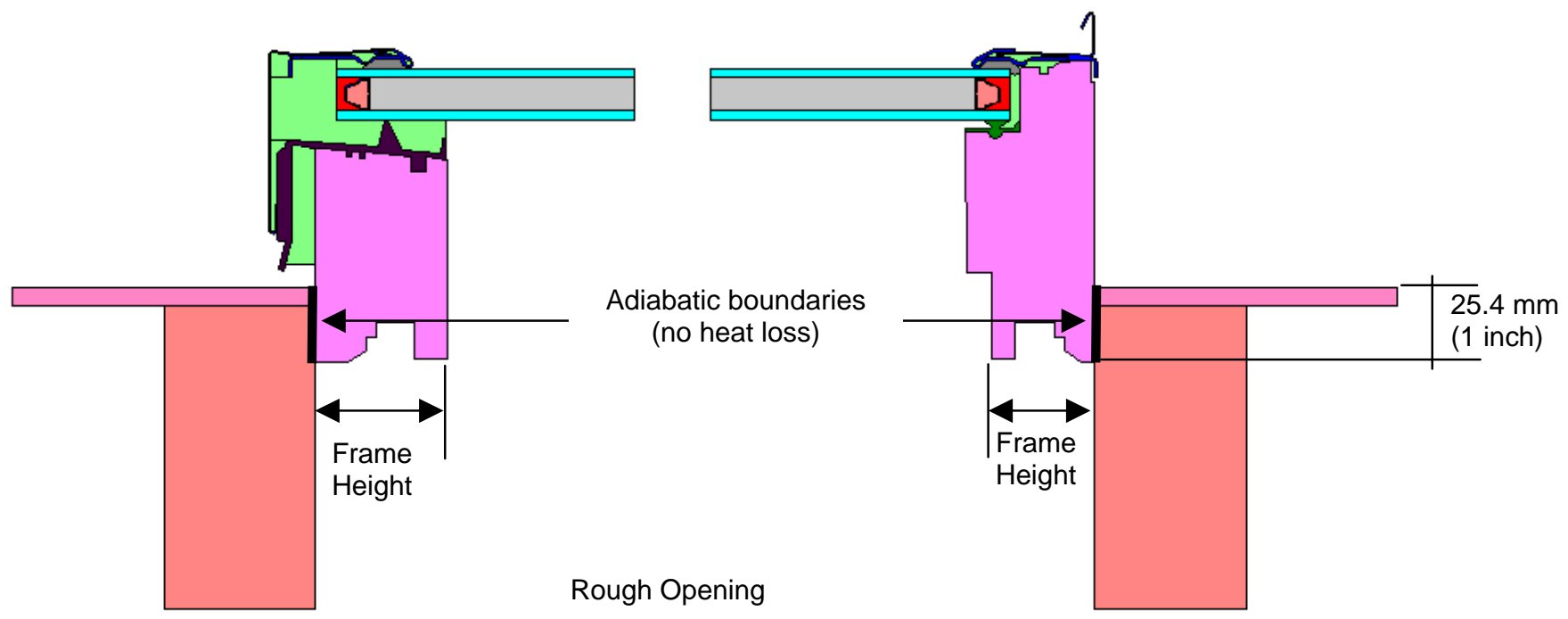

Figure 8-33 A flush-mounted skylight.

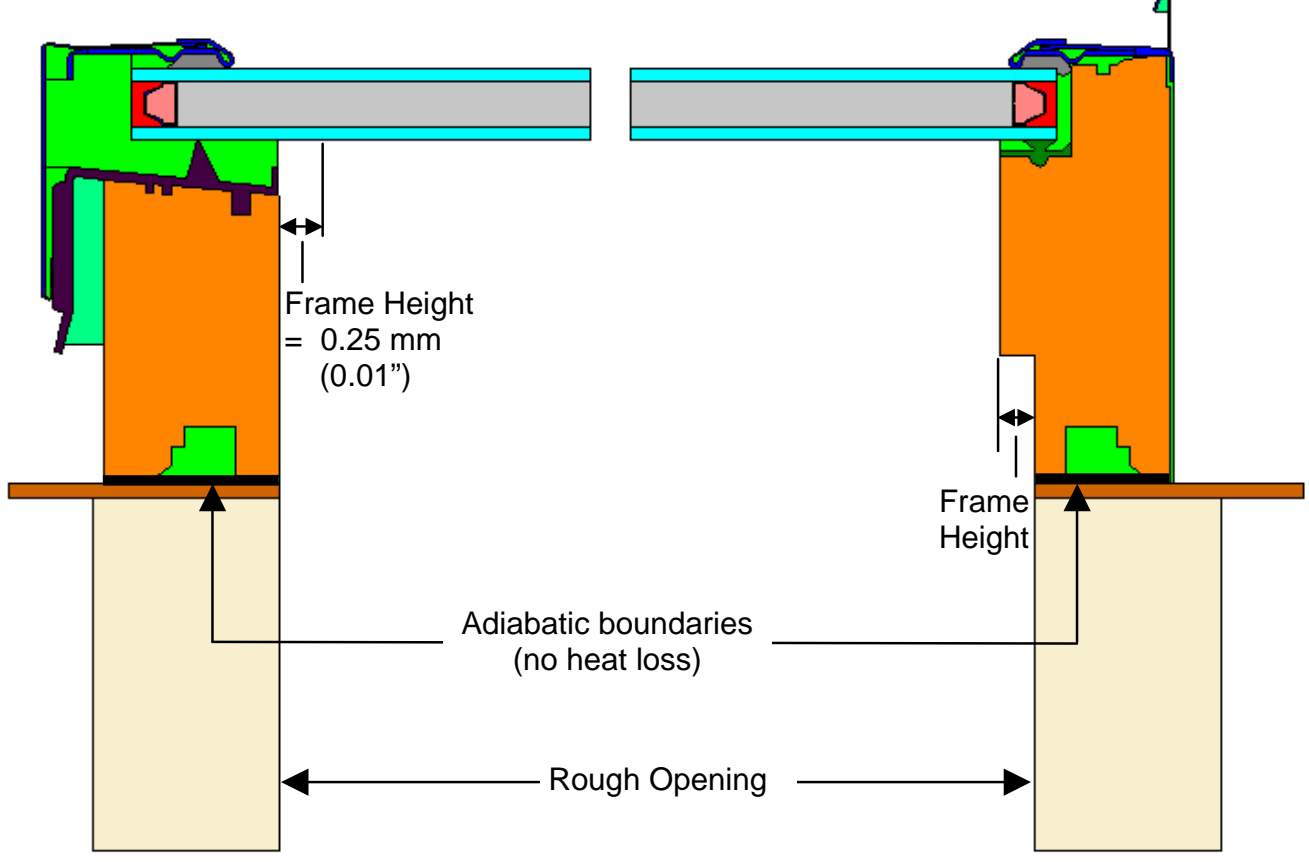

Figure 8-34 A curb-mounted skylight. 


\subsubsection{Example Flush Mounted Skylight Problem}

This example assumes a flush-mounted skylight.

\section{In WINDOW:}

1. Glazing System Library: Make a glazing system with a tilt of $20^{\circ}$ off horizontal. In this example, the glazing system is called Skylight Double Glz and is made up of generic glass layers.

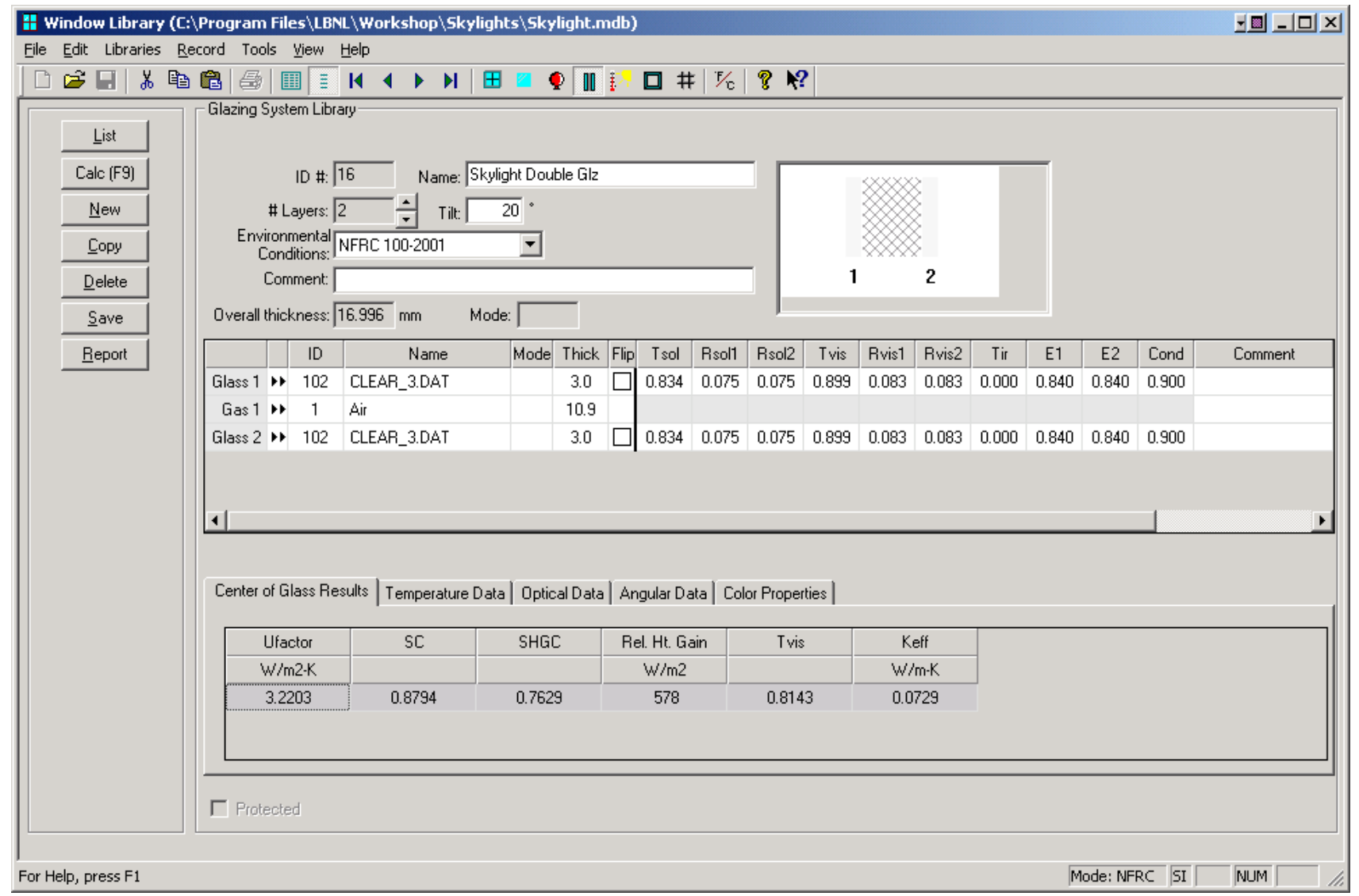

Figure 8-35 Make new glazing system in the Glazing System Library with Tilt $=20$ degrees.

2. Save the file: Make sure to save the glazing system (Record menu, Save choice.). 


\section{In THERM, for Sill:}

1. Draw the appropriate cross sections for the Sill.

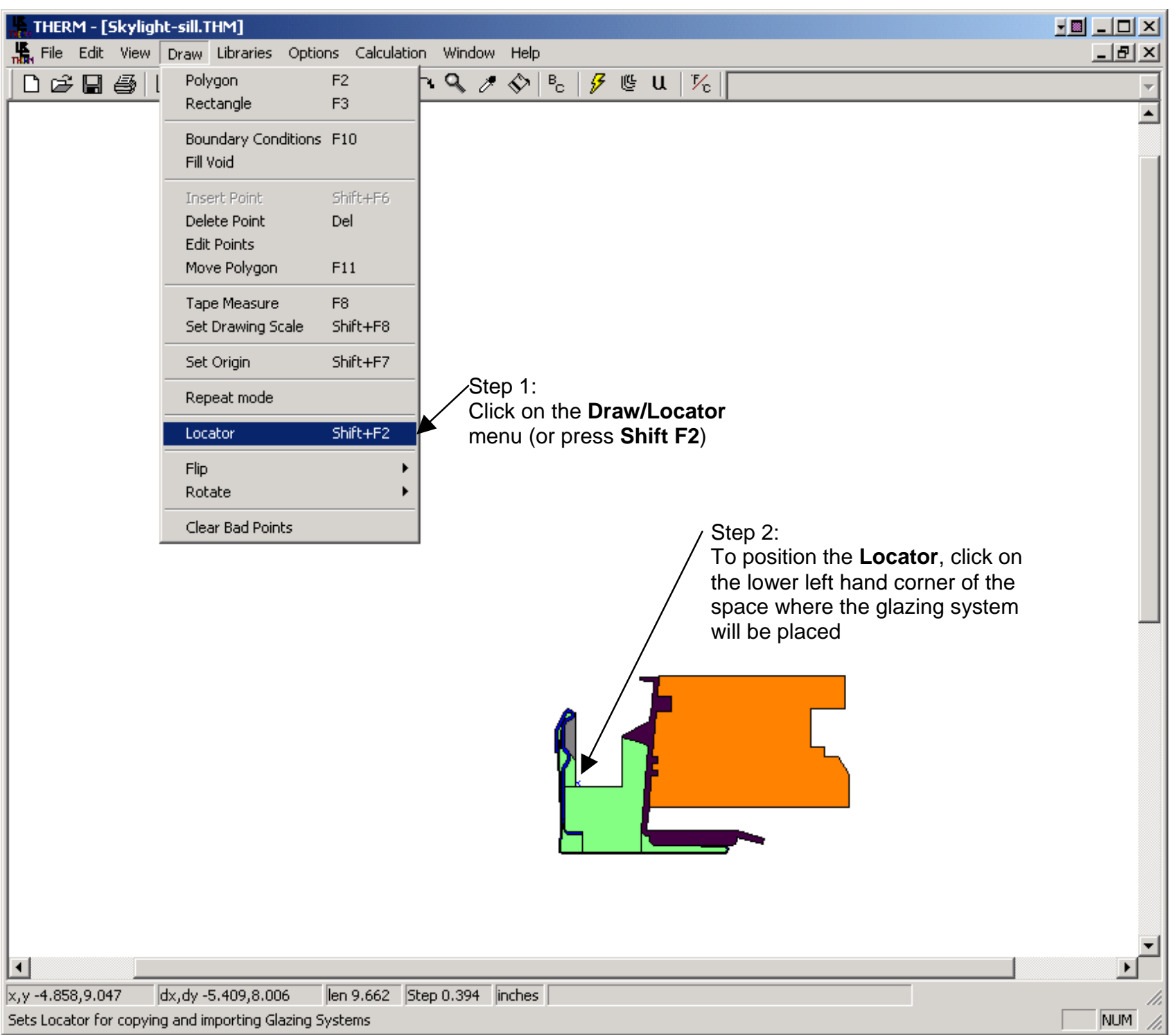

Figure 8-36 Position the locator so that the first glazing section can be inserted. 
2. Insert the glazing system for the Sill, with the following settings:

- Orientation $=U p$

- Cavity height $=1000 \mathrm{~mm}$

- Sight line to bottom of glass = measure this value with the tape measure or get from dimensioned drawings

- Spacer height $=$ measure this value with the tape measure or get from dimensioned drawings

- Edge of Glass Dimension $=150 \mathrm{~mm}$ (6.0 inches)

- Draw spacer $=$ not checked

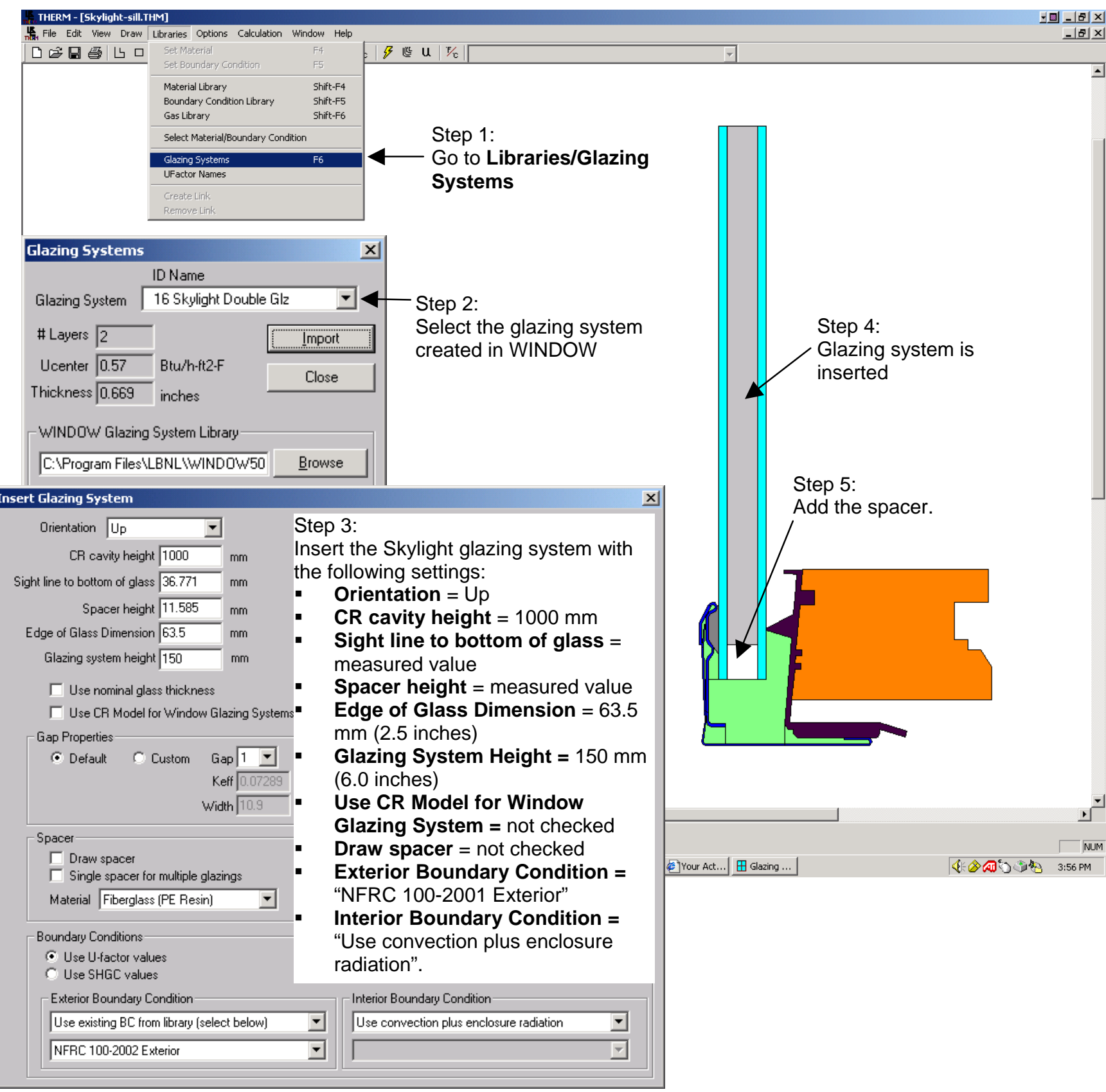

Figure 8-37 Insert the glazing system . 
3. Assign Boundary Conditions and U-factor tags: Click on the Boundary Conditions (BC) toolbar button and correct any problems encountered with the geometry (see Section 6.5.3, "Voids, Overlaps, and Bad Points" in this manual).

4. Tilt the cross section to be 20 degrees off the horizontal plane. For this example sill cross section, click on the Draw menu, Rotate/Degree choice, and enter 70 degrees Clockwise.

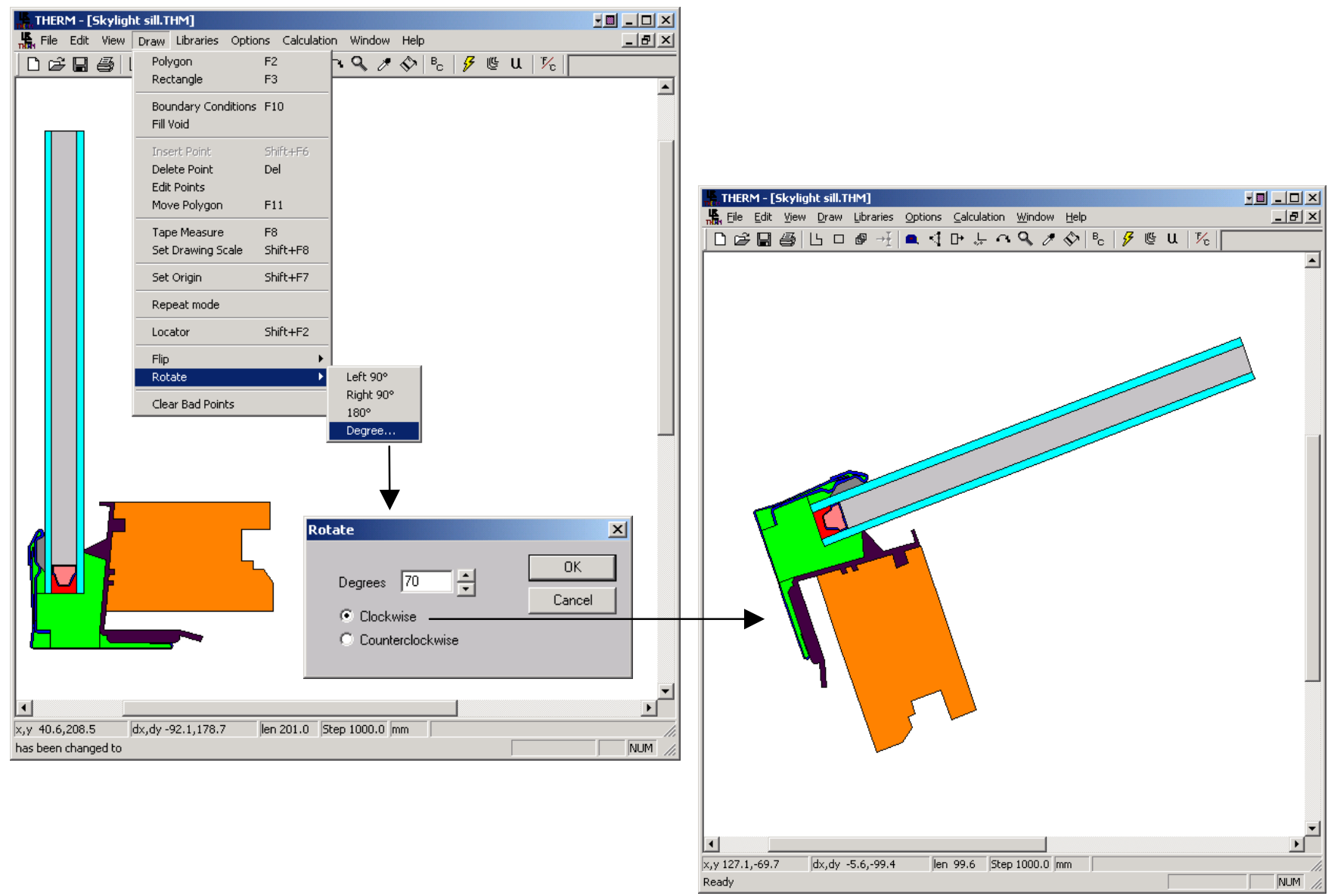

Figure 8-39 Rotate the sill cross section AFTER assigning Boundary Conditions. 


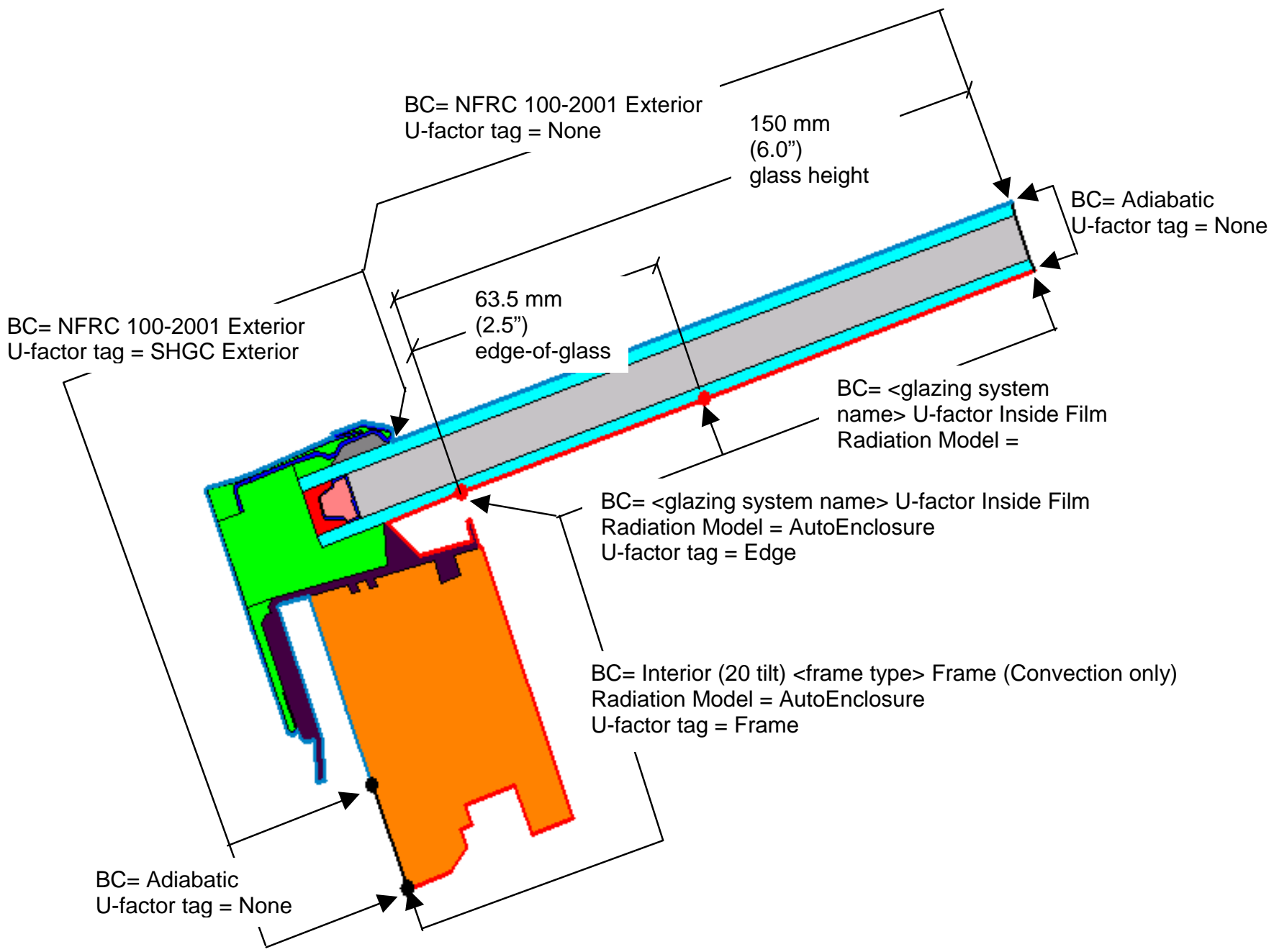

Figure 8-40 Boundary condition and U-factor tag settings for inset skylight Sill example.

6. Check the Gravity Vector for the Sill cross section (View/Gravity Arrow), which should point down.

7. Simulate the file. 
8. Click on the Show U-factors button to view the U-factors dialog box. Make sure that the projection is set to "Projected in Glass Plane" which will allow the program to calculate the correct projected frame dimensions with a tilted cross section.

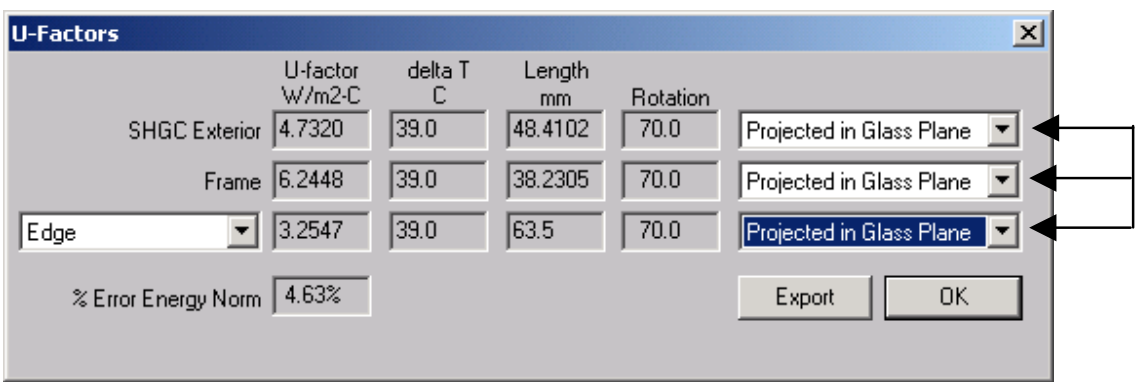

Select Projected in Glass Plane from the pulldown list to replace Projected $Y$ for Frame, Edge and

Figure 8-41 Select the Projected in Glass Plane for the projected frame dimension calculation. 


\section{In THERM, for Head:}

1. Create the cross section for the Head, set the Cross Section Type to "Head", and import the glazing system facing Down (in order to get the Gravity Vector pointing in the proper direction).

2. Assign the Boundary Conditions as shown in the figure below.

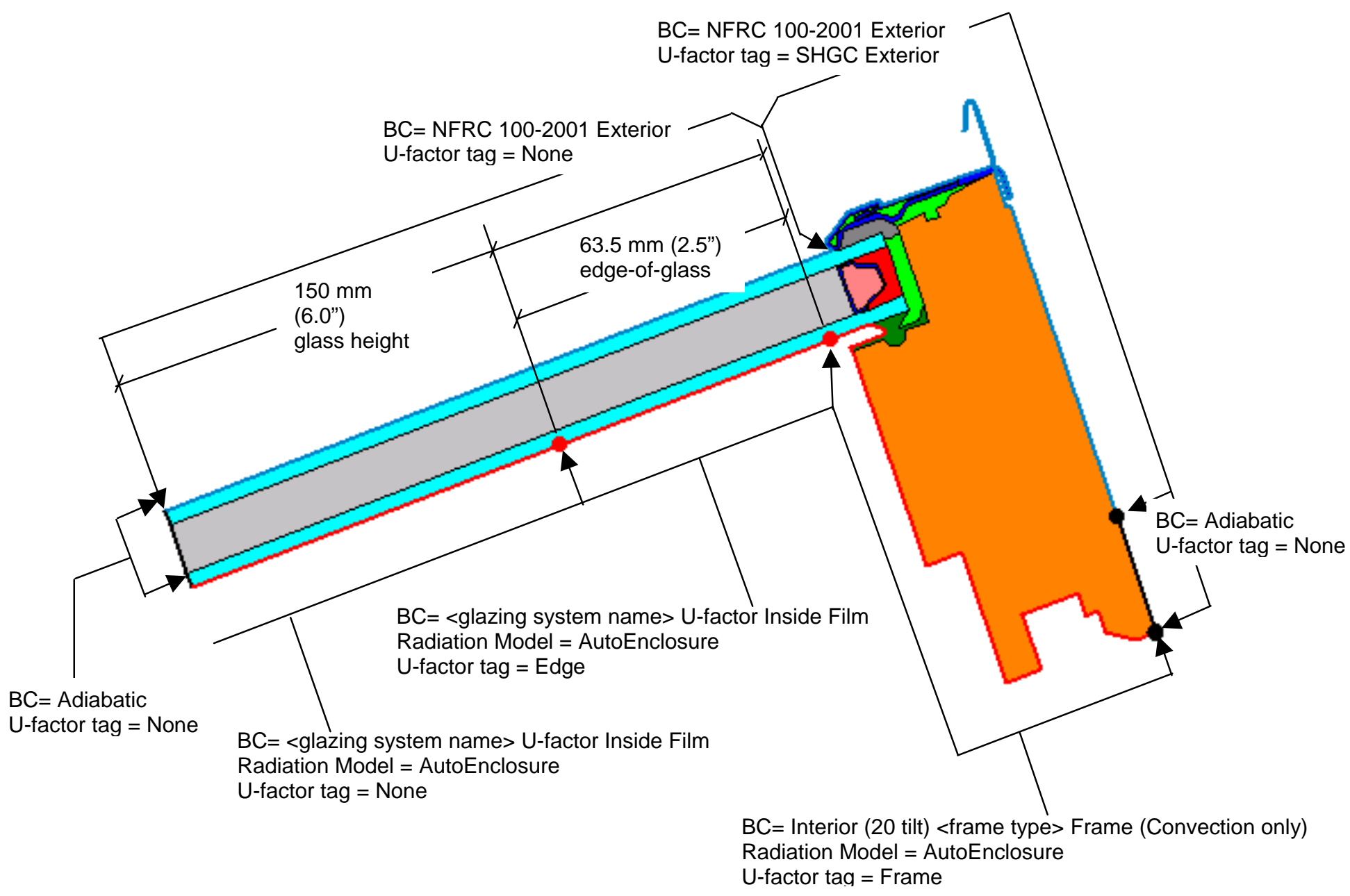

3. Tilt the Head cross section so that it is 20 degrees off horizontal (click on the Draw menu, Rotate/Degree choice, and enter $\mathbf{7 0}$ degrees Clockwise).

4. Check the Gravity Vector (View/Gravity Arrow), which should be pointed down.

Figure 8-43 Boundary condition and U-factor tag settings for inset skylight Head example.

5. Simulate the file. 
6. Click on the Show U-factors button to view the U-factors dialog box. Make sure that the projection is set to "Projected in Glass Plane" which will allow the program to calculate the correct projected frame dimensions with a tilted cross section.

Figure 8-44 Select the Projected in Glass Plane for the projected

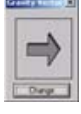

frame dimension calculation.

\section{In THERM, for Jamb:}

1. Create the cross-section for the Jamb. The steps are similar to modeling the head and sill, except for the following:

- Jambs are modeled in the vertical direction

- The Cross Section Type is set to "Sill"

- The glazing system is oriented " $U p$ "

- The gravity vector is set by hand to "Right"

2. Simulate the file.

Because the cross section is not rotated, the projection in the U-factor dialog box can be set to either "Projected Y" or "Projected in Glass Plane"; both settings will result in the same answer. Figure 8-46 The projection can be set to either "Projected Y" or "Projected in Glass Plane"; both will result in the same answer.
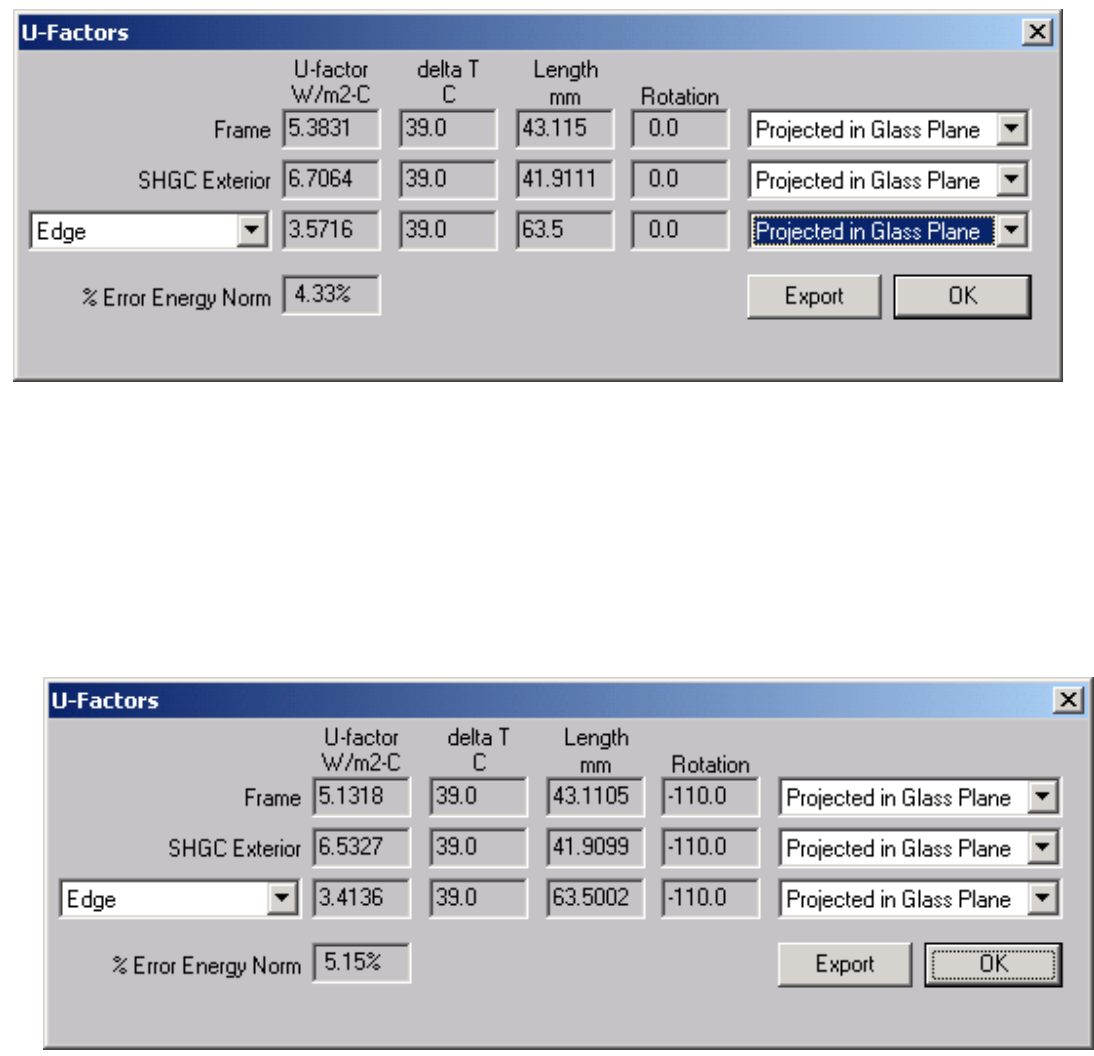


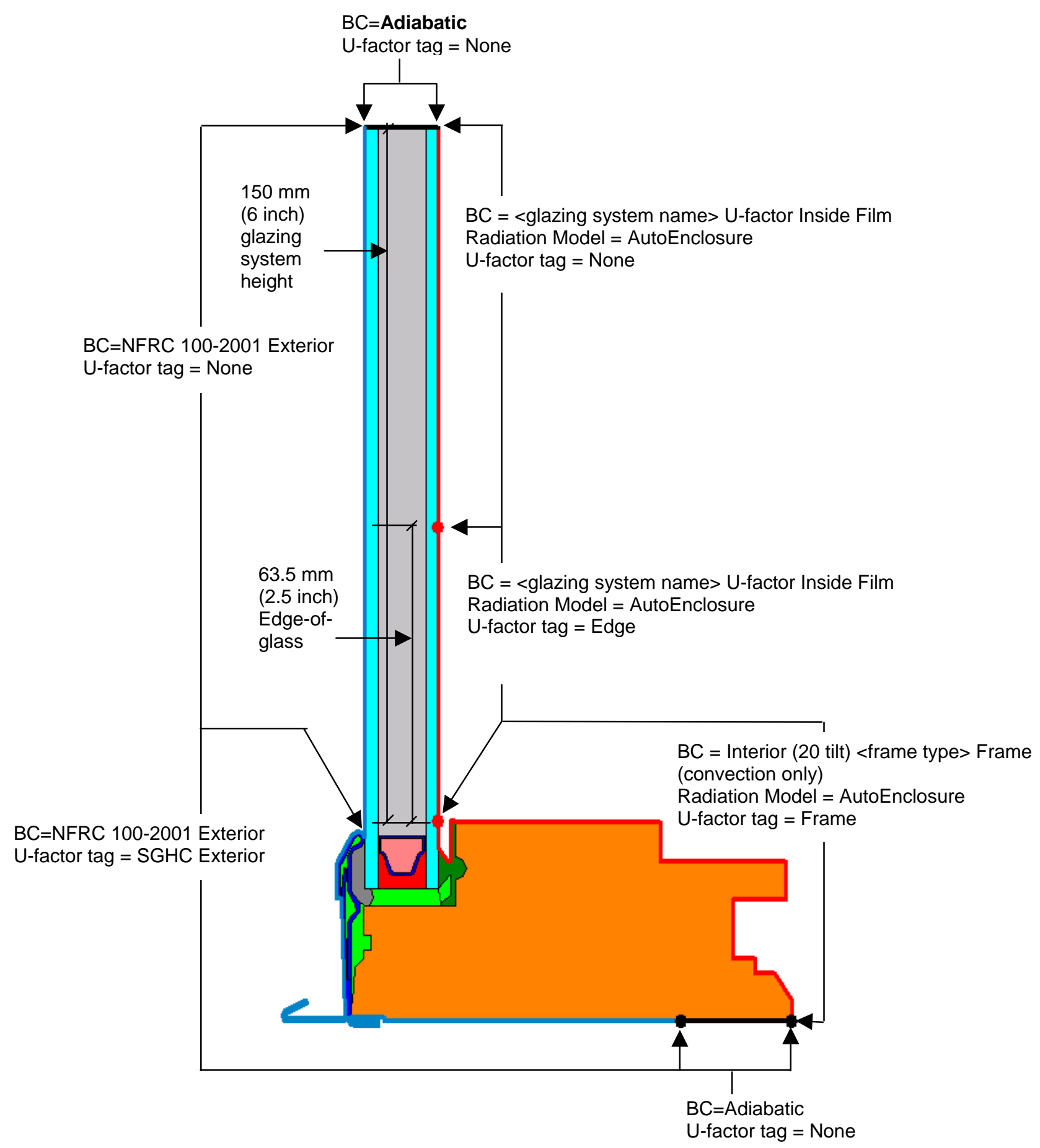

Figure 8-48 Boundary condition and U-factor tag settings for inset skylight jamb example. 


\section{In WINDOW, Calculate the Total Product U-factor:}

1. In the WINDOW Frame Library, import the THERM files for the Head, Sill, Jamb and any other needed cross sections that were modeled.

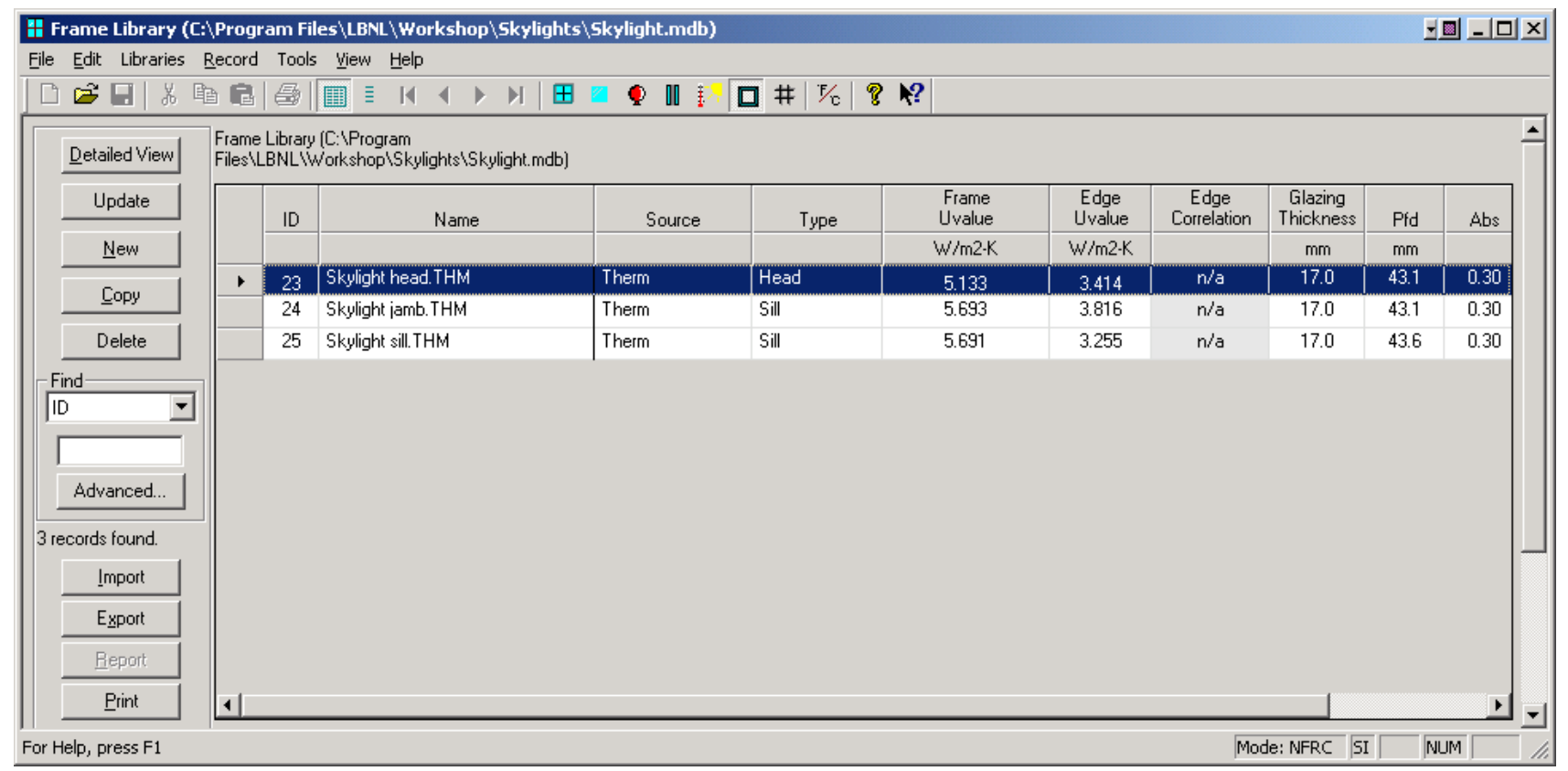

Figure 8-49 Import the skylight THERM files into the WINDOW Frame Library.

2. Construct the whole skylight in the WINDOW Window Library by using the THERM files for the frame components and the glazing system for the center of glass.

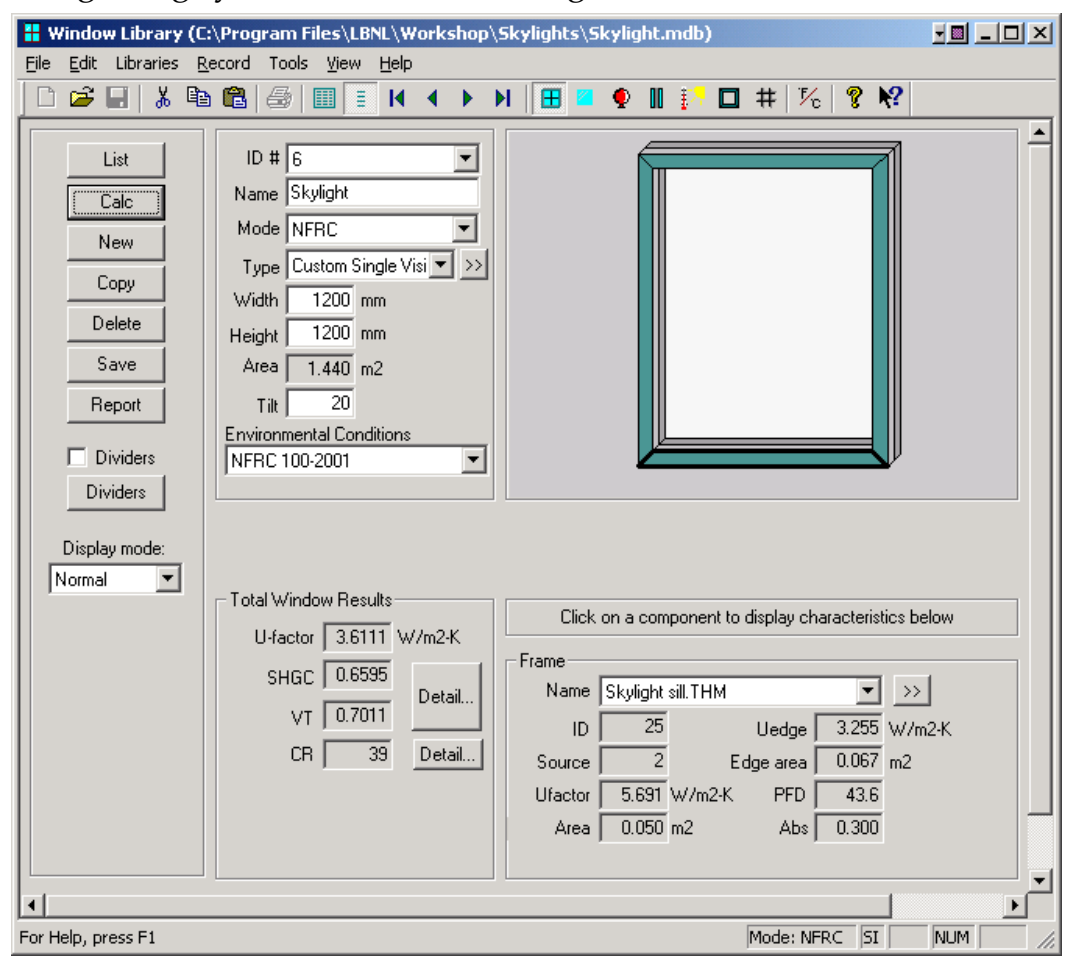

Figure 8-50. Create the whole skylight in the Window Library. 


\subsection{Modeling Tubular Daylighting Devices}

Tubular skylights are a group of products that can loosely be defined as non-standard skylight products. Their primary purpose is to provide daylighting, and not view to the outside. For this reason, there are some arguments whether these products can be considered fenestration at all. However, because they penetrate the building envelope and provide some of the essential functionality of a fenestration system (i.e., daylight) they are considered to be a fenestration product.

The assumptions and methodology for modeling these products differs considerably from typical fenestration products. The following is a list of standardized assumptions to be used when modeling tubular daylighting devices:

- $\mathrm{D}=$ Shaft Diameter $=350 \mathrm{~mm}(14$ in.)

- $\mathrm{L}=$ Shaft Length $=750 \mathrm{~mm}(30 \mathrm{in}$.)

- Standard dome mounted on $350 \mathrm{~mm}$ (14 in.) shaft

- Exterior boundary conditions are applied on the exterior side of the dome

- Standard ASHRAE Attic boundary conditions are applied to the exposed surfaces of the shaft,

- Bottom of the shaft is mounted in a $250 \mathrm{~mm}$ (10 in.) thick surround panel (standard surround panel material, such as EPS),

- Bottom of tubular skylight is covered with light diffusing plate (manufacturer supplied).

The first step is to draw the geometry of the tubular daylighting device in THERM, per the manufacturers' drawings and using the assumptions above (see Figure 8-46). Material properties, other than frame cavities, should be assigned from the THERM material library.

Next calculate the effective conductivity of the shaft and dome cavity. The set of equations and assumptions required to calculate effective conductivity of this cavity is detailed in Curcija (2001). A custom spreadsheet is also designed to facilitate this calculation and is available on request from NFRC (tubes_keff.xls). The information necessary to calculate $k_{\text {eff }}$ of this cavity are the average temperatures and emissivities of the inside surface of the diffuser plate at the bottom of the cavity, and inside surface of the dome at the top of the cavity. Initially these temperatures need to be estimated, a reasonable starting point being $-2^{\circ} \mathrm{C}(28.4 \mathrm{o})$ for the diffuser plate and $-17^{\circ} \mathrm{C}\left(1.4^{\circ} \mathrm{F}\right)$ for the dome (when the dome is single glazed). After the THERM simulation is calculated with the keff determined from these estimated temperatures, find the average temperatures for the diffuser plate and dome surfaces using the THERM tape measure tool. If the resulting average temperatures differ by more than $1^{\circ} \mathrm{C}(2 \mathrm{oF})$ from the estimated values, a new keff shall be calculated and the THERM simulation repeated with the new keff. This process should be repeated until the criterion of $1{ }^{\circ} \mathrm{C}(2$ $\circ$ F) temperature difference is satisfied. In many cases, one iteration is enough, but the temperatures shall be checked to make sure that this has been met for the particular case. In the THERM file, fill the shaft/dome cavity with a solid material which has the conductivity equal to the calculated $k_{\text {eff. }}$ Fill the other small frame cavities with the "Frame Cavity NFRC 100-2001" frame cavity material, which will automatically calculate effective conductivity of them. 
Next the boundary conditions need to be defined and assigned as shown in Figure 8-47. The exterior and adiabatic boundary conditions can be used from the THERM library, while the attic and indoor side of the diffuser plate must be defined in the THERM Boundary Condition Library. The following values should be used for the boundary conditions:

- Exterior: NFRC 100-2001 Exterior

$h_{0}=30 \mathrm{~W} / \mathrm{m}^{2} \cdot \mathrm{K} ; T_{0}=-18^{\circ} \mathrm{C}$

$\left(h_{0}=5.3 \mathrm{Btu} / \mathrm{hr} \cdot \mathrm{ft}^{2} .^{\circ} \mathrm{F} ; \mathrm{T}_{o}=0{ }^{\circ} \mathrm{F}\right)$

- Adiabatic: Adiabatic

$q=0 \mathrm{~W} / \mathrm{m}^{2}$

$\left(q=0 \mathrm{Btu} / \mathrm{hr} \cdot \mathrm{ft}^{2}\right)$

- Attic: User defined

$h_{a}=12.5 \mathrm{~W} / \mathrm{m}^{2} \cdot \mathrm{K} ; T_{a}=-18^{\circ} \mathrm{C}$

$\left(h_{a}=2.2 \mathrm{Btu} / \mathrm{hr} \cdot \mathrm{ft}^{2} \cdot{ }^{\circ} \mathrm{F} ; \mathrm{T}_{a}=0{ }^{\circ} \mathrm{F}\right)$

- Indoor Side of diffuser plate: User defined

$h_{i}=9 \mathrm{~W} / \mathrm{m}^{2} \cdot \mathrm{K} ; T_{i}=21^{\circ} \mathrm{C}\left(1.582 \mathrm{Btu} / \mathrm{hr} \cdot \mathrm{ft}^{2} \cdot{ }^{\circ} \mathrm{F} ; \mathrm{T}_{i}=70^{\circ} \mathrm{F}\right)$

Note: The height of the shaft/dome cavity represents area weighted equivalent height, $L_{\text {eqv }}$, and is set to 1.041 $\mathrm{m}$ (41 in.) for domed top diffuser products and $750 \mathrm{~mm}(30 \mathrm{in}$.) for flat top diffuser products. 


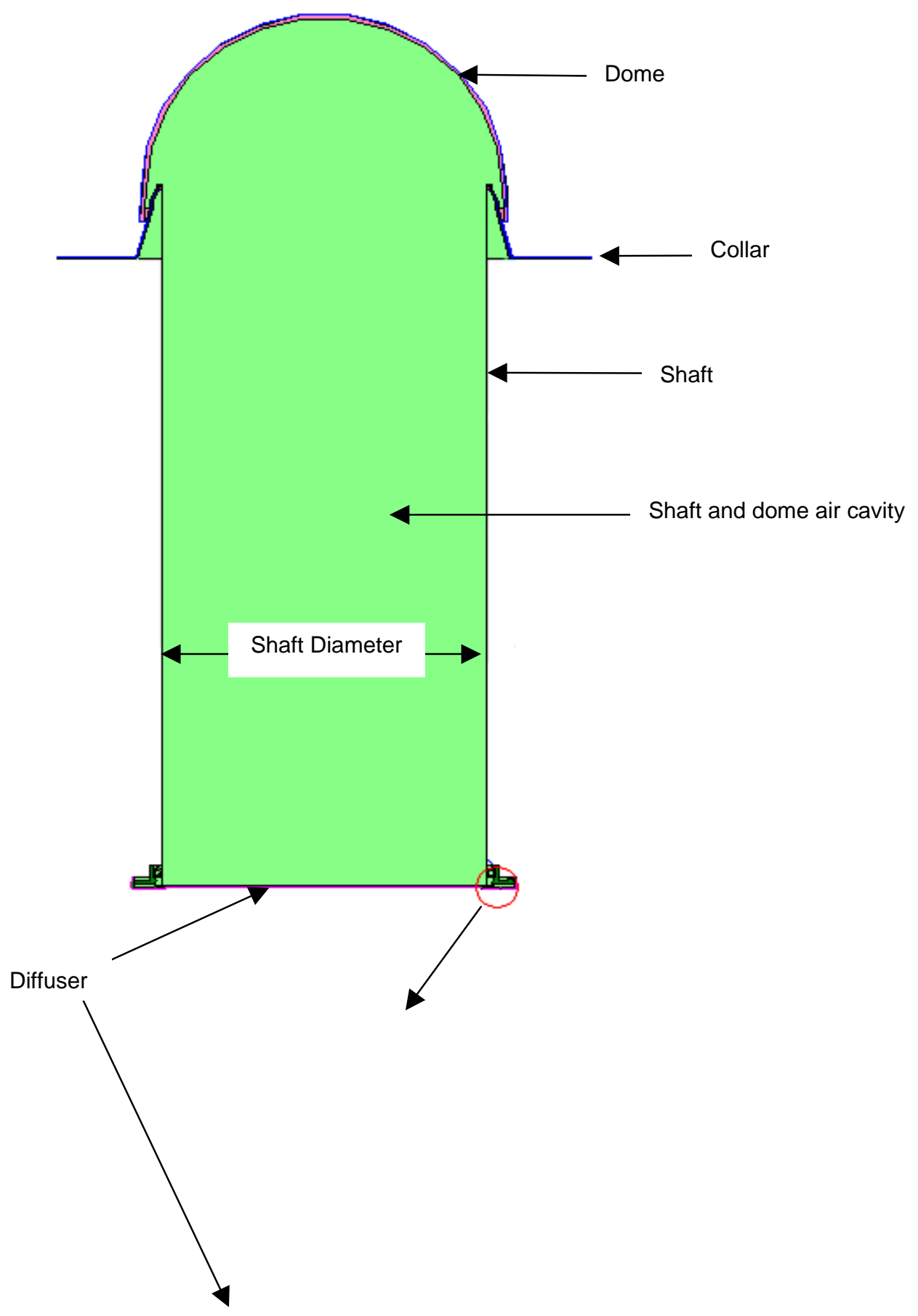

Figure 8-5

$M$. 

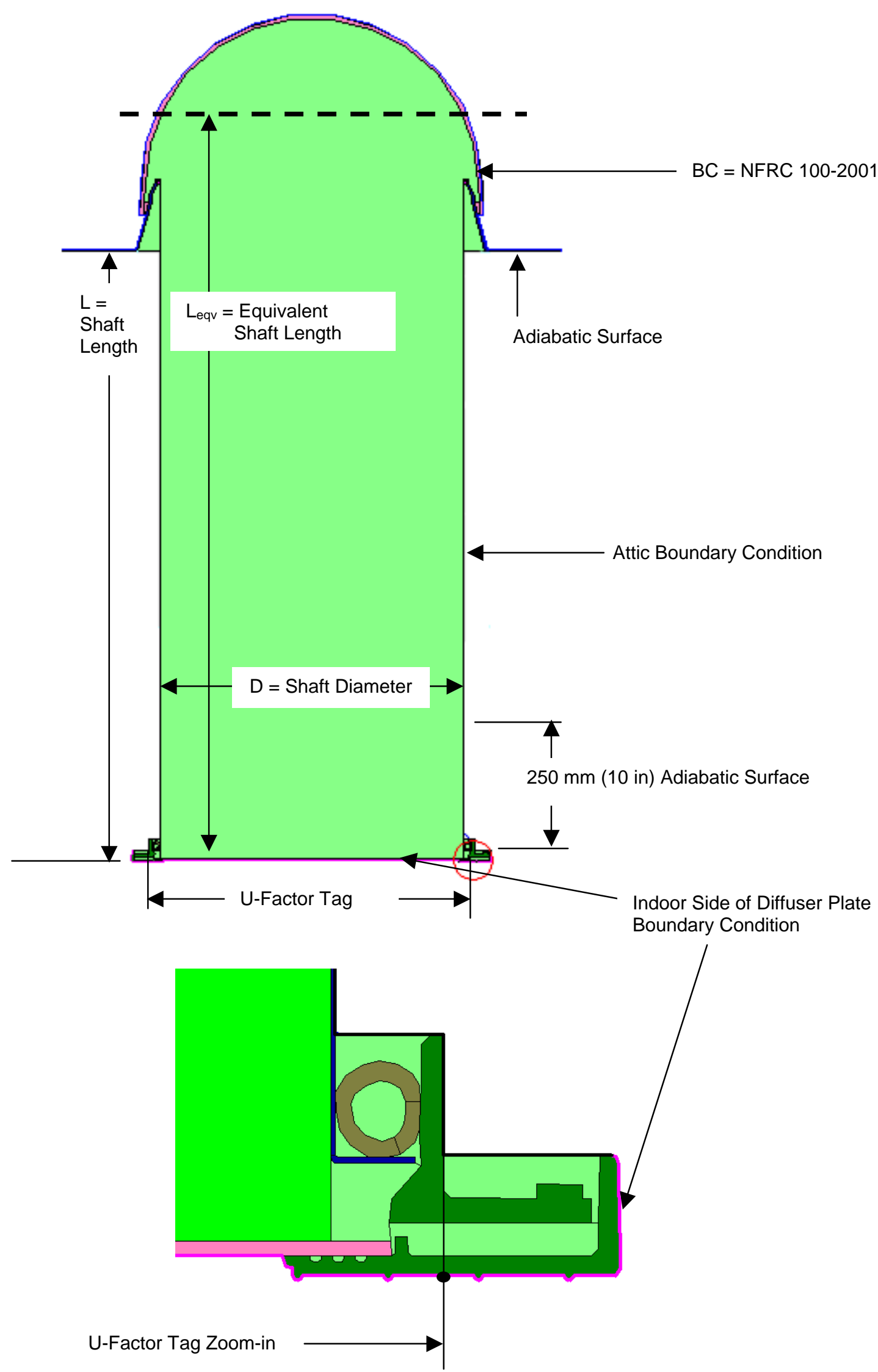

Figure 8-52 Boundary Conditions and location of U-factor tag for Tubular Daylighting Device. 
The last step before simulating the problem is to define the U-factor tag. This tag is defined as shown in Figure 8-50. After the calculation is done, the U-factor obtained represents the total product performance.

\subsubsection{Example Tubular Devices Problem}

This example assumes that the bottom diffuser plate is made up of a single layer. For multiple layer plates, additional instructions are given at the end of this example. For single layer plates, it is not necessary to do calculations with WINDOW.

Begin by drawing geometry in THERM either by using DXF file underlay as shown below or by using a dimensioned drawing.

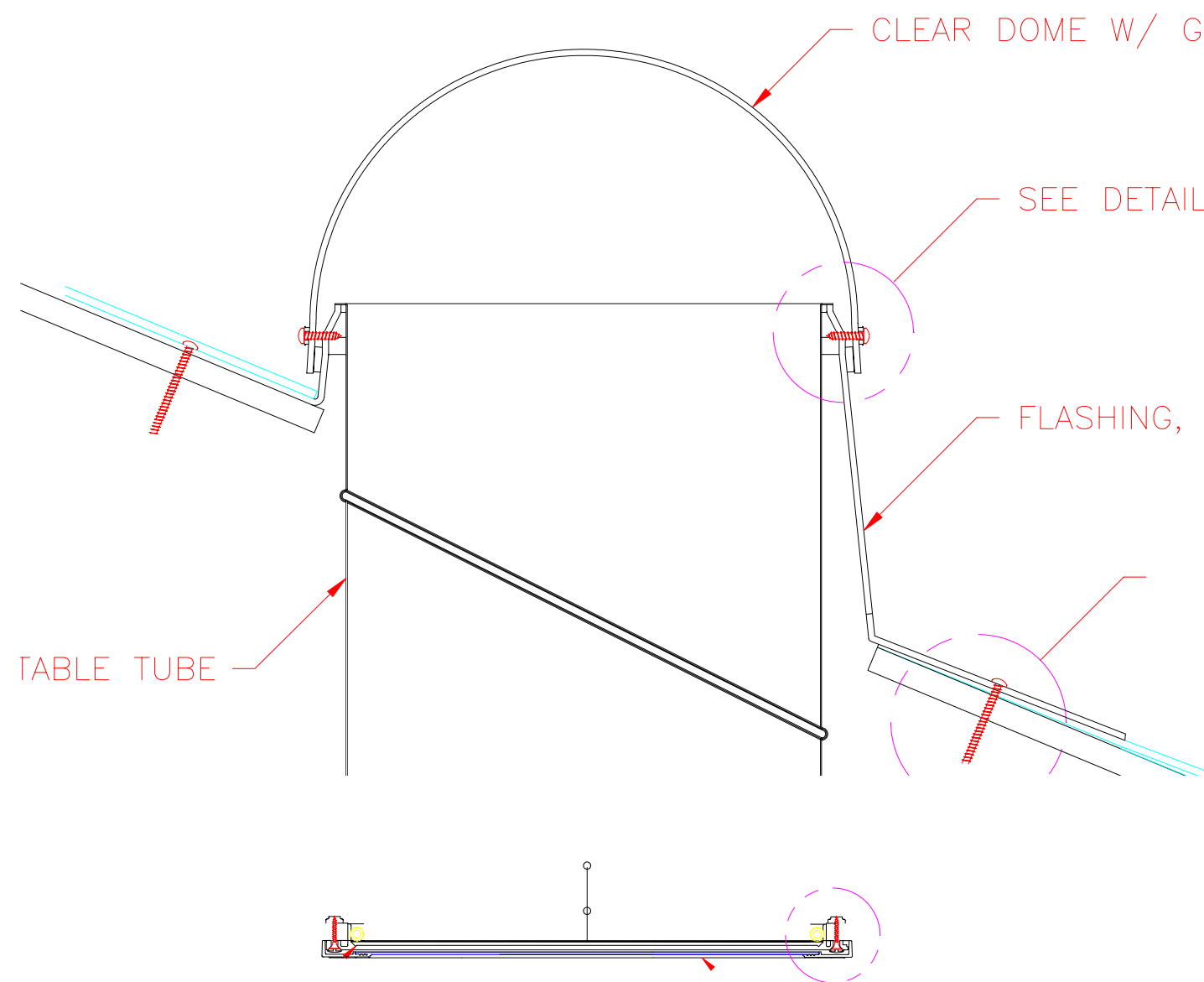

Figure 8-53 DXF File for Use as an Underlay in THERM. 


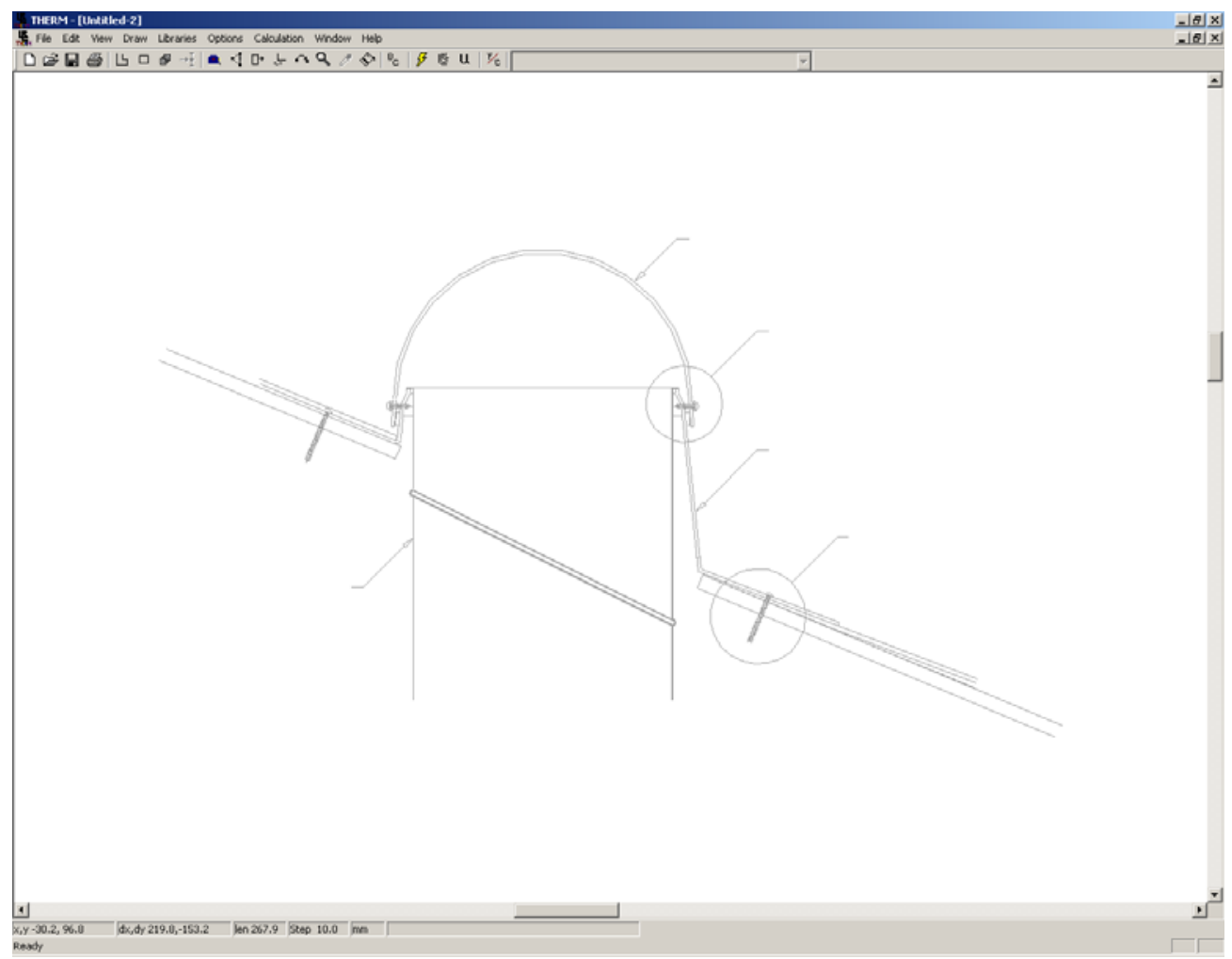

Figure 8-54 Underlay of the Top Part of the Tubular Daylighting Device.

Draw the geometry of all solid pieces of the tubular skylight, making sure that the shaft is $350 \mathrm{~mm}$ (14 in.) wide. The width of the dome should be adjusted to fit over such a shaft but thickness of the dome material shall not be changed. Include all of the details of sealing as per the manufacturers' drawings and specifications. The figure below shows the completed dome, collar, and one side of the shaft wall, along with gaskets.

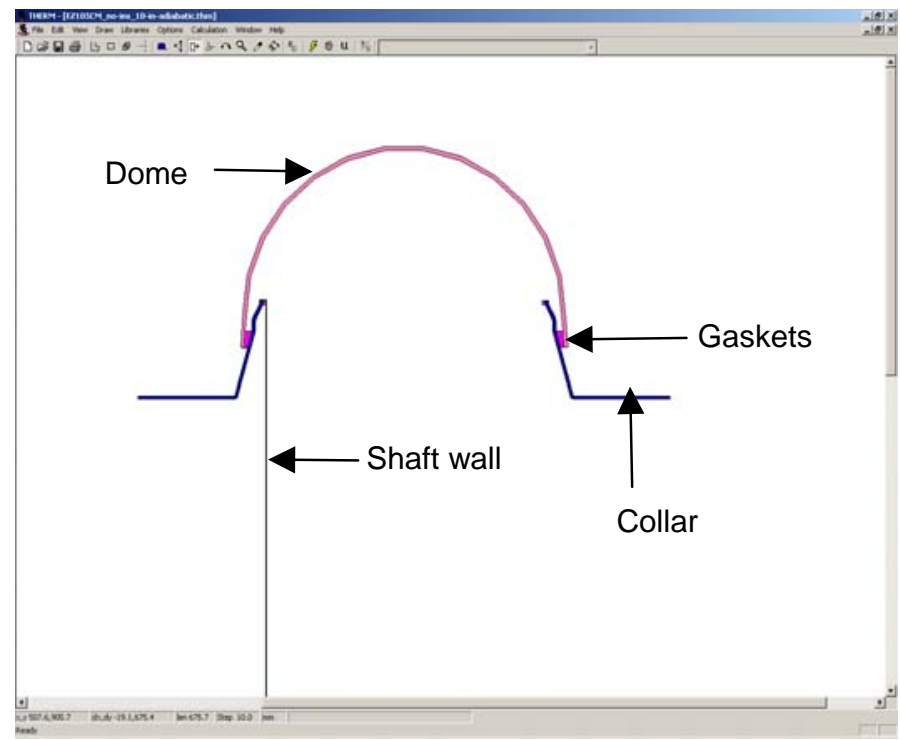

Figure 8-55 THERM drfwing of the dome, collar, gaskets and one section of the shaft wall. 
The figure below shows the completed solid sections without any frame cavity polygons defined.

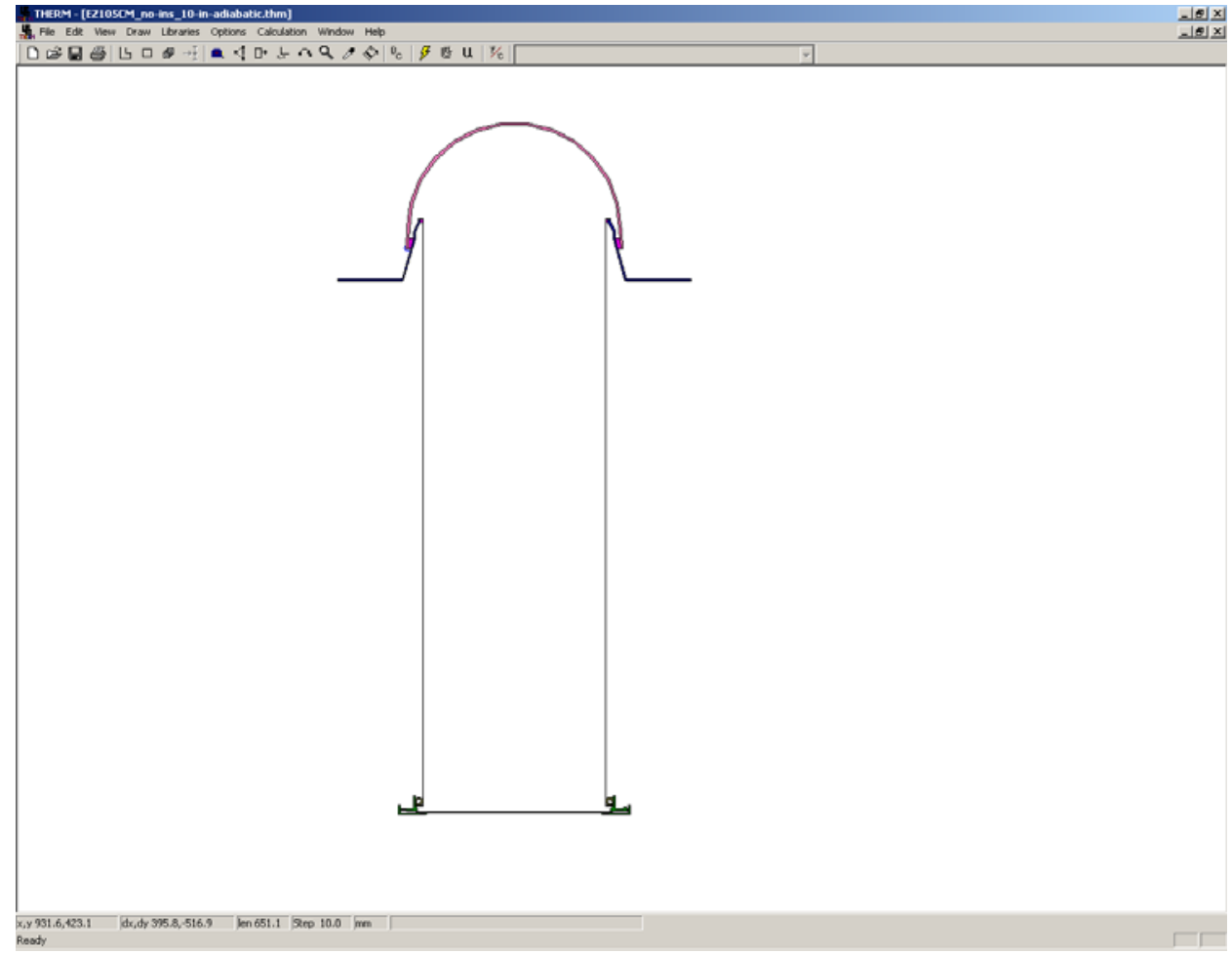

Figure 8-56 THERM drawing of all of solid sections.

Create polygons (using the Fill tool where possible) and assign the "Frame Cavity NFRC 100-2001" material for all the frame cavities except the large central shaft/dome cavity, as shown in the figure below.

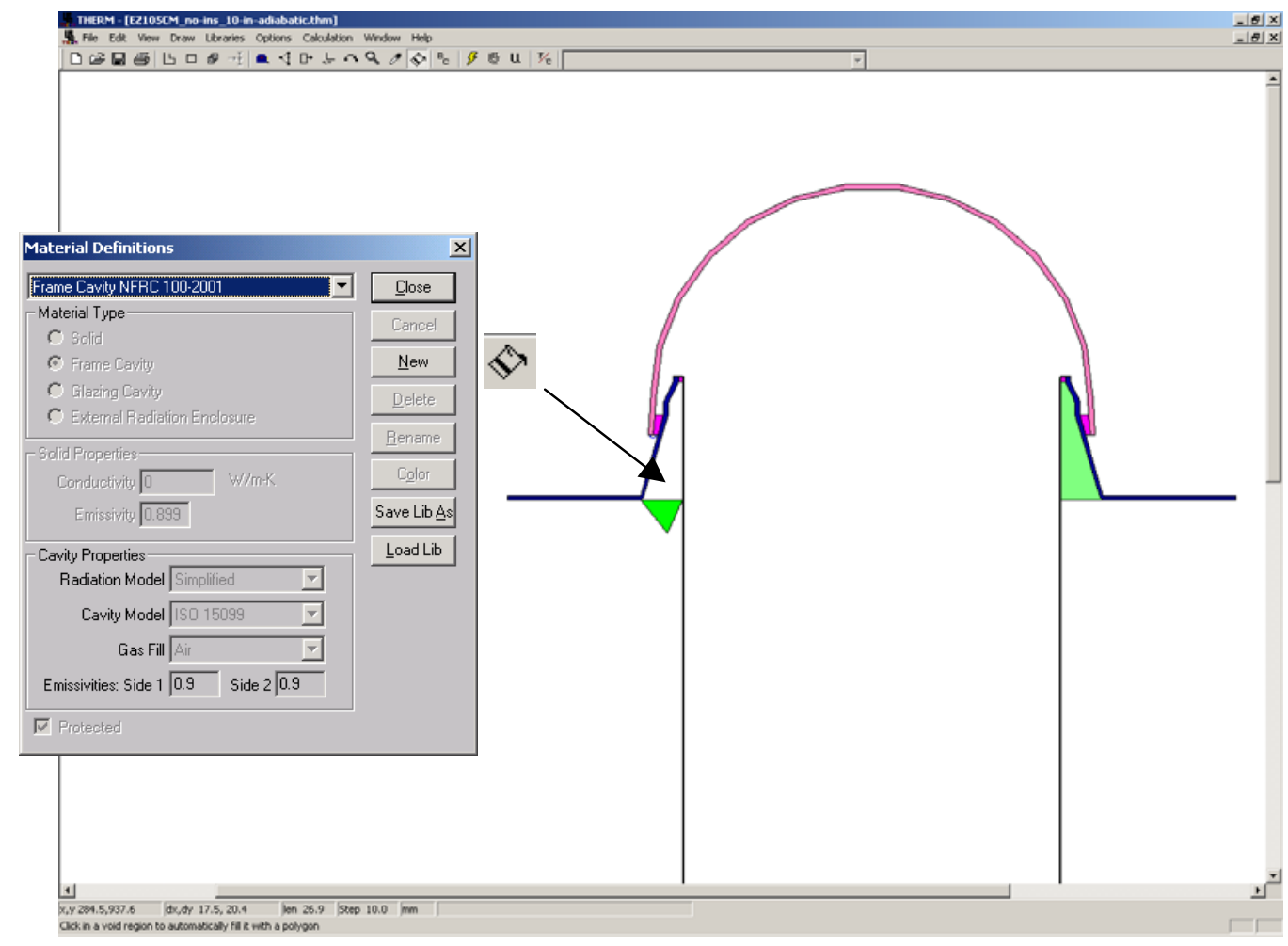

Figure 8-57 Fill all cavities, except for shaft/dome, with the "Frame Cavity NFRC 100-2001" material. 


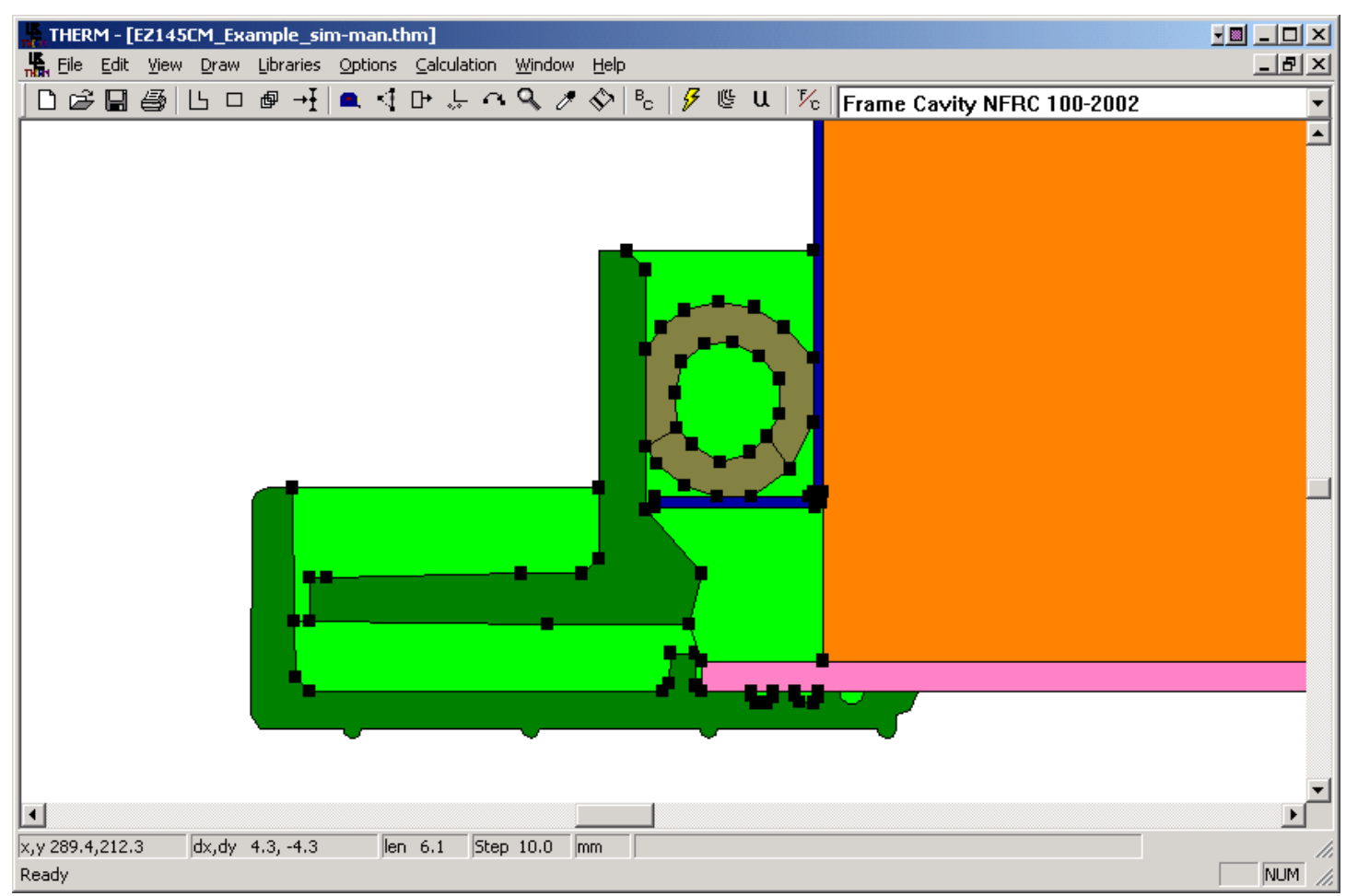

Figure 8-58 Small Frame Cavities Around the Edges of the Diffuser set to "Frame Cavity NFRC 100-2001" material.

Before the large shaft/dome cavity can be filled, it is first necessary to calculate $k_{\text {eff }}$ for this cavity. Open spreadsheet Tubes_keff.xls and input the four yellow highlighted fields that are available for inputting data as shown in the figure below:

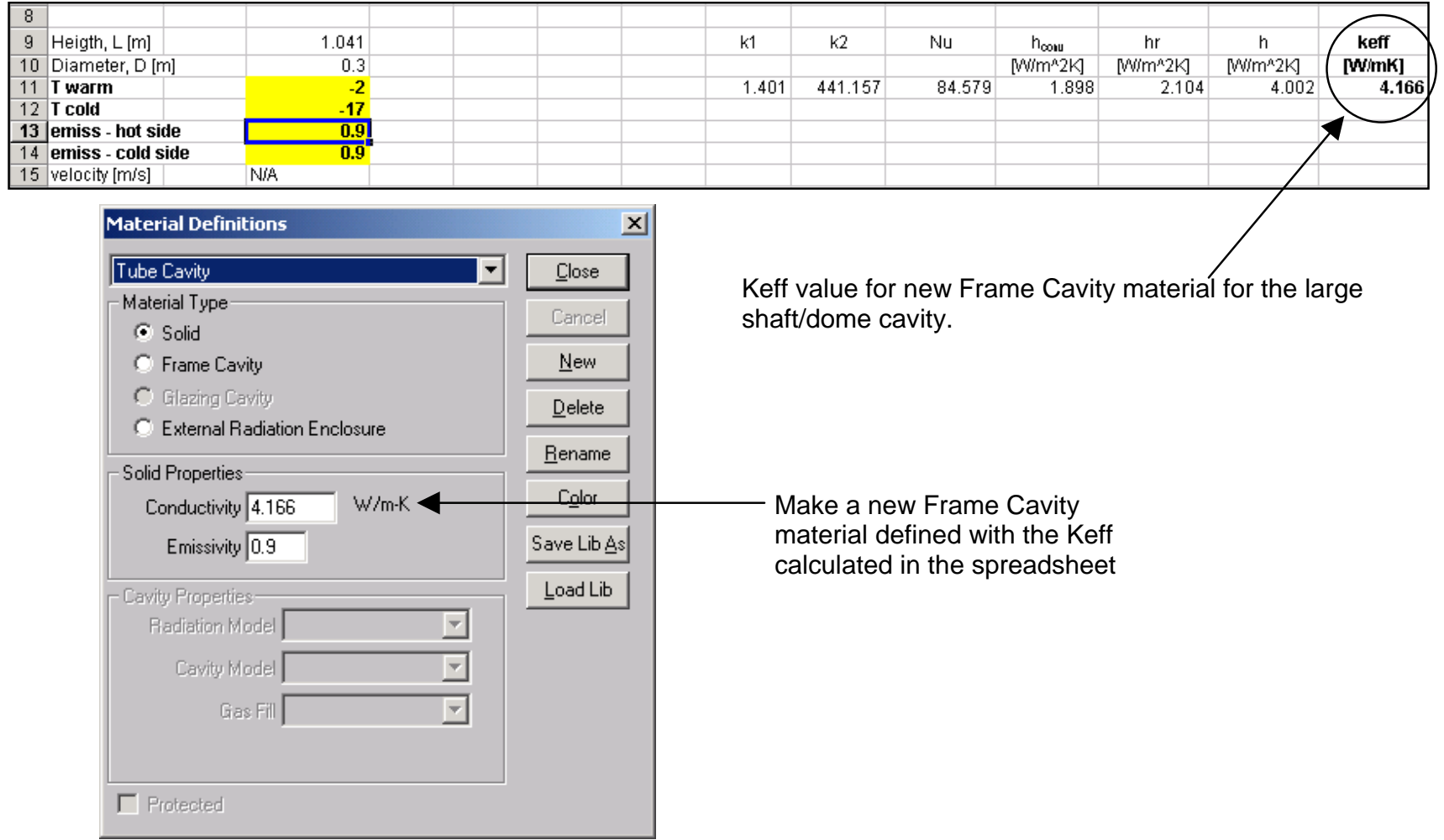

Figure 8-59 Tubes_keff.xls spreadsheet with input data highlighted in yellow 
Temperatures of the inside surface of a bottom diffuser plate $\left(\mathrm{T}_{\text {warm }}\right)$ and top dome $\left(\mathrm{T}_{\text {cold }}\right)$ shall be estimated by finding respective average temperatures.

For the bottom diffuser plate, the average temperature can be estimated simply by stretching tape measure across the inside surface of the bottom diffuser plate from the left side of the shaft/dome cavity to the right side, as shown in the figure below.

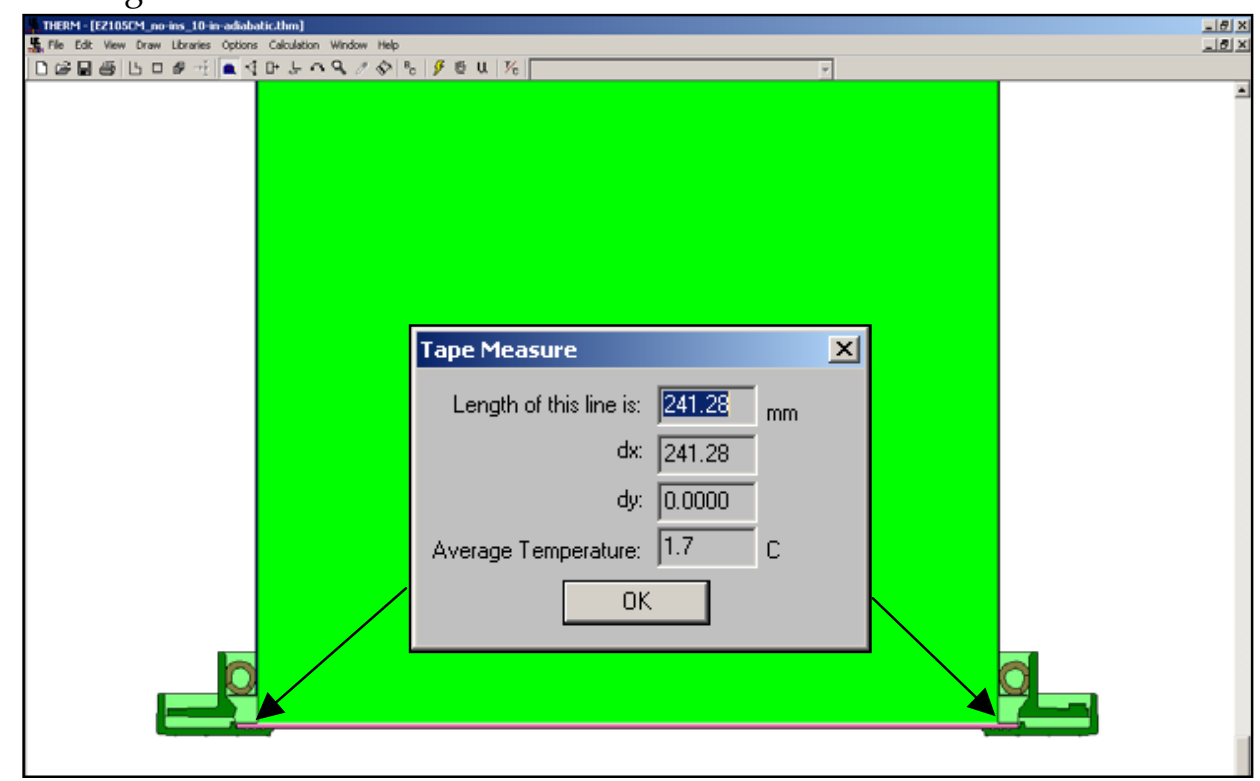

Figure 8-60. Estimate of the average temperature of the inside surface of a bottom diffuser plate

For the dome, the average temperature should be calculated in increments, because the surface consists of several straight line increments. Because the lines are of approximately the same length, the average can be estimated by summing the temperatures for all the segments and dividing by the number of segments, as shown in the figure below. As discussed at the beginning of this section, this is an iterative process, and once the model has been simulated, find the average temperatures for the diffuser plate and dome surfaces using the tape measuring tool, and if the resulting average temperatures differ by more than $1^{\circ} \mathrm{C}\left(2{ }^{\circ} \mathrm{F}\right)$ from the estimated values, the new Keff shall be calculated and the simulation repeated until the criterion of $1^{\circ} \mathrm{C}\left(2{ }^{\circ} \mathrm{F}\right)$ is met. Emissivities are input from the surface emissivities of the inside surface of the bottom diffuser plate (emiss - hot) and inside surface of the top dome (emiss - cold). The resulting $k_{\text {eff }}$ value is calculated using the spreadsheet, and a new material shall be made with that conductivity and assigned to the shaft/dome cavity. 


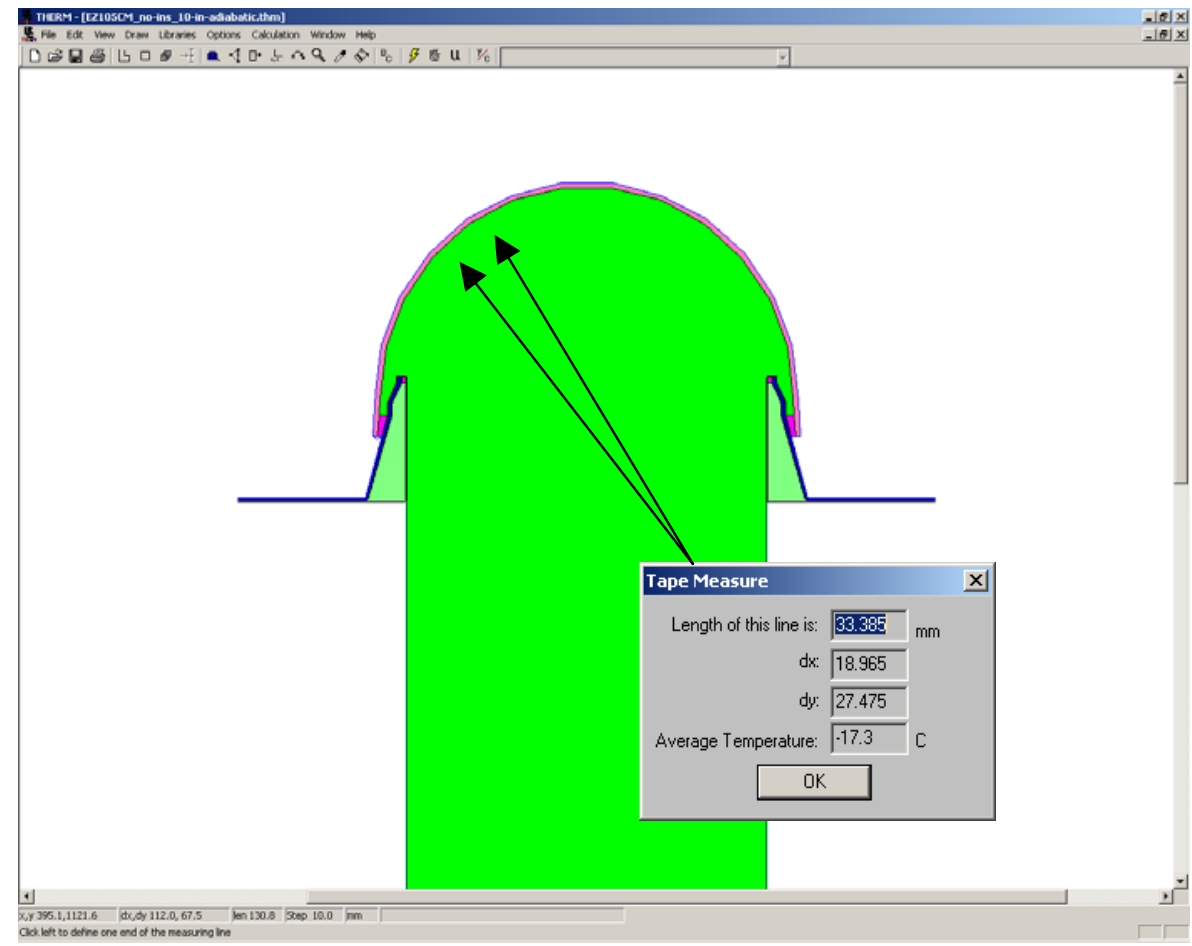

Figure 8-61. Estimate of the average temperature of the inside surface of a bottom diffuser platetemperatures of segments (usually at $15^{\circ}$ increments)

The next step is to define the boundary conditions. The outside surface of the dome and collar should be assigned the standard "NFRC 100-2001 Exterior" boundary condition. The bottom of the collar shall have standard "Adiabatic" boundary condition, as shown in the figure below.

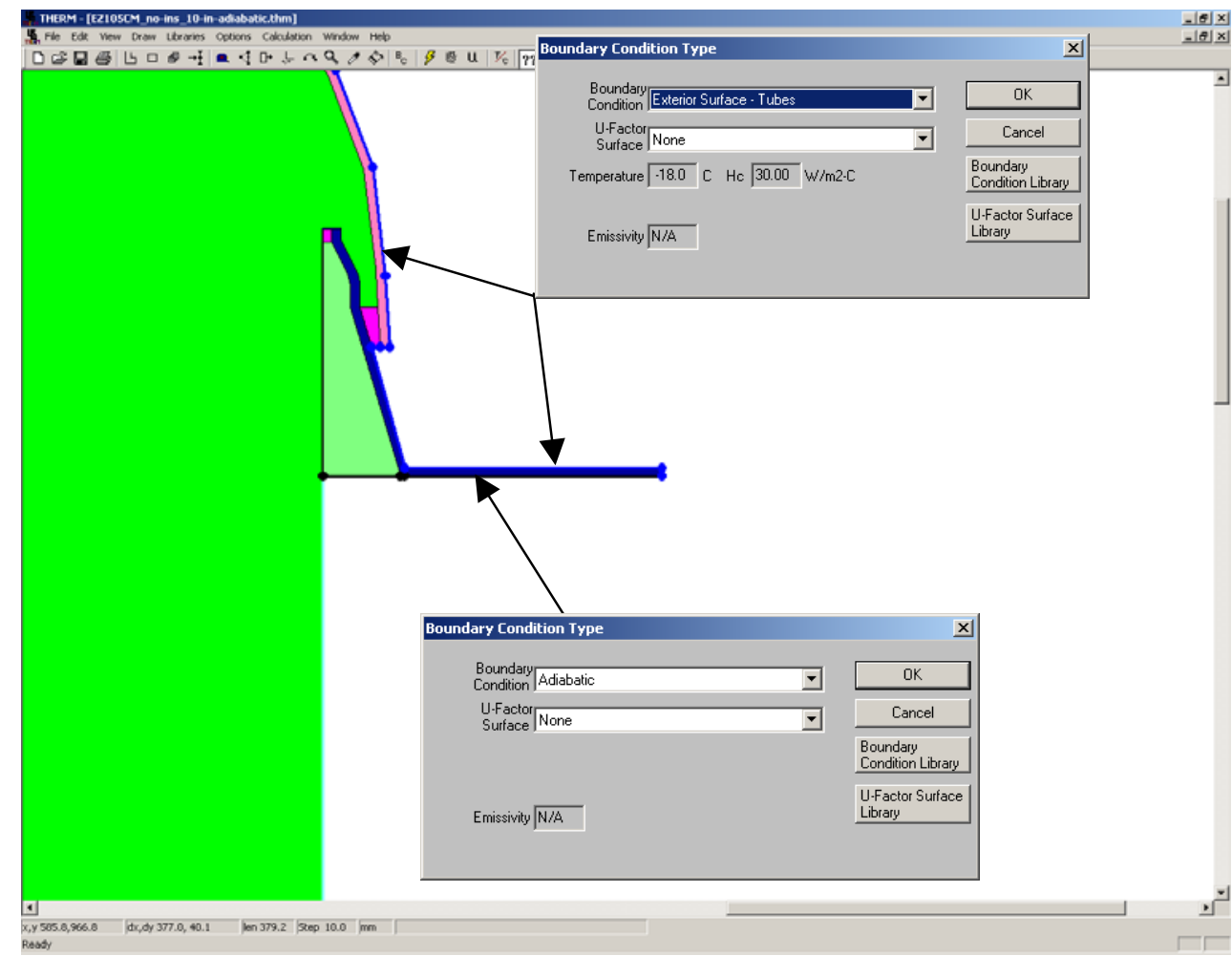

Figure 8-62. Exterior and adiabatic boundary conditions near the top dome 
The outside surfaces of the shaft walls, except for the bottom $250 \mathrm{~mm}$ (10 inches) should be assigned a user defined "Attic boundary condition" (see description of all boundary conditions at the beginning of this section). The bottom $250 \mathrm{~mm}$ (10 in.) of the shaft walls and portion of the diffuser plate edge assembly shall have an "Adiabatic" boundary condition as shown in the figure below.

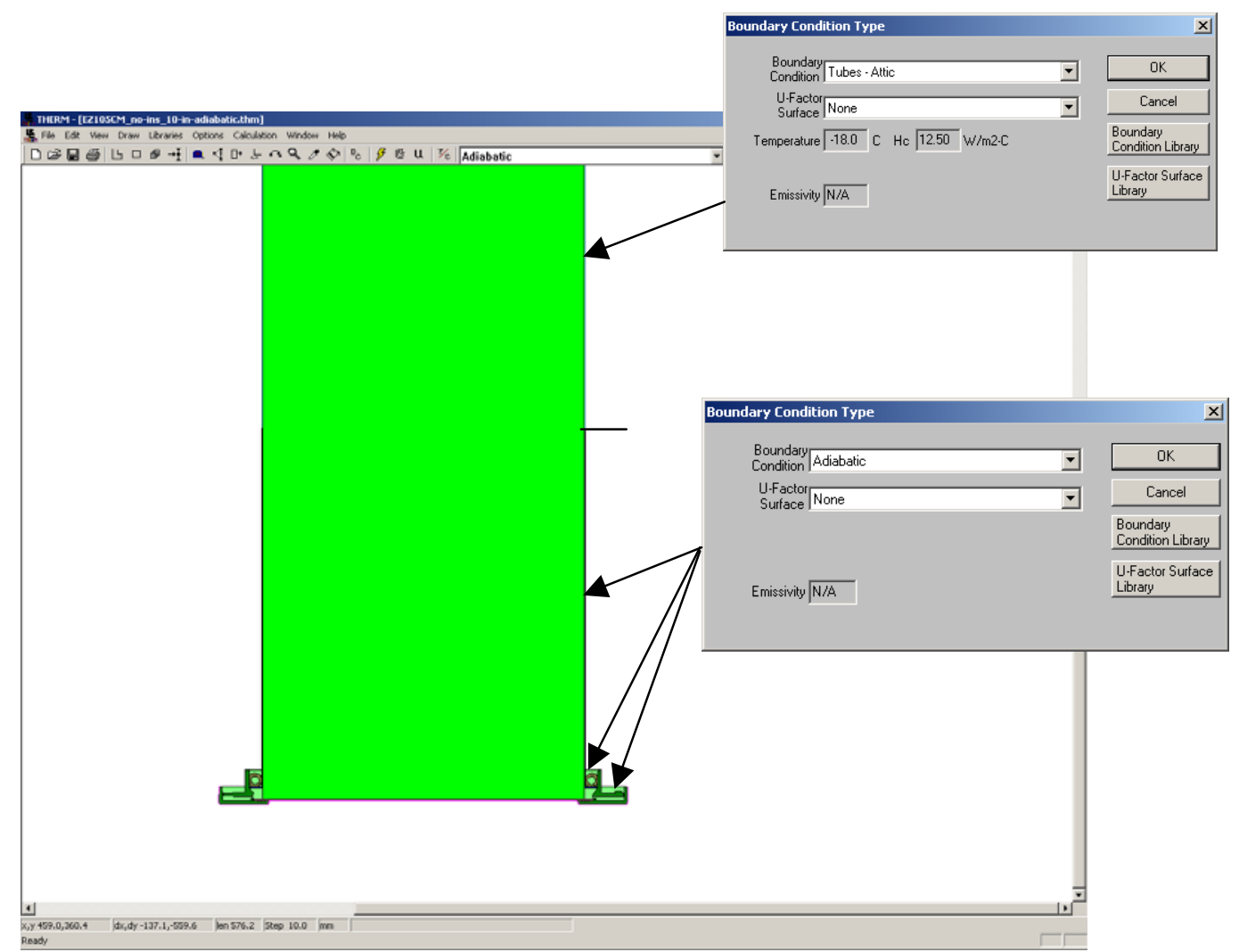

Figure 8-63. Attic and adiabatic boundary conditions on the shaft wall and near the bottom diffuser 
The remaining boundary condition, "Indoor Side of Diffuser Plate" shall be applied to the exposed surfaces of the bottom diffuser plate and edge assemblies up to the point where adiabatic boundary condition ends, as shown in the figure below.

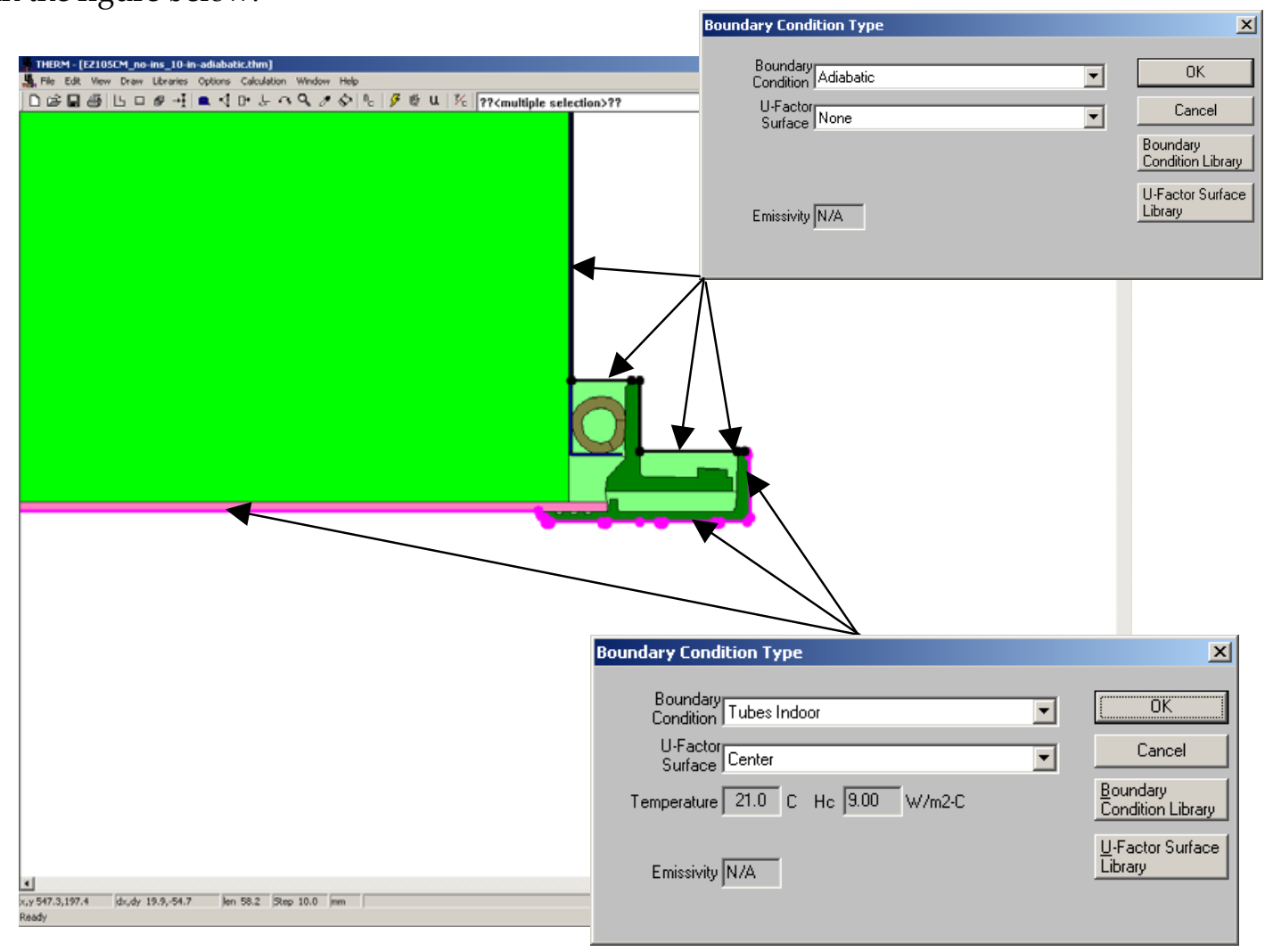

Figure 8-64. Indoor boundary condition on the exposed surfaces of the diffuser plate

After all boundary conditions are defined, the remaining task left is to define the U-factor tag. The U-factor shall be calculated for the area corresponding to the rough opening in the ceiling, which is defined on Figure 8-50. Select bottom diffuser plate and insert points on both sides of the model and define U-factor tag "Center" (or some other name if desired) for the surface between those two points.

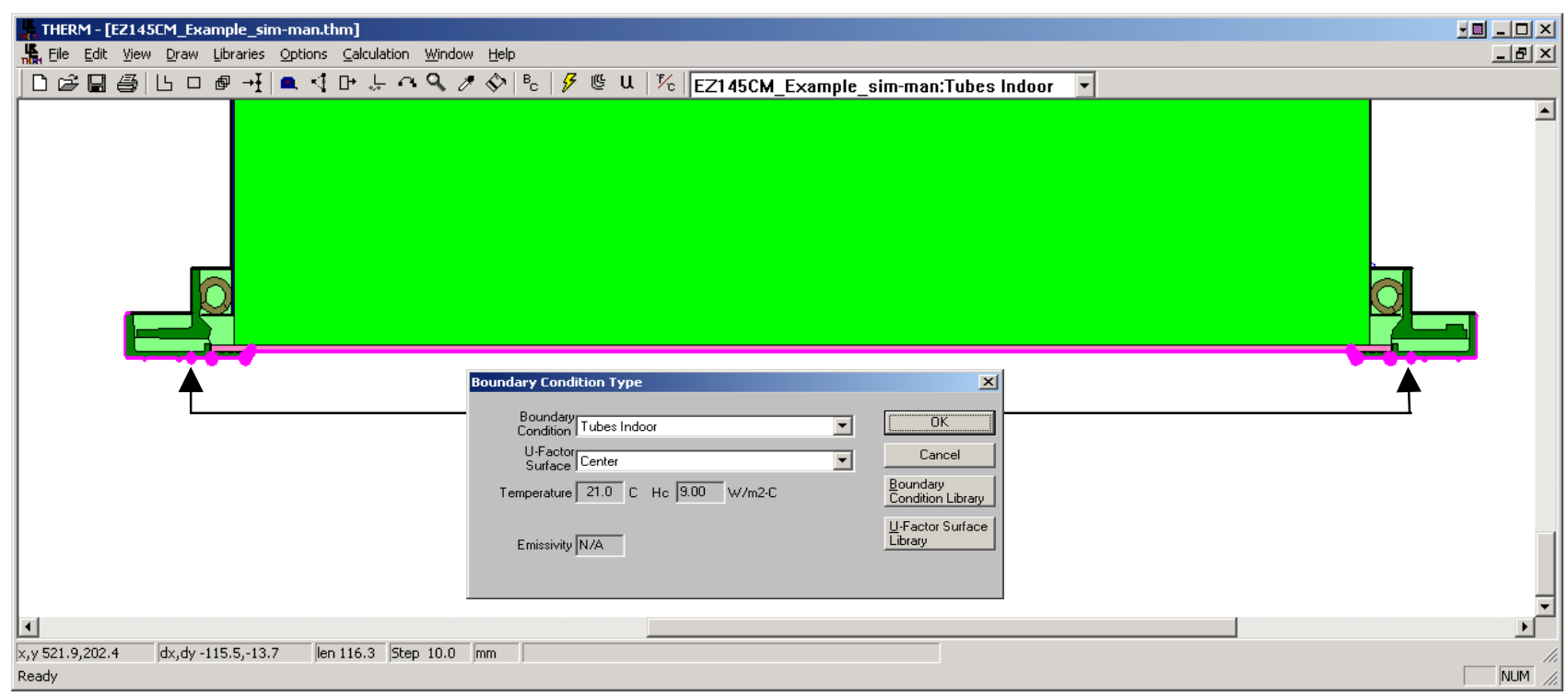

Figure 8-65. Definition of U-factor tag 
This completes the definition of the model. The final step is to simulate the problem. As discussed at the beginning of this section, it is an iterative process to obtain the Keff value for the material defined for the shaft/dome cavity. Once the model has been simulated, find the average temperatures for the diffuser plate and dome surfaces using the tape measuring tool, and if the resulting average temperatures differ by more than $1^{\circ} \mathrm{C}$ from the estimated values, the new Keff shall be calculated and the simulation repeated until the criterion of $1^{\circ} \mathrm{C}$ is met. The resulting $\mathrm{U}$-factor is the overall product $\mathrm{U}$-factor.

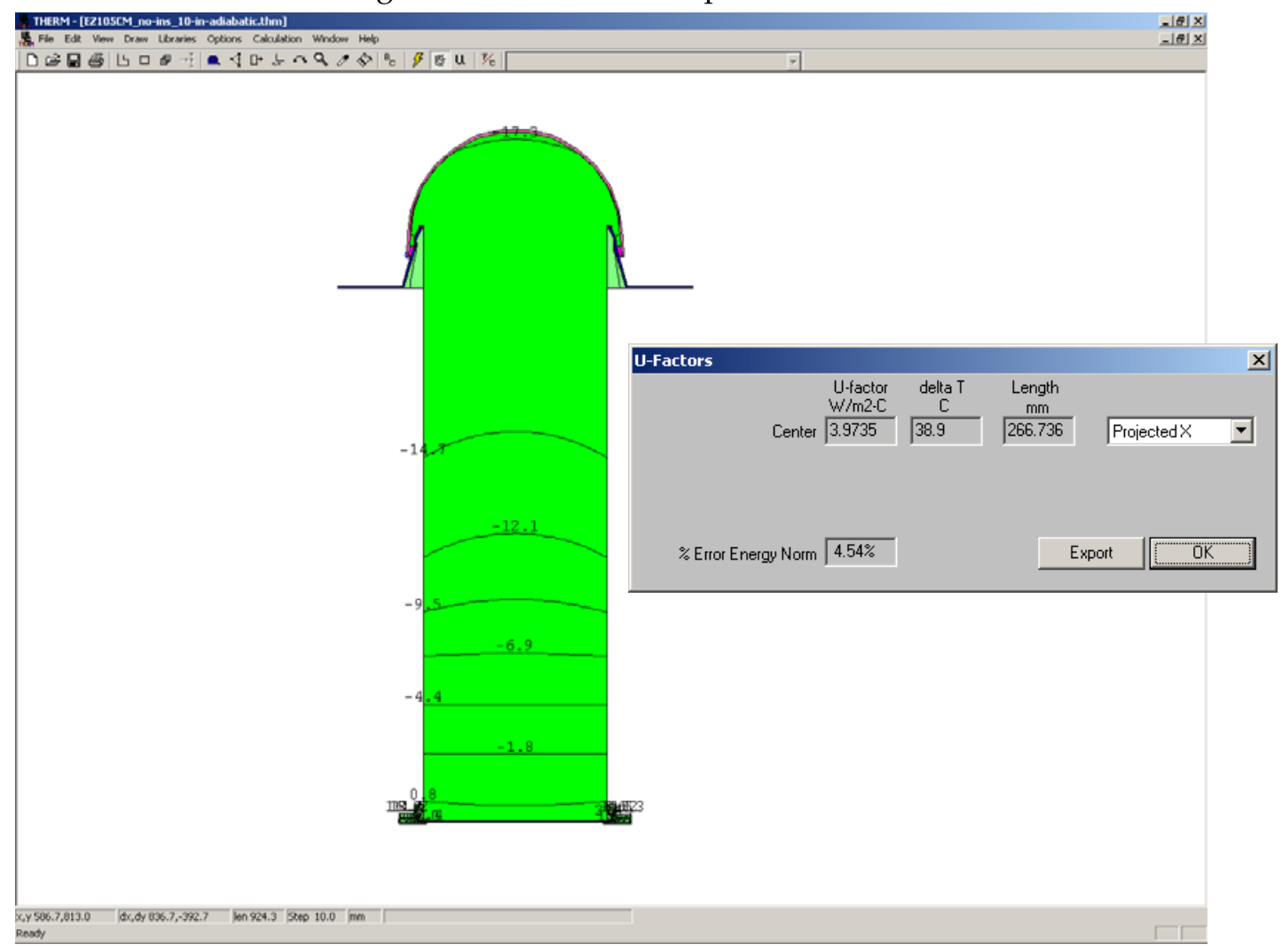

Figure 8-66. Temperature Contour plot and U-factor results 


\subsubsection{Example: Tubular Device Problem With the Double-Glazed Diffuser Plate}

Using a double glazed diffuser plate is a variation to the design presented in the first example. This case can be modeled by first using WINDOW to calculate the effective conductivity of the gap space in a diffuser and then specifying this conductivity in the THERM model.

In WINDOW create a special boundary conditions for this case (i.e., tubes diffuser) by copying the NFRC 1002001 record in the Environmental Conditions Library to a new record, and set outdoor wind speed to 0 (this is the closest approximation to convection and radiation heat transfer inside shaft/dome cavity that borders cold side of this double layer diffuser). Name the new environmental condition something that makes it clear how it is to be used, such as "NFRC Tubular Skylight", as shown in the figure below.

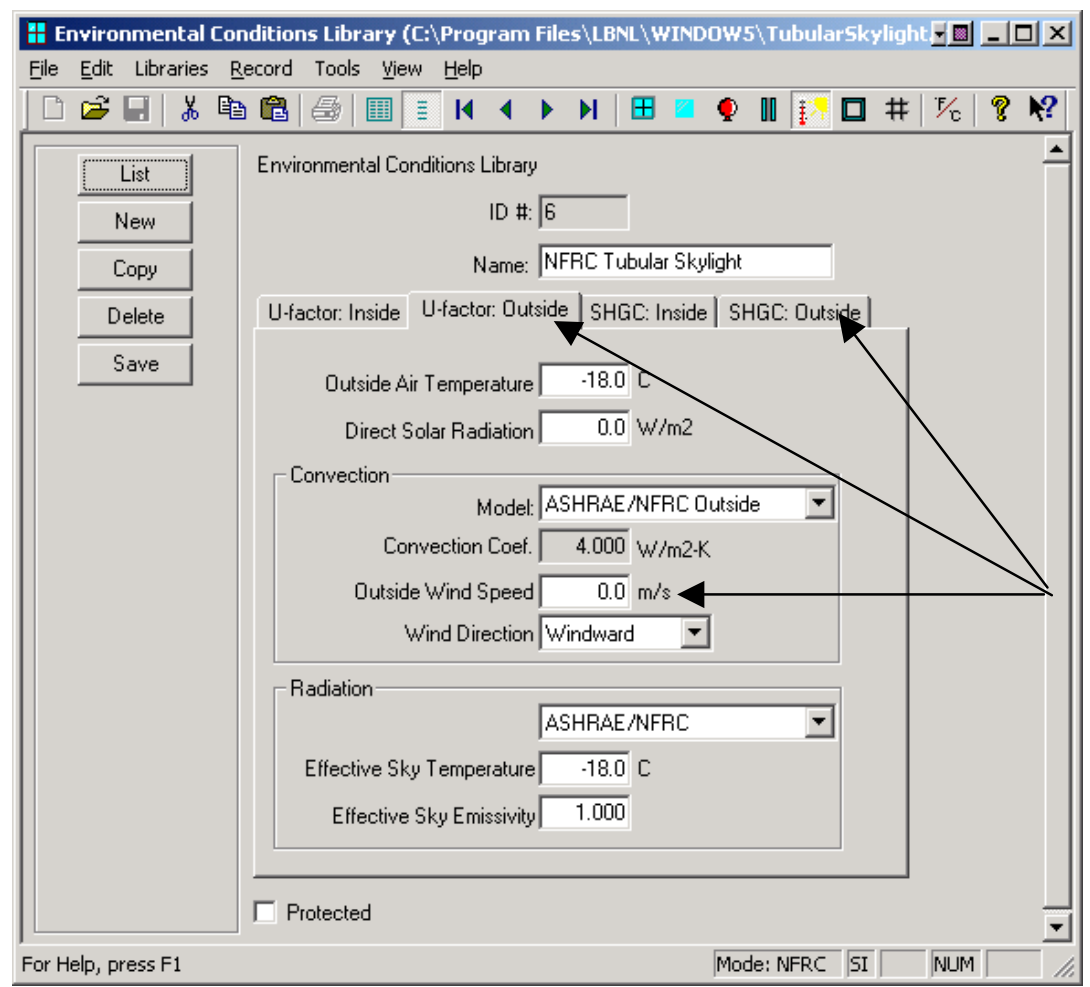

Set outside wind speed to 0 in both the U-factor Outside and SHGC Outside tabs.

Figure 8-67. New environmental conditions for calculating center of glass performance of the diffuser plate. 
In the glass library, create the new glazing layer, naming it appropriately to the material used (called Lexan in this example) and specify the thickness per the manufacturer drawings. Set the conductivity and emissivity by copying the values from the library of material thermo-physical propertiesor value derived from NFRC 101.

Figure 8-68. Glass layer definition in Glass Library for the double-layer diffuser plate 
In the Glazing System Library, create a new double glazed system using the newly defined entries in the Glass Library, and reference the new environmental condition, "NFRC Tubular Skylight". Set the tilt to 0 degrees, and set the gap thickness and gap gas according to the manufacturer's specifications. After the calculation is done, make note of the effective conductivity (Keff value under the Center of Glass Results tab) for later use in THERM.

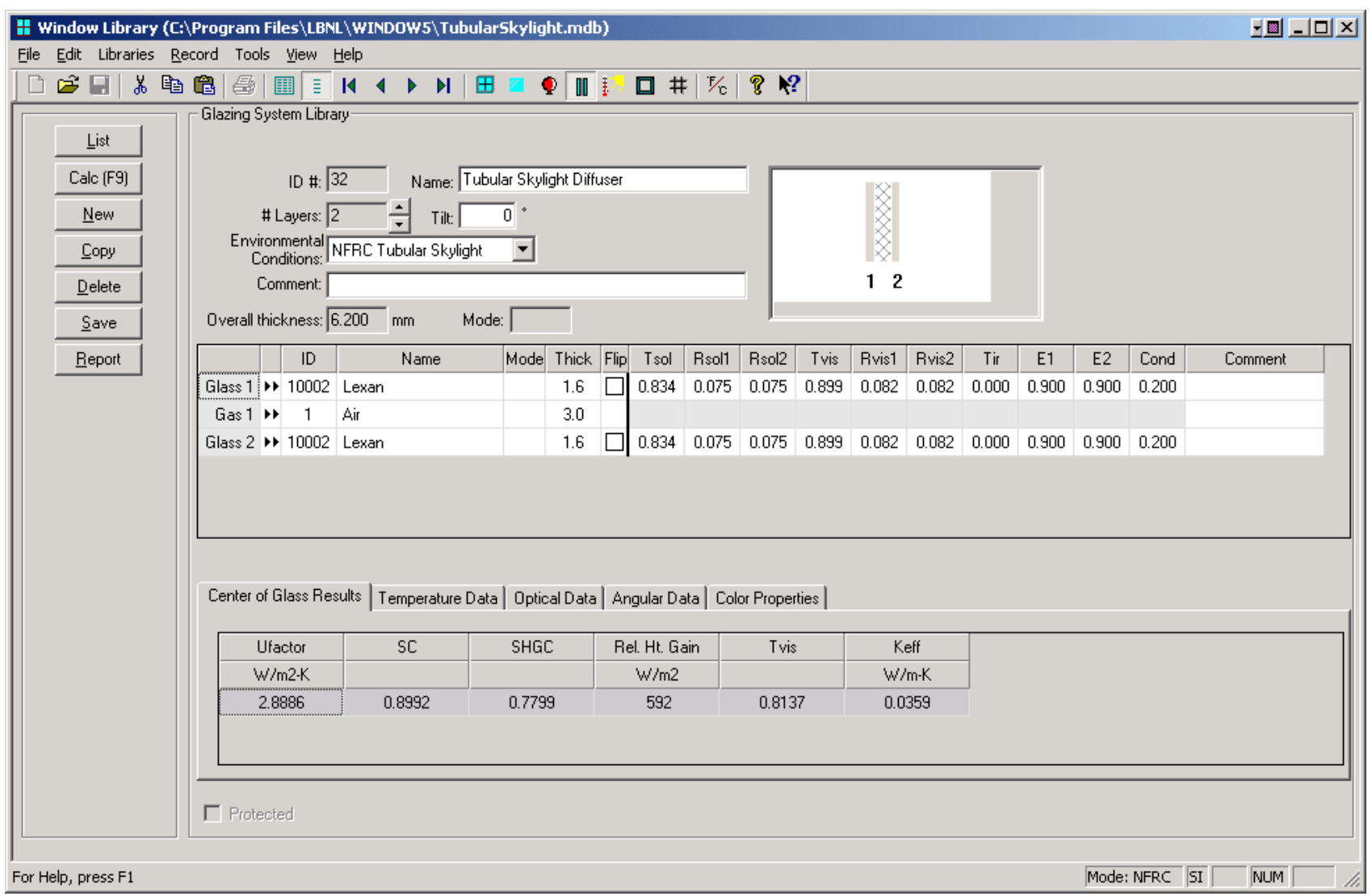

Figure 8-69. Glazing system for the double-layer diffuser plate

These calculations are only for U-factor; the SHGC results will not be valid because the layers were created without spectral data. When performing a calculation on this glazing system, the following message will appear, indicating that there is not spectral data associated with the glazing layers.

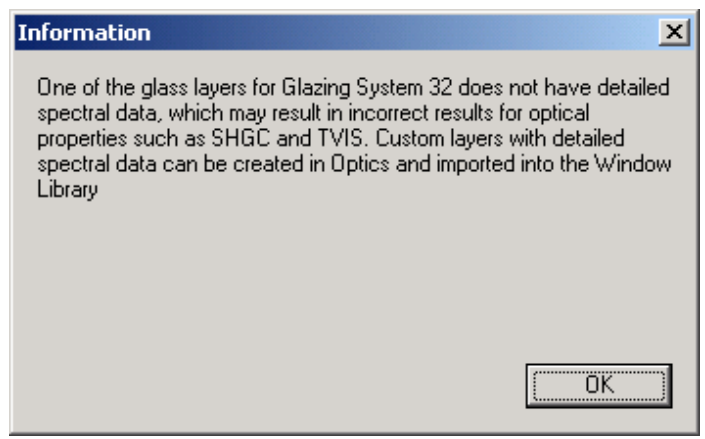

Figure 8-70. Message at calculation time, indicating that there is not spectral data for the glazing layers. Therefore, the SHGC value will not be accurate. 
In THERM draw the geometry of the double-layer diffuser plate, including the detail of spacer and draw the rest of the geometry as per original example.

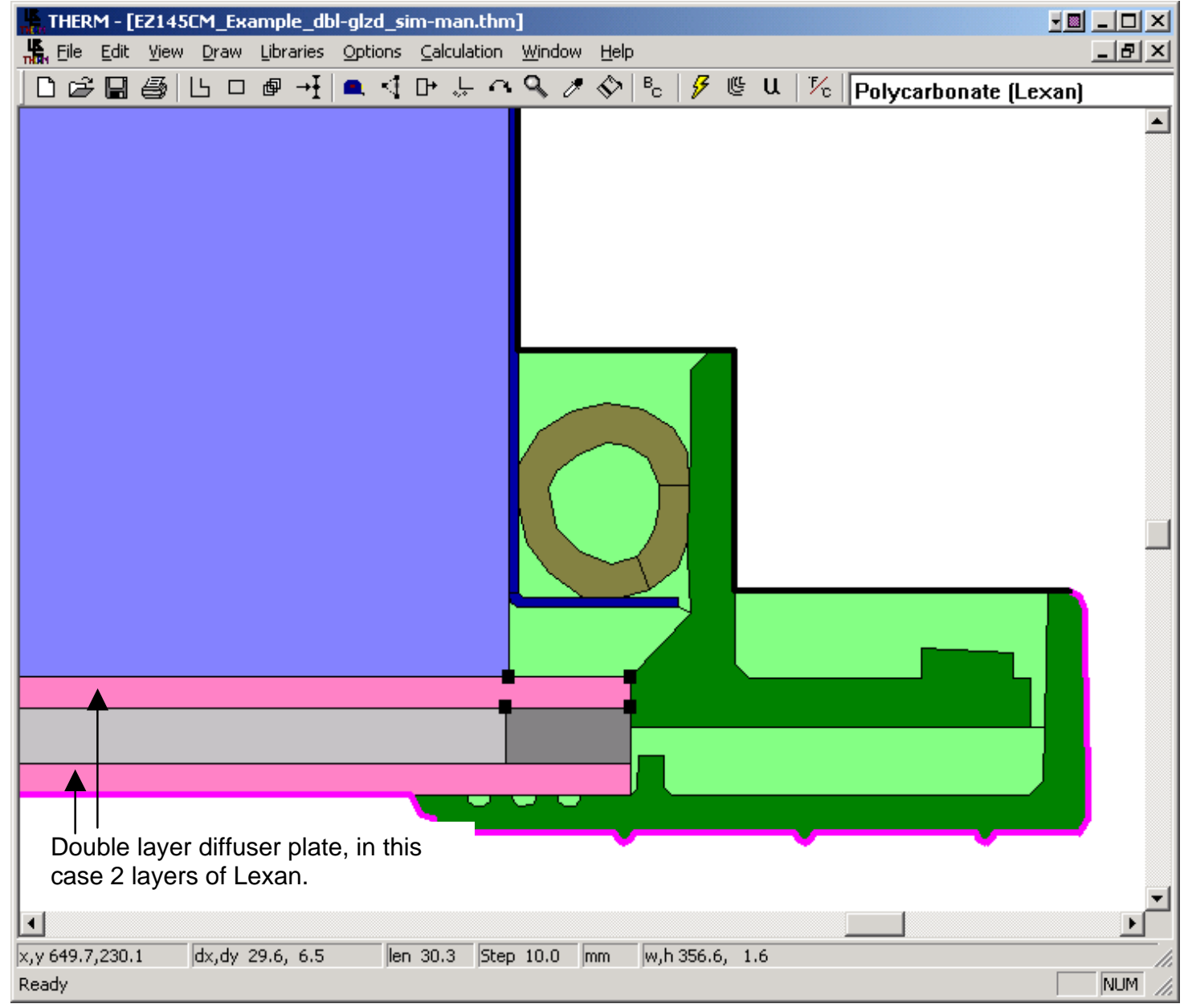

Figure 8-71. THERM model of double-layer diffuser plate 
Define a new material with the conductivity equal to the effective conductivity (Keff) calculated in WINDOW and fill the cavity with that material. As discussed at the beginning of this section, it is an iterative process to obtain the Keff value for the material defined for the shaft/dome cavity. Once the model has been simulated, find the average temperatures for the diffuser plate and dome surfaces using the tape measuring tool, and if the resulting average temperatures differ by more than $1^{\circ} \mathrm{C}\left(2{ }^{\circ} \mathrm{F}\right)$ from the estimated values, the new Keff shall be calculated and the simulation repeated until the criterion of $1^{\circ} \mathrm{C}\left(2{ }^{\circ} \mathrm{F}\right)$ difference is met.

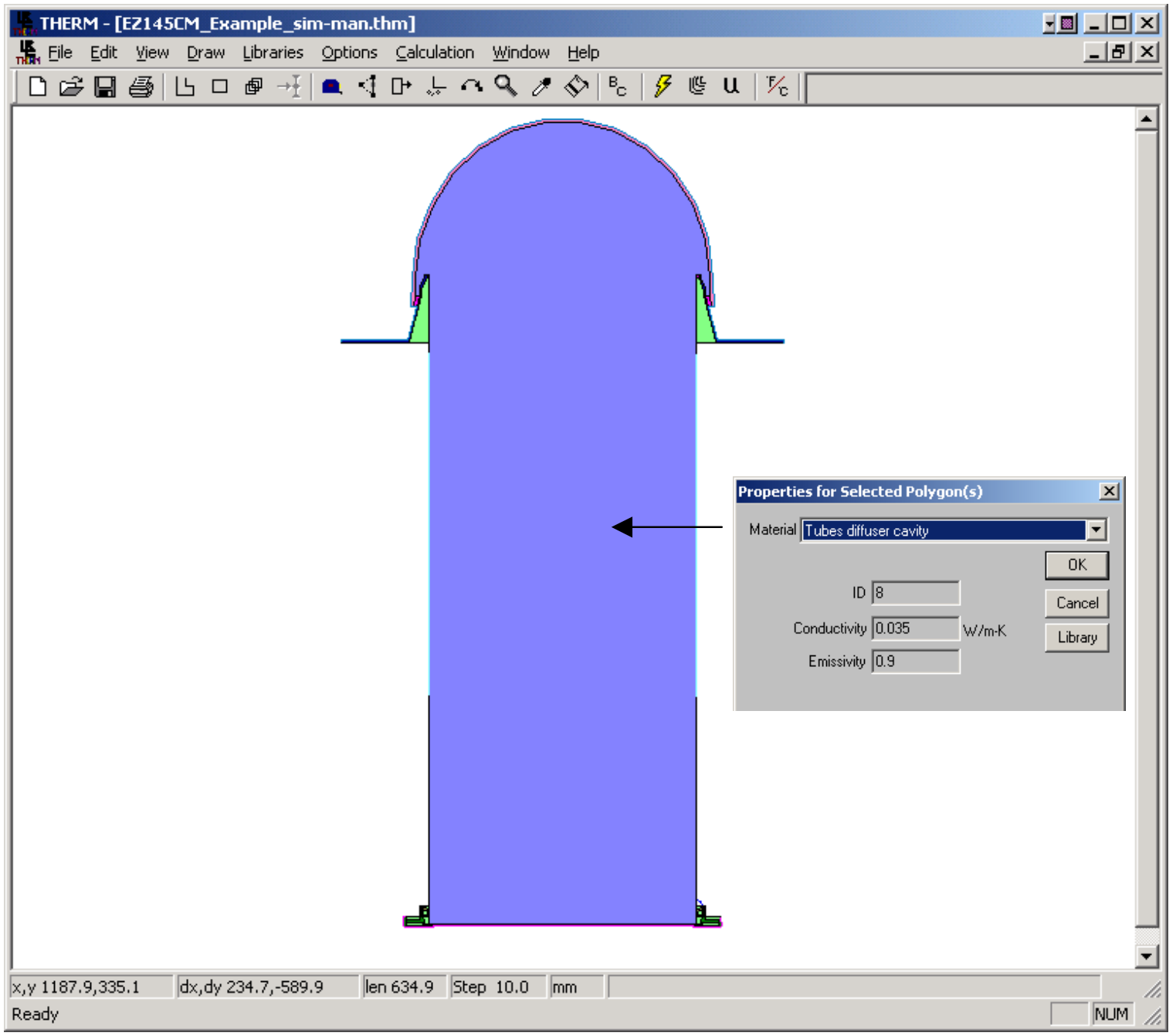

Figure 8-72. THERM model of double-layer diffuser plate 
Define the same set of boundary conditions as in previous example and perform calculation. The following are results for an example where the two layers of Polycarbonate diffuser plates, separated by $3 \mathrm{~mm}(0.1181$ inches) of air space and butyl spacer, are used.

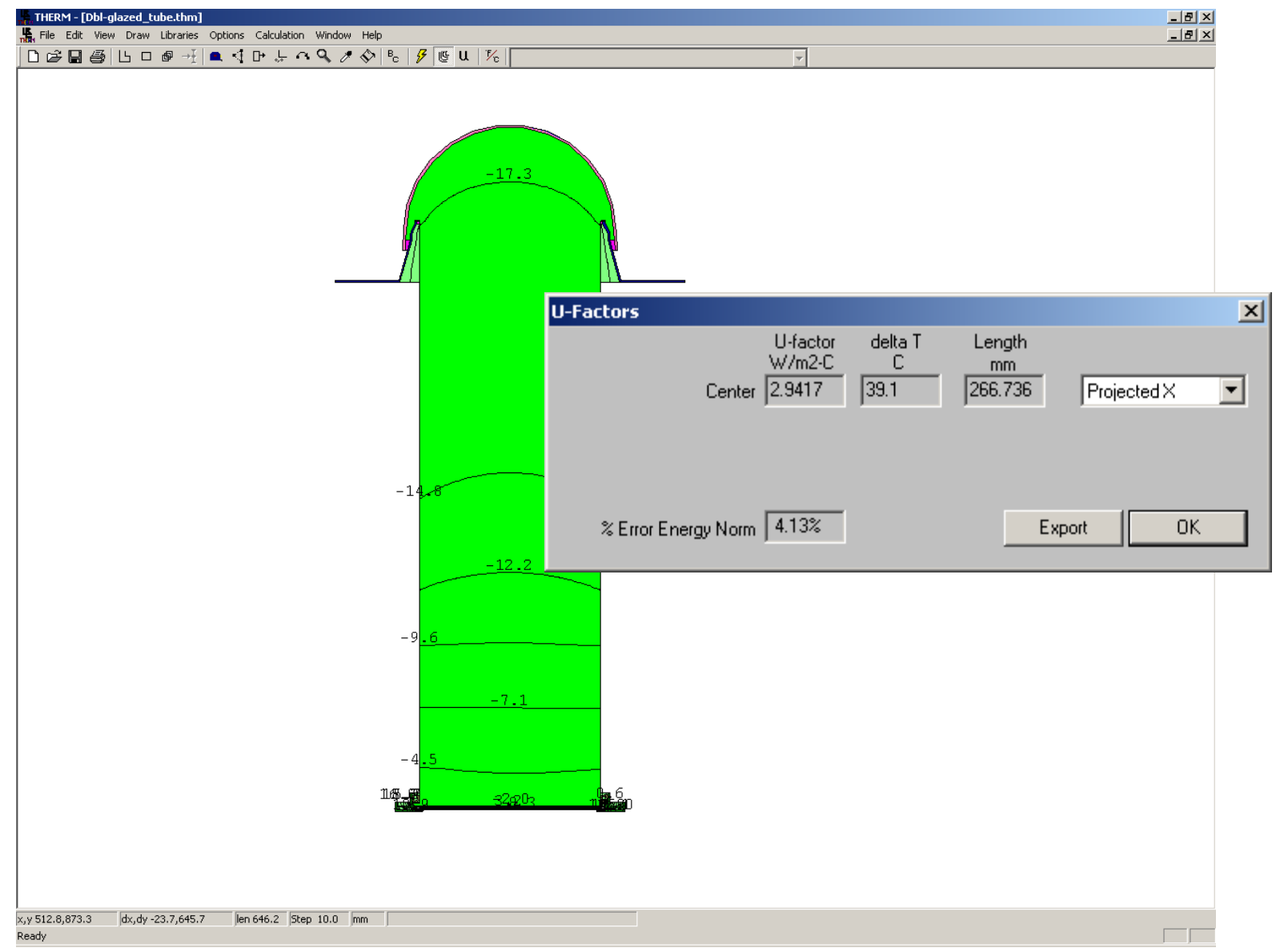

Figure 8-73. Heat Transfer Results for the Tubular Daylighting Device, Incorporating Double-Layer Diffuser Plate

Note that the overall U-factor has been reduced from $3.97 \mathrm{~W} / \mathrm{m}^{2}-^{\circ} \mathrm{C}\left(0.699 \mathrm{Btu} / \mathrm{h}-\mathrm{ft}^{2} \mathrm{-}^{\circ} \mathrm{F}\right)$ to $2.94 \mathrm{~W} / \mathrm{m}^{2}-^{\circ} \mathrm{C}$ $\left(0.518 \mathrm{Btu} / \mathrm{h}-\mathrm{ft}^{2}\right.$-oF $)$, by using double-layer configuration for the diffuser plate instead of the original single layer. This analysis does not include solar optical properties or Solar Heat Gain Coefficient calculation, which will also be affected by the introduction of double-glazed diffuser plate. It is likely that the daylighting performance would be negatively affected due to the presence of an additional diffuser plate, which will reduce overall visible transmittance $(\mathrm{VT})$. 


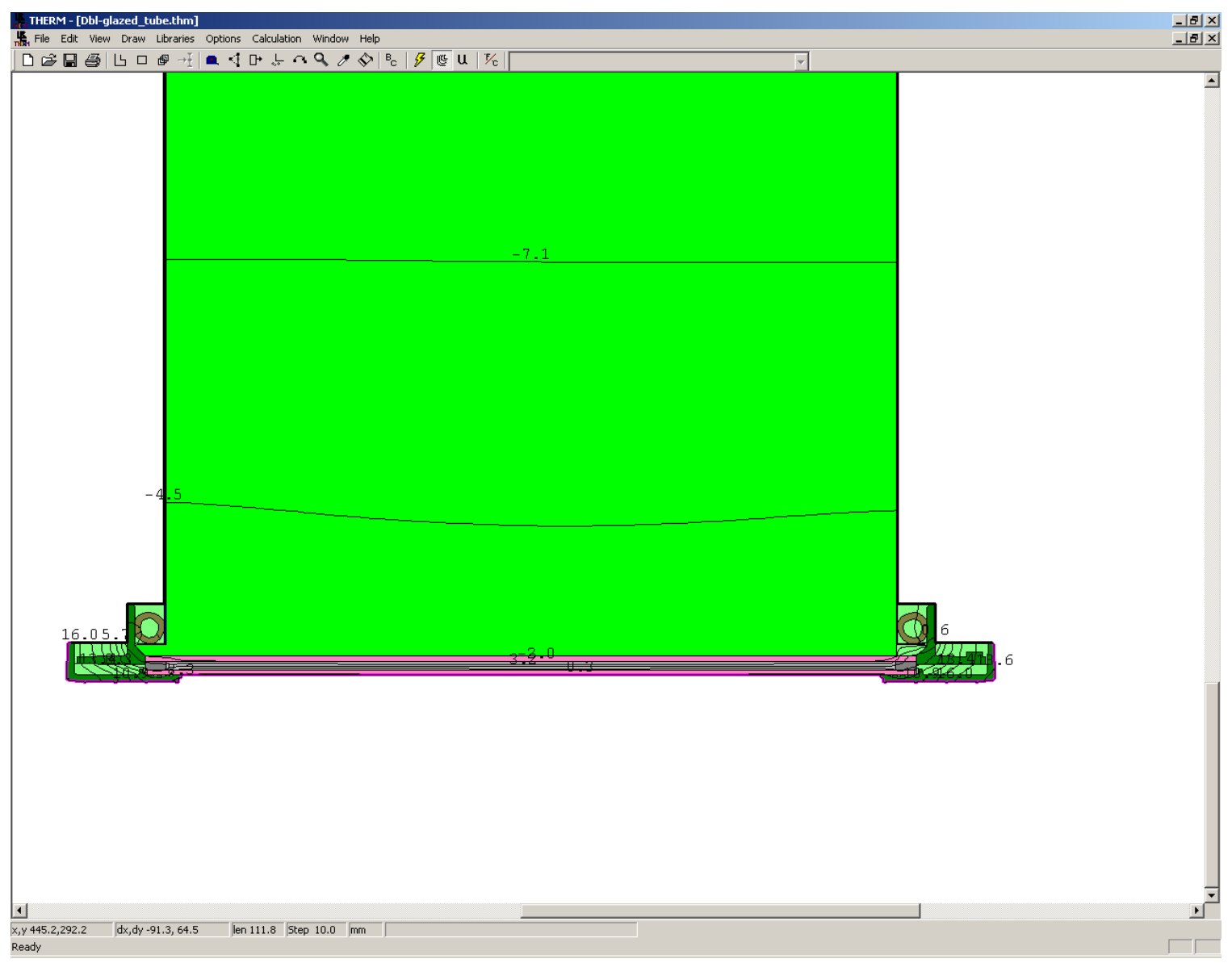

Figure 8-74. Zoomed-in Region Near the Diffuser Plate

\section{References:}

Curcija, D.C. 2001. "Proposed Methodology for Modeling Tubular Skylights For NFRC Rating Purposes." CEERE Technical Report. June, 2001. 


\subsection{Doors}

Swinging entry doors are modeled differently than window products because there are more opaque sections to be modeled in THERM. The procedures for modeling doors are included in NFRC 100: and that document should be reviewed in detail before modeling any entry door systems.

NFRC has defined nine regions within a door that need to be modeled. These regions include:

- Head

- Sill

- Hinge Jamb

- Lock Jamb

- Panel

- Edge of panel

- Lite frame

- Center of lite

- Edge of lite

- Divider or caming

- Edge of divider or caming

NFRC 100 contains several figures which illustrate the location of the door sections to be modeled in THERM. When modeling glazing options with caming, the NFRC default caming can be used.

A spreadsheet must be used to do the door area-weighting from the THERM files, because the current version of WINDOW does not area-weight doors. In THERM, the U-factor Surface Tags can have any name and as many U-factor Surface Tags can be defined as are needed to accurately describe the model. (See Section 6.2.4, "Define U-factor Surface Tags in the THERM User's Manual), so define as many U-factor Surface Tags as needed and name them descriptively.

Chapter 9 contains a door example, which describes in detail the THERM modeling steps. 


\subsection{Spacers}

\subsubsection{Overview}

THERM has the capability to model spacers in great detail, so that modeling of spacer effective conductivity is no longer needed. Spacer models can be easily reshaped in THERM, and the program's cut and paste feature allows spacers to be copied into each cross section as needed. A library of spacer models can be produced for each spacer type. See the THERM User's Manual, Section 3.5, "Adding a Custom Spacer". A sample spacer, spacer.thm, is included on the THERM installation CD.

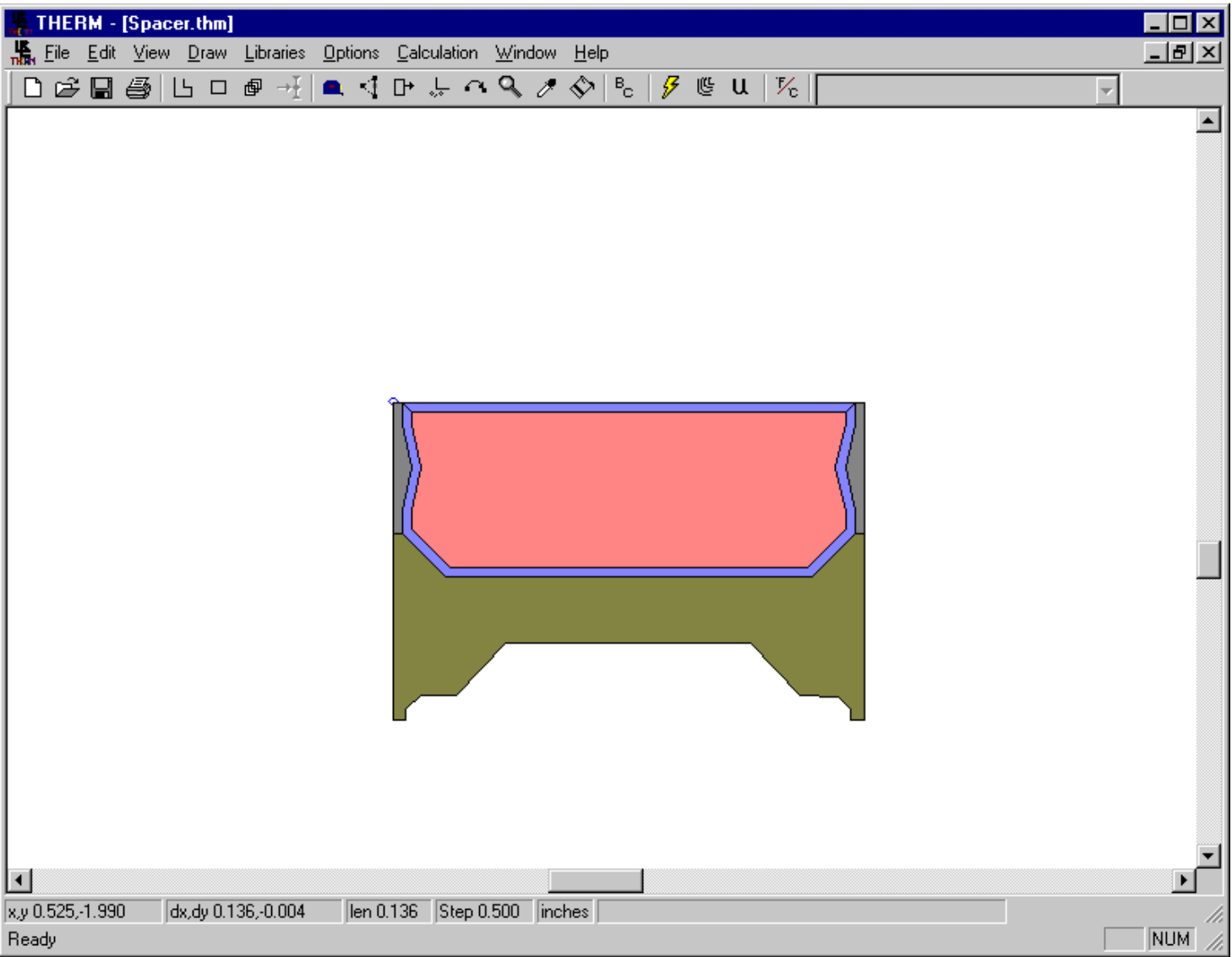

Figure 8-75. spacer.thm sample file. 


\subsubsection{Linking Glazing Cavity properties (imported from WINDOW) for Open Spacers}

The properties of a glazing cavity can be linked to another polygon in order to properly model spacers that are open to the glazing system cavity. Section 5.11.5, "Linking Materials Properties of Polygons" in the THERM User Manual explains this methodology in detail.

To Link the properties of two materials, follow these steps:

- Select the polygon that is to linked to another polygon

- Select the Libraries/Create Link menu choice.

- The cursor will become an Eye Dropper. Click the Eye Dropper cursor in the polygon to be linked to. The material properties of the first polygon are not linked to the material properties of the second polygon.

When using the multiple glazing calculation option, THERM will automatically use the glazing system cavity properties for each glazing option for the linked polygon.

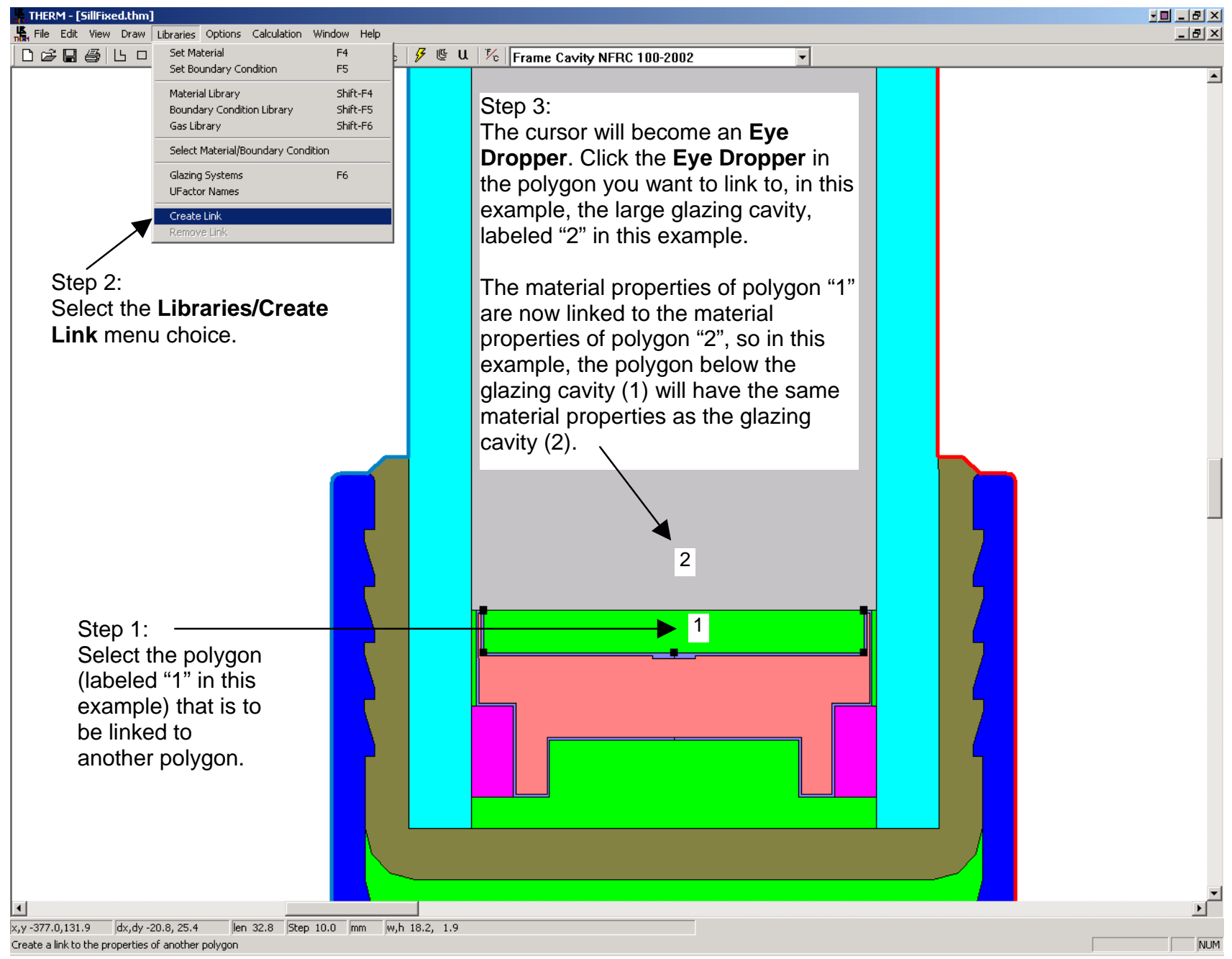

Figure 8-76. Link the open spacer cavity to the glazing system cavity using the Library/Create Link feature . 


\subsection{Non Continuous Thermal Bridge Elements}

Bolts skip and debridge thermal break, including partially de-bridged thermal break material, and thermally slotted cross section shall be included in the model using the concept of isothermal planes. The isothermal planes methodology calculates an effective conductivity of the bridging material based on area weighting the sections of the product with and without thermal bridging material based on the bridging material spacing dimensions. This method is also valid for other regularly spaced thermal bridges such as skip-and-debridged systems.

The effect on the performance of a curtain wall system due to bolts is explained in detail in an ASHRAE paper published in 1998 entitled "The Significance of Bolts in the Thermal Performance of Curtain-Wall Frames for Glazed Facades", by Brent Griffith, Elizabeth Finlayson, Mehrangiz Yazdanian and Dariush Arasteh.

The THERM model to be simulated for the final result is one in which the actual materials of the thermal bridging elements are replaced with a user-defined material having an effective conductivity which represents the area-weighted value that combines the bridging and non-bridging elements.

Figure 8-74 below illustrates an example of a curtain wall system which would require that the thermal bridging elements, in this case the bolts, be modeled using the isothermal planes method.

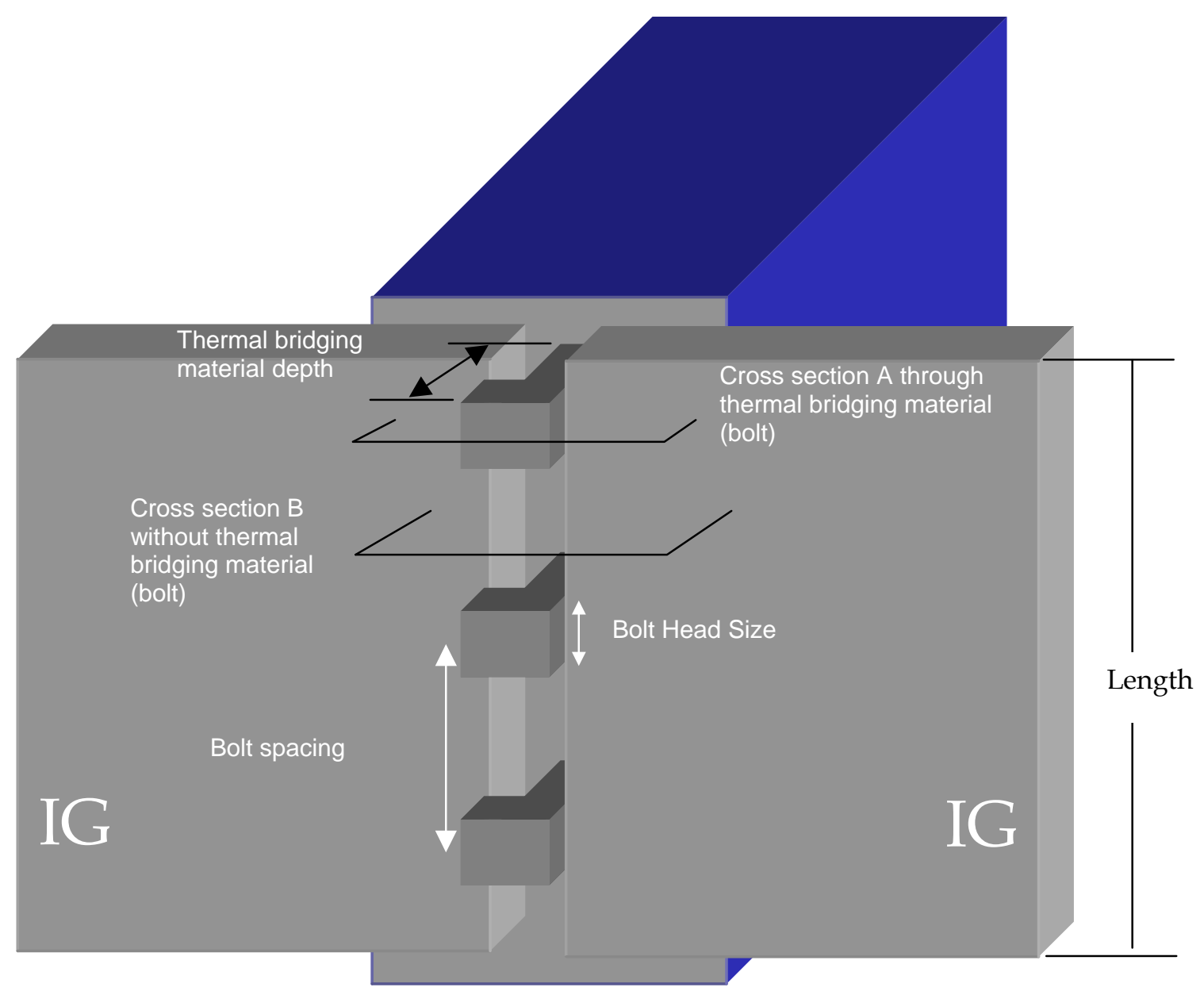

Figure 8-77. Example of a curtain wall system with regularly spaced bolts which act as thermal bridges. 


\subsubsection{Modeling Steps}

The steps for constructing the final THERM model to be simulated are the following:

1. Draw the THERM model without the thermal bridging material.

2. Determine the conductivities of the materials that the thermal bridging material replaces.

- Conductivities of materials can be obtained from the THERM Material Library

- Conductivities of air-filled cavities (such as frame cavities) are assumed to be $0.024 \mathrm{~W} / \mathrm{m}-\mathrm{K}$ (or 0.014 $\mathrm{Btu} / \mathrm{hr}-\mathrm{ft}-\mathrm{o}$ F).

3. Using a cross-section that contains the non-thermal bridging material, measure the depths of each element of the non-thermal bridging material that will have a different thermal conductivity in the nonbridging cross section.

4. Use the conductivities of the non-thermal bridging materials and depths of the non-thermal bridging materials in Equation 2 below to determine the Resistance $(\mathrm{R})$ for each non-thermal bridging element.

5. Sum the resistances (Rt) and divide by the total depth of the non-thermal bridging elements to obtain Kn, as shown in Equation 3, to calculate the conductivity of the non-thermal bridging elements

6. Calculate the fraction of thermal and non-thermal bridging material along the length of the façade using Equations 4 and 5.

7. Calculate the final effective conductivity value for the thermal bridging elements using Equation 1.

8. In THERM, define a new material with the Keff value derived in Step 7, and assign it to the cross section polygons that represent the thermal bridging elements.

9. Simulate the model. 


\subsubsection{Equations}

Calculate the effective conductivity of thermal bridging elements (e.g., bolts, screws, etc.)

$$
\mathrm{Keff}=\mathrm{Fb}^{*} \mathrm{~Kb}+\mathrm{Fn} * \mathrm{Kn}
$$

Equation 1

where

$\mathrm{Fb} \quad=$ Fraction of the Length which contains the thermal bridging elements (see equation 4 below)

Fn $\quad=$ the fraction of the Length which contains non-thermal bridging elements(see equation 5 below)

$\mathrm{Kb} \quad=$ conductivity of the thermal bridging elements

$\mathrm{Kn} \quad=$ conductivity of the non-thermal-bridging elements

(from the sum of the resistances, Rt, of individual elements from Equation 2 below) Assume a default value of $0.24 \mathrm{~W} / \mathrm{m}-\mathrm{K}$ for air cavities.

This methodology should be applied with the following caveats:

- If less than $1 \%$ (to obtain percentage, multiply fraction by a 100) of the Length is made of thermal bridging elements (such as stainless steel), ie, $\mathrm{Fb}<0.01$, do not model the thermal bridging elements.

- If between $1 \%$ and $5 \%$ of the Length is made of thermal bridging elements $(0.01<=\mathrm{Fb}<=0.05)$ and if the conductivity of the thermal bridging elements is more than 10 times the conductivity of the thermal break, then model the thermal bridging elements. Then model the thermal bridging elements using the keff calculated in Equation 1.

- If more than $5 \%$ of the length is made of thermal bridging elements ( $\mathrm{Fb}>0.05)$, always model the thermal bridging elements. Then model the thermal bridging elements using the keff calculated in Equation 1.

Calculate the total resistance of the non-thermal bridging elements, $\mathrm{Rt}$, by summing individual resistances (non-thermal bridging element conductivity) for each non-thermal bridging element using the formula:

$$
\mathrm{Rt}=\Sigma(\mathrm{D} / \mathrm{k}) \quad \text { Equation } 2
$$

Where:

$\mathrm{Rt}=$ Sum of the thermal resistances of the individual non-thermal bridging material. Units: $\mathrm{m}^{2} \cdot \mathrm{K} / \mathrm{W}$ (SI), or hr.ft ${ }^{2}{ }^{\circ} \mathrm{F} / \mathrm{Btu}(\mathrm{IP})$

$\mathrm{D}=$ Depth of the individual non-thermal bridging elements that will be substituted by the calculated effective conductivity. Units: $\mathrm{m}(\mathrm{SI})$, or $\mathrm{ft}(\mathrm{IP})$, or (in) (alternate IP) $\mathrm{k}=$ conductivity of the individual non-thermal bridging elements that will be substituted. Units: $\mathrm{W} / \mathrm{m} \cdot \mathrm{K}(\mathrm{SI})$, or Btu $/ \mathrm{hr} \cdot \mathrm{in} \cdot{ }^{\circ} \mathrm{F}(\mathrm{IP})$, or $\mathrm{Btu} \cdot \mathrm{in} / \mathrm{hr} \cdot \mathrm{ft}^{2} .^{\circ} \mathrm{F}$ (alternate IP)

Therefore:

$$
\mathrm{Kn}=\mathrm{Dt} / \mathrm{Rt}
$$

\section{Equation 3}

Where:

$\mathrm{Dt}=$ Total depth, which is the sum of the depths of the individual non-thermal bridging elements Calculate the fraction of thermal bridging material to non thermal bridging material as follows:
$\mathrm{Fb}=\mathrm{Wb} / \mathrm{Sb} \quad(\% \mathrm{Fb}=\mathrm{Fb} \cdot 100)$
Equation 4
$\mathrm{Fn}=1-\mathrm{Fb}$
Equation 5 
Where:

$$
\begin{aligned}
& \mathrm{Wb}=\text { Bridging material width } \\
& \mathrm{Sb}=\text { Bridging material spacing }
\end{aligned}
$$

\subsubsection{Example 1: Bolts in Curtain Wall}

The following figures show two cross sections of the curtain wall in Figure 8-74. Figure 8-75 represents the cross section of the curtain wall where the bolt occurs (screw threads should be averaged and not drawn explicitly), and Figure 8-76 represents the cross section of the curtain wall where the bolt does not occur. The geometry of the cross-section in Figure 8-75 would be used for the final THERM run, and the conductivity of the materials used to define the bolt would be changed to the value derived from the methodology explained in this section. The geometry in Figure 8-76 is drawn only to obtain the conductivity values for calculating the conductivity of this "averaged" material.

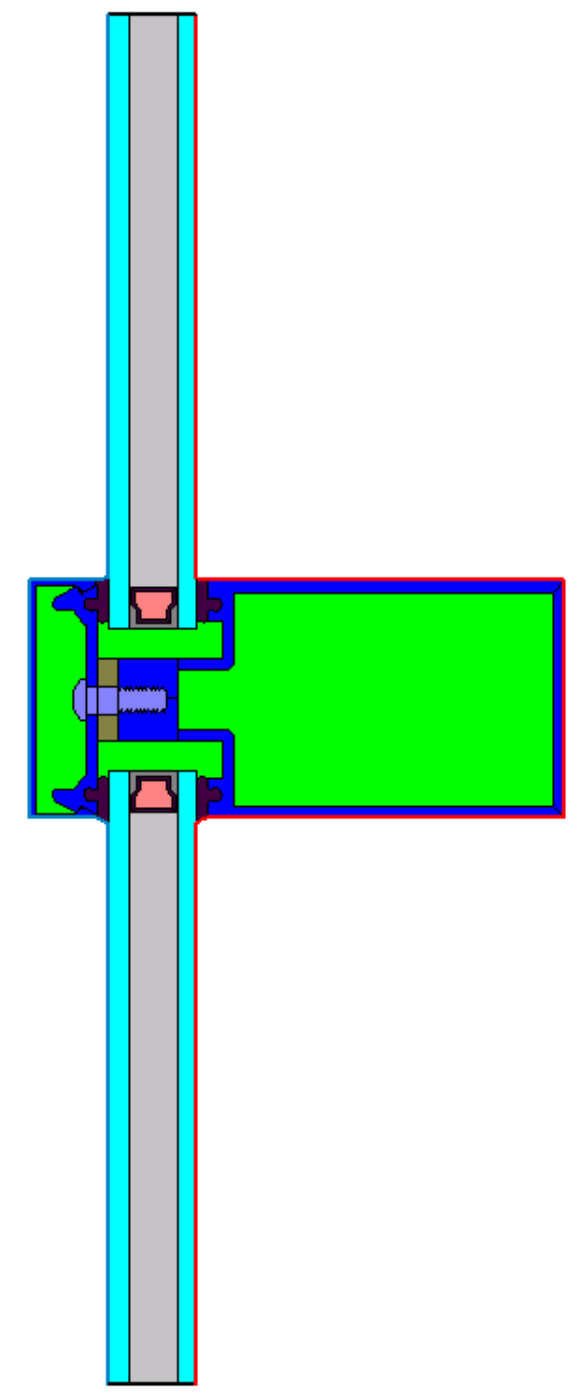

Figure 8-78. THERM cross section where the bolt occurs.

Figure 8-76 shows the conductivity values for the four materials that must be obtained for the calculation. Material 1 and 4 are air cavities, and the conductivity is assumed to be $0.024 \mathrm{~W} / \mathrm{m}-\mathrm{K}$. 


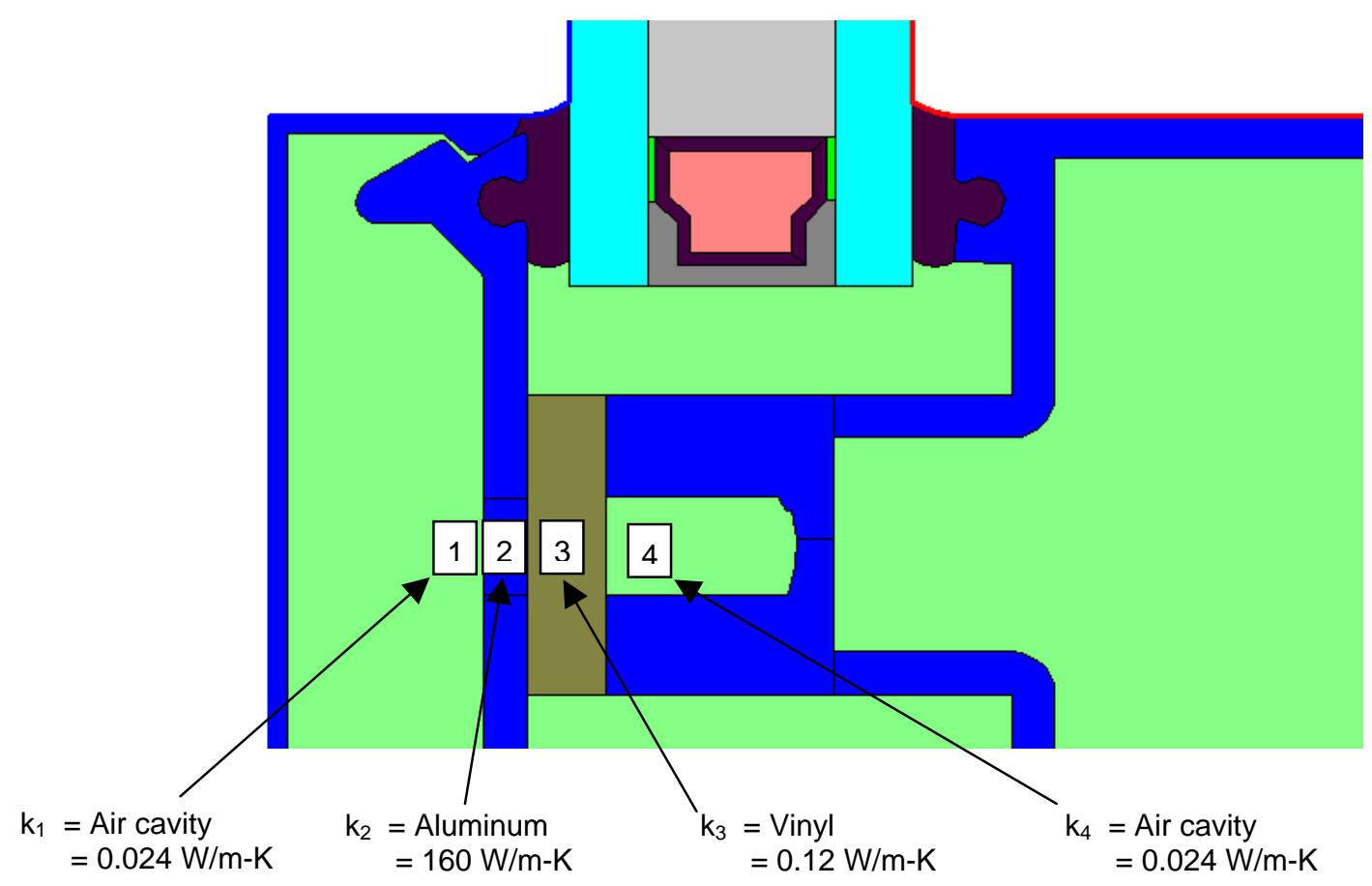

Figure 8-79. Materials in the non-bridging material cross section for which conductivities must be obtained.

Figure 8-77 shows the depths of each of the thermal bridging elements that are used in the Keff calculation.

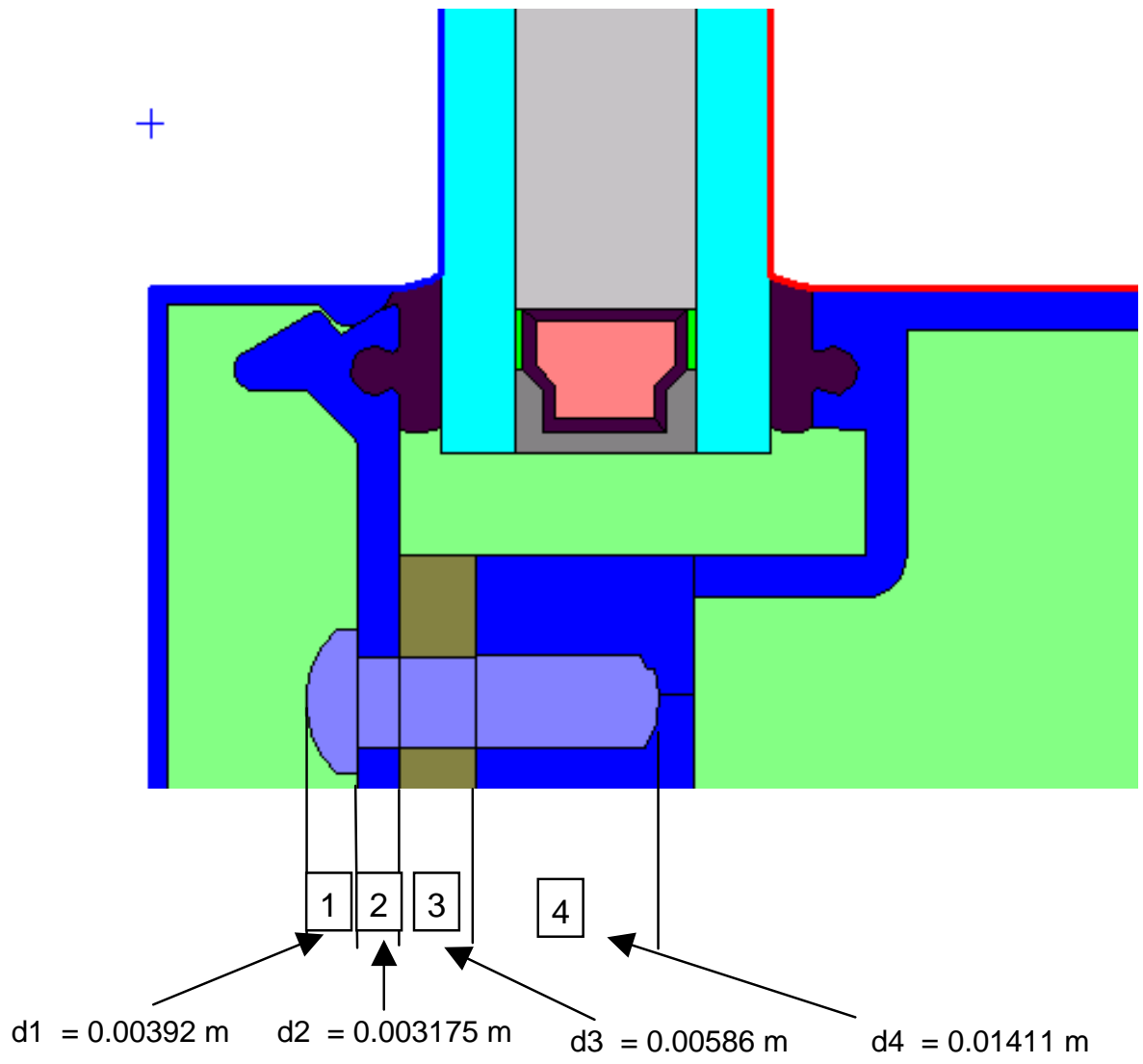

Figure 8-80. Material depths for the thermal bridging materials. 
Table 8-1 shows the conductivity and depth values used to calculate the $\mathrm{R}$ for each non-thermal bridging element using Equation 2.

Table 8-1

\begin{tabular}{|l|l|l|l|l|}
\hline $\begin{array}{l}\text { Cross } \\
\text { Section } \\
\text { Element }\end{array}$ & Material & $\begin{array}{l}\text { Conductivity } \\
{[\mathbf{W} / \mathbf{m K}]}\end{array}$ & $\begin{array}{l}\text { Depth } \\
(\mathbf{m})\end{array}$ & $\begin{array}{l}\mathbf{R} \\
{[\mathbf{m} 2 K /} \\
\mathbf{W}]\end{array}$ \\
\hline 1 & Air cavity (default value) & 0.024 & 0.00392 & 0.16333 \\
\hline 2 & $\begin{array}{l}\text { Aluminum } \\
\text { (conductivity from THERM Material Library) }\end{array}$ & 160 & 0.003175 & 0.0000198 \\
\hline 3 & $\begin{array}{l}\text { Vinyl } \\
\text { (conductivity from THERM Material Library) }\end{array}$ & 0.12 & 0.00586 & 0.049 \\
\hline 4 & Air cavity (default value) & 0.024 & 0.01411 & 0.587917 \\
\hline & Total & & $\mathbf{0 . 0 2 7 0 6}$ & $\mathbf{0 . 8 0 0 1 0 3}$ \\
\hline
\end{tabular}

Calculate Rt as follows:

$$
\begin{array}{rll}
\mathrm{R}_{\mathrm{t}} & =\Sigma(\mathrm{d} / \mathrm{k}) \\
& =\quad\left(\mathrm{d}_{1} / \mathrm{k}_{1}\right)+\left(\mathrm{d}_{2} / \mathrm{k}_{2}\right)+\left(\mathrm{d}_{3} / \mathrm{k}_{3}\right)+\left(\mathrm{d}_{4} / \mathrm{k}_{4}\right) \\
& =(0.00392 / 0.024)+(0.003175 / 160)+(0.00586 / 0.12)+(0.01411 / 0.024) \\
& =0.800103 \mathrm{~m}^{2} \mathrm{~K} / \mathrm{W} \\
\mathrm{D}_{\mathrm{t}} & =0.00392 \mathrm{~m}+0.003175 \mathrm{~m}+0.00586 \mathrm{~m}+0.01411 \mathrm{~m} \\
& =0.02706 \mathrm{~m}
\end{array}
$$

Calculate the conductivities as follows:

$$
\begin{array}{rll}
\mathrm{Kn} & = & \mathrm{D}_{\mathrm{t}} / \mathrm{R}_{\mathrm{t}} \\
& = & 0.02706 / 0.800103 \\
& = & 0.033821 \mathrm{~W} / \mathrm{m} \cdot \mathrm{K} \\
\mathrm{Kb} & = & 14.3 \mathrm{~W} / \mathrm{m} \cdot \mathrm{K} \text { (stainless steel) }
\end{array}
$$

Calculate the fraction of bolt to no bolt as follows:

$\begin{array}{rll}\mathrm{Wb} & = & \text { Bolt head width } \\ & = & 11.1 \mathrm{~mm} \\ \mathrm{Sb} & = & \text { Bolt spacing 12" } \\ & = & 304.8 \mathrm{~mm} \\ \mathrm{Fb} & = & \mathrm{Wb} / \mathrm{Sb} \\ & =11.1 \mathrm{~mm} / 304.8 \mathrm{~mm} \\ & =0.036(\% \mathrm{Fb}=0.036 \cdot 100=3.6 \%) \\ \mathrm{Fn} & =1-\mathrm{Fb} \\ & =1-0.036 \\ & =0.964\end{array}$


Calculate the new Keff, which will be used in THERM as follows:

$$
\begin{array}{rlrl}
\text { Keff } & = & \mathrm{Fb}^{*} \mathrm{~Kb}+\mathrm{Fn}{ }^{*} \mathrm{Kn} \\
\text { Keff } & =(0.036 * 14.3)+(0.964 * 0.033827) \\
& =0.55 \mathrm{~W} / \mathrm{m} \cdot \mathrm{K}
\end{array}
$$

In THERM, create a new material in the Material Library with this Keff. In the THERM cross section, the bolt material should be changed from Stainless Steel to this new material. The resulting cross section is a 2-D thermal equivalent of the cross section with and without the thermal bridging material.

Figure 8-81. Final THERM model with boundary conditions defined.

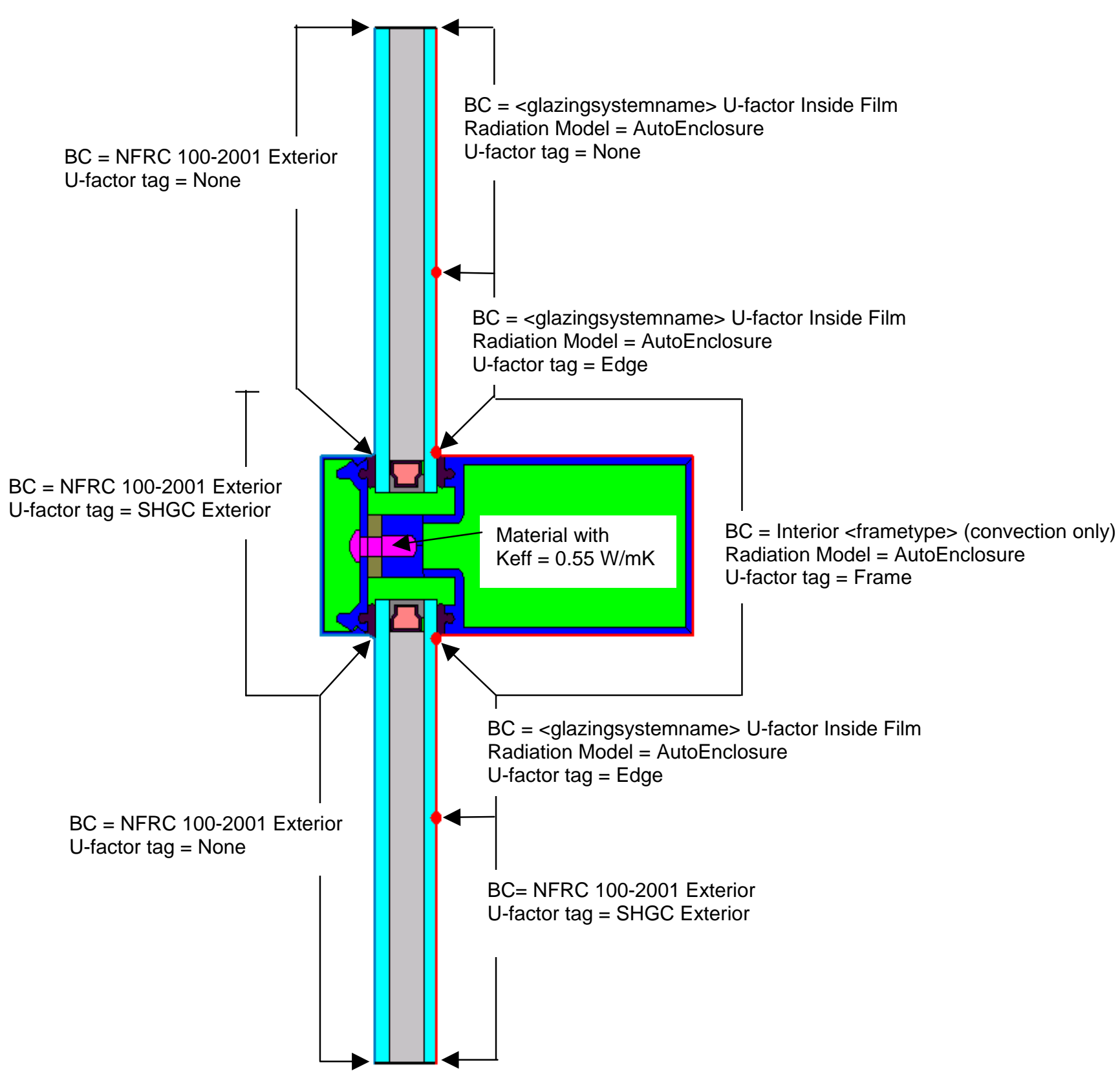




\subsubsection{Example 2: Thermally slotted cross-section}

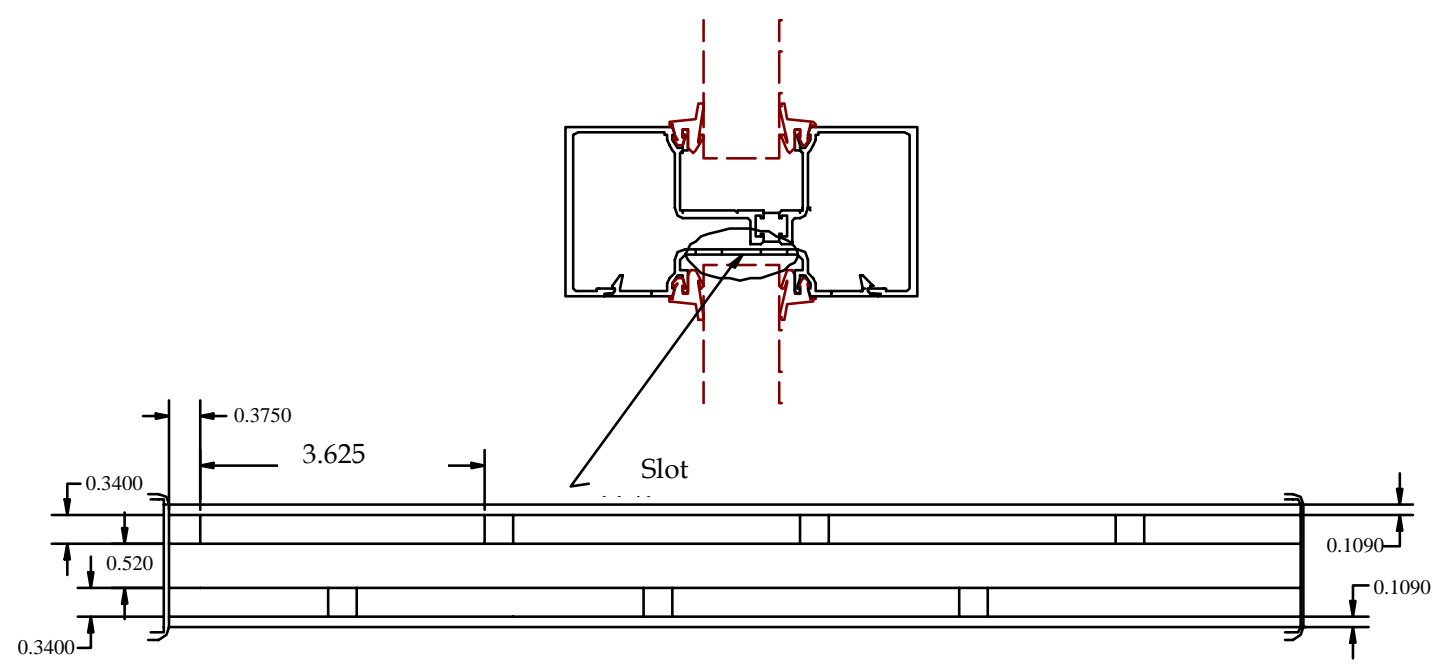

Figure 8-82. DXF for thermally slotted cross section.

\section{Step I}

$\begin{array}{ll}\text { Skip } & =0.375 \mathrm{in} .(0.009525 \mathrm{~m}) \\ \text { Slot (Air) } & =3.625 \mathrm{in} .(0.092075 \mathrm{~m}) \\ \text { Interval } & =3.625 \mathrm{in} .+0.375 \mathrm{in} . \\ & =4 \text { in. }(0.009525 \mathrm{~m}+0.092075 \mathrm{~m}) \\ & =0.1016 \mathrm{~m}) \\ & =0.009525 \mathrm{~m} / 0.1016 \mathrm{~m} \\ & =0.094 \\ \text { Fn } \quad B C=1 & =1-F b \\ & =1-0.094 \\ & =0.906\end{array}$

Percent of thermal bridge $\quad=(\mathrm{Fb}) * 100$

$$
=(0.094) * 100
$$$$
=9.4 \%
$$

Because the thermal bridge is $9.4 \%$ of the length of the façade, the skip-and-debridge needs to be calculated using the isothermal plane procedure. 
$\mathrm{Kb}=160 \mathrm{~W} / \mathrm{m}-\mathrm{K}$ (conductivity of skipped debridge, in this case Aluminum)

Rt $\quad=\sum$ (Depth $/$ conductivity)

$=\mathrm{D}_{\mathrm{d}} / \mathrm{k}_{\mathrm{d}}$

$=(0.0086 \mathrm{~m} / 0.024 \mathrm{~W} / \mathrm{m}-\mathrm{K})$

$=0.35833 \mathrm{~m}^{2}-\mathrm{K} / \mathrm{W}$

where Depth is length of thermal bridge in a direction of heat flow, and the air is assumed to have the conductivity of $0.024 \mathrm{~W} / \mathrm{m}-\mathrm{K}$

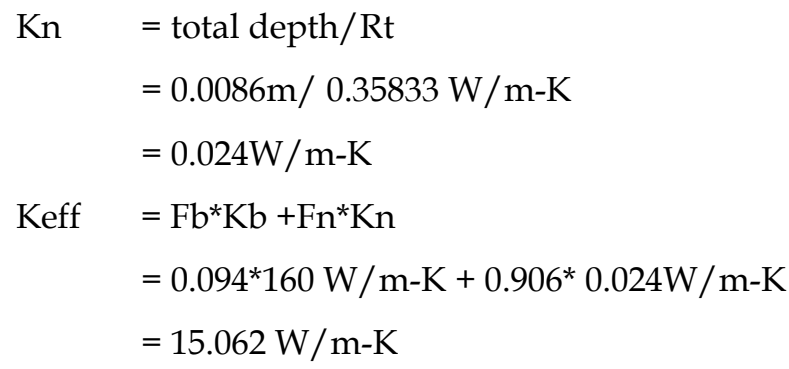

To convert to IP:

Keff $\quad=15.062 \mathrm{~W} / \mathrm{m}-\mathrm{K} * 0.57782$

$=8.703 \mathrm{Btu} / \mathrm{hr}-\mathrm{ft}-\mathrm{o} F$

or in alternative IP units,

Keff $=15.062 * 0.57782 * 12 \mathrm{in} / \mathrm{ft}$

$=104.436$ Btu-in $/ \mathrm{hr}_{-} \mathrm{ft}^{2}{ }^{-} \mathrm{o} F$ 


\section{Step-2}

Replace the strip of air-aluminum-air with new keff material of $15.078 \mathrm{~W} / \mathrm{m}-\mathrm{K}$

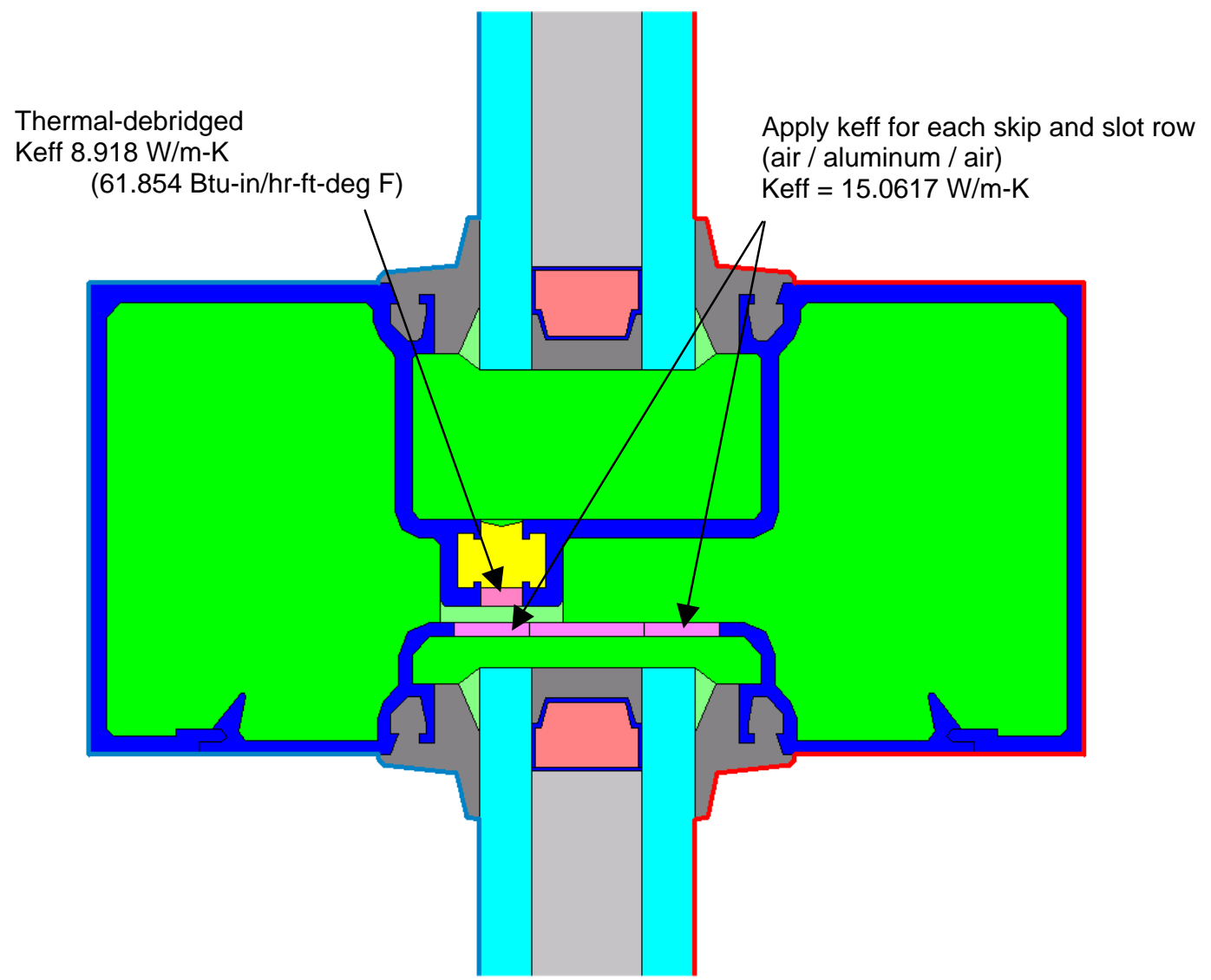

Figure 8-83. New Keff assigned to each skip and debridged row. 


\section{Step 3}

Define the Boundary condition and run the model to calculate the U-factor for frame and edge-of-glass.

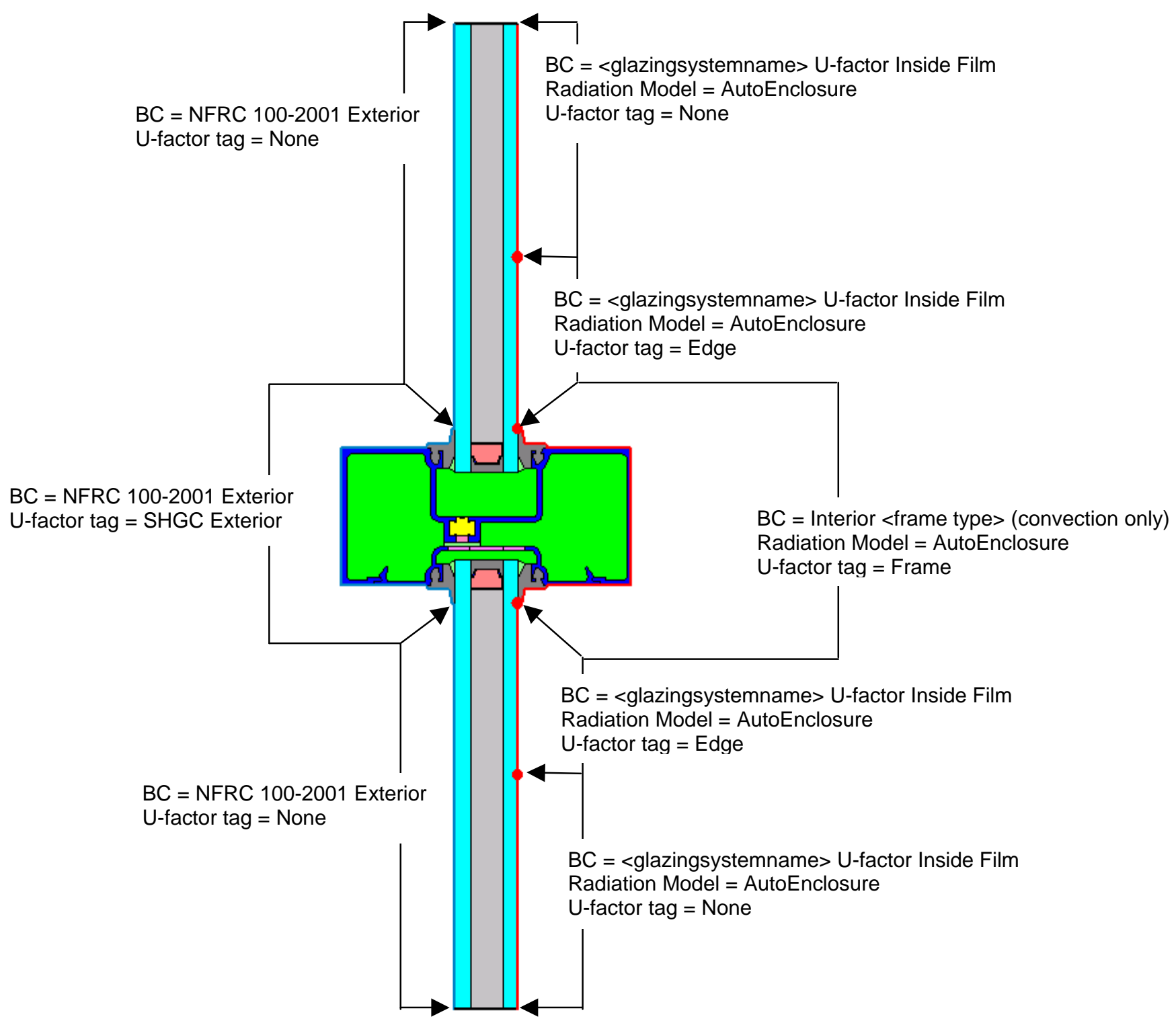

Figure 8-84. Final THERM model with boundary conditions defined. 


\subsubsection{Example 3: Skip-and-debridge:}
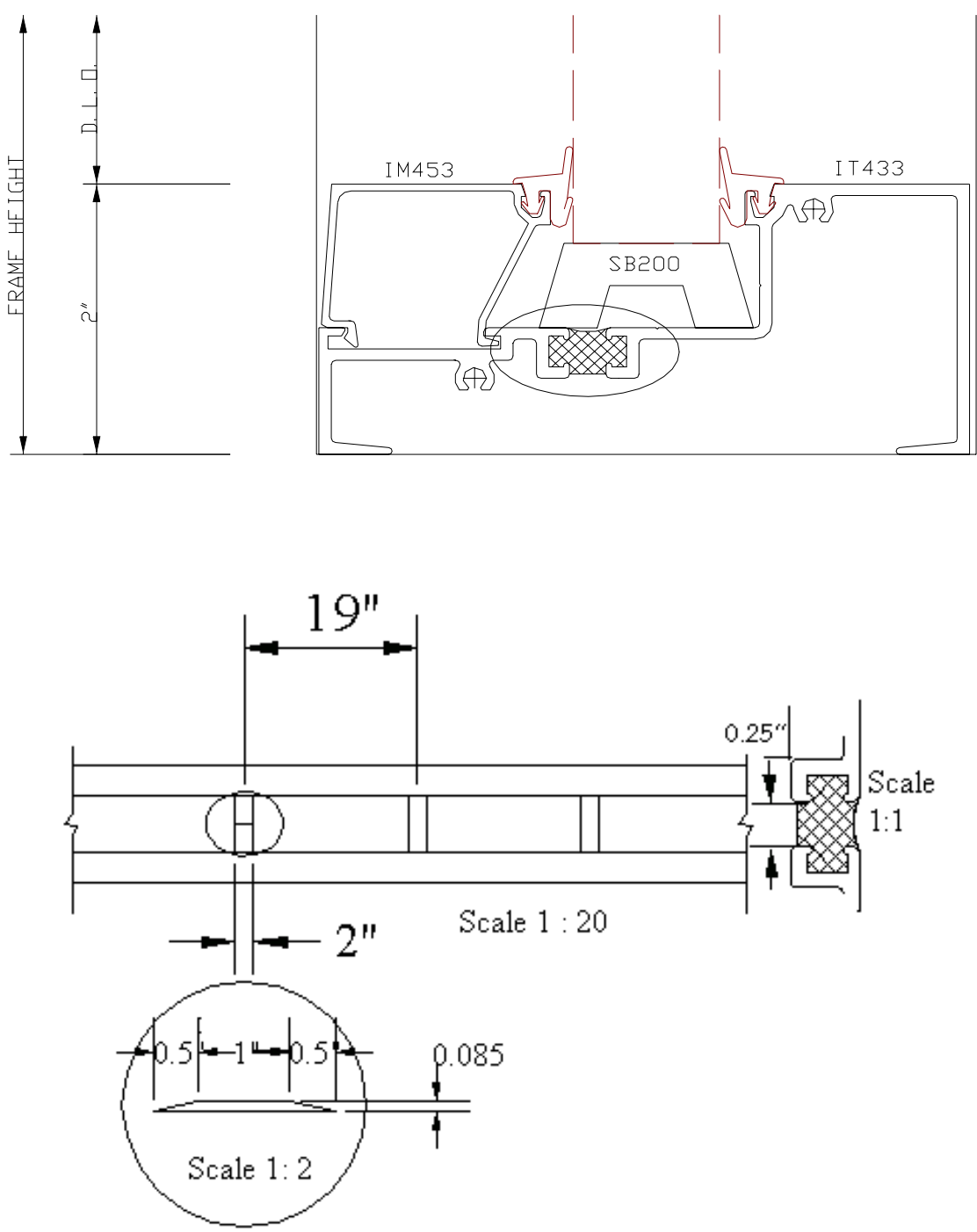

Note: the skip trapezoid shall be treated as a rectangle equal to the total length of the base of the trapezoid.

Figure 8-85. Drawings for Example 3 Skip and Debridge. 


\section{STEP 1}

Skip $=0.0508 \mathrm{~m}$

Debridge (Air) $=0.4318 \mathrm{~m}$

Interval $=0.508 \mathrm{~m}+0.4318 \mathrm{~m}=0.4826 \mathrm{~m}$

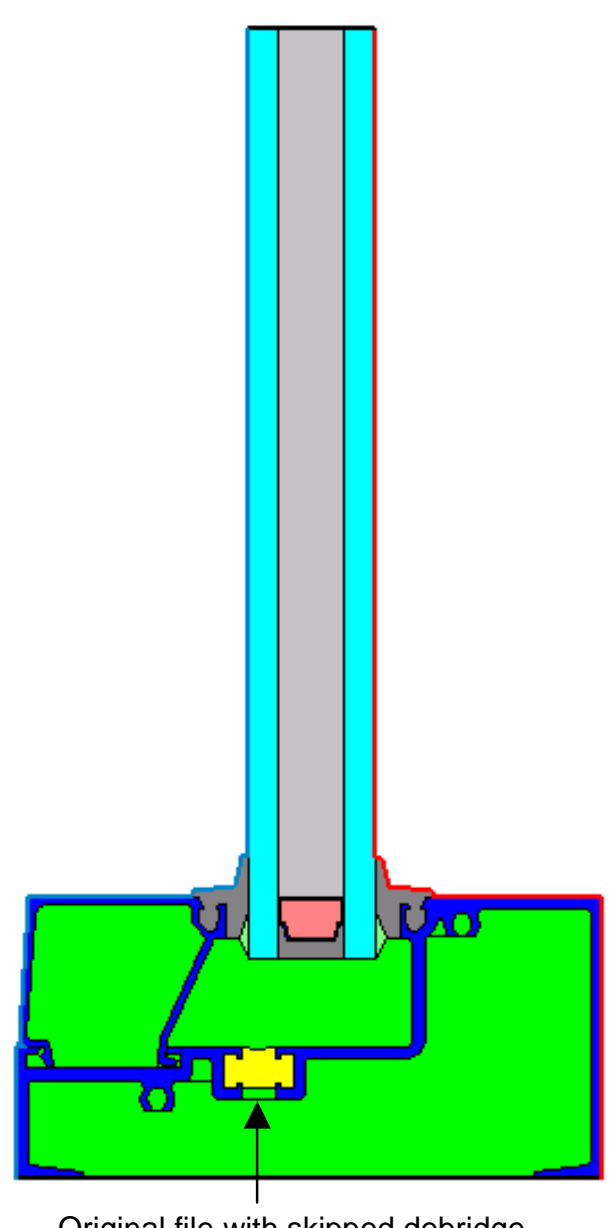

Original file with skipped debridge

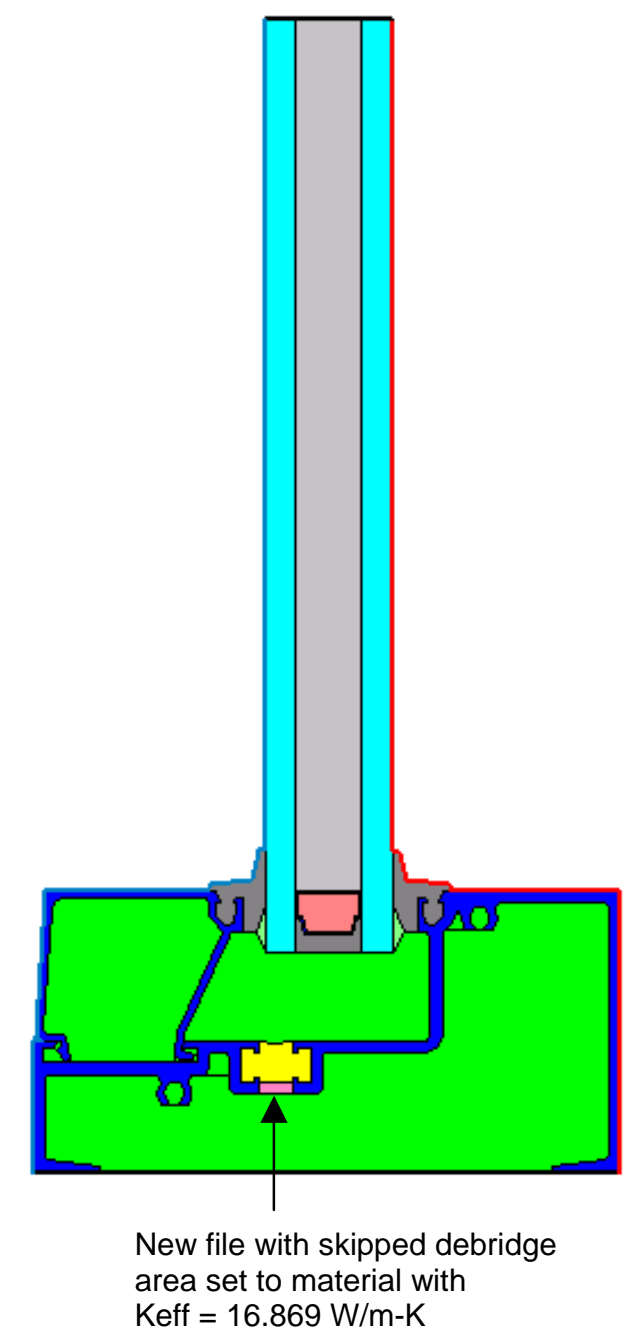

Figure 8-86. Original THERM model and new model with new Keff for skipped debridge area.

$\mathrm{Fb} \quad=0.0508 \mathrm{~m} / 0.4826 \mathrm{~m}=0.1053$

$\mathrm{Fn} \quad=1-\mathrm{Fb}$

$=1-0.1053=0.4947$

$\% \mathrm{Fb} \quad=(\mathrm{Fb}) \cdot 100$

$=(0.1053) \cdot 100$

$=10.53 \%$ (Skip-and-debridge needs to be calculated using Isothermal plane procedure).

$\mathrm{Kb}=160 \mathrm{~W} / \mathrm{m}-\mathrm{K}$ (conductivity of skipped debridge, in this case aluminum) 
Rt $\quad=\sum$ Length $/$ conductivity

$=(0.00635 \mathrm{~m} / 0.024 \mathrm{~W} / \mathrm{m}-\mathrm{K})$

$=0.2646 \mathrm{~m}^{2}-\mathrm{K} / \mathrm{W}$

The length is the length of material in a direction of heat flow i.e. 0.25 " as shown in the figure. (The air effective conductivity calculated using THEM)

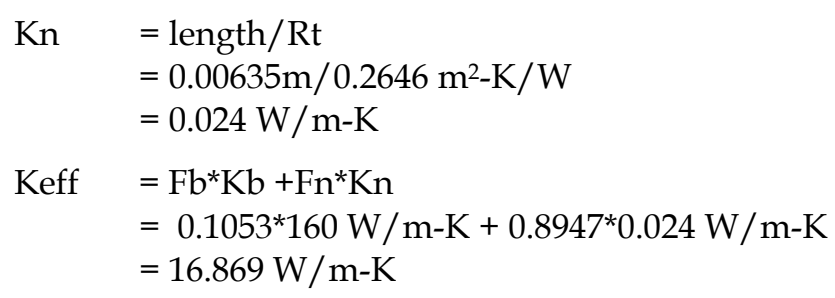

To convert to IP:

Keff $=16.869 * 0.57782=9.747 \mathrm{Btu} / \mathrm{hr}-\mathrm{ft}-\mathrm{F}$ (or in alternative IP Units: $\left.116.97 \mathrm{Btu}-\mathrm{in} / \mathrm{hr}-\mathrm{ft}^{2}-\mathrm{F}\right)$ 



\section{SAMPLE PROBLEMS}

\subsection{Overview}

There are four sample problems:

- Problem 1: Vinyl fixed window

page 9-2

- Problem 2: Aluminum horizontal sliding window

page $9-16$

- Problem 3: Skylight

page $9-50$

- Problem 4: Door:

page 9-59

These sample problems may contain boundary conditions, frame cavity conditions and modeling techniques that do not conform to the NFRC modeling rules. If this is the case, the NFRC modeling rules always take precedence over what is shown in these example problems. Also, the results shown in these examples may not correspond exactly to results obtained with the WINDOW and THERM programs. 


\subsection{Problem 1: Vinyl Fixed}

For this fixed vinyl window product, calculate the U-factor, SHGC, VT, and CR values.

\subsubsection{Description}

\begin{tabular}{|c|c|}
\hline Window Type & Fixed picture window \\
\hline Frame Material & $\begin{array}{l}\text { PVC frame; the same geometry can be used for the head, sill and jambs. The wall } \\
\text { thickness is } 3.175 \mathrm{~mm}\left(0.125^{\prime \prime}\right) \text {. }\end{array}$ \\
\hline Glazing System & $\begin{array}{l}\text { Double glazing, } 19.05 \mathrm{~mm}(0.750 ") \text { overall I.G. thickness. The outboard lite is double- } \\
\text { strength clear glass }(3.277 \mathrm{~mm}(0.129 ") \text { thick), the inboard lite is double-strength clear } \\
\text { glass with a PPG Sungate100 low-E coating on surface three, and the glazing cavity (12.5 } \\
\mathrm{mm}\left(0.492^{\prime \prime}\right) \text { ) is air filled. }\end{array}$ \\
\hline Spacer type & Intercept Spacer with PIB primary seal and hot-melt butyl secondary seal \\
\hline Glazing Tape & Foam rubber tape, $3.175 \mathrm{~mm}(0.125 ")$ thick \\
\hline Dividers & $\begin{array}{l}\text { Aluminum grille pattern, painted white, between the glass. The grille pattern for the } \\
\text { window is three by four. }\end{array}$ \\
\hline Cross Sections & See Section 9.2.7 for drawings of this product. \\
\hline
\end{tabular}

\subsubsection{Glazing Matrix}

The window is offered by the manufacturer both with and without dividers. The drawings indicate that there is less than $3.0 \mathrm{~mm}(0.118 ")$ between the glass and the divider, dividers, so the glazing matrix must include both a case with and without the dividers. 


\subsubsection{Center-of-glazing Modeling (WINDOW)}

Model the glazing system in WINDOW with double strength glass, a $12.5 \mathrm{~mm}\left(0.492^{\prime \prime}\right)$ air space, air filled, and Sungate100 Low-e.

The figure below shows the WINDOW Glazing System Library for this glazing system.

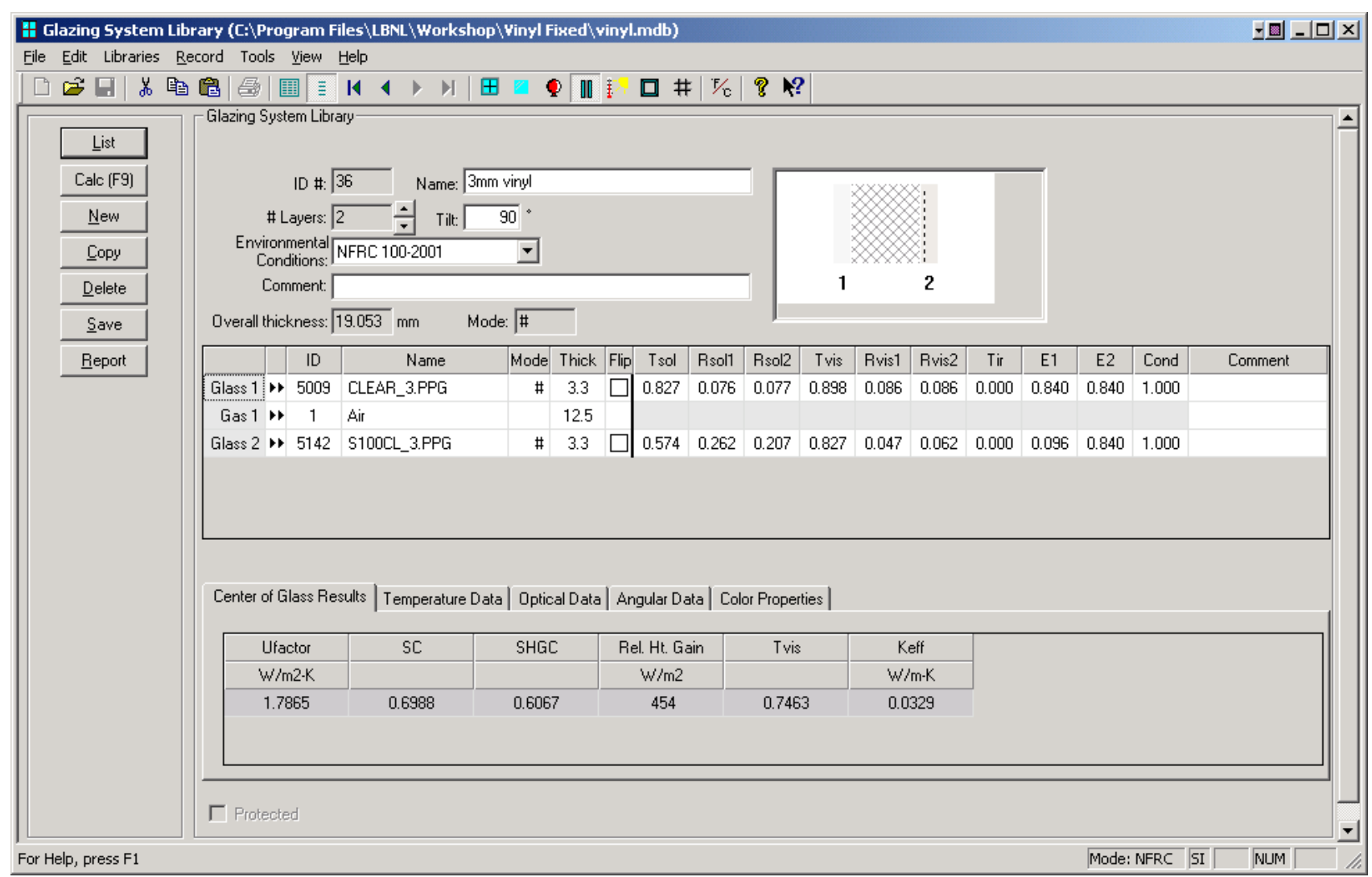

Figure 9-1. WINDOW 4.1 Glazing System Library for vinyl window

The results for the center-of-glazing U-factor are shown in the following table:

Table 9-1. Center-of-glazing U-factor results from WINDOW

\begin{tabular}{|c|c|c|c|}
\hline & \multicolumn{2}{|c|}{ Center-of-glazing U-factor } \\
\hline & $\begin{array}{c}\text { Glazing Options ( } 0.75 " \text { overall } \\
\text { thickness) }\end{array}$ & $\mathrm{W} / \mathrm{m}^{2}-{ }^{\circ} \mathrm{C}$ & $\begin{array}{l}\text { (Btu/hr- } \\
\left.\mathrm{ft}^{2}-{ }^{\circ} \mathrm{F}\right)\end{array}$ \\
\hline 1 & Clear (3 mm), Air, Low-e (3mm) & 1.7865 & 0.314 \\
\hline
\end{tabular}

This glazing system will be used in THERM to calculate the edge-of-glazing and frame U-factors, and also in WINDOW to calculate the overall product U-factor. 


\subsubsection{Edge-of-glazing and Frame Modeling (THERM) for U-factor}

Although this is a fixed window, and the head, sill and jamb have the same geometry, it is necessary to create a different cross section for each component type, because of the ISO 15099 modeling assumptions for gravity vectors as well as for Condensation Resistance modeling. The cross sections for each component will be the same, but the orientation of the glazing system and the properties will be different for each.

Table 9-3 shows the files that are on the CD for this example.

Table 9-2. Files associated with the vinyl window example

\begin{tabular}{|l|l|l|}
\hline Cross Section & DXF Filename & \multicolumn{1}{c|}{ THERM file } \\
\hline Sill (model with CR on) & Vinylp.dxf & Vinyl-Sill.thm \\
\hline Head (model with CR on) & Vinylp.dxf & Vinyl-Head.thm \\
\hline Jamb (model with CR off) & Vinylp.dxf & Vinyl-Jamb.thm \\
\hline Divider (model as vertical divider with CR off) & & Vinyl-Div.thm \\
\hline
\end{tabular}

Table 9-4 shows the resulting U-factors the vinyl frame and the divider cross sections.

Table 9-3. THERM results for vinyl window cross sections

\begin{tabular}{|c|c|c|c|c|}
\hline & \multicolumn{2}{|c|}{ Frame U-factor } & \multicolumn{2}{|c|}{ Edge U-factor } \\
\hline Cross Section & $\left(\mathrm{W} / \mathrm{m}^{2}-^{\circ} \mathrm{C}\right)$ & (Btu/hr-ft $\left.{ }^{2}-{ }^{\circ} \mathrm{F}\right)$ & $\left(\mathrm{W} / \mathrm{m}^{2}-{ }^{\circ} \mathrm{C}\right)$ & (Btu/hr-ft $\left.{ }^{2}{ }^{\circ} F\right)$ \\
\hline Sill & 1.6147 & 0.2821 & 2.0806 & 0.3663 \\
\hline Head & 1.6024 & 0.2822 & 2.0828 & 0.3668 \\
\hline Jamb & 1.7250 & 0.3038 & 2.0708 & 0.3647 \\
\hline Divider & 2.7018 & 0.4758 & 2.0076 & 0.3535 \\
\hline
\end{tabular}

Figures 9-2 and 9-3 show the THERM cross sections and U-factor results for this window. 
Sill

Cross Section Type $=$ Sill Gravity Vector $=$ Down

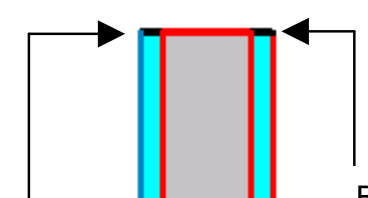

$\mathrm{BC}=3 \mathrm{~mm}$ Vinyl U-factor Inside Film Radiation Model $=$ AutoEnclosure U-factor tag $=$ None

$\mathrm{BC}=$ NFRC 100-2001 Exterior Radiation Model $=$ Blackbody U-factor tag $=$ None

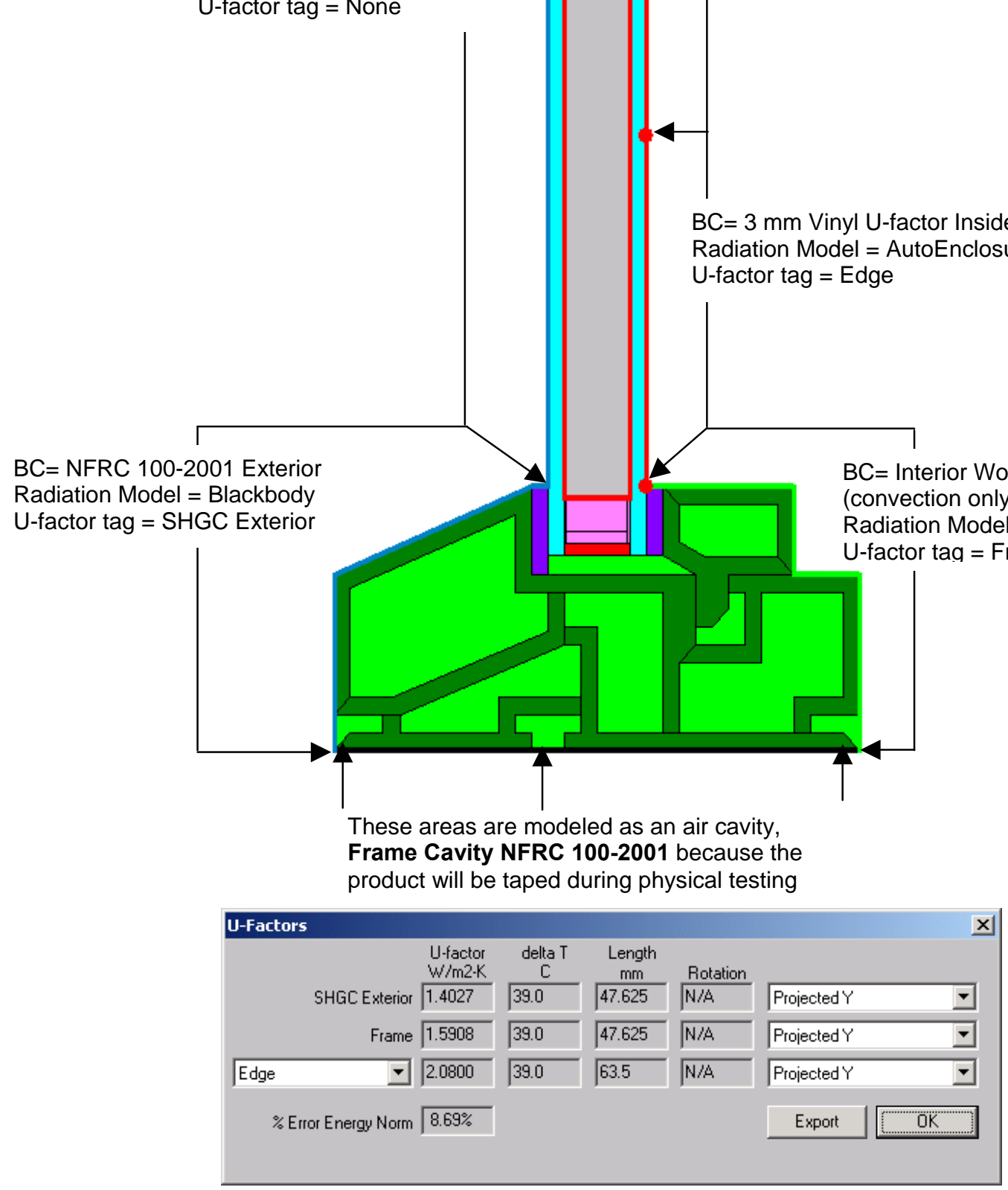

Figure 9-2. THERM cross section and U-factor for the vinyl fixed window sill. 


\section{Jamb}

Modeling Assumptions:

- $\quad$ Cross Section Type = Jamb

- Gravity Vector = Into the Screen

- Jambs are modeled without the Condensation Resistance Model
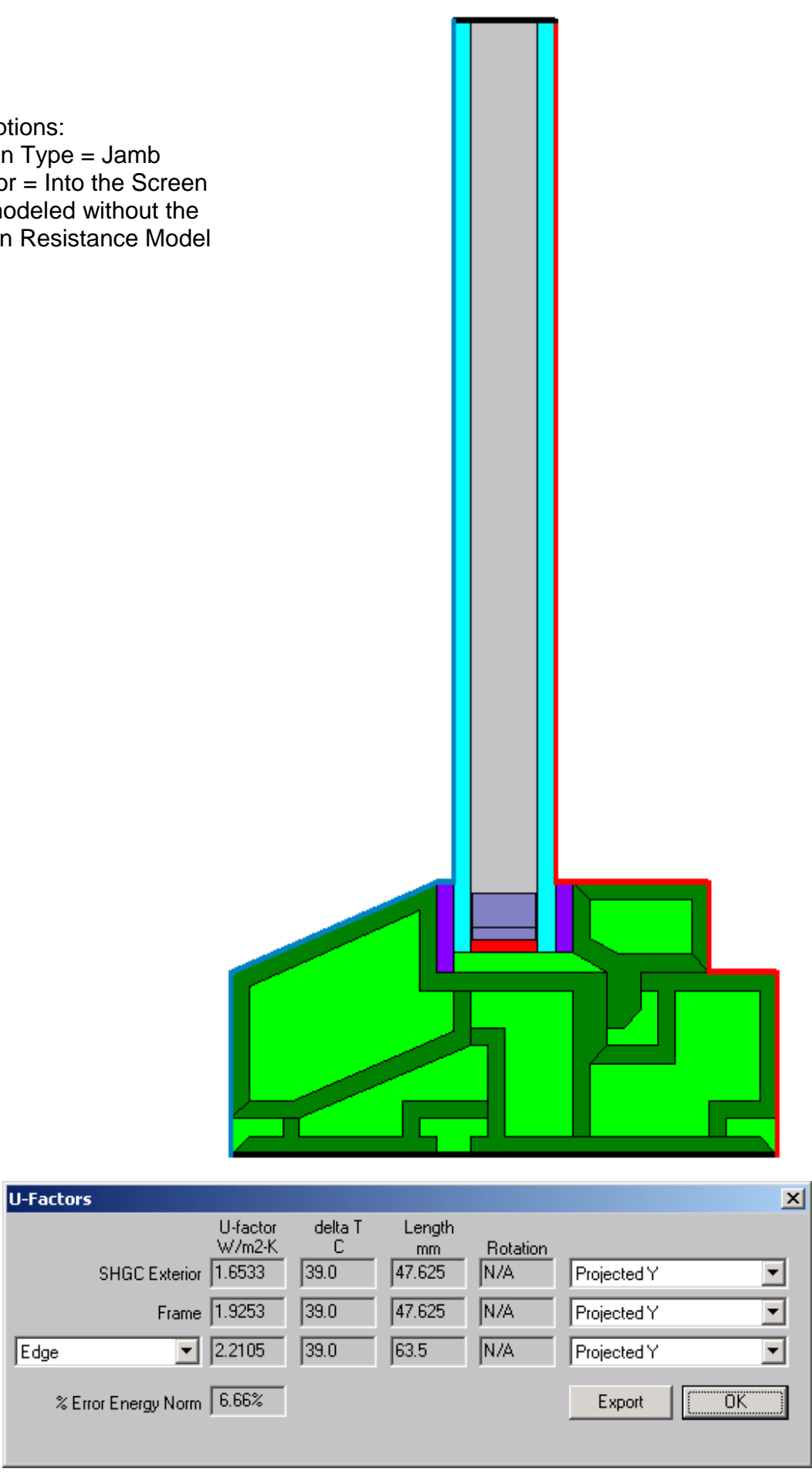

Figure 9-3. THERM cross section and U-factor for the vinyl fixed window jamb 


\section{Head}

Modeling Assumptions:

- Cross Section Type = Head

- Gravity Vector = Down
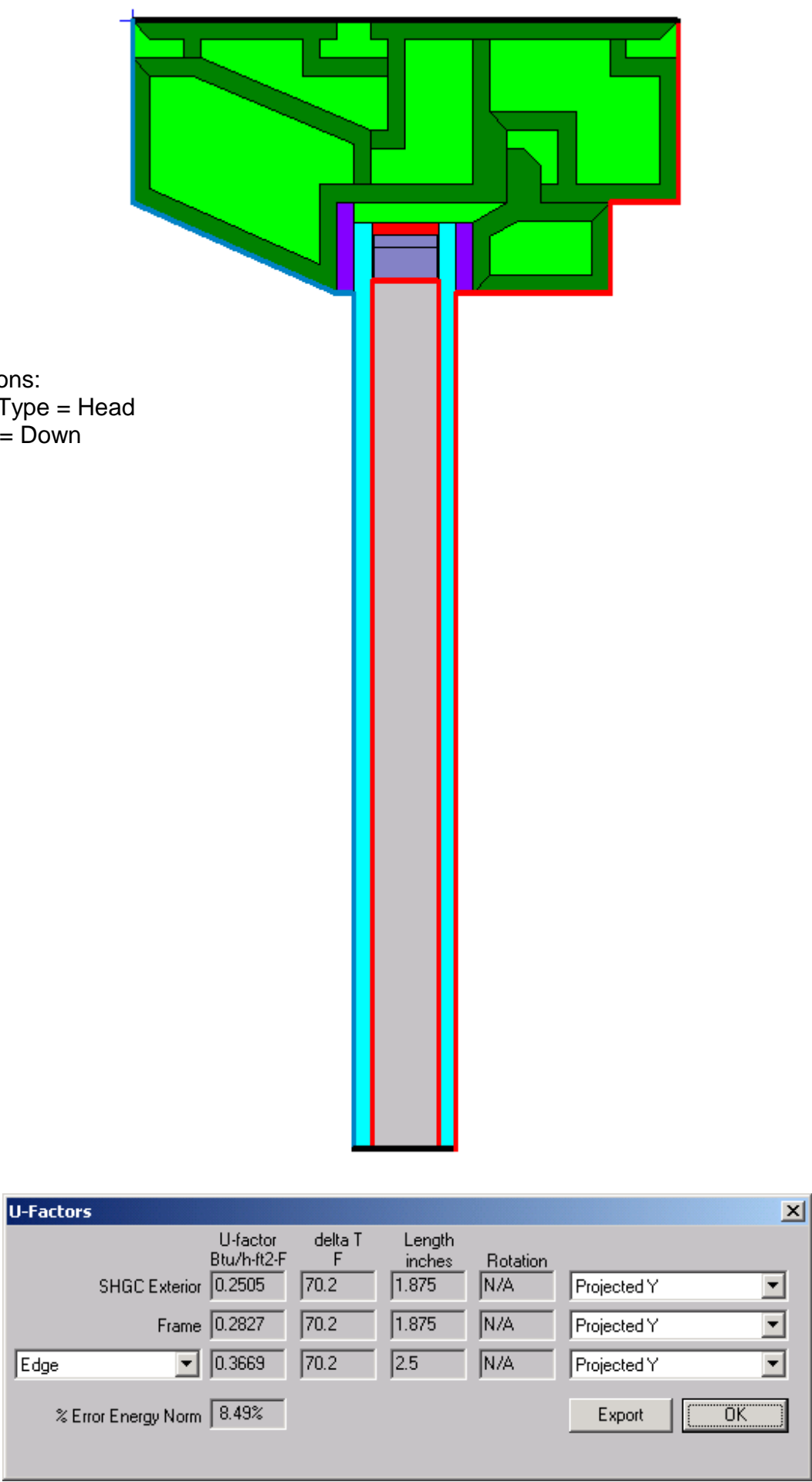

Figure 9-4. THERM cross section and $U$-factor for the vinyl fixed window head. 


\section{Divider}

- $\quad$ Cross Section Type $=$ Vertical Divider

- (model both vertical and horizontal dividers as Vertical)

- Do not use the CR model with a Vertical Divider cross section.

- Gravity Vector = Into the Screen

- It is not necessary to apply the SHGC Exterior U-factor tag in this case because the interior projected frame dimension and the exterior wetted length are the same (but it should be added for consistency).

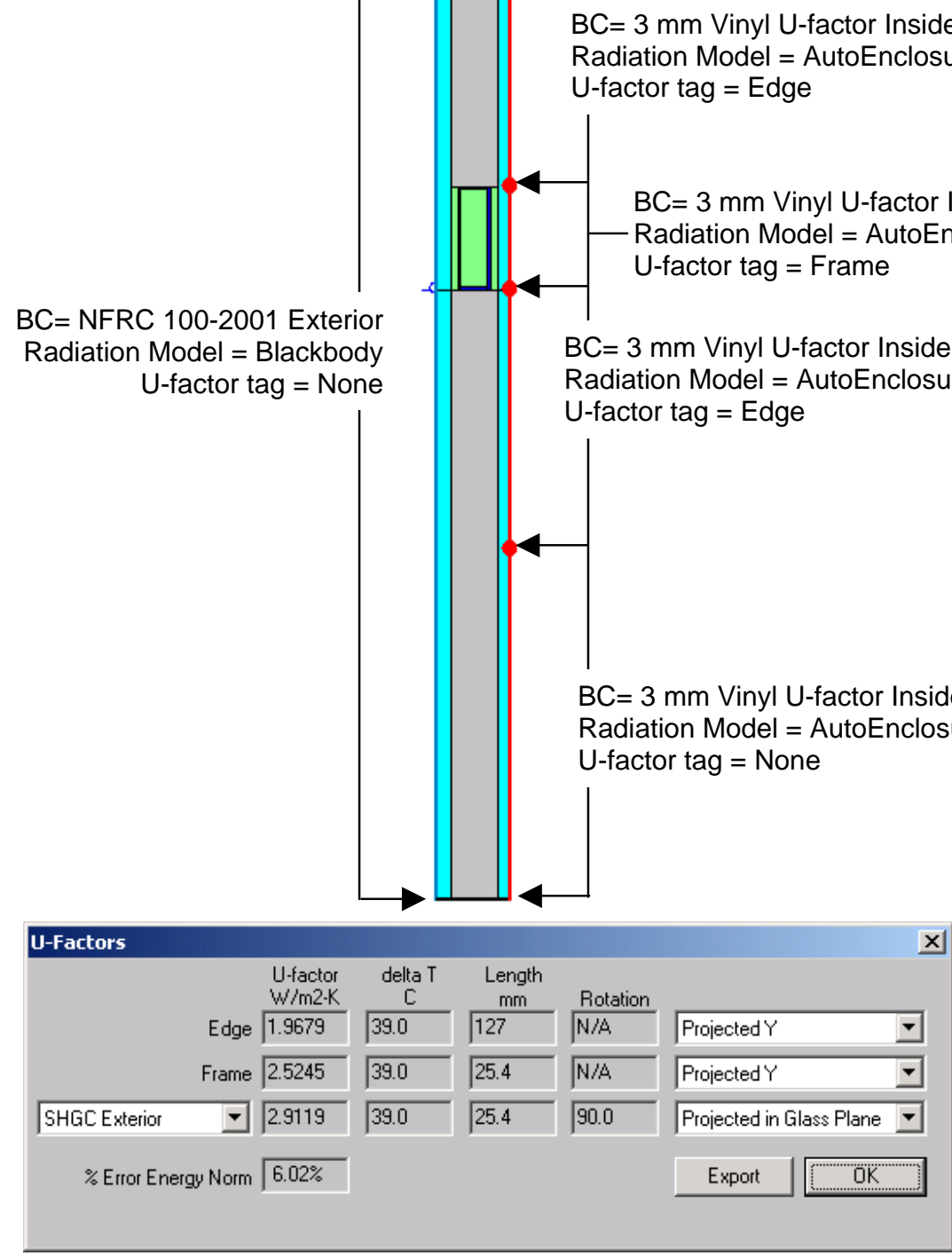

Figure 9-5. THERM cross section and U-factor for the vinyl fixed window divider. 


\subsubsection{Total Product U-Factor}

In WINDOW, import the THERM cross sections into the Frame Library.

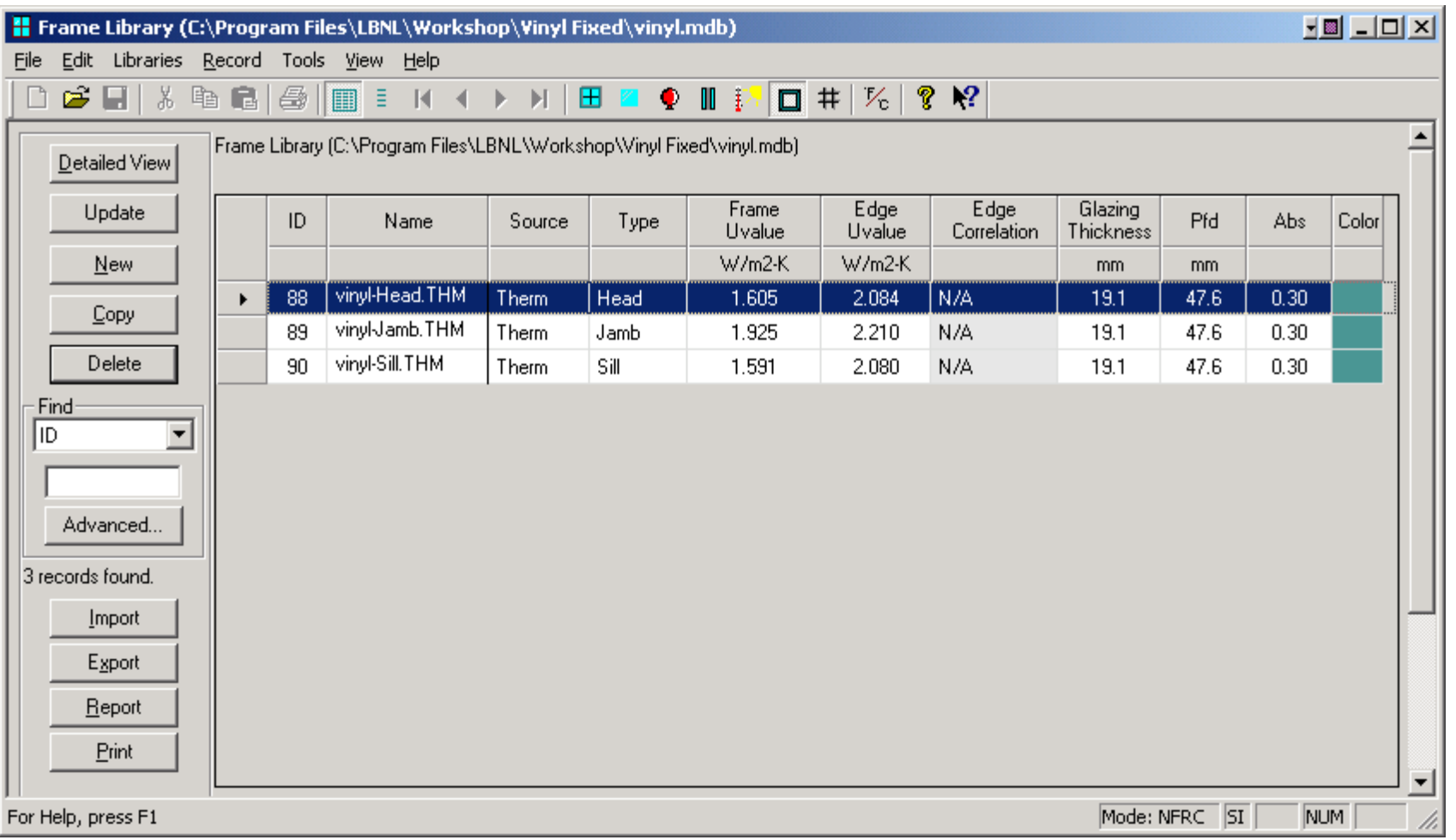

Figure 9-6. THERM files imported into the Frame Library.

In the WINDOW Window Library, two records are created for the U-factor calculation, one without dividers and one with the manufacturer supplied dividers, as shown in the figure below.

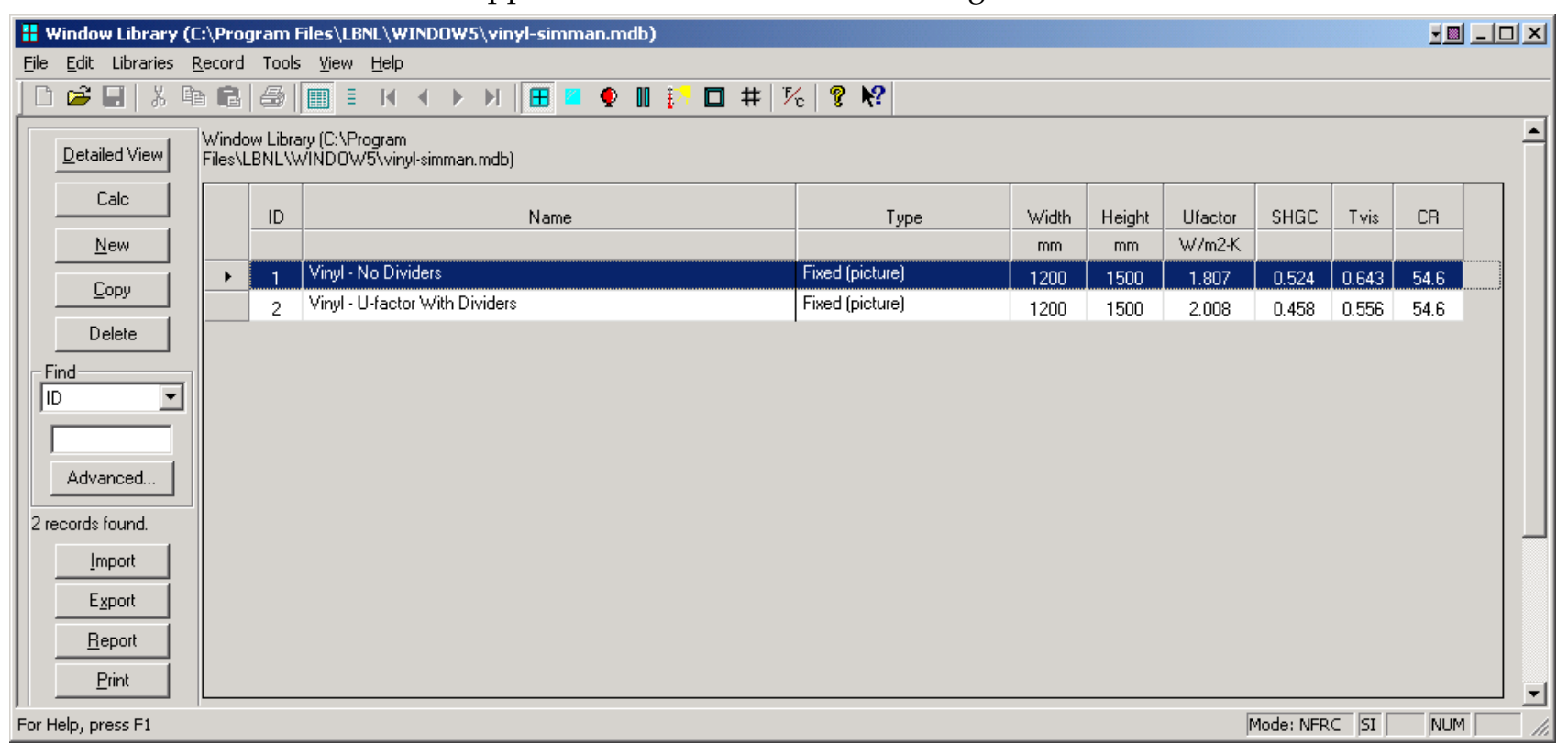

Figure 9-7. Window Library records for the product with and without dividers for the U-factor calculation. 


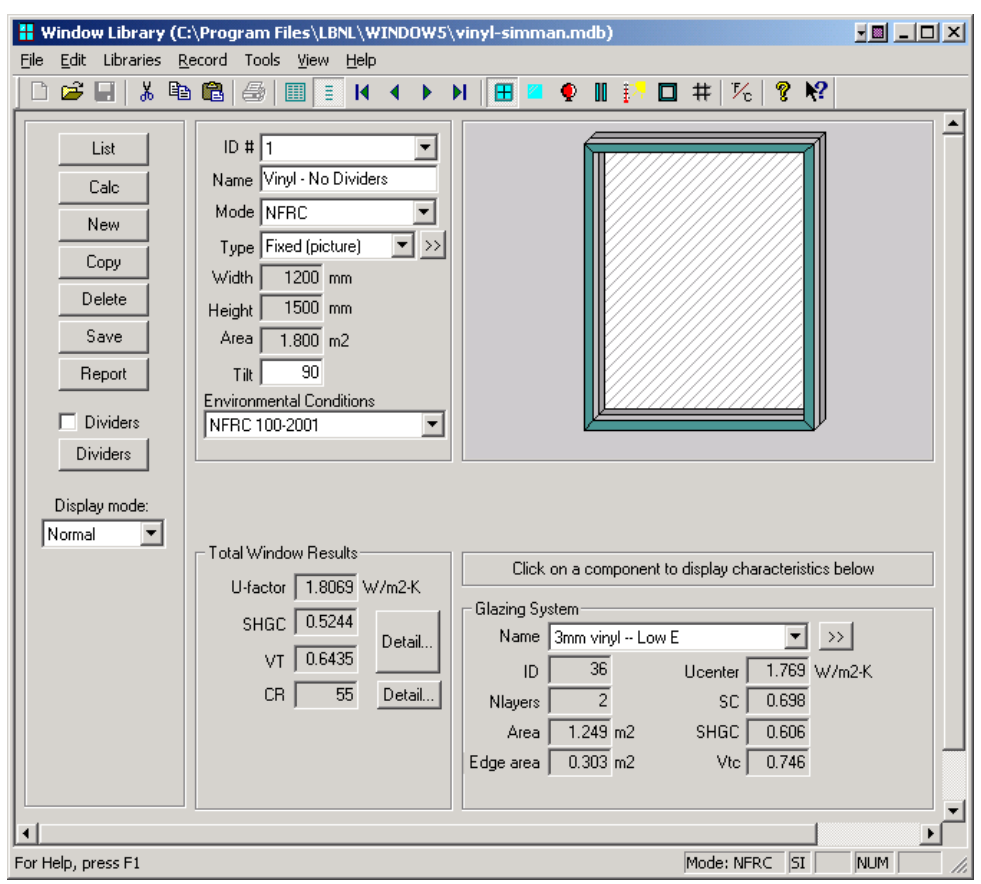

Figure 9-8. WINDOW total product $U$-factor calculation without dividers

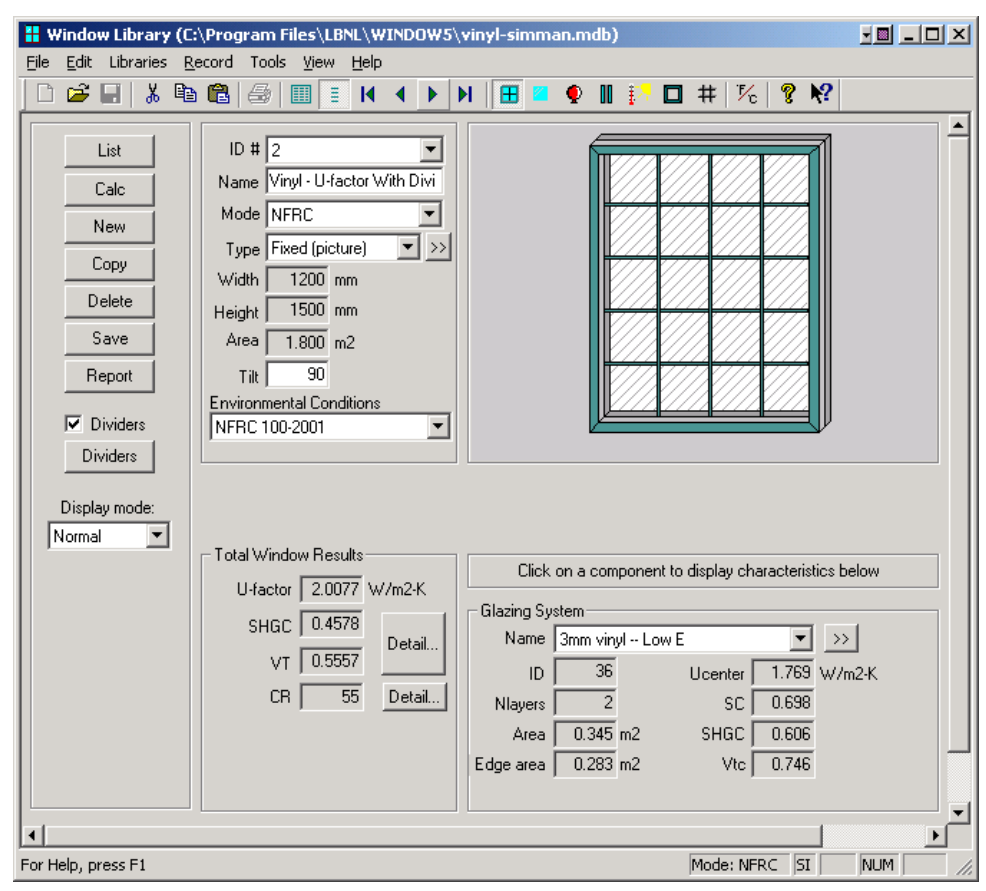

Figure 9-9. WINDOW total product U-factor calculation with dividers

The following table shows the overall product U-factor, from WINDOW, with and without dividers.

Table 9-4. Total Product U-factors

\begin{tabular}{|c|c|c|c|}
\hline \multirow{2}{*}{\multicolumn{2}{|c|}{$\begin{array}{c}\text { Glazing Options } \\
\text { (19.0 mm overall thickness) }\end{array}$}} & \multicolumn{2}{|c|}{ Total Product U-factor } \\
\hline & & $\left(\mathrm{W} / \mathrm{m}^{2}-{ }^{\circ} \mathrm{C}\right)$ & (Btu/hr-ft $\left.{ }^{2}-{ }^{\circ} \mathrm{F}\right)$ \\
\hline 1 & Clear, Air, Low-e, Without dividers & 1.8069 & 0.3182 \\
\hline 2 & Clear, Air, Low-e, With dividers & 2.0077 & 0.3536 \\
\hline
\end{tabular}




\subsubsection{Individual Product SHGC and VT using SHGC 0 \& 1 and VT 0 \& 1}

The methodology for determining the Solar Heat Gain Coefficient (SHGC) and Visible Transmittance (VT) for products is outlined in NFRC 200 using values of $\mathrm{SHGC}_{0}, \mathrm{SHGC}_{1}, \mathrm{VT}_{0}, \mathrm{VT}_{1}$. These values are calculated in WINDOW for the best glazing option modeled with the highest frame and edge U-factor frame, as outlined in NFRC 200, Section 6.1.1 (a). The values calculated from that one case are then used to calculate the SHGC for any other glazing options using Equations 3 and 4 in NFRC 200 -- do not use the $\mathrm{SHGC}_{0}, \mathrm{SHGC}_{1}, \mathrm{VT}_{0}, \mathrm{VT}_{1}$ from WINDOW5 for every glazing option, just for the best glazing option.

WINDOW5 automatically calculates the the $\mathrm{SHGC}_{0}, \mathrm{SHGC}_{1}, \mathrm{VT}_{0}, \mathrm{VT}_{1}$ values for any product in the Window Library, for the three cases outlined in NFRC 200:

- No Dividers

- Dividers $<25.4 \mathrm{~mm}$

- $\quad$ Dividers $\geq 25.4 \mathrm{~mm}$

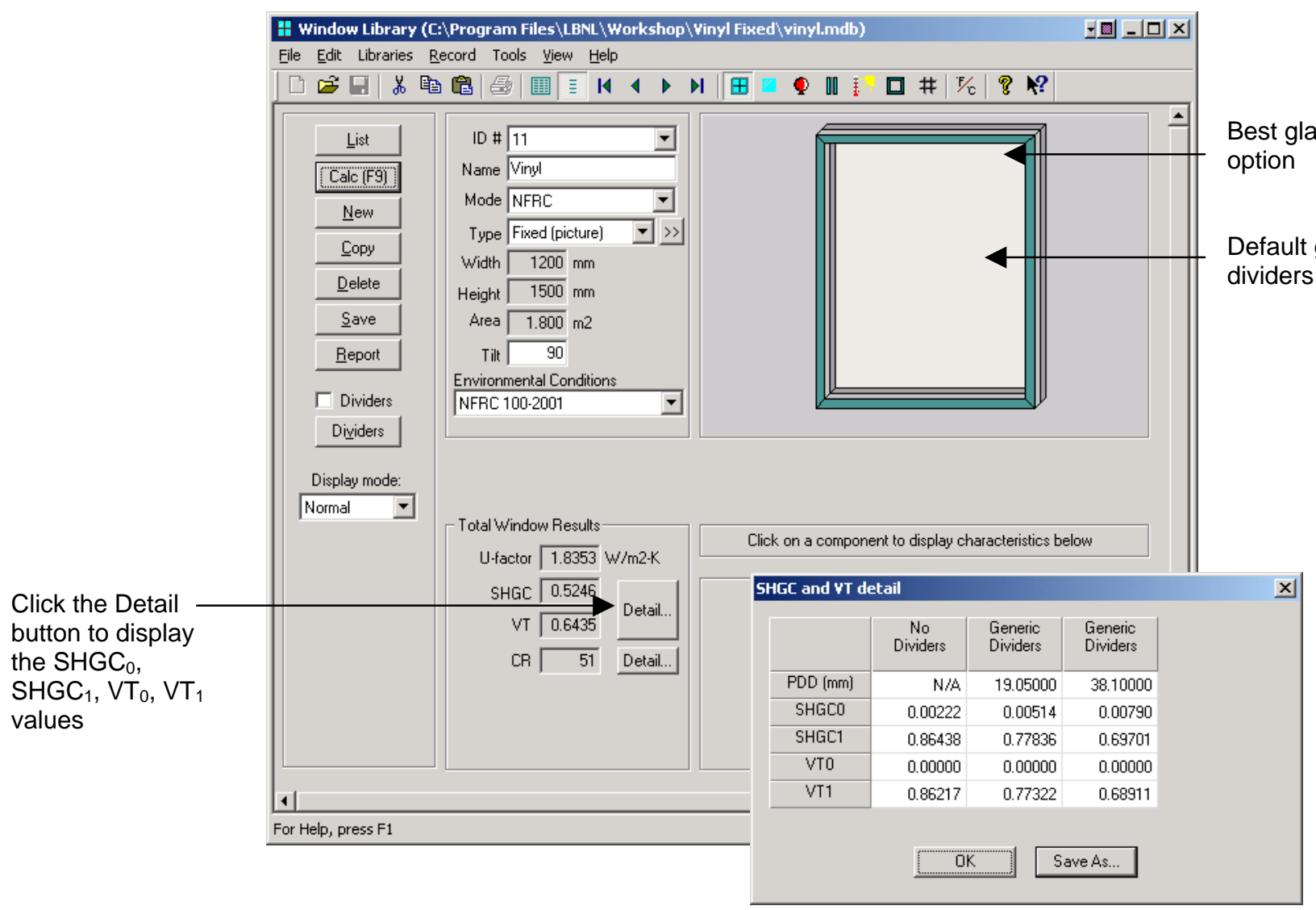

Table 9-5. SHGC $C_{0} S H G C_{1} V T_{0}, V T_{1}$ for the three cases with the best glazing option for this product line, Clear, Air, Low-E.

\begin{tabular}{|c|l|l|l|}
\hline & No Dividers & $\begin{array}{c}\text { Dividers } \\
\mathbf{2} \mathbf{2 5 . 4} \mathbf{~ m m} \\
\mathbf{( 1 . 0} \text { inches })\end{array}$ & $\begin{array}{c}\text { Dividers } \\
\mathbf{2} \mathbf{2 5 . 4} \mathbf{~ m m} \\
\mathbf{( 1 . 5} \text { inches })\end{array}$ \\
\hline $\mathbf{S H G C}_{\mathbf{0}}$ & 0.00222 & 0.00514 & 0.00790 \\
\hline $\mathbf{S H G C}_{\mathbf{1}}$ & 0.86438 & 0.77836 & 0.69701 \\
\hline $\mathbf{V T}_{\mathbf{0}}$ & 0.000 & 0.000 & 0.000 \\
\hline $\mathbf{V T}_{\mathbf{1}}$ & 0.86217 & 0.77320 & 0.68911 \\
\hline
\end{tabular}


Figure 9-10. Window Library record for $S H G C_{0}, S H G C_{1}, V T_{0}, V T_{1}$ calculation.

\subsubsection{Drawings Vinyl Fixed Window}

The following pages contain detailed drawings for this window.

\section{Head, Sill and Jamb}
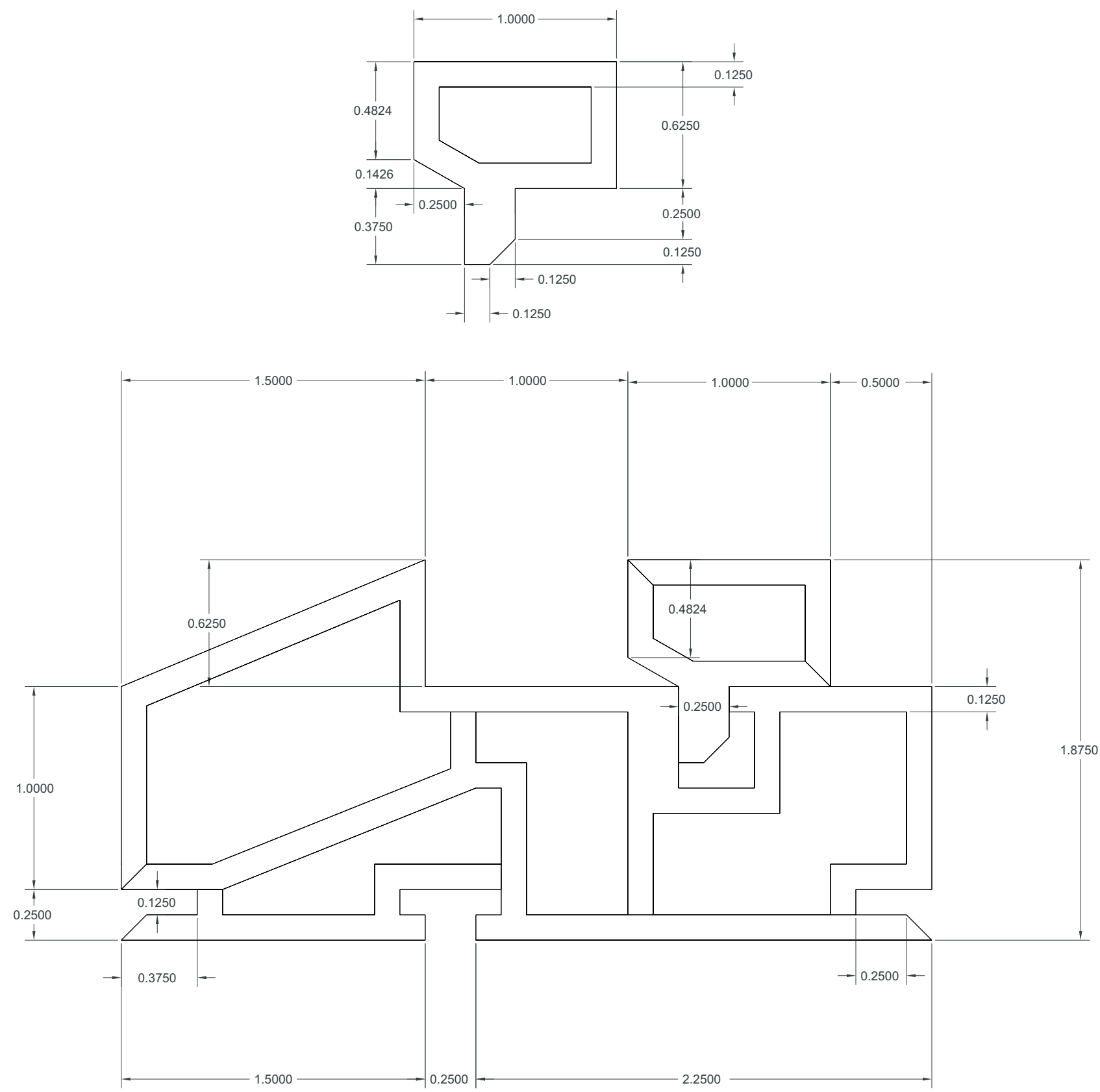

Figure 9-11. Dimensioned drawing for vinyl fixed window. 


\section{Spacer}

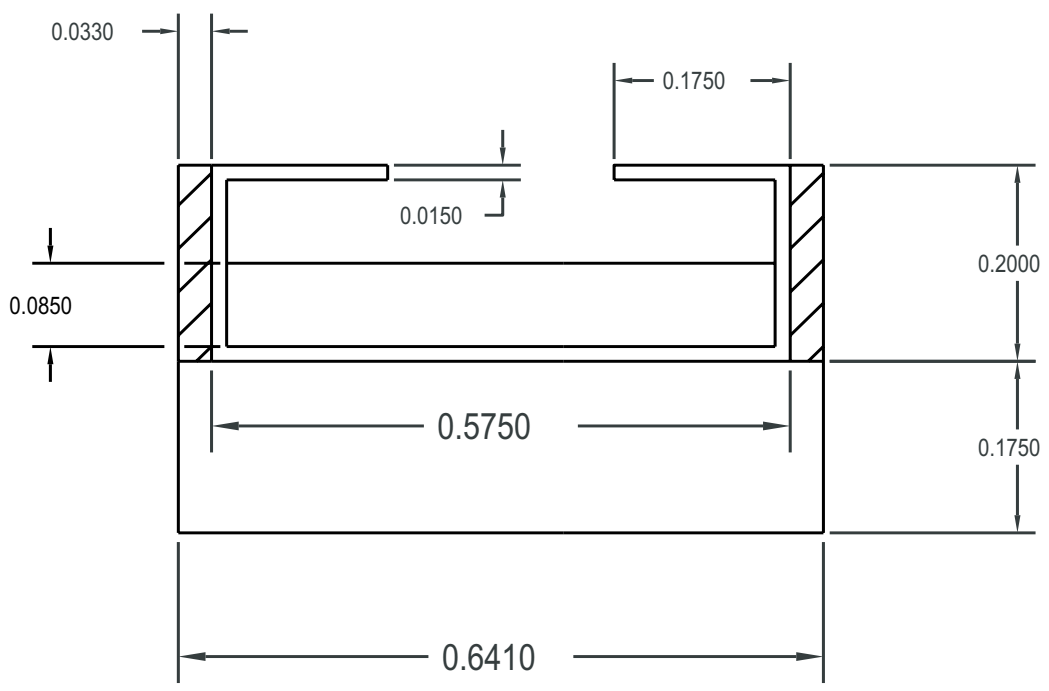

Figure 9-12. Dimensioned drawing for spacer.

\section{Divider}

\section{0-A}

Page 21

\section{Divider type A}

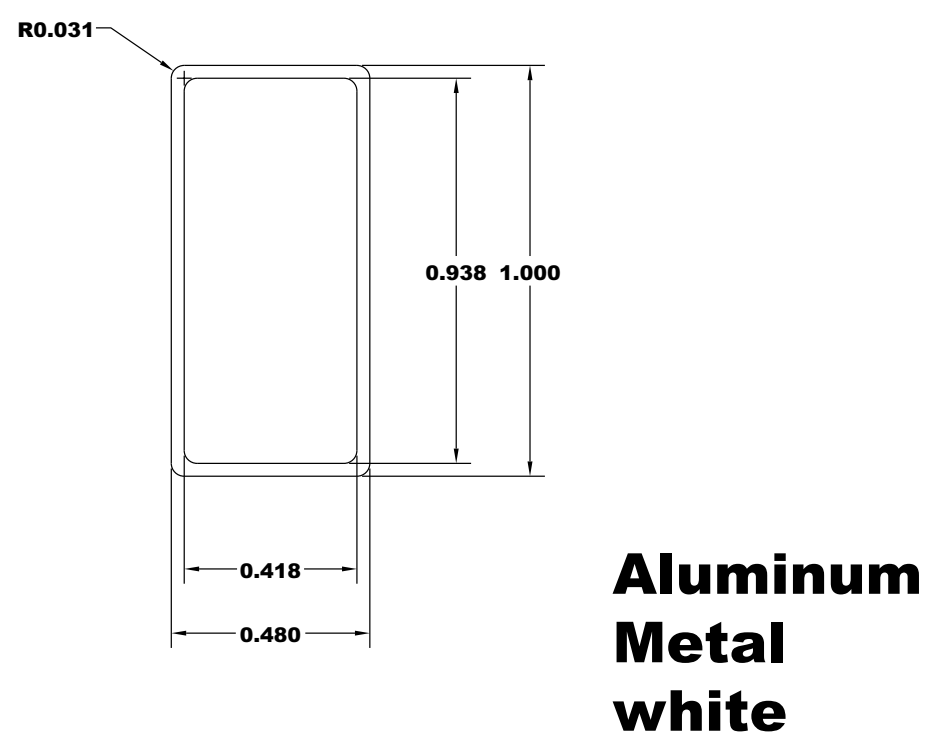

Figure 9-13. Dimensioned drawing for divider. 


\subsection{Problem 2: Aluminum Horizontal Slider Window}

For this example problem, there are 4 glazing options. Calculate the total product U-factor, SHGC, VT and the specialty products table.

\subsubsection{Description}

Window Type Horizontal Slider

Frame Material Aluminum painted white. Thermal breaks as indicated in the drawing assembly. The manufacturer indicated that the de-bridge width is 0.250 " for all the cross sections. Thermal break material is poured in place polyurethane.

Overall Size $\quad$ Width $=1500 \mathrm{~mm}$; Height $=600 \mathrm{~mm}$

Spacer type See drawings in Section 9.3.7.

Weather Strip See drawings in Section 9.3.7.

Cross Sections Section 9.3.7 contains the drawings for this example.

Dividers $\quad$ Aluminum painted white. See drawing for dimensions.

Manufacturer provides standard 12" on center or less horizontal and vertical grid pattern for his products.

Based on the drawings and the glazing cavity thickness, the dividers do not need to be modeled because the gap between the divider and the glass is greater than $3.0 \mathrm{~mm}$ $\left(0.118^{\prime \prime}\right)$.

Glazing system The manufacturer uses the following glass type options depending on the market availability and price factor. The manufacturer uses clear and Low-e coated glass from the same manufacturer.

All clear glass having nominal thickness of $3 \mathrm{~mm}, 4 \mathrm{~mm}, 5 \mathrm{~mm}$, and $6 \mathrm{~mm}$ from PPG or CIG.

Low-e coated clear glass having emissivity range from $0.100-0.096$. Thicknesses are nominal $3 \mathrm{~mm}, 4 \mathrm{~mm}, 5 \mathrm{~mm}$ and $6 \mathrm{~mm}$ from PPG (SUNGATE 100, clear glass) or CIG (LoE SUN45 coating, clear glass)

\subsubsection{Glazing Matrix}

The following table shows the glazing matrix that is to be simulated for this window. However, for this example, only glazing option 1 (the $3 \mathbf{~ m m}$ case) will be modeled using CIG glass.

Table 9-6. Matrix of Glazing Options for Problem 2

\begin{tabular}{|c|c|l|l|}
\hline & \multicolumn{1}{|c|}{$\begin{array}{c}\text { Glazing Options (1.0" overall } \\
\text { thickness) }\end{array}$} & \multicolumn{1}{|c|}{ Grid Option } & $\begin{array}{c}\text { Manufacture } \\
\mathrm{r}\end{array}$ \\
\hline$* 1$ & Clear (3 mm), Argon (95\%), Low-e $(3 \mathrm{~mm})$ & $\begin{array}{l}\text { Grids do not need to be } \\
\text { modeled (see note } \\
\text { above in divider } \\
\text { description) }\end{array}$ & CIG, PPG \\
\hline 2 & Clear (3 mm), Argon (95\%), Clear $(3 \mathrm{~mm})$ & $\begin{array}{l}\text { Grids do not need to be } \\
\text { modeled (see note } \\
\text { above in divider } \\
\text { description) }\end{array}$ & CIG, PPG \\
\hline
\end{tabular}




\subsubsection{Center-of-glazing Modeling (WINDOW)}

In WINDOW, create the glazing systems needed for the Glazing Matrix in Section 9.3.2. An example of one of the four glazing systems is shown in the figure below.

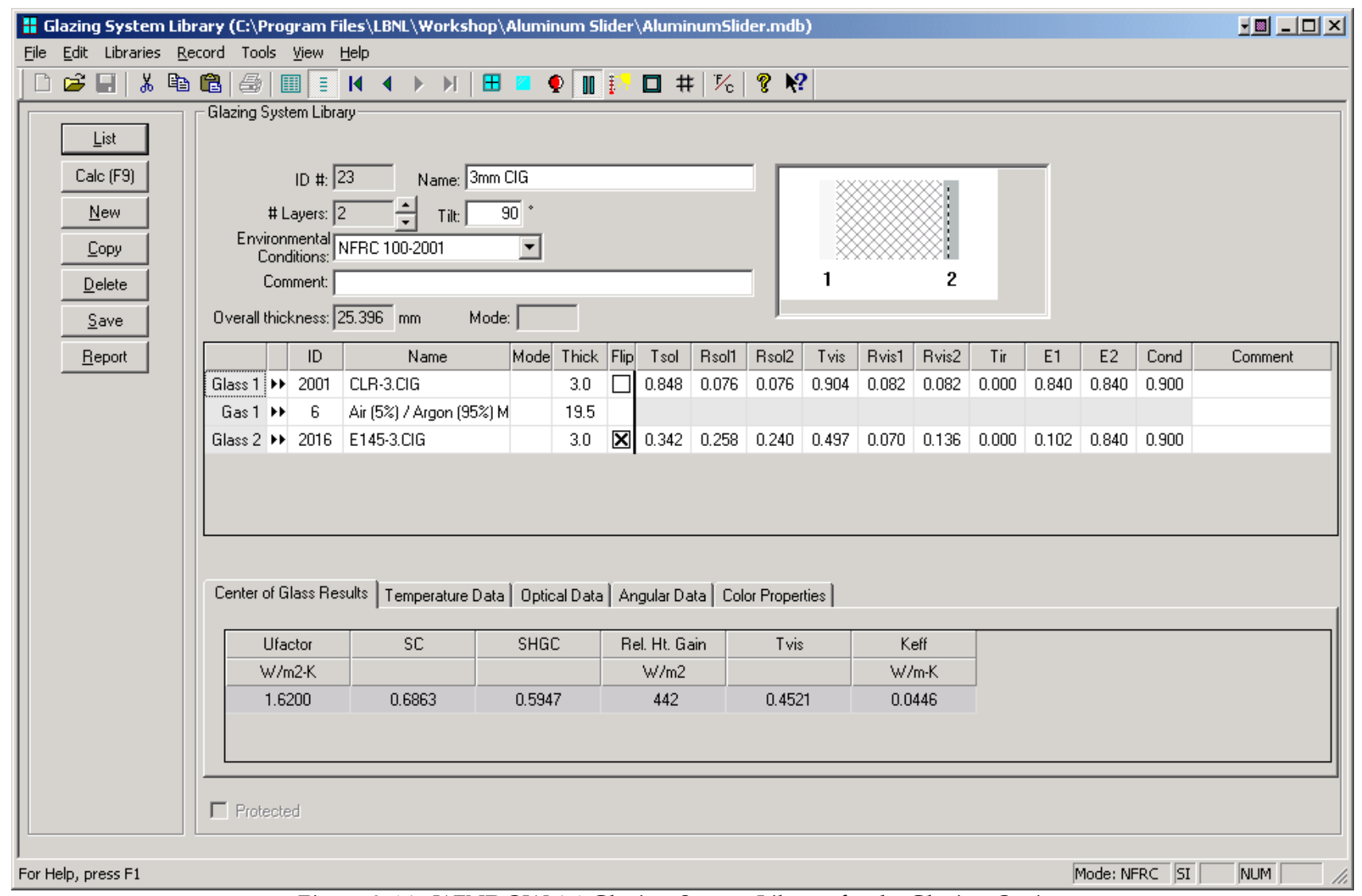

Figure 9-14. WINDOW 4.1 Glazing System Library for the Glazing Options

The results for the center-of-glazing U-factor are shown in the following table:

Table 9-7. Center-of-glazing U-factor Results for the Matrix of Glazing Options

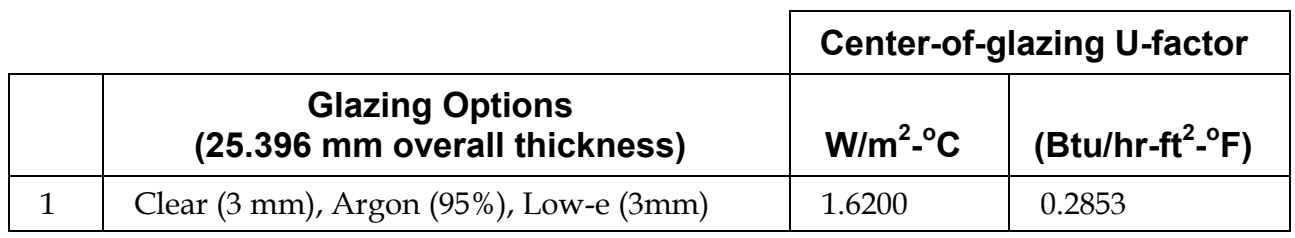

These glazing systems will be used in THERM to calculate the frame and edge-of-glazing U-factors, and also in WINDOW to calculate the overall product U-factor. 


\subsubsection{Edge-of-glazing and Frame Modeling (THERM)}

There are seven cross-sections that must be modeled for this product, listed in Table 9-10. The THERM files listed in this table can be found on the CD.

Table 9-8. Cross sections to be modeled with their associated $d x$ and THERM files

\begin{tabular}{|l|l|l|}
\hline Cross Section & \multicolumn{1}{|c|}{ DXF Filename } & THERM file for $\mathbf{3} \mathbf{~ m m}$ \\
\hline Sill Vent & 530vtsl.dxf & sv_01.thm \\
\hline Sill Fixed & 530fxsl.dxf & sf_01.thm \\
\hline Jamb Vent & 530vtjb.dxf & jv_01.thm \\
\hline Jamb Fixed & 530fxjb.dxf & jf_01.thm \\
\hline Head Vent & 530vthd.dxf & hv_01.thm \\
\hline Head Fixed & 530fxhd.dxf & hf_01.thm \\
\hline Meeting Rail & 530mtrl.dxf & mr_01.thm \\
\hline Spacer & Cig30.dxf & Spacer-3mm.thm \\
\hline
\end{tabular}

In addition to the DXF files provided on $C D$, detailed drawings of these cross sections are in Section 9.3.7 of this manual.

Make a THERM file for each of the cross sections using the following steps:

- Use the DXF files as an underlay for each file. The files were not created in a manner that THERM can autoconvert, but the underlay can be used to trace the cross sections. (See Chapter 5, "Drawing CrossSection Geometry" in the THERM User's Manual).

- As the polygons for each cross section are being drawn, assign the correct material properties to them. (See Chapter 5, "Drawing Cross-Section Geometry" in the THERM User's Manual.)

- Insert the glazing system created in WINDOW 5. (See Section 5.9, "Inserting a Glazing System" in the THERM User's Manual.) Notice that the spacer is open to the glazing cavity, so use the technique discussed in Section 8.7.3, "Modifying Glazing Cavities (imported from WINDOW) for Open Spacers."

- Assign the boundary conditions to the entire cross section. (See Chapter 6, "Defining Boundary Conditions" in the THERM User's Manual.)

- Calculate the U-factor and CR temperatures for the cross section. (See Chapter 7, "Calculating Results" in the THERM User's Manual.) 
- Table 9-11 shows the resulting U-factors from each cross section for the $3 \mathrm{~mm}$ glazing case. Note the result for the Grid case, which will be imported into the WINDOW Divider Library.

Table 9-9. Frame and Edge THERM U-factor Results for Glazing Option 1: $3 \mathrm{~mm}$

\begin{tabular}{|c|c|c|c|c|}
\hline & \multicolumn{2}{|c|}{ Frame U-factor } & \multicolumn{2}{|c|}{ Edge U-factor } \\
\hline Cross Section & $\left(\mathrm{W} / \mathrm{m}^{2}-^{\circ} \mathrm{C}\right)$ & $\left(B t u / h r-\mathrm{ft}^{2}-{ }^{\circ} \mathrm{F}\right)$ & $\left(\mathrm{W} / \mathrm{m}^{2}-{ }^{\circ} \mathrm{C}\right)$ & $\left(B t u / h r-\mathrm{ft}^{2}-{ }^{\circ} \mathrm{F}\right)$ \\
\hline Sill Vent & 5.696 & 1.003 & 2.097 & 0.369 \\
\hline Sill Fixed & 8.411 & 1.481 & 2.011 & 0.354 \\
\hline Jamb Vent & 4.916 & 0.866 & 2.071 & 0.365 \\
\hline Jamb Fixed & 7.546 & 1.329 & 2.036 & 0.359 \\
\hline Head Vent & 5.315 & 0.936 & 2.031 & 0.358 \\
\hline Head Fixed & 6.848 & 1.206 & 2.053 & 0.362 \\
\hline Meeting Rail & 4.862 & 0.856 & 2.110 & 0.372 \\
\hline
\end{tabular}

- Table 9-12 shows the resulting U-factors from each cross-section for the $6 \mathrm{~mm}$ glazing case.

The figures on the following pages show THERM files and U-factor results screens for each of the crosssections with the $3 \mathrm{~mm}$ glazing system. 


\section{Sill Vent Cross Section}

(sv_01.thm)
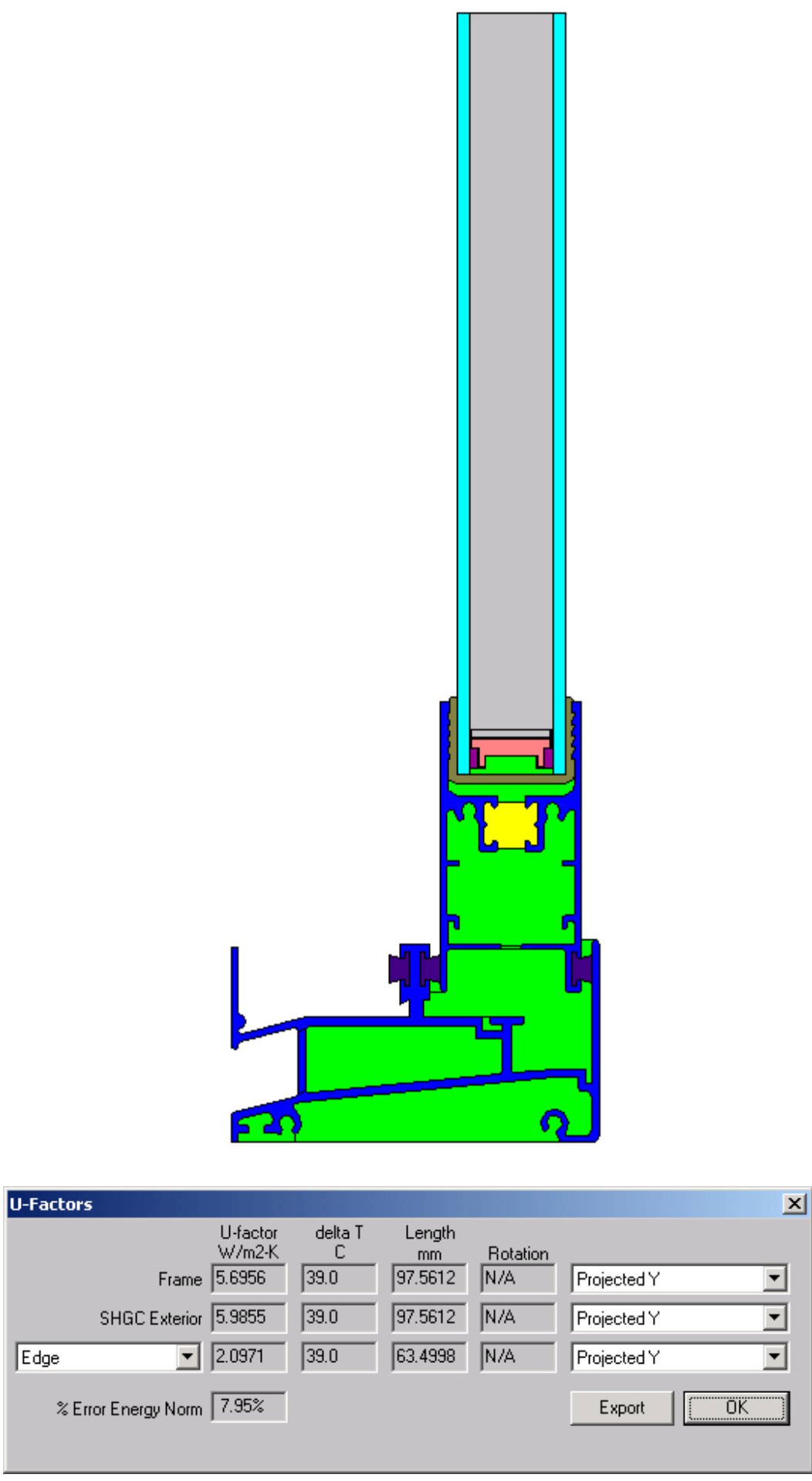

Figure 9-15. THERM file and U-factors for Sill Vent Cross Section. 


\section{Sill Fixed Cross Section}

(sf_01.thm)
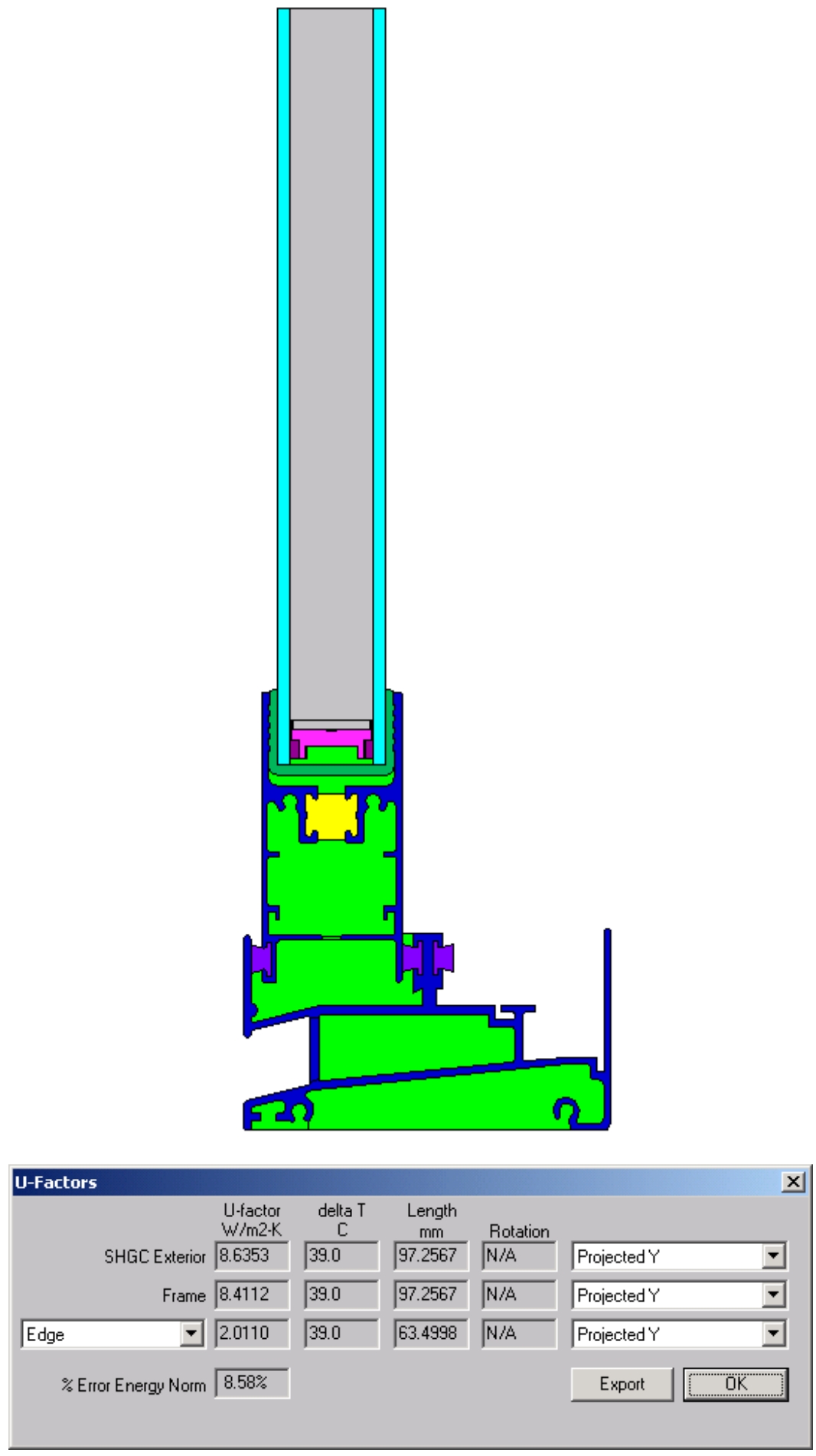

Figure 9-16. THERM file and U-factors for Sill Fixed Cross Section. 


\section{Jamb Vent Cross Section}

(jv_01.thm)
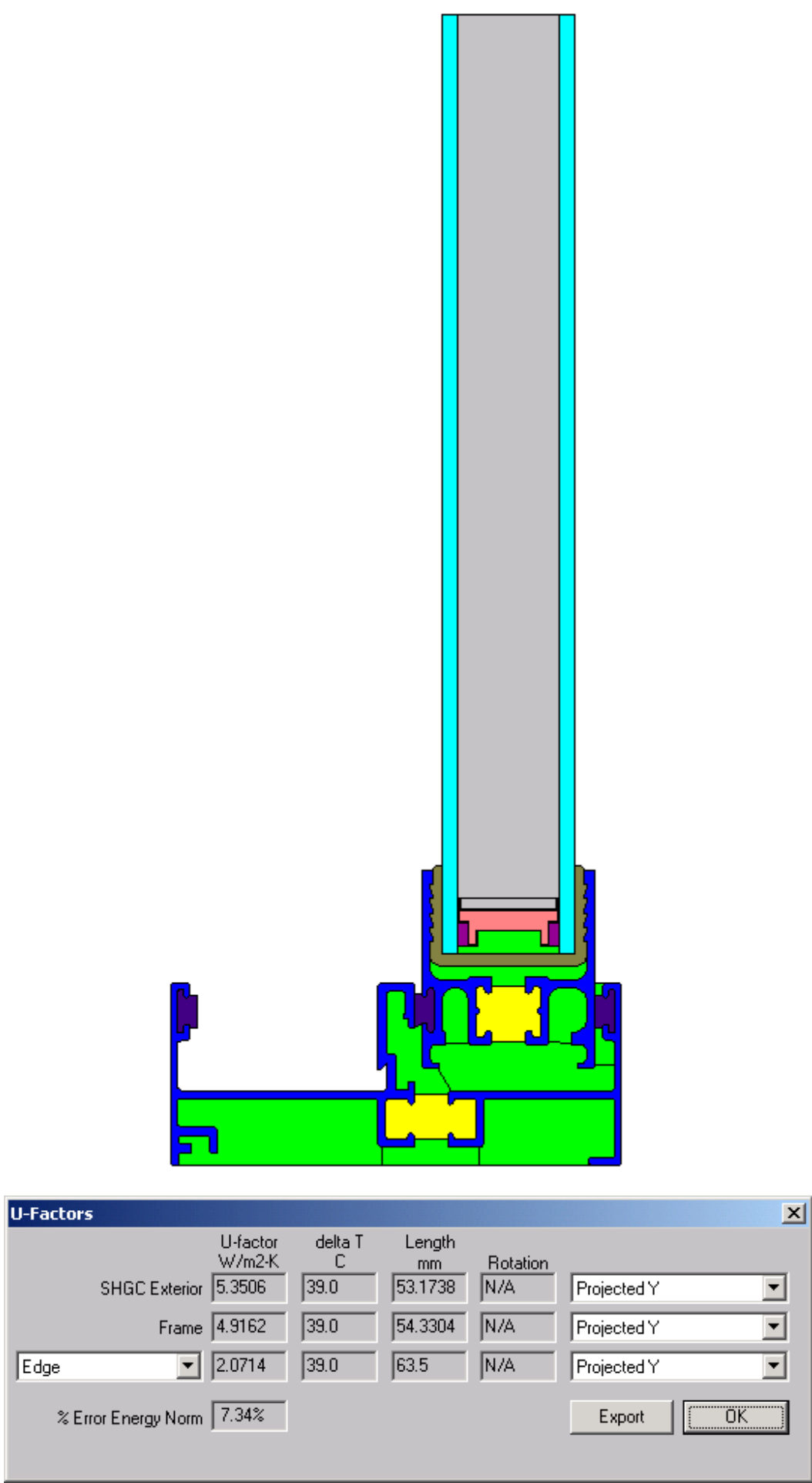

Figure 9-17. THERM file and U-factors for Jamb Vent Cross Section. 
Jamb Fixed Cross Section

(jf_01.thm)
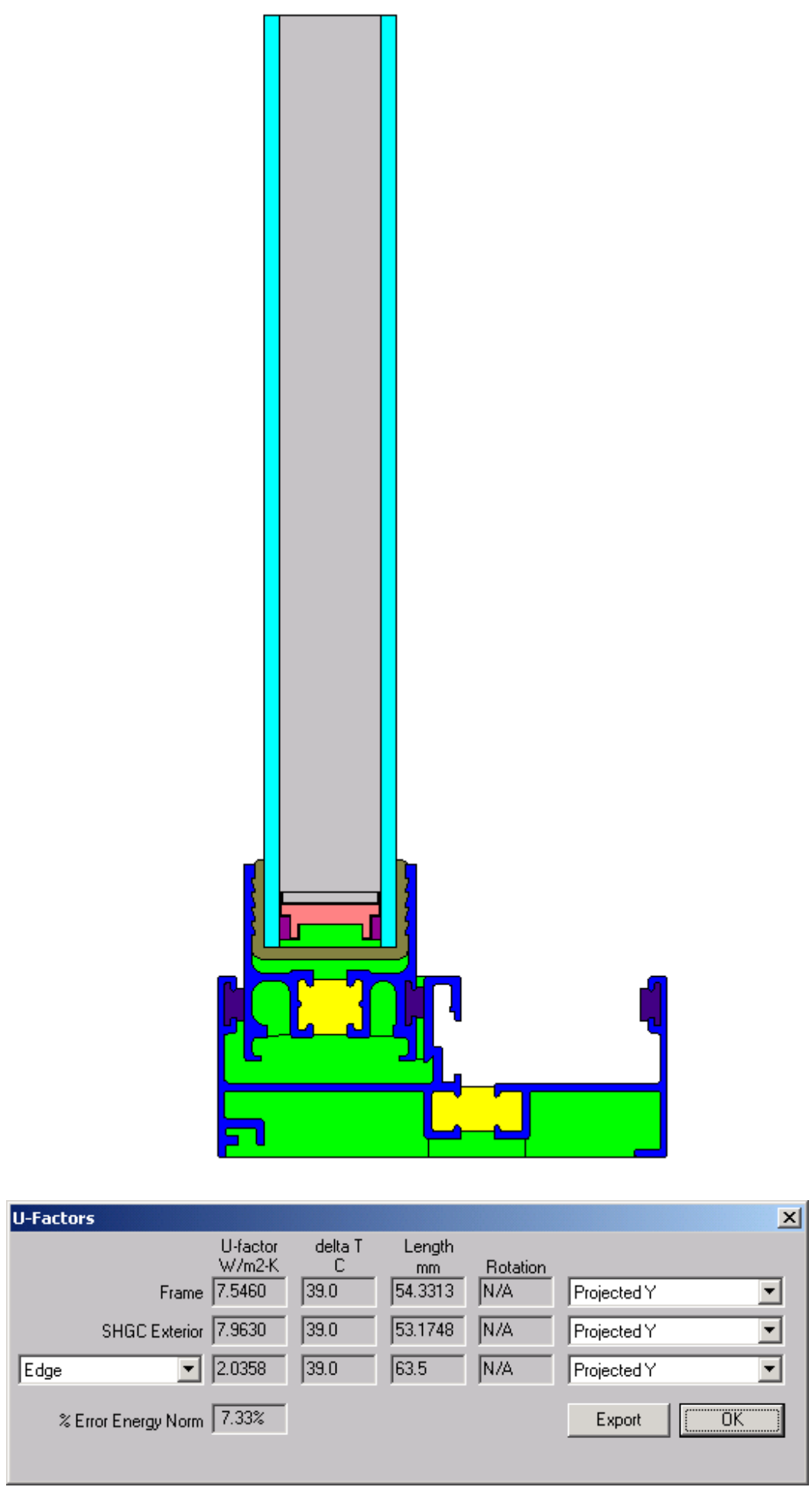

Figure 9-18. THERM file and U-factors for Jamb Fixed Cross Section. 
Head Vent Cross Section

(hv_01. thm)
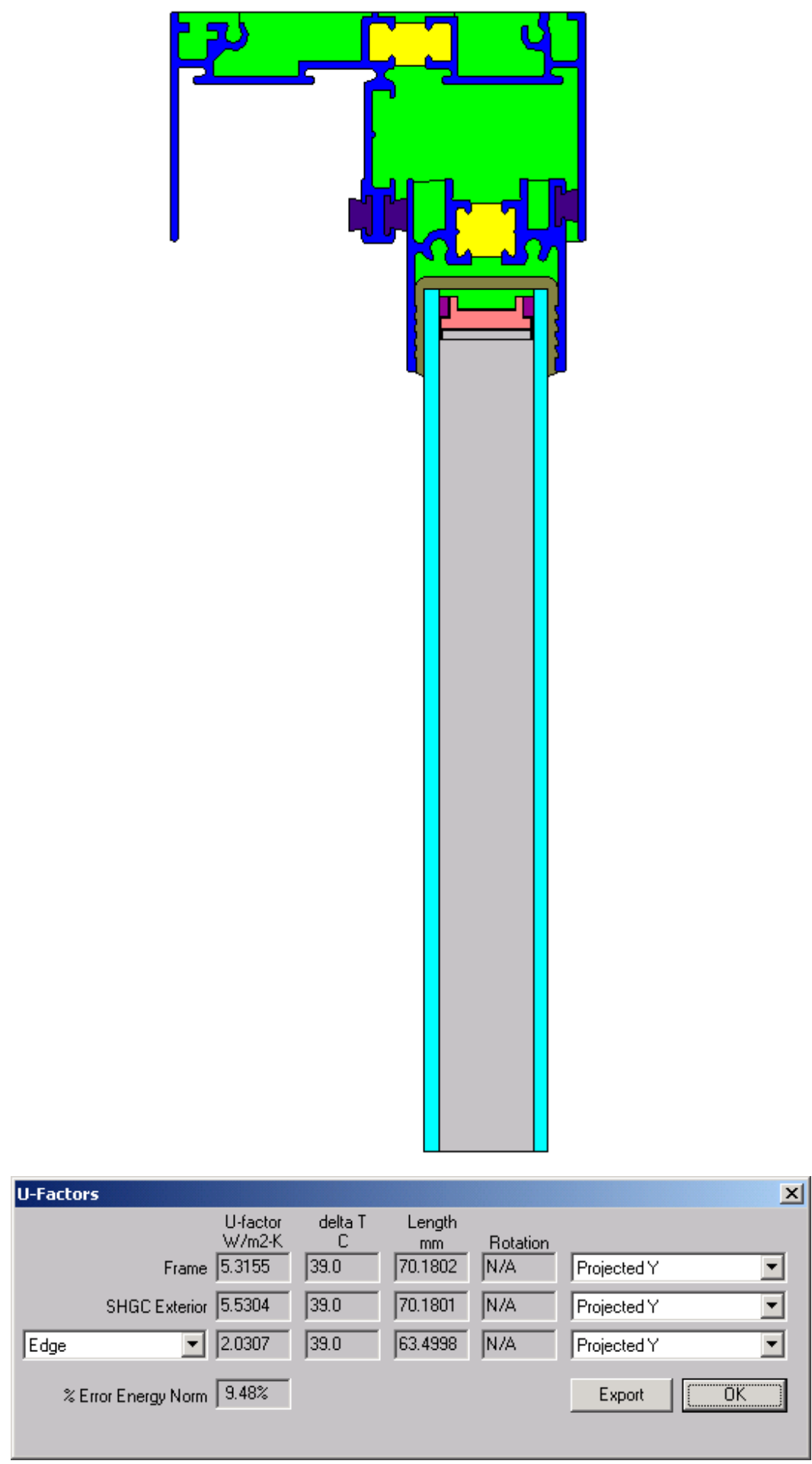

Figure 9-19. THERM file and U-factors for Head Vent Cross Section. 


\section{Head Fixed Cross Section}

(hf_01.thm)
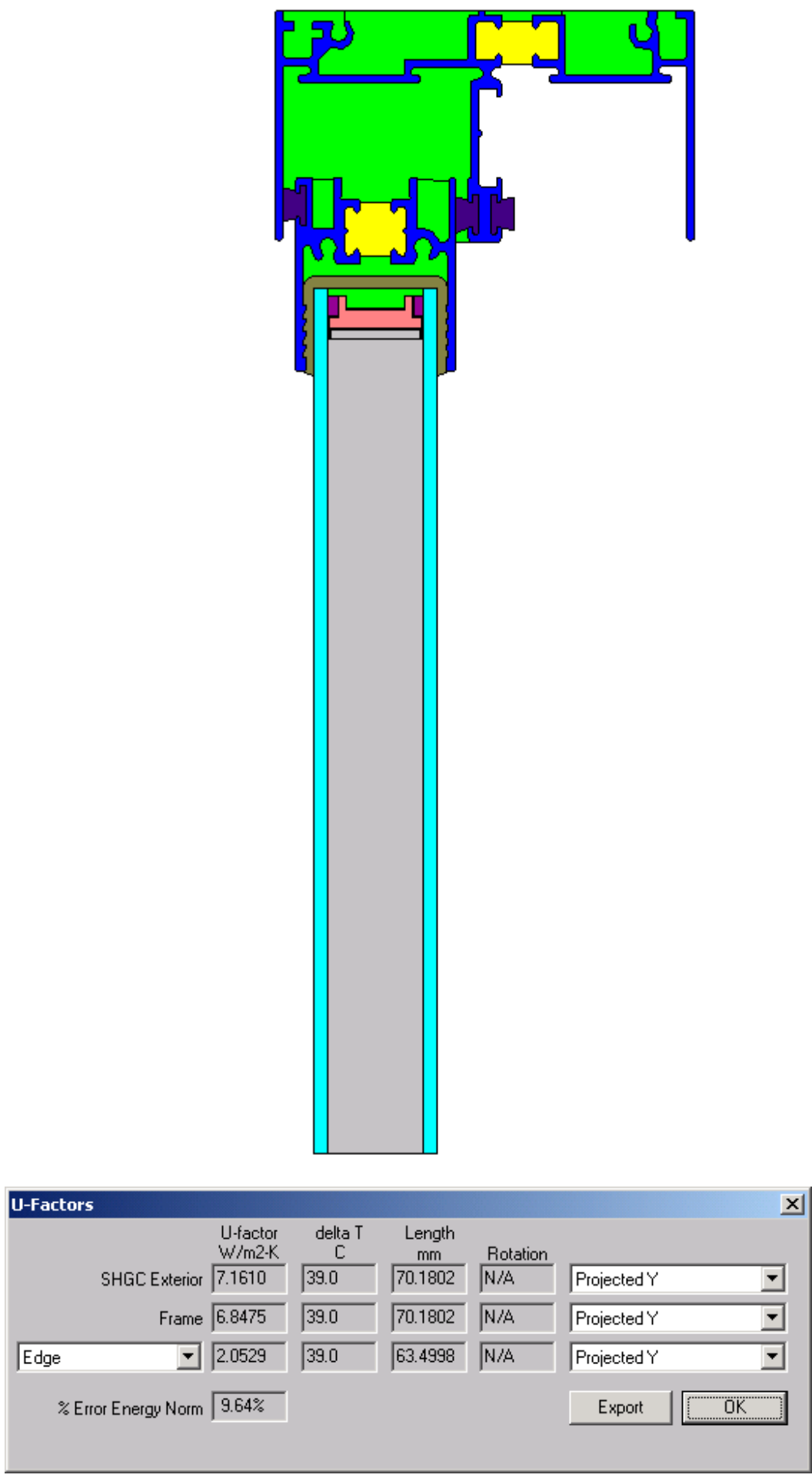

Figure 9-20. THERM file and U-factors for Head Fixed Cross Section. 


\section{Meeting Rail Cross Section}

(mr_01.thm)
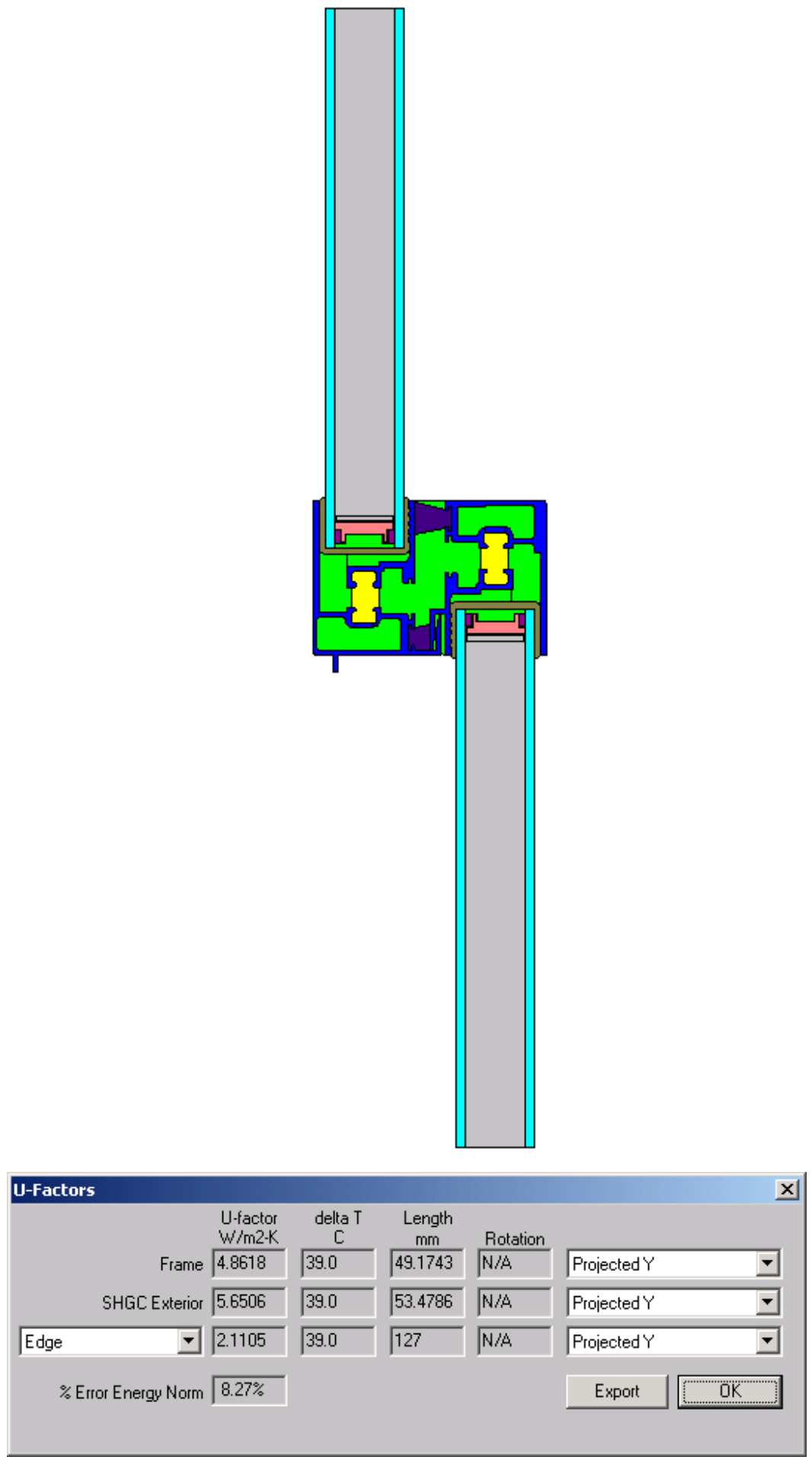

Figure 9-21. THERM file and U-factors for Meeting Rail Cross Section. 


\subsubsection{Total Product U-Factor}

In WINDOW, import the THERM cross sections into the Frame Library.

\begin{tabular}{|c|c|c|c|c|c|c|c|c|c|c|c|c|c|}
\hline \multicolumn{11}{|c|}{ 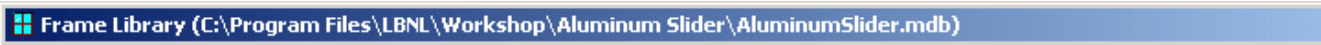 } & \multicolumn{3}{|c|}{ 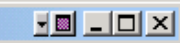 } \\
\hline \multicolumn{14}{|c|}{ File Edit Libraries Record Tools Yiew Help } \\
\hline \multicolumn{14}{|c|}{ 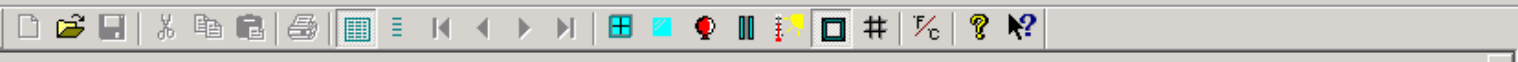 } \\
\hline & 슨 \\
\hline Update & & ID & Name & Source & Type & $\begin{array}{c}\text { Frame } \\
\text { Uvalue }\end{array}$ & $\begin{array}{l}\text { Edge } \\
\text { Uvalue }\end{array}$ & $\begin{array}{c}\text { Edge } \\
\text { Correlation }\end{array}$ & \begin{tabular}{|c|} 
Glazing \\
Thickness
\end{tabular} & Pfd & Abs & Color & \\
\hline New & & & & & & W/m2-K & $\mathrm{W} / \mathrm{m} 2 \cdot \mathrm{K}$ & & $\mathrm{mm}$ & $\mathrm{mm}$ & & & \\
\hline & • & 24 & HE_01.thm & Therm & Head & 6.848 & 2.053 & $\mathrm{~N} / \mathrm{A}$ & 25.4 & 70.2 & 0.30 & & \\
\hline Lopy & & 25 & hv_01.thm & Therm & Head & 5.315 & 2.031 & N/A & 25.4 & 70.2 & 0.30 & & \\
\hline Delete & & 26 & J__01.thm & Therm & Jamb & 7.546 & 2.036 & $\mathrm{~N} / \mathrm{A}$ & 25.4 & 54.3 & 0.30 & & \\
\hline Find & & 27 & Jv_01.thm & Therm & Jamb & 4.916 & 2.071 & $\mathrm{~N} / \mathrm{A}$ & 25.4 & 54.3 & 0.30 & & \\
\hline ID & & 28 & Mr_01.thm & Therm & Vertical Me & 4.862 & 2.110 & $\mathrm{~N} / \mathrm{A}$ & 25.4 & 49.2 & 0.30 & & \\
\hline & & 29 & Sf_01.thm & Therm & Sill & 8.411 & 2.011 & $\mathrm{~N} / \mathrm{A}$ & 25.4 & 97.3 & 0.30 & & \\
\hline the & & 30 & Sv_01.thm & Therm & Sill & 5.696 & 2.097 & $\mathrm{~N} / \mathrm{A}$ & 25.4 & 97.6 & 0.30 & & \\
\hline \multicolumn{14}{|l|}{7 records found. } \\
\hline \multicolumn{14}{|l|}{ Import } \\
\hline \multicolumn{14}{|l|}{ Export } \\
\hline \multicolumn{14}{|l|}{ Report } \\
\hline \multicolumn{14}{|l|}{ Print } \\
\hline For Help, press F1 & & & & & & & & & Mode: $\mathrm{N}$ & $\mathrm{RC}$ SI & NUM & & \\
\hline
\end{tabular}

Figure 9-22. THERM files imported into the Frame Library.

In the WINDOW Window Library, create a record using the THERM files imported into the Frame Library, and the glazing system created for the product, as shown in the figure below.

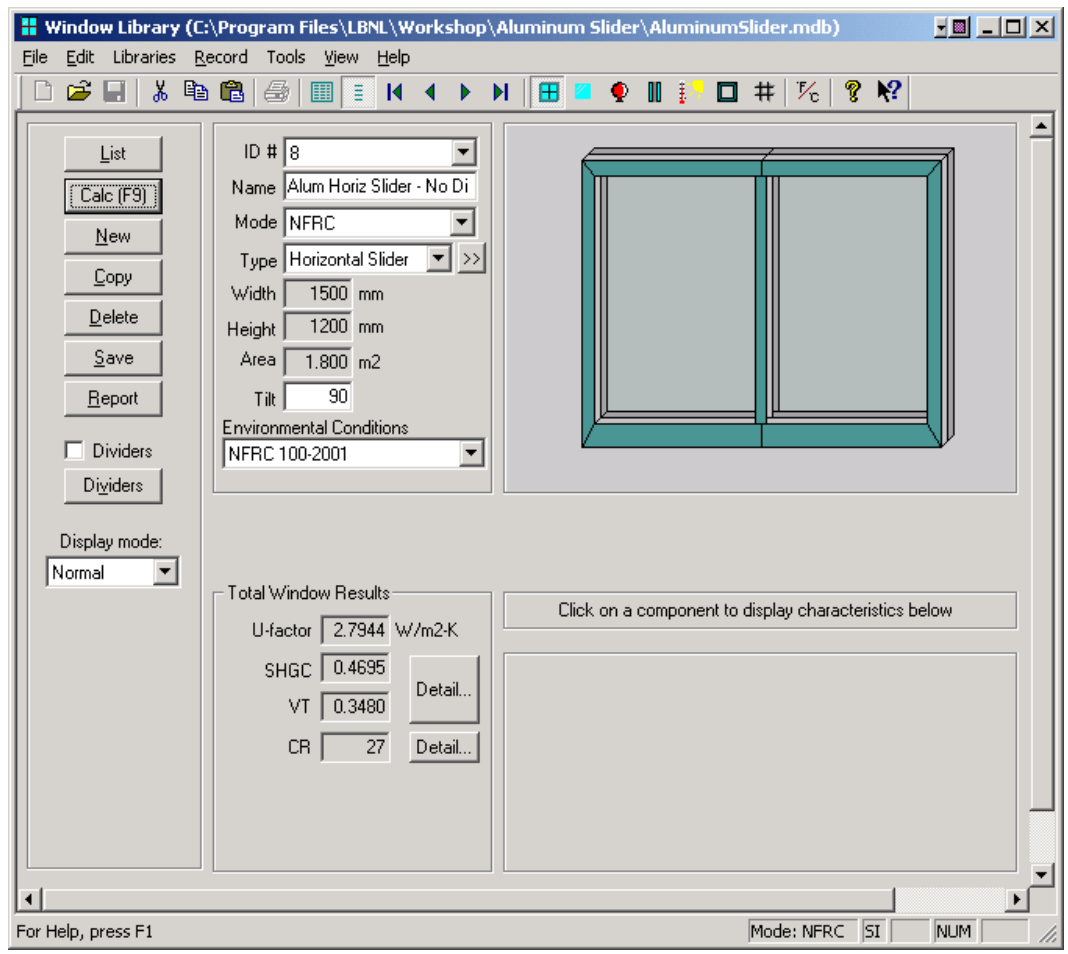

Figure 9-23. WINDOW total product U-factor calculation. The following table shows the overall product U-factor, from WINDOW, with and without dividers. 
Table 9-10. Total Product U-factors

\begin{tabular}{|c|l|c|c|}
\hline \multicolumn{2}{|c|}{$\begin{array}{c}\text { Glazing Options } \\
\text { (19.0 } \mathbf{~ m m} \text { overall thickness) }\end{array}$} & \multicolumn{2}{c|}{ Total Product U-factor } \\
\cline { 3 - 4 } & $\left(\mathbf{W} / \mathbf{m}^{\mathbf{2}}{ }^{-}{ }^{\circ} \mathbf{C}\right)$ & $\left(\right.$ Btu/hr-ft $\left.{ }^{2}{ }^{\circ} \mathbf{F}\right)$ \\
\hline 1 & Clear, Argon, Low-e, Without dividers & 2.7944 & 0.4921 \\
\hline
\end{tabular}

\subsubsection{Individual Product SHGC and VT using SHGC 0 \& 1 and VT 0 \& 1}

The methodology for determining the Solar Heat Gain Coefficient (SHGC) and Visible Transmittance (VT) for products is outlined in NFRC 200 using values of $\mathrm{SHGC}_{0}, \mathrm{SHGC}_{1}, \mathrm{VT}_{0}, \mathrm{VT}_{1}$. These values are calculated in WINDOW for the best glazing option modeled with the highest frame and edge U-factor frame, as outlined in NFRC 200, Section 6.1.1 (a).

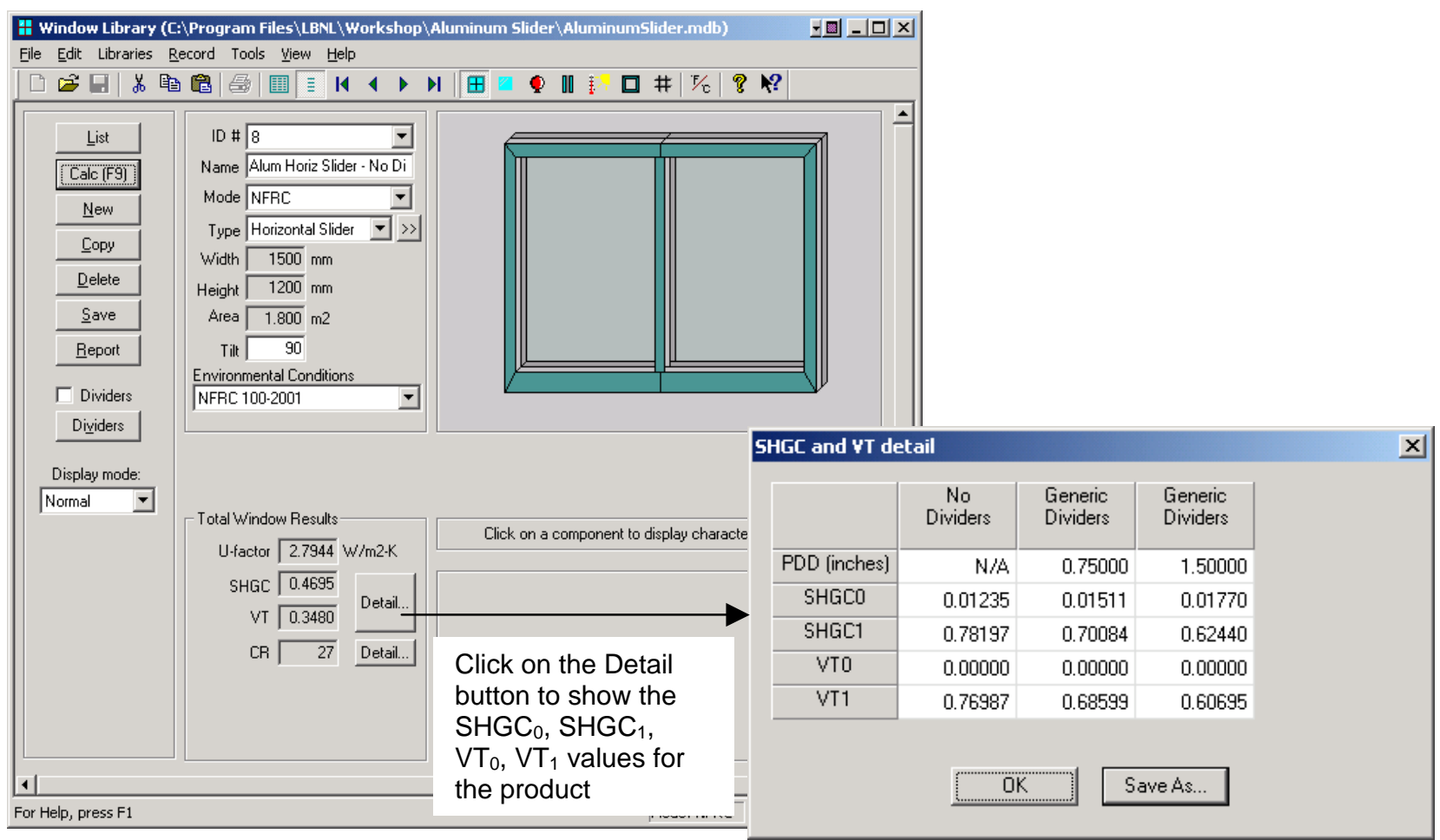

Figure 9-24. Dimensioned drawing for vinyl fixed window. 


\subsubsection{Drawings for Aluminum Horizontal Slider Window}

The following pages contain detailed drawings for this window:

\section{Sliding Head} (NFRC-VT-HD)

\begin{tabular}{|l|c|}
\hline $\begin{array}{l}\text { Glass } \\
\text { Thickness }\end{array}$ & $A$ \\
\hline $3 \mathrm{~mm}$ & $0.120_{-0.005}^{+0.010}$ \\
\hline $4 \mathrm{~mm}$ & $0.155_{-0.005}^{+0.005}$ \\
\hline $5 \mathrm{~mm}$ & $0.185_{-0.005}^{+0.005}$ \\
\hline $6 \mathrm{~mm}$ & $0.225_{-0.005}^{+0.005}$ \\
\hline
\end{tabular}

Note : All Weather Strippings are Type-I
Page 1

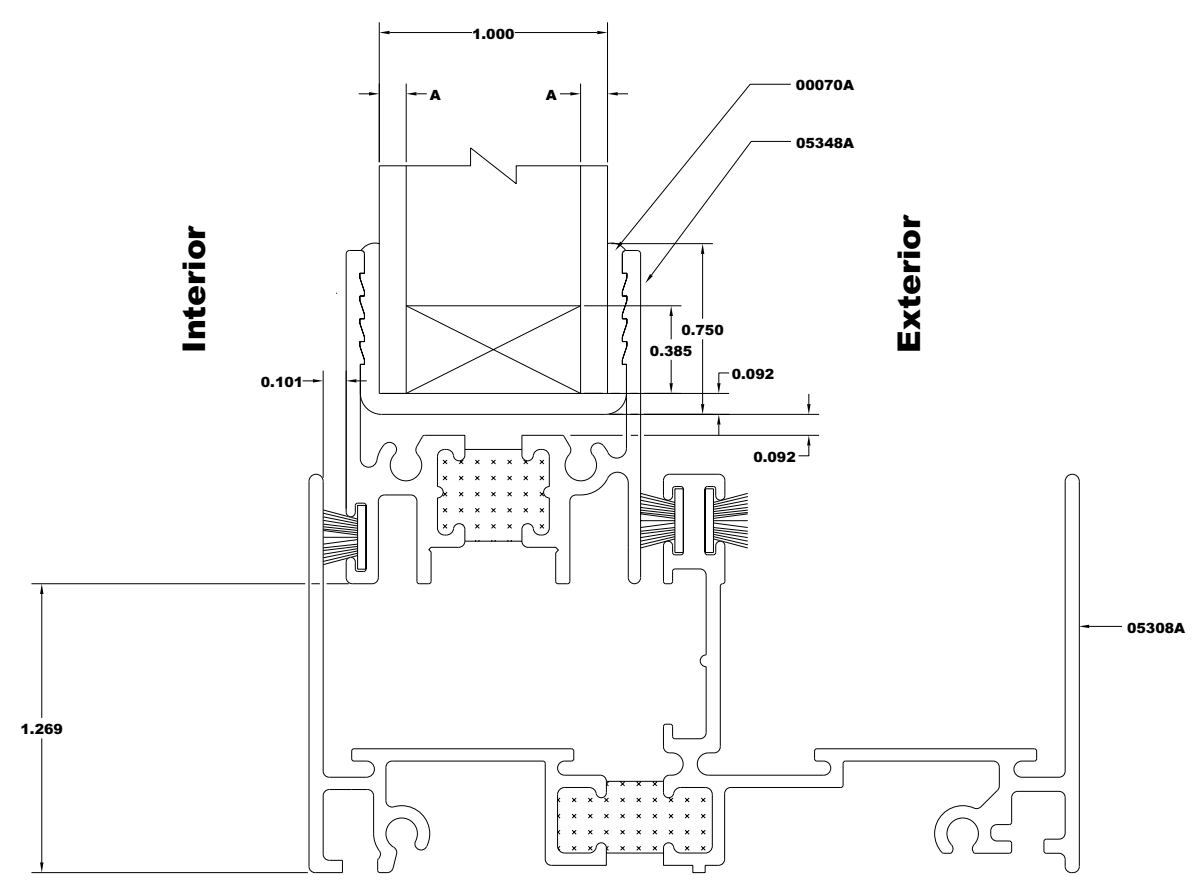

Figure 9-25. Vent Head Drawing for Horizontal Aluminum Slider. 
Page 2

\section{Fix Jamb}

\begin{tabular}{|c|c|}
\hline $\begin{array}{l}\text { Glass } \\
\text { Thickness }\end{array}$ & $A$ \\
\hline $3 \mathrm{~mm}$ & $0.120_{-0.005}^{+0.010}$ \\
\hline $4 \mathrm{~mm}$ & $0.155_{-0.005}^{+0.005}$ \\
\hline $5 \mathrm{~mm}$ & $0_{-0.005}^{+0.005}$ \\
\hline $6 \mathrm{~mm}$ & $0.225_{-0.005}^{+0.005}$ \\
\hline
\end{tabular}

(NFRC-FX-JB)

Note: All Weather Strippings are Type-I

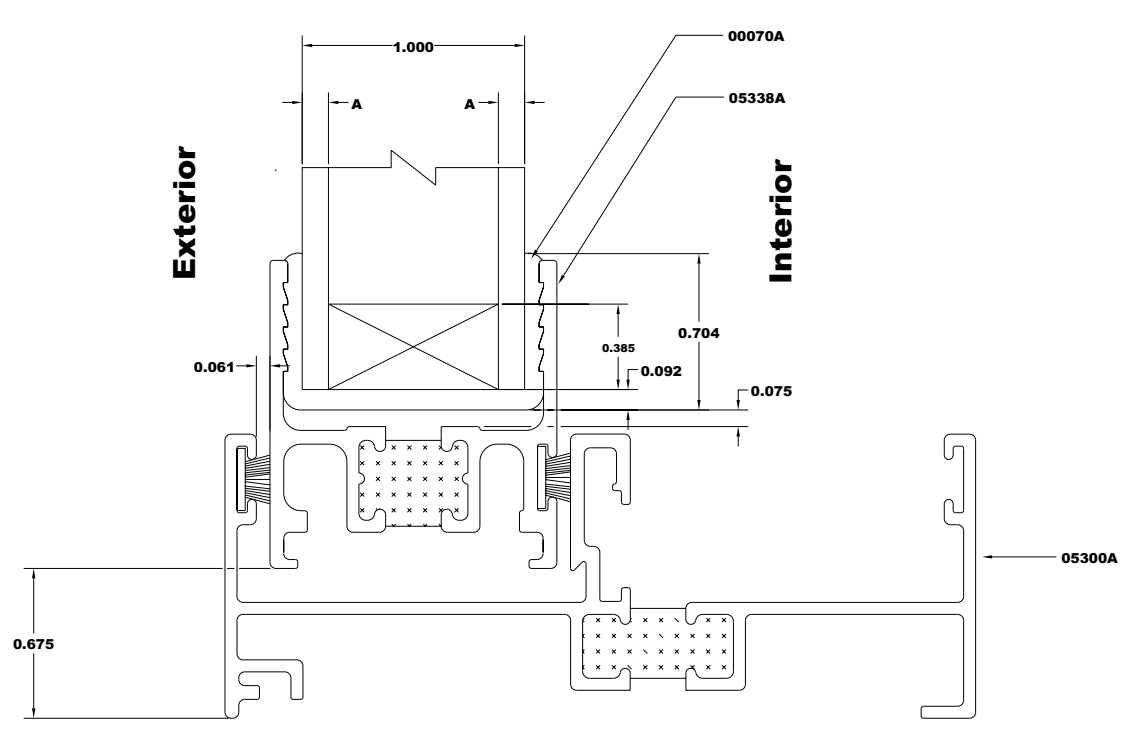

Figure 9-26. Fixed Jamb Drawing for Horizontal Aluminum Slider. 


\section{Sliding Sill}

(NFRC-VT-SL)

\begin{tabular}{|c|c|}
\hline $\begin{array}{c}\text { Glass } \\
\text { Thickness }\end{array}$ & $A$ \\
\hline $3 \mathrm{~mm}$ & $0.120_{-0.005}^{+0.010}$ \\
\hline $4 \mathrm{~mm}$ & $0.155_{-0.005}^{+0.005}$ \\
\hline $5 \mathrm{~mm}$ & $0.185_{-0.005}^{+0.005}$ \\
\hline $6 \mathrm{~mm}$ & $0.225_{-0.005}^{+0.005}$ \\
\hline
\end{tabular}

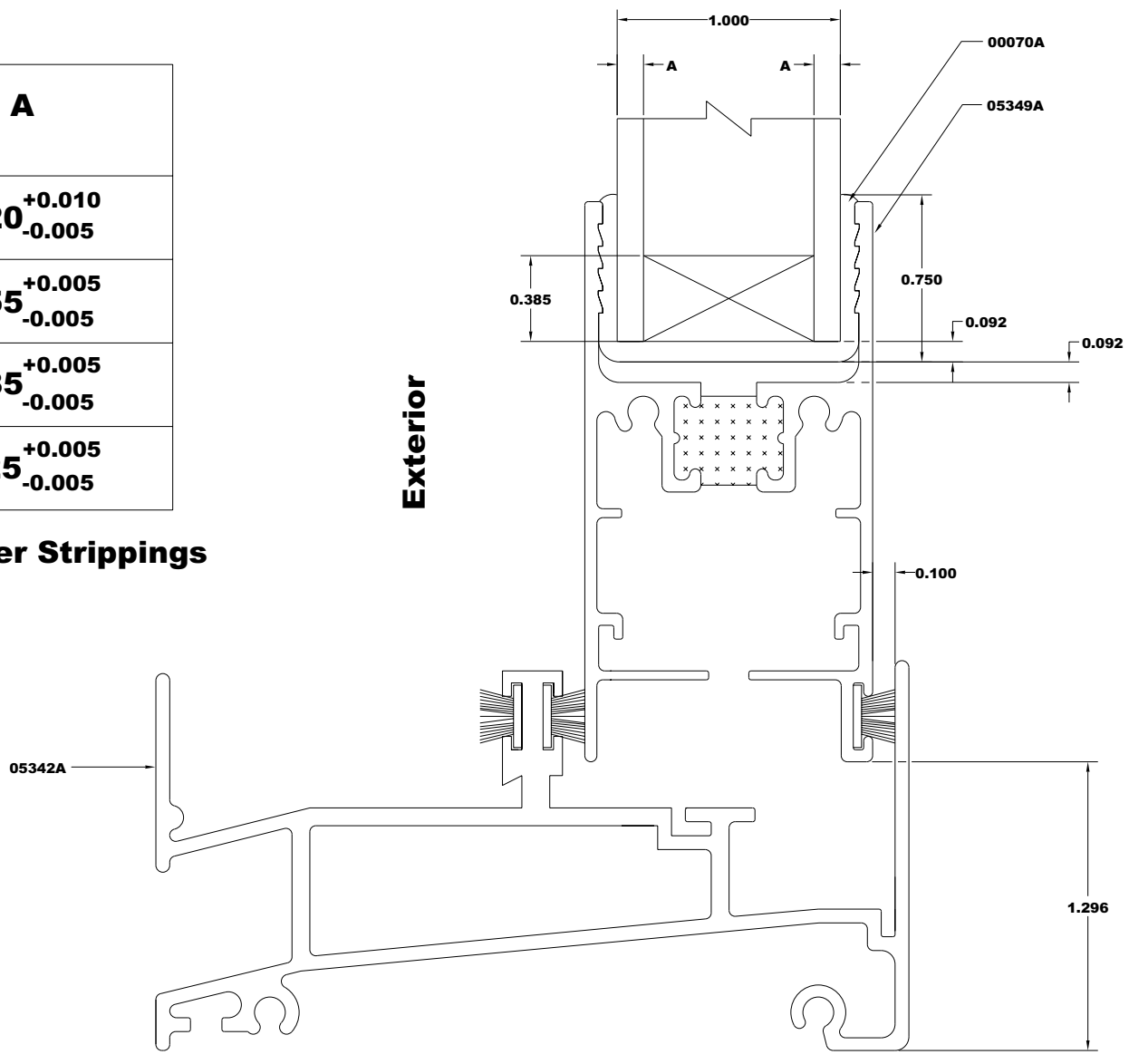

Note : All Weather Strippings are Type-I

Figure 9-27. Vent Sill Drawing for Horizontal Aluminum Slider. 
Fix Head

(NFRC-FX-HD)

\begin{tabular}{|c|c|}
\hline $\begin{array}{l}\text { Glass } \\
\text { Thickness }\end{array}$ & A \\
\hline $3 \mathrm{~mm}$ & $0.120_{-0.005}^{+0.010}$ \\
\hline $4 \mathrm{~mm}$ & $0.155_{-0.005}^{+0.005}$ \\
\hline $5 \mathrm{~mm}$ & $0.185_{-0.005}^{+0.005}$ \\
\hline $6 \mathrm{~mm}$ & $0.225_{-0.005}^{+0.005}$ \\
\hline
\end{tabular}

Note : All Weather Strippings are Type-I
Page 4

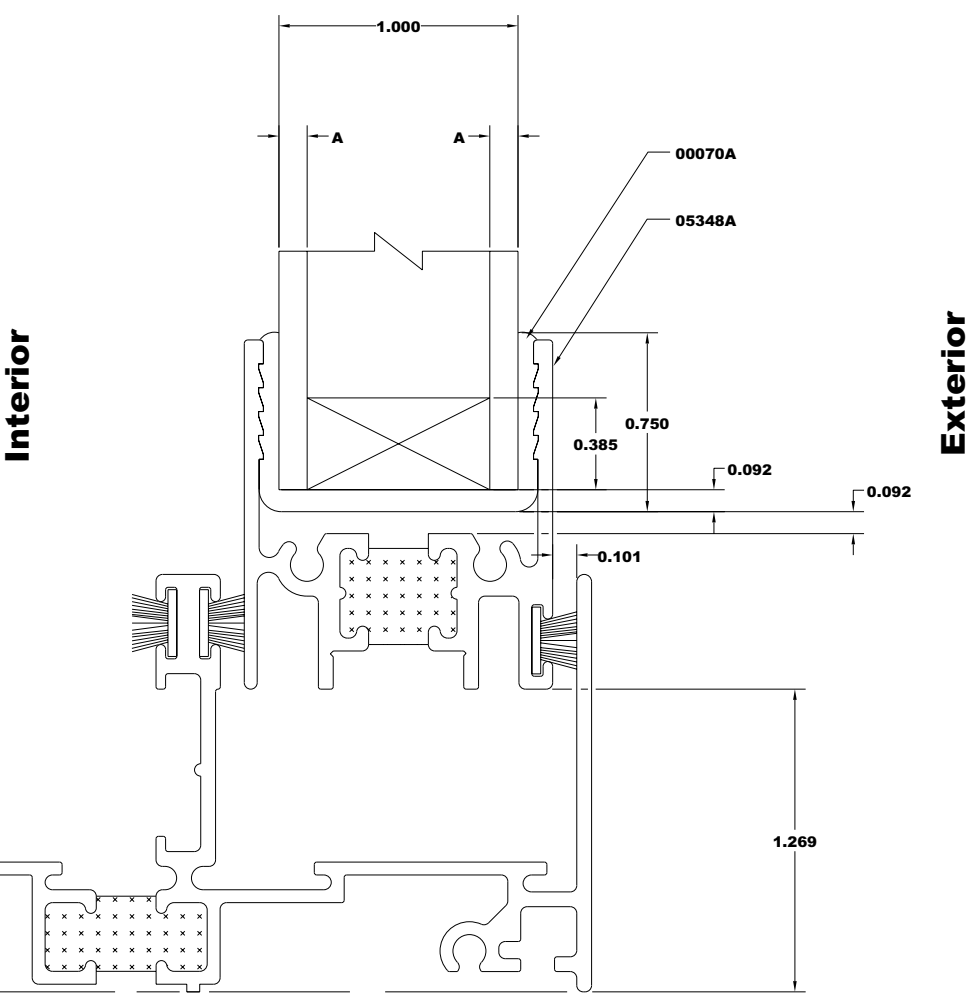

Figure 9-28. Fixed Head Drawing for Horizontal Aluminum Slider 


\section{Page 5}

\section{Sliding Jamb}

(NFRC-VT-JB)

\begin{tabular}{|l|c|}
\hline $\begin{array}{l}\text { Glass } \\
\text { Thickness }\end{array}$ & $A$ \\
\hline $3 \mathrm{~mm}$ & $0.120_{-0.005}^{+0.010}$ \\
\hline $4 \mathrm{~mm}$ & $0.155_{-0.005}^{+0.005}$ \\
\hline $5 \mathrm{~mm}$ & $0_{-185_{-0.005}^{+0.005}}$ \\
\hline $6 \mathrm{~mm}$ & ${0.225_{-0.005}^{+0.005}}$ \\
\hline
\end{tabular}

Note : All Weather Strippings are Type-I

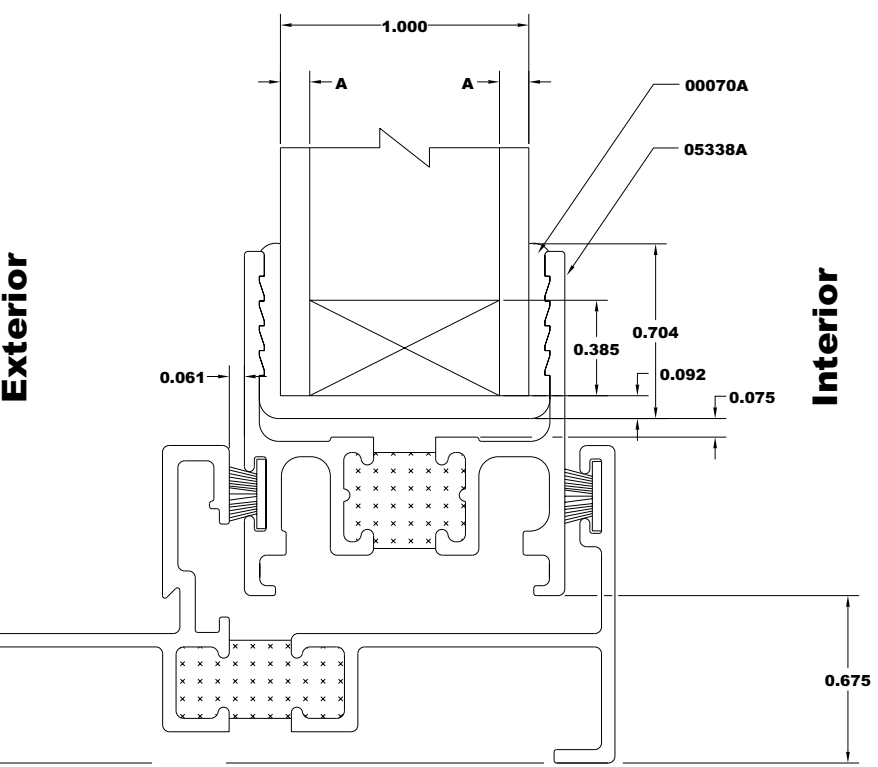

Figure 9-29. Vent Jamb Drawing for Horizontal Aluminum Slider. 
Fix Sill

Page 6

(NFRC-FX-SL)

\begin{tabular}{|l|c|}
\hline $\begin{array}{l}\text { Glass } \\
\text { Thickness }\end{array}$ & $A$ \\
\hline $3 \mathrm{~mm}$ & $0.120_{-0.005}^{+0.010}$ \\
\hline $4 \mathrm{~mm}$ & $0.155_{-0.005}^{+0.005}$ \\
\hline $5 \mathrm{~mm}$ & $0.185_{-0.005}^{+0.005}$ \\
\hline $6 \mathrm{~mm}$ & $0.225_{-0.005}^{+0.005}$ \\
\hline
\end{tabular}

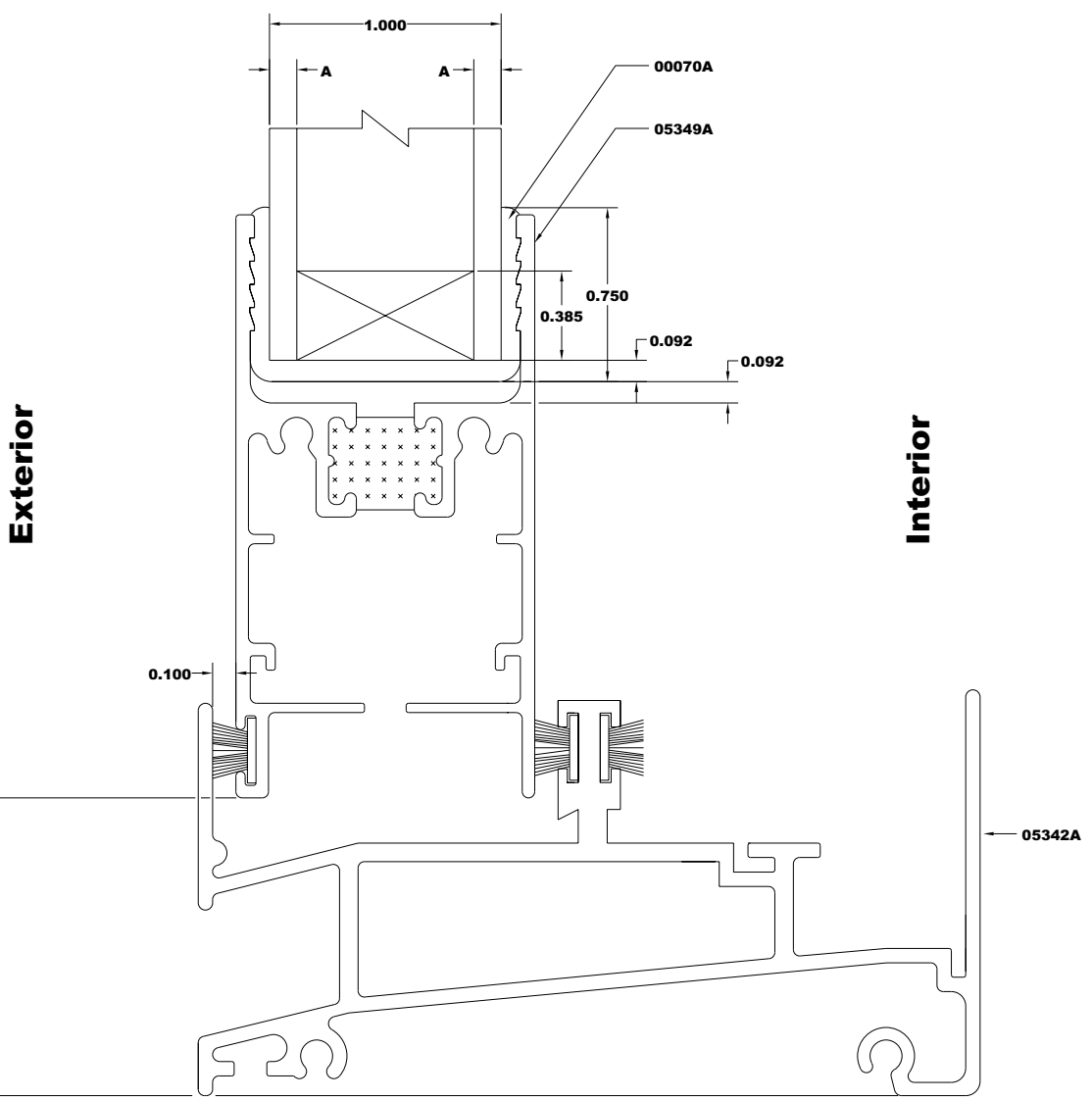

Note : All Weather Strippings are Type-I

Figure 9-30. Fixed Sill Drawing for Horizontal Aluminum Slider. 
Page 7

\section{Meeting Rail}

\begin{tabular}{|l|c|}
\hline $\begin{array}{l}\text { Glass } \\
\text { Thickness }\end{array}$ & $A$ \\
\hline $3 \mathrm{~mm}$ & $0.120_{-0.005}^{+0.010}$ \\
\hline $4 \mathrm{~mm}$ & $0.155_{-0.005}^{+0.005}$ \\
\hline $5 \mathrm{~mm}$ & $0.185_{-0.005}^{+0.005}$ \\
\hline $6 \mathrm{~mm}$ & $0.225_{-0.005}^{+0.005}$ \\
\hline
\end{tabular}

\section{(NFRC-MTG)}

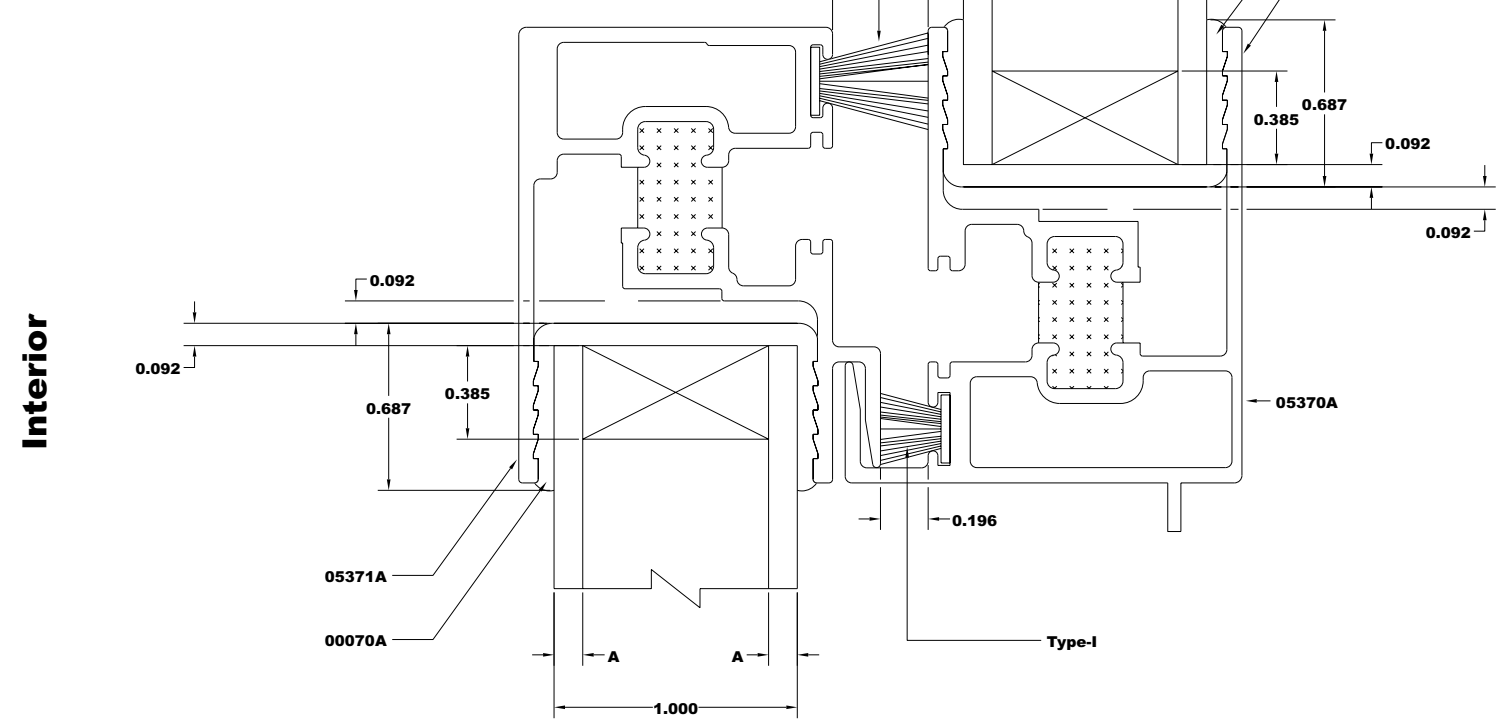

Figure 9-31. Meeting Rail Drawing for Horizontal Aluminum Slider. 


\section{0-A}

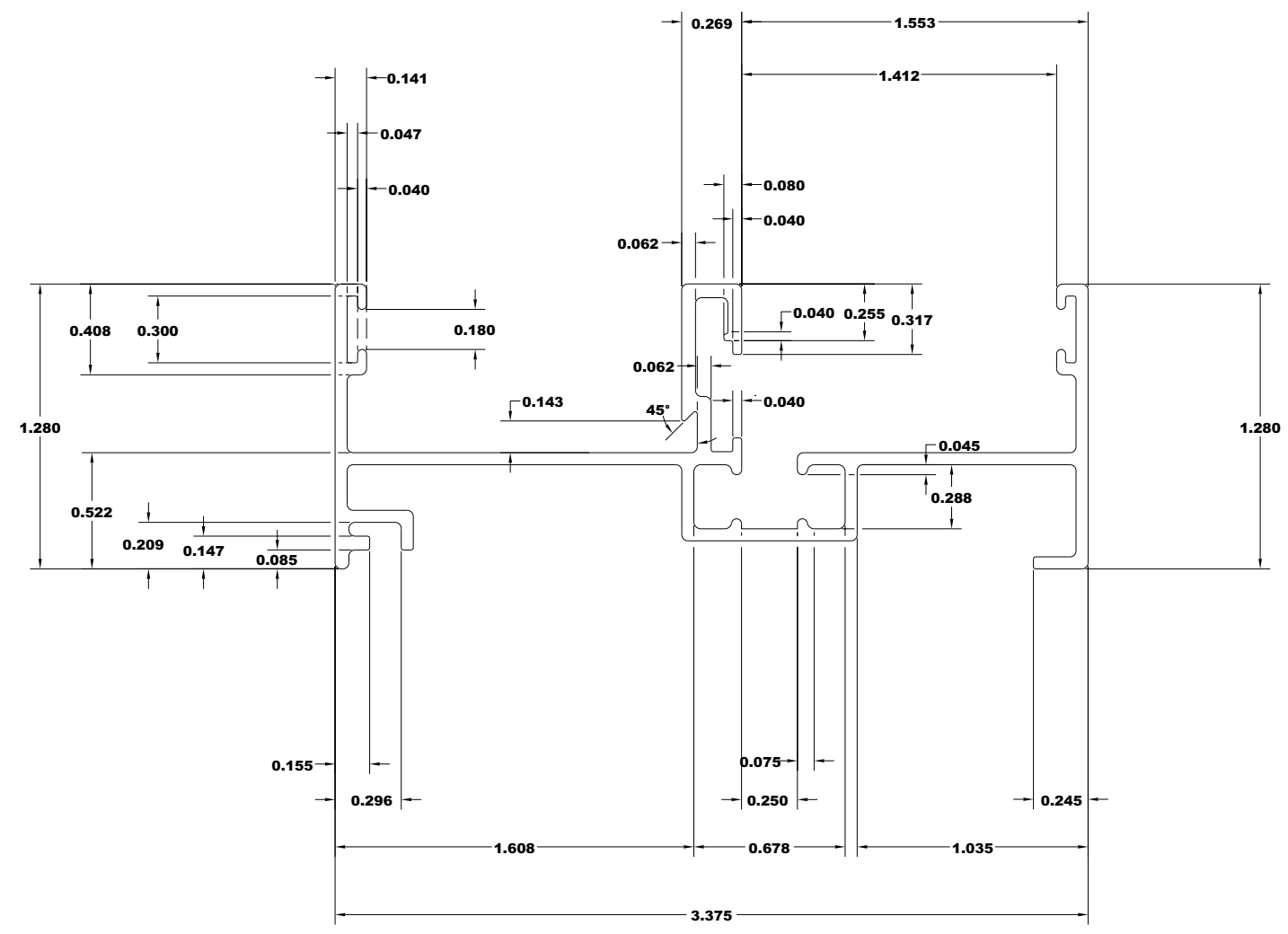

Figure 9-32. Fixed Jamb Drawing for Horizontal Aluminum Slider. 


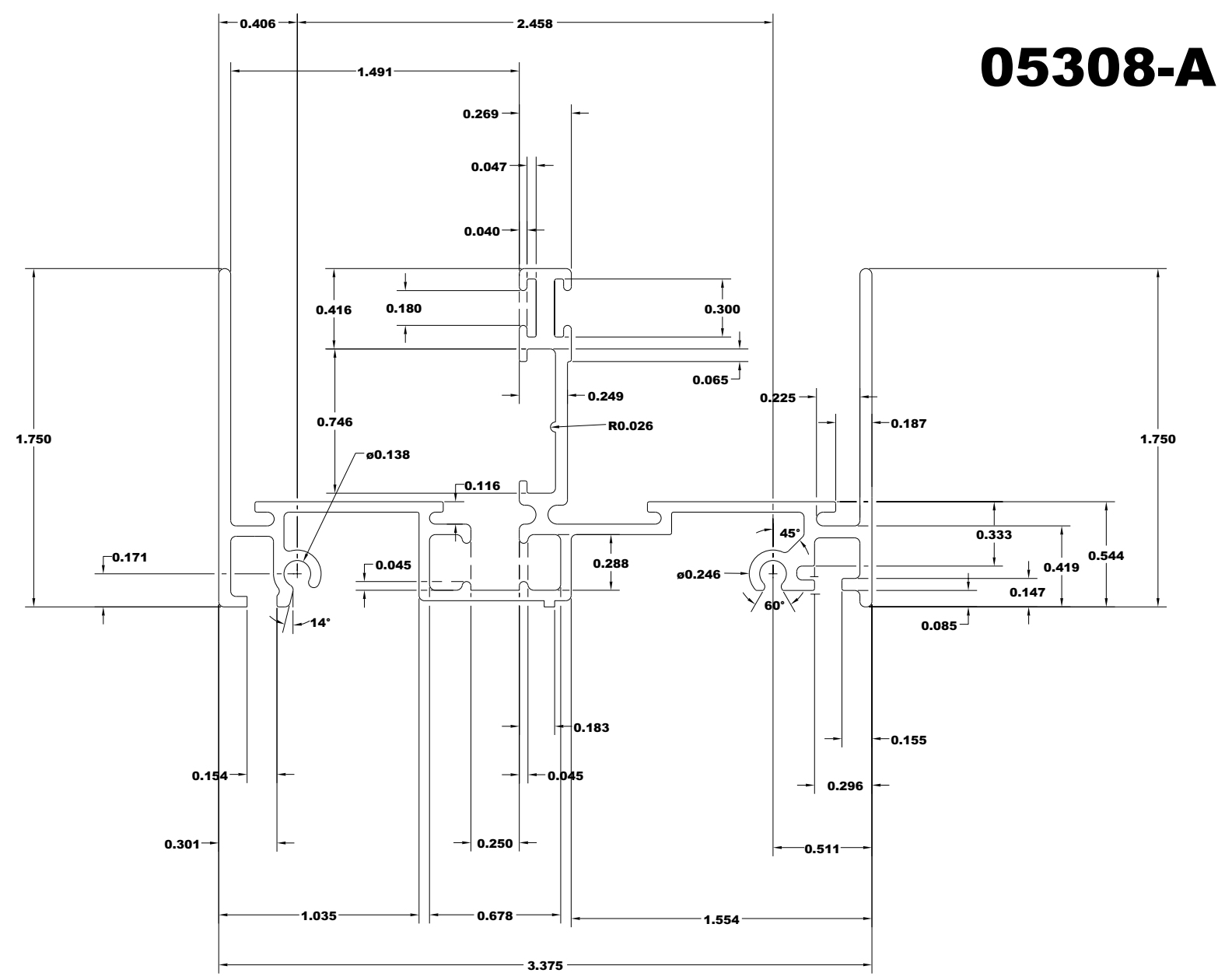

Figure 9-33. Head Drawing for Horizontal Aluminum Slider. 


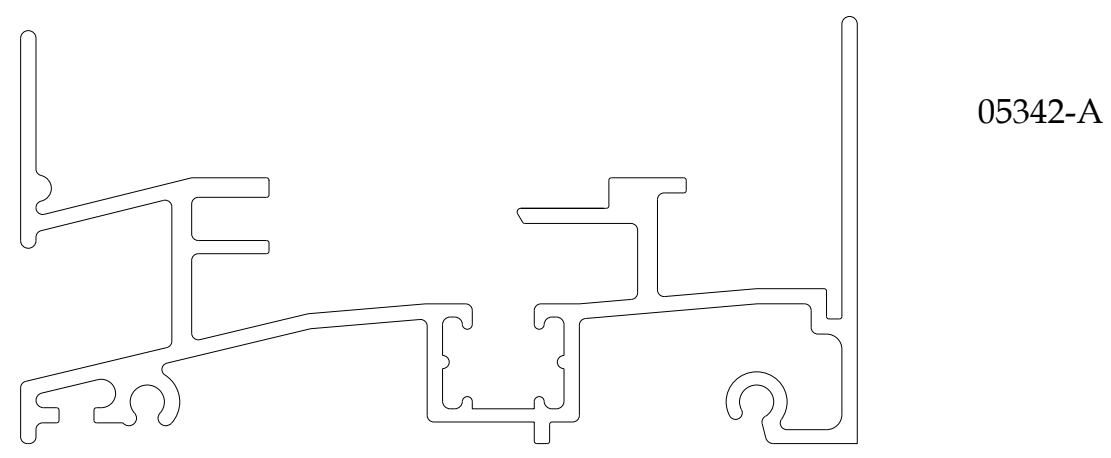

Figure 9-34. Sill Drawing for Horizontal Aluminum Slider.

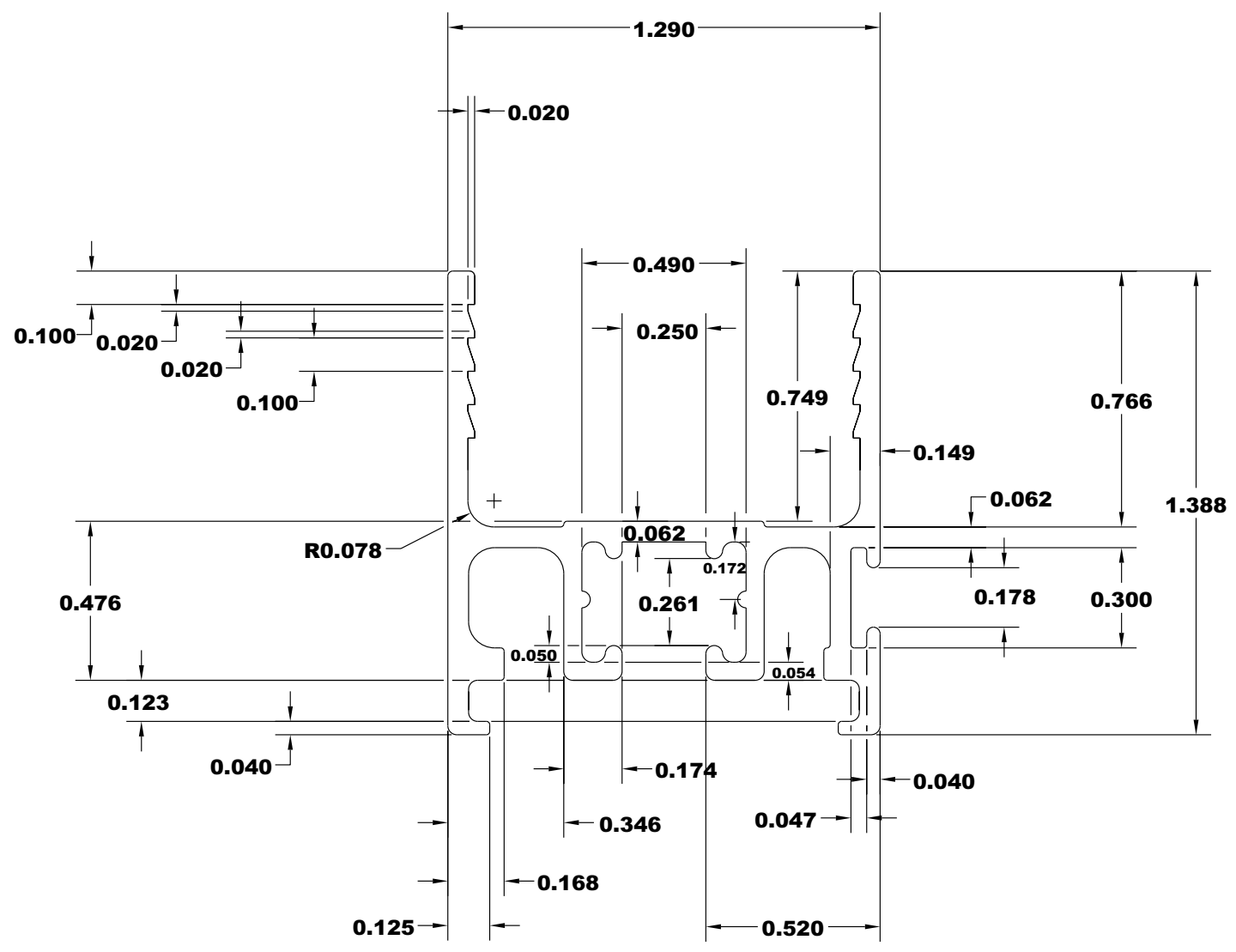

Figure 9-35. Stile-Fixed Drawing for Horizontal Aluminum Slider. 


\section{8-A}

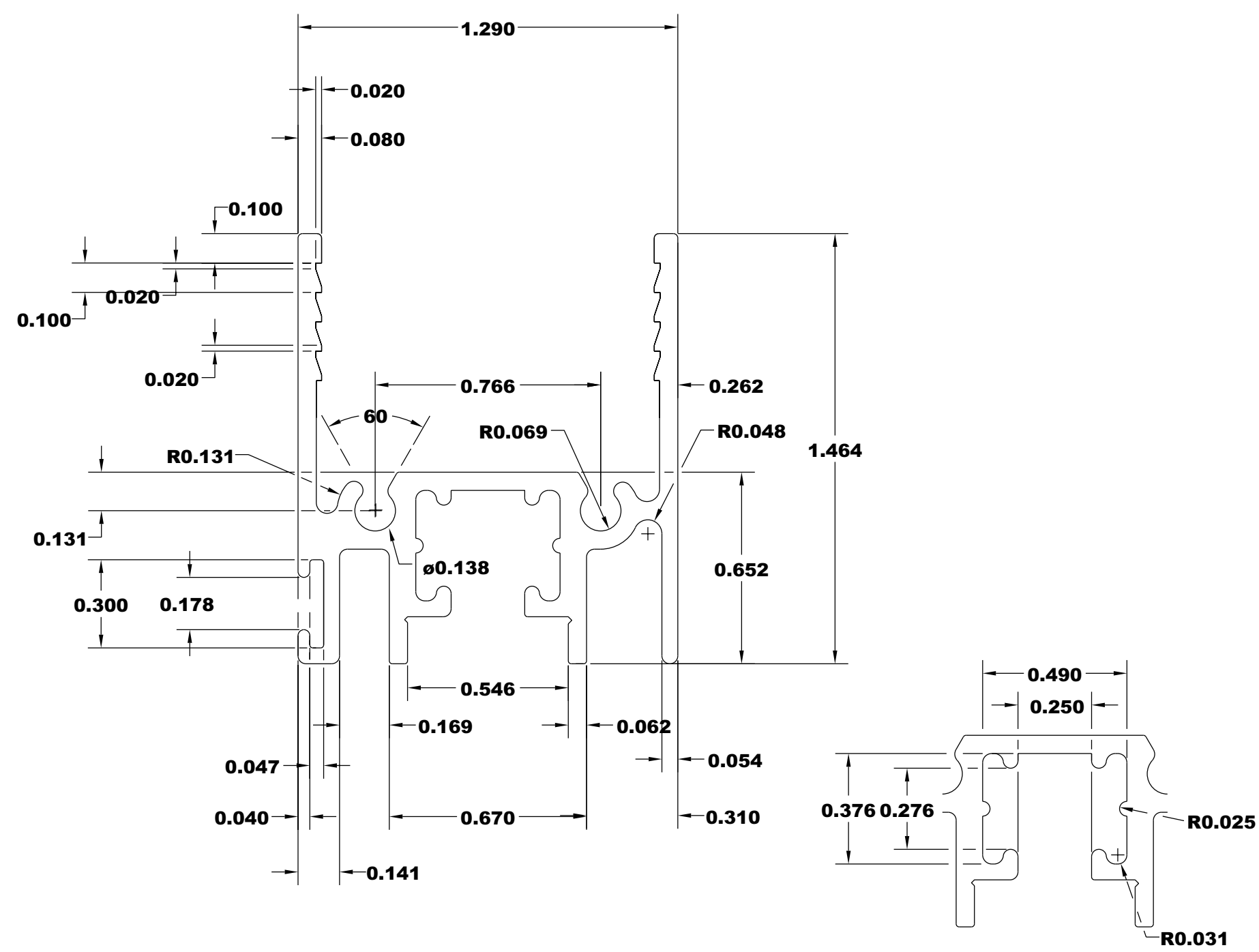

Figure 9-36. Stile-Fixed Drawing for Horizontal Aluminum Slider. 


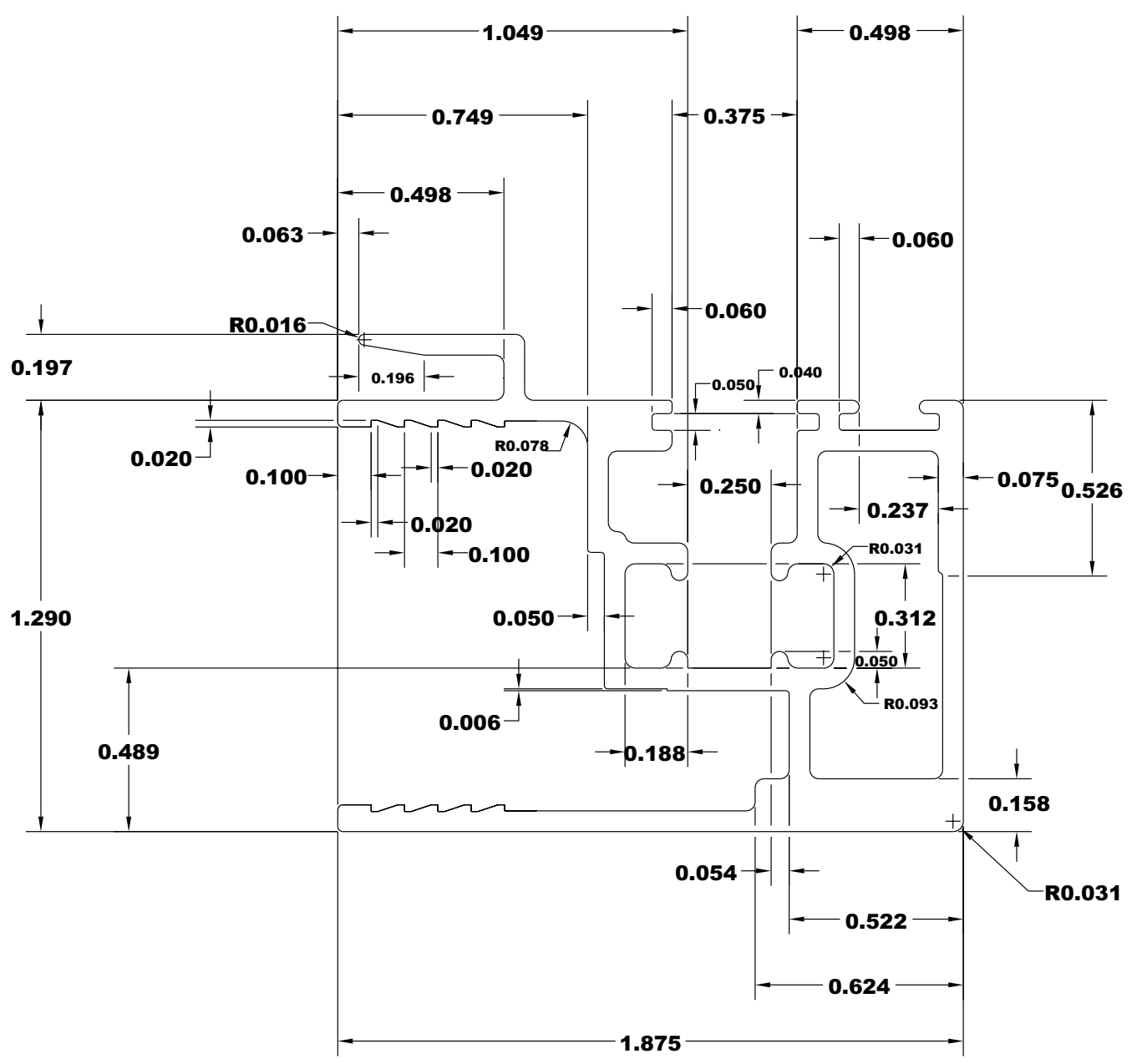

Figure 9-37. Stile-Slide / Silt - Fix Drawing for Horizontal Aluminum Slider. 


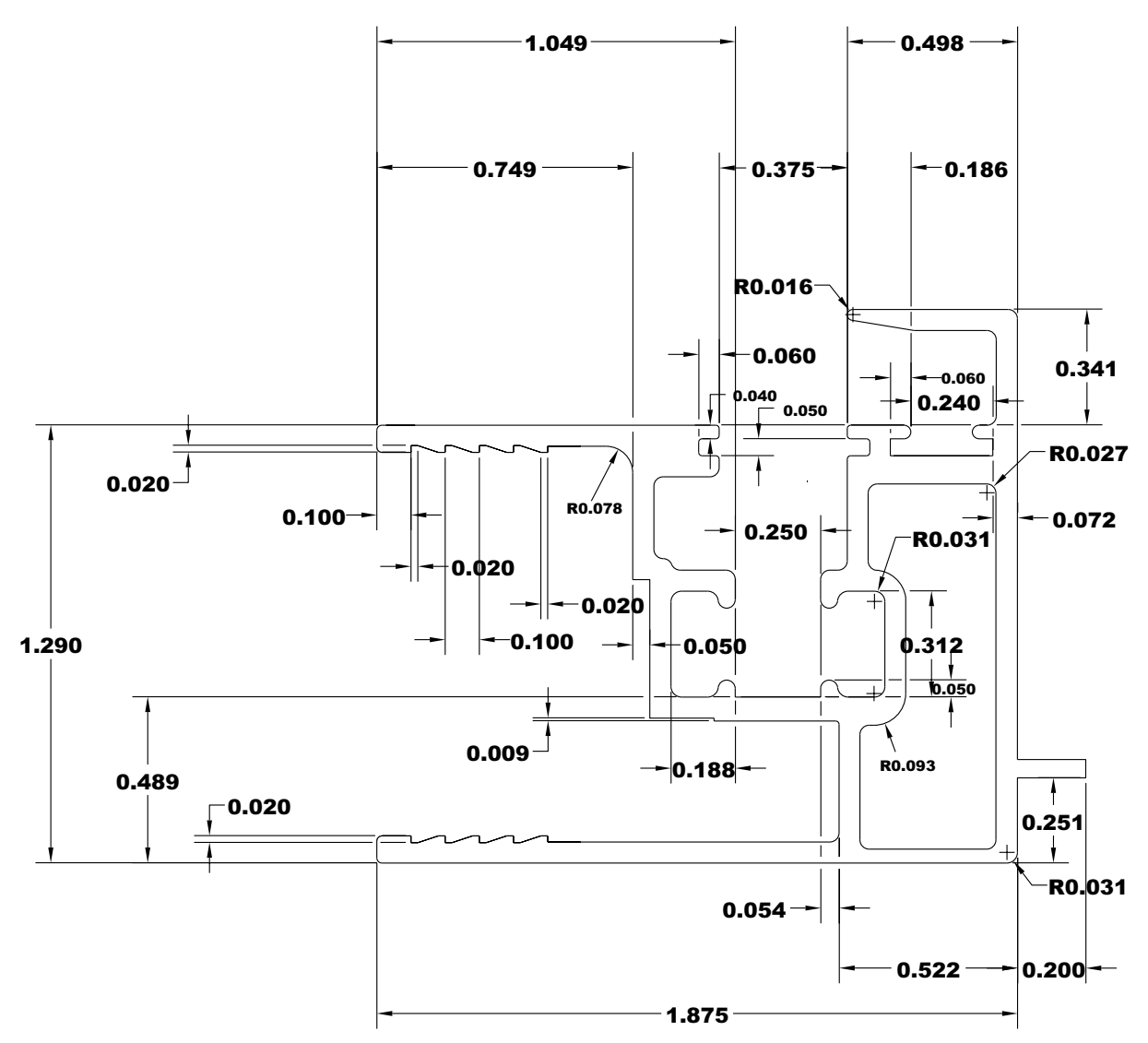

Figure 9-38. Stile-Fixed Drawing for Horizontal Aluminum Slider. 


\section{0-A}

Page 16
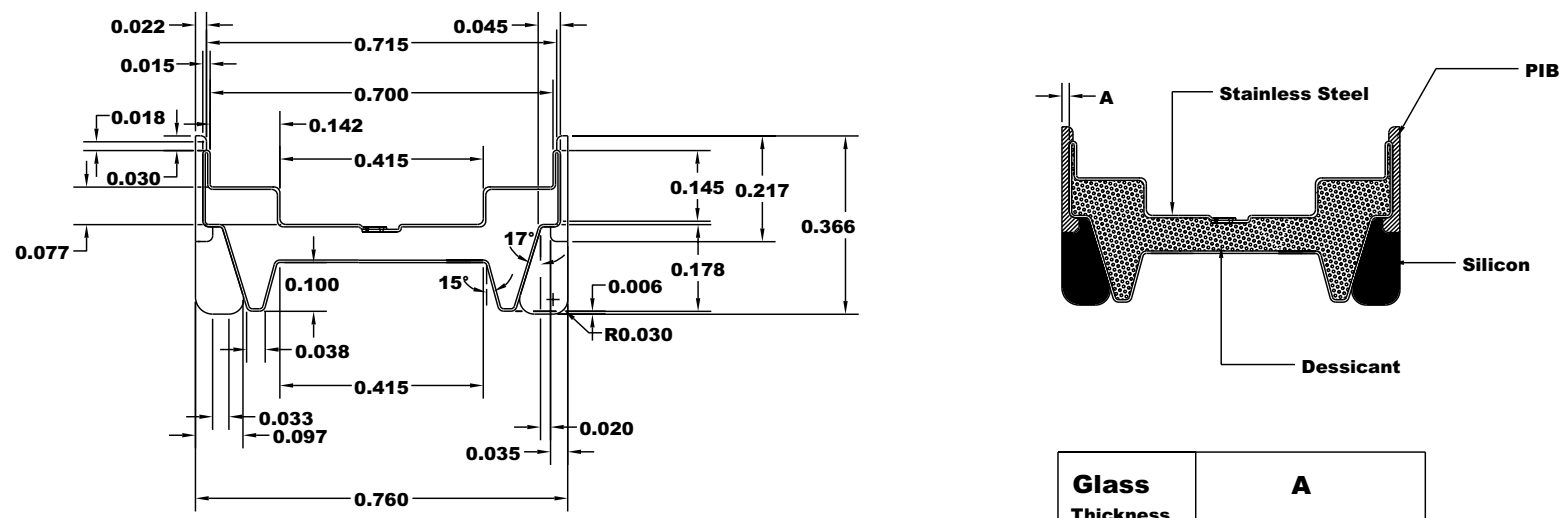

\begin{tabular}{|c|c|}
\hline $\begin{array}{l}\text { Glass } \\
\text { Thickness }\end{array}$ & $A$ \\
\hline $3 \mathrm{~mm}$ & $0.015_{-0.005}^{+0.010}$ \\
\hline $4 \mathrm{~mm}$ & $0.015_{-0.005}^{+0.005}$ \\
\hline $5 \mathrm{~mm}$ & $0.015_{-0.005}^{+0.005}$ \\
\hline $6 \mathrm{~mm}$ & $0.015_{-0.005}^{+0.005}$ \\
\hline
\end{tabular}

Figure 9-39. Spacer Horizontal Aluminum Slider.

\section{0-A}
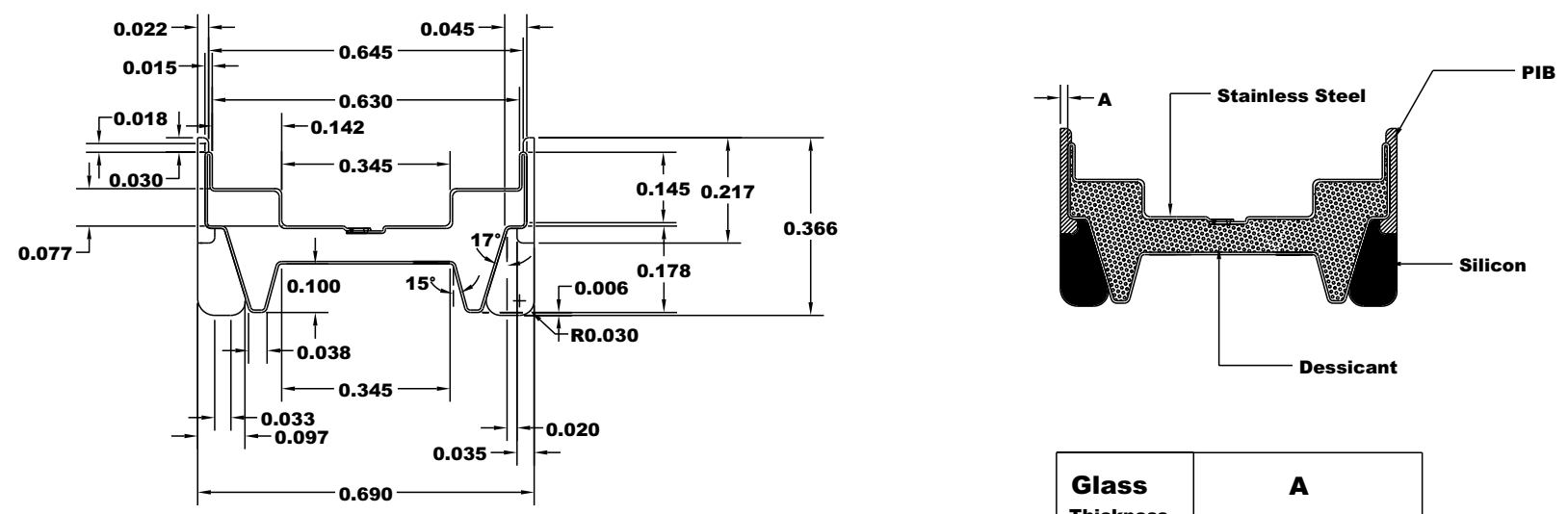

\begin{tabular}{|l|c|}
\hline $\begin{array}{l}\text { Glass } \\
\text { Thickness }\end{array}$ & $A$ \\
\hline $3 \mathrm{~mm}$ & $0.015_{-0.005}^{+0.010}$ \\
\hline $4 \mathrm{~mm}$ & $0.015_{-0.005}^{+0.005}$ \\
\hline $5 \mathrm{~mm}$ & $0.015_{-0.005}^{+0.005}$ \\
\hline $6 \mathrm{~mm}$ & $0.015_{-0.005}^{+0.005}$ \\
\hline
\end{tabular}

Figure 9-40. Spacer for Horizontal Aluminum Slider. 


\section{0-A}

Page 18
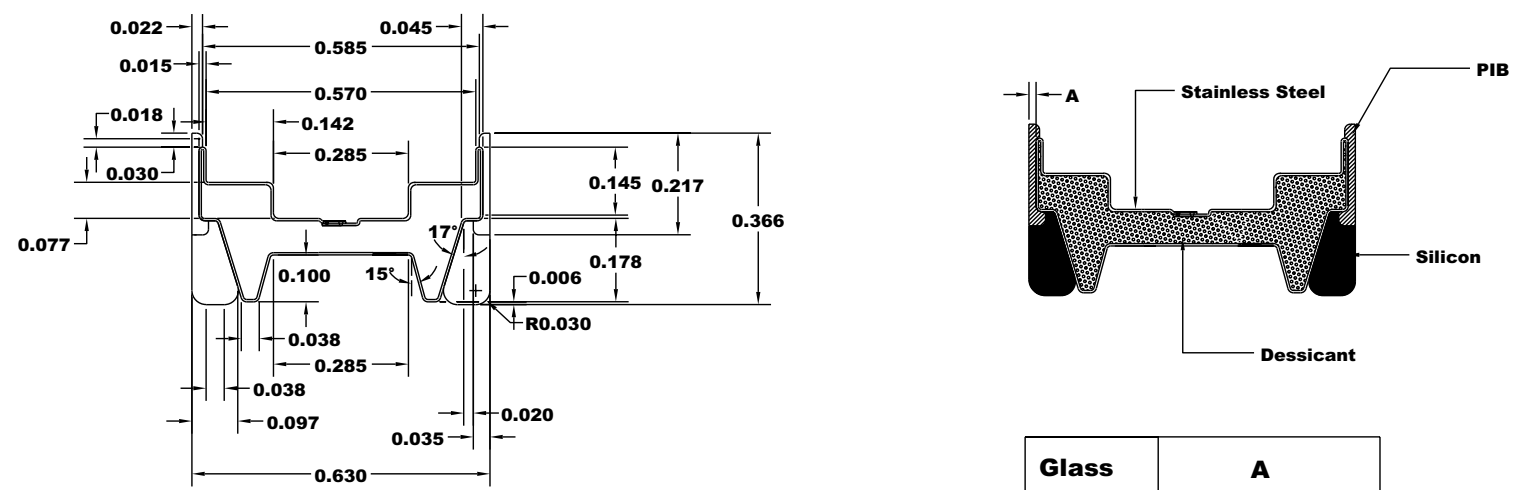

\begin{tabular}{|c|c|}
\hline $\begin{array}{l}\text { Glass } \\
\text { Thickness }\end{array}$ & $A$ \\
\hline $3 \mathrm{~mm}$ & $0.015_{-0.005}^{+0.010}$ \\
\hline $4 \mathrm{~mm}$ & $0.015_{-0.005}^{+0.005}$ \\
\hline $5 \mathrm{~mm}$ & $0.015_{-0.005}^{+0.005}$ \\
\hline $6 \mathrm{~mm}$ & $0.015_{-0.005}^{+0.005}$ \\
\hline
\end{tabular}

Figure 9-41. Spacer Horizontal Aluminum Slider.

\section{0-A}

Page 19
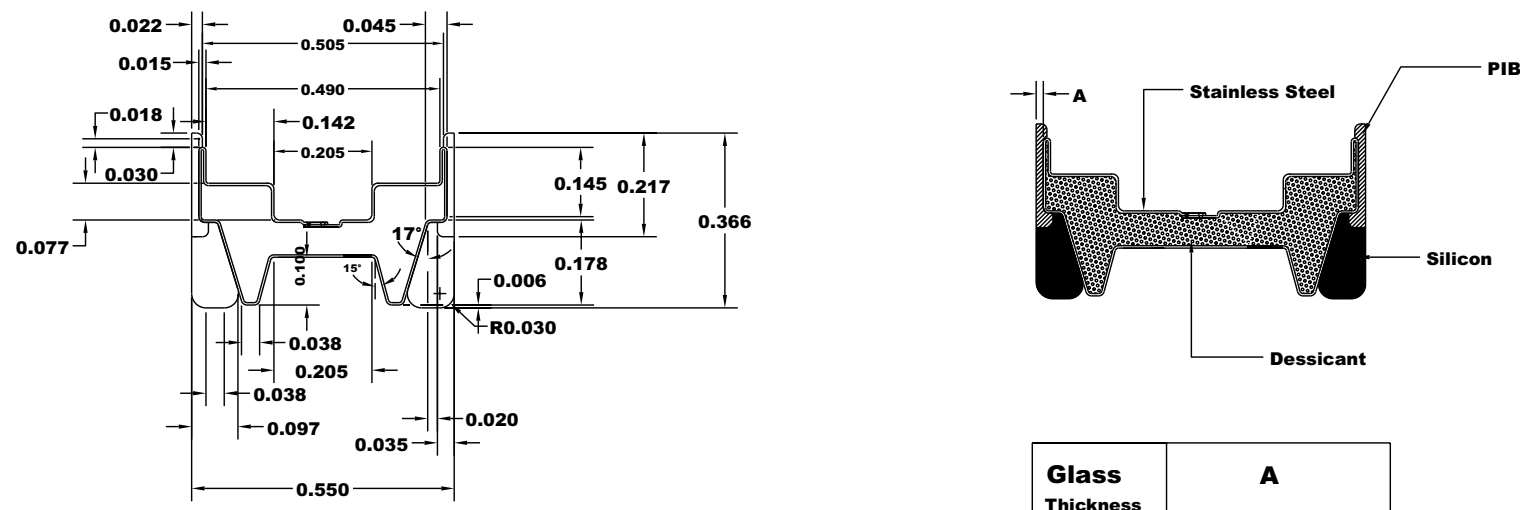

\begin{tabular}{|l|c|}
\hline $\begin{array}{l}\text { Glass } \\
\text { Thickness }\end{array}$ & $A$ \\
\hline $3 \mathrm{~mm}$ & $0.015_{-0.005}^{+0.010}$ \\
\hline $4 \mathrm{~mm}$ & $0.015_{-0.005}^{+0.005}$ \\
\hline $5 \mathrm{~mm}$ & $0.015_{-0.005}^{+0.005}$ \\
\hline $6 \mathrm{~mm}$ & $0.015_{-0.005}^{+0.005}$ \\
\hline
\end{tabular}

Figure 9-42. Spacer Horizontal Aluminum Slider. 


\section{0-A}

Page 21

Divider type A

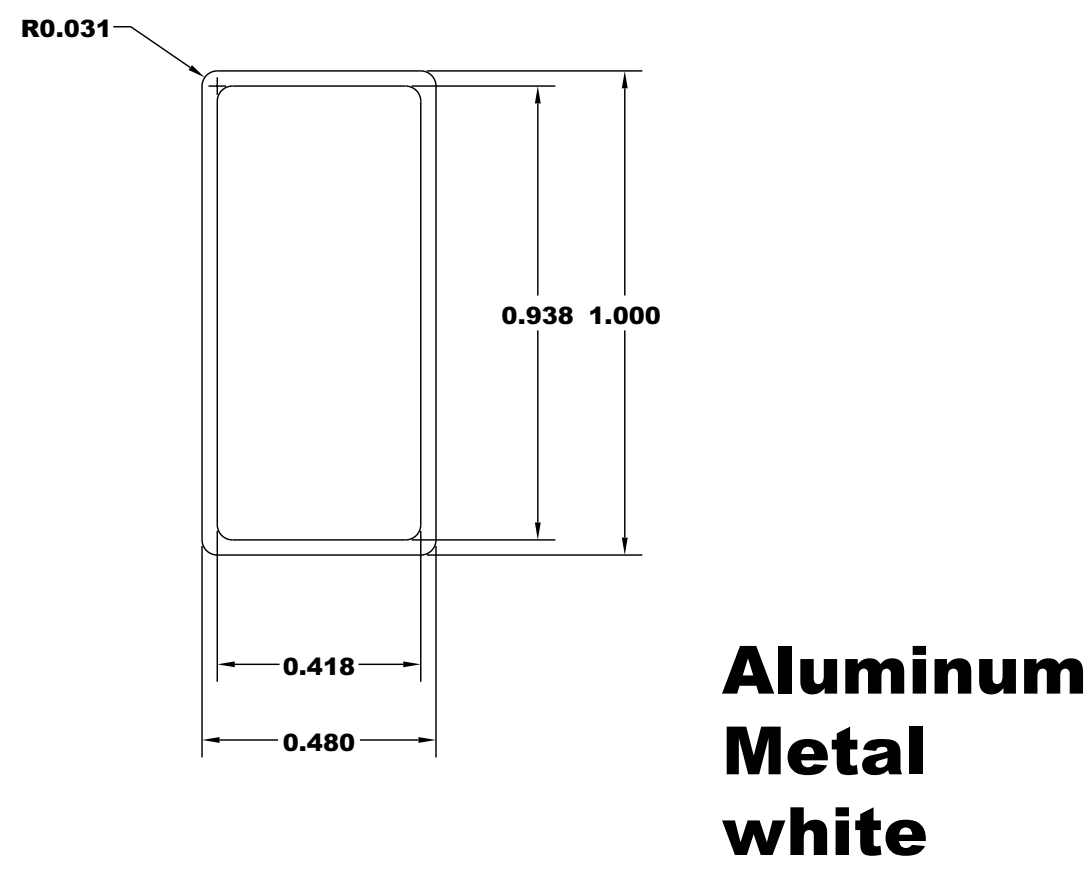

Figure 9-43Divider for Horizontal Slider

\section{0-A}

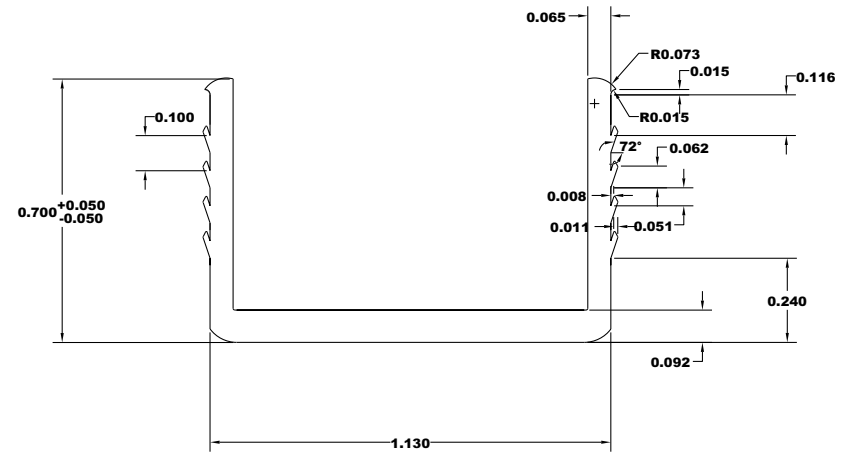

U-Chanel
Figure 9-44. U-channel for Horizontal Slider 


\subsection{Problem 3: Skylight}

\subsubsection{Description}

$\begin{array}{ll}\text { Window Type } & \text { Skylight } \\ \text { Frame Material } & \text { Wood } \\ \text { Overall Size } & 1181 \mathrm{~mm}\left(46.5^{\prime \prime}\right) \text { by } 1181 \mathrm{~mm}\left(46.5^{\prime \prime}\right) \\ \text { Spacer type } & \text { Aluminum folded spacer. } \\ \text { Weather strip } & \text { Not applicable. } \\ \text { Cross Sections } & \text { Head, sill, and jambs - the jambs are each the same so only one needs to be modeled } \\ \text { Dividers } & \text { Not applicable } \\ \text { Glazing System } & \text { See the glazing matrix in Table 9-14. }\end{array}$

\subsubsection{Glazing Matrix}

The following table shows the glazing matrix that is to be simulated for this window.

Table 9-11. Center-of-glazing U-factor Results for the Matrix of Glazing Options

\begin{tabular}{|c|c|l|l|}
\hline & \multicolumn{1}{|c|}{ Glazing Options } & \multicolumn{1}{|c|}{$\begin{array}{c}\text { Grid } \\
\text { Option }\end{array}$} & Manufacturer \\
\hline 1 & Clear $(3 \mathrm{~mm})$, Air, Clear $(3 \mathrm{~mm})$ & N/A & Generic \\
\hline
\end{tabular}




\subsubsection{Center-of-glazing Modeling (WINDOW)}

In WINDOW, create the glazing system(s) needed for the Glazing Matrix, making sure to set the Tilt to $20^{\circ}$ (from horizontal). The center-of-glass U-factor is displayed in the U-factor column of the Center-of-glazing Results tab in the WINDOW Glazing System Library. This glazing system will be brought into the THERM cross-sections.

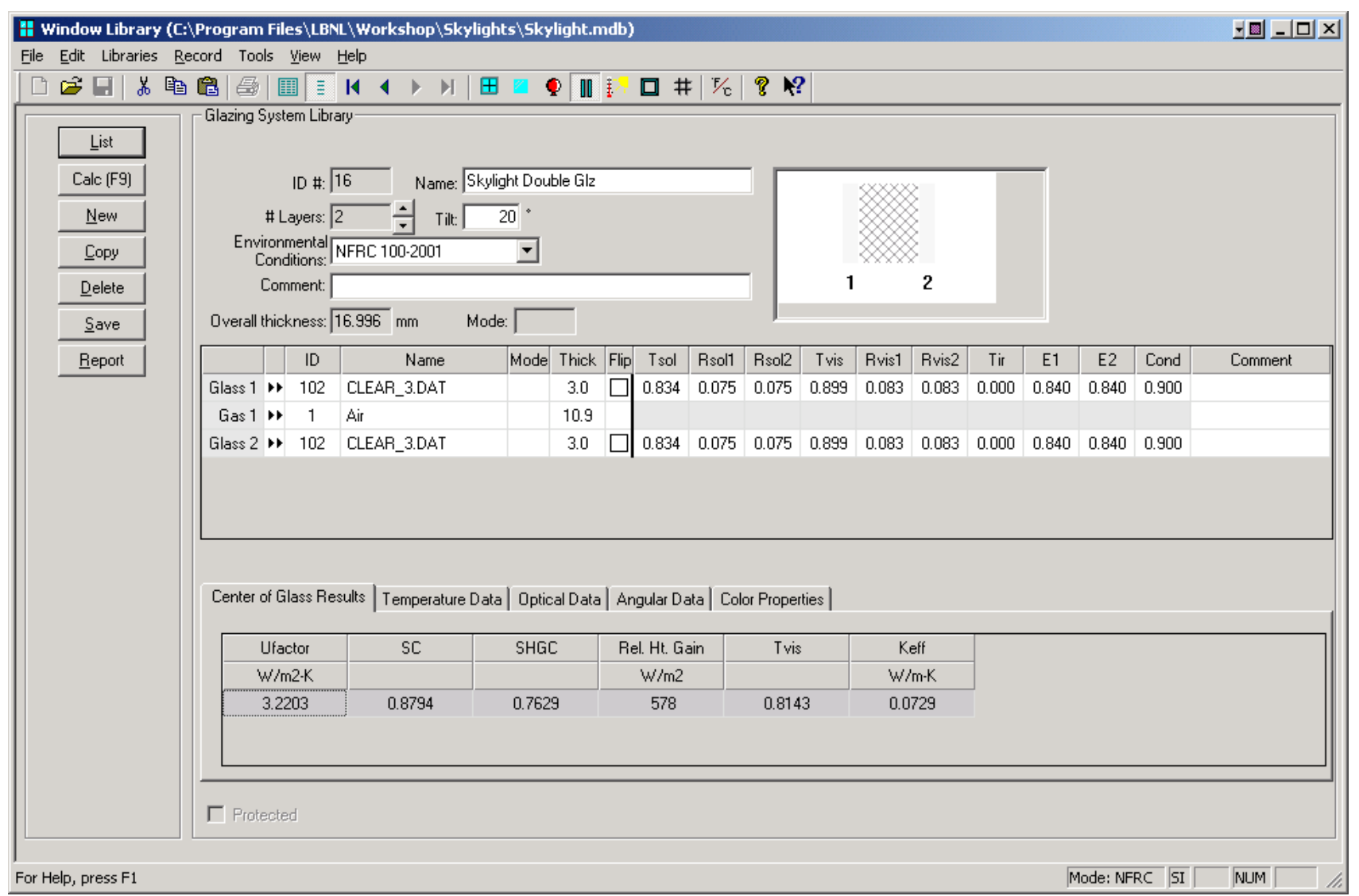

Figure 9-45. WINDOW Glazing System Library for the glazing option.

The results for the WINDOW center-of-glazing U-factor are shown in the following table.

Table 9-12. WINDOW Center-of-glazing U-factor Results for the Matrix of Glazing Options

\begin{tabular}{|c|c|c|c|}
\hline & \multicolumn{2}{|c|}{ Center-of-glazing U-factor } \\
\hline & $\begin{array}{c}\text { Glazing Options (1.0" overall } \\
\text { thickness) }\end{array}$ & $\mathrm{W} / \mathrm{m}^{2}-{ }^{\circ} \mathrm{C}$ & $\left(\right.$ Btu/hr-ft $\left.{ }^{2}-{ }^{\circ} F\right)$ \\
\hline 1 & Clear (3 mm), Air, Clear (3mm) & 3.220 & 0.5671 \\
\hline
\end{tabular}




\subsubsection{Edge-of-glazing and Frame Modeling (THERM)}

Model skylights in the same manner as other products, with separate THERM files for Sill, Head, Jamb and so forth, and use the AutoEnclosure radiation model for each cross section. See Section 8-5 of this manual for detailed instructions about modeling skylights.

Table 9-16 lists the cross sections to be modeled, and any associated files (DXF and THERM files) that are found on the CD.

Table 9-13. Cross sections to be modeled with their associated $d x f$ and THERM files

\begin{tabular}{|l|l|l|}
\hline Cross Section & DXF Filename & \multicolumn{1}{|c|}{ THERM file } \\
\hline Sill & N/A & Skylight Sill.thm \\
\hline Head & N/A & Skylight Head.thm \\
\hline Jamb & N/A & Skylight Jamb.thm \\
\hline
\end{tabular}

Table 9-17 shows the resulting U-factors from each cross section.

Table 9-14. Frame and Edge THERM U-factor Results

\begin{tabular}{|c|c|c|c|c|}
\hline & \multicolumn{2}{|c|}{ Frame U-factor } & \multicolumn{2}{|c|}{ Edge U-factor } \\
\hline Cross Section & $\left(\mathrm{W} / \mathrm{m}^{2}-{ }^{\circ} \mathrm{C}\right)$ & (Btu/hr-ft $\left.{ }^{2}-{ }^{\circ} \mathrm{F}\right)$ & $\left(\mathrm{W} / \mathrm{m}^{2}-^{\circ} \mathrm{C}\right)$ & (Btu/hr-ft $\left.{ }^{2}-{ }^{\circ} \mathrm{F}\right)$ \\
\hline Head & 5.133 & 0.904 & 3.414 & 0.601 \\
\hline Sill & 5.691 & 1.002 & 3.255 & 0.672 \\
\hline Jamb & 5.693 & 1.003 & 3.816 & 0.573 \\
\hline
\end{tabular}

Figures 9-52 and 9-54 on the following pages show THERM files and U-factor results screens for each of the cross sections.

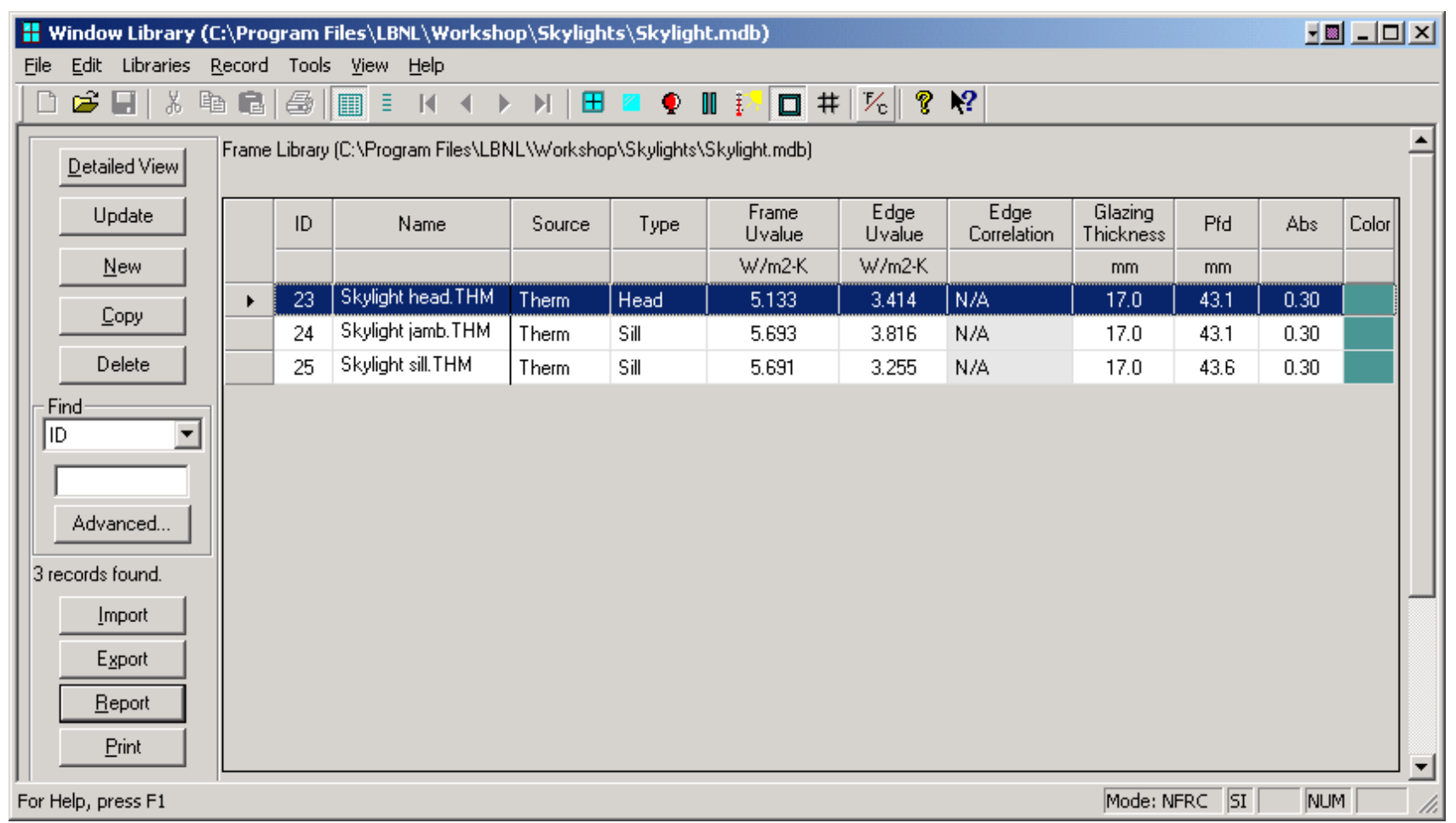

Figure 9-46. WINDOW Frame Library. 


\section{Head}
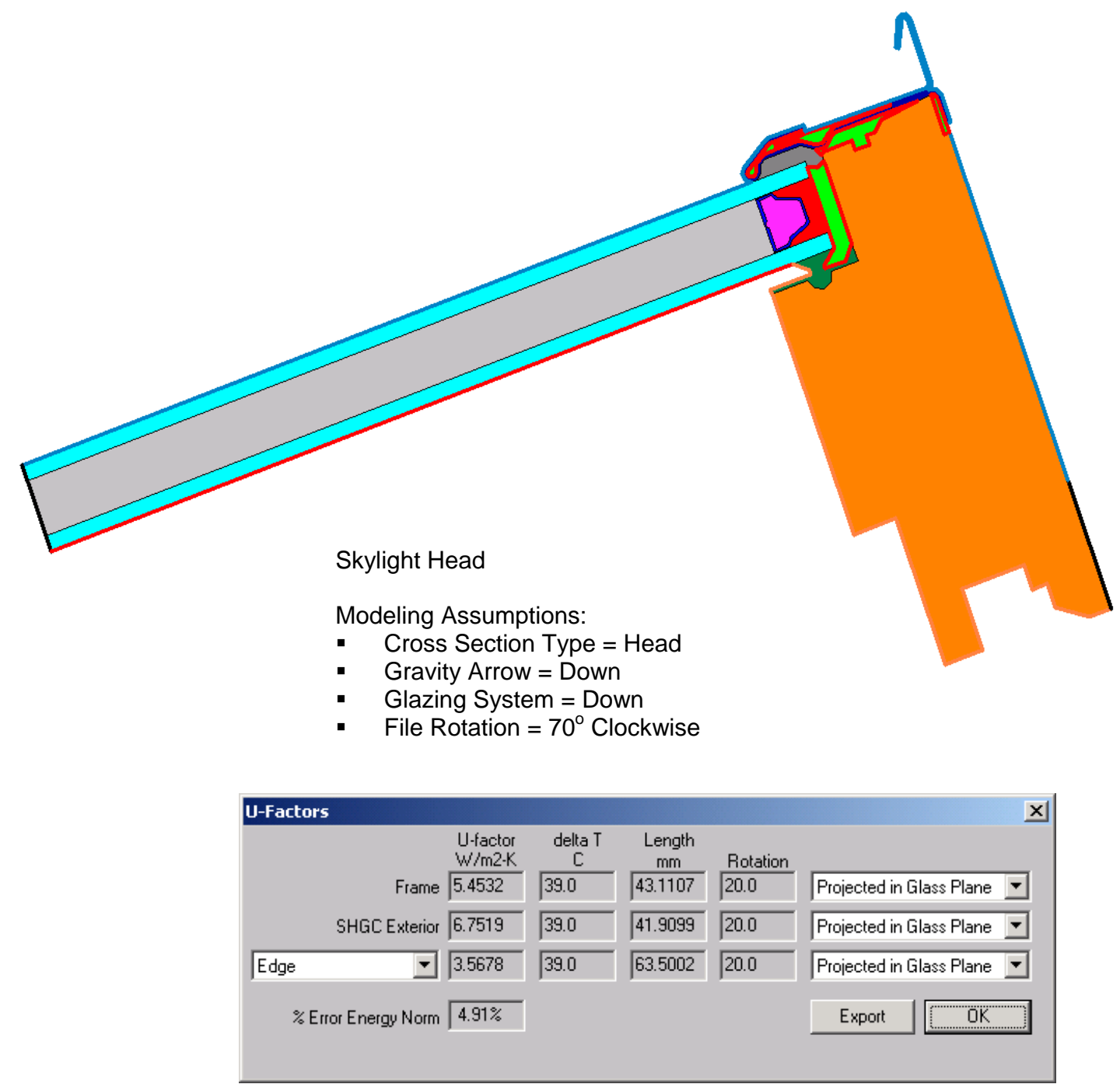

Figure 9-47. THERM file for Head and Sill inset mount skylight cross section. 


\section{Sill}

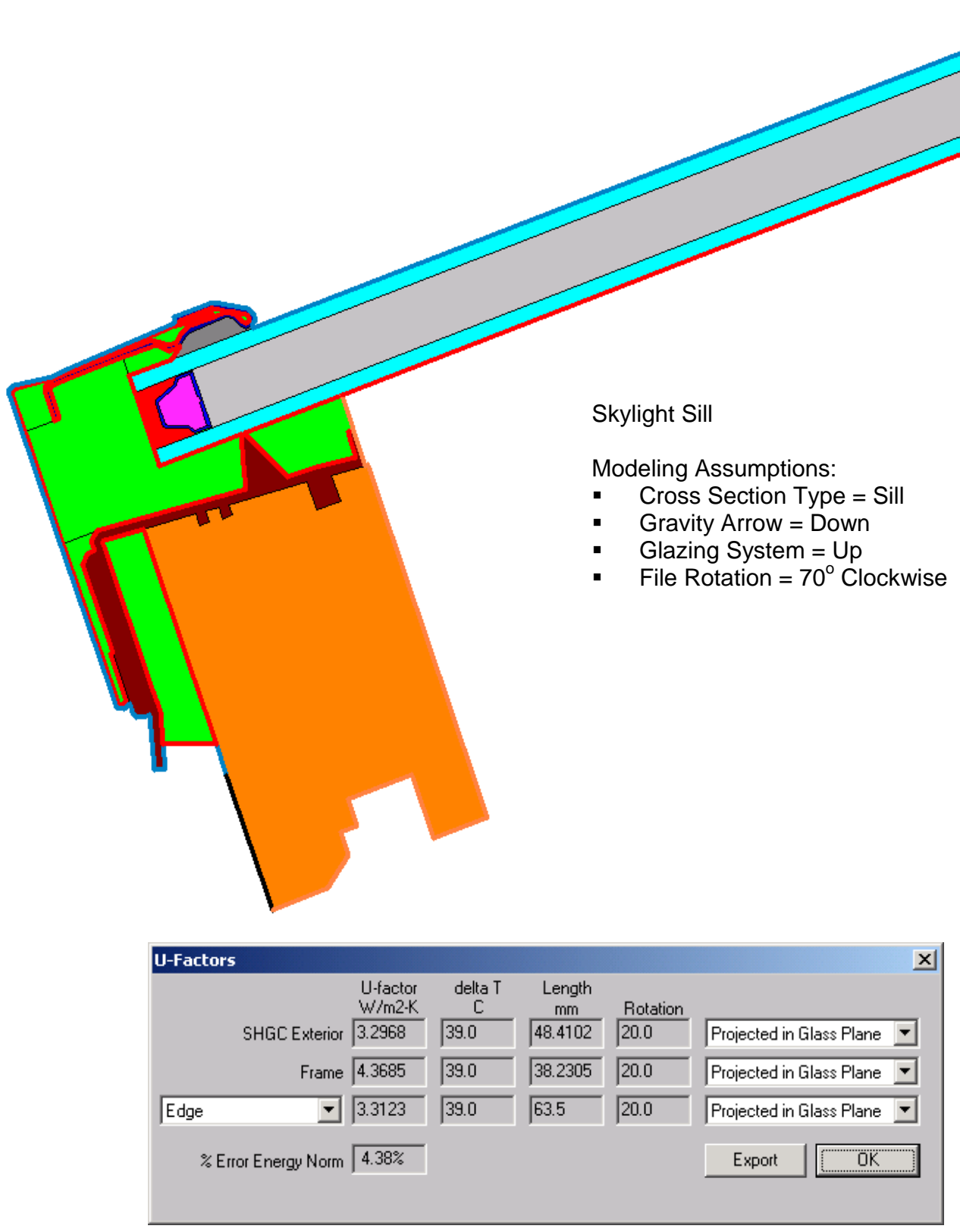

Figure 9-48. THERM file for Head and Sill inset mount skylight cross section. 


\section{Jamb}

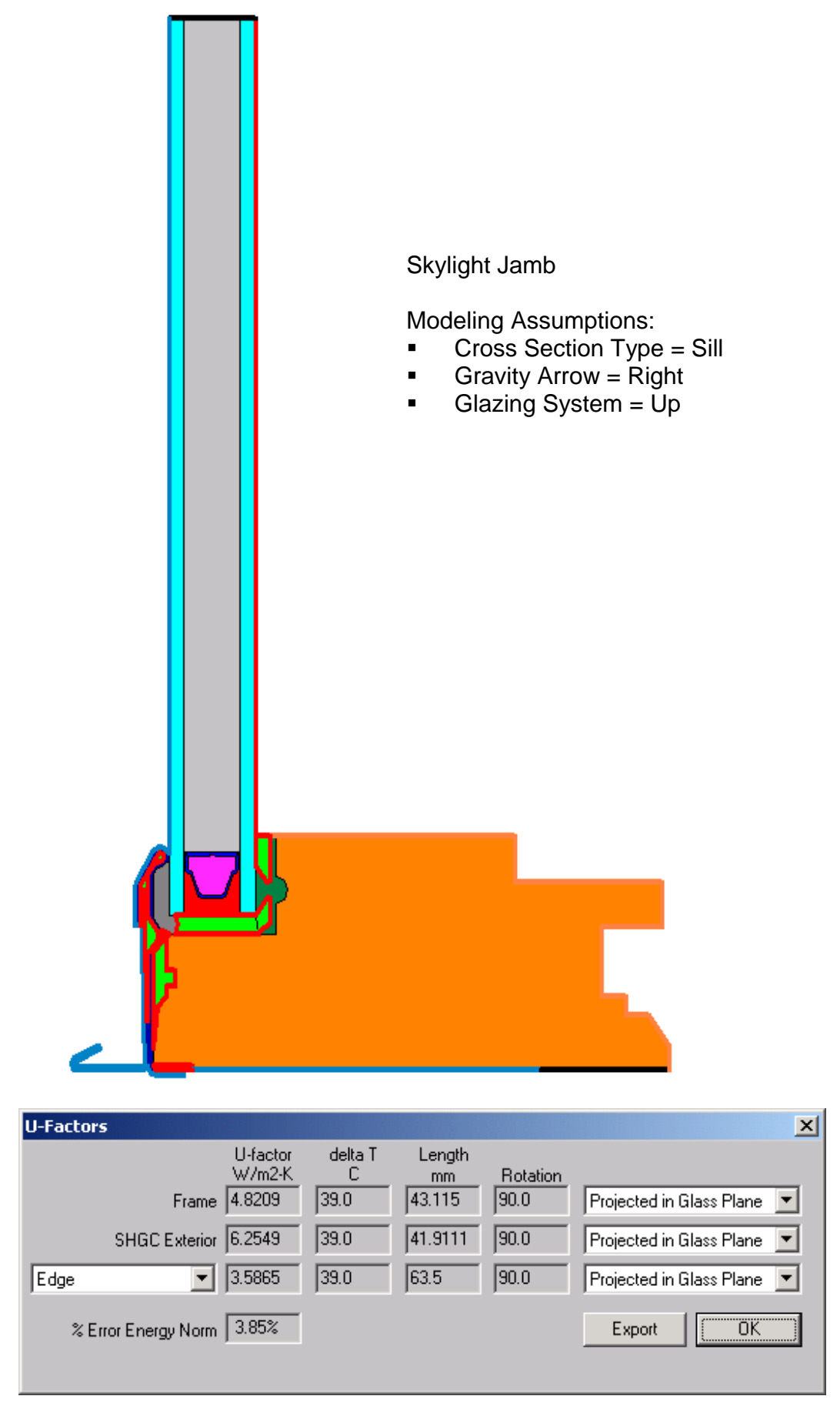

Figure 9-49. THERM file for Jamb inset mount skylight cross section. 


\subsubsection{Total Product U-Factor}

In WINDOW, import the THERM cross sections into the Frame Library.

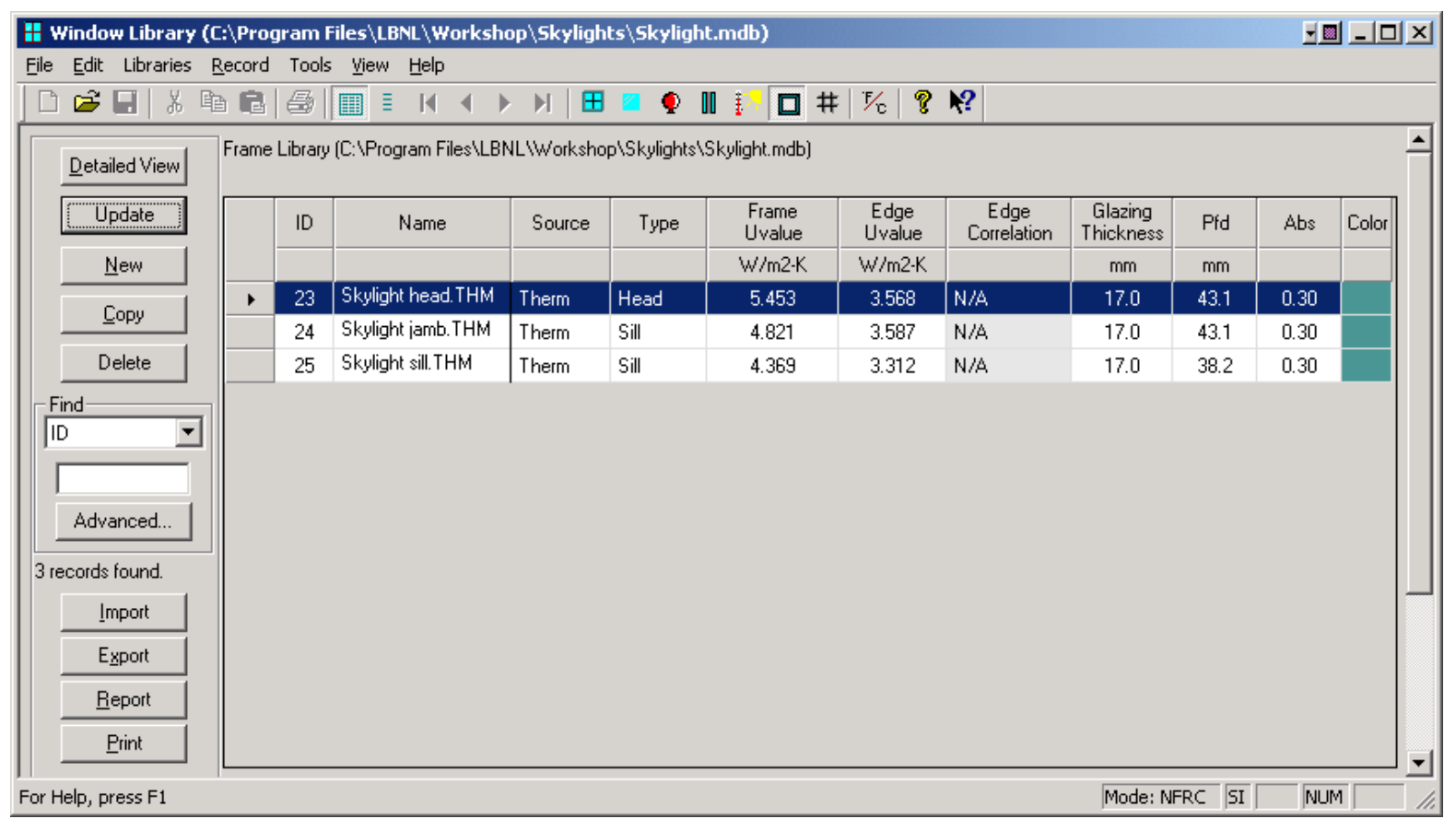

Figure 9-50. THERM files imported into the Frame Library.

In the WINDOW Window Library, one record is created.

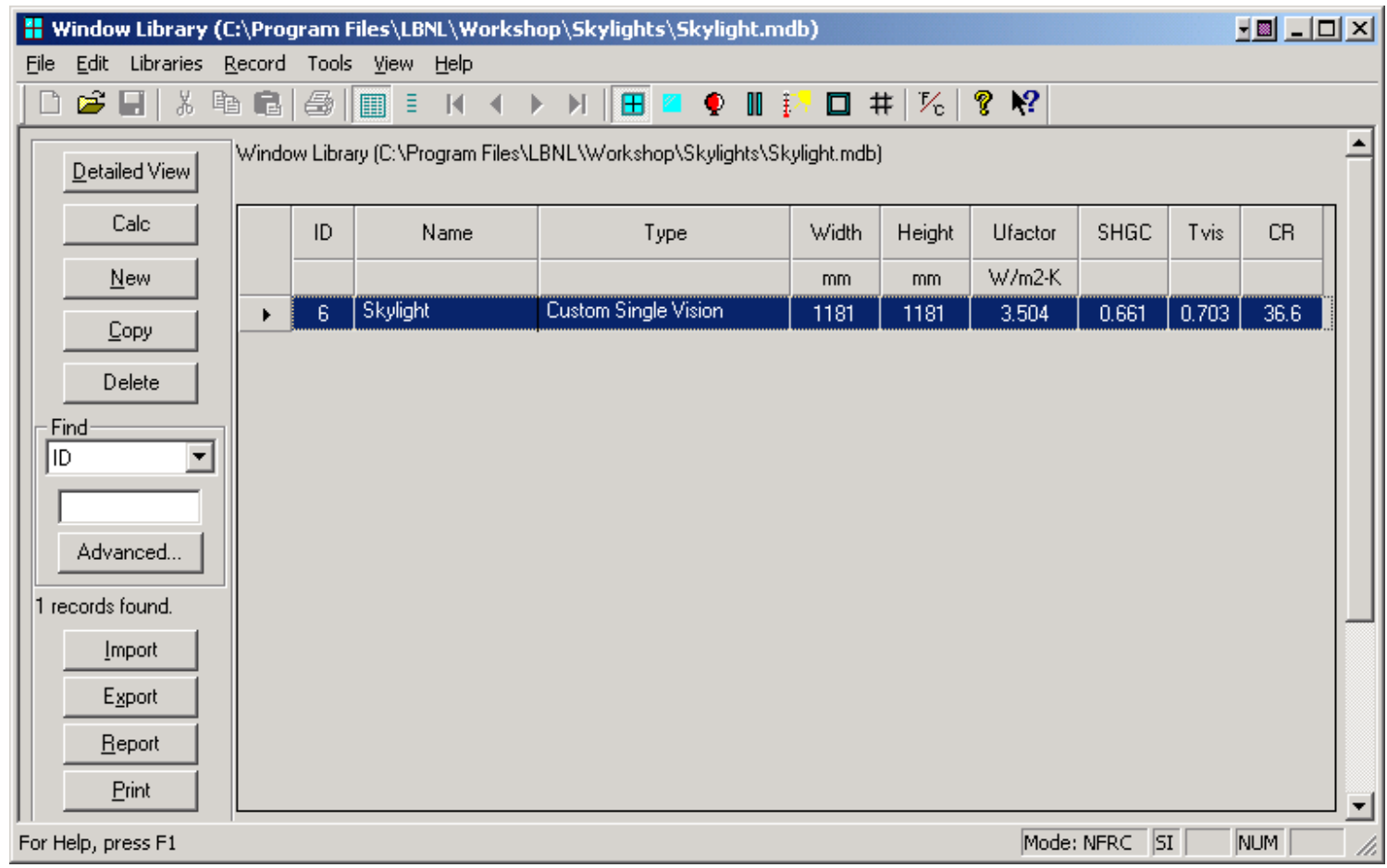

Figure 9-51. Window Library skylight record. 
Table 9-20 shows the overall product U-factor from the Window Library.

Table 9-15. Total Product U-factors for skylight

\begin{tabular}{|l|l|l|l|}
\hline \multicolumn{2}{|c|}{ Glazing Options } & \multicolumn{2}{c|}{ Total Product U-factor } \\
\cline { 3 - 4 } & $\left(\mathbf{W} / \mathbf{m}^{2}{ }^{-}{ }^{\circ} \mathbf{C}\right)$ & $\left(\mathbf{B t u}_{\left.\mathbf{h r}-\mathrm{ft}^{2}{ }^{\circ}{ }^{\circ} \mathbf{F}\right)}\right.$ \\
\hline 1 & Clear (3 mm), Air, Clear (3mm), No Dividers & 3.504 & 0.617 \\
\hline
\end{tabular}




\subsubsection{Individual Product SHGC and VT using SHGC 0 \& 1 and VT 0 \& 1}

The methodology for determining the Solar Heat Gain Coefficient (SHGC) and Visible Transmittance (VT) for products is outlined in NFRC 200 using values of $\mathrm{SHGC}_{0}, \mathrm{SHGC}_{1}, \mathrm{VT}_{0}, \mathrm{VT}_{1}$. These values are calculated in WINDOW for the best glazing option modeled with the highest frame and edge U-factor frame, as outlined in NFRC 200, Section 6.1.1 (a).

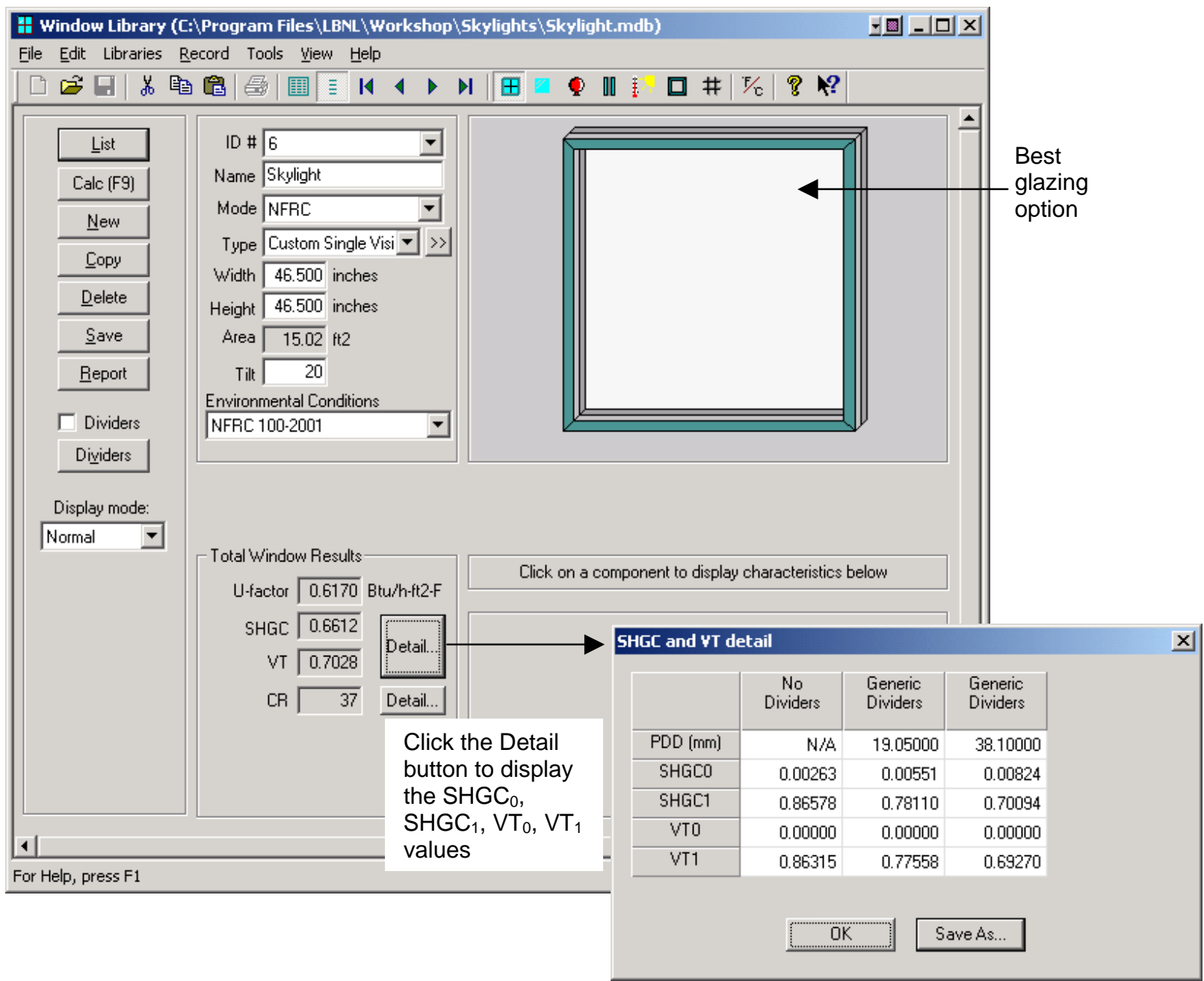

Figure 9-52. Window Library Detail button calculates $S H G C_{0}, S H G C_{1}, V T_{0}, V T_{1}$.

\subsubsection{Skylight Drawings.}

Drawings for the skylight are not available. 


\subsection{Problem 4: Door}

For this wood stile and rail door, calculate the U-factor, SHGC and VT.

\subsubsection{Description}

\begin{tabular}{|c|c|}
\hline Door Type & Wood stile and rail door \\
\hline Frame Material & Wood with a thermal-break aluminum sill \\
\hline Overall Size & $1000 \mathrm{~mm}$ x $2000 \mathrm{~mm}\left(39^{\prime \prime} \times 79^{\prime \prime}\right)$ door slab \\
\hline Glazing System & $\begin{array}{l}\text { Double glazing, } 0.500^{\prime \prime} \text { overall IG thickness. There are four individual glass options all } \\
\text { with two layers of double-strength PPG glass ( } 0.129^{\prime \prime} \text { thickness). The first option is two } \\
\text { layers of clear glass air filled, the second is two layers of clear glass argon filled ( } 90 \% \\
\text { two probe filling), the third is two layers of clear glass with Solarban } 60 \text { on surface } 2 \text { and } \\
\text { air filled, the fourth option is two layers of clear glass with Solarban } 60 \text { on surface } 2 \text { and } \\
\text { argon filled. }\end{array}$ \\
\hline Spacer Type & Intercept $\mathrm{TM}^{\mathrm{TM}}$ tin-plate with butyl sealant on three sides \\
\hline Glazing Method & Wet glazed with Silicone on both sides \\
\hline Dividers & There are no dividers in this product \\
\hline Drawings & $\begin{array}{l}\text { There are detailed drawings for this door shown in Section 9.5.7. Also included on the } \\
\text { CD are the DXF files of the assembly drawings for this door. }\end{array}$ \\
\hline
\end{tabular}

\subsubsection{Glazing Matrix}

The table below lists the four glazing options for the door.

Table 9-16. Glazing Options Matrix

\begin{tabular}{|l|}
\hline Glass Option \\
\hline 1. Clear_Air_Clear \\
\hline 2. Clear_Argon $(95 \%)$ _Clear \\
\hline 3. Low-e_Air_Clear \\
\hline 4. Low-e_Argon $(95 \%)$ Clear \\
\hline
\end{tabular}




\subsubsection{Center-of-Glazing Modeling (WINDOW)}

The four IG units were modeled in WINDOW with double-strength glass, 0.242 " air space, $95 \%$ argon (where appropriate), and Solarban 60 (where appropriate).

Figure 9-58 shows the WINDOW Glazing System Library with all four IG units.

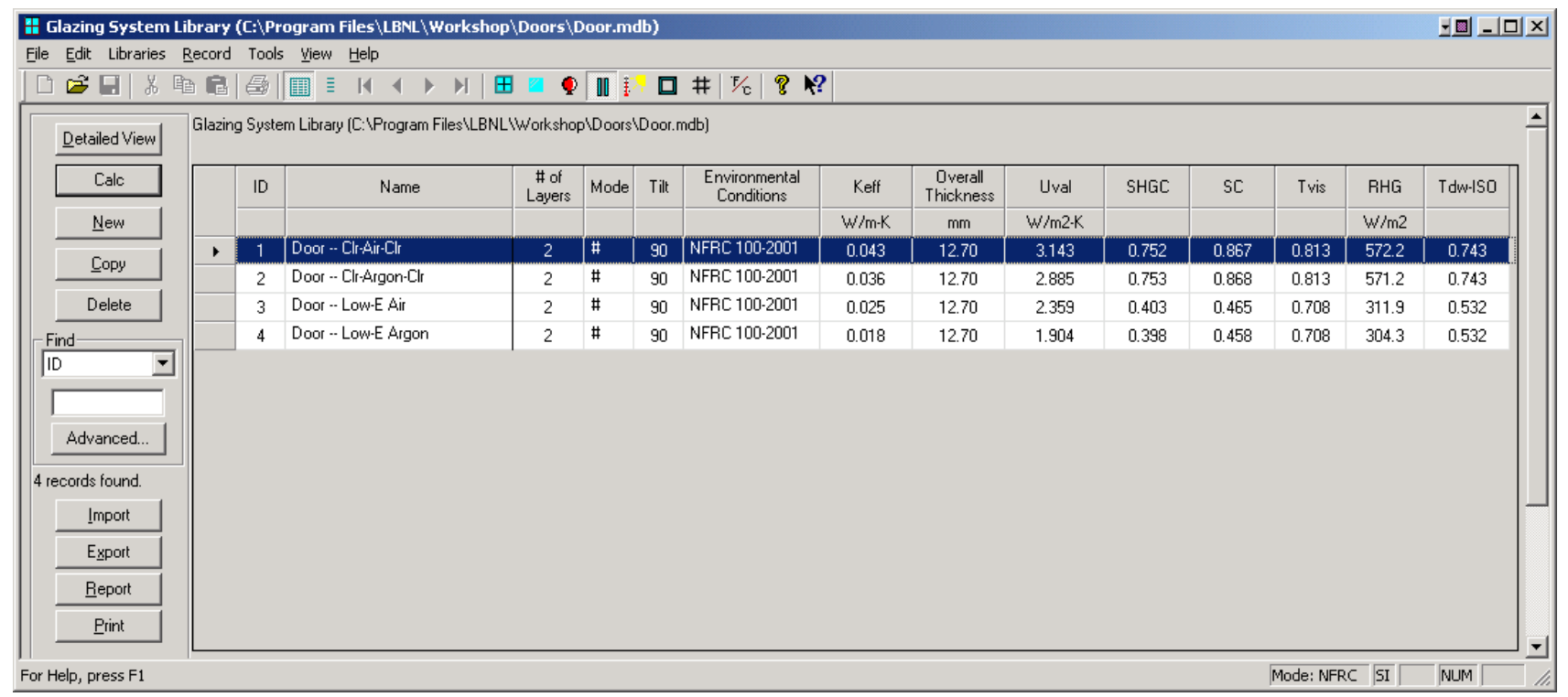

Figure 9-53. WINDOW 5 Glazing System Library for the door lites.

The results for the center-of-glazing U-factor are shown in the following table:

Table 9-17. Center-of-glazing U-factor results from WINDOW

\begin{tabular}{|c|c|c|c|}
\hline \multirow{2}{*}{\multicolumn{2}{|c|}{ Glazing Options }} & \multicolumn{2}{|c|}{ Center-of-glazing U-factor } \\
\hline & & \multirow{2}{*}{$\frac{\left(\mathrm{W} / \mathrm{m}^{2}-{ }^{\circ} \mathrm{C}\right)}{3.143}$} & \multirow{2}{*}{$\frac{\left(\mathbf{B t u} / \mathbf{h r}-\mathrm{ft}^{\mathbf{2}} \mathbf{}^{\circ} \mathbf{F}\right)}{0.554}$} \\
\hline 1 & $\begin{array}{l}\text { Clear }\left(3.277 \mathrm{~mm}, 0.129^{\prime \prime}\right) \text {, Air }(6.2 \mathrm{~mm} \text {, } \\
\left.0.242^{\prime \prime}\right) \text {, Clear }\left(3.277 \mathrm{~mm}, 0.129^{\prime \prime}\right)\end{array}$ & & \\
\hline 2 & $\begin{array}{l}\text { Clear (3.277 mm 0.129"), Argon (95\%, } 6.2 \\
\left.\text { mm, 0.242"), Clear ( } 3.277 \mathrm{~mm}, 0.129^{\prime \prime}\right)\end{array}$ & 2.885 & 0.508 \\
\hline 3 & 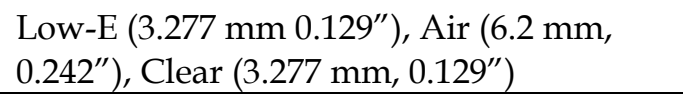 & 2.359 & 0.416 \\
\hline 4 & $\begin{array}{l}\text { Low-E (3.277 mm, 0.129"), Argon (95\%, } 6.2 \\
\left.\text { mm, 0.242"), Clear ( } 3.277 \mathrm{~mm}, 0.129^{\prime \prime}\right)\end{array}$ & 1.904 & 0.335 \\
\hline
\end{tabular}

These U-factors will be used with the edge-of-glazing and frame values from THERM to calculate the overall U-factor for the door in Section 9.5. 


\subsubsection{Edge-of-Glazing and Frame Modeling (THERM)}

An entry door requires eight THERM models to completely model the heat flow through the door. The eight models are:

- Head

- Lock jamb

- Hinge jamb

- Sill

- Panel

- Door lite

- Panel core

- Door core

This door design has identical sections for the head, lock jamb, and hinge jamb so only one THERM model will be required to represent jamb sections. The door core model has been added to the head model and the panel core model has been added to the panel model. Four door lite models are required, one for each glass option.

Table 9-24 shows the files that are included on the CD for this example problem. The Door Lite base case file (Lite.thm) has glazing option 1, and has Glazing System Options defined for the other three glazing options. When Lite.thm is simulated, it will automatically create the four door lite option files, lite_01.thm through lite_04.thm.

Table 9-18. Files associated with the wood door example

\begin{tabular}{|l|l|}
\hline Cross Section & \multicolumn{1}{c|}{ THERM file } \\
\hline Head & head.thm \\
\hline Lock Jamb, Hinge Jamb & jamb.thm \\
\hline Sill & sill.thm \\
\hline Panel & panel-edge.thm \\
\hline $\begin{array}{l}\text { Door Lite Base Case } \\
\text { (with 4 glazing options) }\end{array}$ & Lite.thm \\
\hline Door Lite Option 1 & lite_001.thm \\
\hline Door Lite Option 2 & lite_002.thm \\
\hline Door Lite Option 3 & lite_003.thm \\
\hline Door Lite Option 4 & lite_004.thm \\
\hline
\end{tabular}

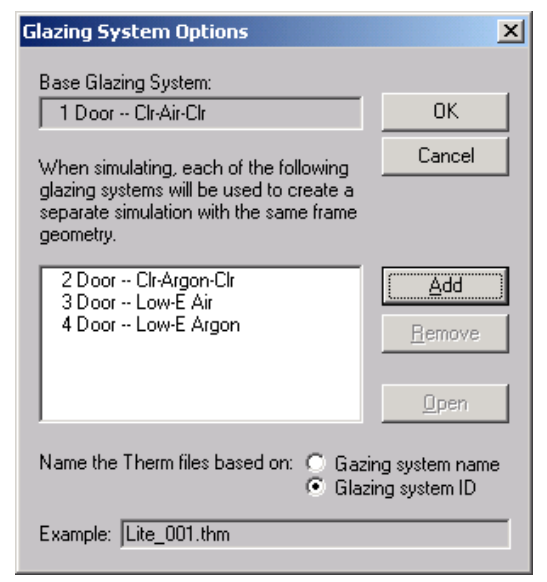

Figure 9-54. Glazing System Options for Lite.thm 
Table 9-25 shows the resulting U-factors from each cross section for the $3 \mathrm{~mm}$ (with dividers) glazing case.

Table 9-19. THERM results for vinyl window cross sections

\begin{tabular}{|c|c|c|c|c|c|c|c|c|}
\hline \multirow[b]{2}{*}{ Cross Section } & \multicolumn{2}{|c|}{ Frame U-factor } & \multicolumn{2}{|c|}{ Edge U-factor } & \multicolumn{2}{|c|}{$\begin{array}{l}\text { Frame } \\
\text { Height (in) }\end{array}$} & \multicolumn{2}{|c|}{$\begin{array}{l}\text { Edge } \\
\text { Height (in) }\end{array}$} \\
\hline & $\begin{array}{l}\left(W / m^{2}-\right. \\
\left.{ }^{\circ} \mathrm{C}\right)\end{array}$ & $\begin{array}{l}\text { (Btu/hr } \\
\left.-\mathrm{ft}^{2}-{ }^{\circ} \mathrm{F}\right)\end{array}$ & $\begin{array}{l}\left(W / m^{2}-\right. \\
\left.{ }^{\circ} \mathrm{C}\right)\end{array}$ & $\begin{array}{l}\text { (Btu/hr } \\
\left.-\mathrm{ft}^{2}-{ }^{\circ} \mathrm{F}\right)\end{array}$ & $\mathrm{mm}$ & inch & $\mathrm{mm}$ & inch \\
\hline Head & 1.7682 & 0.3114 & 1.9437 & 0.3423 & 22.225 & 0.875 & 63.500 & 2.500 \\
\hline $\begin{array}{l}\text { Lock Jamb, } \\
\text { Hinge Jamb }\end{array}$ & 1.8431 & 0.3246 & 1.9581 & 0.3448 & 22.225 & 0.875 & 63.500 & 2.500 \\
\hline $\begin{array}{l}\text { Door Core } \\
\text { (from the head model) }\end{array}$ & 2.0440 & 0.3600 & $\mathrm{~N} / \mathrm{A}$ & $\mathrm{N} / \mathrm{A}$ & $\mathrm{N} / \mathrm{A}$ & $\mathrm{N} / \mathrm{A}$ & $\mathrm{N} / \mathrm{A}$ & N/A \\
\hline Sill & 0.7763 & 0.7763 & 2.0855 & 0.3673 & 39.696 & 1.563 & 63.4919 & 2.500 \\
\hline Panel & 2.0076 & 0.3535 & 2.9372 & 0.5172 & 25.400 & 1.000 & 76.597 & 3.0156 \\
\hline $\begin{array}{l}\text { Panel Core (from the } \\
\text { panel model) }\end{array}$ & 2.5847 & 0.4552 & N/A & N/A & $\mathrm{N} / \mathrm{A}$ & $\mathrm{N} / \mathrm{A}$ & $\mathrm{N} / \mathrm{A}$ & N/A \\
\hline Door Lite Option 1 & 2.799 & 0.493 & 3.260 & 0.574 & 42.063 & 1.656 & 63.500 & 2.500 \\
\hline Door Lite Option 2 & 2.780 & 0.490 & 3.051 & 0.537 & 42.063 & 1.656 & 63.500 & 2.500 \\
\hline Door Lite Option 3 & 2.744 & 0.483 & 2.632 & 0.464 & 42.063 & 1.656 & 63.500 & 2.500 \\
\hline Door Lite Option 4 & 2.716 & 0.478 & 2.277 & 0.401 & 42.063 & 1.656 & 63.500 & 2.500 \\
\hline
\end{tabular}




\section{Head}

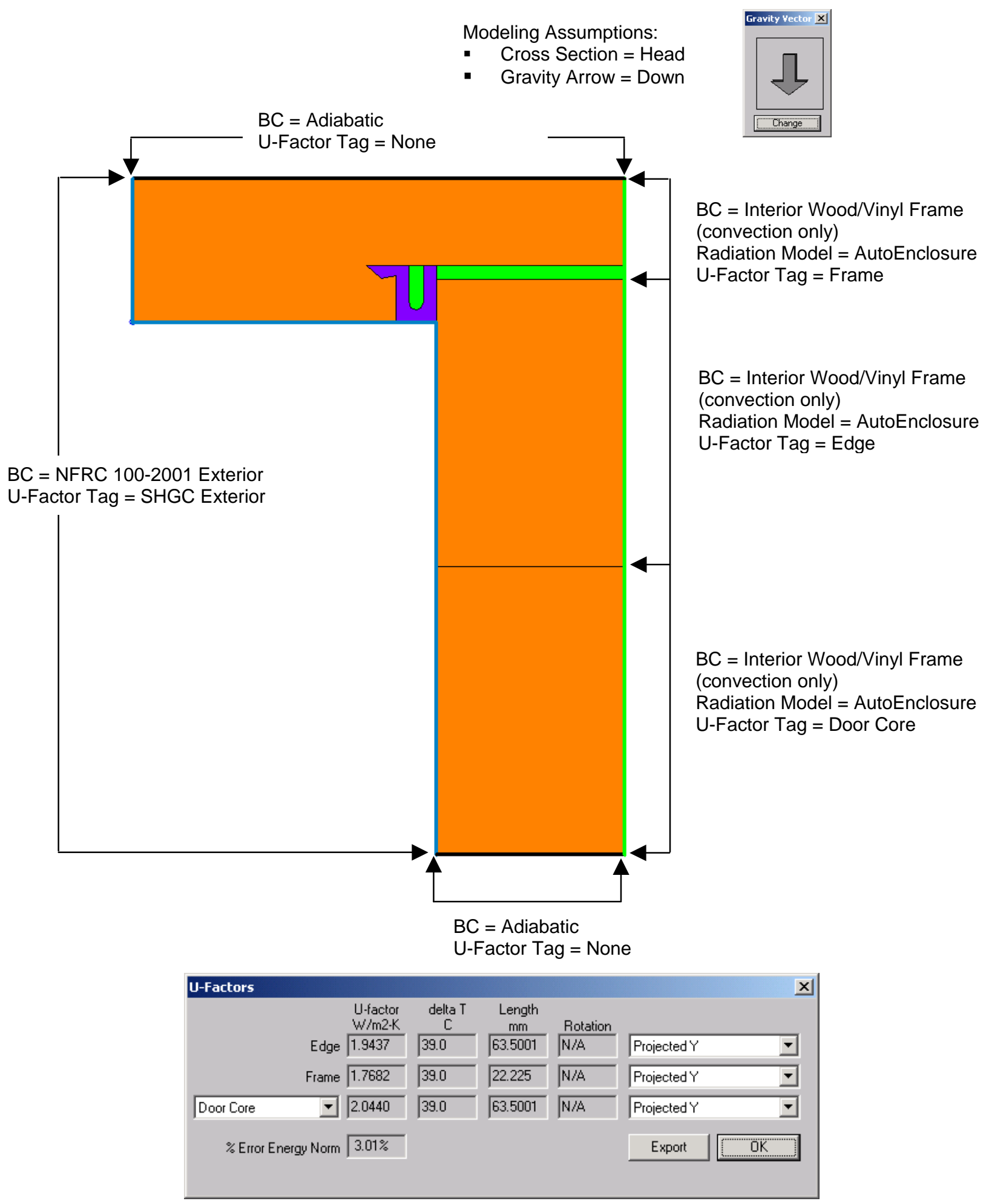

Figure 9-55. Door Head THERM file. 


\section{Jamb}

Modeling Assumptions:

- Cross Section = Jamb

- Gravity Arrow = Into the screen
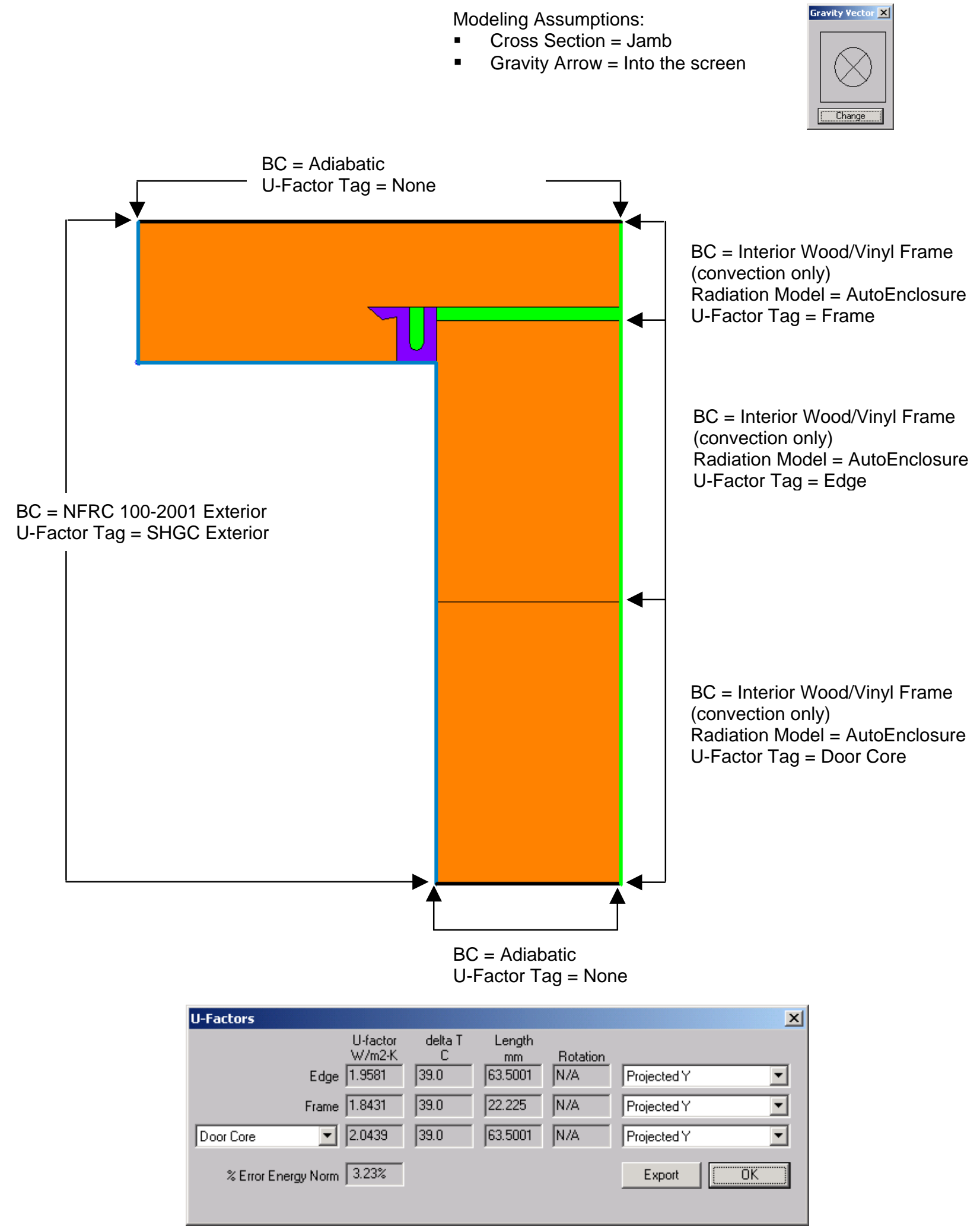

Figure 9-56. Door Jamb THERM file. 


\section{Sill}

Modeling Assumptions:

- $\quad$ Cross Section = Sill

- Gravity Arrow = Down

Modeling Assumptions:

- Cross Section = Sill

$\mathrm{BC}=$ Adiabatic

- Gravitv Arrow = Down

U-Factor Surface tag $=$ None
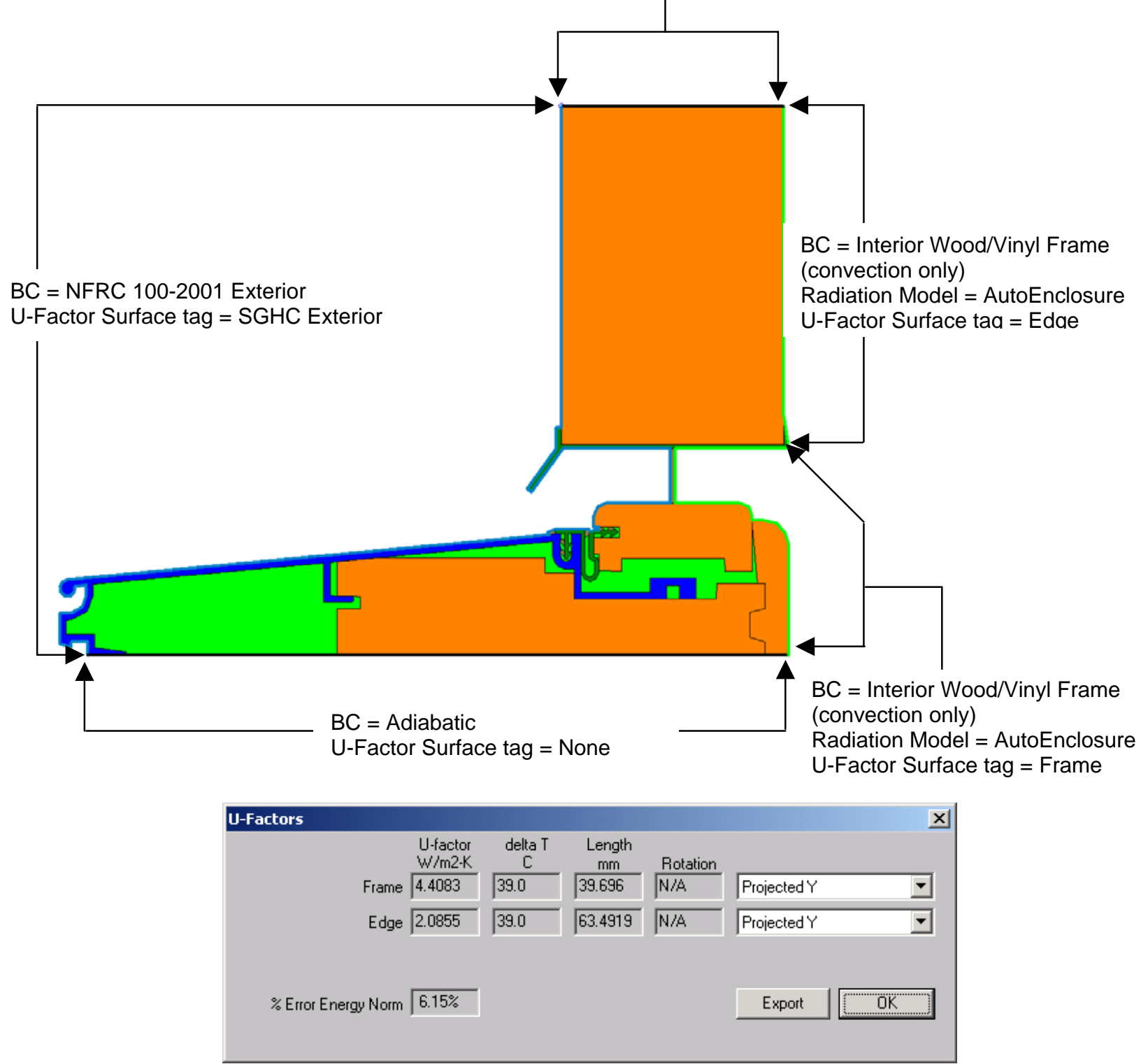

Figure 9-57. Door Sill THERM file. 


\section{Panel-Edge}

Modeling Assumptions:

- $\quad$ Cross Section = Head

- Gravity Arrow = Down
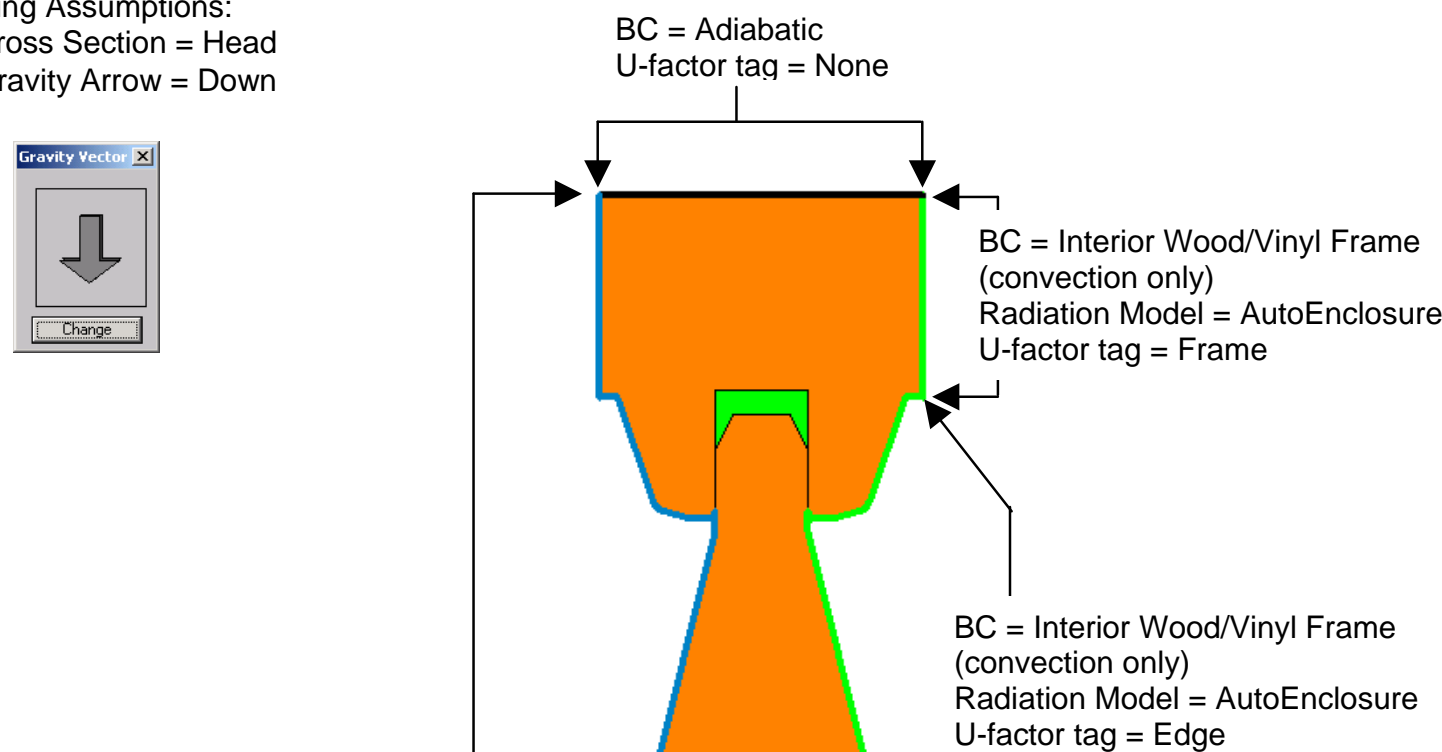

$\mathrm{BC}=\mathrm{NFRC}$ 100-2001 Exterior $\mathrm{U}$-factor tag $=$ SHGC Exterior

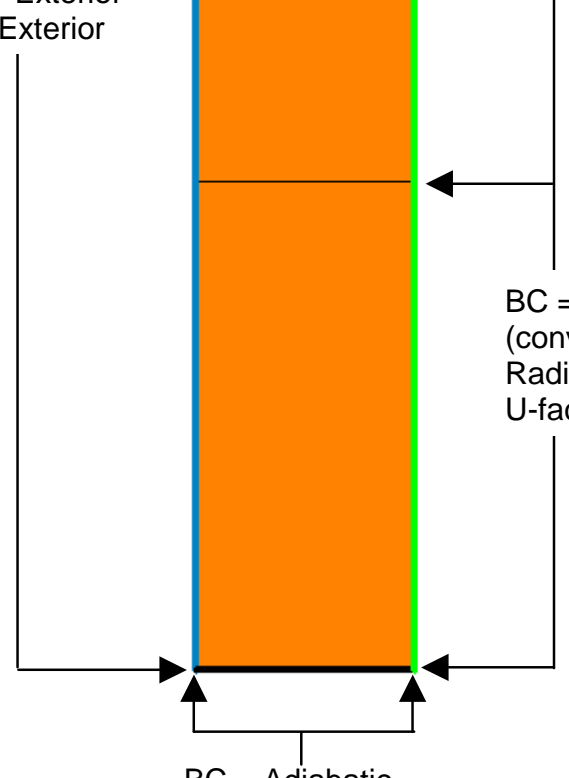

$\mathrm{BC}=$ Adiabatic

U-factor tag $=$ None

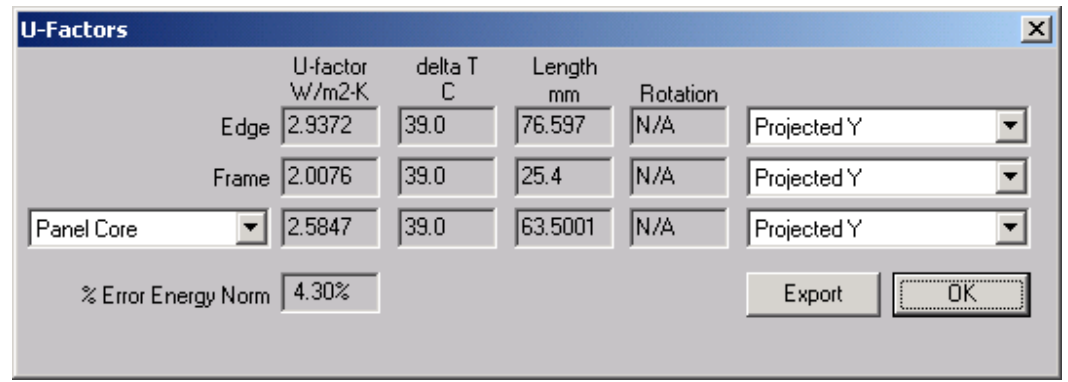

Figure 9-58. Door Panel Edge THERM file. 


\section{Door Lite}

Modeling Assumptions:

- Cross Section = Sill

- Gravity Arrow = Down

$\mathrm{BC}=$ Adiabatic

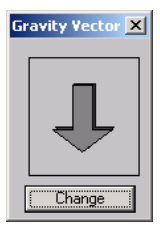

U-Factor tag $=$ None

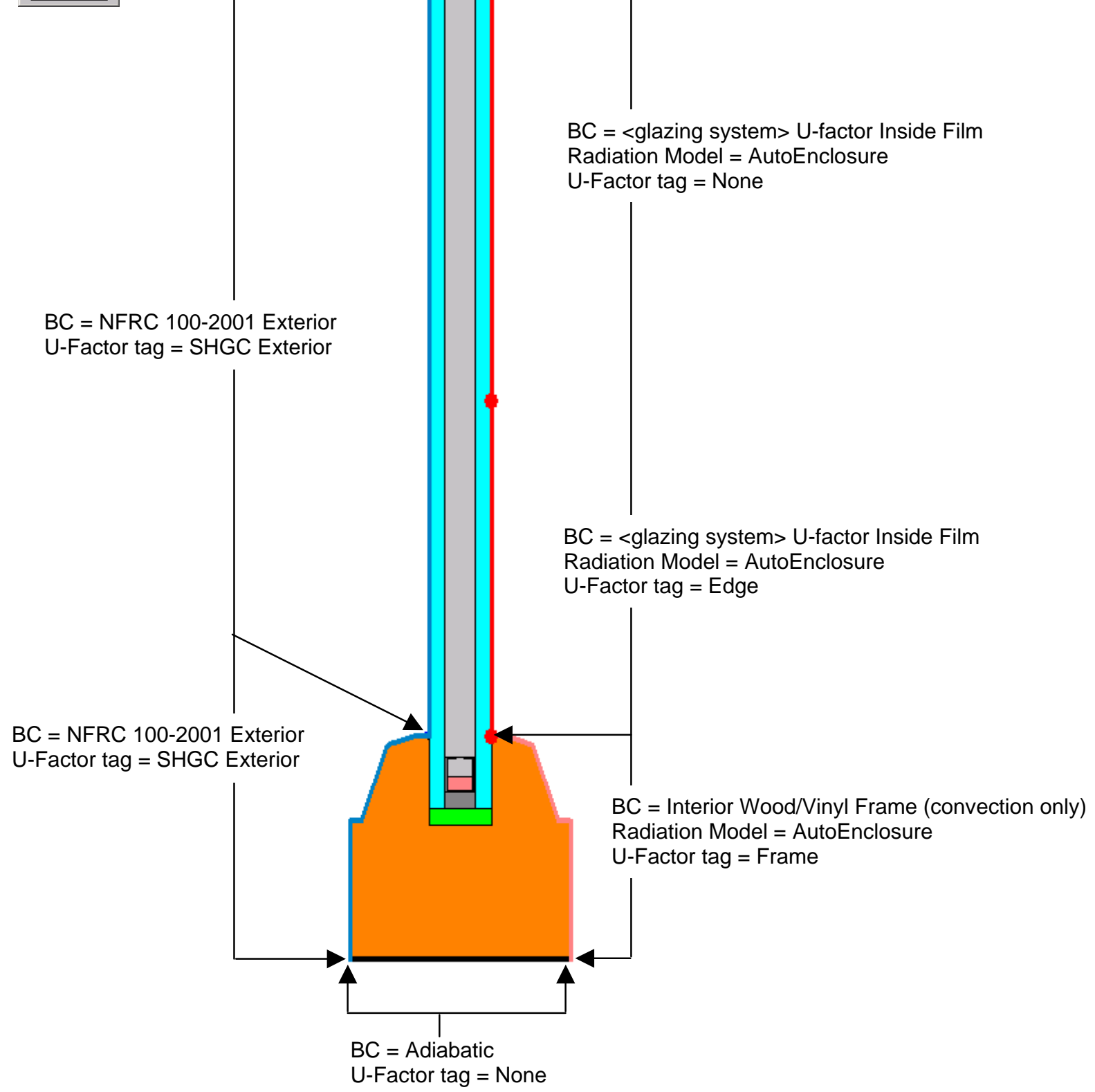

Figure 9-59. Door Lite THERM file. 


\subsubsection{Total Product U-Factor, SHGC and VT}

The results from these THERM and WINDOW runs can be used in a spreadsheet to calculate the total product U-factor, SHGC and VT. That spreadsheet calculation is outside the scope of this manual and is not included.

\subsubsection{Wood Stile and Rail Door Drawings.}

Note that some of the drawings provided (indicated with an asterisk on the following pages) are patented drawings and shall not be used by anyone without the written consent from NFRC. 


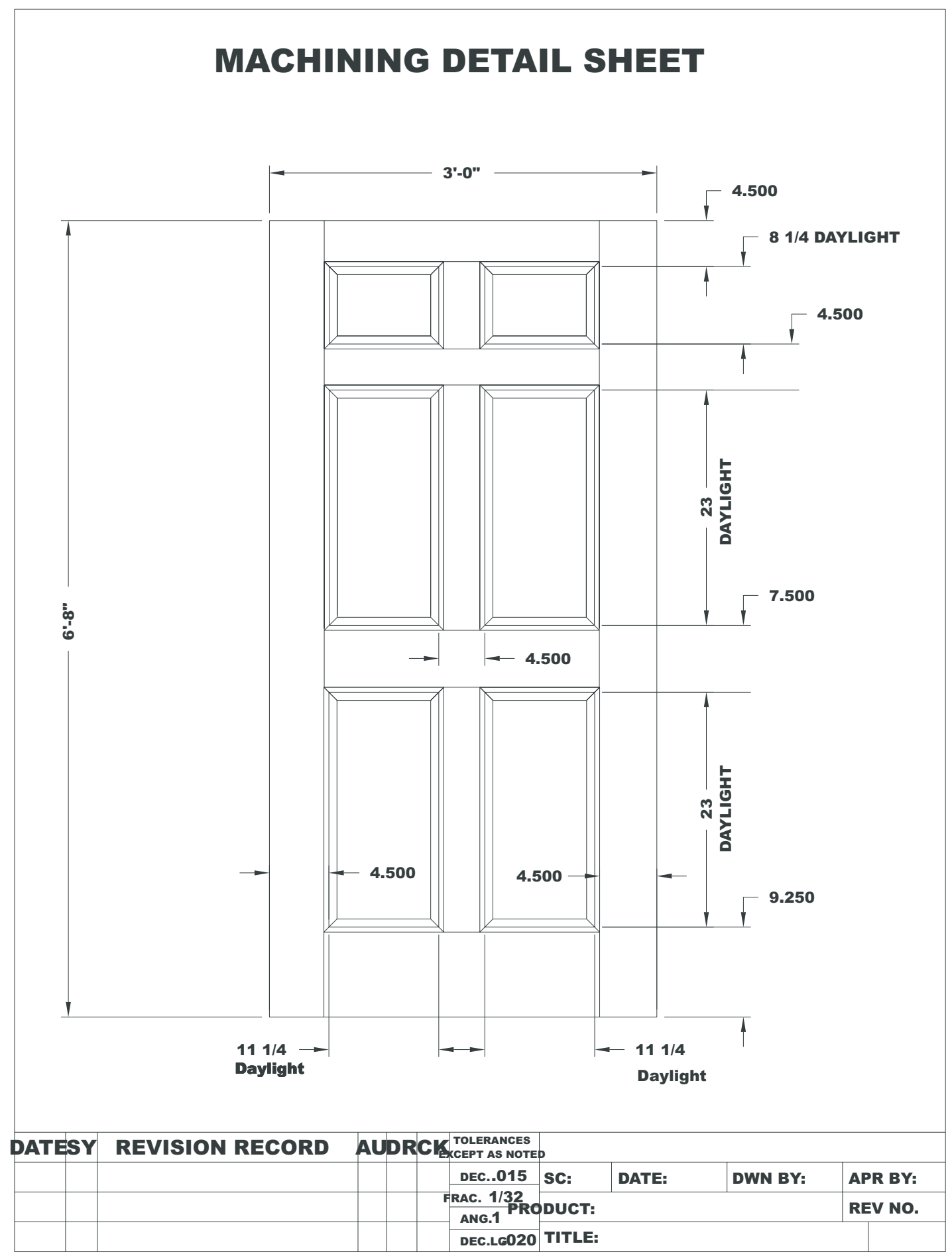

Figure 9-60. 6-Panel Panel Layout.dwg 


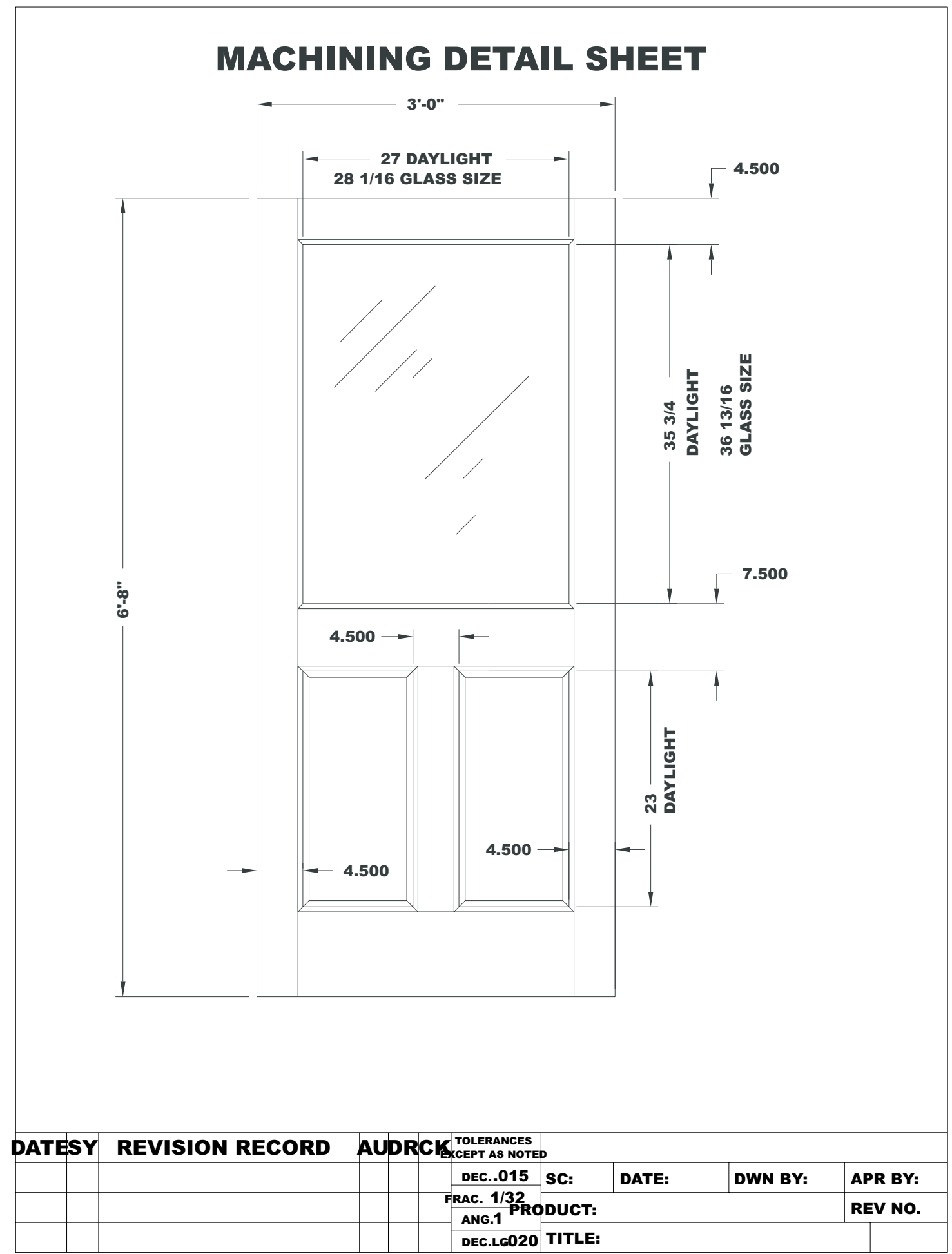

Figure 9-61. Half Panel with Glass Layout.dwg 


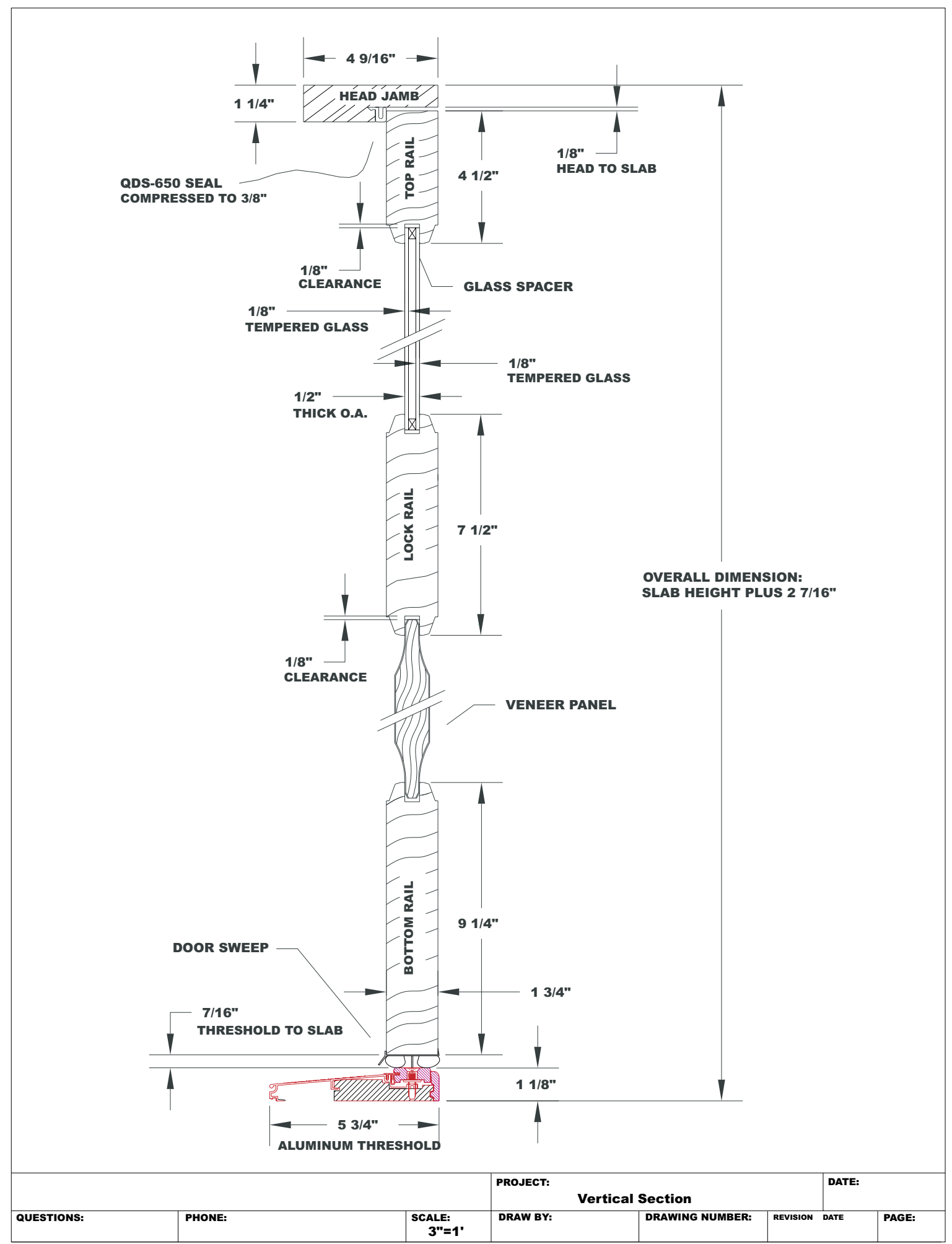

Figure 9-62. Half Panel with Glass Vert Sec.dwg 


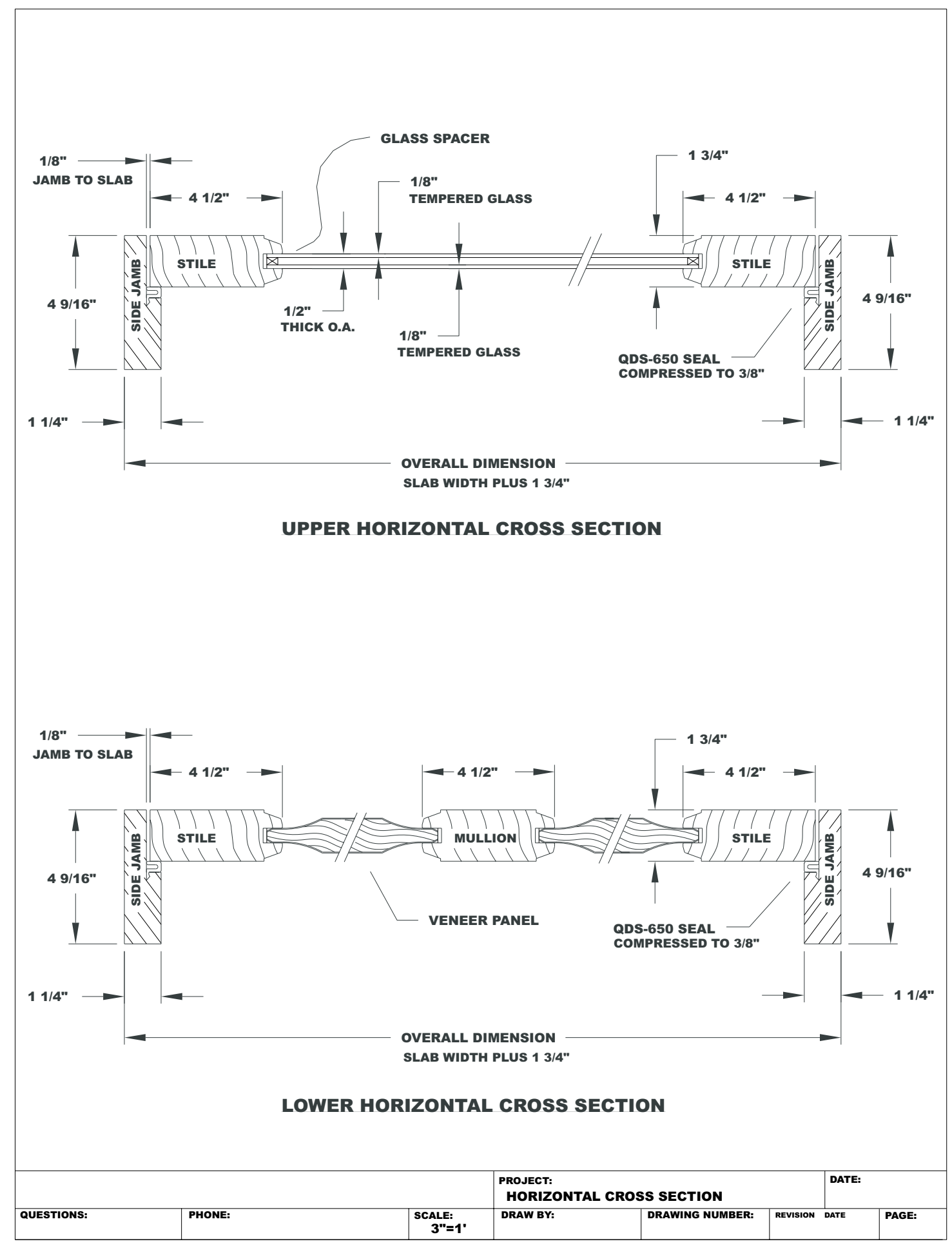

Figure 9-63. Half Panel with Glass Horz Sec.dwg 


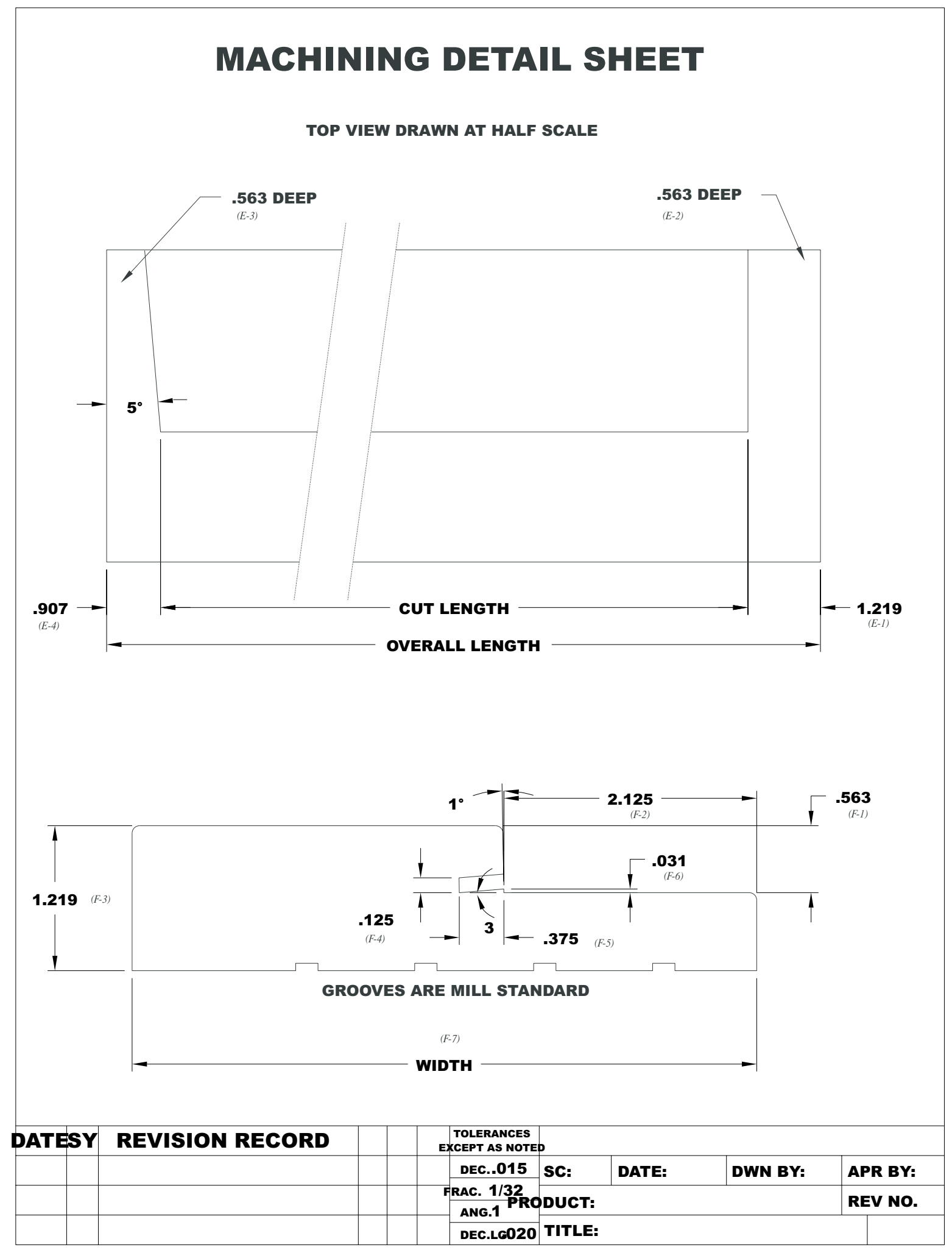

Figure 9-64. 6-Panel Jamb.dwg 


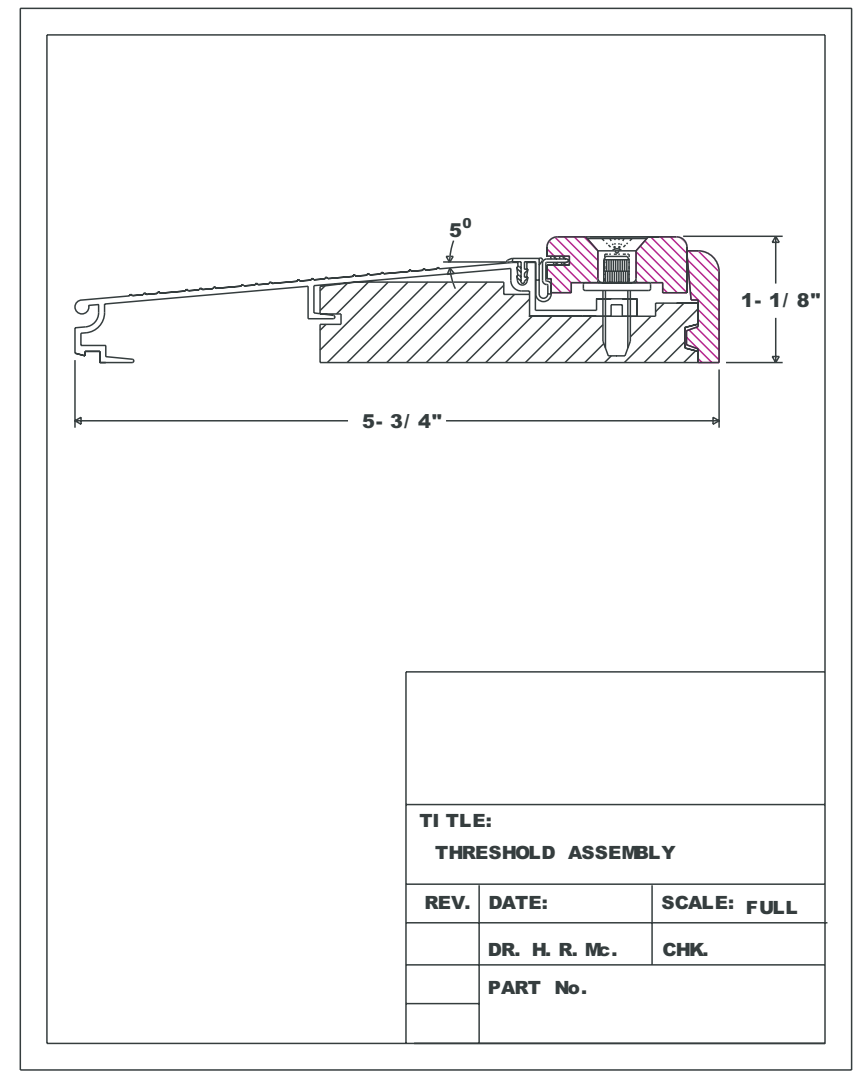

Figure 9-65. 6-Panel Sill.dwg ( ${ }^{*}$ PATENTED DRAWING)

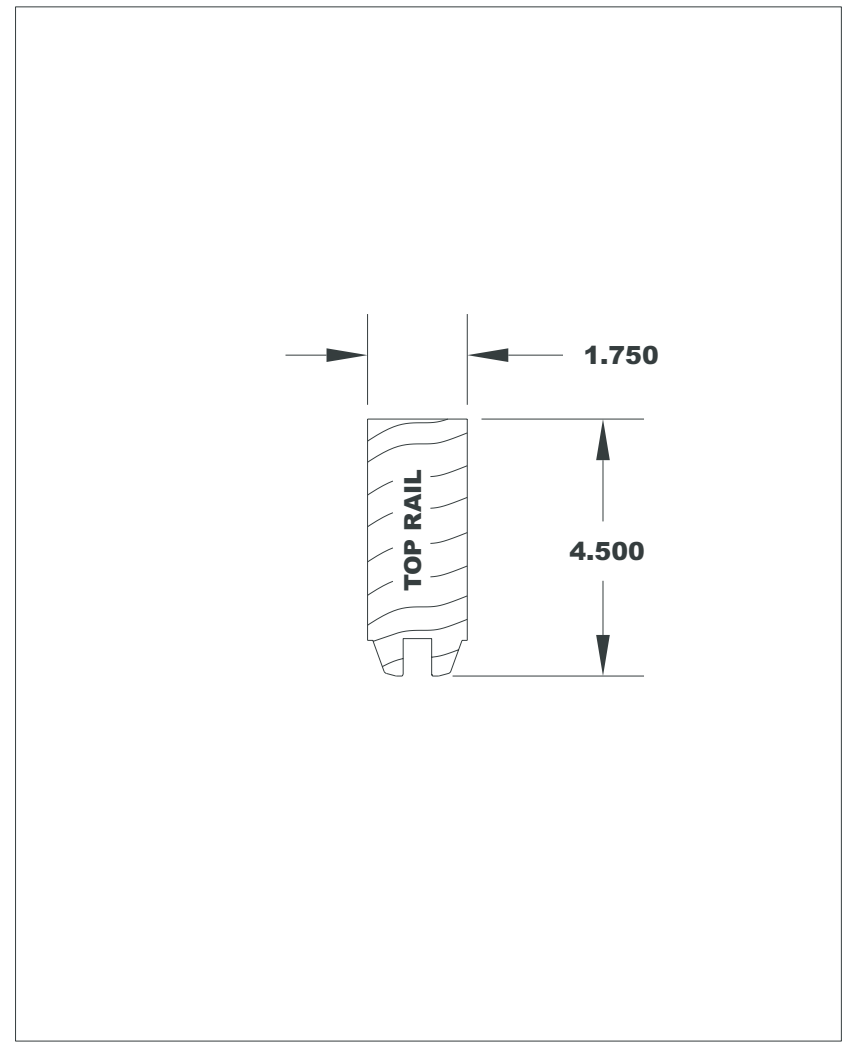

Figure 9-66. 6-Panel Top Rail.dwg 


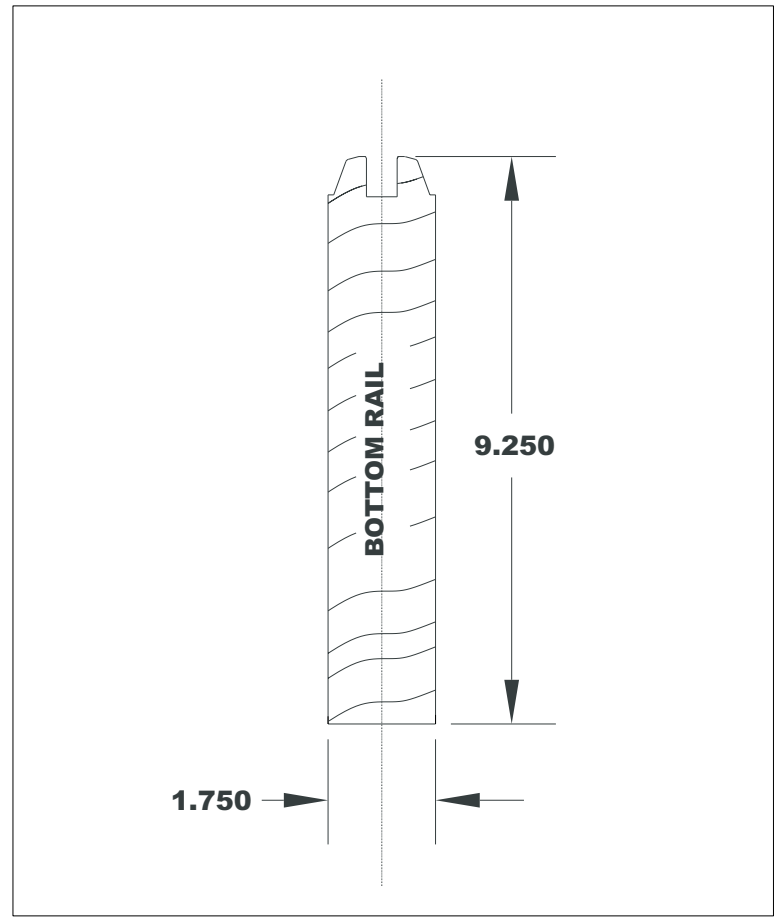

Figure 9-67. 6-Panel Bottom Rail.dwg

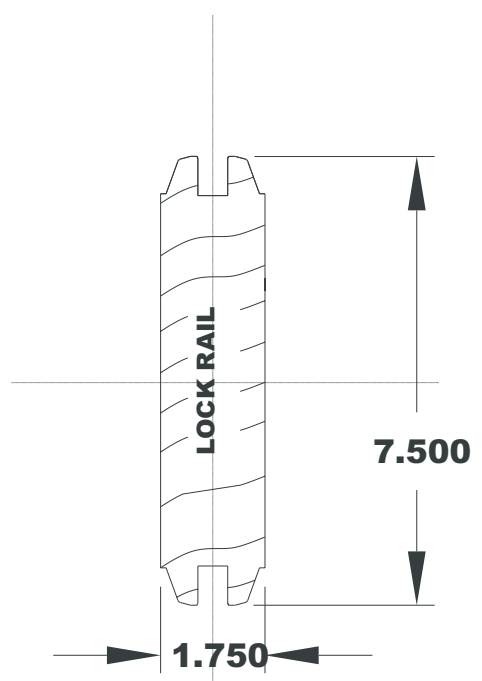

Figure 9-68. 6-Panel Lock Rail.dwg 


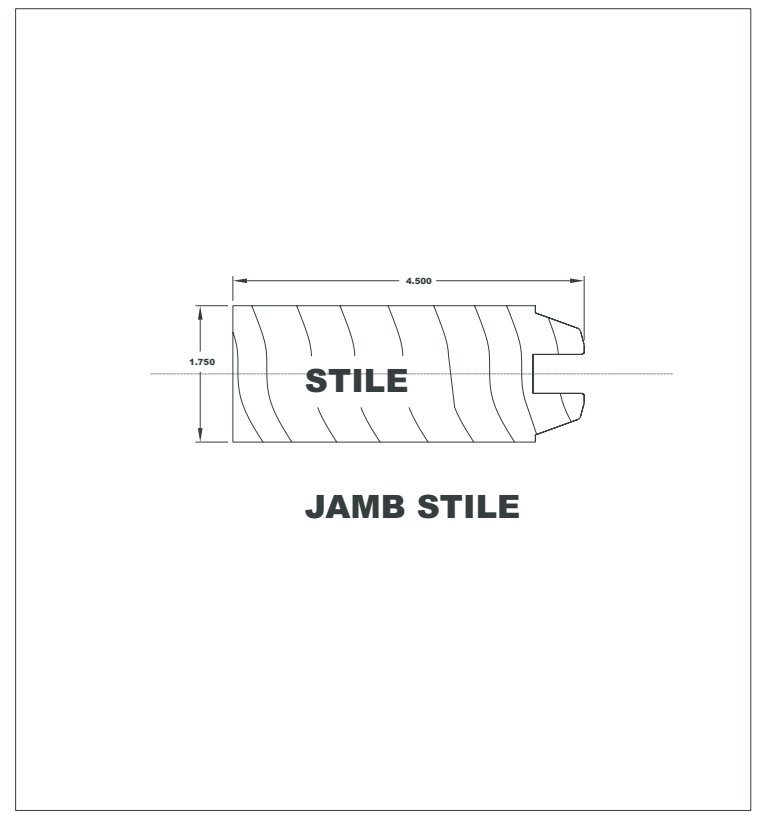

Figure 9-69. 6-Panel Stile.dwg

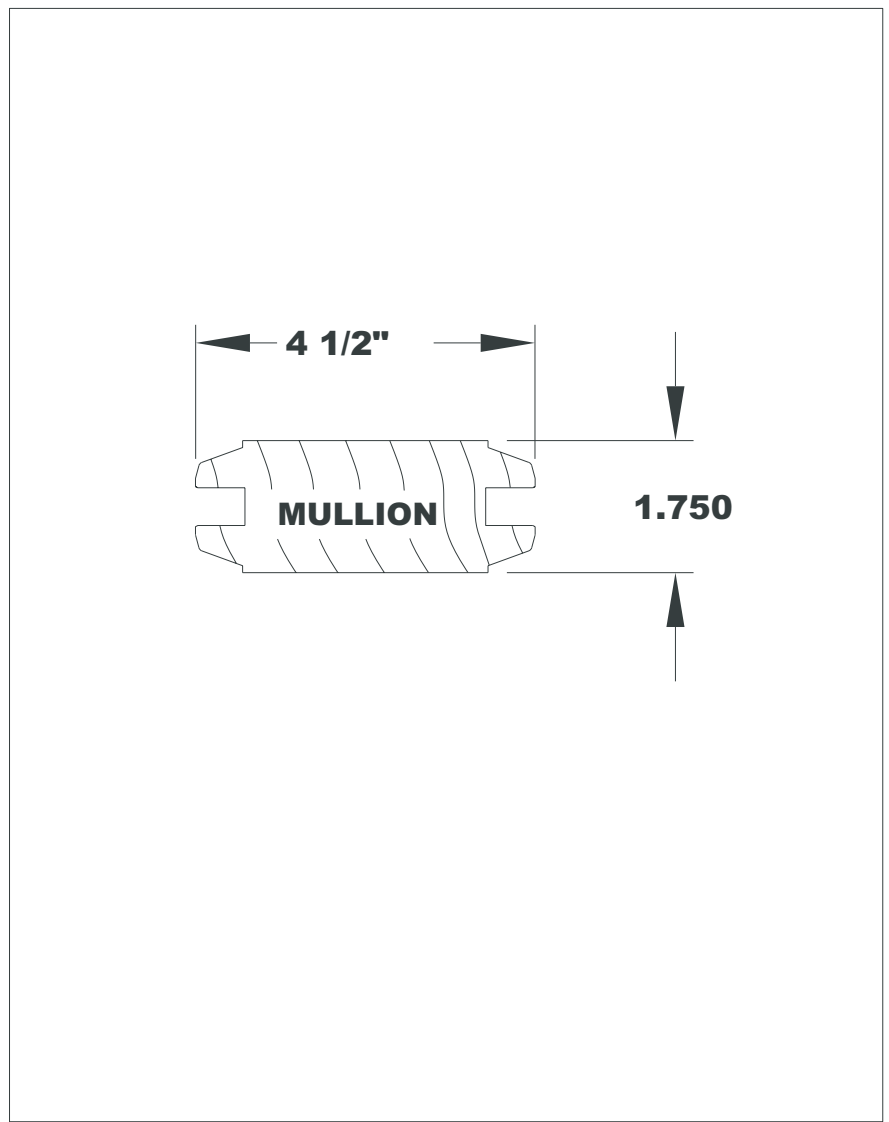

Figure 9-70. 6-Panel Mullion.dwg 


\section{MACHINING DETAIL SHEET}

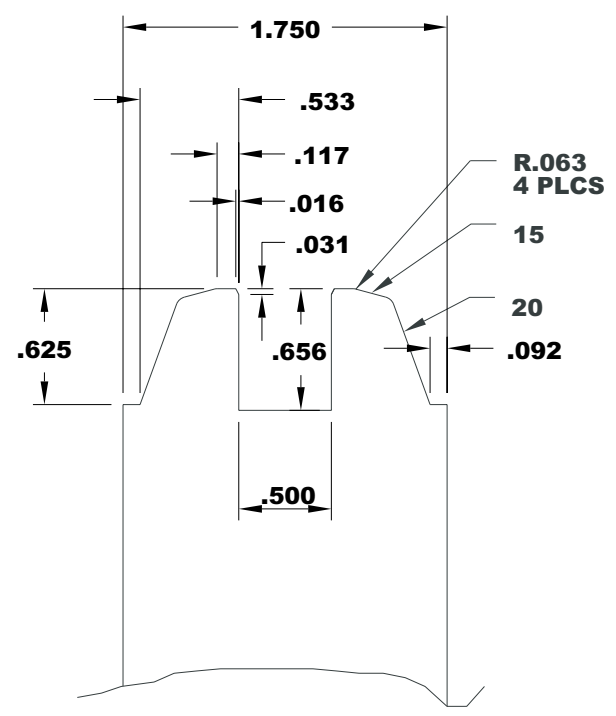

\begin{tabular}{|l|l|l|l|l|l|l|l|l|}
\hline DATESY & REVISION RECORD & AUDRCK TOLERANCES & \multicolumn{4}{|l|}{} \\
\hline & & & & DEC..015 & SC: & DATE: & DWN BY: & APR BY: \\
\hline & & & & FRAC. 1/32 & ANG.1 PRODUCT: & Milling Detail & REV NO. \\
\hline & & & & DEC.LG020 & TITLE: & & & \\
\hline
\end{tabular}

Figure 9-71. 6-Panel Milling Detail.dwg 


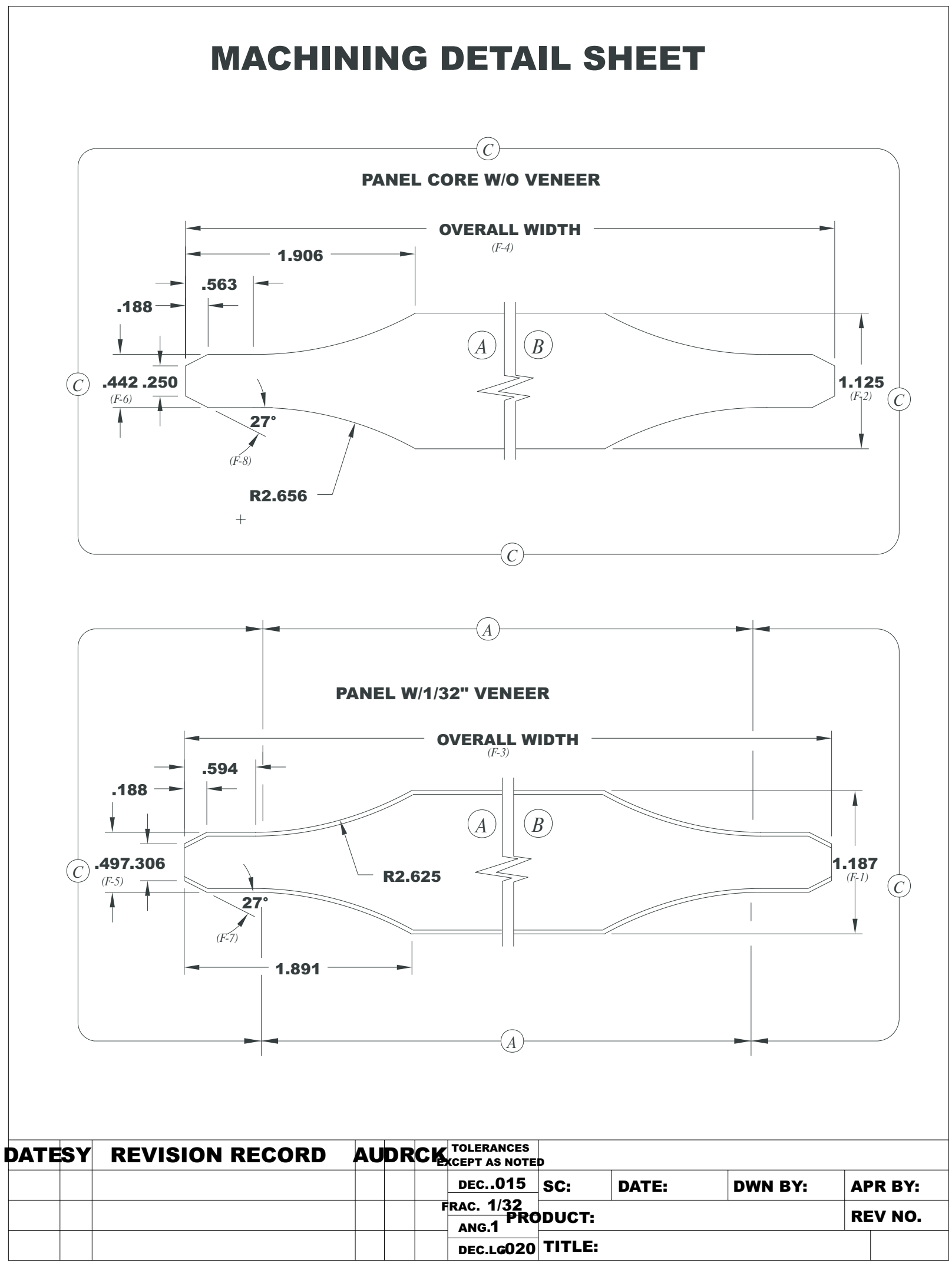

Figure 9-72. 6-Panel Panel.dwg 


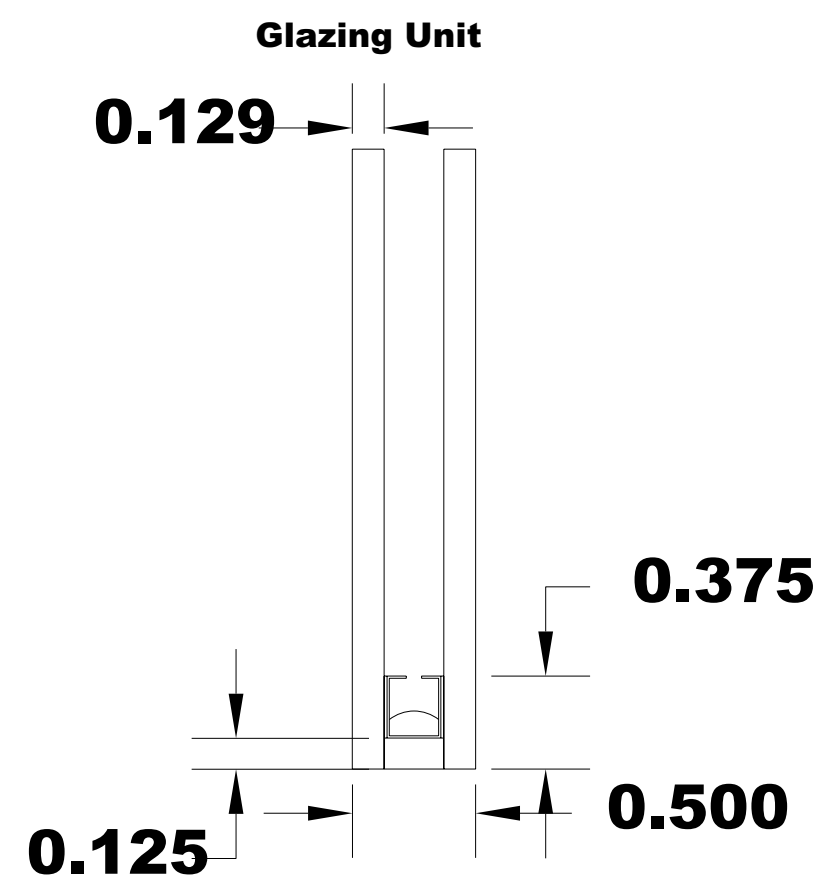

Figure 9-73. 6-Panel Glazing.dwg

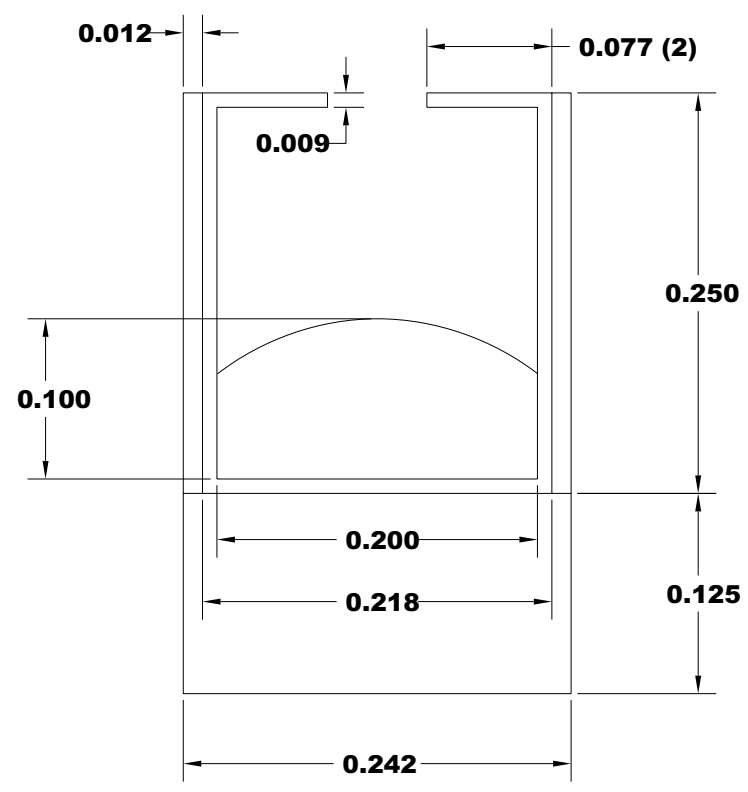

\section{Intercept Spacer}

Figure 9-74. 6-Panel Spacer.dwg 


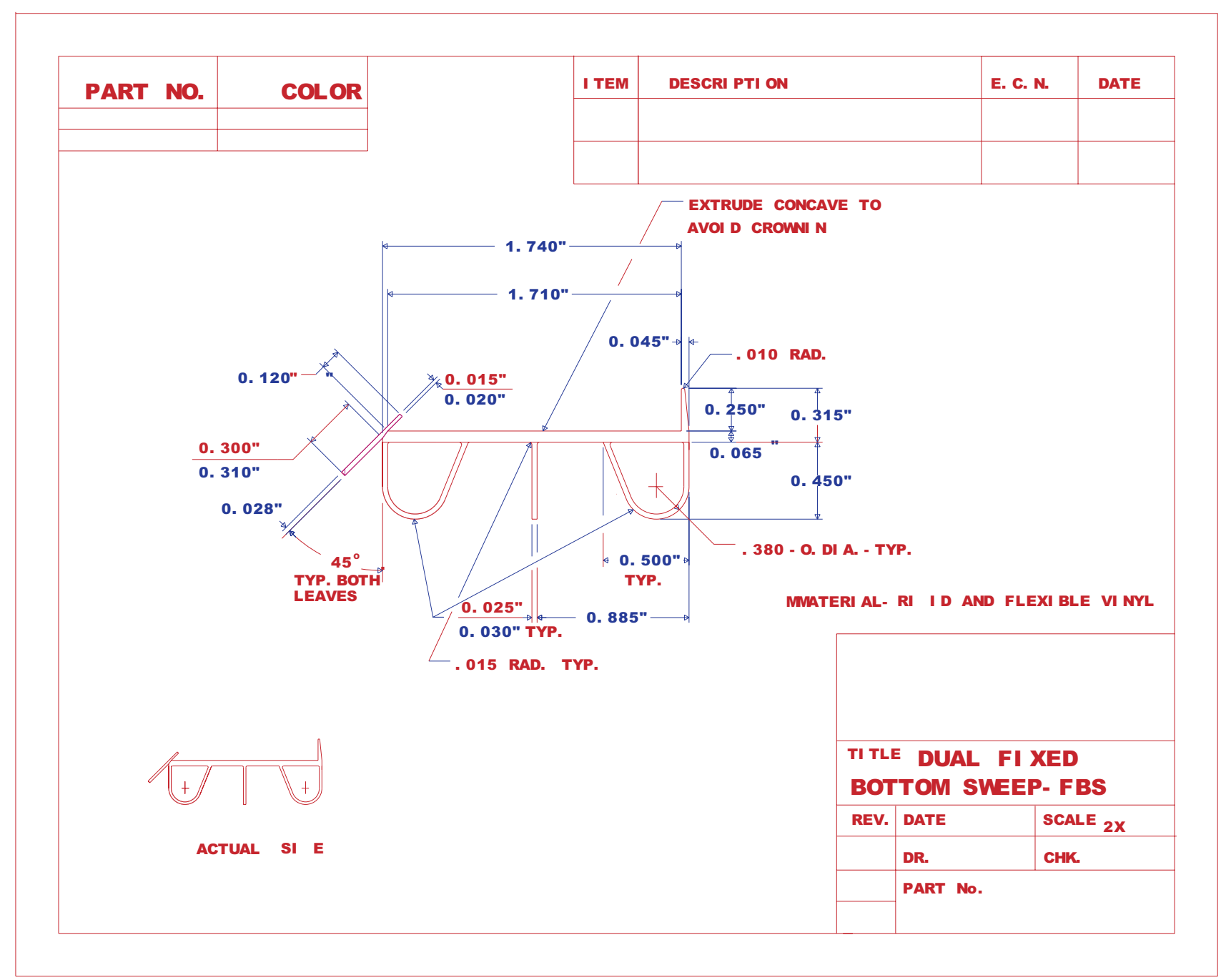

Figure 9-75. 6-Panel Bottom Sweep.dwg (*PATENTED DRAWING) 


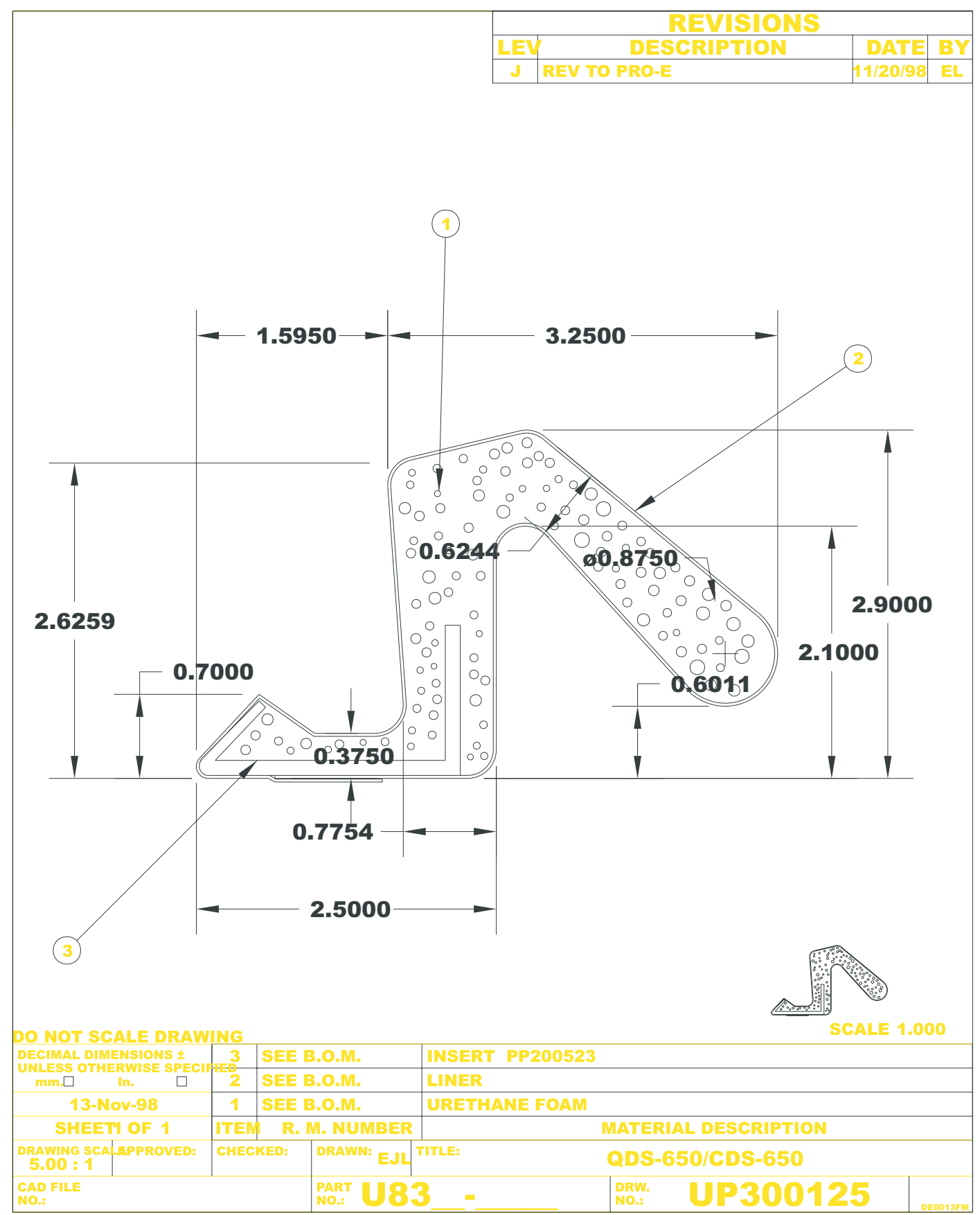

Figure 9-76. 6-Panel Weather Strip.dwg (* PATENTED DRAWING) 


\section{ACKNOWLEDGEMENTS}

\section{Acknowledgements}

This work was supported by the Assistant Secretary for Energy Efficiency and Renewable Energy, Office of Building Technology, State and Community Programs, Office of Building Systems of the U.S. Department of Energy under Contract No. DE-AC03-76SF00098. The program authors wish to thank the window manufacturers who supplied data and drawings on their products for use in this manual; Jeff Baker, Marcia Falke, Randy Van Voorst, Ross DePaola and Bipin Shah for their review of draft versions; the many members of the NFRC community who contributed suggestions and ideas; and Pat Ross for all her many daily contributions including the final production of the program and manual. 



\section{APPENDIX A. The Application of ISO 15099 to NFRC 100 and 200}

Software utilized in computing NFRC U-factors (NFRC 100-02) and SHGCs (NFRC 200-02) will be based on the recently completed ISO 15099 document (FDIS, September 21, 2001). In several cases, ISO 15099 suggests that individual national standards will need to be more specific and in other cases the ISO document gives users the choice of two options. This document clarifies these specific issues as they are to be implemented in NFRC approved software/algorithms:

1) Section 4.1: For calculating the overall U-factor, ISO 15099 offers a choice between the linear thermal transmittance (4.1.2) and the area weighted method (4.1.3). The area weighted method (4.1.3) shall be used.

2) Frame and divider SHGC's shall be calculated in accordance with Section 4.2.2. The alternate approach in section 8.6 shall not be used. [Note: current research is aimed at assessing which method is more accurate; at some point in the future, this recommendation may be revised.]

3) Section 6.4 refers the issue of material properties to national standards. Material conductivities and emissivities shall be determined in accordance with the NFRC Simulation Manual or more currently adopted NFRC standard on this topic.

4) Section 7 on Shading Systems is currently excluded from NFRC procedures.

5) Section 8.2 addresses environmental conditions. The following are defined by NFRC:

For U-factor calculations:

$$
\begin{aligned}
& T_{\text {in }}=21^{\circ} \mathrm{C} \\
& T_{\text {out }}=-18^{\circ} \mathrm{C} \\
& V=5.5 \mathrm{~m} / \mathrm{s} \\
& T_{r \mathrm{~m}, \text { out }}=T_{\text {out }} \\
& T_{r \mathrm{~m}, \text { in }}=T_{\text {in }} \\
& I_{s}=0 \mathrm{~W} / \mathrm{m}^{2}
\end{aligned}
$$

For SHGC calculations:

$$
\begin{aligned}
& T_{\text {in }}=24{ }^{\circ} \mathrm{C} \\
& T_{\text {out }}=32{ }^{\circ} \mathrm{C} \\
& V=2.75 \mathrm{~m} / \mathrm{s} \\
& T_{r \mathrm{~m}, \text { out }}=T_{\text {out }} \\
& T_{r \mathrm{~m}, \text { in }}=T_{\text {in }} \\
& I_{s}=783 \mathrm{~W} / \mathrm{m}^{2}
\end{aligned}
$$


6) Section 8.3 addresses convective film coefficients on the interior and exterior of the window product. In section 8.3.1, NFRC simulations shall use the convective heat transfer coefficient based on the center of glass temperature and the entire window height; this film coefficient shall be used on all glass and edge of glass indoor surfaces. Frame section indoor convective film coefficients shall be constants which depend on frame material type; these shall be determined using the algorithms in ISO15099 and using representative frame surface temperatures for each frame material type (to be included in the Simulation Manual). In section 8.3.2, the formula from this section shall be applied to all outdoor exposed surfaces.

7) Section 8.4.2 presents two possible approaches for incorporating the impacts of self-viewing surfaces on interior radiative heat transfer calculations. NFRC shall use the method in section 8.4.2.1 (TwoDimensional Element To Element View Factor Based Radiation Heat Transfer Calculation). This is also the method referenced in NFRC 500 for CI calculations. Furthermore, in the interests of consistency and accuracy, this method shall be used for all products, including planar products, for U-factor calculations as well as CI calculations. The use of this method makes the use of Slightly or Partially Ventilated cavities (see section 6.7.1) on the interior of frame surfaces redundant. The standard frame convective film coefficients (hc) referenced in 6) above will thus be applied to all interior frame surfaces. 


\section{APPENDIX B. REFERENCES}

1997 ASHRAE Handbook of Fundamentals, American Society of Heating, Refrigerating and Air-Conditioning Engineers, Inc., Atlanta, GA.

Arasteh, D. K. Finlayson, E. U., and Huizenga, C. 1994. "WINDOW 4.1: A PC Program for Analyzing Window Thermal Performance in Accordance with Standard NFRC Procedures." LBL Report 35298.

Berkeley, CA.

ASHRAE Standard 142P, Standard Method for Determining and Expressing the Heat Transfer and Total Optical Properties of Fenestration Products, Public Review Draft, October 1996, American Society of Heating, Refrigerating and Air-Conditioning Engineers, Inc., Atlanta, GA.

Baehmann, P. L. Wittchen, S.L., Shephard, M.S., Grice, K.R. and Yerry, M.A., 1987. "Robust, Geometrically Based, Automatic Two-Dimensional Mesh Generation." International Journal for Numerical Methods in Engineering. 24, 1043 - 1078.

Curcija, D.; Power, J. P.; and Goss, W. P. 1995. "CONRAD: A Finite Element Method Based Computer Program Module for Analyzing 2-D Conductive and Radiative Heat Transfer in Fenestration Systems". Draft Report, University of Massachusetts at Amherst.

Curcija; D., and Goss, W. P. The 'Variable-h' Model For Improved Prediction of Surface Temperatures in Fenestration Systems, DRAFT June 1998 University of Massachusetts Report.

Finlayson, E. U., Arasteh, D. K., Huizenga, C., Rubin, and M. D., Reilly, M. S., 1993. "WINDOW 4.0:

Documentation of Calculation Procedures." LBL Report 33943. Berkeley CA.

Finlayson, E.U., Mitchell, R., Arasteh, D., Huizenga, C., Curcija, D.. 1998. "THERM 2.0: Program Description, A PC Program for Analyzing the Two-Dimensional Heat Transfer Through Building Products". LBL-37371 Rev 2.

George, P.L. Automatic Mesh Generation, Application to Finite Element Methods. John Wiley \& Sons.

Pepper, P. W. and Heinrich, J. C. 1992. The Finite Element Method Basic Concepts and Applications. Hemisphere Publishing Corporation, Washington.

Carmody, J., Selkowitz, Arasteh, D., S., Heschong, L., Residential Windows: A Guide to New Technologies and Energy Performance, Second Edition, 2000, W. W. Norton \& Company.

Rohsenow, W. M., Harnett, J. P., and Ganic, E. N., 1985. Handbook of Heat Transfer Fundamentals, 2nd Edition, McGraw Hill.

Shapiro, A. B. 1986. "TOPAZ2D - A Two-Dimensional Finite Element Code for Heat Transfer Analysis, Electrostatic, and Magnetostatic Problems" Lawrence Livermore National Laboratory, Rept. UCID-20824.

Shapiro, A. B. 1990. "TOPAZ2D Heat Transfer Code Users Manual and Thermal Property Data Base", Lawrence Livermore National Laboratory, Rept. UCRL-ID-104558, May 1990.

Shapiro, A. B., 1983. FACET - A Radiation View Factor Computer Code for Axisymetric, 2D Planar, and 3D Geometries with Shadowing. Lawrence Livermore National Laboratory, Rept. UCID-19887.

Thermal Performance of Windows, Doors and Shading Devices - Detailed Calculations, ISO/FDIS 15099, ISO TC 163. Zhao, Y., Curcija, D., and Goss, W. P., 1996, Condensation Resistance Validation Project - Detailed Computer Simulations Using Finite - Element Methods. PP 508-515, ASHRAE Transactions, Vol 102, Part 2. 
Zienkiewicz, O. C.; and Taylor, R. L. 1989. The Finite Element Method. 4th ed. Vol. 1. McGraw Hill, Maidenhead, UK.

Zienkiewicz, O.C. and Zhu, J.Z. 1992a. "The Superconvergent Patch Recovery and A Posteriori Error Estimates. Part 1: The Recovery Technique." International Journal for Numerical Methods in Engineering. Vol 33. pp. 1331-1364.

Zienkiewicz, O.C. and Zhu, J.Z., 1992b. "The superconvergent patch recovery and a posteriori error estimates. Part 2. The error estimates and adaptivity." International Journal for Numerical Methods in Engineering. Vol 33. pp. 1365-1382. 